\title{
Diethylzinc-Mediated Radical 1,2-Addition of Alkenes and Alkynes
}

Xin $\mathrm{Li}^{+}{ }^{+}$Songtao $\mathrm{He}^{+}{ }^{+}$and Qiuling Song ${ }^{*},+,+$

'Institute of Next Generation Matter Transformation, College of Materials Science \& Engineering, Huaqiao University, 668 Jimei Blvd, Xiamen 361021, Fujian, China

łState Key Laboratory of Organometallic Chemistry and Key Laboratory of Organofluorine Chemistry, Shanghai Institute of Organic Chemistry, Chinese Academy of Sciences, Shanghai 200032

*qsong@hqu.edu.cn

\section{Supporting Information}

\section{Table of Contents}

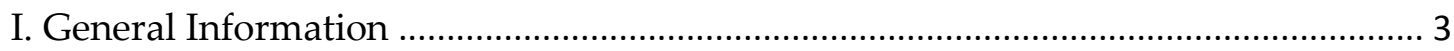

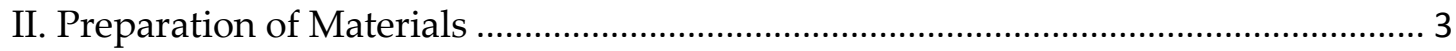

2.1. Synthesis of Benzoate Substrates........................................................................... 3

2.2 Synthesis of Tosylate Substrates............................................................................. 4

2.3 Synthesis of Mesylate Substrate …...................................................................... 4

2.4 Synthesis of Benzyl-Protected Alcohol Substrates.................................................. 5

2.5 Synthesis of TBS-Protected Alcohol Substrates..................................................... 6

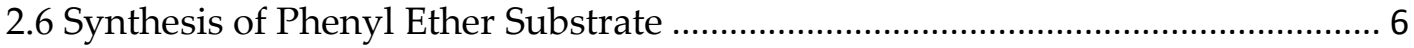

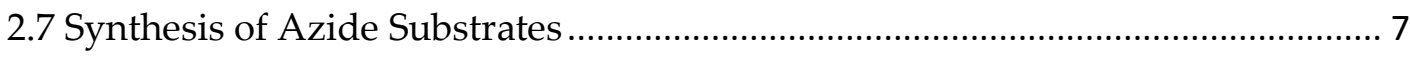

2.8 Synthesis of N-Phth-Protected Amine Substrate ................................................ 8

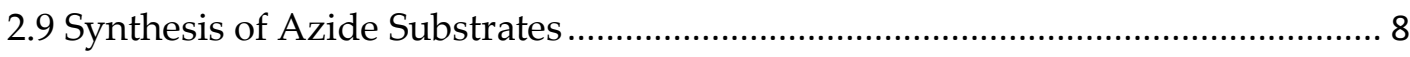

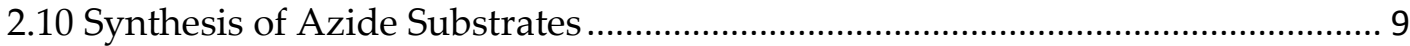

2.11. Synthesis of Camphorsulfonic Acid Derivative ................................................. 9

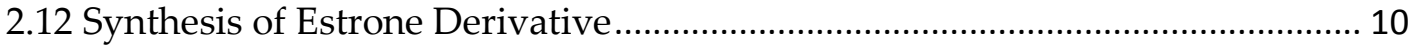

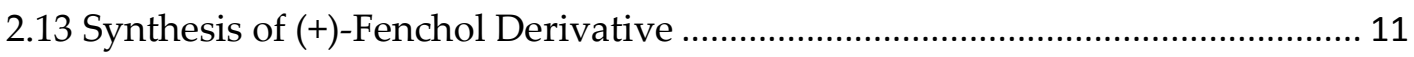

2.14. Synthesis of Ibuprofen Derivative ................................................................... 11

2.15 Synthesis of Dihydrocholesterol Derivative ..................................................... 12

2.16 Synthesis of (+)-Dehydroabietylamine Derivative.......................................... 13

III. Screening of Reaction Conditions ....................................................................... 14

IV. General Procedure for the 1,2-Addition of Perfluoroalkyl Iodides to Alkenes and

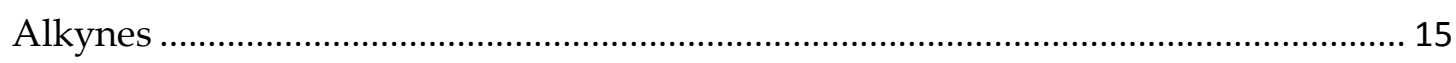

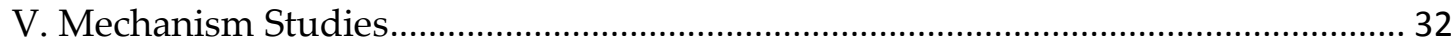

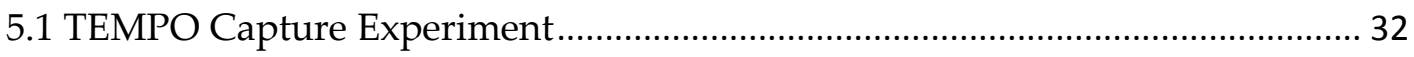


5.2 Radical Ring-Opening Experiment

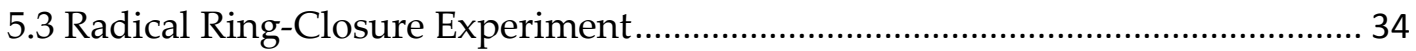

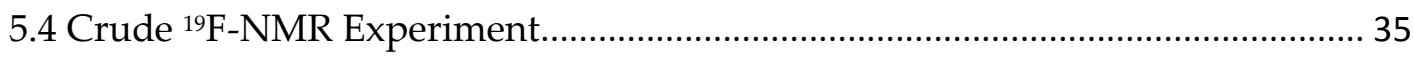

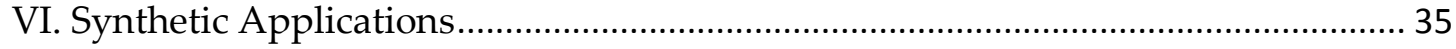

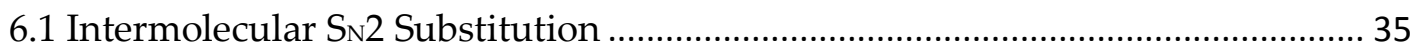

6.2 Reduction \& Intramolecular SN2 Substitution .................................................. 36

6.3 Palladium-Catalyzed Sonogashira Coupling .................................................... 37

6.4 Cobalt-Catalyzed Kumada Coupling ……........................................................... 37

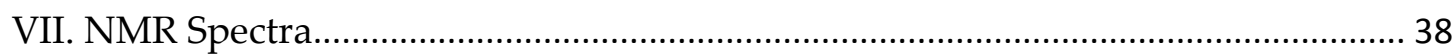




\section{General Information}

All air or moisture sensitive reactions were conducted in oven-dried glassware under nitrogen atmosphere. Ethyl iododifluoroacetate and diethylzinc $(1.0 \mathrm{~mol} / \mathrm{L}$ in hexane, EnergySeal) were purchased from Energy Chemical and used as received. Acetonitrile (99.9\%, for HPLC) was purchased from Sigma Aldrich and used as received. Unless otherwise stated, chemicals and reagents were used as received. Flash column chromatography was performed over silica gel (200-300 mesh) purchased from Qindao Bangkai Co., China. ${ }^{1} \mathrm{H},{ }^{19} \mathrm{~F}$ and ${ }^{13} \mathrm{C}$ NMR spectra were recorded on a Bruker AV $500 \mathrm{MHz}$ NMR spectrometer using residue solvent peaks as an internal standard $\left({ }^{1} \mathrm{H} N M R: \mathrm{CHCl}_{3}\right.$ at $7.26 \mathrm{ppm},{ }^{13} \mathrm{C} \mathrm{NMR:} \mathrm{CDCl}_{3}$ at $\left.77.0 \mathrm{ppm}\right)$. HRMS were recorded on an Agilent 6545 Q-TOF LC/MS instrument with electrospray ionization (ESI) technique.

\section{Preparation of Materials}

\subsection{Synthesis of Benzoate Substrates}

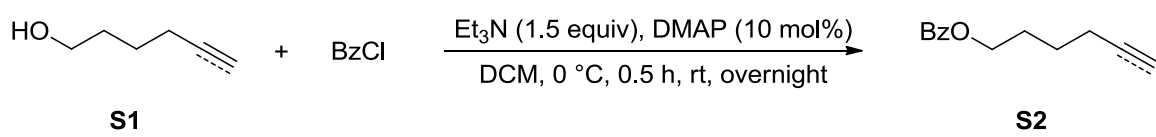

To a solution of $\mathbf{S 1}(3.0 \mathrm{mmol})$, DAMP ( $37 \mathrm{mg}, 0.3 \mathrm{mmol}, 0.1$ equiv), and triethylamine (455 mg, $4.5 \mathrm{mmol}, 1.5$ equiv) in anhydrous $\mathrm{CH}_{2} \mathrm{Cl}_{2}$ was added benzoyl chloride (525 mg, $3.75 \mathrm{mmol}, 1.25$ equiv) dropwise at $0{ }^{\circ} \mathrm{C}$. The resulting mixture was stirred at $0{ }^{\circ} \mathrm{C}$ for 0.5 hour, and then at room temperature overnight. After completion, the reaction was quenched with water and extracted with $\mathrm{CH}_{2} \mathrm{Cl}_{2}$. The combined organic layers were dried with $\mathrm{Na}_{2} \mathrm{SO}_{4}$, filtered, and concentrated under reduced pressure. The residue was purified by silica gel column chromatography (petroleum ether/ethyl acetate $=40 / 1$ ) to afford the corresponding pure product.

\section{Hex-5-en-1-yl benzoate (S2a)}

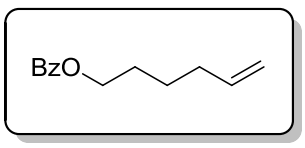

Colorless oil, $610 \mathrm{mg}, 100 \%$ yield.

${ }^{1} \mathrm{H}$ NMR $\left(500 \mathrm{MHz}, \mathrm{CDCl}_{3}\right) \delta 8.12-8.03(\mathrm{~m}, 2 \mathrm{H}), 7.61-7.55(\mathrm{~m}, 1 \mathrm{H})$, $7.49-7.42(\mathrm{~m}, 2 \mathrm{H}), 5.85(\mathrm{ddt}, J=16.9,10.1,6.6 \mathrm{~Hz}, 1 \mathrm{H}), 5.06(\mathrm{dq}, J=$ 17.1, $1.7 \mathrm{~Hz}, 1 \mathrm{H}), 5.01(\mathrm{ddt}, J=10.2,2.3,1.3 \mathrm{~Hz}, 1 \mathrm{H}), 4.35(\mathrm{t}, J=6.6 \mathrm{~Hz}, 2 \mathrm{H}), 2.21-2.12(\mathrm{~m}$, $2 \mathrm{H}), 1.81(\mathrm{dq}, J=8.5,6.6 \mathrm{~Hz}, 2 \mathrm{H}), 1.58(\mathrm{tt}, J=9.9,6.5 \mathrm{~Hz}, 2 \mathrm{H})$.

${ }^{13} \mathrm{C}$ NMR $\left(125 \mathrm{MHz}, \mathrm{CDCl}_{3}\right) \delta$ 166.6, 138.3, 132.8, 130.5, 129.5, 128.3, 114.8, 64.9, 33.3, 28.2, 25.3.

HRMS (ESI) m/z: [M+Na] calcd for $\mathrm{C}_{13} \mathrm{H}_{16} \mathrm{O}_{2} \mathrm{Na}$ : 227.1043; found: 227.1042.

\section{Hex-5-yn-1-yl benzoate (S2b)}

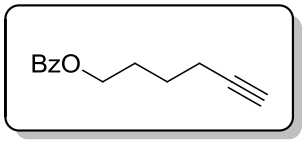

Colorless oil, $605 \mathrm{mg}, 100 \%$ yield.

${ }^{1} \mathbf{H}$ NMR (500 MHz, $\left.\mathrm{CDCl}_{3}\right) \delta 8.11-8.01(\mathrm{~m}, 2 \mathrm{H}), 7.59-7.53(\mathrm{~m}, 1 \mathrm{H})$, $7.49-7.40(\mathrm{~m}, 2 \mathrm{H}), 4.36(\mathrm{t}, J=6.5 \mathrm{~Hz}, 2 \mathrm{H}), 2.29(\mathrm{td}, J=7.0,2.7 \mathrm{~Hz}$, 
2H), $2.00(\mathrm{t}, J=2.6 \mathrm{~Hz}, 1 \mathrm{H}), 1.91(\mathrm{ddt}, J=9.6,8.2,6.4 \mathrm{~Hz}, 2 \mathrm{H}), 1.77-1.66(\mathrm{~m}, 2 \mathrm{H})$.

${ }^{13} \mathrm{C}$ NMR $\left(125 \mathrm{MHz}, \mathrm{CDCl}_{3}\right) \delta$ 166.4, 132.8, 130.2, 129.4, 128.2, 83.7, 68.7, 64.3, 27.7, 25.0, 18.0.

HRMS (ESI) m/z: [M+Na] calcd for $\mathrm{C}_{13} \mathrm{H}_{14} \mathrm{O}_{2} \mathrm{Na}$ : 225.0886; found: 225.0883 .

\subsection{Synthesis of Tosylate Substrates}

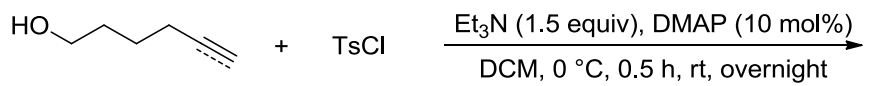

S1

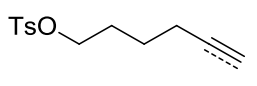

S3

To a solution of $\mathbf{S 1}$ ( $3.0 \mathrm{mmol}$ ), DAMP ( $37 \mathrm{mg}, 0.3 \mathrm{mmol}, 0.1$ equiv), and triethylamine (455 mg, $4.5 \mathrm{mmol}, 1.5$ equiv) in anhydrous $\mathrm{CH}_{2} \mathrm{Cl}_{2}$ was added $p$-toluenesulfonyl chloride (715 mg, $3.75 \mathrm{mmol}, 1.25$ equiv) in potions at $0{ }^{\circ} \mathrm{C}$. The resulting mixture was stirred at $0{ }^{\circ} \mathrm{C}$ for 0.5 hour, and then at room temperature overnight. After completion, the reaction was quenched with water and extracted with $\mathrm{CH}_{2} \mathrm{Cl}_{2}$. The combined organic layers were dried with $\mathrm{Na}_{2} \mathrm{SO}_{4}$, filtered, and concentrated under reduced pressure. The residue was purified by silica gel column chromatography (petroleum ether/ethyl acetate $=3 / 1$ ) to afford the corresponding pure product.

\section{Hex-5-en-1-yl 4-methylbenzenesulfonate (S3a)}

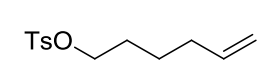

Colorless oil, $501 \mathrm{mg}$, 66\% yield.

${ }^{1} \mathrm{H}$ NMR $\left(500 \mathrm{MHz}, \mathrm{CDCl}_{3}\right) \delta 7.80(\mathrm{~d}, J=8.1 \mathrm{~Hz}, 2 \mathrm{H}), 7.36(\mathrm{~d}, J=8.1$ $\mathrm{Hz}, 2 \mathrm{H}), 5.73(\mathrm{ddt}, J=16.9,10.2,6.7 \mathrm{~Hz}, 1 \mathrm{H}), 5.03-4.90(\mathrm{~m}, 2 \mathrm{H}), 4.04$

$(\mathrm{t}, J=6.4 \mathrm{~Hz}, 2 \mathrm{H}), 2.46(\mathrm{~s}, 3 \mathrm{H}), 2.06-1.97(\mathrm{~m}, 2 \mathrm{H}), 1.66(\mathrm{dq}, J=8.4,6.5 \mathrm{~Hz}, 2 \mathrm{H}), 1.47-1.38$ $(\mathrm{m}, 2 \mathrm{H})$.

${ }^{13} \mathrm{C}$ NMR (125 MHz, $\left.\mathrm{CDCl}_{3}\right) \delta$ 144.6, 137.8, 133.2, 129.8, 127.8, 115.0, 70.4, 32.8, 28.1, 24.5, 21.6.

HRMS (ESI) m/z: [M+Na] $]^{+}$calcd for $\mathrm{C}_{13} \mathrm{H}_{18} \mathrm{O}_{3} \mathrm{SNa}$ : 277.0869; found: 277.0867.

Hex-5-yn-1-yl 4-methylbenzenesulfonate (S3b)

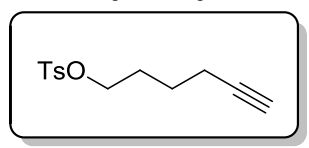

Colorless oil, $433 \mathrm{mg}$, 57\% yield.

${ }^{1} \mathbf{H}$ NMR $\left(500 \mathrm{MHz}, \mathrm{CDCl}_{3}\right) \delta 7.81(\mathrm{~d}, J=8.1 \mathrm{~Hz}, 2 \mathrm{H}), 7.37(\mathrm{~d}, J=8.1$ $\mathrm{Hz}, 2 \mathrm{H}), 4.07(\mathrm{t}, J=6.3 \mathrm{~Hz}, 2 \mathrm{H}), 2.47(\mathrm{~s}, 3 \mathrm{H}), 2.18(\mathrm{td}, J=6.9,2.6 \mathrm{~Hz}$,

$2 \mathrm{H}), 1.94(\mathrm{t}, J=2.7 \mathrm{~Hz}, 1 \mathrm{H}), 1.85-1.75(\mathrm{~m}, 2 \mathrm{H}), 1.57(\mathrm{p}, J=7.1 \mathrm{~Hz}, 2 \mathrm{H})$.

${ }^{13} \mathrm{C}$ NMR (125 MHz, $\left.\mathrm{CDCl}_{3}\right) \delta$ 144.7, 133.1, 129.8, 127.8, 83.4, 69.9, 68.9, 27.7, 24.2, 21.6, 17.7.

HRMS (ESI) m/z: [M+Na] ${ }^{+}$calcd for $\mathrm{C}_{13} \mathrm{H}_{16} \mathrm{O}_{3} \mathrm{SNa}$ : 275.0712; found: 275.0711.

\subsection{Synthesis of Mesylate Substrate}

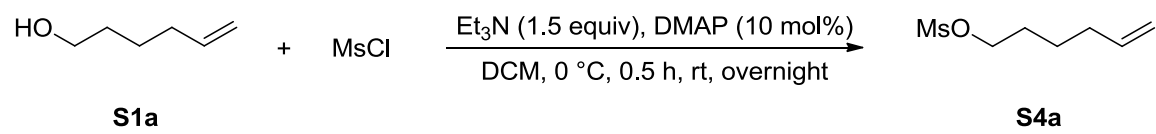

To a solution of S1a (1000 mg, $10 \mathrm{mmol})$, DAMP (122 mg, $1 \mathrm{mmol}, 0.1$ equiv), and triethylamine (1515 mg, $15 \mathrm{mmol}, 1.5$ equiv) in anhydrous $\mathrm{CH}_{2} \mathrm{Cl}_{2}$ was added 
methylsulfonyl chloride ( $1432 \mathrm{mg}, 12.5 \mathrm{mmol}, 1.25$ equiv) dropwise at $0{ }^{\circ} \mathrm{C}$. The resulting mixture was stirred at $0{ }^{\circ} \mathrm{C}$ for 0.5 hour, and then at room temperature overnight. After completion, the reaction was quenched with water and extracted with $\mathrm{CH}_{2} \mathrm{Cl}_{2}$. The combined organic layers were dried with $\mathrm{Na}_{2} \mathrm{SO}_{4}$, filtered, and concentrated under reduced pressure. The residue was purified by silica gel column chromatography (petroleum ether/ethyl acetate $=3 / 1$ ) to afford the corresponding pure product.

Hex-5-en-1-yl methanesulfonate (S4a)

MsO

Pale yellow oil, $1770 \mathrm{mg}$, 99\% yield.

${ }^{1} \mathbf{H}$ NMR $\left(500 \mathrm{MHz}, \mathrm{CDCl}_{3}\right) \delta 5.80(\mathrm{ddt}, J=16.9,10.2,6.7 \mathrm{~Hz}, 1 \mathrm{H})$, $5.12-4.96(\mathrm{~m}, 2 \mathrm{H}), 4.25(\mathrm{t}, J=6.5 \mathrm{~Hz}, 2 \mathrm{H}), 3.02(\mathrm{~s}, 3 \mathrm{H}), 2.16-2.08(\mathrm{~m}$, $2 \mathrm{H}), 1.83-1.73(\mathrm{~m}, 2 \mathrm{H}), 1.53(\mathrm{tt}, J=10.1,6.5 \mathrm{~Hz}, 2 \mathrm{H})$.

${ }^{13} \mathrm{C}$ NMR $\left(125 \mathrm{MHz}, \mathrm{CDCl}_{3}\right) \delta$ 137.8, 115.2, 69.9, 37.3, 32.9, 28.5, 24.6.

HRMS (ESI) m/z: [M+Na] ${ }^{+}$calcd for $\mathrm{C}_{7} \mathrm{H}_{14} \mathrm{O}_{3} \mathrm{SNa}$ : 201.0556; found: 201.0554 .

\subsection{Synthesis of Benzyl-Protected Alcohol Substrates}

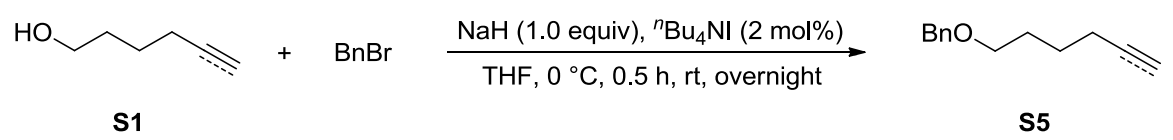

To a solution of $\mathbf{S} 1$ (2.2 mmol, 1.1 equiv) and ${ }^{n} \mathrm{Bu} 4 \mathrm{NI}$ ( $15 \mathrm{mg}, 0.04 \mathrm{mmol}, 0.02$ equiv) in anhydrous THF was added $\mathrm{NaH}\left(80 \mathrm{mg}, 2 \mathrm{mmol}, 1.0\right.$ equiv) in potions at $0{ }^{\circ} \mathrm{C}$. After stirring at $0{ }^{\circ} \mathrm{C}$ for 10 minutes, benzyl bromide ( $376 \mathrm{mg}, 2.2 \mathrm{mmol}, 1.1$ equiv) was added dropwise. The resulting mixture was stirred at $0{ }^{\circ} \mathrm{C}$ for additional 20 minutes, and then at room temperature overnight. After completion, the reaction was quenched with saturated $\mathrm{NH}_{4} \mathrm{Cl}$ and extracted with diethyl ether. The combined organic layers were dried with $\mathrm{Na}_{2} \mathrm{SO}_{4}$, filtered, and concentrated under reduced pressure. The residue was purified by silica gel column chromatography (petroleum ether/ethyl acetate $=40 / 1$ ) to afford the corresponding pure product.

((Hex-5-en-1-yloxy)methyl)benzene (S5a)

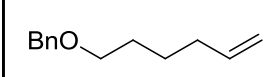

Colorless oil, $357 \mathrm{mg}$, $94 \%$ yield.

${ }^{1} \mathrm{H}$ NMR (500 MHz, $\left.\mathrm{CDCl}_{3}\right) \delta 7.42-7.35(\mathrm{~m}, 4 \mathrm{H}), 7.34-7.29(\mathrm{~m}, 1 \mathrm{H})$, $5.84(\mathrm{ddt}, J=16.9,10.2,6.7 \mathrm{~Hz}, 1 \mathrm{H}), 5.04(\mathrm{dq}, J=17.1,1.7 \mathrm{~Hz}, 1 \mathrm{H})$, $4.98(\mathrm{ddt}, J=10.2,2.3,1.2 \mathrm{~Hz}, 1 \mathrm{H}), 4.54(\mathrm{~s}, 2 \mathrm{H}), 3.51(\mathrm{t}, J=6.6 \mathrm{~Hz}, 2 \mathrm{H}), 2.15-2.06(\mathrm{~m}, 2 \mathrm{H})$, $1.71-1.64(\mathrm{~m}, 2 \mathrm{H}), 1.55-1.47(\mathrm{~m}, 2 \mathrm{H})$.

${ }^{13} \mathrm{C}$ NMR $\left(125 \mathrm{MHz}, \mathrm{CDCl}_{3}\right) \delta$ 138.74, 138.66, 128.3, 127.6, 127.5, 114.5, 72.9, 70.2, 33.5, 29.2, 25.5.

HRMS (ESI) m/z: [M+Na] $]^{+}$calcd for $\mathrm{C}_{13} \mathrm{H}_{18} \mathrm{ONa}$ : 213.1250; found: 213.1245.

((Hex-5-yn-1-yloxy)methyl)benzene (S5b)

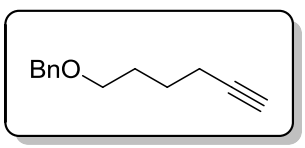

Colorless oil, $332 \mathrm{mg}$, $88 \%$ yield.

${ }^{1} \mathrm{H}$ NMR $\left(500 \mathrm{MHz}, \mathrm{CDCl}_{3}\right) \delta 7.42-7.35(\mathrm{~m}, 4 \mathrm{H}), 7.34-7.26(\mathrm{~m}, 1 \mathrm{H})$, $4.54(\mathrm{~s}, 2 \mathrm{H}), 3.53(\mathrm{t}, J=6.3 \mathrm{~Hz}, 2 \mathrm{H}), 2.25(\mathrm{td}, J=7.0,2.7 \mathrm{~Hz}, 2 \mathrm{H}), 1.98$

$(\mathrm{t}, J=2.7 \mathrm{~Hz}, 1 \mathrm{H}), 1.82-1.73(\mathrm{~m}, 2 \mathrm{H}), 1.67(\mathrm{tdd}, J=10.0,4.8,2.0 \mathrm{~Hz}, 2 \mathrm{H})$.

${ }^{13} \mathrm{C}$ NMR $\left(125 \mathrm{MHz}, \mathrm{CDCl}_{3}\right) \delta 138.5,128.3,127.6,127.5,84.3,72.9,69.7,68.4,28.7,25.2$, 18.2 . 
HRMS (ESI) m/z: [M+Na $]^{+}$calcd for $\mathrm{C}_{13} \mathrm{H}_{16} \mathrm{ONa}$ : 211.1093; found: 211.1089.

\subsection{Synthesis of TBS-Protected Alcohol Substrates}

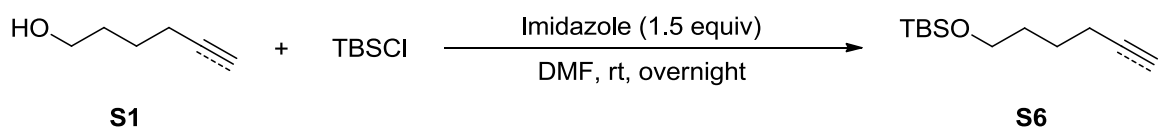

To a solution of $\mathbf{S 1}(3.0 \mathrm{mmol})$ and imidazole $(306 \mathrm{mg}, 4.5 \mathrm{mmol}, 1.5$ equiv) in anhydrous DMF was added TBSCl (543 mg, $3.6 \mathrm{mmol}, 1.2$ equiv) in one potion. After stirring at room temperature overnight, the reaction was quenched with water and extracted with diethyl ether. The combined organic layers were washed with saturated $\mathrm{NH}_{4} \mathrm{Cl}$ for 5 times, dried with $\mathrm{Na}_{2} \mathrm{SO}_{4}$, filtered, and concentrated under reduced pressure. The residue was purified by silica gel column chromatography (petroleum ether) to afford the corresponding pure product.

tert-butyl(hex-5-en-1-yloxy)dimethylsilane (S6a)

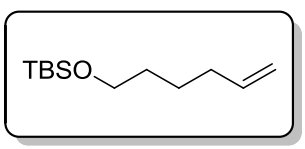

Colorless oil, $579 \mathrm{mg}$, $90 \%$ yield.

${ }^{1} \mathrm{H}$ NMR $\left(500 \mathrm{MHz}, \mathrm{CDCl}_{3}\right) \delta 5.83$ (ddt, $\left.J=16.9,10.1,6.6 \mathrm{~Hz}, 1 \mathrm{H}\right)$,

$5.03(\mathrm{dq}, J=17.1,1.7 \mathrm{~Hz}, 1 \mathrm{H}), 4.96(\mathrm{ddt}, J=10.2,2.3,1.2 \mathrm{~Hz}, 1 \mathrm{H}), 2.11$

- $2.06(\mathrm{~m}, 2 \mathrm{H}), 1.59-1.52(\mathrm{~m}, 2 \mathrm{H}), 1.49-1.41(\mathrm{~m}, 2 \mathrm{H}), 0.92(\mathrm{~s}, 9 \mathrm{H}), 0.07(\mathrm{~s}, 6 \mathrm{H})$.

${ }^{13} \mathrm{C}$ NMR $\left(125 \mathrm{MHz}, \mathrm{CDCl}_{3}\right) \delta 138.9,114.3,63.1,33.5,32.3,26.0,25.2,18.4,-5.3$.

HRMS (ESI) m/z: [M+K] $]^{+}$calcd for $\mathrm{C}_{12} \mathrm{H}_{26} \mathrm{OSiK}$ : 253.1385; found: 253.1405.

tert-butyl(hex-5-yn-1-yloxy)dimethylsilane (S6b)

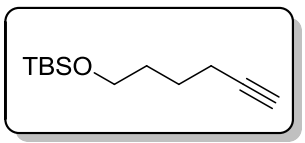

Colorless oil, $525 \mathrm{mg}$, 83\% yield.

${ }^{1} \mathrm{H}$ NMR $\left(500 \mathrm{MHz}, \mathrm{CDCl}_{3}\right) \delta 3.65(\mathrm{t}, J=6.0 \mathrm{~Hz}, 2 \mathrm{H}), 2.23(\mathrm{td}, J=6.8$, $2.6 \mathrm{~Hz}, 2 \mathrm{H}), 1.96(\mathrm{t}, J=2.7 \mathrm{~Hz}, 1 \mathrm{H}), 1.71-1.53(\mathrm{~m}, 4 \mathrm{H}), 0.91(\mathrm{~s}, 9 \mathrm{H})$,

$0.07(\mathrm{~s}, 6 \mathrm{H})$.

${ }^{13} \mathrm{C}$ NMR $\left(125 \mathrm{MHz}, \mathrm{CDCl}_{3}\right) \delta$ 8 84.5, 68.2, 62.6, 31.8, 26.0, 25.0, 18.3, 18.2, -5.3.

HRMS (ESI) m/z: [M+Na] ${ }^{+}$calcd for $\mathrm{C}_{12} \mathrm{H}_{24} \mathrm{OSiNa}$ : 235.1489; found: 235.1493.

\subsection{Synthesis of Phenyl Ether Substrate}

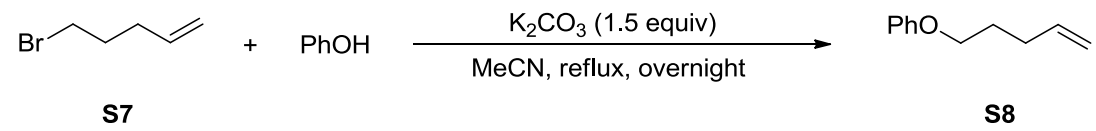

A mixture of $\mathbf{S 7}$ ( $581 \mathrm{mg}, 3.9 \mathrm{mmol}, 1.3$ equiv), phenol ( $282 \mathrm{mg}, 3 \mathrm{mmol}, 1.0$ equiv), and $\mathrm{K}_{2} \mathrm{CO}_{3}$ (621 mg, $4.5 \mathrm{mmol}, 1.5$ equiv) in anhydrous acetonitrile was heated to reflux in an oil bath. After refluxing overnight, the reaction mixture was cooled to room temperature, concentrated under reduced pressure, diluted with saturated $\mathrm{NH}_{4} \mathrm{Cl}$ and extracted with ethyl acetate. The combined organic layers were dried with $\mathrm{Na}_{2} \mathrm{SO}_{4}$, filtered, and concentrated under reduced pressure. The residue was purified by silica gel column chromatography (petroleum ether) to afford the corresponding pure product.

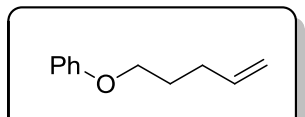

(pent-4-en-1-yloxy)benzene (S8)

Colorless oil, $403 \mathrm{mg}$, 83\% yield. 
${ }^{1} \mathrm{H}$ NMR $\left(500 \mathrm{MHz}, \mathrm{CDCl}_{3}\right) \delta 7.33(\mathrm{dd}, J=8.6,7.2 \mathrm{~Hz}, 2 \mathrm{H}), 7.01-6.97(\mathrm{~m}, 1 \mathrm{H}), 6.97-6.94$ $(\mathrm{m}, 2 \mathrm{H}), 5.91(\mathrm{ddt}, J=16.9,10.1,6.6 \mathrm{~Hz}, 1 \mathrm{H}), 5.12(\mathrm{dq}, J=17.1,1.9 \mathrm{~Hz}, 1 \mathrm{H}), 5.06(\mathrm{ddt}, J=$ $10.2,2.1,1.2 \mathrm{~Hz}, 1 \mathrm{H}), 4.02(\mathrm{t}, J=6.4 \mathrm{~Hz}, 2 \mathrm{H}), 2.37-2.23(\mathrm{~m}, 2 \mathrm{H}), 1.99-1.90(\mathrm{~m}, 2 \mathrm{H})$.

${ }^{13} \mathrm{C}$ NMR (125 MHz, $\left.\mathrm{CDCl}_{3}\right) \delta$ 159.0, 137.8, 129.4, 120.5, 115.1, 114.5, 67.0, 30.1, 28.5.

HRMS (ESI) m/z: [M+Na] calcd for $\mathrm{C}_{11} \mathrm{H}_{14} \mathrm{ONa}$ : 185.0937; found: 185.0935.

\subsection{Synthesis of Azide Substrates}

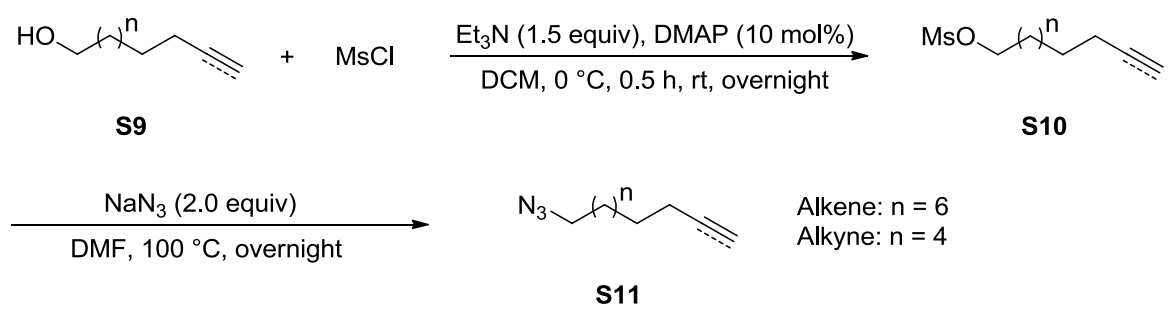

To a solution of $\mathbf{S 9}$ ( $3.0 \mathrm{mmol})$, DAMP (37 $\mathrm{mg}, 0.3 \mathrm{mmol}, 0.1$ equiv), and triethylamine (455 mg, $4.5 \mathrm{mmol}, 1.5$ equiv) in anhydrous $\mathrm{CH}_{2} \mathrm{Cl}_{2}$ was added methylsulfonyl chloride (429 mg, $3.75 \mathrm{mmol}, 1.25$ equiv) dropwise at $0{ }^{\circ} \mathrm{C}$. The resulting mixture was stirred at $0{ }^{\circ} \mathrm{C}$ for 0.5 hour, and then at room temperature overnight. After completion, the reaction was quenched with water and extracted with $\mathrm{CH}_{2} \mathrm{Cl}_{2}$. The combined organic layers were dried with $\mathrm{Na}_{2} \mathrm{SO}_{4}$, filtered, and concentrated under reduced pressure. The residue was used for the next step without further purification.

A mixture of $\mathbf{S 1 0}$ and sodium azide (390 $\mathrm{mg}, 6 \mathrm{mmol}, 2.0$ equiv) in anhydrous DMF was heated at $100{ }^{\circ} \mathrm{C}$ in an oil bath overnight. After cooling to room temperature, the reaction was quenched with water and extracted with diethyl ether. The combined organic layers were washed with saturated $\mathrm{NH}_{4} \mathrm{Cl}$ for 5 times, dried with $\mathrm{Na}_{2} \mathrm{SO}_{4}$, filtered, and concentrated under reduced pressure. The residue was purified by silica gel column chromatography (petroleum ether) to afford the corresponding pure product.

11-azidoundec-1-ene (S11a)

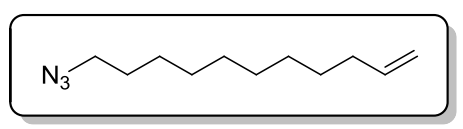

Colorless oil, $490 \mathrm{mg}$, $84 \%$ yield.

${ }^{1} \mathbf{H}$ NMR $\left(500 \mathrm{MHz}, \mathrm{CDCl}_{3}\right) \delta 5.83(\mathrm{ddt}, J=16.9,10.2,6.7$ $\mathrm{Hz}, 1 \mathrm{H}), 5.02(\mathrm{dq}, J=17.2,1.8 \mathrm{~Hz}, 1 \mathrm{H}), 4.95(\mathrm{dq}, J=10.3,1.4$

$\mathrm{Hz}, 1 \mathrm{H}), 3.28(\mathrm{t}, J=7.0 \mathrm{~Hz}, 2 \mathrm{H}), 2.12-2.01(\mathrm{~m}, 2 \mathrm{H}), 1.68-1.56(\mathrm{~m}, 2 \mathrm{H}), 1.46-1.25(\mathrm{~m}$, $12 \mathrm{H})$.

${ }^{13} \mathrm{C}$ NMR (125 MHz, $\left.\mathrm{CDCl}_{3}\right) \delta$ 139.1, 114.1, 51.5, 33.8, 29.4, 29.3, 29.1, 29.0, 28.9, 28.8, 26.7.

HRMS (ESI) m/z: [M+Na] calcd for $\mathrm{C}_{11} \mathrm{H}_{21} \mathrm{~N}_{3} \mathrm{Na}$ : 218.1628; found: 218.1626.

9-azidonon-1-yne (S11b)

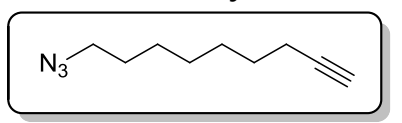

Colorless oil, $393 \mathrm{mg}$, 79\% yield.

${ }^{1} \mathrm{H}$ NMR (500 MHz, $\left.\mathrm{CDCl}_{3}\right) \delta 3.28(\mathrm{t}, J=6.9 \mathrm{~Hz}, 2 \mathrm{H}), 2.21(\mathrm{td}, J$ $=7.3,2.9 \mathrm{~Hz}, 2 \mathrm{H}), 1.96(\mathrm{t}, J=2.6 \mathrm{~Hz}, 1 \mathrm{H}), 1.62(\mathrm{p}, J=6.9 \mathrm{~Hz}, 2 \mathrm{H})$,

$1.55(\mathrm{p}, J=7.0 \mathrm{~Hz}, 2 \mathrm{H}), 1.49-1.32(\mathrm{~m}, 6 \mathrm{H})$,

${ }^{13} \mathrm{C}$ NMR (125 MHz, $\left.\mathrm{CDCl}_{3}\right) \delta 84.5,68.2,51.4,28.8,28.6,28.5,28.3,26.6,18.3$.

HRMS (ESI) m/z: [M+Na] $]^{+}$calcd for $\mathrm{C}_{9} \mathrm{H}_{15} \mathrm{~N}_{3} \mathrm{Na}$ : 188.1158; found: 188.1155 . 


\subsection{Synthesis of N-Phth-Protected Amine Substrate}

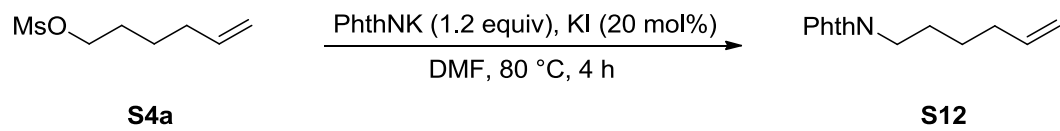

A mixture of S4a(356 mg, $2 \mathrm{mmol}, 2.0 \mathrm{mmol}), \mathrm{KI}$ (33.2 mg, $0.2 \mathrm{mmol}, 0.1$ equiv), and potassium phthalimide (445 mg, $2.4 \mathrm{mmol}, 1.2$ equiv) in anhydrous DMF was heated at $80{ }^{\circ} \mathrm{C}$ in an oil bath for 4 hours. After cooling to room temperature, the reaction was quenched with water and extracted with diethyl ether. The combined organic layers were washed with saturated $\mathrm{NH}_{4} \mathrm{Cl}$ for 5 times, dried with $\mathrm{Na}_{2} \mathrm{SO}_{4}$, filtered, and concentrated under reduced pressure. The residue was purified by silica gel column chromatography (petroleum ether/ethyl acetate $=8 / 1$ ) to afford the corresponding pure product.

\section{2-(hex-5-en-1-yl)isoindoline-1,3-dione (S12)}

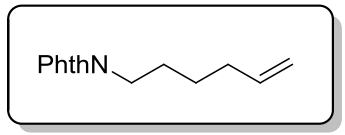

Colorless oil, $428 \mathrm{mg}$, 93\% yield.

${ }^{1} \mathbf{H}$ NMR $\left(500 \mathrm{MHz}, \mathrm{CDCl}_{3}\right) \delta 7.85(\mathrm{dd}, J=5.4,3.0 \mathrm{~Hz}, 2 \mathrm{H}), 7.72$ $(\mathrm{dd}, J=5.5,3.0 \mathrm{~Hz}, 2 \mathrm{H}), 5.79(\mathrm{ddt}, J=16.9,10.2,6.7 \mathrm{~Hz}, 1 \mathrm{H}), 5.02$ $(\mathrm{dq}, J=17.1,1.7 \mathrm{~Hz}, 1 \mathrm{H}), 4.96(\mathrm{ddt}, J=10.2,2.3,1.2 \mathrm{~Hz}, 1 \mathrm{H}), 3.70(\mathrm{t}, J=7.3 \mathrm{~Hz}, 2 \mathrm{H}), 2.11$ $(\mathrm{dtd}, J=7.3,5.9,1.4 \mathrm{~Hz}, 2 \mathrm{H}), 1.81-1.66(\mathrm{~m}, 2 \mathrm{H}), 1.52-1.41(\mathrm{~m}, 2 \mathrm{H})$.

${ }^{13} \mathrm{C}$ NMR $\left(125 \mathrm{MHz}, \mathrm{CDCl}_{3}\right) \delta 168.4,138.2,133.8,132.1,123.1,114.8,37.8,33.2,28.0,26.1$.

HRMS (ESI) m/z: [M+Na] ${ }^{+}$calcd for $\mathrm{C}_{14} \mathrm{H}_{15} \mathrm{O}_{2} \mathrm{Na}$ : 252.0995; found: 252.0994 .

\subsection{Synthesis of Azide Substrates}

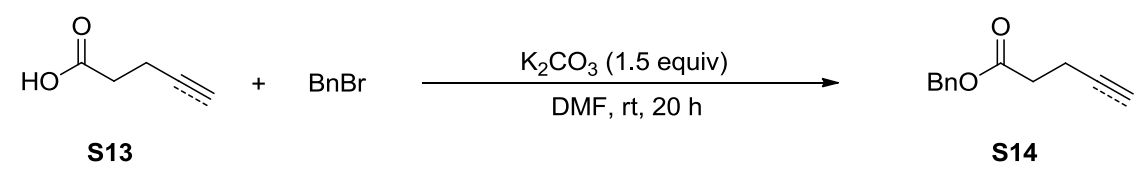

To a mixture of $\mathbf{S} 13$ (3.0 mmol) and $\mathrm{K}_{2} \mathrm{CO}_{3}(621 \mathrm{mg}$, $4.5 \mathrm{mmol}, 1.5$ equiv) in anhydrous DMF was added benzyl bromide ( $616 \mathrm{mg}, 3.6 \mathrm{mmol}, 1.2$ equiv) dropwise. After stirring at room temperature for 20 hours, the reaction was quenched with water and extracted with diethyl ether. The combined organic layers were washed with saturated $\mathrm{NH}_{4} \mathrm{Cl}$ for 5 times, dried with $\mathrm{Na}_{2} \mathrm{SO}_{4}$, filtered, and concentrated under reduced pressure. The residue was purified by silica gel column chromatography (petroleum ether/ethyl acetate $=40 / 1$ ) to afford the corresponding pure product.

\section{Benzyl pent-4-enoate (S14a)}

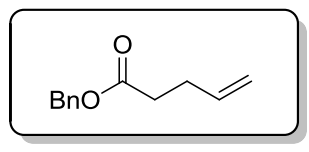

Pale yellow oil, $489 \mathrm{mg}$, $86 \%$ yield.

${ }^{1} \mathrm{H}$ NMR $\left(500 \mathrm{MHz}, \mathrm{CDCl}_{3}\right) \delta 7.42-7.38(\mathrm{~m}, 4 \mathrm{H}), 7.38-7.34(\mathrm{~m}, 1 \mathrm{H})$, $5.86(\mathrm{ddt}, J=16.6,10.2,6.3 \mathrm{~Hz}, 1 \mathrm{H}), 5.16(\mathrm{~s}, 2 \mathrm{H}), 5.09(\mathrm{dq}, J=17.2,1.7$

$\mathrm{Hz}, 1 \mathrm{H}), 5.04(\mathrm{dq}, J=10.2,1.4 \mathrm{~Hz}, 1 \mathrm{H}), 2.50(\mathrm{ddd}, J=7.9,6.6,1.6 \mathrm{~Hz}, 2 \mathrm{H}), 2.47-2.40(\mathrm{~m}$, $2 \mathrm{H})$.

${ }^{13} \mathrm{C}$ NMR $\left(125 \mathrm{MHz}, \mathrm{CDCl}_{3}\right) \delta$ 172.7, 136.5, 136.0, 128.5, 128.1 (two peaks), 115.5, 66.1, 33.5, 28.8 .

HRMS (ESI) m/z: [M+Na] calcd for $\mathrm{C}_{12} \mathrm{H}_{14} \mathrm{O}_{2} \mathrm{Na}$ : 213.0886; found: 213.0881 . 


\section{Benzyl pent-4-ynoate (S14b)}

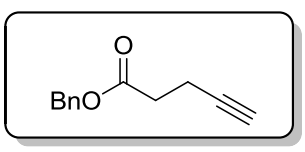

Colorless oil, $506 \mathrm{mg}$, 90\% yield.

${ }^{1} \mathrm{H}$ NMR $\left(500 \mathrm{MHz}, \mathrm{CDCl}_{3}\right) \delta 7.44-7.32(\mathrm{~m}, 5 \mathrm{H}), 5.18(\mathrm{~s}, 2 \mathrm{H}), 2.66-$ $2.60(\mathrm{~m}, 2 \mathrm{H}), 2.58-2.53(\mathrm{~m}, 2 \mathrm{H}), 2.00(\mathrm{t}, J=2.6 \mathrm{~Hz}, 1 \mathrm{H})$.

${ }^{13} \mathrm{C}$ NMR $\left(125 \mathrm{MHz}, \mathrm{CDCl}_{3}\right) \delta$ 171.5, 135.7, 128.5, 128.3, 128.2, 82.4, 69.1, 66.5, 33.3, 14.3.

HRMS (ESI) m/z: [M+Na] calcd for $\mathrm{C}_{12} \mathrm{H}_{12} \mathrm{O}_{2} \mathrm{Na}$ : 211.0730; found: 211.0732.

\subsection{Synthesis of Azide Substrates}

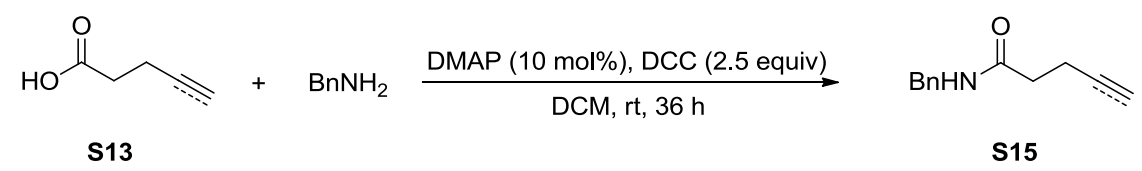

To a solution of S13 (3.0 mmol, 1.5 equiv), DAMP ( $24 \mathrm{mg}, 0.2 \mathrm{mmol}, 0.1$ equiv), and benzylamine (214 mg, $2 \mathrm{mmol}, 1.0$ equiv) in anhydrous $\mathrm{CH}_{2} \mathrm{Cl}_{2}$ was added DCC (1030 mg, $5 \mathrm{mmol}, 2.5$ equiv) in one potion. After stirring at room temperature for 36 hours, the mixture was filtered through a pad of Celite, washed with $\mathrm{CH}_{2} \mathrm{Cl}_{2}$. The combined organic layers were washed with water, dried with $\mathrm{Na}_{2} \mathrm{SO}_{4}$, filtered, and concentrated under reduced pressure. The residue was purified by silica gel column chromatography (petroleum ether/ethyl acetate $=1 / 1$ ) to afford the corresponding pure product.

\section{$\mathrm{N}$-benzylpent-4-enamide (S15a)}

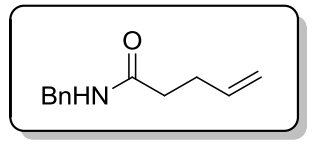

White amorphous solid, $483 \mathrm{mg}$, $85 \%$ yield.

${ }^{1} \mathbf{H}$ NMR $\left(500 \mathrm{MHz}, \mathrm{CDCl}_{3}\right) \delta 7.34(\mathrm{dd}, J=8.4,6.4 \mathrm{~Hz}, 2 \mathrm{H}), 7.31-$ $7.25(\mathrm{~m}, 3 \mathrm{H}), 6.01(\mathrm{~s}, 1 \mathrm{H}), 5.84(\mathrm{ddt}, J=16.8,10.2,6.4 \mathrm{~Hz}, 1 \mathrm{H}), 5.08$ $(\mathrm{dq}, J=17.1,1.7 \mathrm{~Hz}, 1 \mathrm{H}), 5.02(\mathrm{dt}, J=10.3,1.5 \mathrm{~Hz}, 1 \mathrm{H}), 4.44(\mathrm{~d}, J=5.7 \mathrm{~Hz}, 2 \mathrm{H}), 2.47-2.38$ $(\mathrm{m}, 2 \mathrm{H}), 2.35-2.27(\mathrm{~m}, 2 \mathrm{H})$.

${ }^{13} \mathrm{C}$ NMR (125 MHz, $\left.\mathrm{CDCl}_{3}\right) \delta$ 172.0, 138.3, 137.0, 128.7, 127.8, 127.5, 115.6, 43.6, 35.9, 29.6.

HRMS (ESI) m/z: [M+Na $]^{+}$calcd for $\mathrm{C}_{12} \mathrm{H}_{15} \mathrm{NONa}$ : 212.1046; found: 212.1052.

\section{$\mathrm{N}$-benzylpent-4-ynamide (S15b)}

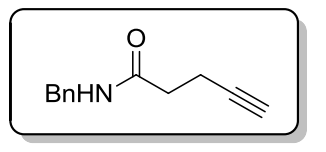

Pale yellow amorphous solid, $385 \mathrm{mg}, 69 \%$ yield.

${ }^{1} \mathbf{H}$ NMR (500 MHz, $\left.\mathrm{CDCl}_{3}\right) \delta 7.38-7.34(\mathrm{~m}, 2 \mathrm{H}), 7.31(\mathrm{dd}, J=7.2$, $1.7 \mathrm{~Hz}, 3 \mathrm{H}), 5.97(\mathrm{~s}, 1 \mathrm{H}), 4.69-4.17(\mathrm{~m}, 2 \mathrm{H}), 2.59(\mathrm{tt}, J=7.3,6.4,2.2$

$\mathrm{Hz}, 2 \mathrm{H}), 2.46(\mathrm{td}, J=7.1,1.8 \mathrm{~Hz}, 2 \mathrm{H}), 2.01(\mathrm{td}, J=2.6,0.9 \mathrm{~Hz}, 1 \mathrm{H})$.

${ }^{13} \mathrm{C}$ NMR $\left(125 \mathrm{MHz}, \mathrm{CDCl}_{3}\right) \delta$ 170.7, 138.1, 128.7, 127.8, 127.5, 82.9, 69.4, 43.7, 35.4, 14.9.

HRMS (ESI) m/z: [M+Na] ${ }^{+}$calcd for $\mathrm{C}_{12} \mathrm{H}_{13} \mathrm{NONa}$ : 210.0899; found: 210.0890 .

\subsection{Synthesis of Camphorsulfonic Acid Derivative}
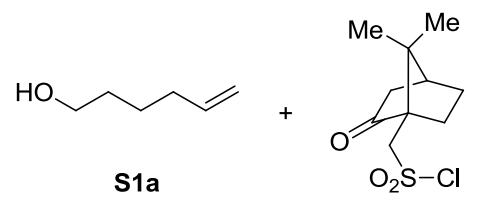

S16

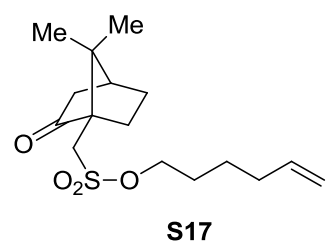

S17 
To a solution of S1a (300 mg, $3.0 \mathrm{mmol}$ ), DAMP (37 mg, $0.3 \mathrm{mmol}, 0.1$ equiv), and triethylamine (455 mg, $4.5 \mathrm{mmol}, 1.5$ equiv) in anhydrous $\mathrm{CH}_{2} \mathrm{Cl}_{2}$ was added camphorsulfonyl chloride $\left(900 \mathrm{mg}, 3.6 \mathrm{mmol}, 1.2\right.$ equiv) in potions at $0{ }^{\circ} \mathrm{C}$. The resulting mixture was stirred at $0{ }^{\circ} \mathrm{C}$ for 0.5 hour, and then at room temperature for 36 hours. After completion, the reaction was quenched with water and extracted with $\mathrm{CH}_{2} \mathrm{Cl}_{2}$. The combined organic layers were dried with $\mathrm{Na}_{2} \mathrm{SO}_{4}$, filtered, and concentrated under reduced pressure. The residue was purified by silica gel column chromatography (petroleum ether/ethyl acetate $=4 / 1$ ) to afford the corresponding pure product as a colorless oil, $837 \mathrm{mg}$, $89 \%$ yield.

Hex-5-en-1-yl ((1S,4S)-7,7-dimethyl-2-oxobicyclo[2.2.1]heptan-1-yl)methanesulfonate (S17)

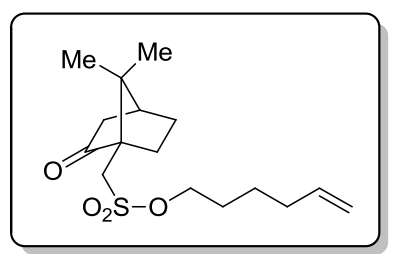

${ }^{1} \mathrm{H}$ NMR (500 MHz, $\left.\mathrm{CDCl}_{3}\right) \delta 5.80(\mathrm{ddt}, J=16.9,10.2,6.7 \mathrm{~Hz}$, $1 \mathrm{H}), 5.03(\mathrm{dq}, J=17.2,1.7 \mathrm{~Hz}, 1 \mathrm{H}), 4.99(\mathrm{dq}, J=10.2,1.4 \mathrm{~Hz}, 1 \mathrm{H})$, 4.30 (qt, $J=9.7,6.6 \mathrm{~Hz}, 2 \mathrm{H}), 3.61(\mathrm{~d}, J=15.1 \mathrm{~Hz}, 1 \mathrm{H}), 3.00(\mathrm{~d}, J=$ $15.1 \mathrm{~Hz}, 1 \mathrm{H}), 2.51(\mathrm{ddd}, J=14.9,11.8,4.0 \mathrm{~Hz}, 1 \mathrm{H}), 2.40(\mathrm{ddd}, J=$ $18.5,4.7,3.3 \mathrm{~Hz}, 1 \mathrm{H}), 2.16-2.05(\mathrm{~m}, 4 \mathrm{H}), 1.97(\mathrm{~d}, J=18.6 \mathrm{~Hz}$, $1 \mathrm{H}), 1.77(\mathrm{dt}, J=15.2,6.7 \mathrm{~Hz}, 2 \mathrm{H}), 1.67(\mathrm{ddd}, J=14.1,9.4,4.7 \mathrm{~Hz}, 1 \mathrm{H}), 1.57-1.50(\mathrm{~m}, 2 \mathrm{H})$, $1.46(\mathrm{ddt}, J=13.1,9.4,4.4 \mathrm{~Hz}, 1 \mathrm{H}), 1.13(\mathrm{~s}, 3 \mathrm{H}), 0.89(\mathrm{~s}, 3 \mathrm{H})$. ${ }^{13} \mathrm{C}$ NMR $\left(125 \mathrm{MHz}, \mathrm{CDCl}_{3}\right) \delta 214.5,137.9,115.1,70.4,57.9,47.9,46.6,42.7,42.5,33.0,28.6$, 26.8, 24.8, 24.6, 19.8, 19.7.

HRMS (ESI) m/z: [M+Na] ${ }^{+}$calcd for $\mathrm{C}_{16} \mathrm{H}_{26} \mathrm{O}_{4} \mathrm{SNa}$ : 337.1444; found: 337.1445.

\subsection{Synthesis of Estrone Derivative}

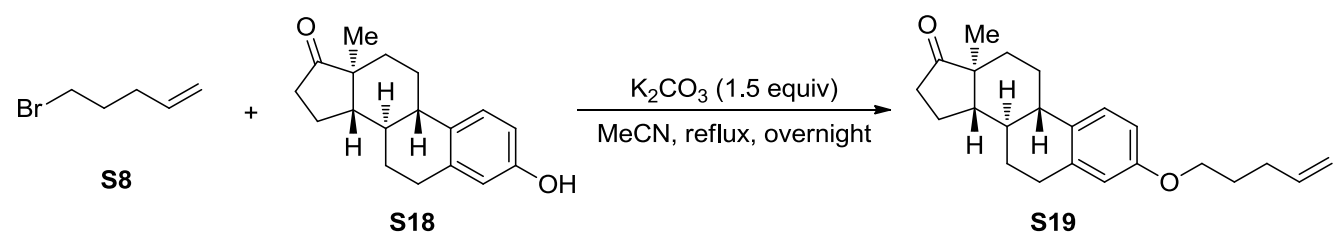

A mixture of $\mathbf{S 8}$ ( $373 \mathrm{mg}, 2.5 \mathrm{mmol}, 1.25$ equiv), Estrone (540 mg, $2 \mathrm{mmol}, 1.0$ equiv), and $\mathrm{K}_{2} \mathrm{CO}_{3}$ (414 mg, $3 \mathrm{mmol}, 1.5$ equiv) in anhydrous acetonitrile was heated to reflux in an oil bath. After refluxing overnight, the reaction mixture was cooled to room temperature, concentrated under reduced pressure, diluted with saturated $\mathrm{NH}_{4} \mathrm{Cl}$ and extracted with ethyl acetate. The combined organic layers were dried with $\mathrm{Na}_{2} \mathrm{SO}_{4}$, filtered, and concentrated under reduced pressure. The residue was purified by silica gel column chromatography (petroleum ether/ethyl acetate $=3 / 1$ ) to afford the corresponding pure product as a white amorphous solid, $580 \mathrm{mg}, 97 \%$ yield.

(8R,9S,13S,14S)-13-methyl-3-(pent-4-en-1-yloxy)-7,8,9,11,12,13,15,16-octahydro-6H-cyclo penta[a]phenanthren-17(14H)-one (S19)

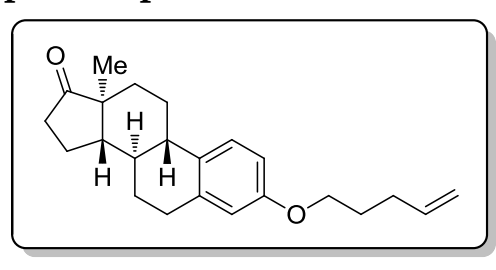

${ }^{1} \mathrm{H}$ NMR $\left(500 \mathrm{MHz}, \mathrm{CDCl}_{3}\right) \delta 7.22(\mathrm{dd}, J=8.7,1.1 \mathrm{~Hz}$, $1 \mathrm{H}), 6.74(\mathrm{dd}, J=8.6,2.8 \mathrm{~Hz}, 1 \mathrm{H}), 6.67(\mathrm{~d}, J=2.7 \mathrm{~Hz}, 1 \mathrm{H})$, $5.88(\mathrm{ddt}, J=16.9,10.2,6.6 \mathrm{~Hz}, 1 \mathrm{H}), 5.09(\mathrm{dq}, J=17.1,1.7$ $\mathrm{Hz}, 1 \mathrm{H}), 5.02(\mathrm{ddt}, J=10.2,2.2,1.3 \mathrm{~Hz}, 1 \mathrm{H}), 3.97(\mathrm{t}, J=6.4$ $\mathrm{Hz}, 2 \mathrm{H}), 2.92(\mathrm{td}, J=6.9,2.0 \mathrm{~Hz}, 2 \mathrm{H}), 2.53(\mathrm{ddd}, J=18.9$, 
8.8, $0.9 \mathrm{~Hz}, 1 \mathrm{H}), 2.45-2.38(\mathrm{~m}, 1 \mathrm{H}), 2.32-2.22(\mathrm{~m}, 3 \mathrm{H}), 2.22-2.14(\mathrm{~m}, 1 \mathrm{H}), 2.12-1.94(\mathrm{~m}$, $3 \mathrm{H}), 1.93-1.85(\mathrm{~m}, 2 \mathrm{H}), 1.71-1.41(\mathrm{~m}, 6 \mathrm{H}), 0.93(\mathrm{~s}, 3 \mathrm{H})$.

${ }^{13} \mathrm{C}$ NMR $\left(125 \mathrm{MHz}, \mathrm{CDCl}_{3}\right) \delta 157.1,137.9,137.7,131.9,126.3,115.1,114.6,112.1,67.1,50.4$, 48.0, 44.0, 38.4, 35.9, 31.6, 30.1, 29.6, 28.5, 26.6, 25.9, 21.6, 13.8 .

HRMS (ESI) m/z: [M+K] $]^{+}$calcd for $\mathrm{C}_{23} \mathrm{H}_{30} \mathrm{O}_{2} \mathrm{~K}: 377.1877$; found: 377.1870 .

\subsection{Synthesis of (+)-Fenchol Derivative}
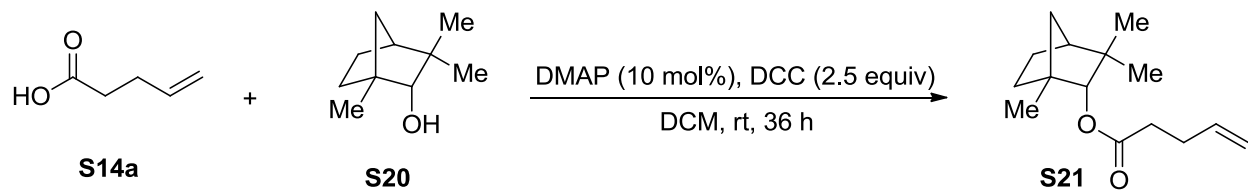

To a solution of S14a (300 mg, 3 mmol, 1.5 equiv), (+)-Fenchol (308 mg, 2 mmol, 1.0 equiv), and DAMP (24 mg, $0.2 \mathrm{mmol}, 0.1$ equiv) in anhydrous $\mathrm{CH}_{2} \mathrm{Cl}_{2}$ was added DCC (1030 mg, $5 \mathrm{mmol}, 2.5$ equiv) in one potion. After stirring at room temperature for 36 hours, the mixture was filtered through a pad of Celite, washed with $\mathrm{CH}_{2} \mathrm{Cl}_{2}$. The combined organic layers were washed with water, dried with $\mathrm{Na}_{2} \mathrm{SO}_{4}$, filtered, and concentrated under reduced pressure. The residue was purified by silica gel column chromatography (petroleum ether/ethyl acetate $=5 / 1$ ) to afford the corresponding pure product as a yellow oil, $454 \mathrm{mg}, 96 \%$ yield.

(1S,2R,4S)-1,3,3-trimethylbicyclo[2.2.1]heptan-2-yl pent-4-enoate (S21)

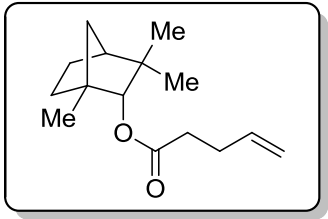

$0.79(\mathrm{~s}, 3 \mathrm{H})$.

${ }^{13} \mathrm{C}$ NMR $\left(125 \mathrm{MHz}, \mathrm{CDCl}_{3}\right) \delta 173.4,136.8,115.4,86.1,48.3,48.2,41.4,39.4,33.7,29.7,29.0$, 26.6, 25.8, 20.2, 19.4 .

HRMS (ESI) m/z: [M+Na] calcd for $\mathrm{C}_{15} \mathrm{H}_{24} \mathrm{O}_{2} \mathrm{Na}$ : 259.1669; found: 259.1668.

\subsection{Synthesis of Ibuprofen Derivative}

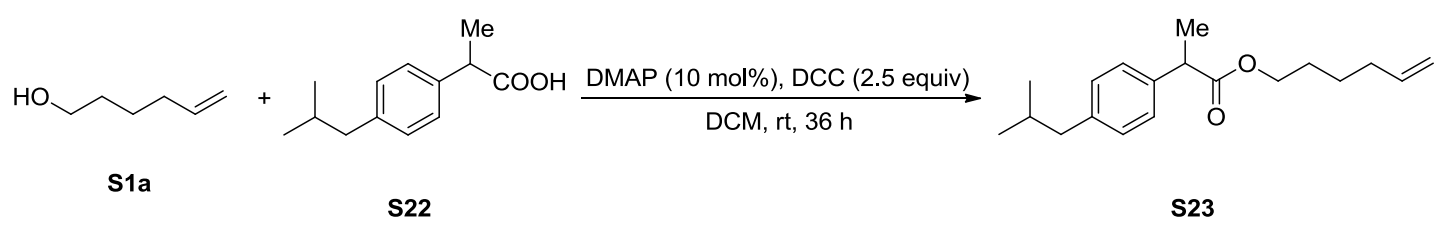

To a solution of S1a (300 mg, $3 \mathrm{mmol}, 1.5$ equiv), Ibuprofen (412 mg, $2 \mathrm{mmol}, 1.0$ equiv), and DAMP (24 mg, $0.2 \mathrm{mmol}, 0.1$ equiv) in anhydrous $\mathrm{CH}_{2} \mathrm{Cl}_{2}$ was added DCC (1030 mg, $5 \mathrm{mmol}, 2.5$ equiv) in one potion. After stirring at room temperature for 36 hours, the mixture was filtered through a pad of Celite, washed with $\mathrm{CH}_{2} \mathrm{Cl}_{2}$. The combined organic layers were washed with water, dried with $\mathrm{Na}_{2} \mathrm{SO}_{4}$, filtered, and concentrated under 
reduced pressure. The residue was purified by silica gel column chromatography (petroleum ether/ethyl acetate $=30 / 1$ ) to afford the corresponding pure product as a colorless oil, $510 \mathrm{mg}$, 895 yield.

\section{Hex-5-en-1-yl 2-(4-isobutylphenyl)propanoate (S23)}

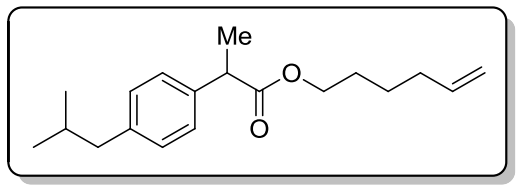

${ }^{1} \mathbf{H}$ NMR $\left(500 \mathrm{MHz}, \mathrm{CDCl}_{3}\right) \delta 7.24(\mathrm{~d}, J=8.1 \mathrm{~Hz}, 2 \mathrm{H})$, $7.13(\mathrm{~d}, J=8.1 \mathrm{~Hz}, 2 \mathrm{H}), 5.77(\mathrm{ddt}, J=16.9,10.1,6.6 \mathrm{~Hz}$, $1 \mathrm{H}), 5.06-4.93(\mathrm{~m}, 2 \mathrm{H}), 4.10(\mathrm{td}, J=6.6,1.8 \mathrm{~Hz}, 2 \mathrm{H})$, $3.72(\mathrm{q}, J=7.1 \mathrm{~Hz}, 1 \mathrm{H}), 2.49(\mathrm{~d}, J=7.2 \mathrm{~Hz}, 2 \mathrm{H}), 2.09-$ $2.00(\mathrm{~m}, 2 \mathrm{H}), 1.89(\mathrm{dp}, J=13.5,6.7 \mathrm{~Hz}, 1 \mathrm{H}), 1.68-1.57(\mathrm{~m}, 2 \mathrm{H}), 1.53(\mathrm{~d}, J=7.2 \mathrm{~Hz}, 3 \mathrm{H})$, $1.39(\mathrm{tt}, J=9.8,6.5 \mathrm{~Hz}, 2 \mathrm{H}), 0.94(\mathrm{~d}, J=6.7 \mathrm{~Hz}, 6 \mathrm{H})$.

${ }^{13} \mathrm{C}$ NMR (125 MHz, $\left.\mathrm{CDCl}_{3}\right) \delta$ 174.7, 140.3, 138.2, 137.8, 129.2, 127.1, 114.6, 64.4, 45.1, 45.0, 33.1, 30.1, 27.9, 25.0, 22.3, 18.4.

HRMS (ESI) m/z: [M+Na] $]^{+}$calcd for $\mathrm{C}_{19} \mathrm{H}_{28} \mathrm{O}_{2} \mathrm{Na}$ : 311.1982; found: 311.1986.

\subsection{Synthesis of Dihydrocholesterol Derivative}

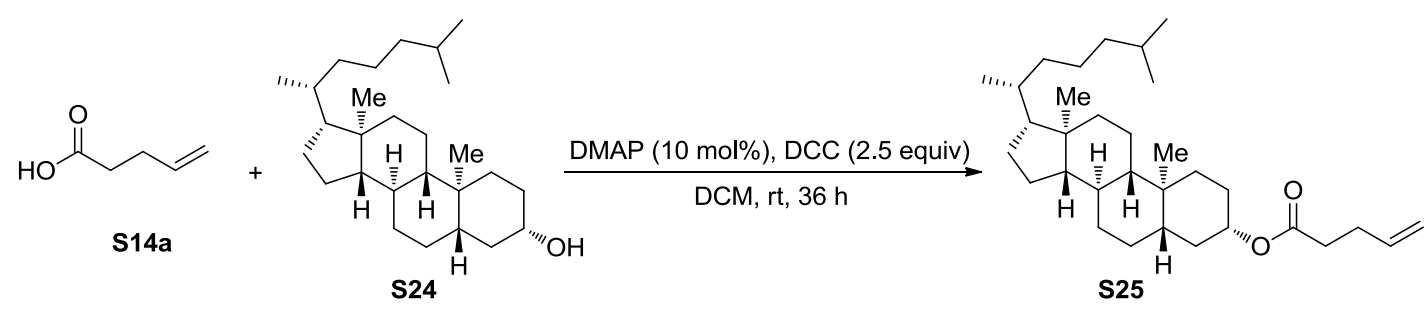

To a solution of S14a (96 mg, $0.96 \mathrm{mmol}, 1.5$ equiv), Dihydrocholesterol (250 mg, 0.64 mmol, 1.0 equiv), and DAMP ( $8 \mathrm{mg}, 0.06 \mathrm{mmol}, 0.1$ equiv) in anhydrous $\mathrm{CH}_{2} \mathrm{Cl}_{2}$ was added DCC (330 mg, $1.6 \mathrm{mmol}, 2.5$ equiv) in one potion. After stirring at room temperature for 36 hours, the mixture was filtered through a pad of Celite, washed with $\mathrm{CH}_{2} \mathrm{Cl}_{2}$. The combined organic layers were washed with water, dried with $\mathrm{Na}_{2} \mathrm{SO}_{4}$, filtered, and concentrated under reduced pressure. The residue was purified by silica gel column chromatography (petroleum ether/ethyl acetate $=5 / 1$ ) to afford the corresponding pure product as a pale yellow amorphous solid, $278 \mathrm{mg}, 92 \%$ yield.

(3S,5S,8R,9S,10S,13R,14S,17R)-10,13-dimethyl-17-((R)-6-methylheptan-2-yl)hexadecahy dro-1H-cyclopenta[ $a]$ phenanthren-3-yl pent-4-enoate (S25)

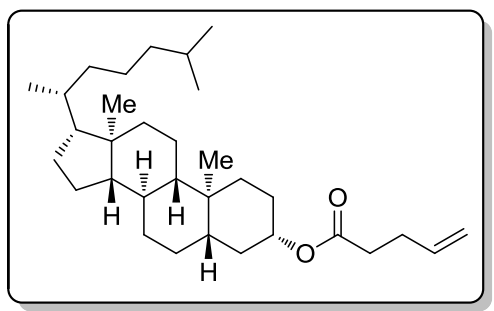

${ }^{1} \mathrm{H}$ NMR $\left(500 \mathrm{MHz}, \mathrm{CDCl}_{3}\right) \delta 5.84(\mathrm{ddq}, J=16.6,9.8,3.3$ $\mathrm{Hz}, 1 \mathrm{H}), 5.07$ (dd, $J=17.0,1.7 \mathrm{~Hz}, 1 \mathrm{H}), 5.01$ (dd, $J=10.2$, $1.7 \mathrm{~Hz}, 1 \mathrm{H}), 4.73(\mathrm{tt}, J=11.3,4.9 \mathrm{~Hz}, 1 \mathrm{H}), 2.42-2.34(\mathrm{~m}$, $1 \mathrm{H}), 2.39(\mathrm{~s}, 3 \mathrm{H}), 1.98(\mathrm{dt}, J=12.6,3.4 \mathrm{~Hz}, 1 \mathrm{H}), 1.82(\mathrm{tdd}$, $J=9.3,5.9,3.6 \mathrm{~Hz}, 2 \mathrm{H}), 1.74(\mathrm{dt}, J=13.4,3.7 \mathrm{~Hz}, 1 \mathrm{H}), 1.67$ $(\mathrm{dq}, J=13.0,3.4 \mathrm{~Hz}, 1 \mathrm{H}), 1.63-1.45(\mathrm{~m}, 6 \mathrm{H}), 1.42-1.22$ $(\mathrm{m}, 10 \mathrm{H}), 1.22-0.96(\mathrm{~m}, 12 \mathrm{H}), 0.95-0.80(\mathrm{~m}, 2 \mathrm{H}), 0.92$ $(\mathrm{d}, J=6.5 \mathrm{~Hz}, 3 \mathrm{H}), 0.89(\mathrm{~d}, J=2.4 \mathrm{~Hz}, 3 \mathrm{H}), 0.88(\mathrm{~d}, J=2.3 \mathrm{~Hz}, 3 \mathrm{H}), 0.84(\mathrm{~s}, 3 \mathrm{H}), 0.67(\mathrm{~s}, 3 \mathrm{H})$. ${ }^{13} \mathrm{C}$ NMR $\left(125 \mathrm{MHz}, \mathrm{CDCl}_{3}\right) \delta$ 172.6, 136.8, 115.4, 73.7, 56.4, 56.3, 54.2, 44.7, 42.6, 40.0, 39.5, $36.8,36.2,35.8,35.49,35.46,34.1,33.9,32.0,29.0,28.6,28.2,28.0,27.5,24.2,23.8,22.8,22.6$, 21.2, 18.7, 12.2, 12.1 .

HRMS (ESI) m/z: [M+Na] $]^{+}$calcd for $\mathrm{C}_{32} \mathrm{H}_{54} \mathrm{O}_{2} \mathrm{Na}$ : 493.4016; found: 493.4017 . 


\subsection{Synthesis of (+)-Dehydroabietylamine Derivative}

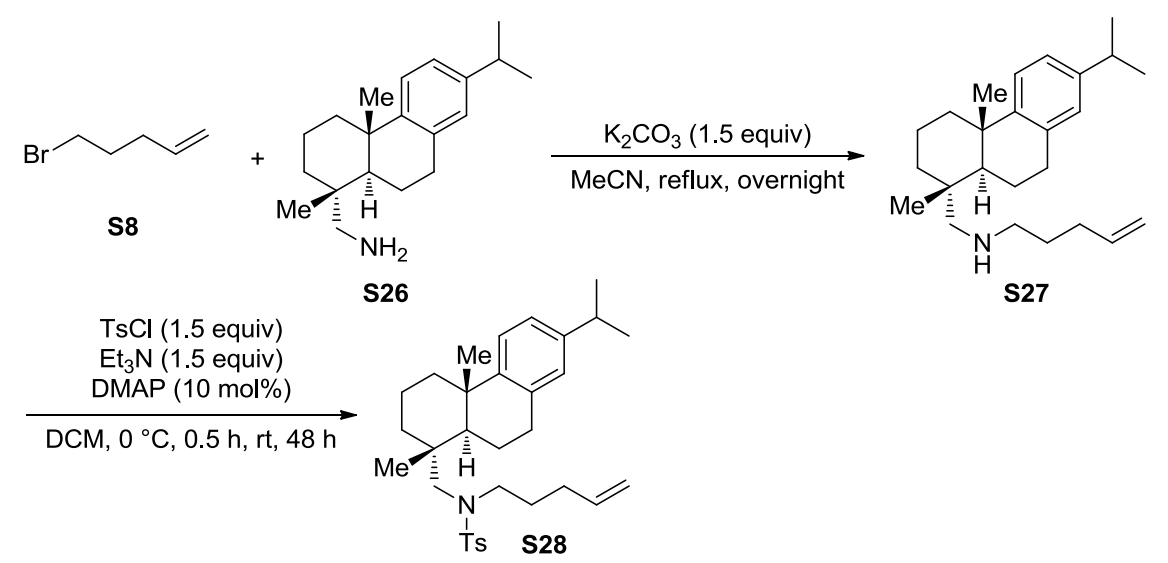

A mixture of $\mathbf{S 8}$ (551 mg, $3.75 \mathrm{mmol}, 1.25$ equiv), (+)-Dehydroabietylamine (856 mg, 3 mmol, 1.0 equiv), and $\mathrm{K}_{2} \mathrm{CO}_{3}(621 \mathrm{mg}$, $4.5 \mathrm{mmol}$, 1.5 equiv) in anhydrous acetonitrile was heated to reflux in an oil bath. After refluxing overnight, the reaction mixture was cooled to room temperature, concentrated under reduced pressure, diluted with saturated $\mathrm{NH}_{4} \mathrm{Cl}$ and extracted with ethyl acetate. The combined organic layers were dried with $\mathrm{Na}_{2} \mathrm{SO}_{4}$, filtered, and concentrated under reduced pressure. The residue was purified by silica gel column chromatography (petroleum ether/ethyl acetate $=5 / 1$ ) to afford the corresponding pure product S27 as a pale yellow oi, $966 \mathrm{mg}$, 91\% yield.

To a solution of S27 (530 mg, $1.5 \mathrm{mmol}, 1.0$ equiv), DAMP (18 mg, $0.15 \mathrm{mmol}, 0.1$ equiv), and triethylamine (227 mg, $2.25 \mathrm{mmol}, 1.5$ equiv) in anhydrous $\mathrm{CH}_{2} \mathrm{Cl}_{2}$ was added $p$-toluenesulfonyl chloride ( $357 \mathrm{mg}, 1.86 \mathrm{mmol}, 1.25$ equiv) in potions at $0{ }^{\circ} \mathrm{C}$. The resulting mixture was stirred at $0{ }^{\circ} \mathrm{C}$ for 0.5 hour, and then at room temperature for 48 hours. After completion, the reaction was quenched with water and extracted with $\mathrm{CH}_{2} \mathrm{Cl}_{2}$. The combined organic layers were dried with $\mathrm{Na}_{2} \mathrm{SO}_{4}$, filtered, and concentrated under reduced pressure. The residue was purified by silica gel column chromatography (petroleum ether/ethyl acetate $=20 / 1$ ) to afford the corresponding pure product as a pale yellow sticky oil, $636 \mathrm{mg}$, $84 \%$ yield.

$\mathrm{N}$-(((1R,4aS,10aR)-7-isopropyl-1,4a-dimethyl-1,2,3,4,4a,9,10,10a-octahydrophenanthren1-yl)methyl)-4-methyl- $N$-(pent-4-en-1-yl)benzenesulfonamide (S28)

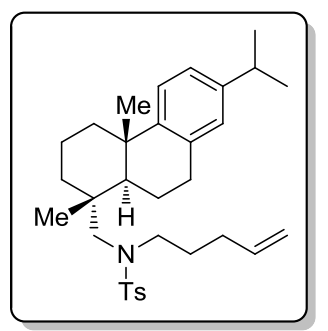

${ }^{1}$ H NMR $\left(500 \mathrm{MHz}, \mathrm{CDCl}_{3}\right) \delta 7.70(\mathrm{~d}, J=8.1 \mathrm{~Hz}, 2 \mathrm{H}), 7.31(\mathrm{~d}, J=8.1$ $\mathrm{Hz}, 2 \mathrm{H}), 7.18(\mathrm{~d}, J=8.2 \mathrm{~Hz}, 1 \mathrm{H}), 7.01(\mathrm{dd}, J=8.2,2.0 \mathrm{~Hz}, 1 \mathrm{H}), 6.91(\mathrm{~d}$, $J=1.9 \mathrm{~Hz}, 1 \mathrm{H}), 5.76-5.59(\mathrm{~m}, 1 \mathrm{H}), 4.96(\mathrm{~d}, J=1.5 \mathrm{~Hz}, 1 \mathrm{H}), 4.93(\mathrm{dq}$, $J=6.0,1.6 \mathrm{~Hz}, 1 \mathrm{H}), 3.17(\mathrm{~d}, J=14.5 \mathrm{~Hz}, 1 \mathrm{H}), 3.14-3.03(\mathrm{~m}, 2 \mathrm{H}), 3.02$ $-2.96(\mathrm{~m}, 1 \mathrm{H}), 2.96-2.88(\mathrm{~m}, 2 \mathrm{H}), 2.85(\mathrm{p}, J=6.9 \mathrm{~Hz}, 1 \mathrm{H}), 2.44(\mathrm{~s}$, $3 \mathrm{H}), 2.31(\mathrm{dq}, J=13.8,2.6,2.1 \mathrm{~Hz}, 1 \mathrm{H}), 1.96-1.84(\mathrm{~m}, 3 \mathrm{H}), 1.85-$ $1.67(\mathrm{~m}, 4 \mathrm{H}), 1.66-1.57(\mathrm{~m}, 2 \mathrm{H}), 1.51(\mathrm{ddd}, J=13.3,6.8,3.5 \mathrm{~Hz}, 2 \mathrm{H})$,

$1.41(\mathrm{td}, J=12.9,4.4 \mathrm{~Hz}, 1 \mathrm{H}), 1.25(\mathrm{~d}, J=6.9 \mathrm{~Hz}, 6 \mathrm{H}), 1.25(\mathrm{~s}, 3 \mathrm{H}), 1.02(\mathrm{~s}, 3 \mathrm{H})$.

${ }^{13} \mathrm{C}$ NMR $\left(125 \mathrm{MHz}, \mathrm{CDCl}_{3}\right) \delta 147.4,145.6,143.1,137.3,136.7,134.5,129.5,127.5,126.8$, $123.9,123.8,115.3,59.8,51.3,45.5,38.9,38.2,37.6,37.2,33.4,31.2,29.8,27.3,25.6,24.0,24.0$, 21.5, 19.3, 18.9, 18.6 .

HRMS (ESI) m/z: [M+Na] $]^{+}$calcd for $\mathrm{C}_{32} \mathrm{H}_{45} \mathrm{NO}_{2} \mathrm{SNa}$ : 530.3063; found: 530.3063 . 


\section{Screening of Reaction Conditions}

Table S1. Optimization of Reaction Conditions for Alkene

\begin{tabular}{|c|c|c|c|c|c|c|}
\hline & & & & & & \\
\hline entry & solvent & $\mathbf{T}\left({ }^{\circ} \mathrm{C}\right)$ & $\mathrm{R}_{2} \mathrm{Zn}$ & (x mol\%) & $t(h)$ & yield $(\%)$ \\
\hline 1 & THF & 0 & $\mathrm{Me}_{2} \mathrm{Zn}$ & 100 & 20 & 72 \\
\hline 2 & THF & -10 & $\mathrm{Me}_{2} \mathrm{Zn}$ & 100 & 20 & 88 \\
\hline 3 & THF & -20 & $\mathrm{Me}_{2} \mathrm{Zn}$ & 100 & 20 & 90 \\
\hline 4 & hexane & -20 & $\mathrm{Me}_{2} \mathrm{Zn}$ & 100 & 20 & 73 \\
\hline 5 & $\mathrm{MeCN}$ & -20 & $\mathrm{Me}_{2} \mathrm{Zn}$ & 100 & 20 & 96 \\
\hline 6 & DMF & -20 & $\mathrm{Me}_{2} \mathrm{Zn}$ & 100 & 20 & 82 \\
\hline 7 & $\mathrm{DCM}$ & -20 & $\mathrm{Me}_{2} \mathrm{Zn}$ & 100 & 20 & 93 \\
\hline 8 & $\mathrm{MeCN}$ & -20 & $\mathrm{Me}_{2} \mathrm{Zn}$ & 50 & 20 & 97 \\
\hline 9 & $\mathrm{MeCN}$ & -20 & $\mathrm{Me}_{2} \mathrm{Zn}$ & 20 & 20 & $51 \sim 97^{a}$ \\
\hline 10 & $\mathrm{MeCN}$ & -20 & $\mathrm{Me}_{2} \mathrm{Zn}$ & 10 & 20 & 42 \\
\hline 11 & $\mathrm{MeCN}$ & -20 & $\mathrm{Me}_{2} \mathrm{Zn}$ & 5 & 20 & 29 \\
\hline 12 & $\mathrm{MeCN}$ & -20 & $\mathrm{Et}_{2} \mathrm{Zn}$ & 50 & 20 & 96 \\
\hline 13 & $\mathrm{MeCN}$ & -20 & $\mathrm{Et}_{2} \mathrm{Zn}$ & 20 & 20 & $47 \sim 93^{b}$ \\
\hline 14 & $\mathrm{MeCN}$ & -20 & $\mathrm{Et}_{2} \mathrm{Zn}$ & 10 & 20 & 27 \\
\hline 15 & $\mathrm{MeCN}$ & -20 & $\mathrm{Et}_{2} \mathrm{Zn}$ & 5 & 20 & 15 \\
\hline 16 & $\mathrm{MeCN}$ & -20 & $\mathrm{Et}_{2} \mathrm{Zn}$ & 50 & 16 & 99 \\
\hline 17 & $\mathrm{MeCN}$ & -20 & $\mathrm{Et}_{2} \mathrm{Zn}$ & 50 & 8 & 79 \\
\hline 18 & $\mathrm{MeCN}$ & -20 & $\mathrm{Et}_{2} \mathrm{Zn}$ & 50 & 4 & 69 \\
\hline 19 & $\mathrm{MeCN}$ & -20 & $\mathrm{Et}_{2} \mathrm{Zn}$ & 50 & 2 & 51 \\
\hline 20 & $\mathrm{MeCN}$ & -30 & $\mathrm{Et}_{2} \mathrm{Zn}$ & 50 & 16 & 95 \\
\hline
\end{tabular}

a: The yields varied from $51 \%$ to $97 \%$ in 5 runs b: The yields varied from $47 \%$ to $93 \%$ in 5 runs 
Table S2. Optimization of Reaction Conditions for Alkyne

\begin{tabular}{|c|c|c|c|c|c|c|}
\hline entry & solvent & $\mathbf{T}\left({ }^{\circ} \mathrm{C}\right)$ & $\mathrm{R}_{2} \mathrm{Zn}$ & (x mol\%) & $t(h)$ & yield (\%) \\
\hline 1 & THF & -10 & $\mathrm{Me}_{2} \mathrm{Zn}$ & 100 & 20 & 63 \\
\hline 2 & toluene & -10 & $\mathrm{Me}_{2} \mathrm{Zn}$ & 100 & 20 & $<10$ \\
\hline 3 & $\mathrm{MeCN}$ & -10 & $\mathrm{Me}_{2} \mathrm{Zn}$ & 100 & 20 & 66 \\
\hline 4 & DMF & -10 & $\mathrm{Me}_{2} \mathrm{Zn}$ & 100 & 20 & 23 \\
\hline 5 & $\mathrm{DCM}$ & -10 & $\mathrm{Me}_{2} \mathrm{Zn}$ & 100 & 20 & $<5$ \\
\hline 6 & $\mathrm{MeCN}$ & -20 & $\mathrm{Me}_{2} \mathrm{Zn}$ & 100 & 20 & 73 \\
\hline 7 & $\mathrm{MeCN}$ & -20 & $\mathrm{Me}_{2} \mathrm{Zn}$ & 100 & 16 & 74 \\
\hline 8 & $\mathrm{MeCN}$ & -20 & $\mathrm{Me}_{2} \mathrm{Zn}$ & 100 & 8 & 73 \\
\hline 9 & $\mathrm{MeCN}$ & -20 & $\mathrm{Me}_{2} \mathrm{Zn}$ & 100 & 4 & 42 \\
\hline 10 & $\mathrm{MeCN}$ & -20 & $\mathrm{Me}_{2} \mathrm{Zn}$ & 50 & 16 & 65 \\
\hline 11 & $\mathrm{MeCN}$ & -20 & $\mathrm{Me}_{2} \mathrm{Zn}$ & 50 & 8 & 57 \\
\hline 12 & $\mathrm{MeCN}$ & -20 & $\mathrm{Me}_{2} \mathrm{Zn}$ & 50 & 4 & 47 \\
\hline 13 & $\mathrm{MeCN}$ & -20 & $\mathrm{Et}_{2} \mathrm{Zn}$ & 100 & 16 & 85 \\
\hline 14 & $\mathrm{MeCN}$ & -20 & $\mathrm{Et}_{2} \mathrm{Zn}$ & 100 & 8 & 87 \\
\hline 15 & $\mathrm{MeCN}$ & -20 & $\mathrm{Et}_{2} \mathrm{Zn}$ & 50 & 16 & 93 \\
\hline 16 & $\mathrm{MeCN}$ & -20 & $\mathrm{Et}_{2} \mathrm{Zn}$ & 50 & 8 & 99 \\
\hline 17 & $\mathrm{MeCN}$ & -20 & $\mathrm{Et}_{2} \mathrm{Zn}$ & 20 & 8 & 51 \\
\hline 18 & $\mathrm{MeCN}$ & -20 & $\mathrm{Et}_{2} \mathrm{Zn}$ & 10 & 8 & 21 \\
\hline
\end{tabular}

IV. General Procedure for the 1,2-Addition of Perfluoroalkyl Iodides to Alkenes

and Alkynes

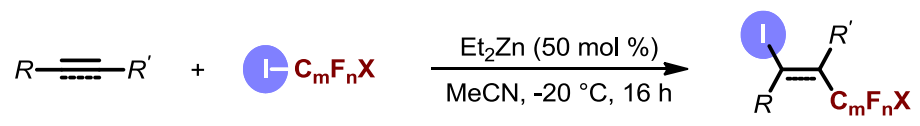

For liquid substrates:

An oven-dried Schlenk tube was charged with a stir bar. The tube was degased and refilled with nitrogen for 3 times, and then a solution of alkenes or alkynes $(0.2 \mathrm{mmol})$ and perfluoroalkyl iodides ( $0.2 \mathrm{mmol}, 1.0$ equiv) in acetonitrile $(2 \mathrm{~mL})$ was injected to the tube via a syringe. The reaction mixture was cooled to the temperature indicated. After stirring for 3 minutes, a solution of diethylzinc ( $1.0 \mathrm{~mol} / \mathrm{L}$ in hexane, $0.1 \mathrm{~mL}, 0.5$ equiv) was injected to the reaction mixture via a syringe. After stirring at the same temperature for the time indicated, the reaction was quenched with saturated $\mathrm{NH}_{4} \mathrm{Cl}$ and extracted with ethyl acetate $(3 \times 50 \mathrm{~mL})$. The combined organic layers were dried with $\mathrm{Na}_{2} \mathrm{SO}_{4}$, filtered through a short pad of silica gel, and concentrated under reduced pressure. The residue was pure enough to submit to NMR experiments or was purified by silica gel 
column chromatography (petroleum ether/ethyl acetate) to afford the pure product if necessary.

For solid substrates:

An oven-dried Schlenk tube was charged with alkenes or alkynes $(0.2 \mathrm{mmol})$ and a stir bar. The tube was degased and refilled with nitrogen for 3 times, and then a solution of perfluoroalkyl iodides $(0.2 \mathrm{mmol}, 1.0$ equiv) in acetonitrile $(2 \mathrm{~mL})$ was injected to the tube via a syringe. The following procedure was the same to that of liquid substrates.

For a $1 \mathrm{mmol}$ scale synthesis of $3 a$ :

An oven-dried Schlenk tube was charged with a stir bar. The tube was degased and refilled with nitrogen for 3 times, and then a solution of $2 \mathbf{a}(118.0 \mathrm{mg}, 1.0 \mathrm{mmol})$ and ethyl iododifluoroacetate $1 \mathrm{a}(249.9 \mathrm{mg}, 1.0 \mathrm{mmol}, 1.0$ equiv) in acetonitrile $(10 \mathrm{~mL})$ was injected to the tube via a syringe. The reaction mixture was cooled to $-20{ }^{\circ} \mathrm{C}$. After stirring for 3 minutes, a solution of diethylzinc $(1.0 \mathrm{~mol} / \mathrm{L}$ in hexane, $0.5 \mathrm{~mL}, 0.5$ equiv) was injected to the reaction mixture via a syringe. After stirring at $-20^{\circ} \mathrm{C}$ for 16 hours, the reaction was quenched with saturated $\mathrm{NH}_{4} \mathrm{Cl}$ and extracted with ethyl acetate $(3 \times$ $100 \mathrm{~mL}$ ). The combined organic layers were dried with $\mathrm{Na}_{2} \mathrm{SO}_{4}$, filtered through a short pad of silica gel, and concentrated under reduced pressure. The residue (colorless oil, $368.7 \mathrm{mg}$, 99\% yield) was pure enough to submit to NMR experiments.

\section{Ethyl 2,2-difluoro-4-iodo-5-phenylpentanoate (3a)}

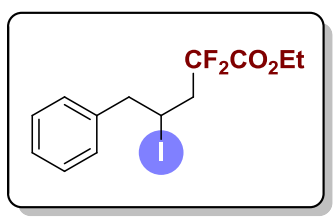

Colorless oil, $73.5 \mathrm{mg}$, $99 \%$ yield.

${ }^{1} \mathbf{H}$ NMR $\left(500 \mathrm{MHz}, \mathrm{CDCl}_{3}\right) \delta 7.40-7.29(\mathrm{~m}, 3 \mathrm{H}), 7.24-7.19(\mathrm{~m}$, $2 \mathrm{H}), 4.42-4.31(\mathrm{~m}, 3 \mathrm{H}), 3.26(\mathrm{qd}, J=14.5,7.3 \mathrm{~Hz}, 2 \mathrm{H}), 2.94(\mathrm{qd}, J=$ $16.5,7.3 \mathrm{~Hz}, 1 \mathrm{H}), 2.87-2.75(\mathrm{~m}, 1 \mathrm{H}), 1.39(\mathrm{t}, J=7.2 \mathrm{~Hz}, 3 \mathrm{H})$.

${ }^{19}$ F NMR $\left(471 \mathrm{MHz}, \mathrm{CDCl}_{3}\right) \delta-101.8(\mathrm{~d}, J=263.5 \mathrm{~Hz}),-106.4(\mathrm{~d}, J=$

263.5 Hz).

${ }^{13} \mathrm{C}$ NMR $\left(125 \mathrm{MHz}, \mathrm{CDCl}_{3}\right) \delta 163.4\left(\mathrm{t},{ }^{2} J_{C-F}=32.3 \mathrm{~Hz}\right), 138.8,129.0,128.6,127.2,115.2(\mathrm{dd}$, $\left.{ }^{1} J_{C-F}=253.5,251.7 \mathrm{~Hz}\right), 63.3,47.2,44.4\left(\mathrm{t},{ }^{2} J_{C-F}=23.5 \mathrm{~Hz}\right), 21.8\left(\mathrm{t},{ }^{3} \mathrm{~J}_{\mathrm{C}-F}=3.9 \mathrm{~Hz}\right), 13.9$.

HRMS (ESI) m/z: [M+Na] $]^{+}$calcd for $\mathrm{C}_{13} \mathrm{H}_{15} \mathrm{~F}_{2} \mathrm{IO}_{2} \mathrm{Na}$ : 390.9977; found: 390.9979.

\section{Ethyl 2,2-difluoro-4-iodo-6-phenylhexanoate (3b)}

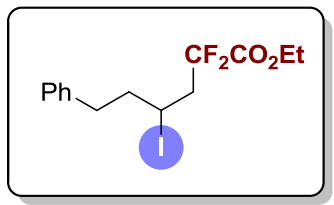

Colorless oil, $74.9 \mathrm{mg}$, $98 \%$ yield.

${ }^{1} \mathrm{H}$ NMR $\left(500 \mathrm{MHz}, \mathrm{CDCl}_{3}\right) \delta 7.36-7.30(\mathrm{~m}, 2 \mathrm{H}), 7.27-7.20(\mathrm{~m}$, $3 \mathrm{H}), 4.33(\mathrm{q}, J=7.1 \mathrm{~Hz}, 2 \mathrm{H}), 4.19(\mathrm{dtd}, J=9.9,6.7,3.9 \mathrm{~Hz}, 1 \mathrm{H}), 3.07$

$7.1 \mathrm{~Hz}, 3 \mathrm{H})$.

${ }^{19}$ F NMR $\left(471 \mathrm{MHz}, \mathrm{CDCl}_{3}\right) \delta-101.7(\mathrm{~d}, J=263.5 \mathrm{~Hz}),-106.4(\mathrm{~d}, J=263.1 \mathrm{~Hz})$.

${ }^{13} \mathrm{C}$ NMR $\left(125 \mathrm{MHz}, \mathrm{CDCl}_{3}\right) \delta 163.3\left(\mathrm{t},{ }^{2}{ }_{\mathrm{C}-F}=32.3 \mathrm{~Hz}\right), 140.1,128.51,128.48,126.3,115.1$

$\left(\mathrm{dd},{ }^{1} J_{\mathrm{C}-F}=253.9,252.6 \mathrm{~Hz}\right), 63.2,45.3\left(\mathrm{t},{ }^{2} J_{\mathrm{C}-F}=23.2 \mathrm{~Hz}\right), 41.8,35.5,22.4\left(\mathrm{t},{ }^{3} J_{\mathrm{C}-F}=4.0 \mathrm{~Hz}\right)$, 13.8 .

HRMS (ESI) m/z: [M+Na $]^{+}$calcd for $\mathrm{C}_{14} \mathrm{H}_{17} \mathrm{~F}_{2} \mathrm{IO}_{2} \mathrm{Na}$ : 405.0134; found: 405.0130.

\section{Ethyl 2,2-difluoro-4-iododecanoate (3c)}

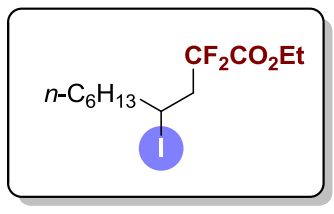

Colorless oil, $71.7 \mathrm{mg}, 99 \%$ yield.

${ }^{1} \mathrm{H}$ NMR $\left(500 \mathrm{MHz}, \mathrm{CDCl}_{3}\right) \delta 4.37(\mathrm{q}, J=7.2 \mathrm{~Hz}, 2 \mathrm{H}), 4.24(\mathrm{ddd}, J=$ $11.2,8.9,5.6 \mathrm{~Hz}, 1 \mathrm{H}), 2.93(\mathrm{dtd}, J=18.3,16.0,6.5 \mathrm{~Hz}, 1 \mathrm{H}), 2.76$ (dddd, $J=17.8,15.6,12.5,7.1 \mathrm{~Hz}, 1 \mathrm{H}), 1.83(\mathrm{dtd}, J=14.3,9.4,4.8$ 
$\mathrm{Hz}, 1 \mathrm{H}), 1.75(\mathrm{ddt}, J=14.7,9.8,4.9 \mathrm{~Hz}, 1 \mathrm{H}), 1.54(\mathrm{dtt}, J=14.7,10.4,5.4 \mathrm{~Hz}, 1 \mathrm{H}), 1.47-1.24$ $(\mathrm{m}, 7 \mathrm{H}), 1.39(\mathrm{t}, J=7.2 \mathrm{~Hz}, 3 \mathrm{H}), 0.91(\mathrm{t}, J=6.7 \mathrm{~Hz}, 3 \mathrm{H})$.

${ }^{19}$ F NMR $\left(471 \mathrm{MHz}, \mathrm{CDCl}_{3}\right) \delta-102.1(\mathrm{~d}, J=262.8 \mathrm{~Hz}),-106.8(\mathrm{~d}, J=262.8 \mathrm{~Hz})$.

${ }^{13} \mathrm{C}$ NMR $\left(125 \mathrm{MHz}, \mathrm{CDCl}_{3}\right) \delta 163.5\left(\mathrm{t},{ }^{2} \mathrm{JC}_{\mathrm{C}-\mathrm{F}}=32.3 \mathrm{~Hz}\right), 115.20\left(\mathrm{dd},{ }^{1} \mathrm{JC}_{\mathrm{C}-\mathrm{F}}=253.5,252.2 \mathrm{~Hz}\right)$, 63.2, $45.4\left(\mathrm{t},{ }^{2} \mathrm{C}_{\mathrm{C}-\mathrm{F}}=23.1 \mathrm{~Hz}\right), 40.5,31.6,29.4,28.2,23.3\left(\mathrm{t},{ }^{3} \mathrm{~J}_{\mathrm{C}-\mathrm{F}}=4.1 \mathrm{~Hz}\right), 22.5,14.0,13.9$.

HRMS (ESI) m/z: [M+Na] $]^{+}$calcd for $\mathrm{C}_{12} \mathrm{H}_{21} \mathrm{~F}_{2} \mathrm{IO}_{2} \mathrm{Na}$ : 385.0447; found: 385.0444.

Ethyl 4-cyclohexyl-2,2-difluoro-4-iodobutanoate (3d)

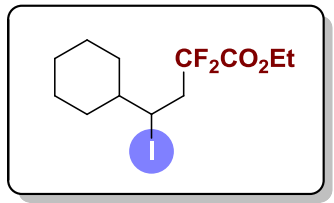

Colorless oil, $69.8 \mathrm{mg}$, 97\% yield.

${ }^{1} \mathbf{H}$ NMR $\left(500 \mathrm{MHz}, \mathrm{CDCl}_{3}\right) \delta 4.36(\mathrm{q}, J=7.2 \mathrm{~Hz}, 2 \mathrm{H}), 4.28-4.21$ $(\mathrm{m}, 1 \mathrm{H}), 2.96-2.70(\mathrm{~m}, 2 \mathrm{H}), 1.86-1.63(\mathrm{~m}, 5 \mathrm{H}), 1.39(\mathrm{t}, J=7.2 \mathrm{~Hz}$, $3 \mathrm{H}), 1.37-1.07(\mathrm{~m}, 5 \mathrm{H}), 0.92-0.81(\mathrm{~m}, 1 \mathrm{H})$.

${ }^{19}$ F NMR $\left(471 \mathrm{MHz}, \mathrm{CDCl}_{3}\right) \delta-102.3(\mathrm{~d}, J=261.6 \mathrm{~Hz}),-107.3(\mathrm{~d}, J=$

261.6 Hz).

${ }^{13} \mathrm{C}$ NMR $\left(125 \mathrm{MHz}, \mathrm{CDCl}_{3}\right) \delta 163.5\left(\mathrm{t},{ }^{2} \mathrm{~J}_{\mathrm{C}-\mathrm{F}}=32.4 \mathrm{~Hz}\right), 115.3\left(\mathrm{dd},{ }^{1} J_{\mathrm{C}-F}=253.6,251.3 \mathrm{~Hz}\right)$, 63.2, 44.5, $42.9\left(\mathrm{t},{ }^{2} J_{C-F}=23.3 \mathrm{~Hz}\right), 33.3,32.8\left(\mathrm{t},{ }^{3} J_{C-F}=3.8 \mathrm{~Hz}\right), 29.9,26.0,25.8,25.6,13.9$.

HRMS (ESI) m/z: [M+Na] $]^{+}$calcd for $\mathrm{C}_{12} \mathrm{H}_{1}{ }_{1} \mathrm{~F}_{2} \mathrm{IO}_{2} \mathrm{Na}$ : 383.0290; found: 383.0289.

Diethyl 2,2,11,11-tetrafluoro-4,9-diiodododecanedioate (3e)

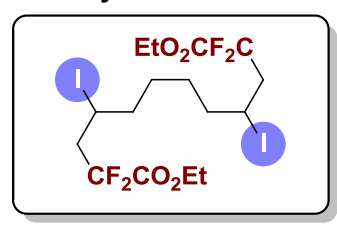

Colorless oil, $120.8 \mathrm{mg}$, $99 \%$ yield.

${ }^{1} \mathrm{H}$ NMR $\left(500 \mathrm{MHz}, \mathrm{CDCl}_{3}\right) \delta 4.35(\mathrm{q}, J=7.2 \mathrm{~Hz}, 4 \mathrm{H}), 4.26-4.18$ $(\mathrm{m}, 2 \mathrm{H}), 3.00-2.84(\mathrm{~m}, 2 \mathrm{H}), 2.74(\mathrm{dddd}, J=18.2,15.6,12.2,7.3 \mathrm{~Hz}$, $2 \mathrm{H}), 1.79(\mathrm{dqd}, J=28.7,9.4,4.7 \mathrm{~Hz}, 4 \mathrm{H}), 1.66-1.40(\mathrm{~m}, 4 \mathrm{H}), 1.37(\mathrm{t}$, $J=7.2 \mathrm{~Hz}, 6 \mathrm{H})$.

${ }^{19}$ F NMR $\left(471 \mathrm{MHz}, \mathrm{CDCl}_{3}\right) \delta-102.12(\mathrm{~d}, J=263.8 \mathrm{~Hz}),-102.13(\mathrm{~d}, J=263.8 \mathrm{~Hz}),-106.73(\mathrm{~d}$, $J=263.8 \mathrm{~Hz}),-106.76(\mathrm{~d}, J=263.8 \mathrm{~Hz})$.

${ }^{13} \mathrm{C}$ NMR $\left(125 \mathrm{MHz}, \mathrm{CDCl}_{3}\right) \delta 163.3\left(\mathrm{t},{ }^{2} J_{\mathrm{C}-\mathrm{F}}=32.3 \mathrm{~Hz}\right), 115.1\left(\mathrm{t},{ }^{1} \mathrm{JC}_{\mathrm{C}-\mathrm{F}}=252.7 \mathrm{~Hz}\right), 63.2,45.21$ $\left(\mathrm{t},{ }^{2} J_{C-F}=23.1 \mathrm{~Hz}\right), 45.15\left(\mathrm{t},{ }^{2} J_{C-F}=23.1 \mathrm{~Hz}\right), 40.0,39.9,28.41,28.38,22.66\left(\mathrm{t},{ }^{3} J_{C-F}=4.3 \mathrm{~Hz}\right)$, $22.62\left(\mathrm{t},{ }^{3}{ }^{\mathrm{C}-\mathrm{F}}=4.3 \mathrm{~Hz}\right), 13.8$.

HRMS (ESI) m/z: [M+Na] $]^{+}$calcd for $\mathrm{C}_{16} \mathrm{H}_{24} \mathrm{~F}_{4} \mathrm{I}_{2} \mathrm{O}_{4} \mathrm{Na}$ : 632.9592; found: 632.9589 .

Ethyl 7-bromo-2,2-difluoro-4-iodoheptanoate (3f)

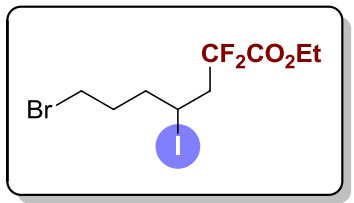

Colorless oil, $78.8 \mathrm{mg}$, 99\% yield.

${ }^{1} \mathrm{H}$ NMR $\left(500 \mathrm{MHz}, \mathrm{CDCl}_{3}\right) \delta 4.36(\mathrm{q}, J=7.2 \mathrm{~Hz}, 2 \mathrm{H}), 4.29-4.20$ $(\mathrm{m}, 1 \mathrm{H}), 3.45(\mathrm{td}, J=5.7,3.0 \mathrm{~Hz}, 2 \mathrm{H}), 2.95(\mathrm{dtd}, J=19.0,15.5,6.3$ $\mathrm{Hz}, 1 \mathrm{H}), 2.84-2.70(\mathrm{~m}, 1 \mathrm{H}), 2.22-2.09(\mathrm{~m}, 1 \mathrm{H}), 2.05-1.91(\mathrm{~m}$,

$3 \mathrm{H}), 1.39(\mathrm{t}, J=7.2 \mathrm{~Hz}, 3 \mathrm{H})$.

${ }^{19}$ F NMR $\left(471 \mathrm{MHz}, \mathrm{CDCl}_{3}\right) \delta-102.3(\mathrm{~d}, J=263.7 \mathrm{~Hz}),-106.6(\mathrm{~d}, J=263.7 \mathrm{~Hz})$.

${ }^{13} \mathrm{C}$ NMR $\left(125 \mathrm{MHz}, \mathrm{CDCl}_{3}\right) \delta 163.3\left(\mathrm{t},{ }^{2} \mathrm{~J}_{\mathrm{C}-\mathrm{F}}=32.2 \mathrm{~Hz}\right), 115.0\left(\mathrm{t},{ }^{1} \mathrm{~J} \mathrm{C}-\mathrm{F}=252.9 \mathrm{~Hz}\right), 63.3,45.3$

$\left(\mathrm{t},{ }^{2} J_{C-F}=23.2 \mathrm{~Hz}\right), 38.7,32.5,32.0,21.1\left(\mathrm{t},{ }^{3} J_{C-F}=3.9 \mathrm{~Hz}\right), 13.9$.

HRMS (ESI) m/z: [M+Na] ${ }^{+}$calcd for $\mathrm{C}_{9} \mathrm{H}_{14} \mathrm{BrF}_{2} \mathrm{IO}_{2} \mathrm{Na}$ : 420.9082 ; found: 420.9087.

Ethyl 5-acetoxy-2,2-difluoro-4-iodopentanoate (3g)

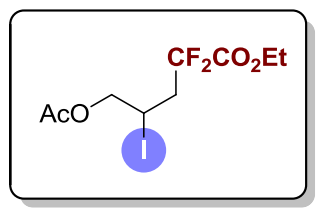

Colorless oil, $65.1 \mathrm{mg}, 93 \%$ yield.

${ }^{1} \mathrm{H}$ NMR $\left(500 \mathrm{MHz}, \mathrm{CDCl}_{3}\right) \delta 4.40-4.30(\mathrm{~m}, 2 \mathrm{H}), 4.36(\mathrm{q}, J=7.2 \mathrm{~Hz}$, $2 \mathrm{H}), 4.29-4.23(\mathrm{~m}, 1 \mathrm{H}), 2.95-2.74(\mathrm{~m}, 2 \mathrm{H}), 2.12(\mathrm{~s}, 3 \mathrm{H}), 1.39(\mathrm{t}, J=$ $7.2 \mathrm{~Hz}, 3 \mathrm{H})$.

${ }^{19}$ F NMR $\left(471 \mathrm{MHz}, \mathrm{CDCl}_{3}\right) \delta-103.1(\mathrm{~d}, J=265.7 \mathrm{~Hz}),-106.0(\mathrm{~d}, J=265.7 \mathrm{~Hz})$. 
${ }^{13} \mathrm{C}$ NMR $\left(125 \mathrm{MHz}, \mathrm{CDCl}_{3}\right) \delta 170.0,163.11\left(\mathrm{t},{ }^{2} \mathrm{C}_{\mathrm{C}-\mathrm{F}}=32.2 \mathrm{~Hz}\right), 114.8\left(\mathrm{t},{ }^{1} \mathrm{JC}_{\mathrm{C}-\mathrm{F}}=252.7 \mathrm{~Hz}\right)$, 68.6, 63.3, $41.7\left(\mathrm{t},{ }^{2} J_{C-F}=23.7 \mathrm{~Hz}\right), 20.7,14.5\left(\mathrm{t},{ }^{3} \mathrm{C}_{\mathrm{C}-\mathrm{F}}=4.1 \mathrm{~Hz}\right), 13.8$.

HRMS (ESI) m/z: [M+Na] $]^{+}$calcd for $\mathrm{C}_{9} \mathrm{H}_{13} \mathrm{~F}_{2} \mathrm{IO}_{4} \mathrm{Na}$ : 372.9719; found: 372.9714 .

\section{8-Ethoxy-7,7-difluoro-5-iodo-8-oxooctyl benzoate ( $3 \mathrm{~h})$}

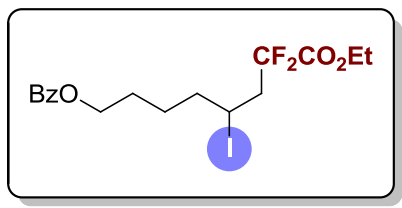

Colorless oil, $87.2 \mathrm{mg}, 96 \%$ yield.

${ }^{1} \mathbf{H}$ NMR $\left(500 \mathrm{MHz}, \mathrm{CDCl}_{3}\right) \delta 8.07(\mathrm{~d}, J=7.1 \mathrm{~Hz}, 2 \mathrm{H}), 7.57(\mathrm{t}, J$ $=7.6 \mathrm{~Hz}, 1 \mathrm{H}), 7.46(\mathrm{t}, J=7.7 \mathrm{~Hz}, 2 \mathrm{H}), 4.40-4.31(\mathrm{~m}, 2 \mathrm{H}), 4.36$ $(\mathrm{q}, J=7.2 \mathrm{~Hz}, 2 \mathrm{H}), 4.27(\mathrm{dtd}, J=10.7,6.9,4.1 \mathrm{~Hz}, 1 \mathrm{H}), 2.95(\mathrm{dtd}$,

$J=18.9,15.7,6.3 \mathrm{~Hz}, 1 \mathrm{H}), 2.77(\mathrm{dddd}, J=18.7,15.5,12.1,7.3 \mathrm{~Hz}, 1 \mathrm{H}), 1.93-1.72(\mathrm{~m}, 5 \mathrm{H})$, $1.66-1.54(\mathrm{~m}, 1 \mathrm{H}), 1.38(\mathrm{t}, J=7.2 \mathrm{~Hz}, 3 \mathrm{H})$.

${ }^{19}$ F NMR $\left(471 \mathrm{MHz}, \mathrm{CDCl}_{3}\right) \delta-102.1(\mathrm{~d}, J=263.0 \mathrm{~Hz}),-106.8(\mathrm{~d}, J=263.0 \mathrm{~Hz})$.

${ }^{13} \mathrm{C}$ NMR $\left(125 \mathrm{MHz}, \mathrm{CDCl}_{3}\right) \delta$ 166.5, $163.3\left(\mathrm{t},{ }^{2} \mathrm{JC}_{\mathrm{C}-\mathrm{F}}=32.2 \mathrm{~Hz}\right), 132.9,130.3,129.5,128.3$, $115.1\left(\mathrm{t},{ }^{1} J_{C-F}=253.2 \mathrm{~Hz}\right), 64.4,63.2,45.3\left(\mathrm{t},{ }^{2} J_{C-F}=23.1 \mathrm{~Hz}\right), 39.9,27.7,26.2,22.5\left(\mathrm{t},{ }^{3} J_{C-F}=4.0\right.$ $\mathrm{Hz}), 13.9$.

HRMS (ESI) m/z: [M+Na $]^{+}$calcd for $\mathrm{C}_{17} \mathrm{H}_{21} \mathrm{~F}_{2} \mathrm{IO}_{4} \mathrm{Na}$ : 477.0345; found: 477.0346 .

\section{Ethyl 2,2-difluoro-4-iodo-8-(tosyloxy)octanoate (3i)}

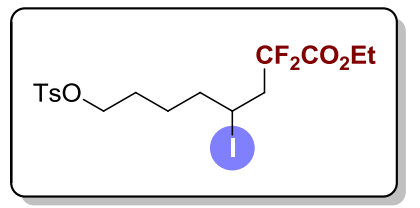

Colorless oil, $93.7 \mathrm{mg}$, $93 \%$ yield.

${ }^{1} \mathbf{H}$ NMR $\left(500 \mathrm{MHz}, \mathrm{CDCl}_{3}\right) \delta 7.80(\mathrm{~d}, J=8.1 \mathrm{~Hz}, 2 \mathrm{H}), 7.37(\mathrm{~d}, J$ $=8.1 \mathrm{~Hz}, 2 \mathrm{H}), 4.35(\mathrm{q}, J=7.2 \mathrm{~Hz}, 2 \mathrm{H}), 4.15(\mathrm{dtd}, J=11.0,6.9$, $4.1 \mathrm{~Hz}, 1 \mathrm{H}), 4.05(\mathrm{t}, J=6.3 \mathrm{~Hz}, 2 \mathrm{H}), 2.89(\mathrm{dtd}, J=19.1,15.7,6.4$ Hz, 1H), 2.69 (dddd, $J=18.8,15.6,12.0,7.3 \mathrm{~Hz}, 1 \mathrm{H}), 2.46(\mathrm{~s}, 3 \mathrm{H}), 1.79-1.63(\mathrm{~m}, 4 \mathrm{H}), 1.56$ $(\mathrm{dtt}, J=20.1,10.2,5.3 \mathrm{~Hz}, 1 \mathrm{H}), 1.47-1.36(\mathrm{~m}, 1 \mathrm{H}), 1.38(\mathrm{t}, J=7.2 \mathrm{~Hz}, 3 \mathrm{H})$.

${ }^{19}$ F NMR $\left(471 \mathrm{MHz}, \mathrm{CDCl}_{3}\right) \delta-102.2(\mathrm{~d}, J=263.2 \mathrm{~Hz}),-106.9(\mathrm{~d}, J=263.2 \mathrm{~Hz})$.

${ }^{13} \mathrm{C}$ NMR $\left(125 \mathrm{MHz}, \mathrm{CDCl}_{3}\right) \delta 163.3\left(\mathrm{t},{ }^{2} \mathrm{~J}_{\mathrm{C}-\mathrm{F}}=32.3 \mathrm{~Hz}\right), 144.8,133.0,129.8,127.8,115.0(\mathrm{t}$, $\left.{ }^{1} J_{C-F}=252.7 \mathrm{~Hz}\right), 70.0,63.2,45.1\left(\mathrm{t},{ }^{2} J_{C-F}=23.1 \mathrm{~Hz}\right), 39.5,27.8,25.6,22.0\left(\mathrm{t},{ }^{3} J_{C-F}=3.9 \mathrm{~Hz}\right)$, 21.6, 13.8 .

HRMS (ESI) m/z: [M+Na] ${ }^{+}$calcd for $\mathrm{C}_{17} \mathrm{H}_{23} \mathrm{~F}_{2} \mathrm{IO}_{5} \mathrm{SNa}$ : 527.0171; found: 527.0173.

Ethyl 2,2-difluoro-4-iodo-8-((methylsulfonyl)oxy)octanoate (3j)

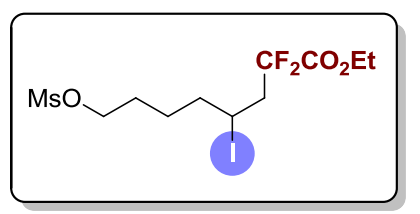

Colorless oil, $74.7 \mathrm{mg}, 92 \%$ yield.

${ }^{1} \mathrm{H}$ NMR $\left(500 \mathrm{MHz}, \mathrm{CDCl}_{3}\right) \delta 4.36(\mathrm{q}, J=7.2 \mathrm{~Hz}, 2 \mathrm{H}), 4.29-$ $4.20(\mathrm{~m}, 1 \mathrm{H}), 4.25(\mathrm{t}, J=6.3 \mathrm{~Hz}, 2 \mathrm{H}), 3.03(\mathrm{~s}, 3 \mathrm{H}), 3.00-2.86(\mathrm{~m}$, $1 \mathrm{H}), 2.83-2.69(\mathrm{~m}, 1 \mathrm{H}), 1.92-1.75(\mathrm{~m}, 4 \mathrm{H}), 1.75-1.64(\mathrm{~m}$, $1 \mathrm{H}), 1.60-1.49(\mathrm{~m}, 1 \mathrm{H}), 1.38(\mathrm{t}, J=7.2 \mathrm{~Hz}, 3 \mathrm{H})$.

${ }^{19}$ F NMR $\left(471 \mathrm{MHz}, \mathrm{CDCl}_{3}\right) \delta-102.2(\mathrm{~d}, J=263.1 \mathrm{~Hz}),-106.8(\mathrm{~d}, J=263.1 \mathrm{~Hz})$.

${ }^{13} \mathrm{C}$ NMR $\left(125 \mathrm{MHz}, \mathrm{CDCl}_{3}\right) \delta 163.2\left(\mathrm{t},{ }^{2} J_{\mathrm{C}-\mathrm{F}}=32.2 \mathrm{~Hz}\right), 115.1\left(\mathrm{t},{ }^{1} J_{\mathrm{C}-\mathrm{F}}=252.8 \mathrm{~Hz}\right), 69.4,63.3$, $45.1\left(\mathrm{t},{ }^{2} J_{C-F}=23.1 \mathrm{~Hz}\right), 39.5,37.3,28.0,25.6,22.1\left(\mathrm{t},{ }^{3} J_{C-F}=3.9 \mathrm{~Hz}\right), 13.8$.

HRMS (ESI) m/z: [M+H] $]^{+}$calcd for $\mathrm{C}_{11} \mathrm{H}_{20} \mathrm{~F}_{2} \mathrm{IO} 5 \mathrm{~S}: 429.0033$; found: 429.0033 .

Ethyl 8-(benzyloxy)-2,2-difluoro-4-iodooctanoate (3k)

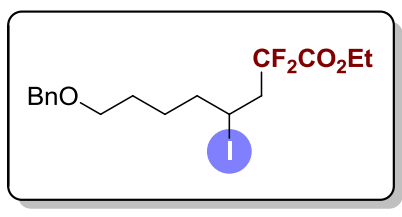

Colorless oil, $82.7 \mathrm{mg}, 94 \%$ yield.

${ }^{1} \mathbf{H}$ NMR $\left(500 \mathrm{MHz}, \mathrm{CDCl}_{3}\right) \delta 7.41-7.35(\mathrm{~m}, 4 \mathrm{H}), 7.33-7.29$ $(\mathrm{m}, 1 \mathrm{H}), 4.53(\mathrm{~s}, 2 \mathrm{H}), 4.36(\mathrm{q}, J=7.2 \mathrm{~Hz}, 2 \mathrm{H}), 4.25(\mathrm{dtd}, J=8.8$, $6.8,4.3 \mathrm{~Hz}, 1 \mathrm{H}), 3.51(\mathrm{t}, J=5.9 \mathrm{~Hz}, 2 \mathrm{H}), 2.94(\mathrm{dtd}, J=18.4,15.8$, $6.5 \mathrm{~Hz}, 1 \mathrm{H}), 2.76$ (dddd, $J=17.9,15.6,12.4,7.0 \mathrm{~Hz}, 1 \mathrm{H}), 1.92-$ $1.75(\mathrm{~m}, 2 \mathrm{H}), 1.74-1.60(\mathrm{~m}, 3 \mathrm{H}), 1.59-1.47(\mathrm{~m}, 1 \mathrm{H}), 1.39(\mathrm{t}, J=7.2 \mathrm{~Hz}, 3 \mathrm{H})$. 
${ }^{19}$ F NMR $\left(471 \mathrm{MHz}, \mathrm{CDCl}_{3}\right) \delta-102.1(\mathrm{~d}, J=263.3 \mathrm{~Hz}),-106.8(\mathrm{~d}, J=263.3 \mathrm{~Hz})$.

${ }^{13} \mathrm{C}$ NMR $\left(125 \mathrm{MHz}, \mathrm{CDCl}_{3}\right) \delta 163.4\left(\mathrm{t},{ }^{2} \mathrm{~J}_{\mathrm{C}-\mathrm{F}}=32.2 \mathrm{~Hz}\right), 138.5,128.3,127.6,127.5,115.1(\mathrm{t}$, $\left.{ }^{1} J_{C-F}=252.6 \mathrm{~Hz}\right), 72.9,69.9,63.2,45.3\left(\mathrm{t},{ }^{2} J_{C-F}=23.2 \mathrm{~Hz}\right), 40.2,28.7,26.3,22.9\left(\mathrm{t},{ }^{3} J_{C-F}=4.0\right.$ $\mathrm{Hz}), 13.9$.

HRMS (ESI) m/z: [M+Na] $]^{+}$calcd for $\mathrm{C}_{17} \mathrm{H}_{23} \mathrm{~F}_{2} \mathrm{IO}_{3} \mathrm{Na}$ : 463.0552; found: 463.0551 .

Ethyl 8-((tert-butyldimethylsilyl)oxy)-2,2-difluoro-4-iodooctanoate (31)

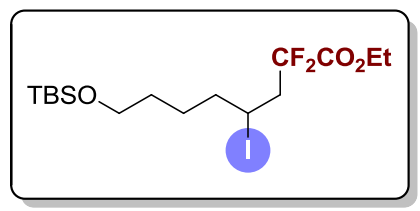

Colorless oil, $84.9 \mathrm{mg}$, $96 \%$ yield.

${ }^{1} \mathrm{H}$ NMR $\left(500 \mathrm{MHz}, \mathrm{CDCl}_{3}\right) \delta 4.36(\mathrm{q}, J=7.2 \mathrm{~Hz}, 2 \mathrm{H}), 4.24$ $(\mathrm{dtd}, J=8.7,6.8,4.2 \mathrm{~Hz}, 1 \mathrm{H}), 3.64(\mathrm{t}, J=6.0 \mathrm{~Hz}, 2 \mathrm{H}), 2.93(\mathrm{dtd}$, $J=18.4,15.9,6.5 \mathrm{~Hz}, 1 \mathrm{H}), 2.75(\mathrm{dddd}, J=18.0,15.6,12.4,7.0$

$\mathrm{Hz}, 1 \mathrm{H}), 1.92-1.72(\mathrm{~m}, 2 \mathrm{H}), 1.68-1.44(\mathrm{~m}, 4 \mathrm{H}), 1.39(\mathrm{t}, J=7.2 \mathrm{~Hz}, 3 \mathrm{H}), 0.91(\mathrm{~s}, 9 \mathrm{H}), 0.07(\mathrm{~s}$, $6 \mathrm{H})$.

${ }^{19}$ F NMR $\left(471 \mathrm{MHz}, \mathrm{CDCl}_{3}\right) \delta-102.1(\mathrm{~d}, J=263.2 \mathrm{~Hz}),-106.9(\mathrm{~d}, J=263.2 \mathrm{~Hz})$.

${ }^{13} \mathrm{C}$ NMR (125 MHz, CDCl$) \delta 163.4\left(\mathrm{t},{ }^{2} J_{C-F}=32.3 \mathrm{~Hz}\right), 115.2\left(\mathrm{t},{ }^{1} J_{C-F}=252.1 \mathrm{~Hz}\right), 63.2,62.7$, $45.4\left(\mathrm{t},{ }^{2} J_{C-F}=23.1 \mathrm{~Hz}\right), 40.2,31.7,26.0,25.9,23.1\left(\mathrm{t},{ }^{3} J_{C-F}=3.9 \mathrm{~Hz}\right), 18.3,13.9,-5.3$.

HRMS (ESI) m/z: [M+H] $]^{+}$calcd for $\mathrm{C}_{16} \mathrm{H}_{32} \mathrm{~F}_{2} \mathrm{IO}_{3} \mathrm{Si}$ : 465.1128; found: 465.1133 .

Ethyl 2,2-difluoro-4-iodo-7-phenoxyheptanoate (3m)

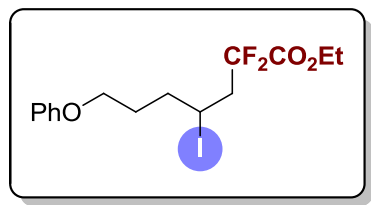

Colorless oil, $74.2 \mathrm{mg}, 90 \%$ yield.

${ }^{1} \mathbf{H}$ NMR $\left(500 \mathrm{MHz}, \mathrm{CDCl}_{3}\right) \delta 7.34-7.27(\mathrm{~m}, 2 \mathrm{H}), 7.00-6.95(\mathrm{~m}$, $1 \mathrm{H}), 6.94-6.89(\mathrm{~m}, 2 \mathrm{H}), 4.40-4.29(\mathrm{~m}, 1 \mathrm{H}), 4.36(\mathrm{q}, J=7.2 \mathrm{~Hz}$, $2 \mathrm{H}), 4.02(\mathrm{t}, J=5.8 \mathrm{~Hz}, 2 \mathrm{H}), 2.99(\mathrm{dtd}, J=18.6,15.8,6.5 \mathrm{~Hz}, 1 \mathrm{H})$, 2.81 (dddd, $J=17.9,15.6,12.4,7.2 \mathrm{~Hz}, 1 \mathrm{H}), 2.13-1.97(\mathrm{~m}, 3 \mathrm{H})$, $1.97-1.88(\mathrm{~m}, 1 \mathrm{H}), 1.39(\mathrm{t}, J=7.2 \mathrm{~Hz}, 3 \mathrm{H})$.

${ }^{19} \mathrm{~F}$ NMR $\left(471 \mathrm{MHz}, \mathrm{CDCl}_{3}\right) \delta-102.1(\mathrm{~d}, J=263.6 \mathrm{~Hz}),-106.7(\mathrm{~d}, J=263.6 \mathrm{~Hz})$.

${ }^{13} \mathrm{C}$ NMR $\left(125 \mathrm{MHz}, \mathrm{CDCl}_{3}\right) \delta 163.4\left(\mathrm{t},{ }^{2} J_{\mathrm{C}-\mathrm{F}}=32.2 \mathrm{~Hz}\right), 158.8,129.4,120.8,115.1\left(\mathrm{t},{ }^{1} J_{\mathrm{C}-F}=\right.$ $252.6 \mathrm{~Hz}), 114.5,66.5,63.3,45.3\left(\mathrm{t},{ }^{2} J_{C-F}=23.2 \mathrm{~Hz}\right), 37.2,29.5,22.4\left(\mathrm{t},{ }^{3} J_{C-F}=3.9 \mathrm{~Hz}\right), 13.9$.

HRMS (ESI) m/z: [M+Na $]^{+}$calcd for $\mathrm{C}_{15} \mathrm{H}_{19} \mathrm{~F}_{2} \mathrm{IO}_{3} \mathrm{Na}$ : 435.0239; found: 435.0235 .

Ethyl 13-azido-2,2-difluoro-4-iodotridecanoate (3n)

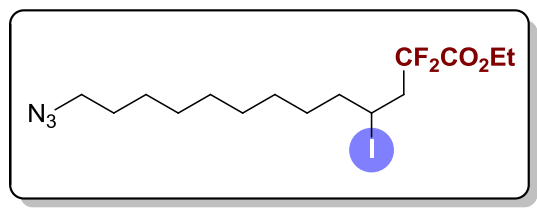

Colorless oil, $87.6 \mathrm{mg}, 95 \%$ yield.

${ }^{1} \mathrm{H}$ NMR $\left(500 \mathrm{MHz}, \mathrm{CDCl}_{3}\right) \delta 4.36(\mathrm{q}, J=7.1 \mathrm{~Hz}, 2 \mathrm{H})$, $4.23(\mathrm{dtd}, J=10.9,6.9,4.2 \mathrm{~Hz}, 1 \mathrm{H}), 3.27(\mathrm{t}, J=7.0 \mathrm{~Hz}$, $2 \mathrm{H}), 2.93(\mathrm{dtd}, J=18.5,15.9,6.5 \mathrm{~Hz}, 1 \mathrm{H}), 2.75(\mathrm{dddd}, J$ $=17.9,15.6,12.4,7.1 \mathrm{~Hz}, 1 \mathrm{H}), 1.82(\mathrm{dtd}, J=14.3,9.4$, $4.8 \mathrm{~Hz}, 1 \mathrm{H}), 1.74(\mathrm{ddt}, J=14.7,9.8,4.9 \mathrm{~Hz}, 1 \mathrm{H}), 1.61(\mathrm{p}, J=7.0 \mathrm{~Hz}, 2 \mathrm{H}), 1.53(\mathrm{td}, J=8.4,3.9$ $\mathrm{Hz}, 1 \mathrm{H}), 1.44-1.26(\mathrm{~m}, 11 \mathrm{H}), 1.39(\mathrm{t}, J=7.1 \mathrm{~Hz}, 3 \mathrm{H})$.

${ }^{19}$ F NMR $\left(471 \mathrm{MHz}, \mathrm{CDCl}_{3}\right) \delta-102.1(\mathrm{~d}, J=262.8 \mathrm{~Hz}),-106.8(\mathrm{~d}, J=262.8 \mathrm{~Hz})$.

${ }^{13} \mathrm{C}$ NMR $\left(125 \mathrm{MHz}, \mathrm{CDCl}_{3}\right) \delta 163.4\left(\mathrm{t},{ }^{2} J_{\mathrm{C}-F}=32.2 \mathrm{~Hz}\right), 115.2\left(\mathrm{t},{ }^{1} J_{\mathrm{C}-F}=252.1 \mathrm{~Hz}\right), 63.2,51.4$, $45.3\left(\mathrm{t},{ }^{2} J_{C-F}=23.2 \mathrm{~Hz}\right), 40.4,29.4,29.3,29.2,29.0,28.8,28.4,26.6,23.2\left(\mathrm{t},{ }^{3} J_{\mathrm{c}-\mathrm{F}}=3.9 \mathrm{~Hz}\right), 13.8$.

HRMS (ESI) m/z: [M+K] $]^{+}$calcd for $\mathrm{C}_{15} \mathrm{H}_{26} \mathrm{~F}_{2} \mathrm{IN}_{3} \mathrm{~K}: 484.0669$; found: 484.0663 .

Ethyl 8-(1,3-dioxoisoindolin-2-yl)-2,2-difluoro-4-iodooctanoate (3o)

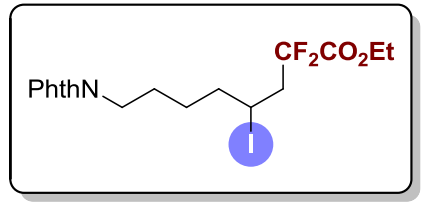

Colorless oil, $93.0 \mathrm{mg}, 97 \%$ yield.

${ }^{1} \mathbf{H}$ NMR $\left(500 \mathrm{MHz}, \mathrm{CDCl}_{3}\right) \delta 7.84(\mathrm{dd}, J=5.5,3.0 \mathrm{~Hz}, 2 \mathrm{H})$, $7.72(\mathrm{dd}, J=5.5,3.0 \mathrm{~Hz}, 2 \mathrm{H}), 4.34(\mathrm{q}, J=7.2 \mathrm{~Hz}, 2 \mathrm{H}), 4.20(\mathrm{dtd}$, $J=8.7,6.8,4.2 \mathrm{~Hz}, 1 \mathrm{H}), 3.70(\mathrm{t}, J=7.2 \mathrm{~Hz}, 2 \mathrm{H}), 2.91(\mathrm{dtd}, J=$ 
18.6, 15.9, 6.5 Hz, 1H), 2.73 (dddd, $J=18.1,15.5,12.3,7.2 \mathrm{~Hz}, 1 \mathrm{H}), 1.90-1.55(\mathrm{~m}, 5 \mathrm{H}), 1.46$ (dddd, $J=15.6,9.9,8.2,4.7 \mathrm{~Hz}, 1 \mathrm{H}), 1.37(\mathrm{t}, J=7.2 \mathrm{~Hz}, 3 \mathrm{H})$.

${ }^{19}$ F NMR $\left(471 \mathrm{MHz}, \mathrm{CDCl}_{3}\right) \delta-102.1(\mathrm{~d}, J=263.3 \mathrm{~Hz}),-106.7(\mathrm{~d}, J=263.3 \mathrm{~Hz})$.

${ }^{13} \mathrm{C}$ NMR $\left(125 \mathrm{MHz}, \mathrm{CDCl}_{3}\right) \delta 168.3,163.3\left(\mathrm{t},{ }^{2} \mathrm{JC}_{\mathrm{C}-\mathrm{F}}=32.2 \mathrm{~Hz}\right), 133.9,132.0,123.1,115.1(\mathrm{t}$, $\left.{ }^{1} J_{C-F}=252.8 \mathrm{~Hz}\right), 63.2,45.2\left(\mathrm{t},{ }^{2} \mathrm{~J}_{\mathrm{C}-\mathrm{F}}=23.2 \mathrm{~Hz}\right), 39.7,37.5,27.4,26.8,22.4\left(\mathrm{t},{ }^{1} \mathrm{JC}_{\mathrm{F}}=3.9 \mathrm{~Hz}\right)$, 13.8.

HRMS (ESI) m/z: [M+Na] ${ }^{+}$calcd for $\mathrm{C}_{18} \mathrm{H}_{20} \mathrm{~F}_{2} \mathrm{INO}_{4} \mathrm{Na}$ : 502.0297; found: 502.0297.

\section{Ethyl 5-cyano-2,2-difluoro-4-iodopentanoate (3p)}

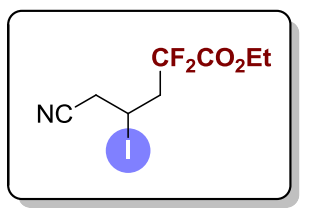

Colorless oil, $62.8 \mathrm{mg}$, $99 \%$ yield.

${ }^{1} \mathrm{H}$ NMR $\left(500 \mathrm{MHz}, \mathrm{CDCl}_{3}\right) \delta 4.44-4.35(\mathrm{~m}, 1 \mathrm{H}), 4.37(\mathrm{t}, J=7.2 \mathrm{~Hz}$, $2 \mathrm{H}), 3.27(\mathrm{dd}, J=17.6,6.3 \mathrm{~Hz}, 1 \mathrm{H}), 3.18(\mathrm{dd}, J=17.6,4.6 \mathrm{~Hz}, 1 \mathrm{H}), 3.04$ $-2.83(\mathrm{~m}, 2 \mathrm{H}), 1.39(\mathrm{t}, J=7.2 \mathrm{~Hz}, 3 \mathrm{H})$.

${ }^{19}$ F NMR $\left(471 \mathrm{MHz}, \mathrm{CDCl}_{3}\right) \delta-103.2(\mathrm{~d}, J=265.4 \mathrm{~Hz}),-106.7(\mathrm{~d}, J=265.4 \mathrm{~Hz})$.

${ }^{13} \mathrm{C}$ NMR $\left(125 \mathrm{MHz}, \mathrm{CDCl}_{3}\right) \delta 162.6\left(\mathrm{t},{ }^{2} \mathrm{~J} \mathrm{C}-\mathrm{F}=31.9 \mathrm{~Hz}\right), 116.8,114.7\left(\mathrm{t},{ }^{1} \mathrm{JC}_{\mathrm{F}}=253.6 \mathrm{~Hz}\right), 63.6$, $44.0\left(\mathrm{t},{ }^{2} \mathrm{C}_{\mathrm{C}-\mathrm{F}}=23.4 \mathrm{~Hz}\right), 30.2,13.8,7.7\left(\mathrm{t},{ }^{3} \mathrm{C}_{\mathrm{C}-\mathrm{F}}=3.6 \mathrm{~Hz}\right)$.

HRMS (ESI) m/z: [M+Na] calcd for $\mathrm{C}_{8} \mathrm{H}_{10} \mathrm{~F}_{2} \mathrm{INO}_{2} \mathrm{Na}$ : 339.9616; found: 339.9619.

7-Benzyl 1-ethyl 2,2-difluoro-4-iodoheptanedioate (3q)

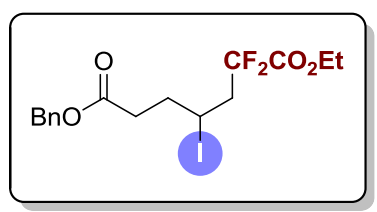

Colorless oil, $81.8 \mathrm{mg}, 93 \%$ yield.

${ }^{1} \mathbf{H}$ NMR (500 MHz, $\left.\mathrm{CDCl}_{3}\right) \delta 7.45-7.32(\mathrm{~m}, 5 \mathrm{H}), 5.16(\mathrm{~s}, 2 \mathrm{H})$, $4.36(\mathrm{q}, J=7.2 \mathrm{~Hz}, 2 \mathrm{H}), 4.30(\mathrm{dtd}, J=10.3,6.8,3.8 \mathrm{~Hz}, 1 \mathrm{H}), 2.97$ $(\mathrm{dtd}, J=18.9,15.5,6.4 \mathrm{~Hz}, 1 \mathrm{H}), 2.77$ (dddd, $J=18.1,15.6,12.1$, $7.3 \mathrm{~Hz}, 1 \mathrm{H}), 2.66(\mathrm{ddd}, J=16.8,8.8,5.4 \mathrm{~Hz}, 1 \mathrm{H}), 2.56(\mathrm{ddd}, J=$ 16.7, 8.9, $6.7 \mathrm{~Hz}, 1 \mathrm{H}), 2.19(\mathrm{dddd}, J=15.3,8.8,6.6,3.7 \mathrm{~Hz}, 1 \mathrm{H}), 2.10(\mathrm{dtd}, J=14.9,9.2,5.4$ $\mathrm{Hz}, 1 \mathrm{H}), 1.39(\mathrm{t}, J=7.2 \mathrm{~Hz}, 3 \mathrm{H})$.

${ }^{19}$ F NMR $\left(471 \mathrm{MHz}, \mathrm{CDCl}_{3}\right) \delta-102.2(\mathrm{~d}, J=264.2 \mathrm{~Hz}),-106.5(\mathrm{~d}, J=264.2 \mathrm{~Hz})$.

${ }^{13} \mathrm{C}$ NMR $\left(125 \mathrm{MHz}, \mathrm{CDCl}_{3}\right) \delta$ 171.8, $163.2\left(\mathrm{t},{ }^{2} \mathrm{~J} C-\mathrm{F}=32.2 \mathrm{~Hz}\right), 135.7,128.5,128.3,128.2$, $115.0\left(\mathrm{t},{ }^{1} \mathrm{JC}_{\mathrm{C}-\mathrm{F}}=252.7 \mathrm{~Hz}\right), 66.5,63.3,45.2\left(\mathrm{t},{ }^{2} \mathrm{~J}_{\mathrm{C}-\mathrm{F}}=23.3 \mathrm{~Hz}\right), 35.2,34.4,21.3\left(\mathrm{t},{ }^{3} \mathrm{~J}_{\mathrm{C}-\mathrm{F}}=4.0 \mathrm{~Hz}\right)$, 13.8 .

HRMS (ESI) m/z: [M+Na $]^{+}$calcd for $\mathrm{C}_{16} \mathrm{H}_{19} \mathrm{~F}_{2} \mathrm{IO}_{4} \mathrm{Na}$ : 463.0188; found: 463.0186 .

Ethyl 7-(benzylamino)-2,2-difluoro-4-iodo-7-oxoheptanoate (3r)

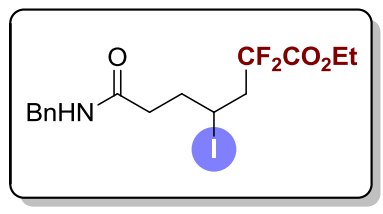

Purified by silica gel column chromatography (petroleum ether/ethyl acetate $=1 / 1$ ), pale yellow oil, $85.9 \mathrm{mg}, 90 \%$ yield.

${ }^{1} \mathbf{H}$ NMR $\left(500 \mathrm{MHz}, \mathrm{CDCl}_{3}\right) \delta 7.41-7.32(\mathrm{~m}, 2 \mathrm{H}), 7.31-7.26(\mathrm{~m}$, $3 \mathrm{H}), 6.01(\mathrm{t}, J=5.7 \mathrm{~Hz}, 1 \mathrm{H}), 4.44(\mathrm{~d}, J=5.7 \mathrm{~Hz}, 2 \mathrm{H}), 4.35(\mathrm{q}, J=$ $7.2 \mathrm{~Hz}, 2 \mathrm{H}), 4.28(\mathrm{dtd}, J=10.3,6.8,3.7 \mathrm{~Hz}, 1 \mathrm{H}), 2.95(\mathrm{dtd}, J=$ 18.2, 15.6, $6.7 \mathrm{~Hz}, 1 \mathrm{H}), 2.77$ (dddd, $J=17.8,15.6,12.5,6.8 \mathrm{~Hz}, 1 \mathrm{H}), 2.49$ (ddd, $J=14.3,8.7$, $5.2 \mathrm{~Hz}, 1 \mathrm{H}), 2.41-2.31(\mathrm{~m}, 1 \mathrm{H}), 2.20(\mathrm{dddd}, J=15.3,8.6,6.8,3.6 \mathrm{~Hz}, 1 \mathrm{H}), 2.08(\mathrm{dtd}, J=14.7$, 9.2, $5.2 \mathrm{~Hz}, 1 \mathrm{H}), 1.38(\mathrm{t}, J=7.2 \mathrm{~Hz}, 3 \mathrm{H})$.

${ }^{19}$ F NMR $\left(471 \mathrm{MHz}, \mathrm{CDCl}_{3}\right) \delta-102.1(\mathrm{~d}, J=264.3 \mathrm{~Hz}),-106.4(\mathrm{~d}, J=264.3 \mathrm{~Hz})$.

${ }^{13} \mathrm{C}$ NMR $\left(125 \mathrm{MHz}, \mathrm{CDCl}_{3}\right) \delta$ 170.8, $163.3\left(\mathrm{t},{ }^{2} \mathrm{~J} C-\mathrm{F}=32.2 \mathrm{~Hz}\right), 138.1,128.7,127.8,127.5$, $115.0\left(\mathrm{t},{ }^{1} \mathrm{JC}_{\mathrm{C}-\mathrm{F}}=252.2 \mathrm{~Hz}\right), 63.3,45.3\left(\mathrm{t},{ }^{2} \mathrm{JC}_{\mathrm{C}-\mathrm{F}}=23.3 \mathrm{~Hz}\right), 43.7,36.4,35.9,22.2\left(\mathrm{t},{ }^{3} \mathrm{JC}_{\mathrm{C}-F}=4.1 \mathrm{~Hz}\right)$, 13.8.

HRMS (ESI) m/z: [M+Na] $]^{+}$calcd for $\mathrm{C}_{16} \mathrm{H}_{20} \mathrm{~F}_{2} \mathrm{INO}_{3} \mathrm{Na}$ : 462.0348; found: 462.0345 .

Ethyl 2,2-difluoro-2-(2-iodocyclopentyl)acetate (3s) 


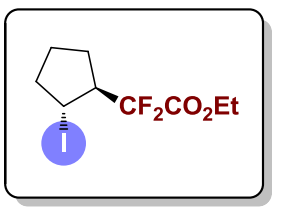

Colorless oil, $63.0 \mathrm{mg}$, $99 \%$ yield.

${ }^{1} \mathbf{H}$ NMR $\left(500 \mathrm{MHz}, \mathrm{CDCl}_{3}\right) \delta 4.37(\mathrm{q}, J=7.2 \mathrm{~Hz}, 2 \mathrm{H}), 4.32(\mathrm{q}, J=6.0$ $\mathrm{Hz}, 1 \mathrm{H}), 3.13$ (dddd, $J=23.8,11.6,9.7,5.9 \mathrm{~Hz}, 1 \mathrm{H}), 2.27-2.18(\mathrm{~m}, 1 \mathrm{H})$, $2.18-2.10(\mathrm{~m}, 1 \mathrm{H}), 1.95(\mathrm{tdd}, J=12.8,6.8,3.3 \mathrm{~Hz}, 1 \mathrm{H}), 1.86(\mathrm{dt}, J=11.9$, $6.8 \mathrm{~Hz}, 1 \mathrm{H}), 1.82-1.75(\mathrm{~m}, 1 \mathrm{H}), 1.75-1.66(\mathrm{~m}, 1 \mathrm{H}), 1.39(\mathrm{t}, J=7.2 \mathrm{~Hz}, 3 \mathrm{H})$. ${ }^{19}$ F NMR $\left(471 \mathrm{MHz}, \mathrm{CDCl}_{3}\right) \delta-111.3(\mathrm{~d}, J=256.6 \mathrm{~Hz}),-113.3(\mathrm{~d}, J=256.6 \mathrm{~Hz})$. ${ }^{13} \mathrm{C}$ NMR $\left(125 \mathrm{MHz}, \mathrm{CDCl}_{3}\right) \delta 163.5\left(\mathrm{t},{ }^{2} \mathrm{JC}_{\mathrm{C}-\mathrm{F}}=32.7 \mathrm{~Hz}\right), 116.0\left(\mathrm{t},{ }^{1} \mathrm{JC}-\mathrm{F}=253.0 \mathrm{~Hz}\right), 63.1,55.1$ $\left(\mathrm{t},{ }^{2} J_{C-F}=22.5 \mathrm{~Hz}\right), 41.2,25.4\left(\mathrm{dd},{ }^{3} J_{C-F}=4.2,2.6 \mathrm{~Hz}\right), 25.2,19.5\left(\mathrm{dd},{ }^{3} J_{C-F}=5.3,2.2 \mathrm{~Hz}\right), 13.9$.

HRMS (ESI) m/z: [M+Na] ${ }^{+}$calcd for $\mathrm{C}_{9} \mathrm{H}_{13} \mathrm{~F}_{2} \mathrm{IO}_{2} \mathrm{Na}$ : 340.9821; found: 340.9822 .

Ethyl 2,2-difluoro-2-(8-iodocyclooct-4-en-1-yl)acetate (3t)

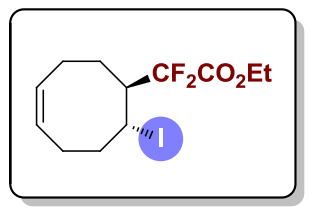

Purified by silica gel column chromatography (petroleum ether/ethyl acetate $=100 / 1)$, colorless oil, $63.7 \mathrm{mg}$, $89 \%$ yield.

${ }^{1} \mathrm{H}$ NMR $\left(500 \mathrm{MHz}, \mathrm{CDCl}_{3}\right) \delta 5.93(\mathrm{dtd}, J=10.2,7.7,1.2 \mathrm{~Hz}, 1 \mathrm{H}), 5.63$ $(\mathrm{tdd}, J=10.2,6.8,1.6 \mathrm{~Hz}, 1 \mathrm{H}), 4.88(\mathrm{t}, J=4.2 \mathrm{~Hz}, 1 \mathrm{H}), 4.36(\mathrm{qq}, J=$ 10.7, $7.1 \mathrm{~Hz}, 2 \mathrm{H}), 2.72(\mathrm{tdd}, J=13.3,10.3,6.5 \mathrm{~Hz}, 1 \mathrm{H}), 2.56-2.45(\mathrm{~m}$, $1 \mathrm{H}), 2.32(\mathrm{dddd}, J=14.9,6.6,4.7,1.7 \mathrm{~Hz}, 1 \mathrm{H}), 2.16-1.99(\mathrm{~m}, 3 \mathrm{H}), 1.93-1.85(\mathrm{~m}, 1 \mathrm{H}), 1.83$ $-1.69(\mathrm{~m}, 2 \mathrm{H}), 1.39(\mathrm{t}, J=7.1 \mathrm{~Hz}, 3 \mathrm{H})$.

${ }^{19}$ F NMR $\left(471 \mathrm{MHz}, \mathrm{CDCl}_{3}\right) \delta-107.2(\mathrm{~d}, J=253.2 \mathrm{~Hz}),-111.2(\mathrm{~d}, J=253.2 \mathrm{~Hz})$.

${ }^{13} \mathrm{C}$ NMR $\left(125 \mathrm{MHz}, \mathrm{CDCl}_{3}\right) \delta 163.7\left(\mathrm{t},{ }^{2} \mathrm{C}_{\mathrm{C}-\mathrm{F}}=32.7 \mathrm{~Hz}\right), 131.5,128.9,117.0\left(\mathrm{dd},{ }^{1} \mathrm{JC}_{\mathrm{C}-\mathrm{F}}=256.7\right.$, $253.3 \mathrm{~Hz}), 63.1,43.3\left(\mathrm{t},{ }^{2} \mathrm{~J}_{\mathrm{C}-\mathrm{F}}=20.7 \mathrm{~Hz}\right), 39.2,31.7\left(\mathrm{dd},{ }^{3} J_{\mathrm{C}-F}=3.8,2.5 \mathrm{~Hz}\right), 30.1\left(\mathrm{t},{ }^{3} J_{\mathrm{C}-F}=2.9\right.$ $\mathrm{Hz}), 25.2,23.0,13.9$.

HRMS (ESI) m/z: [M+Na] $]^{+}$calcd for $\mathrm{C}_{12} \mathrm{H}_{17} \mathrm{~F}_{2} \mathrm{IO}_{2} \mathrm{Na}$ : 381.0134; found: 381.0130 .

Ethyl 4-(cyclohex-3-en-1-yl)-2,2-difluoro-4-iodobutanoate (3u) (1:1 dr)

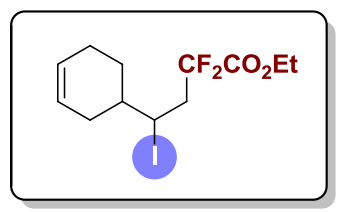

Purified by silica gel column chromatography (petroleum ether/ethyl acetate $=100 / 1)$, colorless oil, $67.2 \mathrm{mg}$, $94 \%$ yield.

${ }^{1} \mathrm{H}$ NMR $\left(500 \mathrm{MHz}, \mathrm{CDCl}_{3}\right) \delta 5.75-5.62(\mathrm{~m}, 2 \mathrm{H}), 4.37(\mathrm{q}, J=7.2$ $\mathrm{Hz}, 2 \mathrm{H}), 4.40-4.29(\mathrm{~m}, 1 \mathrm{H}), 3.04-2.72(\mathrm{~m}, 2 \mathrm{H}), 2.20-1.88(\mathrm{~m}$, $4 \mathrm{H}), 1.83-1.64(\mathrm{~m}, 1 \mathrm{H}), 1.54-1.33(\mathrm{~m}, 1 \mathrm{H}), 1.39(\mathrm{t}, J=7.2 \mathrm{~Hz}, 3 \mathrm{H}), 1.17$ (ddddd, $J=26.6$, $10.8,7.9,5.2,2.7 \mathrm{~Hz}, 1 \mathrm{H})$.

${ }^{19}$ F NMR $\left(471 \mathrm{MHz}, \mathrm{CDCl}_{3}\right) \delta-102.2(\mathrm{~d}, J=261.8 \mathrm{~Hz}),-107.3(\mathrm{~d}, J=261.8 \mathrm{~Hz})$.

${ }^{13} \mathrm{C}$ NMR $\left(125 \mathrm{MHz}, \mathrm{CDCl}_{3}\right) \delta 163.5\left(\mathrm{t},{ }^{2} \mathrm{C}_{\mathrm{C}-\mathrm{F}}=32.2 \mathrm{~Hz}\right), 127.0,125.0,115.3\left(\mathrm{dd},{ }^{1} \mathrm{JC}_{\mathrm{C}-\mathrm{F}}=251.8\right.$, $245.7 \mathrm{~Hz}), 63.2,43.0\left(\mathrm{t},{ }^{2} J_{C-F}=23.3 \mathrm{~Hz}\right), 40.2,32.7\left(\mathrm{t},{ }^{3} J_{\mathrm{C}-\mathrm{F}}=4.3 \mathrm{~Hz}\right), 30.3,28.7,25.0,13.9$.

HRMS (ESI) m/z: [M+Na $]^{+}$calcd for $\mathrm{C}_{12} \mathrm{H}_{17} \mathrm{~F}_{2} \mathrm{IO}_{2} \mathrm{Na}$ : 381.0134; found: 381.0131 .

Ethyl 2,2-difluoro-4-iodo-4-phenylbut-3-enoate (5a)

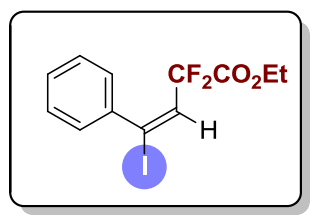

Colorless oil, $69.7 \mathrm{mg}$, 99\% yield.

${ }^{1} \mathrm{H}$ NMR (500 MHz, $\left.\mathrm{CDCl}_{3}\right) \delta 7.39-7.30(\mathrm{~m}, 5 \mathrm{H}), 6.75(\mathrm{t}, J=11.0 \mathrm{~Hz}$, $1 \mathrm{H}), 3.99(\mathrm{q}, J=7.2 \mathrm{~Hz}, 2 \mathrm{H}), 1.22(\mathrm{t}, J=7.2 \mathrm{~Hz}, 3 \mathrm{H})$.

${ }^{19} \mathrm{~F}$ NMR $\left(471 \mathrm{MHz}, \mathrm{CDCl}_{3}\right) \delta-93.8$.

${ }^{13} \mathrm{C}$ NMR $\left(125 \mathrm{MHz}, \mathrm{CDCl}_{3}\right) \delta 162.5\left(\mathrm{t},{ }^{2} \mathrm{C}_{\mathrm{C}-\mathrm{F}}=33.2 \mathrm{~Hz}\right), 140.7,133.0(\mathrm{t}$, $\left.{ }^{2} J_{C-F}=28.4 \mathrm{~Hz}\right), 129.4,128.1,127.8\left(\mathrm{t},{ }^{4} J_{C-F}=2.0 \mathrm{~Hz}\right), 110.9\left(\mathrm{t},{ }^{1} J_{\mathrm{C}-F}=250.2 \mathrm{~Hz}\right), 108.7\left(\mathrm{t},{ }^{3} J_{C-F}=\right.$ $10.1 \mathrm{~Hz}), 63.1,13.6$.

HRMS (ESI) m/z: [M+Na $]^{+}$calcd for $\mathrm{C}_{12} \mathrm{H}_{11} \mathrm{~F}_{2} \mathrm{IO}_{2} \mathrm{Na}$ : 374.9664; found: 374.9660 .

Ethyl 2,2-difluoro-4-iodo-4-(4-methoxyphenyl)but-3-enoate (5b) 


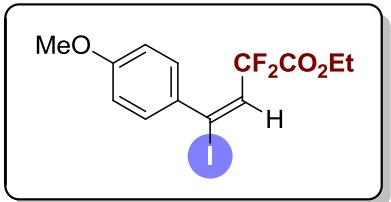

Colorless oil, $75.7 \mathrm{mg}, 99 \%$ yield.

${ }^{1} \mathrm{H}$ NMR $\left(500 \mathrm{MHz}, \mathrm{CDCl}_{3}\right) \delta 7.28(\mathrm{~d}, J=8.8 \mathrm{~Hz}, 2 \mathrm{H}), 6.84(\mathrm{~d}, J$ $=8.8 \mathrm{~Hz}, 2 \mathrm{H}), 6.69(\mathrm{t}, J=10.8 \mathrm{~Hz}, 1 \mathrm{H}), 4.01(\mathrm{q}, J=7.2 \mathrm{~Hz}, 2 \mathrm{H})$, $3.82(\mathrm{~s}, 3 \mathrm{H}), 1.21(\mathrm{t}, J=7.2 \mathrm{~Hz}, 3 \mathrm{H})$.

${ }^{19} \mathrm{~F}$ NMR $\left(471 \mathrm{MHz}, \mathrm{CDCl}_{3}\right) \delta$-93.3.

${ }^{13} \mathrm{C}$ NMR $\left(125 \mathrm{MHz}, \mathrm{CDCl}_{3}\right) \delta 162.6\left(\mathrm{t},{ }^{2} \mathrm{~J}_{\mathrm{C}-\mathrm{F}}=33.3 \mathrm{~Hz}\right), 160.3,133.0,132.5\left(\mathrm{t},{ }^{2} \mathrm{C}_{\mathrm{C}-\mathrm{F}}=28.4 \mathrm{~Hz}\right)$, $129.7\left(\mathrm{t},{ }^{4} \mathrm{~J}_{\mathrm{C}-\mathrm{F}}=2.1 \mathrm{~Hz}\right), 113.4,110.9\left(\mathrm{t},{ }^{1} \mathrm{~J}_{\mathrm{C}-\mathrm{F}}=249.6 \mathrm{~Hz}\right), 109.4\left(\mathrm{t},{ }^{3} J_{C-F}=10.2 \mathrm{~Hz}\right), 63.1,55.3$, 13.7.

HRMS (ESI) m/z: [M+Na $]^{+}$calcd for $\mathrm{C}_{13} \mathrm{H}_{13} \mathrm{~F}_{2} \mathrm{IO}_{3} \mathrm{Na}$ : 404.9763; found: 404.9763 .

Eethyl 2,2-difluoro-4-iodo-4-(4-(pentyloxy)phenyl)but-3-enoate (5c)

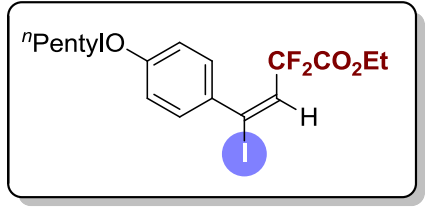

Colorless oil, $86.8 \mathrm{mg}$, $99 \%$ yield.

${ }^{1} \mathrm{H}$ NMR $\left(500 \mathrm{MHz}, \mathrm{CDCl}_{3}\right) \delta 7.27(\mathrm{~d}, J=8.7 \mathrm{~Hz}, 2 \mathrm{H}), 6.82(\mathrm{~d}$, $J=8.7 \mathrm{~Hz}, 2 \mathrm{H}), 6.68(\mathrm{t}, J=10.8 \mathrm{~Hz}, 1 \mathrm{H}), 4.00(\mathrm{q}, J=7.1 \mathrm{~Hz}$, $2 \mathrm{H}), 3.97(\mathrm{q}, J=7.0 \mathrm{~Hz}, 2 \mathrm{H}), 1.80(\mathrm{p}, J=6.7 \mathrm{~Hz}, 2 \mathrm{H}), 1.52-$ $1.35(\mathrm{~m}, 4 \mathrm{H}), 1.21(\mathrm{t}, J=7.1 \mathrm{~Hz}, 3 \mathrm{H}), 0.95(\mathrm{t}, J=7.0 \mathrm{~Hz}, 3 \mathrm{H})$.

${ }^{19}$ F NMR $\left(471 \mathrm{MHz}, \mathrm{CDCl}_{3}\right) \delta$-93.1.

${ }^{13} \mathrm{C}$ NMR $\left(125 \mathrm{MHz}, \mathrm{CDCl}_{3}\right) \delta 162.6\left(\mathrm{t},{ }^{2} J_{\mathrm{C}-\mathrm{F}}=33.3 \mathrm{~Hz}\right), 159.9,132.7,132.4\left(\mathrm{t},{ }^{2} \mathrm{~J}-\mathrm{F}=28.5 \mathrm{~Hz}\right)$, $129.6\left(\mathrm{t},{ }^{4} J_{C-F}=2.0 \mathrm{~Hz}\right), 113.8,110.9\left(\mathrm{t},{ }^{1} J_{C-F}=249.5 \mathrm{~Hz}\right), 109.5\left(\mathrm{t},{ }^{3} J_{C-F}=10.4 \mathrm{~Hz}\right), 68.1,63.0$, 28.8, 28.10, 22.4, 14.0, 13.7 .

HRMS (ESI) m/z: [M+Na] $]^{+}$calcd for $\mathrm{C}_{17} \mathrm{H}_{21} \mathrm{~F}_{2} \mathrm{IO}_{3} \mathrm{Na}$ : 461.0396; found: 461.0399 .

Ethyl 2,2-difluoro-4-iodo-4-(p-tolyl)but-3-enoate (5d)

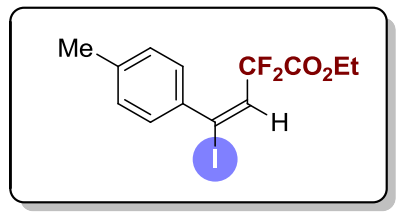

Colorless oil, $72.5 \mathrm{mg}, 99 \%$ yield.

${ }^{1} \mathrm{H}$ NMR $\left(500 \mathrm{MHz}, \mathrm{CDCl}_{3}\right) \delta 7.22(\mathrm{~d}, J=7.9 \mathrm{~Hz}, 2 \mathrm{H}), 7.14(\mathrm{~d}, J$ $=7.9 \mathrm{~Hz}, 2 \mathrm{H}), 6.72(\mathrm{t}, J=10.9 \mathrm{~Hz}, 1 \mathrm{H}), 4.00(\mathrm{q}, J=7.2 \mathrm{~Hz}, 2 \mathrm{H})$, $2.36(\mathrm{~s}, 3 \mathrm{H}), 1.22(\mathrm{t}, J=7.2 \mathrm{~Hz}, 3 \mathrm{H})$.

${ }^{19} \mathrm{~F}$ NMR $\left(471 \mathrm{MHz}, \mathrm{CDCl}_{3}\right) \delta$ - 93.7.

${ }^{13} \mathrm{C}$ NMR $\left(125 \mathrm{MHz}, \mathrm{CDCl}_{3}\right) \delta 162.5\left(\mathrm{t},{ }^{2} \mathrm{~J}-\mathrm{F}=33.3 \mathrm{~Hz}\right), 139.6,137.8,132.7\left(\mathrm{t},{ }^{2} \mathrm{C}-\mathrm{F}=28.4 \mathrm{~Hz}\right)$, 128.6, $127.8\left(\mathrm{t},{ }^{4} J_{C-F}=2.0 \mathrm{~Hz}\right), 110.8\left(\mathrm{t},{ }^{2} \mathrm{~J}_{\mathrm{C}-\mathrm{F}}=250.0 \mathrm{~Hz}\right), 109.2\left(\mathrm{t},{ }^{3} J_{\mathrm{C}-\mathrm{F}}=10.1 \mathrm{~Hz}\right), 63.0,21.3$, 13.6.

HRMS (ESI) m/z: [M+Na] $]^{+}$calcd for $\mathrm{C}_{13} \mathrm{H}_{13} \mathrm{~F}_{2} \mathrm{IO}_{2} \mathrm{Na}$ : 388.9821; found: 388.9819 .

Ethyl 4-(4-(tert-butyl)phenyl)-2,2-difluoro-4-iodobut-3-enoate (5e)

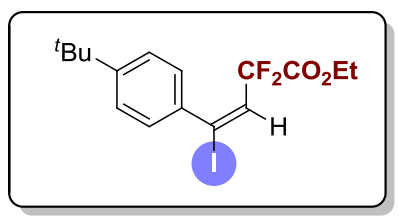

Colorless oil, $80.9 \mathrm{mg}, 99 \%$ yield.

${ }^{1} \mathbf{H}$ NMR $\left(500 \mathrm{MHz}, \mathrm{CDCl}_{3}\right) \delta 7.34(\mathrm{~d}, J=8.2 \mathrm{~Hz}, 2 \mathrm{H}), 7.27(\mathrm{~d}, J$ $=8.2 \mathrm{~Hz}, 2 \mathrm{H}), 6.72(\mathrm{t}, J=10.7 \mathrm{~Hz}, 1 \mathrm{H}), 3.92(\mathrm{q}, J=7.2 \mathrm{~Hz}, 2 \mathrm{H})$, $1.33(\mathrm{~s}, 9 \mathrm{H}), 1.17(\mathrm{t}, J=7.2 \mathrm{~Hz}, 3 \mathrm{H})$.

${ }^{19} \mathrm{~F}$ NMR $\left(471 \mathrm{MHz}, \mathrm{CDCl}_{3}\right) \delta$-93.0.

${ }^{13} \mathrm{C}$ NMR $\left(125 \mathrm{MHz}, \mathrm{CDCl}_{3}\right) \delta 162.5\left(\mathrm{t},{ }^{2} \mathrm{~J} \mathrm{C}-\mathrm{F}=33.2 \mathrm{~Hz}\right), 152.7,137.6,132.7\left(\mathrm{t},{ }^{2} \mathrm{C}-\mathrm{F}=28.8 \mathrm{~Hz}\right)$, $127.8\left(\mathrm{t},{ }^{4} J_{C-F}=1.9 \mathrm{~Hz}\right), 124.9,110.8\left(\mathrm{t},{ }^{1} \mathrm{~J}_{C-F}=249.4 \mathrm{~Hz}\right), 109.3\left(\mathrm{t},{ }^{3} J_{C-F}=10.5 \mathrm{~Hz}\right), 62.9,34.7$, 31.1, 13.6.

HRMS (ESI) m/z: [M+Na] $]^{+}$calcd for $\mathrm{C}_{16} \mathrm{H}_{19} \mathrm{~F}_{2} \mathrm{IO}_{2} \mathrm{Na}$ : 431.0290; found: 431.0287 .

Ethyl 4-([1,1'-biphenyl]-4-yl)-2,2-difluoro-4-iodobut-3-enoate (5f)

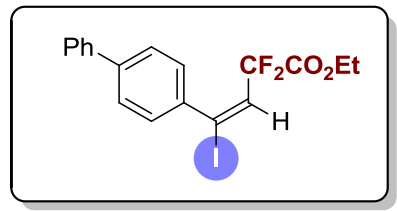

Colorless oil, $83.7 \mathrm{mg}, 98 \%$ yield.

${ }^{1} \mathbf{H}$ NMR $\left(500 \mathrm{MHz}, \mathrm{CDCl}_{3}\right) \delta 7.64-7.56(\mathrm{~m}, 4 \mathrm{H}), 7.48(\mathrm{t}, J=7.5$ $\mathrm{Hz}, 2 \mathrm{H}), 7.44-7.38(\mathrm{~m}, 3 \mathrm{H}), 6.79(\mathrm{t}, J=11.0 \mathrm{~Hz}, 1 \mathrm{H}), 4.04(\mathrm{q}, J=$ 
$7.1 \mathrm{~Hz}, 2 \mathrm{H}), 1.22(\mathrm{t}, J=7.1 \mathrm{~Hz}, 3 \mathrm{H})$.

${ }^{19} \mathrm{~F}$ NMR $\left(471 \mathrm{MHz}, \mathrm{CDCl}_{3}\right) \delta-93.7$.

${ }^{13} \mathrm{C}$ NMR $\left(125 \mathrm{MHz}, \mathrm{CDCl}_{3}\right) \delta 162.6\left(\mathrm{t},{ }^{2} J_{C-F}=33.2 \mathrm{~Hz}\right), 142.3,140.0,139.6,133.0\left(\mathrm{t},{ }^{2} \mathrm{~J}_{\mathrm{C}-F}=\right.$ $28.3 \mathrm{~Hz}), 128.9,128.4\left(\mathrm{t},{ }^{4} \mathrm{~J}_{\mathrm{C}-\mathrm{F}}=1.9 \mathrm{~Hz}\right), 127.9,127.1,126.7,111.0\left(\mathrm{t},{ }^{1} \mathrm{~J}_{\mathrm{C}-\mathrm{F}}=250.4 \mathrm{~Hz}\right), 108.6(\mathrm{t}$, $\left.{ }^{3} J_{C-F}=10.0 \mathrm{~Hz}\right), 63.2,13.7$.

HRMS (ESI) m/z: [M+Na] $]^{+}$calcd for $\mathrm{C}_{18} \mathrm{H}_{15} \mathrm{~F}_{2} \mathrm{IO}_{2} \mathrm{Na}$ : 450.9977; found: 450.9970 .

Ethyl 4-(4-bromophenyl)-2,2-difluoro-4-iodobut-3-enoate (5g)

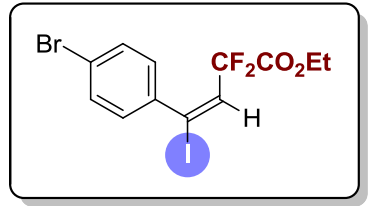

Colorless oil, $84.4 \mathrm{mg}$, $98 \%$ yield.

${ }^{1} \mathrm{H}$ NMR $\left(500 \mathrm{MHz}, \mathrm{CDCl}_{3}\right) \delta 7.48(\mathrm{~d}, J=8.4 \mathrm{~Hz}, 2 \mathrm{H}), 7.20(\mathrm{~d}, J=$ $8.4 \mathrm{~Hz}, 2 \mathrm{H}), 6.73(\mathrm{t}, J=11.3 \mathrm{~Hz}, 1 \mathrm{H}), 4.10(\mathrm{q}, J=7.2 \mathrm{~Hz}, 2 \mathrm{H}), 1.26$ $(\mathrm{t}, J=7.2 \mathrm{~Hz}, 3 \mathrm{H})$.

${ }^{19} \mathrm{~F}$ NMR $\left(471 \mathrm{MHz}, \mathrm{CDCl}_{3}\right) \delta-94.7$.

${ }^{13} \mathrm{C}$ NMR $\left(125 \mathrm{MHz}, \mathrm{CDCl}_{3}\right) \delta 162.5\left(\mathrm{t},{ }^{2} \mathrm{~J}-\mathrm{F}=33.2 \mathrm{~Hz}\right), 139.7,133.4\left(\mathrm{t},{ }^{2} \mathrm{C}_{\mathrm{C}-\mathrm{F}}=27.7 \mathrm{~Hz}\right), 131.2$, $129.3\left(\mathrm{t},{ }^{4} J_{C-F}=2.2 \mathrm{~Hz}\right), 123.6,110.8\left(\mathrm{t},{ }^{1} J_{C-F}=251.2 \mathrm{~Hz}\right), 106.9\left(\mathrm{t},{ }^{3} J_{C-F}=9.4 \mathrm{~Hz}\right), 63.3,13.7$.

HRMS (ESI) m/z: [M+Na] $]^{+}$calcd for $\mathrm{C}_{12} \mathrm{H}_{10} \mathrm{BrF}_{2} \mathrm{IO}_{2} \mathrm{Na}$ : 452.8769; found: 452.8773 .

Ethyl 4-(4-chlorophenyl)-2,2-difluoro-4-iodobut-3-enoate (5h)

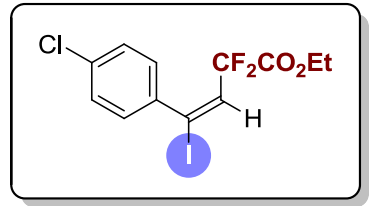

Colorless oil, $76.4 \mathrm{mg}$, 99\% yield.

${ }^{1} \mathrm{H}$ NMR $\left(500 \mathrm{MHz}, \mathrm{CDCl}_{3}\right) \delta 7.32(\mathrm{~d}, J=8.6 \mathrm{~Hz}, 2 \mathrm{H}), 7.26(\mathrm{~d}, J=$ $8.6 \mathrm{~Hz}, 2 \mathrm{H}), 6.73(\mathrm{t}, J=11.3 \mathrm{~Hz}, 1 \mathrm{H}), 4.09(\mathrm{q}, J=7.2 \mathrm{~Hz}, 2 \mathrm{H}), 1.25$ $(\mathrm{t}, J=7.2 \mathrm{~Hz}, 3 \mathrm{H})$.

${ }^{19} \mathrm{~F}$ NMR $\left(471 \mathrm{MHz}, \mathrm{CDCl}_{3}\right) \delta-94.6$

${ }^{13} \mathrm{C}$ NMR $\left(125 \mathrm{MHz}, \mathrm{CDCl}_{3}\right) \delta 162.5\left(\mathrm{t},{ }^{2} \mathrm{~J}_{\mathrm{C}-\mathrm{F}}=33.3 \mathrm{~Hz}\right), 139.2,135.4,133.4\left(\mathrm{t},{ }^{2} \mathrm{C}_{\mathrm{C}-\mathrm{F}}=27.7 \mathrm{~Hz}\right)$, $129.1\left(\mathrm{t},{ }^{4} \mathrm{~J}_{\mathrm{C}-\mathrm{F}}=2.2 \mathrm{~Hz}\right), 128.3,110.8\left(\mathrm{t},{ }^{1} \mathrm{~J}_{\mathrm{C}-\mathrm{F}}=250.0 \mathrm{~Hz}\right), 106.9\left(\mathrm{t},{ }^{3} \mathrm{~J}_{\mathrm{C}-\mathrm{F}}=9.4 \mathrm{~Hz}\right), 63.3,13.7$.

HRMS (ESI) m/z: [M+Na] $]^{+}$calcd for $\mathrm{C}_{12} \mathrm{H}_{10} \mathrm{ClF}_{2} \mathrm{IO}_{2} \mathrm{Na}$ : 408.9274; found: 408.9274 .

Ethyl 2,2-difluoro-4-(4-fluorophenyl)-4-iodobut-3-enoate (5i)

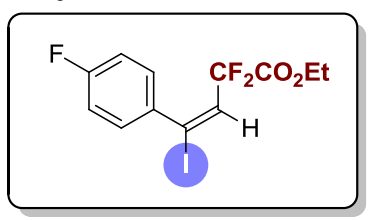

Colorless oil, $73.2 \mathrm{mg}$, $99 \%$ yield.

${ }^{1} \mathbf{H}$ NMR $\left(500 \mathrm{MHz}, \mathrm{CDCl}_{3}\right) \delta 7.32(\mathrm{dd}, J=8.5,5.2 \mathrm{~Hz}, 2 \mathrm{H}), 7.03(\mathrm{t}$, $J=8.5 \mathrm{~Hz}, 2 \mathrm{H}), 6.74(\mathrm{t}, J=11.2 \mathrm{~Hz}, 1 \mathrm{H}), 4.08(\mathrm{q}, J=7.2 \mathrm{~Hz}, 2 \mathrm{H})$, $1.25(\mathrm{t}, J=7.2 \mathrm{~Hz}, 3 \mathrm{H})$.

${ }^{19} \mathrm{~F}$ NMR $\left(471 \mathrm{MHz}, \mathrm{CDCl}_{3}\right) \delta-94.4,-110.6$.

${ }^{13} \mathrm{C}$ NMR (125 MHz, CDCl $) \delta 162.8\left(\mathrm{~d},{ }^{1} J_{\mathrm{C}-\mathrm{F}}=250.0 \mathrm{~Hz}\right), 162.5\left(\mathrm{t},{ }^{2} J_{\mathrm{C}-F}=33.3 \mathrm{~Hz}\right), 136.8(\mathrm{~d}$, $\left.{ }^{4} J_{C-F}=3.5 \mathrm{~Hz}\right), 133.4\left(\mathrm{t},{ }^{2} J_{C-F}=27.8 \mathrm{~Hz}\right), 129.9\left(\mathrm{~d},{ }^{3} J_{C-F}=8.6 \mathrm{~Hz}\right), 115.1\left(\mathrm{~d},{ }^{2} J_{C-F}=22.2 \mathrm{~Hz}\right)$, $110.8\left(\mathrm{t},{ }^{1} J_{C-F}=250.8 \mathrm{~Hz}\right), 107.3\left(\mathrm{t},{ }^{3} J_{C-F}=9.6 \mathrm{~Hz}\right), 63.2,13.7$.

HRMS (ESI) m/z: [M+Na $]^{+}$calcd for $\mathrm{C}_{12} \mathrm{H}_{10} \mathrm{~F}_{3} \mathrm{IO}_{2} \mathrm{Na}$ : 392.9570; found: 392.9570 .

Ethyl 2,2-difluoro-4-(4-formylphenyl)-4-iodobut-3-enoate (5j)

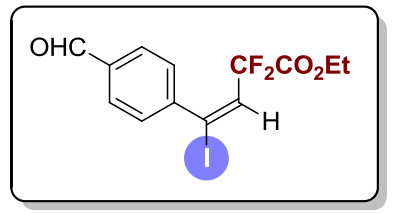

yellow amorphous solid, $70.5 \mathrm{mg}$, 93\% yield.

${ }^{1} \mathrm{H}$ NMR $\left(500 \mathrm{MHz}, \mathrm{CDCl}_{3}\right) \delta 10.04(\mathrm{~s}, 1 \mathrm{H}), 7.61(\mathrm{~d}, J=8.2 \mathrm{~Hz}$, $2 \mathrm{H}), 7.44(\mathrm{~d}, J=8.2 \mathrm{~Hz}, 2 \mathrm{H}), 6.79(\mathrm{t}, J=11.5 \mathrm{~Hz}, 1 \mathrm{H}), 4.11(\mathrm{q}, J=$ $7.1 \mathrm{~Hz}, 2 \mathrm{H}), 1.26(\mathrm{t}, J=7.1 \mathrm{~Hz}, 3 \mathrm{H})$.

${ }^{19} \mathrm{~F}$ NMR $\left(471 \mathrm{MHz}, \mathrm{CDCl}_{3}\right) \delta$-95.6.

${ }^{13} \mathrm{C}$ NMR $\left(125 \mathrm{MHz}, \mathrm{CDCl}_{3}\right) \delta 191.2,162.4\left(\mathrm{t},{ }^{2} \mathrm{~J} C-\mathrm{F}=33.3 \mathrm{~Hz}\right), 146.5,136.4,133.6\left(\mathrm{t},{ }^{2} \mathrm{C}-\mathrm{F}=\right.$ $27.2 \mathrm{~Hz}), 129.3,128.3\left(\mathrm{t},{ }^{4} \mathrm{~J}_{\mathrm{C}-\mathrm{F}}=2.1 \mathrm{~Hz}\right), 110.9\left(\mathrm{t},{ }^{1} \mathrm{~J}_{\mathrm{C}-\mathrm{F}}=252.0 \mathrm{~Hz}\right), 106.1\left(\mathrm{t},{ }^{3} J_{\mathrm{C}-F}=8.8 \mathrm{~Hz}\right), 63.4$, 13.8.

HRMS (ESI) m/z: [M+Na] $]^{+}$calcd for $\mathrm{C}_{13} \mathrm{H}_{11} \mathrm{~F}_{2} \mathrm{IO}_{3} \mathrm{Na}$ : 402.9613; found: 402.9615 . 
Ethyl 2,2-difluoro-4-iodo-4-(4-(trifluoromethyl)phenyl)but-3-enoate (5k)

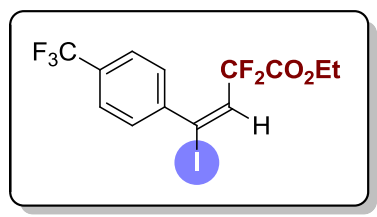

Colorless oil, $80.6 \mathrm{mg}, 96 \%$ yield.

${ }^{1} \mathrm{H}$ NMR $\left(500 \mathrm{MHz}, \mathrm{CDCl}_{3}\right) \delta 7.61(\mathrm{~d}, J=8.2 \mathrm{~Hz}, 2 \mathrm{H}), 7.44(\mathrm{~d}, J=$ $8.2 \mathrm{~Hz}, 2 \mathrm{H}), 6.79(\mathrm{t}, J=11.5 \mathrm{~Hz}, 1 \mathrm{H}), 4.11(\mathrm{q}, J=7.1 \mathrm{~Hz}, 2 \mathrm{H}), 1.26$ $(\mathrm{t}, J=7.1 \mathrm{~Hz}, 3 \mathrm{H})$.

${ }^{19} \mathrm{~F}$ NMR $\left(471 \mathrm{MHz}, \mathrm{CDCl}_{3}\right) \delta-63.0,-95.3$.

${ }^{13} \mathrm{C}$ NMR (125 MHz, CDCl $) \delta 162.4\left(\mathrm{t},{ }^{2} J \mathrm{C}-\mathrm{F}=33.2 \mathrm{~Hz}\right), 144.3,133.8\left(\mathrm{t},{ }^{2} J_{\mathrm{C}-F}=27.3 \mathrm{~Hz}\right), 131.2$ $\left(\mathrm{q},{ }^{2} J_{C-F}=32.8 \mathrm{~Hz}\right), 128.0\left(\mathrm{t},{ }^{4} J_{C-F}=2.2 \mathrm{~Hz}\right), 125.0\left(\mathrm{q},{ }^{3} J_{C-F}=3.8 \mathrm{~Hz}\right), 123.6\left(\mathrm{q},{ }^{1} J_{C-F}=272.4 \mathrm{~Hz}\right)$, $110.9\left(\mathrm{t},{ }^{1} J_{C-F}=251.9 \mathrm{~Hz}\right), 105.9\left(\mathrm{t},{ }^{3} J_{C-F}=9.0 \mathrm{~Hz}\right), 63.4,13.7$.

HRMS (ESI) m/z: [M+Na] $]^{+}$calcd for $\mathrm{C}_{13} \mathrm{H}_{10} \mathrm{~F}_{5} \mathrm{IO}_{2} \mathrm{Na}$ : 442.9538; found: 442.9534 .

Ethyl 4-(3-bromophenyl)-2,2-difluoro-4-iodobut-3-enoate (51)

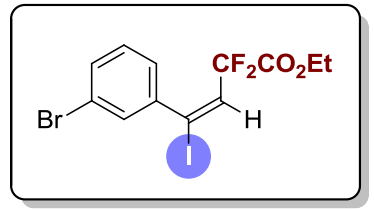

Colorless oil, $80.7 \mathrm{mg}$, 99\% yield.

${ }^{1} \mathbf{H}$ NMR $\left(500 \mathrm{MHz}, \mathrm{CDCl}_{3}\right) \delta 7.55-7.40(\mathrm{~m}, 2 \mathrm{H}), 7.26-7.18(\mathrm{~m}$, $2 \mathrm{H}), 6.74(\mathrm{t}, J=11.2 \mathrm{~Hz}, 1 \mathrm{H}), 4.10(\mathrm{q}, J=7.2 \mathrm{~Hz}, 2 \mathrm{H}), 1.28(\mathrm{t}, J=$ $7.2 \mathrm{~Hz}, 3 \mathrm{H})$.

${ }^{19}$ F NMR $\left(471 \mathrm{MHz}, \mathrm{CDCl}_{3}\right) \delta-94.8$.

${ }^{13} \mathrm{C}$ NMR $\left(125 \mathrm{MHz}, \mathrm{CDCl}_{3}\right) \delta 162.4\left(\mathrm{t},{ }^{2} J_{C-F}=33.2 \mathrm{~Hz}\right), 142.5,133.7\left(\mathrm{t},{ }^{2} \mathrm{~J}_{\mathrm{C}-\mathrm{F}}=27.9 \mathrm{~Hz}\right), 132.3$, $130.4\left(\mathrm{t},{ }^{4} \mathrm{C}_{\mathrm{C}-\mathrm{F}}=2.0 \mathrm{~Hz}\right), 129.5,126.3\left(\mathrm{t},{ }^{4} J_{C-F}=2.1 \mathrm{~Hz}\right), 121.7,110.8\left(\mathrm{t},{ }^{1} J_{C-F}=251.2 \mathrm{~Hz}\right), 105.8(\mathrm{t}$, $\left.{ }^{3} J_{C-F}=9.5 \mathrm{~Hz}\right), 63.3,13.7$.

HRMS (ESI) m/z: [M+H] $]^{+}$calcd for $\mathrm{C}_{12} \mathrm{H}_{11} \mathrm{BrF}_{2} \mathrm{IO}_{2}: 430.8950$; found: 430.8970.

Ethyl 2,2-difluoro-4-iodo-4-(m-tolyl)but-3-enoate (5m)

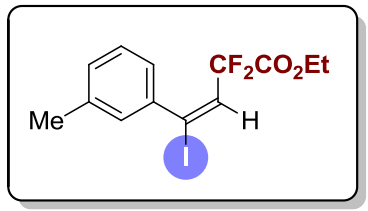

Colorless oil, $72.4 \mathrm{mg}$, 99\% yield.

${ }^{1} \mathbf{H}$ NMR $\left(500 \mathrm{MHz}, \mathrm{CDCl}_{3}\right) \delta 7.22(\mathrm{t}, J=7.8 \mathrm{~Hz}, 1 \mathrm{H}), 7.17-7.10$ $(\mathrm{m}, 3 \mathrm{H}), 6.72(\mathrm{t}, J=10.8 \mathrm{~Hz}, 1 \mathrm{H}), 3.98(\mathrm{q}, J=7.2 \mathrm{~Hz}, 2 \mathrm{H}), 2.36(\mathrm{~s}$, $3 \mathrm{H}), 1.22(\mathrm{t}, J=7.2 \mathrm{~Hz}, 3 \mathrm{H})$.

${ }^{19} \mathrm{~F}$ NMR $\left(471 \mathrm{MHz}, \mathrm{CDCl}_{3}\right) \delta$-93.6.

${ }^{13} \mathrm{C}$ NMR $\left(125 \mathrm{MHz}, \mathrm{CDCl}_{3}\right) \delta 162.5\left(\mathrm{t},{ }^{2} \mathrm{~J}_{\mathrm{C}-\mathrm{F}}=33.3 \mathrm{~Hz}\right), 140.5,137.7,132.8\left(\mathrm{t},{ }^{2} \mathrm{C}_{\mathrm{C}-\mathrm{F}}=28.6 \mathrm{~Hz}\right)$, $130.2,128.3,127.9,124.9\left(\mathrm{t},{ }^{4} \mathrm{~J}_{\mathrm{C}-\mathrm{F}}=2.1 \mathrm{~Hz}\right), 110.8\left(\mathrm{t},{ }^{1} \mathrm{~J}_{\mathrm{C}-\mathrm{F}}=249.8 \mathrm{~Hz}\right), 109.0\left(\mathrm{t},{ }^{3} \mathrm{~J}_{\mathrm{C}-\mathrm{F}}=10.3 \mathrm{~Hz}\right)$, 63.0, 21.2, 13.6.

HRMS (ESI) m/z: [M+Na] $]^{+}$calcd for $\mathrm{C}_{13} \mathrm{H}_{13} \mathrm{~F}_{2} \mathrm{IO}_{2} \mathrm{Na}$ : 388.9821; found: 388.9815 .

Ethyl 2,2-difluoro-4-iodo-4-(3-methoxyphenyl)but-3-enoate (5n)

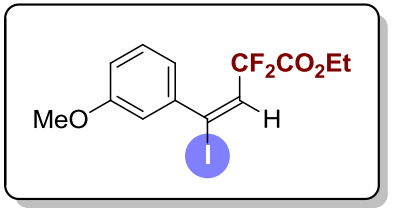

Colorless oil, $75.6 \mathrm{mg}$, 99\% yield.

${ }^{1} \mathrm{H}$ NMR $\left(500 \mathrm{MHz}, \mathrm{CDCl}_{3}\right) \delta 7.25(\mathrm{t}, J=7.9 \mathrm{~Hz}, 1 \mathrm{H}), 6.91(\mathrm{dt}, J$ $=7.6,1.3 \mathrm{~Hz}, 1 \mathrm{H}), 6.89-6.83(\mathrm{~m}, 2 \mathrm{H}), 6.72(\mathrm{t}, J=10.9 \mathrm{~Hz}, 1 \mathrm{H})$, $4.01(\mathrm{q}, J=7.2 \mathrm{~Hz}, 2 \mathrm{H}), 3.83(\mathrm{~s}, 3 \mathrm{H}), 1.22(\mathrm{t}, J=7.2 \mathrm{~Hz}, 3 \mathrm{H})$.

${ }^{19} \mathrm{~F}$ NMR $\left(471 \mathrm{MHz}, \mathrm{CDCl}_{3}\right) \delta$-93.8.

${ }^{13} \mathrm{C}$ NMR $\left(125 \mathrm{MHz}, \mathrm{CDCl}_{3}\right) \delta 162.5\left(\mathrm{t},{ }^{2} \mathrm{~J} \mathrm{C}-\mathrm{F}=33.3 \mathrm{~Hz}\right), 158.8,141.7,133.0\left(\mathrm{t},{ }^{2} \mathrm{C}-\mathrm{F}=28.6 \mathrm{~Hz}\right)$, $129.1,120.3\left(\mathrm{t},{ }^{4} \mathrm{~J}_{C-F}=2.0 \mathrm{~Hz}\right), 115.4,113.1,110.8\left(\mathrm{t},{ }^{1} \mathrm{~J}_{\mathrm{C}-\mathrm{F}}=250.0 \mathrm{~Hz}\right), 108.4\left(\mathrm{t},{ }^{3} J_{C-F}=10.2 \mathrm{~Hz}\right)$, 63.1, 55.3, 13.6 .

HRMS (ESI) m/z: [M+Na] $]^{+}$calcd for $\mathrm{C}_{13} \mathrm{H}_{13} \mathrm{~F}_{2} \mathrm{IO}_{3} \mathrm{Na}$ : 404.9763 ; found: 404.9762 .

Ethyl 4-(2-bromophenyl)-2,2-difluoro-4-iodobut-3-enoate (5o)

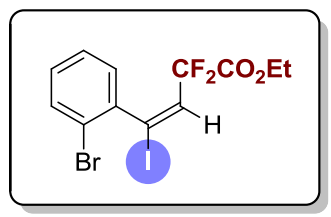

Colorless oil, $85.0 \mathrm{mg}$, 99\% yield.

${ }^{1} \mathrm{H}$ NMR $\left(500 \mathrm{MHz}, \mathrm{CDCl}_{3}\right) \delta 7.57(\mathrm{dd}, J=8.1,1.2 \mathrm{~Hz}, 1 \mathrm{H}), 7.32$ (td, $J=7.5,1.2 \mathrm{~Hz}, 1 \mathrm{H}), 7.25(\mathrm{dd}, J=7.8,1.8 \mathrm{~Hz}, 1 \mathrm{H}), 7.20(\mathrm{td}, J=7.8,1.8$ 
$\mathrm{Hz}, 1 \mathrm{H}), 6.78(\mathrm{t}, J=11.5 \mathrm{~Hz}, 1 \mathrm{H}), 4.19(\mathrm{qd}, J=7.2,2.8 \mathrm{~Hz}, 2 \mathrm{H}), 1.31(\mathrm{t}, J=7.2 \mathrm{~Hz}, 3 \mathrm{H})$.

${ }^{19}$ F NMR $\left(471 \mathrm{MHz}, \mathrm{CDCl}_{3}\right) \delta-97.7(\mathrm{~d}, J=269.0 \mathrm{~Hz}),-98.5(\mathrm{~d}, J=269.0 \mathrm{~Hz})$.

${ }^{13} \mathrm{C}$ NMR $\left(125 \mathrm{MHz}, \mathrm{CDCl}_{3}\right) \delta 162.2\left(\mathrm{t},{ }^{2} \mathrm{JC}_{\mathrm{C}-\mathrm{F}}=33.2 \mathrm{~Hz}\right), 140.7,134.2\left(\mathrm{dd},{ }^{2} \mathrm{CC}_{\mathrm{F}}=28.6,25.9\right.$ $\mathrm{Hz}), 133.0,130.4,128.8\left(\mathrm{t},{ }^{4} \mathrm{~J} C-F=2.2 \mathrm{~Hz}\right), 127.1,121.0,110.8\left(\mathrm{dd},{ }^{1} J_{C-F}=253.3,250.8 \mathrm{~Hz}\right)$, $105.5\left(\mathrm{dd},{ }^{3} \mathrm{C}_{\mathrm{C}-\mathrm{F}}=10.1,8.8 \mathrm{~Hz}\right), 63.3,13.8$.

HRMS (ESI) m/z: [M+Na] calcd for $\mathrm{C}_{12} \mathrm{H}_{10} \mathrm{BrF}_{2} \mathrm{IO}_{2} \mathrm{Na}$ : 452.8769; found: 452.8769.

Ethyl 2,2-difluoro-4-iodo-4-(o-tolyl)but-3-enoate (5p)

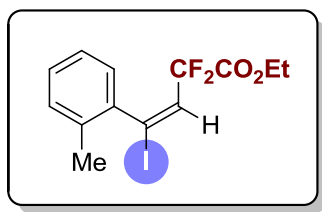

273.1 Hz).

${ }^{13} \mathrm{C}$ NMR $\left(125 \mathrm{MHz}, \mathrm{CDCl}_{3}\right) \delta 162.5\left(\mathrm{t},{ }^{2} \mathrm{~J} \mathrm{C}-\mathrm{F}=33.2 \mathrm{~Hz}\right), 139.7,134.8,133.7\left(\mathrm{t},{ }^{2} \mathrm{C}-\mathrm{F}=28.1 \mathrm{~Hz}\right)$, $130.3,129.4,127.2\left(\mathrm{t},{ }^{4} \mathrm{JC}_{\mathrm{C}-\mathrm{F}}=2.5 \mathrm{~Hz}\right), 125.5,110.7\left(\mathrm{t},{ }^{1} J_{C-F}=250.9 \mathrm{~Hz}\right), 107.9\left(\mathrm{t},{ }^{3} J_{C-F}=9.9 \mathrm{~Hz}\right)$, 63.1, 19.4, 13.6.

HRMS (ESI) m/z: [M+Na $]^{+}$calcd for $\mathrm{C}_{13} \mathrm{H}_{13} \mathrm{~F}_{2} \mathrm{IO}_{2} \mathrm{Na}$ : 388.9821; found: 388.9816 .

Ethyl 2,2-difluoro-4-iodo-4-(thiophen-3-yl)but-3-enoate (5q)

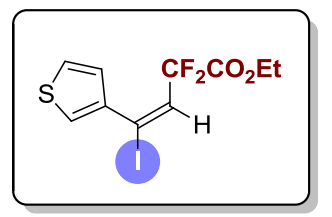

Yellow oil, $64.5 \mathrm{mg}$, 90\% yield.

${ }^{1} \mathrm{H}$ NMR $\left(500 \mathrm{MHz}, \mathrm{CDCl}_{3}\right) \delta 7.43(\mathrm{dd}, J=3.0,1.3 \mathrm{~Hz}, 1 \mathrm{H}), 7.31$ (dd, $J=5.1,3.0 \mathrm{~Hz}, 1 \mathrm{H}), 7.12(\mathrm{dd}, J=5.1,1.3 \mathrm{~Hz}, 1 \mathrm{H}), 6.71(\mathrm{t}, J=10.6 \mathrm{~Hz}$, $1 \mathrm{H}), 4.05(\mathrm{q}, J=7.1 \mathrm{~Hz}, 2 \mathrm{H}), 1.22(\mathrm{t}, J=7.1 \mathrm{~Hz}, 3 \mathrm{H})$.

${ }^{19} \mathrm{~F}$ NMR $\left(471 \mathrm{MHz}, \mathrm{CDCl}_{3}\right) \delta-92.6$.

${ }^{13} \mathrm{C}$ NMR (125 MHz, CDCl $) \delta 162.4\left(\mathrm{t},{ }^{2} \mathrm{~J} \mathrm{C}-\mathrm{F}=33.4 \mathrm{~Hz}\right), 140.1,133.3\left(\mathrm{t},{ }^{2} \mathrm{JC}_{\mathrm{C}-\mathrm{F}}=29.0 \mathrm{~Hz}\right), 128.4$, $125.9\left(\mathrm{t},{ }^{4} \mathrm{~J}_{\mathrm{C}-\mathrm{F}}=2.9 \mathrm{~Hz}\right), 125.6,111.0\left(\mathrm{t},{ }^{1} \mathrm{~J}_{\mathrm{C}-\mathrm{F}}=249.3 \mathrm{~Hz}\right), 102.1\left(\mathrm{t},{ }^{3} \mathrm{JC}_{\mathrm{C}-\mathrm{F}}=10.6 \mathrm{~Hz}\right), 63.2,13.7$.

HRMS (ESI) m/z: [M+Na] ${ }^{+}$calcd for $\mathrm{C}_{10} \mathrm{H}_{9} \mathrm{~F}_{2} \mathrm{IO}_{2} \mathrm{SNa}$ : 380.9228; found: 380.9224 .

Ethyl 2,2-difluoro-4-iodo-5-phenylpent-3-enoate (5r) $(E / Z=3: 1)$

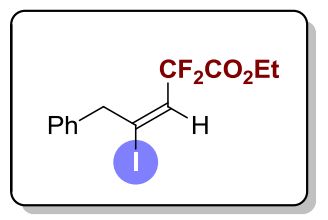

Colorless oil, $69.6 \mathrm{mg}, 95 \%$ yield.

${ }^{1}$ H NMR (500 MHz, $\left.\mathrm{CDCl}_{3}\right) \delta 7.43-7.30(\mathrm{~m}, 3 \mathrm{H}), 7.27-7.20(\mathrm{~m}$, $2 \mathrm{H}), 6.61(\mathrm{t}, J=12.5 \mathrm{~Hz}, 1 \mathrm{H}), 4.38(\mathrm{q}, J=7.1 \mathrm{~Hz}, 2 \mathrm{H}), 4.08(\mathrm{~s}, 2 \mathrm{H})$, $1.39(\mathrm{t}, J=7.1 \mathrm{~Hz}, 3 \mathrm{H})$ (major isomer).

${ }^{19} \mathrm{~F}$ NMR (471 MHz, $\left.\mathrm{CDCl}_{3}\right) \delta$-97.1 (major isomer).

${ }^{13} \mathrm{C}$ NMR $\left(125 \mathrm{MHz}, \mathrm{CDCl}_{3}\right) \delta 163.1\left(\mathrm{t},{ }^{2} \mathrm{~J} \mathrm{C}-\mathrm{F}=34.1 \mathrm{~Hz}\right), 137.0,132.2\left(\mathrm{t},{ }^{2} \mathrm{JC}-\mathrm{F}=26.9 \mathrm{~Hz}\right), 128.9$, 128.6, 127.2, $116.7\left(\mathrm{t},{ }^{3} \mathrm{~J}_{\mathrm{C}-\mathrm{F}}=7.3 \mathrm{~Hz}\right), 111.6\left(\mathrm{t},{ }^{1} \mathrm{~J}_{\mathrm{C}-\mathrm{F}}=252.8 \mathrm{~Hz}\right), 63.5,46.3\left(\mathrm{t},{ }^{4} \mathrm{~J}_{\mathrm{C}-\mathrm{F}}=2.3 \mathrm{~Hz}\right)$, 13.9 (major isomer).

HRMS (ESI) m/z: [M+Na $]^{+}$calcd for $\mathrm{C}_{13} \mathrm{H}_{13} \mathrm{~F}_{2} \mathrm{IO}_{2} \mathrm{Na}$ : 388.9821; found: 388.9813 .

Ethyl 2,2-difluoro-4-iododec-3-enoate $(5 \mathrm{~s})(E / Z=3.3: 1 \mathrm{dr})$

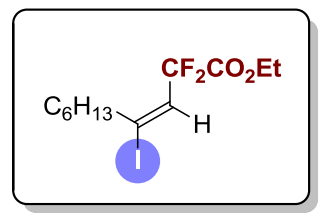

Colorless oil, $71.8 \mathrm{mg}$, $99 \%$ yield.

${ }^{1} \mathbf{H}$ NMR $\left(500 \mathrm{MHz}, \mathrm{CDCl}_{3}\right) \delta 6.42(\mathrm{t}, J=13.2 \mathrm{~Hz}, 1 \mathrm{H}), 4.35(\mathrm{q}, J=7.2$ $\mathrm{Hz}, 2 \mathrm{H}), 2.61(\mathrm{t}, J=7.5 \mathrm{~Hz}, 2 \mathrm{H}), 1.63-1.50(\mathrm{~m}, 2 \mathrm{H}), 1.37(\mathrm{t}, J=7.2$ $\mathrm{Hz}, 3 \mathrm{H}), 1.35-1.26(\mathrm{~m}, 6 \mathrm{H}), 0.91(\mathrm{t}, J=6.6 \mathrm{~Hz}, 3 \mathrm{H})$ (major isomer).

${ }^{19} \mathbf{F}$ NMR (471 MHz, $\left.\mathrm{CDCl}_{3}\right) \delta$-97.7 (major isomer).

${ }^{13} \mathrm{C}$ NMR $\left(125 \mathrm{MHz}, \mathrm{CDCl}_{3}\right) \delta 163.2\left(\mathrm{t},{ }^{2} \mathrm{JC}-\mathrm{F}=34.4 \mathrm{~Hz}\right), 131.2\left(\mathrm{t},{ }^{2} \mathrm{C}-\mathrm{F}=27.0 \mathrm{~Hz}\right), 119.6\left(\mathrm{t},{ }^{3} \mathrm{JC}_{\mathrm{C}-\mathrm{F}}\right.$ $=7.6 \mathrm{~Hz}), 111.5\left(\mathrm{t},{ }^{1} \mathrm{~J}_{\mathrm{C}-\mathrm{F}}=252.3 \mathrm{~Hz}\right), 63.3,40.7\left(\mathrm{t},{ }^{4} \mathrm{~J}_{\mathrm{C}-\mathrm{F}}=2.2 \mathrm{~Hz}\right), 31.5,29.9,28.0,22.5,14.0$, 
13.9 (major isomer).

HRMS (ESI) m/z: [M+Na] $]^{+}$calcd for $\mathrm{C}_{12} \mathrm{H}_{19} \mathrm{~F}_{2} \mathrm{IO}_{2} \mathrm{Na}$ : 383.0290; found: 383.0283

Ethyl 4-cyclopentyl-2,2-difluoro-4-iodobut-3-enoate $(5 t)(E / Z=2: 1)$

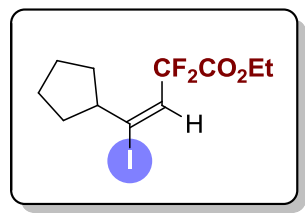

Colorless oil, $68.2 \mathrm{mg}, 99 \%$ yield.

${ }^{1} \mathbf{H}$ NMR $\left(500 \mathrm{MHz}, \mathrm{CDCl}_{3}\right) \delta 6.43(\mathrm{t}, J=13.0 \mathrm{~Hz}, 1 \mathrm{H}), 4.35(\mathrm{q}, J=7.2$ $\mathrm{Hz}, 2 \mathrm{H}), 2.64-2.50(\mathrm{~m}, 1 \mathrm{H}), 1.91-1.82(\mathrm{~m}, 1 \mathrm{H}), 1.81-1.71(\mathrm{~m}, 3 \mathrm{H})$, $1.59-1.50(\mathrm{~m}, 2 \mathrm{H}), 1.50-1.41(\mathrm{~m}, 2 \mathrm{H}), 1.37(\mathrm{t}, J=7.2 \mathrm{~Hz}, 3 \mathrm{H})$ (major isomer).

${ }^{19} \mathbf{F}$ NMR (471 MHz, $\mathrm{CDCl}_{3}$ ) $\delta$-96.8 (major isomer).

${ }^{13} \mathrm{C}$ NMR $\left(125 \mathrm{MHz}, \mathrm{CDCl}_{3}\right) \delta 163.4\left(\mathrm{t},{ }^{2} \mathrm{JC}_{\mathrm{C}-\mathrm{F}}=34.5 \mathrm{~Hz}\right), 131.1\left(\mathrm{t},{ }^{3} \mathrm{C}-\mathrm{F}=27.7 \mathrm{~Hz}\right), 126.4\left(\mathrm{t},{ }^{2} \mathrm{C}_{\mathrm{C}-\mathrm{F}}\right.$ $=29.7 \mathrm{~Hz}), 111.7\left(\mathrm{t},{ }^{1} \mathrm{JC}_{\mathrm{C}-\mathrm{F}}=252.0 \mathrm{~Hz}\right), 63.3,45.9,34.8,25.7,13.9$ (major isomer).

HRMS (ESI) m/z: $\quad[\mathrm{M}+\mathrm{Na}]^{+}$calcd for $\mathrm{C}_{11} \mathrm{H}_{15} \mathrm{~F}_{2} \mathrm{IO}_{2} \mathrm{Na}$ : 366.9977; found: 366.9973 .

Ethyl 2,2-difluoro-4-iodo-5,5-dimethylhex-3-enoate (5u) $(E / Z>20: 1)$

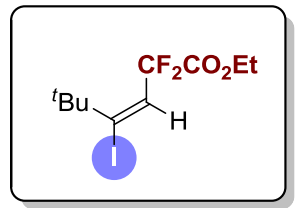

Colorless oil, $65.8 \mathrm{mg}$, $99 \%$ yield.

${ }^{1} \mathrm{H}$ NMR $\left(500 \mathrm{MHz}, \mathrm{CDCl}_{3}\right) \delta 6.44(\mathrm{t}, J=11.7 \mathrm{~Hz}, 1 \mathrm{H}), 4.36(\mathrm{q}, J=7.2$ $\mathrm{Hz}, 2 \mathrm{H}), 1.37(\mathrm{t}, J=7.2 \mathrm{~Hz}, 3 \mathrm{H}), 1.24(\mathrm{~s}, 9 \mathrm{H})$.

${ }^{19} \mathrm{~F}$ NMR $\left(471 \mathrm{MHz}, \mathrm{CDCl}_{3}\right) \delta-96.5$.

${ }^{13} \mathrm{C}$ NMR $\left(125 \mathrm{MHz}, \mathrm{CDCl}_{3}\right) \delta 162.6\left(\mathrm{t},{ }^{2} \mathrm{~J} \mathrm{C}-\mathrm{F}=34.3 \mathrm{~Hz}\right), 131.4\left(\mathrm{t},{ }^{3} \mathrm{~J} \mathrm{C}-\mathrm{F}=\right.$ $9.7 \mathrm{~Hz}), 126.4\left(\mathrm{t},{ }^{2} \mathrm{~J}_{C-F}=30.5 \mathrm{~Hz}\right), 113.0\left(\mathrm{t},{ }^{1} \mathrm{~J}_{C-F}=246.8 \mathrm{~Hz}\right), 63.1,41.4,29.9,13.8$.

HRMS (ESI) m/z: [M+Na] $]^{+}$calcd for $\mathrm{C}_{10} \mathrm{H}_{15} \mathrm{~F}_{2} \mathrm{IO}_{2} \mathrm{Na}$ : 354.9977; found: 354.9981 .

tert-butyl 4-(4-ethoxy-3,3-difluoro-1-iodo-4-oxobut-1-en-1-yl)piperidine-1-carboxylate (5v) $(E / Z=1: 1)$

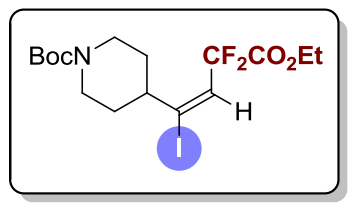

Purified by silica gel column chromatography (petroleum ether/ethyl acetate $=10 / 1)$, colorless oil, $66.0 \mathrm{mg}$, $72 \%$ yield .

${ }^{1} \mathrm{H}$ NMR $\left(500 \mathrm{MHz}, \mathrm{CDCl}_{3}\right) \delta 6.50(\mathrm{t}, J=13.2 \mathrm{~Hz}, 1 \mathrm{H}), 4.36(\mathrm{q}, J=$ $7.3 \mathrm{~Hz}, 2 \mathrm{H}), 4.30-4.11(\mathrm{~m}, 2 \mathrm{H}), 2.97-2.65(\mathrm{~m}, 2 \mathrm{H}), 2.40-2.30(\mathrm{~m}$, $1 \mathrm{H}), 1.91-1.77(\mathrm{~m}, 1 \mathrm{H}), 1.60-1.50(\mathrm{~m}, 3 \mathrm{H}), 1.49(\mathrm{~s}, 9 \mathrm{H}), 1.38(\mathrm{t}, J=7.2 \mathrm{~Hz}, 3 \mathrm{H})$.

${ }^{19}$ F NMR $\left(471 \mathrm{MHz}, \mathrm{CDCl}_{3}\right) \delta$-97.5.

${ }^{13} \mathrm{C}$ NMR (125 MHz, CDCl 3$) \delta 163.2\left(\mathrm{t},{ }^{2} \mathrm{~J}_{\mathrm{C}-\mathrm{F}}=34.2 \mathrm{~Hz}\right), 154.7,131.3\left(\mathrm{t},{ }^{3} \mathrm{~J}_{\mathrm{C}-F}=27.5 \mathrm{~Hz}\right), 127.4$ $\left(\mathrm{t},{ }^{2} J_{C-F}=29.8 \mathrm{~Hz}\right), 112.3\left(\mathrm{t},{ }^{1} J_{\mathrm{C}-F}=247.9 \mathrm{~Hz}\right), 79.7,63.5,50.9,43.0\left(\mathrm{t},{ }^{4} J_{\mathrm{C}-F}=2.3 \mathrm{~Hz}\right), 32.4,28.4$, 13.9.

HRMS (ESI) m/z: [M+Na] $]^{+}$calcd for $\mathrm{C}_{16} \mathrm{H}_{24} \mathrm{~F}_{2} \mathrm{INO}_{4} \mathrm{Na}$ : 482.0610; found: 482.0614 .

Ethyl 2,2-difluoro-4-iodo-3-propylhept-3-enoate (5w) $(E / Z=8: 1)$

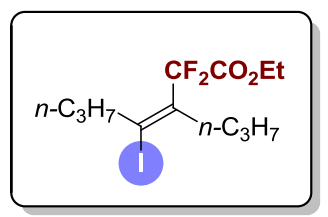

Colorless oil, $49.8 \mathrm{mg}, 69 \%$ yield.

${ }^{1} \mathrm{H}$ NMR $\left(500 \mathrm{MHz}, \mathrm{CDCl}_{3}\right) \delta 4.33(\mathrm{q}, J=7.1 \mathrm{~Hz}, 2 \mathrm{H}), 2.71-2.63(\mathrm{~m}$, $2 \mathrm{H}), 2.48-2.39(\mathrm{~m}, 2 \mathrm{H}), 1.68-1.48(\mathrm{~m}, 4 \mathrm{H}), 1.36(\mathrm{t}, J=7.1 \mathrm{~Hz}, 3 \mathrm{H})$, $0.99(\mathrm{t}, J=7.4 \mathrm{~Hz}, 3 \mathrm{H}), 0.93(\mathrm{t}, J=7.4 \mathrm{~Hz}, 3 \mathrm{H})$.

${ }^{19} \mathrm{~F}$ NMR $\left(471 \mathrm{MHz}, \mathrm{CDCl}_{3}\right) \delta-96.5$.

${ }^{13} \mathrm{C}$ NMR $\left(125 \mathrm{MHz}, \mathrm{CDCl}_{3}\right) \delta 163.7\left(\mathrm{t},{ }^{2} \mathrm{~J} \mathrm{C}-\mathrm{F}=35.2 \mathrm{~Hz}\right), 136.8\left(\mathrm{t},{ }^{2} \mathrm{~J} \mathrm{C}-\mathrm{F}=22.7 \mathrm{~Hz}\right), 119.8\left(\mathrm{t},{ }^{3} \mathrm{~J} \mathrm{C}-\mathrm{F}\right.$ $=6.1 \mathrm{~Hz}), 111.9\left(\mathrm{t},{ }^{1} \mathrm{~J} C-F=256.1 \mathrm{~Hz}\right), 63.2,44.3\left(\mathrm{t},{ }^{4} J_{\mathrm{C}-F}=2.4 \mathrm{~Hz}\right), 41.2\left(\mathrm{t},{ }^{3} \mathrm{C}_{\mathrm{C}-\mathrm{F}}=3.7 \mathrm{~Hz}\right), 23.8$, 21.5, 14.0, 13.9, 12.8 .

HRMS (ESI) m/z: [M+Na $]^{+}$calcd for $\mathrm{C}_{12} \mathrm{H}_{19} \mathrm{~F}_{2} \mathrm{IO}_{2} \mathrm{Na}$ : 383.0290; found: 383.0295 .

Ethyl 2,2-difluoro-3-(iodo(phenyl)methylene)pentanoate (5x) $(E / Z>20: 1)$

Purified by silica gel column chromatography (petroleum ether/ethyl acetate $=25 / 1$ ), 


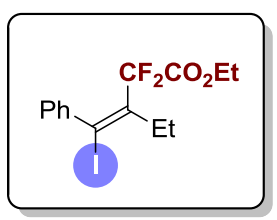

colorless oil, $47.9 \mathrm{mg}$, $63 \%$ yield.

${ }^{1} \mathrm{H}$ NMR $\left(500 \mathrm{MHz}, \mathrm{CDCl}_{3}\right) \delta 7.35-7.26(\mathrm{~m}, 3 \mathrm{H}), 7.25-7.19(\mathrm{~m}, 2 \mathrm{H})$, $3.89(\mathrm{q}, J=7.2 \mathrm{~Hz}, 2 \mathrm{H}), 2.77-2.65(\mathrm{~m}, 2 \mathrm{H}), 1.25(\mathrm{t}, J=7.5 \mathrm{~Hz}, 3 \mathrm{H}), 1.20$ $(\mathrm{t}, J=7.2 \mathrm{~Hz}, 3 \mathrm{H})$.

${ }^{19}$ F NMR $\left(471 \mathrm{MHz}, \mathrm{CDCl}_{3}\right) \delta$-92.6.

${ }^{13} \mathrm{C}$ NMR $\left(125 \mathrm{MHz}, \mathrm{CDCl}_{3}\right) \delta 162.9\left(\mathrm{t},{ }^{2} \mathrm{~J}-\mathrm{F}=33.6 \mathrm{~Hz}\right), 142.6,141.0\left(\mathrm{t},{ }^{2} \mathrm{~J}-\mathrm{F}=23.2 \mathrm{~Hz}\right), 128.7$, $128.5,127.8,110.8\left(\mathrm{t},{ }^{1} J_{C-F}=254.1 \mathrm{~Hz}\right), 108.3\left(\mathrm{t},{ }^{3} \mathrm{JC}_{\mathrm{C}-F}=8.1 \mathrm{~Hz}\right), 62.8,32.3\left(\mathrm{t},{ }^{3} J_{\mathrm{C}-F}=3.2 \mathrm{~Hz}\right)$, 13.6, 12.3 .

HRMS (ESI) m/z: [M+Na] $]^{+}$calcd for $\mathrm{C}_{14} \mathrm{H}_{15} \mathrm{~F}_{2} \mathrm{IO}_{2} \mathrm{Na}$ : 402.9977; found: 402.9973 .

Ethyl 2,2-difluoro-4-iodo-4-(trimethylsilyl)but-3-enoate (5y) $(E / Z=2: 1)$

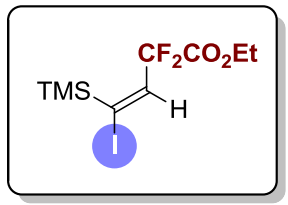

Colorless oil, $69.0 \mathrm{mg}$, 99\% yield.

${ }^{1} \mathbf{H}$ NMR (500 MHz, $\left.\mathrm{CDCl}_{3}\right) \delta 7.39(\mathrm{t}, J=14.7 \mathrm{~Hz}, 1 \mathrm{H}), 4.35$ (q, $J=7.1$

$\mathrm{Hz}, 2 \mathrm{H}), 1.38(\mathrm{t}, J=7.1 \mathrm{~Hz}, 3 \mathrm{H}), 0.33$ (s, 9H) (major isomer).

${ }^{19} \mathrm{~F}$ NMR (471 MHz, $\left.\mathrm{CDCl}_{3}\right) \delta$-97.6 (major isomer).

${ }^{13} \mathrm{C}$ NMR $\left(125 \mathrm{MHz}, \mathrm{CDCl}_{3}\right) \delta 162.8\left(\mathrm{t},{ }^{2} \mathrm{~J} \mathrm{C}-\mathrm{F}=33.3 \mathrm{~Hz}\right), 143.7\left(\mathrm{t},{ }^{2} \mathrm{CC}_{\mathrm{C}-\mathrm{F}}=\right.$ $25.8 \mathrm{~Hz}), 123.5\left(\mathrm{t},{ }^{3} J_{C-F}=7.0 \mathrm{~Hz}\right), 111.7\left(\mathrm{t},{ }^{1} J_{C-F}=252.0 \mathrm{~Hz}\right), 63.4,13.9,-1.9$ (major isomer).

HRMS (ESI) m/z: [M+Na] ${ }^{+}$calcd for $\mathrm{C}_{9} \mathrm{H}_{15} \mathrm{~F}_{2} \mathrm{IO}_{2} \mathrm{SiNa}$ : 370.9746; found: 370.9740 .

\section{8-ethoxy-7,7-difluoro-5-iodo-8-oxooct-5-en-1-yl benzoate $(5 z)(E / Z=5: 1)$}

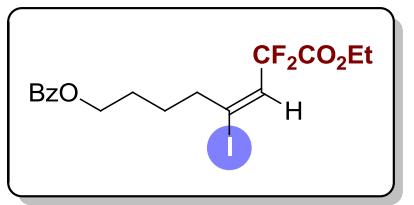

Purified by silica gel column chromatography (petroleum ether/ethyl acetate $=25 / 1$ ), colorless oil, $74.1 \mathrm{mg}, 82 \%$ yield .

${ }^{1} \mathrm{H}$ NMR $\left(500 \mathrm{MHz}, \mathrm{CDCl}_{3}\right) \delta 8.14-7.99(\mathrm{~m}, 2 \mathrm{H}), 7.63-7.54$ $(\mathrm{m}, 1 \mathrm{H}), 7.51-7.42(\mathrm{~m}, 2 \mathrm{H}), 6.47(\mathrm{t}, J=13.2 \mathrm{~Hz}, 1 \mathrm{H}), 4.39-$

$4.33(\mathrm{~m}, 2 \mathrm{H}), 4.34(\mathrm{t}, J=7.2 \mathrm{~Hz}, 2 \mathrm{H}), 2.84-2.62(\mathrm{~m}, 2 \mathrm{H}), 1.88-1.71(\mathrm{~m}, 4 \mathrm{H}), 1.36(\mathrm{t}, J=7.2$ $\mathrm{Hz}, 3 \mathrm{H}$ ) (major isomer).

${ }^{19} \mathrm{~F}$ NMR $\left(471 \mathrm{MHz}, \mathrm{CDCl}_{3}\right) \delta$ - 97.7 (major isomer).

${ }^{13} \mathrm{C}$ NMR $\left(125 \mathrm{MHz}, \mathrm{CDCl}_{3}\right) \delta 166.6,163.1\left(\mathrm{t},{ }^{2} \mathrm{C}_{\mathrm{C}-F}=34.4 \mathrm{~Hz}\right), 132.9,131.9\left(\mathrm{t},{ }^{2} \mathrm{C}-\mathrm{F}=27.0 \mathrm{~Hz}\right)$, 130.3, 129.6, 128.3, $118.6\left(\mathrm{t},{ }^{3} \mathrm{~J}_{\mathrm{C}-\mathrm{F}}=7.5 \mathrm{~Hz}\right), 111.5\left(\mathrm{t},{ }^{1} \mathrm{~J}_{\mathrm{C}-\mathrm{F}}=252.5 \mathrm{~Hz}\right), 64.4,63.4,40.2\left(\mathrm{t},{ }^{4} \mathrm{~J}_{\mathrm{C}-\mathrm{F}}=\right.$ $2.1 \mathrm{~Hz}$ ), 27.4, 26.4, 13.9 (major isomer).

HRMS (ESI) m/z: [M+Na] $]^{+}$calcd for $\mathrm{C}_{17} \mathrm{H}_{19} \mathrm{~F}_{2} \mathrm{IO}_{4} \mathrm{Na}$ : 475.0188; found: 475.0192.

Ethyl 2,2-difluoro-4-iodo-8-(tosyloxy)oct-3-enoate (5aa) $(E / Z=4: 1)$

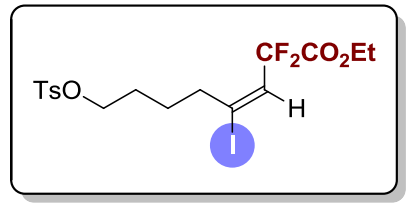

Purified by silica gel column chromatography (petroleum ether/ethyl acetate $=4 / 1)$, colorless oil, $78.0 \mathrm{mg}$, $78 \%$ yield .

${ }^{1} \mathbf{H}$ NMR $\left(500 \mathrm{MHz}, \mathrm{CDCl}_{3}\right) \delta 7.82(\mathrm{~d}, J=8.1 \mathrm{~Hz}, 2 \mathrm{H}), 7.38(\mathrm{~d}, J$ $=8.1 \mathrm{~Hz}, 2 \mathrm{H}), 6.42-6.30(\mathrm{~m}, 1 \mathrm{H}), 4.38(\mathrm{q}, J=7.1 \mathrm{~Hz}, 2 \mathrm{H}), 4.07$ $(\mathrm{t}, J=5.8 \mathrm{~Hz}, 2 \mathrm{H}), 2.57(\mathrm{td}, J=6.2,5.2,2.5 \mathrm{~Hz}, 2 \mathrm{H}), 2.48(\mathrm{~s}, 3 \mathrm{H})$, $1.75-1.55(\mathrm{~m}, 4 \mathrm{H}), 1.39(\mathrm{t}, J=7.1 \mathrm{~Hz}, 3 \mathrm{H})$.

${ }^{19}$ F NMR $\left(471 \mathrm{MHz}, \mathrm{CDCl}_{3}\right) \delta$-98.1.

${ }^{13} \mathrm{C}$ NMR $\left(125 \mathrm{MHz}, \mathrm{CDCl}_{3}\right) \delta 162.5\left(\mathrm{t},{ }^{2} \mathrm{~J}_{\mathrm{C}-\mathrm{F}}=35.3 \mathrm{~Hz}\right), 144.9,133.0,129.9,128.9\left(\mathrm{t},{ }^{2} \mathrm{C}-\mathrm{F}=\right.$ $29.5 \mathrm{~Hz}), 127.9,113.3\left(\mathrm{t},{ }^{3} \mathrm{~J}_{\mathrm{C}-\mathrm{F}}=8.8 \mathrm{~Hz}\right), 111.9\left(\mathrm{t},{ }^{1} \mathrm{~J}_{\mathrm{C}-\mathrm{F}}=248.0 \mathrm{~Hz}\right), 69.8,63.3,45.8,27.2,24.9$, 21.6, 13.9 .

HRMS (ESI) m/z: [M+Na] calcd for $\mathrm{C}_{17} \mathrm{H}_{21} \mathrm{~F}_{2} \mathrm{IO}_{5} \mathrm{SNa}$ : 525.0015; found: 525.0017.

Ethyl 8-((tert-butyldimethylsilyl)oxy)-2,2-difluoro-4-iodooct-3-enoate (5ab) $(E / Z=3: 1)$

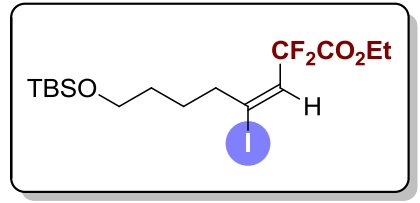

Purified by silica gel column chromatography (petroleum ether/ethyl acetate $=100 / 1)$, colorless oil, $66.0 \mathrm{mg}$, 69\% yield. 
${ }^{1} \mathrm{H}$ NMR $\left(500 \mathrm{MHz}, \mathrm{CDCl}_{3}\right) \delta 6.44(\mathrm{t}, J=13.2 \mathrm{~Hz}, 1 \mathrm{H}), 4.35(\mathrm{q}, J=7.1 \mathrm{~Hz}, 2 \mathrm{H}), 3.64(\mathrm{t}, J=$ $6.1 \mathrm{~Hz}, 2 \mathrm{H}), 2.76-2.58(\mathrm{~m}, 2 \mathrm{H}), 1.71-1.58(\mathrm{~m}, 4 \mathrm{H}), 1.38(\mathrm{t}, J=7.1 \mathrm{~Hz}, 3 \mathrm{H}), 0.92(\mathrm{~s}, 9 \mathrm{H})$, $0.08(\mathrm{~s}, 6 \mathrm{H})$ (major isomer).

${ }^{19} \mathrm{~F}$ NMR $\left(471 \mathrm{MHz}, \mathrm{CDCl}_{3}\right) \delta$ - 97.7 (major isomer).

${ }^{13} \mathrm{C}$ NMR $\left(125 \mathrm{MHz}, \mathrm{CDCl}_{3}\right) \delta 163.2\left(\mathrm{t},{ }^{2} \mathrm{JC}-\mathrm{F}=34.3 \mathrm{~Hz}\right), 131.4\left(\mathrm{t},{ }^{2} \mathrm{~J} \mathrm{C}-\mathrm{F}=27.0 \mathrm{~Hz}\right), 119.4\left(\mathrm{t},{ }^{3} \mathrm{JC}-\mathrm{F}\right.$ $=7.4 \mathrm{~Hz}), 111.6\left(\mathrm{t},{ }^{1} \mathrm{JC}_{\mathrm{C}-\mathrm{F}}=252.4 \mathrm{~Hz}\right), 68.2,62.6,40.4,31.5,26.0,25.0,18.3,13.9$, -5.3 (major isomer).

HRMS (ESI) m/z: [M+K $]^{+}$calcd for $\mathrm{C}_{16} \mathrm{H}_{29} \mathrm{~F}_{2} \mathrm{IO}_{3} \mathrm{SiK}: 501.0530$; found: 501.0538 .

Ethyl 8-(benzyloxy)-2,2-difluoro-4-iodooct-3-enoate (5ac) $(E / Z=3: 1)$

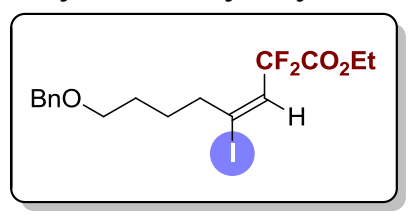

Purified by silica gel column chromatography (petroleum ether/ethyl acetate $=25 / 1)$, colorless oil, $65.7 \mathrm{mg}$, 75\% yield.

${ }^{1} \mathbf{H}$ NMR $\left(500 \mathrm{MHz}, \mathrm{CDCl}_{3}\right) \delta 7.40-7.35(\mathrm{~m}, 4 \mathrm{H}), 7.34-7.29$ $(\mathrm{m}, 1 \mathrm{H}), 6.44(\mathrm{t}, J=13.7 \mathrm{~Hz}, 1 \mathrm{H}), 4.53(\mathrm{~s}, 2 \mathrm{H}), 4.34(\mathrm{q}, J=7.2 \mathrm{~Hz}$ $2 \mathrm{H}), 3.57-3.44(\mathrm{~m}, 2 \mathrm{H}), 2.70-2.61(\mathrm{~m}, 2 \mathrm{H}), 1.72-1.63(\mathrm{~m}, 4 \mathrm{H}), 1.36(\mathrm{t}, J=7.2 \mathrm{~Hz}, 3 \mathrm{H})$ (major isomer).

${ }^{19} \mathbf{F}$ NMR (471 MHz, $\left.\mathrm{CDCl}_{3}\right) \delta$-97.7 (major isomer).

${ }^{13} \mathrm{C}$ NMR $\left(125 \mathrm{MHz}, \mathrm{CDCl}_{3}\right) \delta 163.2\left(\mathrm{t},{ }^{2} \mathrm{~J} \mathrm{C}-\mathrm{F}=34.3 \mathrm{~Hz}\right), 138.5,131.5\left(\mathrm{t},{ }^{2} \mathrm{JC}-\mathrm{F}=27.0 \mathrm{~Hz}\right), 128.3$, 127.6, 127.5, $119.1\left(\mathrm{t},{ }^{3} \mathrm{~J}_{\mathrm{C}-\mathrm{F}}=7.5 \mathrm{~Hz}\right), 111.5\left(\mathrm{t},{ }^{1} \mathrm{~J}_{\mathrm{C}-\mathrm{F}}=252.4 \mathrm{~Hz}\right), 72.9,69.8,63.4,40.4\left(\mathrm{t},{ }^{4} \mathrm{~J}_{\mathrm{C}-\mathrm{F}}=\right.$ $2.2 \mathrm{~Hz}$ ), 28.4, 26.7, 13.9 (major isomer).

HRMS (ESI) m/z: [M+Na] $]^{+}$calcd for $\mathrm{C}_{17} \mathrm{H}_{21} \mathrm{~F}_{2} \mathrm{IO}_{3} \mathrm{Na}$ : 461.0396; found: 461.0392 .

Ethyl 11-azido-2,2-difluoro-4-iodoundec-3-enoate (5ad) $(E / Z=3: 1)$

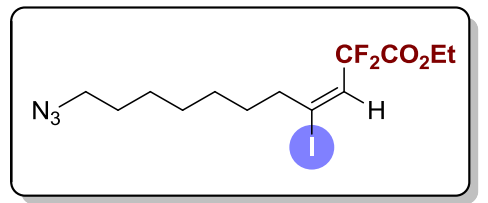

Colorless oil, $78.0 \mathrm{mg}$, $94 \%$ yield.

${ }^{1} \mathbf{H}$ NMR $\left(500 \mathrm{MHz}, \mathrm{CDCl}_{3}\right) \delta 6.42(\mathrm{t}, J=13.2 \mathrm{~Hz}, 1 \mathrm{H}), 4.35$ $(\mathrm{q}, J=7.2 \mathrm{~Hz}, 2 \mathrm{H}), 3.28(\mathrm{t}, J=6.9 \mathrm{~Hz}, 2 \mathrm{H}), 2.66-2.57(\mathrm{~m}$, $2 \mathrm{H}), 1.67-1.54(\mathrm{~m}, 4 \mathrm{H}), 1.44-1.31(\mathrm{~m}, 6 \mathrm{H}), 1.38(\mathrm{t}, J=7.2$

$\mathrm{Hz}, 3 \mathrm{H})$ (major isomer).

${ }^{19} \mathrm{~F}$ NMR (471 MHz, $\left.\mathrm{CDCl}_{3}\right)$-97.7 (major isomer).

${ }^{13} \mathrm{C}$ NMR $\left(125 \mathrm{MHz}, \mathrm{CDCl}_{3}\right) \delta 163.2\left(\mathrm{t},{ }^{2} \mathrm{~J}_{\mathrm{C}-\mathrm{F}}=34.3 \mathrm{~Hz}\right), 131.3\left(\mathrm{t},{ }^{2} \mathrm{C}-\mathrm{F}=27.0 \mathrm{~Hz}\right), 119.4\left(\mathrm{t},{ }^{3} \mathrm{~J} \mathrm{C}-\mathrm{F}\right.$ $=7.6 \mathrm{~Hz}), 111.5\left(\mathrm{t},{ }^{1} J_{\mathrm{C}-\mathrm{F}}=252.4 \mathrm{~Hz}\right), 63.3,51.4,40.6\left(\mathrm{t},{ }^{4} \mathrm{~J}_{\mathrm{C}-\mathrm{F}}=2.2 \mathrm{~Hz}\right), 29.7,28.8,28.7,28.1$, 26.5, 13.9 (major isomer).

HRMS (ESI) m/z: [M+Na] ${ }^{+}$calcd for $\mathrm{C}_{13} \mathrm{H}_{20} \mathrm{~F}_{2} \mathrm{IN}_{3} \mathrm{O}_{2} \mathrm{Na}$ : 438.0460 ; found: 438.0459 .

7-benzyl 1-ethyl 2,2-difluoro-4-iodohept-3-enedioate (5ae) $(E / Z=3: 1)$

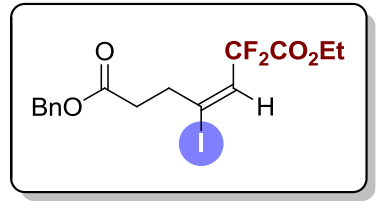

Purified by silica gel column chromatography (petroleum ether/ethyl acetate $=25 / 1)$, colorless oil, $62.9 \mathrm{mg}, 72 \%$ yield.

${ }^{1} \mathrm{H}$ NMR $\left(500 \mathrm{MHz}, \mathrm{CDCl}_{3}\right) \delta 7.46-7.33(\mathrm{~m}, 5 \mathrm{H}), 6.48(\mathrm{t}, J=13.1$ $\mathrm{Hz}, 1 \mathrm{H}), \quad 5.16(\mathrm{~s}, 2 \mathrm{H}), 4.37(\mathrm{q}, J=7.2 \mathrm{~Hz}, 2 \mathrm{H}), 3.12-2.90(\mathrm{~m}$, $2 \mathrm{H}$ ), $2.66(\mathrm{t}, J=7.5 \mathrm{~Hz}, 2 \mathrm{H}$ ), $1.38(\mathrm{t}, J=7.1 \mathrm{~Hz}, 3 \mathrm{H}$ ) (major

isomer).

${ }^{19} \mathrm{~F}$ NMR $\left(471 \mathrm{MHz}, \mathrm{CDCl}_{3}\right) \delta$-98.1 (major isomer).

${ }^{13} \mathrm{C}$ NMR $\left(125 \mathrm{MHz}, \mathrm{CDCl}_{3}\right) \delta 171.0,162.4\left(\mathrm{t},{ }^{2} \mathrm{C}_{\mathrm{c}-F}=34.1 \mathrm{~Hz}\right), 135.6,129.6\left(\mathrm{t},{ }^{2} \mathrm{C}_{\mathrm{c}-F}=29.5 \mathrm{~Hz}\right)$, 128.6, 128.4, 128.3, $111.8\left(\mathrm{t},{ }^{1} \mathrm{~J}_{\mathrm{C}-\mathrm{F}}=248.2 \mathrm{~Hz}\right), 111.1\left(\mathrm{t},{ }^{3} \mathrm{~J}_{\mathrm{C}-\mathrm{F}}=9.8 \mathrm{~Hz}\right), 66.7,63.3,42.0,33.9$, 13.9 (major isomer).

HRMS (ESI) m/z: [M+Na] $]^{+}$calcd for $\mathrm{C}_{16} \mathrm{H}_{17} \mathrm{~F}_{2} \mathrm{IO}_{4} \mathrm{Na}$ : 461.0032; found: 461.0034 .

Eethyl 7-(benzylamino)-2,2-difluoro-4-iodo-7-oxohept-3-enoate (5af) $(E / Z=3: 1)$ 


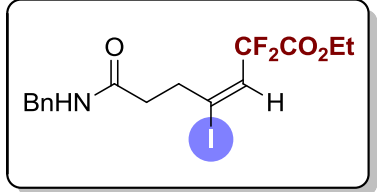

Purified by silica gel column chromatography (petroleum ether/ethyl acetate $=1 / 1)$, colorless oil, $49.8 \mathrm{mg}$, $60 \%$ yield . ${ }^{1} \mathbf{H}$ NMR $\left(500 \mathrm{MHz}, \mathrm{CDCl}_{3}\right) \delta 7.46-7.34(\mathrm{~m}, 2 \mathrm{H}), 7.34-7.26(\mathrm{~m}$, $3 \mathrm{H}), 6.47(\mathrm{t}, J=13.1 \mathrm{~Hz}, 1 \mathrm{H}), 5.82(\mathrm{~s}, 1 \mathrm{H}), 4.47(\mathrm{~d}, J=5.7 \mathrm{~Hz}, 2 \mathrm{H})$, $4.35(\mathrm{q}, J=7.2 \mathrm{~Hz}, 2 \mathrm{H}), 3.14-2.88(\mathrm{~m}, 2 \mathrm{H}), 2.59-2.35(\mathrm{~m}, 2 \mathrm{H}), 1.37(\mathrm{t}, J=7.2 \mathrm{~Hz}, 3 \mathrm{H})$ (major isomer).

${ }^{19} \mathrm{~F}$ NMR $\left(471 \mathrm{MHz}, \mathrm{CDCl}_{3}\right) \delta$-98.0 (major isomer).

${ }^{13} \mathrm{C}$ NMR $\left(125 \mathrm{MHz}, \mathrm{CDCl}_{3}\right) \delta 170.0,163.0\left(\mathrm{t},{ }^{2} \mathrm{~J} \mathrm{C}-\mathrm{F}=34.3 \mathrm{~Hz}\right), 138.0,132.5\left(\mathrm{t},{ }^{2} \mathrm{JC}_{\mathrm{C}-\mathrm{F}}=27.3 \mathrm{~Hz}\right)$, $128.7,127.9,127.6,116.2\left(\mathrm{t},{ }^{3} \mathrm{~J}_{\mathrm{C}-\mathrm{F}}=7.7 \mathrm{~Hz}\right), 111.6\left(\mathrm{t},{ }^{1} \mathrm{JC}_{\mathrm{F}}=252.8 \mathrm{~Hz}\right), 63.6,43.8,36.9\left(\mathrm{t},{ }^{4} \mathrm{~J} C-\mathrm{F}=\right.$ $2.3 \mathrm{~Hz}$ ), 36.4, 13.9 (major isomer).

HRMS (ESI) m/z: [M+Na] ${ }^{+}$calcd for $\mathrm{C}_{16} \mathrm{H}_{18} \mathrm{~F}_{2} \mathrm{INO}_{3} \mathrm{Na}$ : 438.0372; found: 438.0370 .

\section{$(4,4,5,5,6,6,7,7,7-$ nonafluoro-2-iodoheptyl)benzene (6a)}

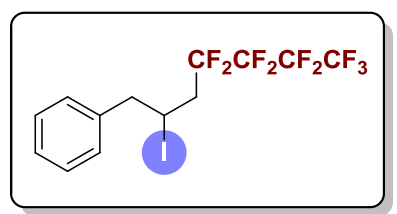

6a is a known product. $^{1}$ Purified by silica gel column chromatography (petroleum ether), colorless oil, $72.3 \mathrm{mg}$, 78\% yield.

${ }^{1} \mathrm{H}$ NMR $\left(500 \mathrm{MHz}, \mathrm{CDCl}_{3}\right) \delta 7.44-7.33(\mathrm{~m}, 3 \mathrm{H}), 7.27-7.22$ $(\mathrm{m}, 2 \mathrm{H}), 4.51(\mathrm{dq}, J=8.9,6.3 \mathrm{~Hz}, 1 \mathrm{H}), 3.34(\mathrm{dd}, J=14.6,5.8 \mathrm{~Hz}$, $1 \mathrm{H}), 3.24(\mathrm{dd}, J=14.6,8.9 \mathrm{~Hz}, 1 \mathrm{H}), 3.06-2.81(\mathrm{~m}, 2 \mathrm{H})$.

${ }^{19} \mathrm{~F}$ NMR $\left(471 \mathrm{MHz}, \mathrm{CDCl}_{3}\right) \delta-81.1(\mathrm{t}, J=9.4 \mathrm{~Hz}, 3 \mathrm{~F}),-112.2(\mathrm{dt}, J=269.4,12.6 \mathrm{~Hz}, 1 \mathrm{~F})$, $-114.1(\mathrm{dt}, J=269.4,12.6 \mathrm{~Hz}, 1 \mathrm{~F}),-124.6(\mathrm{~m}, 2 \mathrm{~F}),-126.0(\mathrm{~m}, 2 \mathrm{~F})$.

${ }^{13} \mathrm{C}$ NMR $\left(125 \mathrm{MHz}, \mathrm{CDCl}_{3}\right) \delta$ 138.6, 128.9, 128.6, 127.3, $121-108(\mathrm{~m}), 47.0,40.8\left(\mathrm{t},{ }^{2} \mathrm{C}-\mathrm{F}=\right.$ $20.9 \mathrm{~Hz}), 19.2$.

LRMS: m/z (EI) 337 (M-I), 148, 117, 103, 91, 77.

$(4,4,5,5,6,6,7,7,8,8,9,9,9$-tridecafluoro-2-iodononyl)benzene (6b)

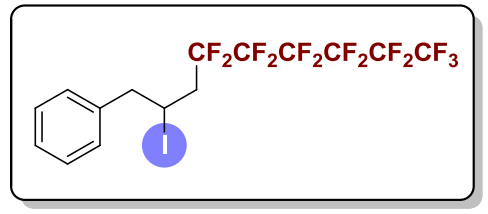

$\mathbf{6 b}$ is a known product. ${ }^{2}$ Purified by silica gel column chromatography (petroleum ether), colorless oil, $69.9 \mathrm{mg}$, $62 \%$ yield.

${ }^{1} \mathbf{H}$ NMR $\left(500 \mathrm{MHz}, \mathrm{CDCl}_{3}\right) \delta 7.41-7.30(\mathrm{~m}, 3 \mathrm{H}), 7.23$ $(\mathrm{dd}, J=6.7,1.8 \mathrm{~Hz}, 2 \mathrm{H}), 4.53-4.43(\mathrm{~m}, 1 \mathrm{H}), 3.33(\mathrm{dd}, J=$ 14.6, $5.8 \mathrm{~Hz}, 1 \mathrm{H}), 3.23(\mathrm{dd}, J=14.6,8.9 \mathrm{~Hz}, 1 \mathrm{H}), 3.03-2.78(\mathrm{~m}, 2 \mathrm{H})$.

${ }^{19} \mathrm{~F}$ NMR $\left(471 \mathrm{MHz}, \mathrm{CDCl}_{3}\right) \delta-80.8(\mathrm{t}, J=10.2 \mathrm{~Hz}, 3 \mathrm{~F}),-112.0(\mathrm{dt}, J=269.8,14.5 \mathrm{~Hz}, 1 \mathrm{~F})$, $-113.8(\mathrm{dt}, J=269.8,14.3 \mathrm{~Hz}, 1 \mathrm{~F}),-121.8(\mathrm{~m}, 2 \mathrm{~F}),-122.9(\mathrm{~m}, 2 \mathrm{~F}),-123.7(\mathrm{~m}, 2 \mathrm{~F}),-126.2(\mathrm{~m}$, $2 \mathrm{~F})$.

${ }^{13} \mathrm{C}$ NMR $\left(125 \mathrm{MHz}, \mathrm{CDCl}_{3}\right) \delta 138.6,128.9,128.6,127.3,120-106(\mathrm{~m}), 47.0,40.8\left(\mathrm{t},{ }^{2} \mathrm{C}-\mathrm{F}=\right.$ $20.9 \mathrm{~Hz}), 19.3$.

LRMS: m/z (EI) 437 (M-I), 178, 148, 117, 103, 91, 69.

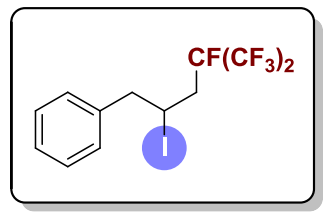

(4,5,5,5-tetrafluoro-2-iodo-4-(trifluoromethyl)pentyl)benzene (6c) 6c is a known product. ${ }^{3}$ Purified by silica gel column chromatography (petroleum ether), colorless oil, $49.6 \mathrm{mg}, 60 \%$ yield.

\footnotetext{
${ }^{1}$ Behrends, I.; Bähr, S.; Czekelius, C. Chem. Eur. J. 2016, 22, 17177-17181.

2 Yajima, T.; Ikegami, M. Eur. J. Org. Chem. 2017, 2126-2129.

${ }^{3}$ Yoshioka, E.; Kohtani, S.; Jichu, T.; Fukazawa, T.; Nagai, T.; Kawashima, A.; Takemoto, Y.; Miyabe, H. J. Org. Chem. 2016, 81, 7217-7229.
} 
${ }^{1} \mathrm{H}$ NMR $\left(500 \mathrm{MHz}, \mathrm{CDCl}_{3}\right) \delta 7.40-7.30(\mathrm{~m}, 3 \mathrm{H}), 7.25-7.17(\mathrm{~m}, 2 \mathrm{H}), 4.52-4.42(\mathrm{~m}, 1 \mathrm{H})$, $3.31(\mathrm{dd}, J=14.6,5.4 \mathrm{~Hz}, 1 \mathrm{H}), 3.17(\mathrm{dd}, J=14.6,9.2 \mathrm{~Hz}, 1 \mathrm{H}), 3.04-2.87(\mathrm{~m}, 2 \mathrm{H})$.

${ }^{19} \mathrm{~F}$ NMR $\left(471 \mathrm{MHz}, \mathrm{CDCl}_{3}\right) \delta-76.2(\mathrm{p}, J=7.1 \mathrm{~Hz}, 3 \mathrm{~F}),-77.2(\mathrm{p}, J=7.1 \mathrm{~Hz}, 3 \mathrm{~F}),-185.4$ (hept, $J=7.1 \mathrm{~Hz}, 1 \mathrm{~F})$.

${ }^{13} \mathrm{C}$ NMR $\left(125 \mathrm{MHz}, \mathrm{CDCl}_{3}\right) \delta 138.6,128.9,128.6,127.3,122-119(\mathrm{~m}), 93-90(\mathrm{~m}), 47.5$, $38.9\left(\mathrm{~d},{ }^{2} \mathrm{~J}-\mathrm{F}=18.3 \mathrm{~Hz}\right), 21.7$.

(4,5,5,6,6,6-hexafluoro-2-iodo-4-(trifluoromethyl)hexyl)benzene (6d) (2.6:1 dr)

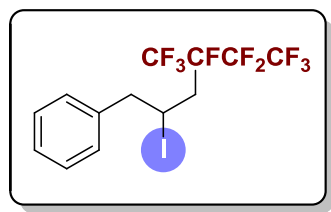

Purified by silica gel column chromatography (petroleum ether), colorless oil, $52.8 \mathrm{mg}, 57 \%$ yield.

${ }^{1} \mathbf{H}$ NMR $\left(500 \mathrm{MHz}, \mathrm{CDCl}_{3}\right) \delta 7.40-7.30(\mathrm{~m}, 3 \mathrm{H}), 7.26-7.17(\mathrm{~m}$, $2 \mathrm{H}), 4.46(\mathrm{tt}, J=13.6,5.9 \mathrm{~Hz}, 1 \mathrm{H}), 3.37-3.20(\mathrm{~m}, 1 \mathrm{H}), 3.14-2.99(\mathrm{~m}$, $2 \mathrm{H}), 2.92(\mathrm{td}, J=17.9,17.4,6.2 \mathrm{~Hz}, 1 \mathrm{H})$.

${ }^{19} \mathrm{~F}$ NMR $\left(471 \mathrm{MHz}, \mathrm{CDCl}_{3}\right) \delta-76.2(\mathrm{tq}, J=14.1,7.0 \mathrm{~Hz}, 3 \mathrm{~F}),-80.0(\mathrm{dq}, J=13.1,6.9 \mathrm{~Hz}, 3 \mathrm{~F})$, -120.9 (pent, $J=7.8 \mathrm{~Hz}, 1 \mathrm{~F}$ ), -121.1 (qd, $J=11.5,4.4 \mathrm{~Hz}, 1 \mathrm{~F}),-186.0(\mathrm{td}, J=13.2,6.3 \mathrm{~Hz}, 1 \mathrm{~F})$. ${ }^{13} \mathrm{C}$ NMR $\left(125 \mathrm{MHz}, \mathrm{CDCl}_{3}\right) \delta$ 138.6, 128.9, 128.6, 127.4, 48.0, $38.8\left(\mathrm{~d},{ }^{2} J_{\mathrm{C}-\mathrm{F}}=18.9 \mathrm{~Hz}\right), 21.0$. LRMS: m/z (EI) 337 (M-I), 281, 253, 207, 191, 126, 97, 72 (100).

Eethyl 8-(((1S,4S)-7,7-dimethyl-2-oxobicyclo[2.2.1]heptan-1-yl)sulfonyl)oxy)-2,2difluoro-4-iodooctanoate (7a) (1:1 dr)

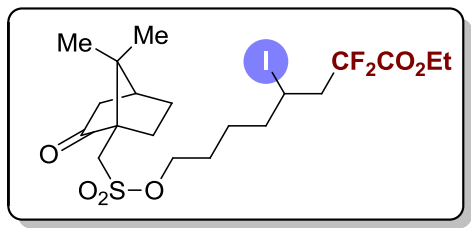

Colorless oil, $111.7 \mathrm{mg}$, 99\% yield.

${ }^{1} \mathrm{H}$ NMR $\left(500 \mathrm{MHz}, \mathrm{CDCl}_{3}\right) \delta 4.35(\mathrm{q}, J=7.1 \mathrm{~Hz}, 2 \mathrm{H}), 4.35$ $-4.19(\mathrm{~m}, 3 \mathrm{H}), 3.60(\mathrm{~d}, J=15.1 \mathrm{~Hz}, 1 \mathrm{H}), 3.00(\mathrm{~d}, J=15.1 \mathrm{~Hz}$, $1 \mathrm{H}), 2.99-2.85(\mathrm{~m}, 1 \mathrm{H}), 2.74$ (dddd, $J=18.8,15.6,12.0,7.3$ $\mathrm{Hz}, 1 \mathrm{H}), 2.49(\mathrm{ddd}, J=14.8,11.9,4.0 \mathrm{~Hz}, 1 \mathrm{H}), 2.39(\mathrm{dt}, J=$ 18.5, 4.1 Hz, 1H), $2.13(\mathrm{t}, J=4.6 \mathrm{~Hz}, 1 \mathrm{H}), 2.07(\mathrm{tp}, J=12.2,4.2,3.8 \mathrm{~Hz}, 1 \mathrm{H}), 1.97(\mathrm{~d}, J=18.5$ $\mathrm{Hz}, 1 \mathrm{H}), 1.90-1.73(\mathrm{~m}, 4 \mathrm{H}), 1.73-1.63(\mathrm{~m}, 2 \mathrm{H}), 1.59-1.49(\mathrm{~m}, 1 \mathrm{H}), 1.45$ (ddd, $J=13.0,9.4$, $4.0 \mathrm{~Hz}, 1 \mathrm{H}), 1.38(\mathrm{t}, J=7.1 \mathrm{~Hz}, 3 \mathrm{H}), 1.12(\mathrm{~s}, 3 \mathrm{H}), 0.88(\mathrm{~s}, 3 \mathrm{H})$.

${ }^{19} \mathrm{~F}$ NMR $\left(471 \mathrm{MHz}, \mathrm{CDCl}_{3}\right) \delta-102.1(\mathrm{~d}, J=263.2 \mathrm{~Hz}),-106.8(\mathrm{~d}, J=263.2,3.4 \mathrm{~Hz})$.

${ }^{13} \mathrm{C}$ NMR $\left(125 \mathrm{MHz}, \mathrm{CDCl}_{3}\right) \delta$ 214.6, $163.3\left(\mathrm{t},{ }^{2} \mathrm{~J}_{\mathrm{C}-\mathrm{F}}=32.2 \mathrm{~Hz}\right), 115.1\left(7,{ }^{1} \mathrm{~J}_{\mathrm{C}-\mathrm{F}}=253.1 \mathrm{~Hz}\right), 70.0$, 63.3, 57.9, 48.0, 46.7, $45.2\left(\mathrm{t},{ }^{2} \mathrm{~J}_{\mathrm{C}-\mathrm{F}}=23.2 \mathrm{~Hz}\right), 42.7,42.5,39.6,28.2,26.8,25.7,24.8,22.2,19.7$, 19.6, 13.9 .

HRMS (ESI) m/z: [M+Na $]^{+}$calcd for $\mathrm{C}_{20} \mathrm{H}_{31} \mathrm{~F}_{2} \mathrm{IO}_{6} \mathrm{Na}$ : 587.0746; found: 587.0750.

Ethyl 2,2-difluoro-4-iodo-7-(((8R,9S,13S,14S)-13-methyl-17-oxo-7,8,9,11,12,13,14,15,16,17 -decahydro-6H-cyclopenta[a]phenanthren-3-yl)oxy)heptanoate (7b)

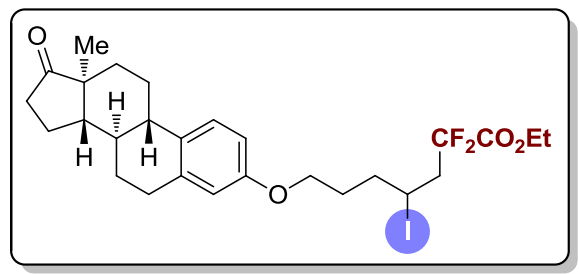

Colorless oil, $116.4 \mathrm{mg}, 99 \%$ yield.

${ }^{1} \mathrm{H}$ NMR $\left(500 \mathrm{MHz}, \mathrm{CDCl}_{3}\right) \delta 7.21(\mathrm{~d}, J=8.6 \mathrm{~Hz}$, $1 \mathrm{H}), 6.72(\mathrm{dd}, J=8.6,2.8 \mathrm{~Hz}, 1 \mathrm{H}), 6.66(\mathrm{~d}, J=2.8 \mathrm{~Hz}$, $1 \mathrm{H}), 4.36(\mathrm{q}, J=7.2 \mathrm{~Hz}, 2 \mathrm{H}), 4.31(\mathrm{dq}, J=6.9,4.0 \mathrm{~Hz}$, $1 \mathrm{H}), 3.98(\mathrm{q}, J=6.4 \mathrm{~Hz}, 2 \mathrm{H}), 3.05-2.86(\mathrm{~m}, 3 \mathrm{H})$, 2.80 (dddd, $J=18.1,15.7,12.4,7.1 \mathrm{~Hz}, 1 \mathrm{H}), 2.52(\mathrm{dd}$, $J=19.0,8.7 \mathrm{~Hz}, 1 \mathrm{H}), 2.44-2.39(\mathrm{~m}, 1 \mathrm{H}), 2.27(\mathrm{td}, J=10.8,4.6 \mathrm{~Hz}, 1 \mathrm{H}), 2.16(\mathrm{dt}, J=18.6,8.9$ $\mathrm{Hz}, 1 \mathrm{H}), 2.11-1.95(\mathrm{~m}, 6 \mathrm{H}), 1.93-1.86(\mathrm{~m}, 1 \mathrm{H}), 1.70-1.59(\mathrm{~m}, 2 \mathrm{H}), 1.59-1.49(\mathrm{~m}, 3 \mathrm{H})$, $1.48-1.42(\mathrm{~m}, 1 \mathrm{H}), 1.39(\mathrm{t}, J=7.2 \mathrm{~Hz}, 3 \mathrm{H}), 0.93(\mathrm{~s}, 3 \mathrm{H})$.

${ }^{19}$ F NMR $\left(471 \mathrm{MHz}, \mathrm{CDCl}_{3}\right) \delta-102.1(\mathrm{~d}, J=263.5 \mathrm{~Hz}),-106.8(\mathrm{~d}, J=263.5 \mathrm{~Hz})$.

${ }^{13} \mathrm{C}$ NMR $\left(125 \mathrm{MHz}, \mathrm{CDCl}_{3}\right) \delta 163.4\left(\mathrm{t},{ }^{2} \mathrm{JC}_{\mathrm{C}-\mathrm{F}}=32.3 \mathrm{~Hz}\right), 156.8,137.7,132.1,126.3,115.09(\mathrm{t}$, 
$\left.{ }^{1} J_{C-F}=253.3 \mathrm{~Hz}\right), 115.06,114.5,112.1,66.6,63.2,50.4,48.0,45.3\left(\mathrm{t},{ }^{2} J_{C-F}=23.2 \mathrm{~Hz}\right), 43.9,38.3$, $37.2,35.8,31.5,29.6,29.5,26.5,25.9,22.4\left(\mathrm{t},{ }^{3}{ }_{\mathrm{C}-\mathrm{F}}=4.0 \mathrm{~Hz}\right), 21.5,13.9,13.8$.

HRMS (ESI) m/z: $[\mathrm{M}+\mathrm{H}]^{+}$calcd for $\mathrm{C}_{27} \mathrm{H}_{36} \mathrm{~F}_{2} \mathrm{IO}_{4}$ : 589.1621; found: 589.1605 .

1-Ethyl 7-((1S,2R,4S)-1,3,3-trimethylbicyclo[2.2.1]heptan-2-yl) 2,2-difluoro-4-iodoheptanedioate $(7 \mathrm{c})(1: 1 \mathrm{dr})$

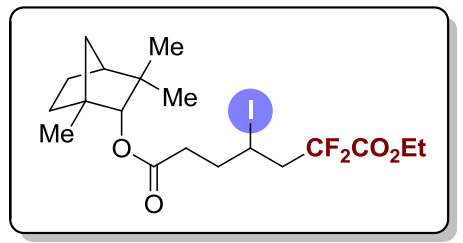

Colorless oil, $96.3 \mathrm{mg}$, $99 \%$ yield.

${ }^{1} \mathbf{H}$ NMR $\left(500 \mathrm{MHz}, \mathrm{CDCl}_{3}\right) \delta 4.38-4.28(\mathrm{~m}, 2 \mathrm{H}), 4.35$ (q, $J$ $=7.2 \mathrm{~Hz}, 2 \mathrm{H}), 3.06-2.89(\mathrm{~m}, 1 \mathrm{H}), 2.77(\mathrm{dddd}, J=18.7,15.6$, 12.1, $7.2 \mathrm{~Hz}, 1 \mathrm{H}), 2.67-2.57(\mathrm{~m}, 1 \mathrm{H}), 2.53(\mathrm{dddd}, J=16.2$, 8.6, 6.8, $3.9 \mathrm{~Hz}, 1 \mathrm{H}), 2.15$ (dddt, $J=12.6,10.2,5.7,2.8 \mathrm{~Hz}$, $1 \mathrm{H}), 2.11-2.02(\mathrm{~m}, 1 \mathrm{H}), 1.84-1.67(\mathrm{~m}, 4 \mathrm{H}), 1.59(\mathrm{dt}, J=10.3,2.1 \mathrm{~Hz}, 1 \mathrm{H}), 1.46(\mathrm{tt}, J=12.3$, $4.3 \mathrm{~Hz}, 1 \mathrm{H}), 1.38(\mathrm{t}, J=7.2 \mathrm{~Hz}, 3 \mathrm{H}), 1.20(\mathrm{dd}, J=10.3,1.5 \mathrm{~Hz}, 1 \mathrm{H}), 1.14-1.03(\mathrm{~m}, 1 \mathrm{H}), 1.11$ $(\mathrm{s}, 3 \mathrm{H}), 1.05(\mathrm{~s}, 3 \mathrm{H}), 0.78(\mathrm{~s}, 3 \mathrm{H})$.

${ }^{19}$ F NMR $\left(471 \mathrm{MHz}, \mathrm{CDCl}_{3}\right) \delta-102.0(\mathrm{~d}, J=264.2 \mathrm{~Hz}),-106.5(\mathrm{~d}, J=264.2 \mathrm{~Hz})$.

${ }^{13} \mathrm{C}$ NMR $\left(125 \mathrm{MHz}, \mathrm{CDCl}_{3}\right) \delta 172.5,163.3\left(\mathrm{t},{ }^{2} \mathrm{~J}-\mathrm{F}=32.2 \mathrm{~Hz}\right), 115.0\left(\mathrm{t},{ }^{1} \mathrm{JC}_{\mathrm{C}-\mathrm{F}}=252.8 \mathrm{~Hz}\right), 86.6$, 63.3, 48.3, 48.2, $45.3\left(\mathrm{t},{ }^{2} \mathrm{~J}_{\mathrm{C}-\mathrm{F}}=23.3 \mathrm{~Hz}\right), 41.3,39.4,35.5,34.6,29.6,26.6,25.7,21.5\left(\mathrm{t},{ }^{3} \mathrm{~J}_{\mathrm{C}-\mathrm{F}}=4.1\right.$ $\mathrm{Hz}), 20.1,19.3,13.8$.

HRMS (ESI) m/z: [M+K] $]^{+}$calcd for $\mathrm{C}_{19} \mathrm{H}_{22} \mathrm{~F}_{2} \mathrm{IO}_{4} \mathrm{~K}$ : 525.0710; found: 525.0677.

Ethyl 2,2-difluoro-4-iodo-8-((2-(4-isobutylphenyl)propanoyl)oxy)octanoate (7d)

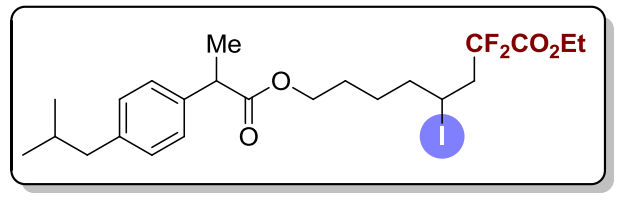

Purified by silica gel column chromatography (petroleum ether/ethyl acetate $=20 / 1$ ), colorless oil, $86.1 \mathrm{mg}, 82 \%$ yield.

${ }^{1} \mathbf{H}$ NMR $\left(500 \mathrm{MHz}, \mathrm{CDCl}_{3}\right) \delta 7.23(\mathrm{~d}, J=8.1 \mathrm{~Hz}$, 2H), $7.12(\mathrm{~d}, J=8.1 \mathrm{~Hz}, 2 \mathrm{H}), 4.37(\mathrm{q}, J=7.2 \mathrm{~Hz}, 2 \mathrm{H}), 4.18(\mathrm{dtd}, J=8.7,6.8,4.1 \mathrm{~Hz}, 1 \mathrm{H}), 4.12$ $-4.07(\mathrm{~m}, 2 \mathrm{H}), 3.71(\mathrm{q}, J=7.2 \mathrm{~Hz}, 1 \mathrm{H}), 2.91(\mathrm{dtdd}, J=18.8,15.8,6.4,1.8 \mathrm{~Hz}, 1 \mathrm{H}), 2.79-2.64$ $(\mathrm{m}, 1 \mathrm{H}), 2.47(\mathrm{~d}, J=7.2 \mathrm{~Hz}, 2 \mathrm{H}), 1.87(\mathrm{dp}, J=13.4,6.6 \mathrm{~Hz}, 1 \mathrm{H}), 1.82-1.53(\mathrm{~m}, 5 \mathrm{H}), 1.51(\mathrm{~d}$, $J=7.2 \mathrm{~Hz}, 3 \mathrm{H}), 1.45-1.32(\mathrm{~m}, 1 \mathrm{H}), 1.40(\mathrm{t}, J=7.2 \mathrm{~Hz}, 3 \mathrm{H}), 0.92(\mathrm{~d}, J=6.6 \mathrm{~Hz}, 6 \mathrm{H})$.

${ }^{19}$ F NMR $\left(471 \mathrm{MHz}, \mathrm{CDCl}_{3}\right) \delta-102.0(\mathrm{~d}, J=263.2 \mathrm{~Hz}),-107.0(\mathrm{~d}, J=263.2 \mathrm{~Hz})$.

${ }^{13} \mathrm{C}$ NMR $\left(125 \mathrm{MHz}, \mathrm{CDCl}_{3}\right) \delta$ 174.7, $163.4\left(\mathrm{t},{ }^{2} \mathrm{~J} C-\mathrm{F}=32.2 \mathrm{~Hz}\right), 140.5,137.8,129.3,127.1$, $115.1\left(t,{ }^{1} J_{C-F}=252.7 \mathrm{~Hz}\right), 64.1,63.2,45.3\left(\mathrm{t},{ }^{2} \mathrm{~J}_{C-F}=23.2 \mathrm{~Hz}\right), 45.2,45.0,39.8,30.2,27.5,26.0$, $22.5\left(\mathrm{t},{ }^{3} \mathrm{C}_{\mathrm{C}-\mathrm{F}}=3.5 \mathrm{~Hz}\right), 22.4,18.4,13.9$.

HRMS (ESI) m/z: [M+K] $]^{+}$calcd for $\mathrm{C}_{23} \mathrm{H}_{33} \mathrm{~F}_{2} \mathrm{IO}_{4} \mathrm{~K}$ : 577.1023; found: 577.1045.

7-((3S,5S,8R,9S,10S,13R,14S,17R)-10,13-dimethyl-17-((R)-6-methylheptan-2-yl)hexadeca hydro-1H-cyclopenta[a]phenanthren-3-yl) 1-ethyl 2,2-difluoro-4-iodoheptanedioate (7e) (1:1 dr)

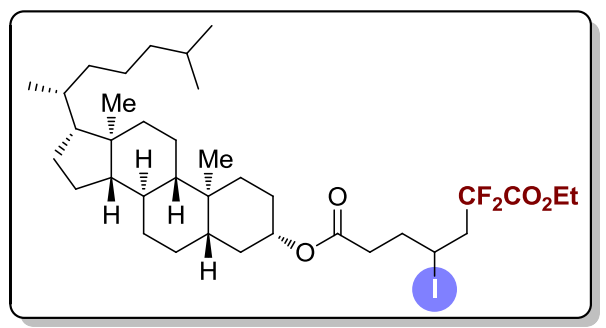

Purified by silica gel column chromatography (petroleum ether/ethyl acetate $=3 / 1$ ), pale yellow amorphous solid, $36.6 \mathrm{mg}$, 51\% yield.

${ }^{1} \mathrm{H}$ NMR $\left(500 \mathrm{MHz}, \mathrm{CDCl}_{3}\right) \delta^{1} \mathrm{H}$ NMR $(500 \mathrm{MHz}$, Chloroform- $d$ ) $\delta 4.73(\mathrm{tt}, J=11.0,4.9 \mathrm{~Hz}, 1 \mathrm{H}), 4.37$ $(\mathrm{q}, J=7.1 \mathrm{~Hz}, 2 \mathrm{H}), 4.30(\mathrm{dtd}, J=10.4,6.8,3.7 \mathrm{~Hz}$, $1 \mathrm{H}), 2.97(\mathrm{dtd}, J=18.9,15.5,6.4 \mathrm{~Hz}, 1 \mathrm{H}), 2.77$ (dddd, $J=18.8,15.5,12.2,7.3 \mathrm{~Hz}, 1 \mathrm{H}), 2.56(\mathrm{ddd}, J=16.5,8.6,5.5 \mathrm{~Hz}, 1 \mathrm{H}), 2.46(\mathrm{dt}, J=16.2$, $7.7 \mathrm{~Hz}, 1 \mathrm{H}), 2.15(\mathrm{ddt}, J=15.1,7.8,3.8 \mathrm{~Hz}, 1 \mathrm{H}), 2.06(\mathrm{ddt}, J=14.8,9.6,4.6 \mathrm{~Hz}, 1 \mathrm{H}), 1.98(\mathrm{dt}$, 
$J=12.6,3.4 \mathrm{~Hz}, 1 \mathrm{H}), 1.84(\mathrm{tdd}, J=14.9,8.2,4.3 \mathrm{~Hz}, 2 \mathrm{H}), 1.75(\mathrm{dt}, J=13.3,3.7 \mathrm{~Hz}, 1 \mathrm{H}), 1.67$ $(\mathrm{dq}, J=13.0,3.4 \mathrm{~Hz}, 1 \mathrm{H}), 1.64-1.46(\mathrm{~m}, 6 \mathrm{H}), 1.40(\mathrm{t}, J=7.2 \mathrm{~Hz}, 3 \mathrm{H}), 1.40-1.23(\mathrm{~m}, 10 \mathrm{H})$, $1.22-0.96(\mathrm{~m}, 10 \mathrm{H}), 0.92(\mathrm{~d}, J=6.6 \mathrm{~Hz}, 3 \mathrm{H}), 0.89(\mathrm{~d}, J=2.4 \mathrm{~Hz}, 3 \mathrm{H}), 0.88(\mathrm{~d}, J=2.4 \mathrm{~Hz}, 3 \mathrm{H})$, $0.84(\mathrm{~s}, 3 \mathrm{H}), 0.67(\mathrm{~s}, 3 \mathrm{H})$.

${ }^{19}$ F NMR $\left(471 \mathrm{MHz}, \mathrm{CDCl}_{3}\right) \delta-102.2(\mathrm{~d}, J=264.2 \mathrm{~Hz}),-106.7(\mathrm{~d}, J=264.2 \mathrm{~Hz})$.

${ }^{13} \mathrm{C}$ NMR $\left(125 \mathrm{MHz}, \mathrm{CDCl}_{3}\right) \delta 171.6,163.3\left(\mathrm{t},{ }^{2} \mathrm{C}_{\mathrm{C}-F}=32.3 \mathrm{~Hz}\right), 115.0\left(\mathrm{t},{ }^{1} \mathrm{JC}_{\mathrm{C}-\mathrm{F}}=252.8 \mathrm{~Hz}\right), 74.2$, 63.3, 56.4, 56.3, 54.2, $45.4\left(\mathrm{t},{ }^{2} \mathrm{~J} \mathrm{C}-\mathrm{F}=23.3 \mathrm{~Hz}\right), 44.7,42.6,40.0,39.5,36.7,36.2,35.8,35.52$, $35.46,34.9,34.01,33.98,32.0,28.6,28.2,28.0,27.4,24.2,23.8,22.8,22.6,21.5,21.2,18.7,13.9$, $12.2,12.1$.

HRMS (ESI) m/z: [M+H] $]^{+}$calcd for $\mathrm{C}_{36} \mathrm{H}_{60} \mathrm{~F}_{2} \mathrm{IO}_{4}: 721.3499$; found: 721.3436 .

Ethyl 2,2-difluoro-4-iodo-7-(N-(((1R,4aS,10aR)-7-isopropyl-1,4a-dimethyl-1,2,3,4,4a,9,10, 10a-octahydrophenanthren-1-yl)methyl)-4-methylphenylsulfonamido)heptanoate (7f) (1:1 dr)

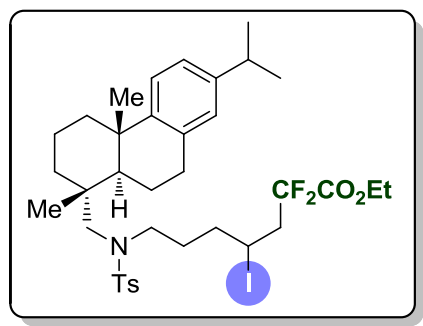

Purified by silica gel column chromatography (petroleum ether/ethyl acetate $=3 / 1$ ), pale yellow amorphous solid, 131.7 $\mathrm{mg}, 87 \%$ yield.

${ }^{1} \mathbf{H}$ NMR $\left(500 \mathrm{MHz}, \mathrm{CDCl}_{3}\right) \delta 7.71(\mathrm{~d}, J=7.9 \mathrm{~Hz}, 2 \mathrm{H}), 7.33(\mathrm{~d}$, $J=7.9 \mathrm{~Hz}, 2 \mathrm{H}), 7.18(\mathrm{~d}, J=8.1 \mathrm{~Hz}, 1 \mathrm{H}), 7.00(\mathrm{~d}, J=8.1 \mathrm{~Hz}, 1 \mathrm{H})$, $6.91(\mathrm{~s}, 1 \mathrm{H}), 4.36(\mathrm{q}, J=7.1 \mathrm{~Hz}, 2 \mathrm{H}), 4.11(\mathrm{p}, J=6.5 \mathrm{~Hz}, 1 \mathrm{H})$, $3.27-2.75(\mathrm{~m}, 8 \mathrm{H}), 2.62(\mathrm{dddd}, J=18.4,15.1,11.6,7.0 \mathrm{~Hz}$,

$1 \mathrm{H}), 2.44(\mathrm{~s}, 3 \mathrm{H}), 2.31(\mathrm{~d}, J=12.8 \mathrm{~Hz}, 1 \mathrm{H}), 1.95-1.69(\mathrm{~m}, 6 \mathrm{H}), 1.66-1.51(\mathrm{~m}, 6 \mathrm{H}), 1.39(\mathrm{t}, J$ $=7.1 \mathrm{~Hz}, 3 \mathrm{H}), 1.25(\mathrm{~d}, J=3.3 \mathrm{~Hz}, 6 \mathrm{H}), 1.24(\mathrm{~s}, 3 \mathrm{H}), 1.03(\mathrm{~s}, 3 \mathrm{H})$.

${ }^{19}$ F NMR $\left(471 \mathrm{MHz}, \mathrm{CDCl}_{3}\right) \delta-102.1(\mathrm{~d}, J=263.0 \mathrm{~Hz}),-107.0(\mathrm{~d}, J=263.0 \mathrm{~Hz})$.

${ }^{13} \mathrm{C}$ NMR $\left(125 \mathrm{MHz}, \mathrm{CDCl}_{3}\right) \delta 163.3\left(\mathrm{t},{ }^{2} \mathrm{JC}-\mathrm{F}=32.4 \mathrm{~Hz}\right), 147.3,145.6,143.3,136.5,134.4$, $129.7,127.5,126.8,123.9,123.8,115.0\left(\mathrm{t},{ }^{1} \mathrm{JC}_{\mathrm{C}-\mathrm{F}}=252.8 \mathrm{~Hz}\right), 63.3,59.6,50.5,45.3,44.9\left(\mathrm{t},{ }^{2} \mathrm{~J} C-\mathrm{F}=\right.$ $24.0 \mathrm{~Hz}), 39.0,38.1,37.8,37.6,37.2,33.4,29.8,28.1,25.6,24.0,21.5,21.4\left(\mathrm{t},{ }^{4} \mathrm{~J} C-F=4.2 \mathrm{~Hz}\right)$, $19.3,18.9,18.6,13.9$.

HRMS (ESI) m/z: [M+H] $]^{+}$calcd for $\mathrm{C}_{36} \mathrm{H}_{50} \mathrm{~F}_{2} \mathrm{INO}_{4} \mathrm{~S}$ : 758.2546; found: 758.2562.

\section{Mechanism Studies}

\subsection{TEMPO Capture Experiment}

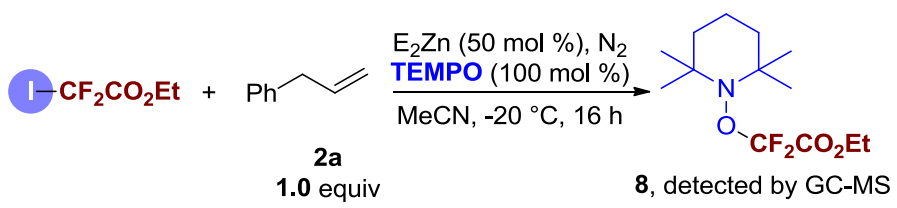

An oven-dried Schlenk tube was charged with a stir bar. The tube was degased and refilled with nitrogen for 3 times, and then a solution of $2 \mathrm{a}(23.6 \mathrm{mg}, 0.2 \mathrm{mmol})$, ethyl iododifluoroacetate 1a (49.9 mg, $0.2 \mathrm{mmol}, 1.0$ equiv) and TEMPO (31.2 mg, $0.2 \mathrm{mmol}, 1.0$ equiv) in acetonitrile $(2 \mathrm{~mL})$ was injected to the tube via a syringe. The reaction mixture was cooled to $-20^{\circ} \mathrm{C}$. After stirring for 3 minutes, a solution of diethylzinc $(1.0 \mathrm{~mol} / \mathrm{L}$ in 
hexane, $0.1 \mathrm{~mL}, 0.5$ equiv) was injected to the reaction mixture via a syringe. After stirring at $-20{ }^{\circ} \mathrm{C}$ for 16 hours, the reaction was quenched with saturated $\mathrm{NH}_{4} \mathrm{Cl}$ and extracted with ethyl acetate $(3 \times 100 \mathrm{~mL})$. The combined organic layers were dried with $\mathrm{Na}_{2} \mathrm{SO}_{4}$, filtered through a short pad of silica gel, and concentrated under reduced pressure. The residue was submitted to GC-MS analysis.

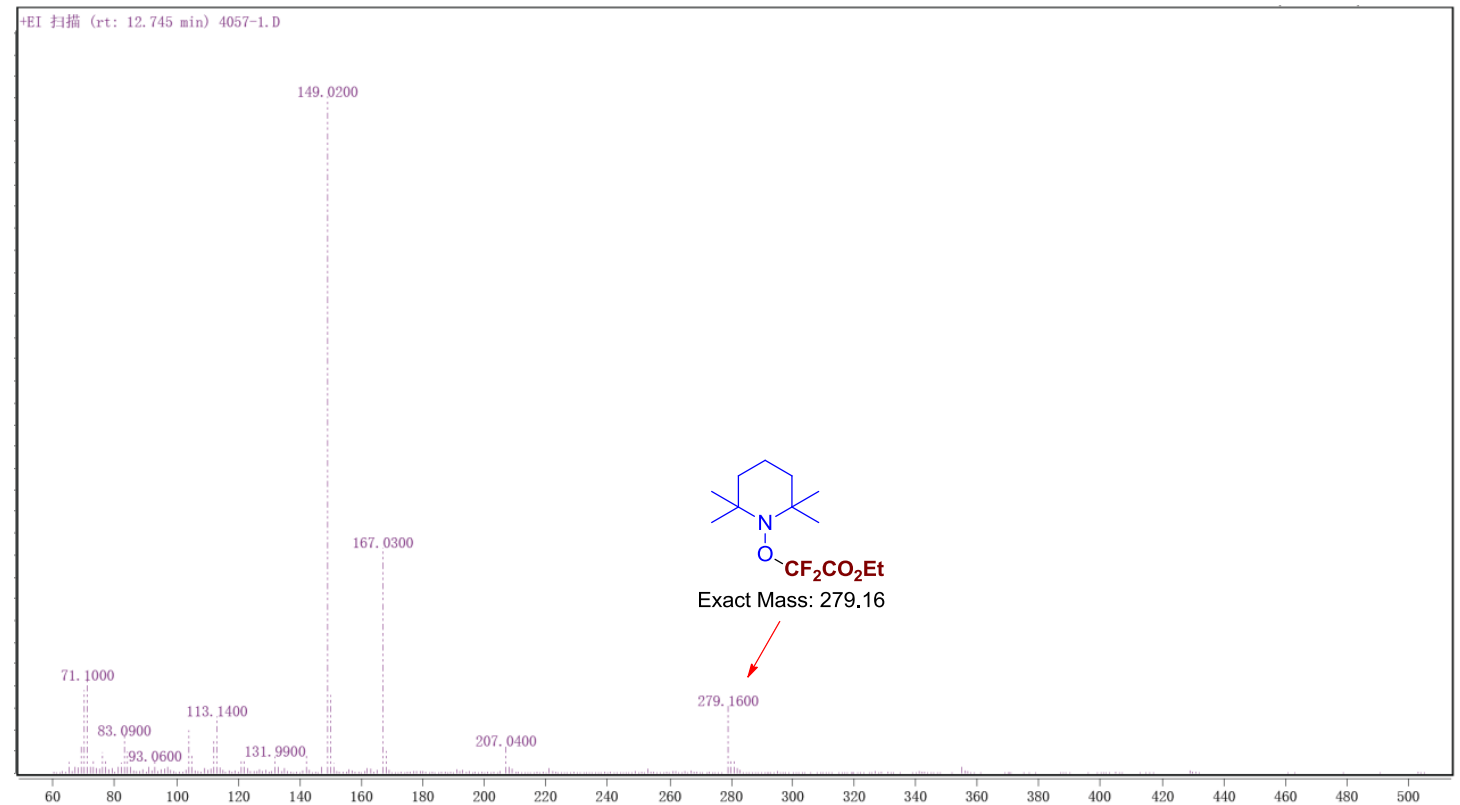

\subsection{Radical Ring-Opening Experiment}

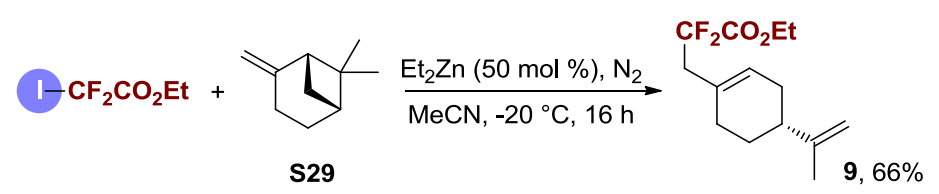

An oven-dried Schlenk tube was charged with a stir bar. The tube was degased and refilled with nitrogen for 3 times, and then a solution of $\alpha$-Pinene $\mathbf{S 2 9}(27.2 \mathrm{mg}, 0.2 \mathrm{mmol})$ and ethyl iododifluoroacetate $\mathbf{1 a}(49.9 \mathrm{mg}, 0.2 \mathrm{mmol}, 1.0$ equiv) in acetonitrile ( $2 \mathrm{~mL}$ ) was injected to the tube via a syringe. The reaction mixture was cooled to $-20{ }^{\circ} \mathrm{C}$. After stirring for 3 minutes, a solution of diethylzinc ( $1.0 \mathrm{~mol} / \mathrm{L}$ in hexane, $0.1 \mathrm{~mL}, 0.5$ equiv) was injected to the reaction mixture via a syringe. After stirring at $-20^{\circ} \mathrm{C}$ for 16 hours, the reaction was quenched with saturated $\mathrm{NH}_{4} \mathrm{Cl}$ and extracted with ethyl acetate $(3 \times$ $100 \mathrm{~mL}$ ). The combined organic layers were dried with $\mathrm{Na}_{2} \mathrm{SO}_{4}$, filtered through a short pad of silica gel, and concentrated under reduced pressure. The residue was purified by silica gel column chromatography (petroleum ether/ethyl acetate $=100 / 1$ ) to afford the corresponding pure product as a colorless oil, $33.9 \mathrm{mg}, 66 \%$ yield.

(R)-ethyl 2,2-difluoro-3-(4-(prop-1-en-2-yl)cyclohex-1-en-1-yl)propanoate (9)

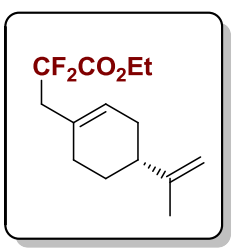

${ }^{1} \mathrm{H}$ NMR $\left(500 \mathrm{MHz}, \mathrm{CDCl}_{3}\right) \delta 5.70-5.59(\mathrm{~m}, 1 \mathrm{H}), 4.74(\mathrm{t}, J=1.8 \mathrm{~Hz}, 1 \mathrm{H})$, $4.71(\mathrm{dt}, J=1.8,0.9 \mathrm{~Hz}, 1 \mathrm{H}), 4.33(\mathrm{q}, J=7.1 \mathrm{~Hz}, 2 \mathrm{H}), 2.74(\mathrm{t}, J=16.6 \mathrm{~Hz}$, $2 \mathrm{H}), 2.24-2.06(\mathrm{~m}, 4 \mathrm{H}), 2.02-1.91(\mathrm{~m}, 1 \mathrm{H}), 1.83(\mathrm{ddt}, J=12.7,5.2,2.5 \mathrm{~Hz}$, $1 \mathrm{H}), 1.74(\mathrm{~s}, 3 \mathrm{H}), 1.53-1.41(\mathrm{~m}, 1 \mathrm{H}), 1.36(\mathrm{t}, J=7.1 \mathrm{~Hz}, 3 \mathrm{H})$.

${ }^{19} \mathrm{~F}$ NMR $\left(471 \mathrm{MHz}, \mathrm{CDCl}_{3}\right) \delta-102.8(\mathrm{~d}, J=254.0 \mathrm{~Hz}),-103.5(\mathrm{~d}, J=254.0$ 
$\mathrm{Hz})$.

${ }^{13} \mathrm{C}$ NMR $\left(125 \mathrm{MHz}, \mathrm{CDCl}_{3}\right) \delta 164.2\left(\mathrm{t},{ }^{2} J_{\mathrm{C}-F}=32.8 \mathrm{~Hz}\right), 149.5,128.3,128.2\left(\mathrm{t},{ }^{3} \mathrm{~J}_{\mathrm{C}-\mathrm{F}}=3.9 \mathrm{~Hz}\right)$, $116.1\left(\mathrm{t},{ }^{1} J_{C-F}=252.4 \mathrm{~Hz}\right), 108.7,62.6,42.7\left(\mathrm{t},{ }^{2} \mathrm{C}_{\mathrm{C}-\mathrm{F}}=23.2 \mathrm{~Hz}\right), 40.4,30.9,29.5,27.7,20.7,14.0$.

HRMS (ESI) m/z: [M+H] $]^{+}$calcd for $\mathrm{C}_{14} \mathrm{H}_{21} \mathrm{~F}_{2} \mathrm{O}_{2}:$ 259.1504; found: 259.1502 .

\subsection{Radical Ring-Closure Experiment}

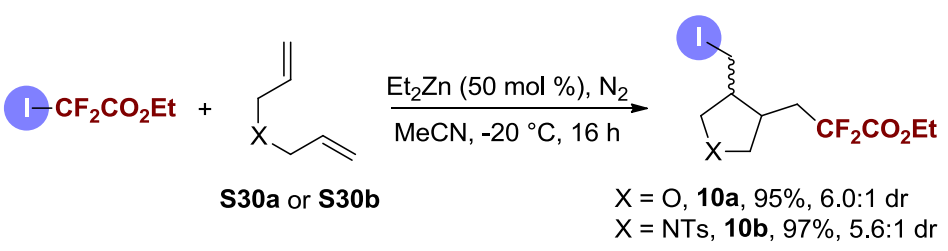

An oven-dried Schlenk tube was charged with a stir bar. The tube was degased and refilled with nitrogen for 3 times, and then a solution of $\mathbf{S 3 0}(27.2 \mathrm{mg}, 0.2 \mathrm{mmol})$ and ethyl iododifluoroacetate $1 \mathrm{a}(49.9 \mathrm{mg}, 0.2 \mathrm{mmol}, 1.0$ equiv) in acetonitrile $(2 \mathrm{~mL})$ was injected to the tube via a syringe. The reaction mixture was cooled to $-20{ }^{\circ} \mathrm{C}$. After stirring for 3 minutes, a solution of diethylzinc $(1.0 \mathrm{~mol} / \mathrm{L}$ in hexane, $0.1 \mathrm{~mL}, 0.5$ equiv) was injected to the reaction mixture via a syringe. After stirring at $-20^{\circ} \mathrm{C}$ for 16 hours, the reaction was quenched with saturated $\mathrm{NH}_{4} \mathrm{Cl}$ and extracted with ethyl acetate $(3 \times$ $100 \mathrm{~mL}$ ). The combined organic layers were dried with $\mathrm{Na}_{2} \mathrm{SO}_{4}$, filtered through a short pad of silica gel, and concentrated under reduced pressure. The residue was purified by silica gel column chromatographyto afford the corresponding pure product.

Ethyl 2,2-difluoro-3-(4-(iodomethyl)tetrahydrofuran-3-yl)propanoate (10a) (6:1 dr)

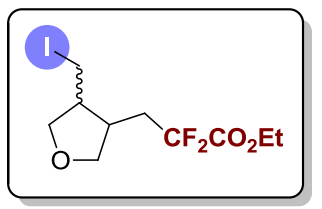

Purified by silica gel column chromatography (petroleum ether/ethyl acetate $=3 / 1)$, colorless oil, $66.1 \mathrm{mg}$, 95\% yield.

${ }^{1}$ H NMR $\left(500 \mathrm{MHz}, \mathrm{CDCl}_{3}\right) \delta 4.34(\mathrm{q}, J=7.1 \mathrm{~Hz}, 2 \mathrm{H}), 4.03-3.93(\mathrm{~m}$, $2 \mathrm{H}), 3.73(\mathrm{dd}, J=9.0,4.5 \mathrm{~Hz}, 1 \mathrm{H}), 3.60(\mathrm{t}, J=8.4 \mathrm{~Hz}, 1 \mathrm{H}), 3.23(\mathrm{ddd}, J$ $=9.8,5.1,1.1 \mathrm{~Hz}, 1 \mathrm{H}), 3.06(\mathrm{dd}, J=10.8,9.7 \mathrm{~Hz}, 1 \mathrm{H}), 2.76(\mathrm{tdd}, J=$ 11.1, 6.5, $4.9 \mathrm{~Hz}, 1 \mathrm{H}), 2.55(\mathrm{dqd}, J=9.6,7.4,4.7 \mathrm{~Hz}, 1 \mathrm{H}), 2.30(\mathrm{dtd}, J=22.7,14.5,4.7 \mathrm{~Hz}$, $1 \mathrm{H}), 2.06$ (dddd, $J=21.6,14.8,11.0,9.5 \mathrm{~Hz}, 1 \mathrm{H}), 1.36(\mathrm{t}, J=7.1 \mathrm{~Hz}, 3 \mathrm{H})$.

${ }^{19}$ F NMR $\left(471 \mathrm{MHz}, \mathrm{CDCl}_{3}\right) \delta-104.2(\mathrm{~d}, J=261.0 \mathrm{~Hz}),-106.3(\mathrm{~d}, J=261.0 \mathrm{~Hz})$.

${ }^{13} \mathrm{C}$ NMR $\left(125 \mathrm{MHz}, \mathrm{CDCl}_{3}\right) \delta 163.7\left(\mathrm{t},{ }^{2} J_{\mathrm{C}-F}=32.5 \mathrm{~Hz}\right), 115.5\left(\mathrm{t},{ }^{1} \mathrm{JC}_{\mathrm{C}-\mathrm{F}}=251.5 \mathrm{~Hz}\right), 73.4,71.5$ $\left(\mathrm{t},{ }^{3} \mathrm{C}_{C-F}=2.1 \mathrm{~Hz}\right), 63.1,45.1,36.5,31.8\left(\mathrm{t},{ }^{2} \mathrm{C}_{\mathrm{C}-\mathrm{F}}=23.3 \mathrm{~Hz}\right), 13.9,3.4$.

HRMS (ESI) m/z: [M+H] $]^{+}$calcd for $\mathrm{C}_{10} \mathrm{H}_{15} \mathrm{~F}_{2} \mathrm{IO}_{3}: 349.0107$; found: 349.0100 .

Ethyl 2,2-difluoro-3-(4-(iodomethyl)-1-tosylpyrrolidin-3-yl)propanoate (10b) (5.6:1 dr)

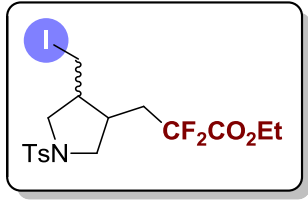

Purified by silica gel column chromatography (petroleum ether/ethyl acetate $=3 / 1)$, colorless oil, $97.3 \mathrm{mg}$, 97\% yield.

${ }^{1} \mathrm{H}$ NMR $\left(500 \mathrm{MHz}, \mathrm{CDCl}_{3}\right) \delta 7.73(\mathrm{~d}, J=8.2 \mathrm{~Hz}, 2 \mathrm{H}), 7.35(\mathrm{~d}, J=8.2$ $\mathrm{Hz}, 2 \mathrm{H}), 4.31(\mathrm{q}, J=7.2 \mathrm{~Hz}, 2 \mathrm{H}), 3.48-3.40(\mathrm{~m}, 2 \mathrm{H}), 3.36(\mathrm{dd}, J=$ 10.6, $3.9 \mathrm{~Hz}, 1 \mathrm{H}), 3.11(\mathrm{dd}, J=10.2,8.1 \mathrm{~Hz}, 1 \mathrm{H}), 3.08-3.04(\mathrm{~m}, 1 \mathrm{H})$, $2.62(\mathrm{dd}, J=10.8,9.3 \mathrm{~Hz}, 1 \mathrm{H}), 2.59-2.53(\mathrm{~m}, 1 \mathrm{H}), 2.46-2.38(\mathrm{~m}, 1 \mathrm{H}), 2.44(\mathrm{~s}, 3 \mathrm{H}), 2.14$ (dddd, $J=22.6,14.9,13.4,5.0 \mathrm{~Hz}, 1 \mathrm{H}), 1.97-1.83(\mathrm{~m}, 1 \mathrm{H}), 1.34(\mathrm{t}, J=7.2 \mathrm{~Hz}, 3 \mathrm{H})$.

${ }^{19} \mathrm{~F}$ NMR $\left(471 \mathrm{MHz}, \mathrm{CDCl}_{3}\right) \delta-104.3(\mathrm{~d}, J=262.4 \mathrm{~Hz}),-106.2(\mathrm{~d}, J=262.4 \mathrm{~Hz})$.

${ }^{13} \mathrm{C}$ NMR $\left(125 \mathrm{MHz}, \mathrm{CDCl}_{3}\right) \delta 163.4\left(\mathrm{t},{ }^{2} \mathrm{~J}_{\mathrm{C}-\mathrm{F}}=32.3 \mathrm{~Hz}\right), 143.7,133.4,129.8,127.3,115.2(\mathrm{t}$, 
$\left.{ }^{1} J_{C-F}=251.9 \mathrm{~Hz}\right), 63.2,52.8,50.9,44.7,35.6\left(\mathrm{t},{ }^{3} \mathrm{C}_{\mathrm{C}-F}=2.9 \mathrm{~Hz}\right), 31.9\left(\mathrm{t},{ }^{2} J_{C-F}=23.3 \mathrm{~Hz}\right), 21.5$, 13.8, 2.4.

HRMS (ESI) m/z: [M+H] $]^{+}$calcd for $\mathrm{C}_{17} \mathrm{H}_{23} \mathrm{~F}_{2} \mathrm{INO}_{4} \mathrm{~S}$ : 502.0355; found: 502.0354 .

\subsection{Crude ${ }^{19} \mathrm{~F}-\mathrm{NMR}$ Experiment}

$$
\text { - } \left.-\mathrm{CF}_{2} \mathrm{CO}_{2} \mathrm{Et} \frac{\mathrm{Et} 2 \mathrm{Zn}\left(1 \text { equiv), } \mathrm{N}_{2}\right.}{\mathrm{CD}_{3} \mathrm{CN},-20^{\circ} \mathrm{C}, 8 \mathrm{~h}} \underset{\mathbf{I}}{\underset{\mathrm{EtZn}}{\mathrm{C}}-\mathrm{CF}_{2} \mathrm{CO}_{2} \mathrm{Et}}\right] \stackrel{\mathrm{H}_{2} \mathrm{O}}{\longrightarrow} \underset{11}{\mathrm{HCF}_{2} \mathrm{CO}_{2} \mathrm{Et}}
$$

An oven-dried Schlenk tube was charged with a stir bar. The tube was degased and refilled with nitrogen for 3 times, and then a solution of ethyl iododifluoroacetate 1a (12.5 $\mathrm{mg}, 0.05 \mathrm{mmol})$ in $\mathrm{CD}_{3} \mathrm{CN}(0.5 \mathrm{~mL})$ was injected to the tube via a syringe. The reaction mixture was cooled to $-20^{\circ} \mathrm{C}$. After stirring for 3 minutes, a solution of diethylzinc $(1.0$ $\mathrm{mol} / \mathrm{L}$ in hexane, $0.05 \mathrm{~mL}, 1.0$ equiv) was injected to the reaction mixture via a syringe. After stirring at $-20{ }^{\circ} \mathrm{C}$ for 8 hours, the reaction was quenched with water ( $2.7 \mathrm{mg}, 3.0$ equiv), and the solution was submitted to crude ${ }^{19} \mathrm{~F}-\mathrm{NMR}$ experiment.

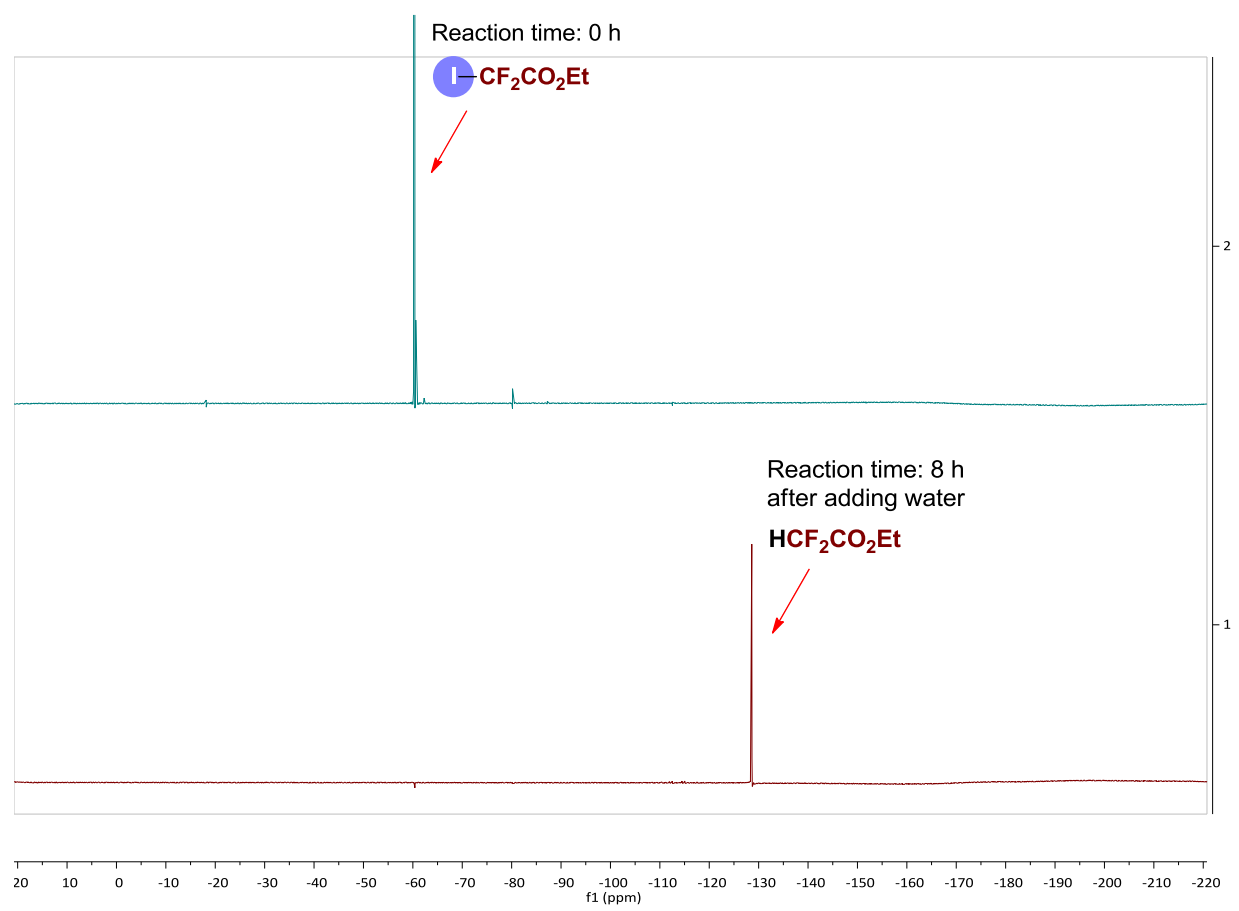

\section{Synthetic Applications}

\subsection{Intermolecular $S_{N} 2$ Substitution}

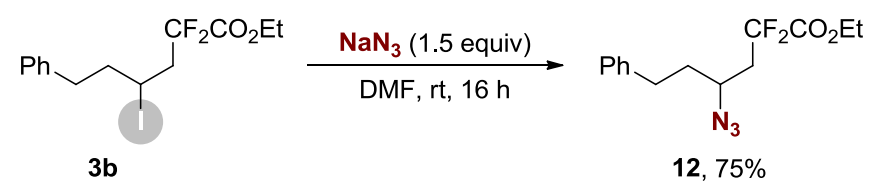


A mixture of $3 \mathbf{b}$ ( $38.2 \mathrm{mg}, 0.1 \mathrm{mmol}$ ) and sodium azide $(9.8 \mathrm{mg}, 0.15 \mathrm{mmol}, 1.5$ equiv) in anhydrous DMF was stirred at room temperature overnight. After completion, the reaction was quenched with water and extracted with diethyl ether. The combined organic layers were washed with saturated $\mathrm{NH}_{4} \mathrm{Cl}$ for 5 times, dried with $\mathrm{Na}_{2} \mathrm{SO}_{4}$, filtered, and concentrated under reduced pressure. The residue was purified by silica gel column chromatography (petroleum ether/ethyl acetate $=25 / 1$ ) to afford the corresponding pure product as a colorless oil, $22.3 \mathrm{mg}$, 75\% yield.

\section{Ethyl 4-azido-2,2-difluoro-6-phenylhexanoate (12)}

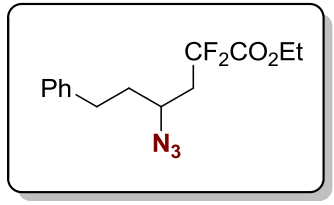

${ }^{1} \mathrm{H}$ NMR $\left(500 \mathrm{MHz}, \mathrm{CDCl}_{3}\right) \delta 7.33(\mathrm{t}, J=7.5 \mathrm{~Hz}, 2 \mathrm{H}), 7.25(\mathrm{~d}, J=7.5$ $\mathrm{Hz}, 1 \mathrm{H}), 7.22(\mathrm{~d}, J=7.5 \mathrm{~Hz}, 2 \mathrm{H}), 4.36(\mathrm{q}, J=7.2 \mathrm{~Hz}, 2 \mathrm{H}), 3.62(\mathrm{dq}, J$ $=9.3,4.6 \mathrm{~Hz}, 1 \mathrm{H}), 2.89-2.80(\mathrm{~m}, 1 \mathrm{H}), 2.74(\mathrm{dt}, J=13.8,8.2 \mathrm{~Hz}, 1 \mathrm{H})$, $2.40(\mathrm{dtd}, J=19.8,14.1,9.3 \mathrm{~Hz}, 1 \mathrm{H}), 2.27(\mathrm{qd}, J=15.1,3.6 \mathrm{~Hz}, 1 \mathrm{H})$, $1.99-1.87(\mathrm{~m}, 2 \mathrm{H}), 1.38(\mathrm{t}, J=7.2 \mathrm{~Hz}, 3 \mathrm{H})$.

${ }^{19}$ F NMR $\left(471 \mathrm{MHz}, \mathrm{CDCl}_{3}\right) \delta-102.3(\mathrm{~d}, J=264.2 \mathrm{~Hz}),-107.2(\mathrm{~d}, J=264.2 \mathrm{~Hz})$.

${ }^{13} \mathrm{C}$ NMR $\left(125 \mathrm{MHz}, \mathrm{CDCl}_{3}\right) \delta 163.7\left(\mathrm{t},{ }^{2} J_{\mathrm{C}-F}=32.2 \mathrm{~Hz}\right), 140.3,128.6,128.4,126.3,114.8(\mathrm{dd}$, $\left.{ }^{1} J_{C-F}=252.6,249.7 \mathrm{~Hz}\right), 63.2,56.1\left(\mathrm{dd},{ }^{3} J_{C-F}=6.1,3.0 \mathrm{~Hz}\right), 39.4\left(\mathrm{t},{ }^{2} J_{C-F}=23.5 \mathrm{~Hz}\right), 36.7,31.9$, 13.9.

HRMS (ESI) m/z: [M+Na] calcd for $\mathrm{C}_{14} \mathrm{H}_{17} \mathrm{~F}_{2} \mathrm{~N}_{3} \mathrm{O}_{2} \mathrm{Na}$ : 320.1181; found: 320.1182 .

\subsection{Reduction \& Intramolecular $S_{\mathrm{N}} 2$ Substitution}

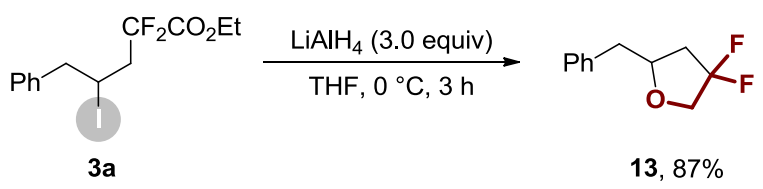

To a solution of $3 \mathrm{a}(36.8 \mathrm{mg}, 0.1 \mathrm{mmol})$ in anhydrous THF was added $\mathrm{LiAlH}_{4}(11.4 \mathrm{mg}$, $0.3 \mathrm{mmol}, 3.0$ equiv) at $0{ }^{\circ} \mathrm{C}$. The resulting mixture was stirred at $0{ }^{\circ} \mathrm{C}$ for 3 hour. After completion, the reaction was quenched with saturated $\mathrm{NH}_{4} \mathrm{Cl}$ and extracted with diethyl ether. The combined organic layers were dried with $\mathrm{Na}_{2} \mathrm{SO}_{4}$, filtered, and concentrated under reduced pressure. The residue was purified by silica gel column chromatography (petroleum ether/ethyl acetate $=5 / 1$ ) to afford the corresponding pure product as a colorless oil, $17.3 \mathrm{mg}$, $87 \%$ yield.

\section{2-benzyl-4,4-difluorotetrahydrofuran (13)}

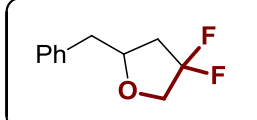

${ }^{1} \mathbf{H}$ NMR $\left(500 \mathrm{MHz}, \mathrm{CDCl}_{3}\right) \delta 7.38-7.31(\mathrm{~m}, 3 \mathrm{H}), 7.27-7.22(\mathrm{~m}, 2 \mathrm{H})$, $4.48(\mathrm{dq}, J=8.9,6.4 \mathrm{~Hz}, 1 \mathrm{H}), 3.90-3.71(\mathrm{~m}, 2 \mathrm{H}), 3.33(\mathrm{dd}, J=14.6,5.8$ $\mathrm{Hz}, 1 \mathrm{H}), 3.23(\mathrm{dd}, J=14.6,8.8 \mathrm{~Hz}, 1 \mathrm{H}), 2.92-2.64(\mathrm{~m}, 2 \mathrm{H})$.

${ }^{19}$ F NMR $\left(471 \mathrm{MHz}, \mathrm{CDCl}_{3}\right) \delta-104.2(\mathrm{~d}, J=253.1 \mathrm{~Hz}),-108.5(\mathrm{~d}, J=$

253.1 Hz).

${ }^{13} \mathrm{C}$ NMR (125 MHz, $\left.\mathrm{CDCl}_{3}\right) \delta$ 139.2, 129.0, 128.5, 127.1, $122.4\left(\mathrm{t},{ }^{1} \mathrm{C}_{\mathrm{C}-\mathrm{F}}=244.4 \mathrm{~Hz}\right), 64.2(\mathrm{t}$, $\left.{ }^{2} J_{C-F}=31.9 \mathrm{~Hz}\right), 47.4,43.6\left(\mathrm{t},{ }^{2} J_{C-F}=23.7 \mathrm{~Hz}\right), 23.7\left(\mathrm{t},{ }^{3} J_{C-F}=3.5 \mathrm{~Hz}\right)$.

HRMS (ESI) m/z: [M+Na] $]^{+}$calcd for $\mathrm{C}_{11} \mathrm{H}_{12} \mathrm{~F}_{2} \mathrm{ONa}$ : 221.0748; found: 221.0759. 


\subsection{Palladium-Catalyzed Sonogashira Coupling}

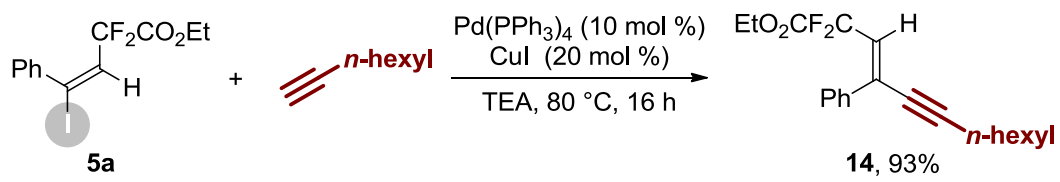

Compound 14 was synthesized according to a literature reported procedure. ${ }^{4}$

An oven-dried Schlenk tube was charged with $\mathrm{Pd}\left(\mathrm{PPh}_{3}\right)_{4}(16.4 \mathrm{mg}, 0.014 \mathrm{mmol}, 10$ $\mathrm{mmol} \%)$, $\mathrm{CuI}$ (5.4 $\mathrm{mg}, 0.028 \mathrm{mmol}, 10 \mathrm{mmol} \%$ ) and a stir bar. The tube was degased and refilled with nitrogen for 3 times, and then a solution of $5 \mathrm{a}(50.0 \mathrm{mg}, 0.14 \mathrm{mmol})$ and 1-octyne (35.8 $\mathrm{mg}, 0.325 \mathrm{mmol}, 2.3$ equiv) in a triethylamine $(1 \mathrm{~mL})$ was injected into the tube via a syringe. The resulting mixture was heated at $60{ }^{\circ} \mathrm{C}$ in an oil bath for 16 hours. After completion, the mixture was filtered through a pad of Celite, washed with diethyl ether. The combined organic layers were concentrated under reduced pressure. The residue was purified by silica gel column chromatography (petroleum ether/ethyl acetate $=20 / 1$ ) to afford the corresponding pure product as a colorless oil, $43.5 \mathrm{mg}$, $93 \%$ yield .

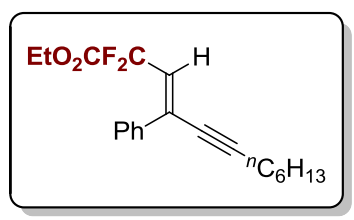

${ }^{19}$ F NMR (471 MHz, $\left.\mathrm{CDCl}_{3}\right) \delta$-91.6.

${ }^{13} \mathrm{C}$ NMR $\left(125 \mathrm{MHz}, \mathrm{CDCl}_{3}\right) \delta 163.0\left(\mathrm{t},{ }^{2} \mathrm{~J}-\mathrm{F}=33.6 \mathrm{~Hz}\right), 136.1,134.3\left(\mathrm{t},{ }^{3} \mathrm{JC}-\mathrm{F}=10.2 \mathrm{~Hz}\right), 128.9$, $128.5\left(\mathrm{t},{ }^{4} \mathrm{~J}_{\mathrm{C}-\mathrm{F}}=2.3 \mathrm{~Hz}\right), 128.0,125.9\left(\mathrm{t},{ }^{2} \mathrm{~J}_{\mathrm{C}-\mathrm{F}}=28.2 \mathrm{~Hz}\right), 112.0\left(\mathrm{t},{ }^{1} \mathrm{~J}_{\mathrm{C}-\mathrm{F}}=245.0 \mathrm{~Hz}\right), 95.8,80.9(\mathrm{t}$, $\left.{ }^{4} J_{C-F}=2.7 \mathrm{~Hz}\right), 62.8,31.2,28.5,28.3,22.5,19.5,14.0,13.6$.

HRMS (ESI) m/z: [M+H] $]^{+}$calcd for $\mathrm{C}_{20} \mathrm{H}_{25} \mathrm{~F}_{2} \mathrm{O}_{2}: 335.1817$; found: 335.1824 .

\subsection{Cobalt-Catalyzed Kumada Coupling}

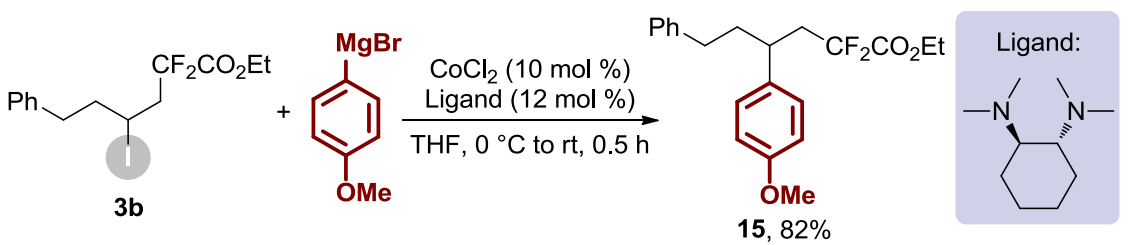

Compound 15 was synthesized according to a literature reported procedure. ${ }^{5}$

An oven-dried Schlenk tube was charged with anhydrous cobalt (II) chloride (1.3 mg, $0.01 \mathrm{mmol}, 10 \mathrm{~mol} \%$ ) and a stir bar. The tube was heated with a hair-dryer in vacuo for 2 minutes. After the color of the cobalt salt became blue, a solution of $(1 R, 2 R)-N, N, N^{\prime}, N^{\prime}$-tetramethylcyclohexane-1,2-diamine $(2.2 \mathrm{mg}, 0.012 \mathrm{mmol}, 12 \mathrm{~mol} \%)$ in anhydrous THF $(1 \mathrm{~mL})$ was injected into the tube via a syringe. The mixture was stirred for 3 minutes at room temperature. Then, $3 \mathbf{b}(38.2 \mathrm{mg}$, $0.10 \mathrm{mmol})$ was added and

\footnotetext{
${ }^{4}$ T. Xu, C. W. Cheung, X. Hu, Angew. Chem. Int. Ed. 2014, 53, 4910-4914

${ }^{5}$ H. Ohmiya, H. Yorimitsu, K. Oshima, J. Am. Chem. Soc. 2006, 128, 1886-1889
} 
the tube was placed to an ice-water bath. 4-Methoxyphenylmagnesium bromide (1.0 M THF solution, $0.12 \mathrm{~mL}, 0.12 \mathrm{mmol}, 1.2$ equiv) was injected into the tube via a syringe at $0{ }^{\circ} \mathrm{C}$. After stirring at $0{ }^{\circ} \mathrm{C}$ for 5 minutes and at room temperature for 20 minutes, the reaction was quenched with saturated $\mathrm{NH}_{4} \mathrm{Cl}$ and extracted with diethyl ether. The combined organic layers were dried with $\mathrm{Na}_{2} \mathrm{SO}_{4}$, filtered, and concentrated under reduced pressure. The residue was purified by silica gel column chromatography (petroleum ether/ethyl acetate $=15 / 1$ ) to afford the corresponding pure product as a colorless oil, $29.4 \mathrm{mg}$, $82 \%$ yield.

Ethyl 2,2-difluoro-4-(4-methoxyphenyl)-6-phenylhexanoate (15)

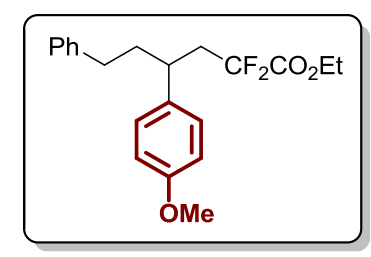

${ }^{1} \mathrm{H}$ NMR $\left(500 \mathrm{MHz}, \mathrm{CDCl}_{3}\right) \delta 7.31-7.25(\mathrm{~m}, 3 \mathrm{H}), 7.19(\mathrm{t}, J=7.4$ $\mathrm{Hz}, 1 \mathrm{H}), 7.13-7.08(\mathrm{~m}, 3 \mathrm{H}), 6.88(\mathrm{~d}, J=8.6 \mathrm{~Hz}, 2 \mathrm{H}), 4.02-3.91$ $(\mathrm{m}, 2 \mathrm{H}), 3.83(\mathrm{~s}, 3 \mathrm{H}), 2.86(\mathrm{tt}, J=9.9,5.0 \mathrm{~Hz}, 1 \mathrm{H}), 2.59-2.34(\mathrm{~m}$, $4 \mathrm{H}), 2.04(\mathrm{dtd}, J=16.9,8.4,7.9,4.9 \mathrm{~Hz}, 1 \mathrm{H}), 1.92(\mathrm{ddt}, J=13.6,9.2$, $7.0 \mathrm{~Hz}, 1 \mathrm{H}), 1.21(\mathrm{t}, J=7.1 \mathrm{~Hz}, 3 \mathrm{H})$.

${ }^{19}$ F NMR $\left(471 \mathrm{MHz}, \mathrm{CDCl}_{3}\right) \delta-100.1(\mathrm{~d}, J=259.9 \mathrm{~Hz}),-106.6(\mathrm{~d}, J=259.9 \mathrm{~Hz})$.

${ }^{13} \mathrm{C}$ NMR $\left(125 \mathrm{MHz}, \mathrm{CDCl}_{3}\right) \delta 163.9\left(\mathrm{t},{ }^{2} \mathrm{JC}-\mathrm{F}=33.2 \mathrm{~Hz}\right), 158.4,141.7,134.7,128.8,128.4$, $128.3,125.8,115.8\left(\mathrm{t},{ }^{1} J_{C-F}=251.9 \mathrm{~Hz}\right), 113.8,62.6,55.3,41.6\left(\mathrm{t},{ }^{2} J_{C-F}=22.5 \mathrm{~Hz}\right), 38.9,38.4(\mathrm{t}$, $\left.{ }^{3} J_{C-F}=3.2 \mathrm{~Hz}\right), 33.3,13.7$.

HRMS (ESI) m/z: [M+Na] $]^{+}$calcd for $\mathrm{C}_{21} \mathrm{H}_{24} \mathrm{~F}_{2} \mathrm{O}_{3} \mathrm{Na}$ : 385.1586; found: 385.1588.

\section{NMR Spectra}

Please refer to next pages 
BzO

${ }^{1} \mathbf{H}$ NMR-spectrum $\left(500 \mathrm{MHz}, \mathrm{CDCl}_{3}\right)$ of $\mathbf{S 2 a}$

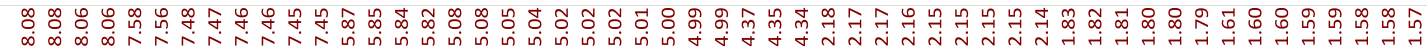

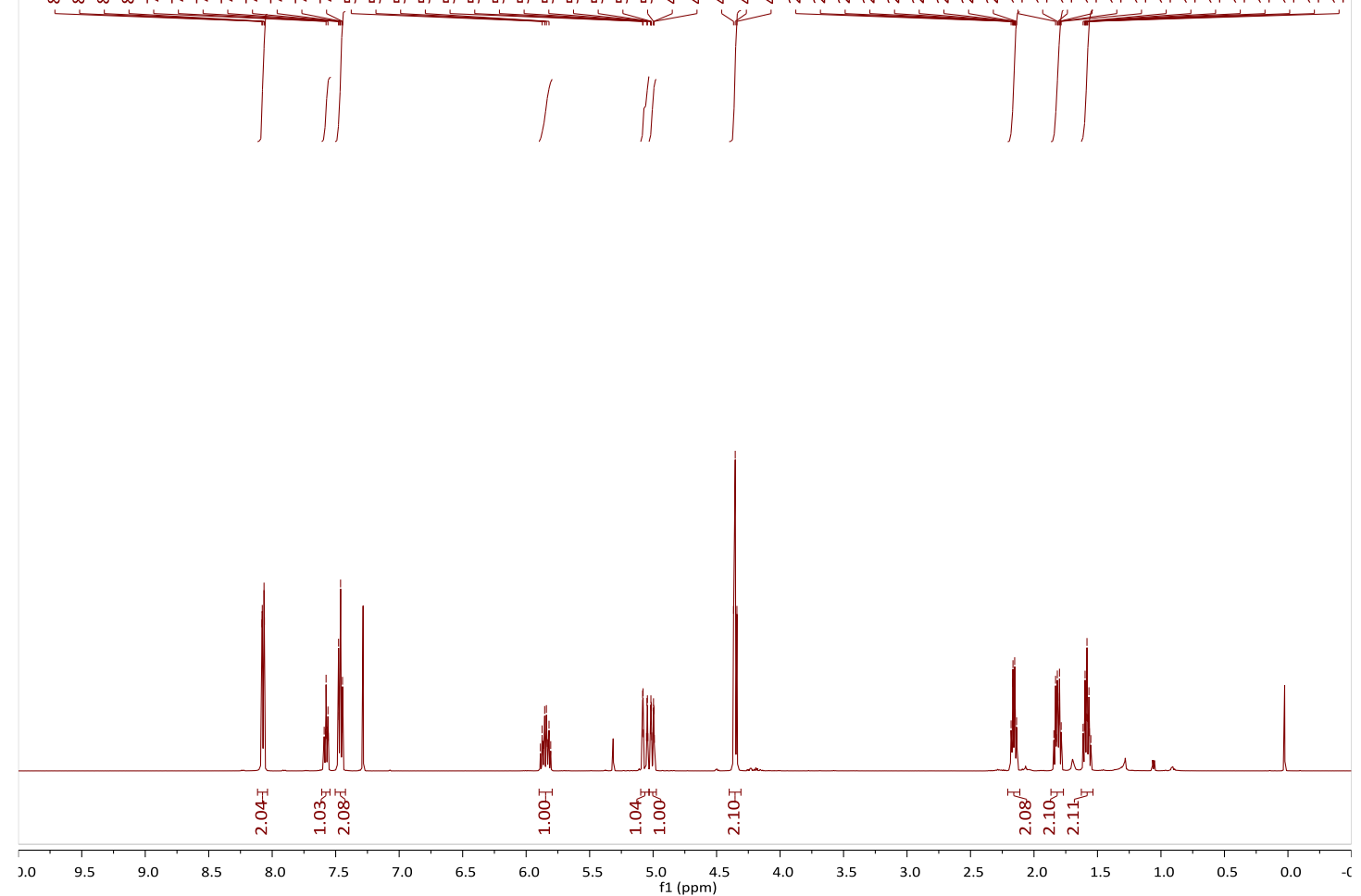

${ }^{13} \mathbf{C}$ NMR-spectrum (125 MHz, $\left.\mathrm{CDCl}_{3}\right)$ of $\mathbf{S 2 a}$

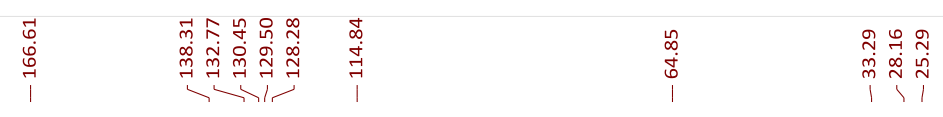

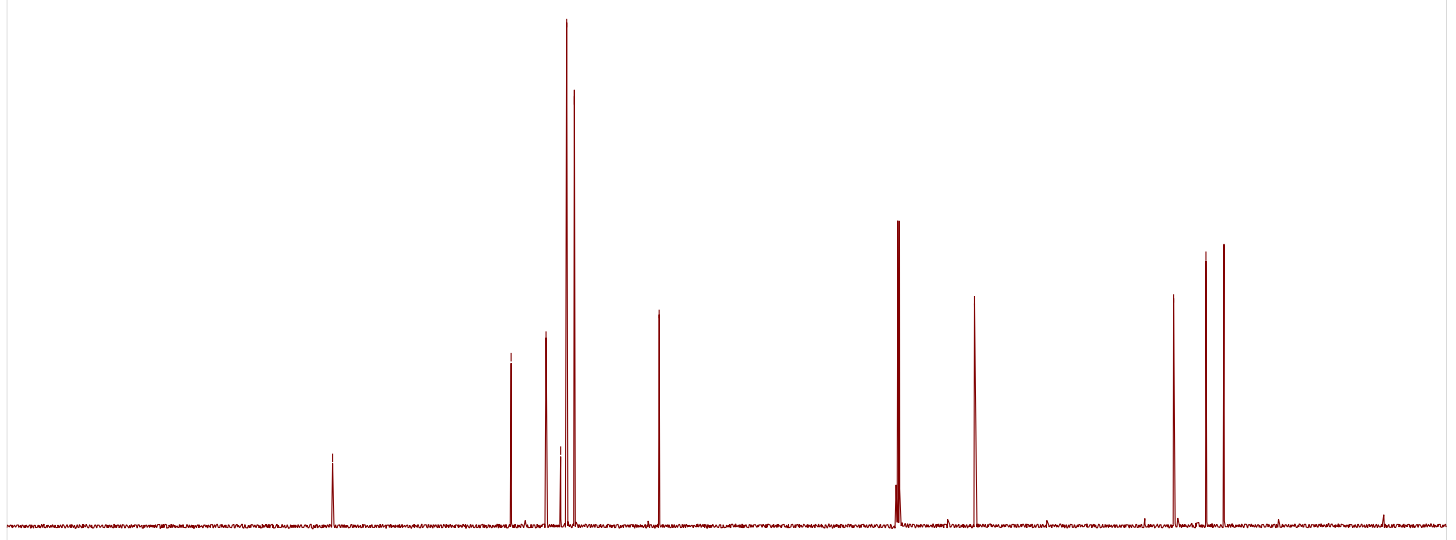

$\begin{array}{llllllllllllllllllllll}210 & 200 & 190 & 180 & 170 & 160 & 150 & 140 & 130 & 120 & 110 & 100 & 90 & 80 & 70 & 60 & 50 & 40 & 30 & 20 & 10 & 0\end{array}$ 
BzO

${ }^{\mathbf{1}} \mathbf{H}$ NMR-spectrum (500 $\left.\mathbf{M H z}, \mathrm{CDCl}_{3}\right)$ of $\mathbf{S 2 b}$

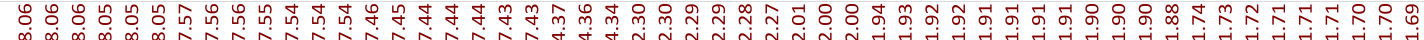

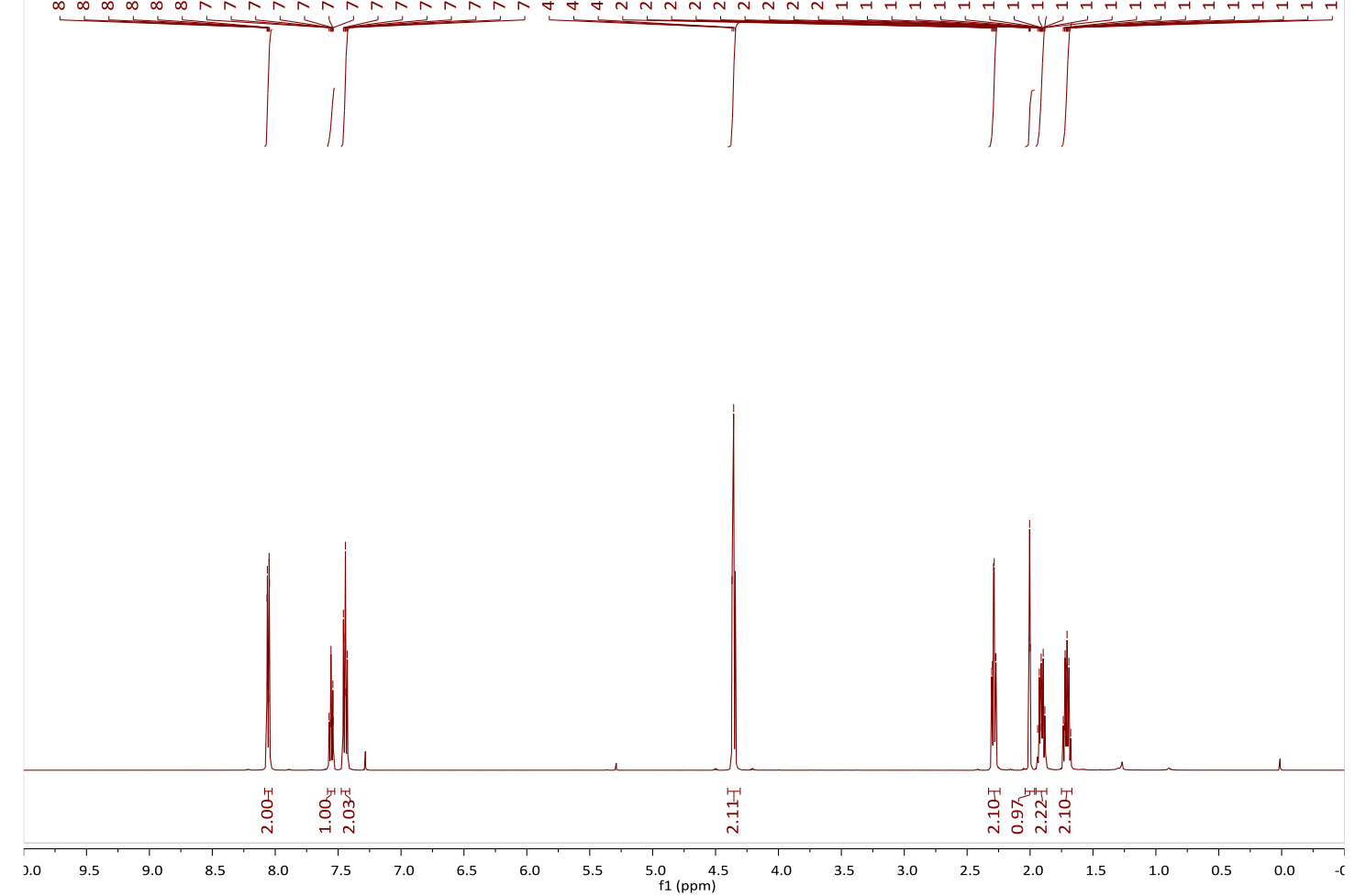

${ }^{13} \mathbf{C}$ NMR-spectrum (125 MHz, $\left.\mathrm{CDCl}_{3}\right)$ of $\mathbf{S 2 b}$

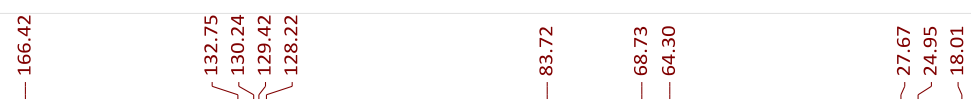

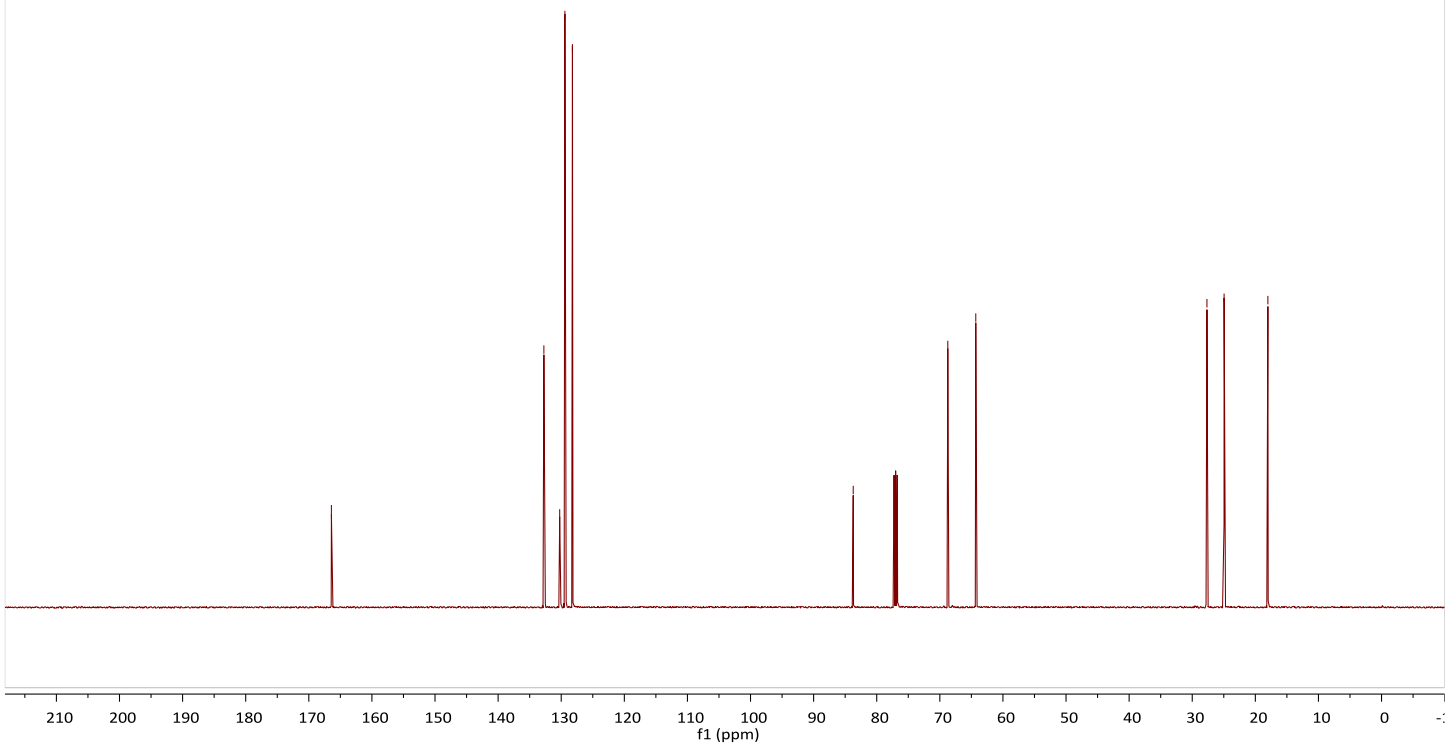


TsO

${ }^{1} \mathbf{H}$ NMR-spectrum $\left(500 \mathrm{MHz}, \mathrm{CDCl}_{3}\right)$ of S3a

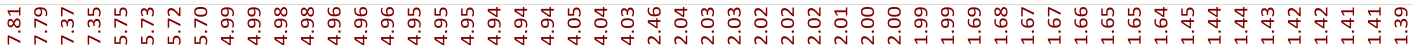

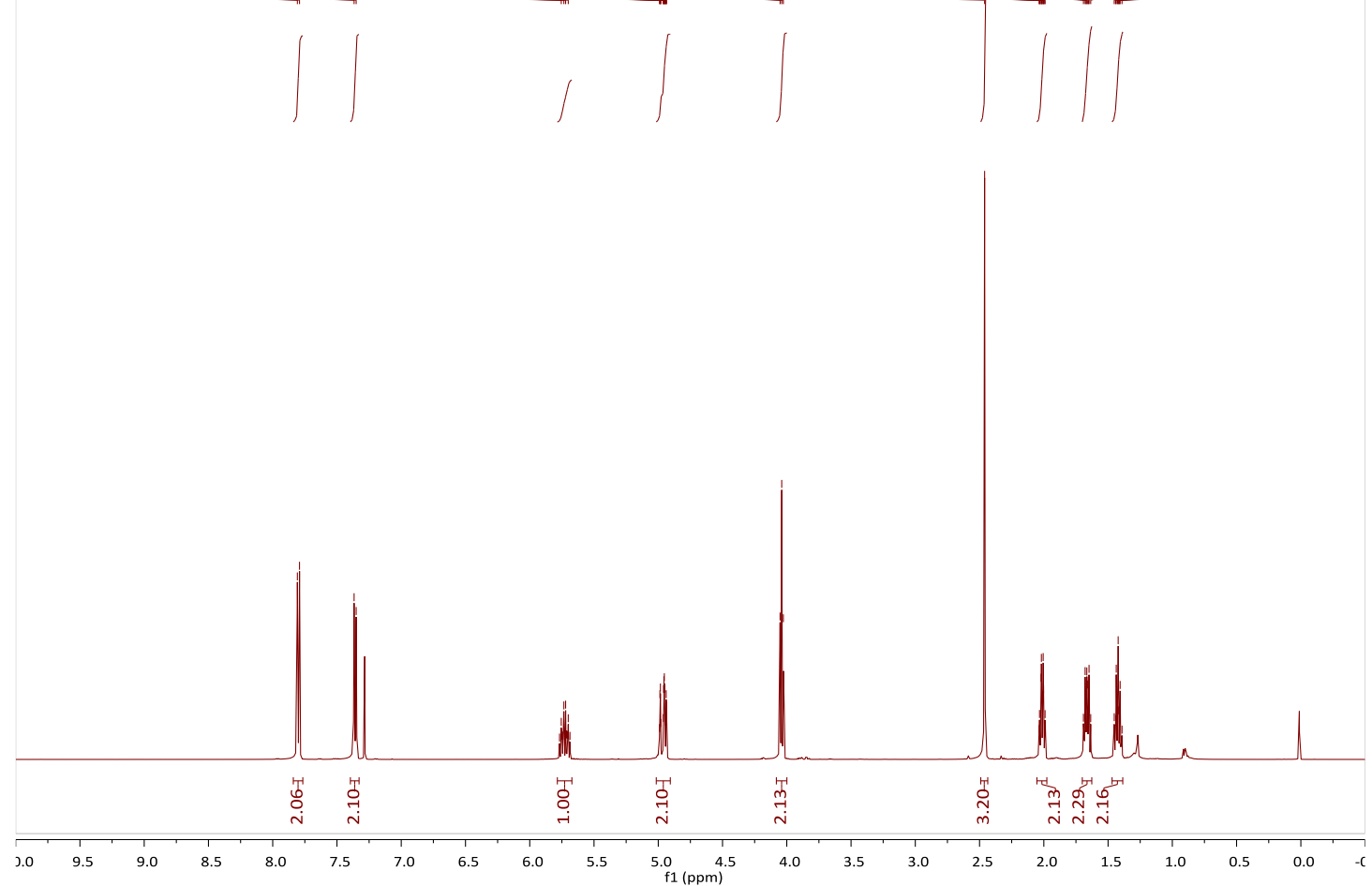

${ }^{13} \mathbf{C}$ NMR-spectrum (125 MHz, $\left.\mathrm{CDCl}_{3}\right)$ of $\mathbf{S 3 a}$

둥

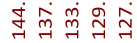

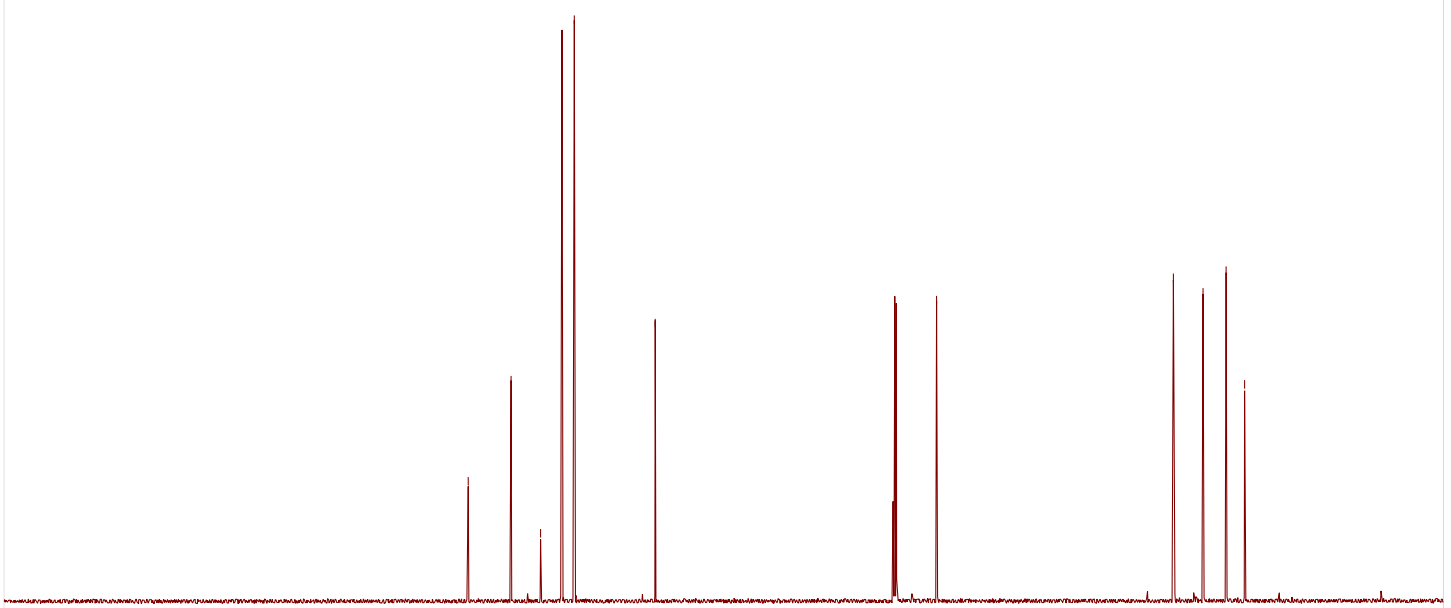

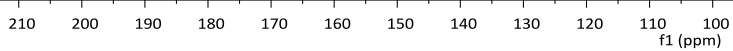


TsO

${ }^{\mathbf{1}} \mathbf{H} \mathbf{N M R}$-spectrum (500 $\left.\mathrm{MHz}, \mathrm{CDCl}_{3}\right)$ of $\mathbf{S 3 b}$

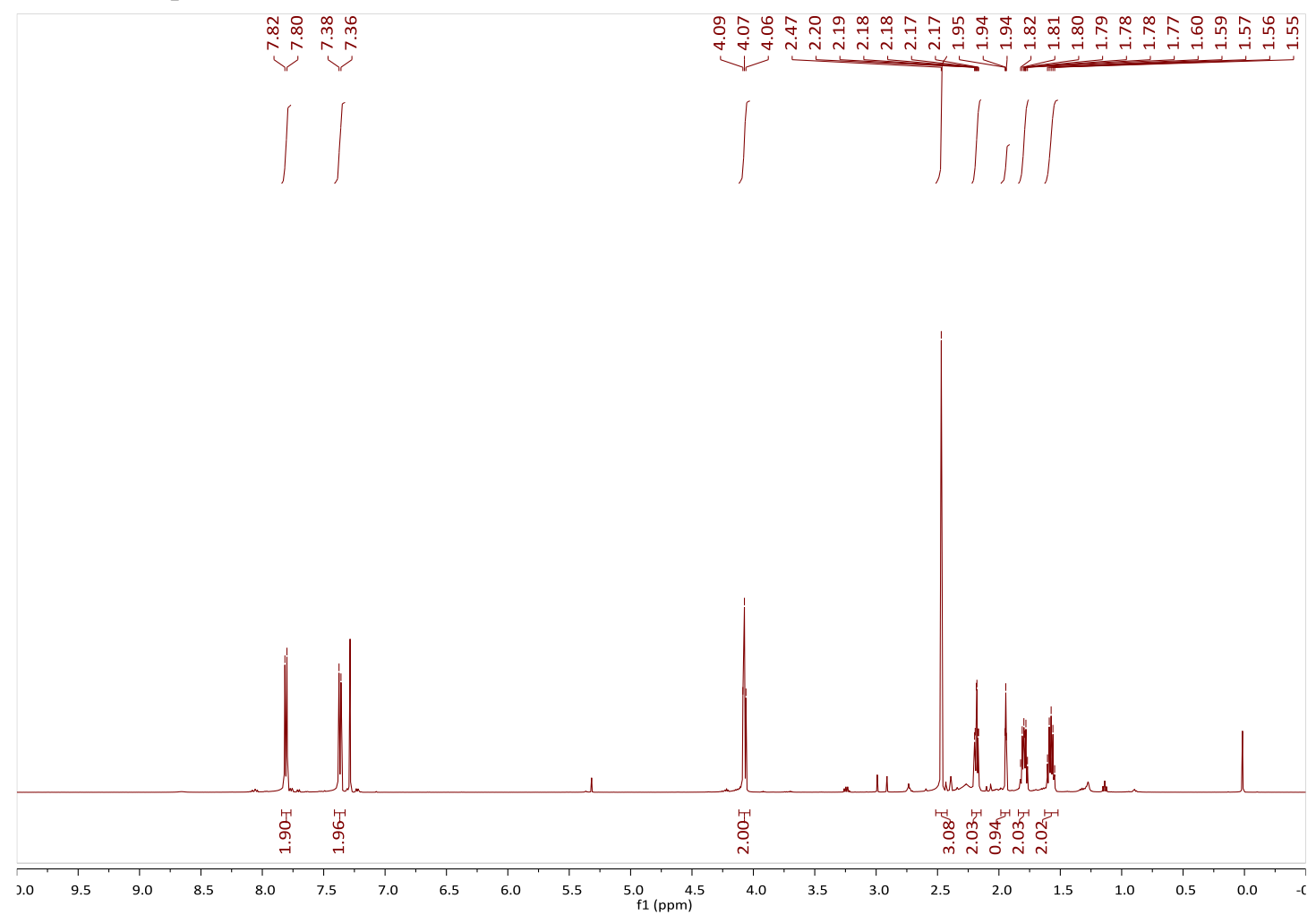

${ }^{13} \mathbf{C}$ NMR-spectrum (125 MHz, $\left.\mathrm{CDCl}_{3}\right)$ of $\mathbf{S 3 b}$

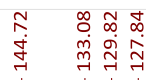

$\begin{array}{lll}\infty & \infty \\ \infty & \infty & 0 \\ \infty & 0 & 0 \\ \infty & 0 & 0 \\ 1 & 0 & 1 \\ 1 & 1\end{array}$

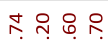

in 4 i

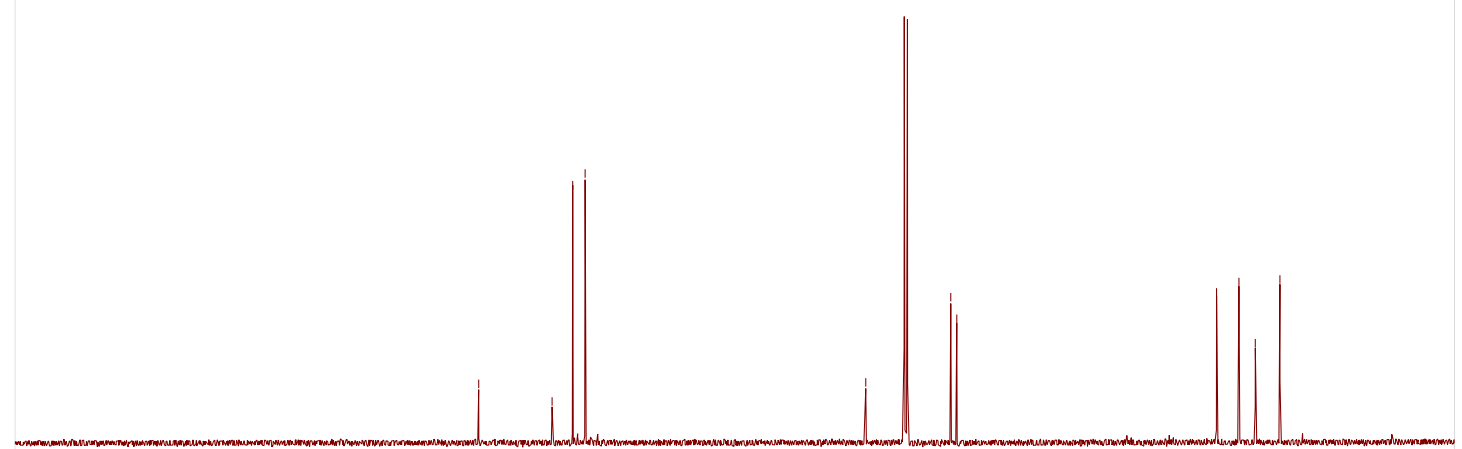

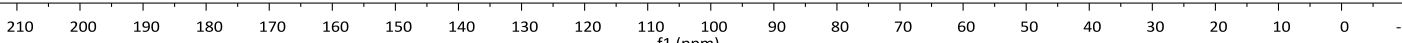


$\mathrm{MsO}^{2}$

${ }^{1} \mathbf{H}$ NMR-spectrum $\left(500 \mathrm{MHz}, \mathrm{CDCl}_{3}\right)$ of $\mathbf{S 4 a}$

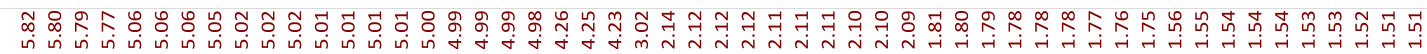

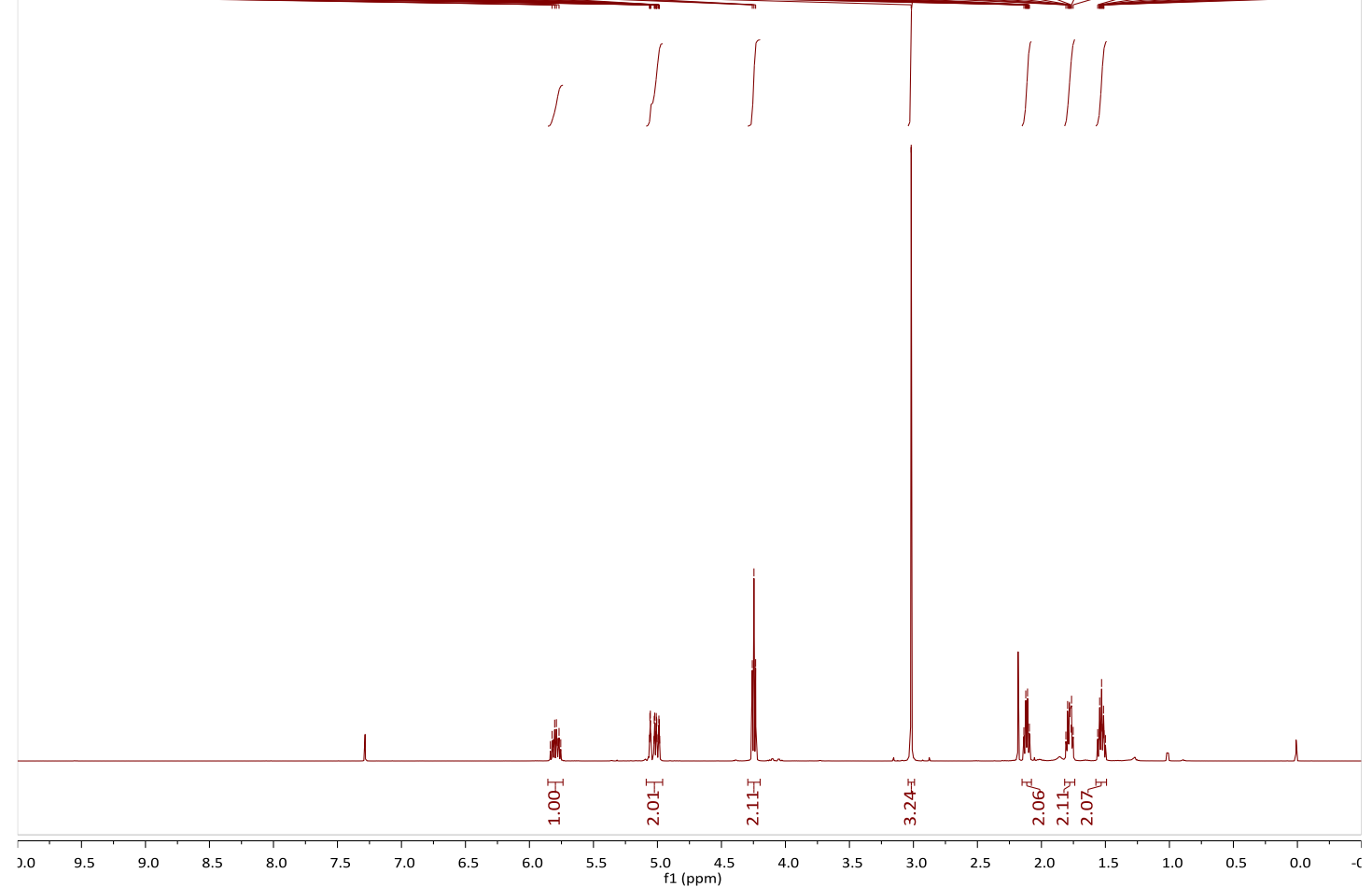

${ }^{13} \mathbf{C}$ NMR-spectrum (125 MHz, $\left.\mathrm{CDCl}_{3}\right)$ of $\mathbf{S 4 a}$

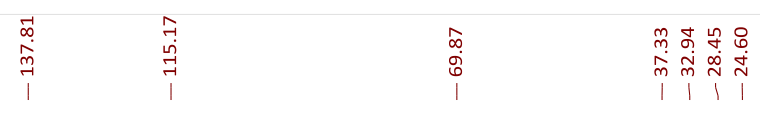

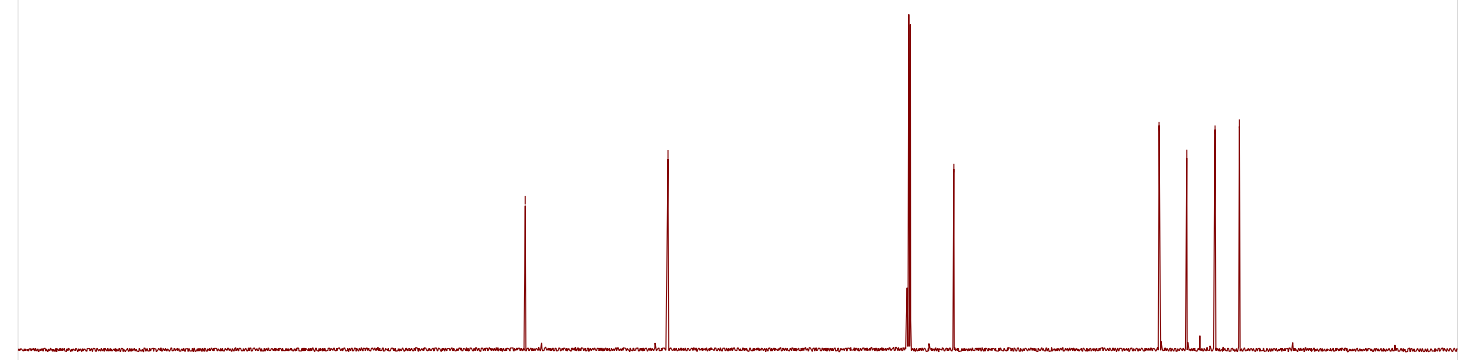

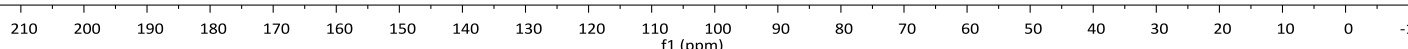


BnO

${ }^{1} \mathbf{H}$ NMR-spectrum $\left(500 \mathrm{MHz}, \mathrm{CDCl}_{3}\right)$ of $\mathbf{S 5 a}$

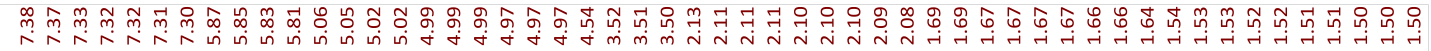

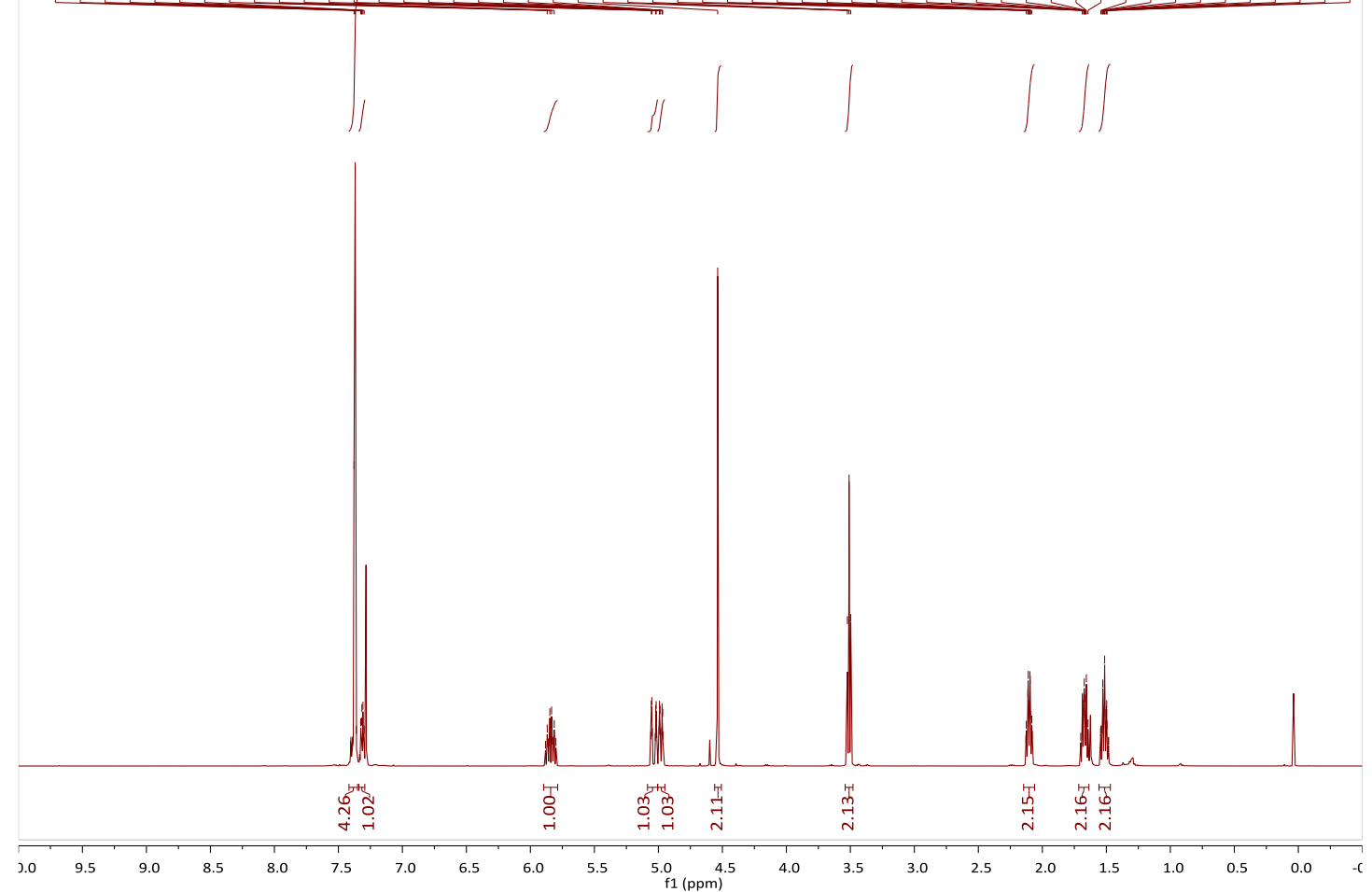

${ }^{13} \mathbf{C}$ NMR-spectrum (125 MHz, $\left.\mathrm{CDCl}_{3}\right)$ of $\mathbf{S 5 a}$

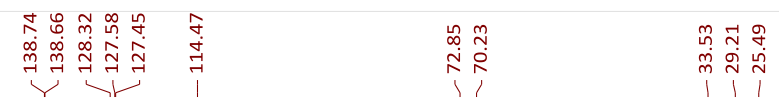

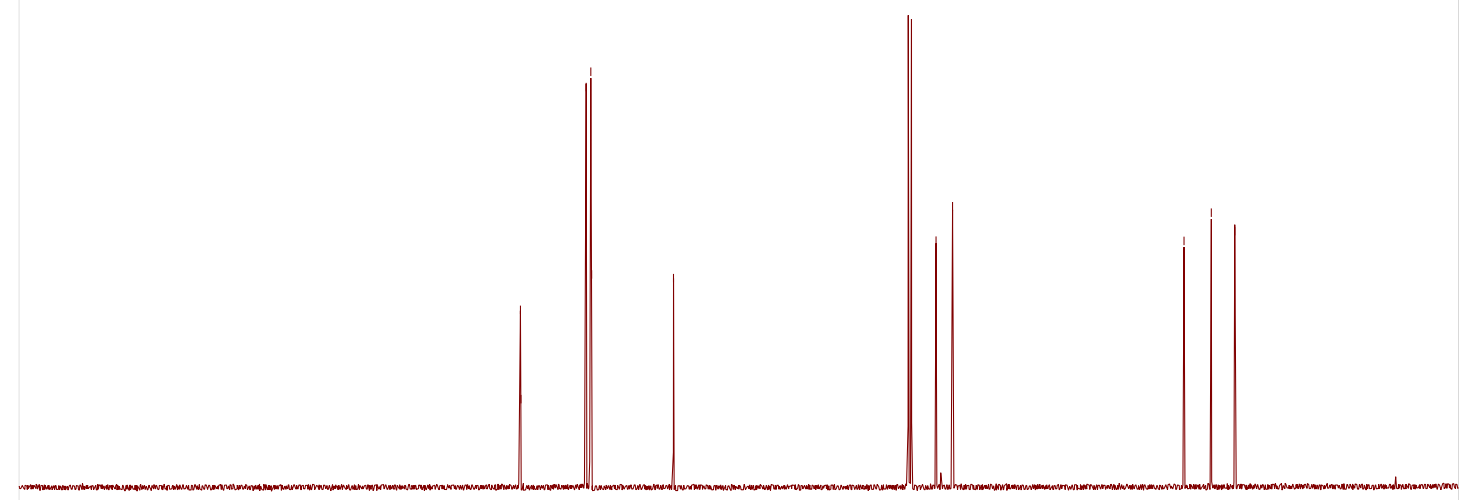

$\begin{array}{llllllllllllllllllllll}210 & 200 & 190 & 180 & 170 & 160 & 150 & 140 & 130 & 120 & \begin{array}{c}110 \\ \mathrm{f} 1(\mathrm{pmm})\end{array} & 90 & 80 & 70 & 60 & 50 & 40 & 30 & 20 & 10 & 0\end{array}$ 
BnO

${ }^{\mathbf{1}} \mathbf{H} \mathbf{N M R}$-spectrum (500 $\left.\mathbf{M H z}, \mathrm{CDCl}_{3}\right)$ of $\mathbf{S} 5 \mathbf{b}$

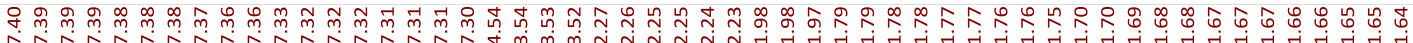

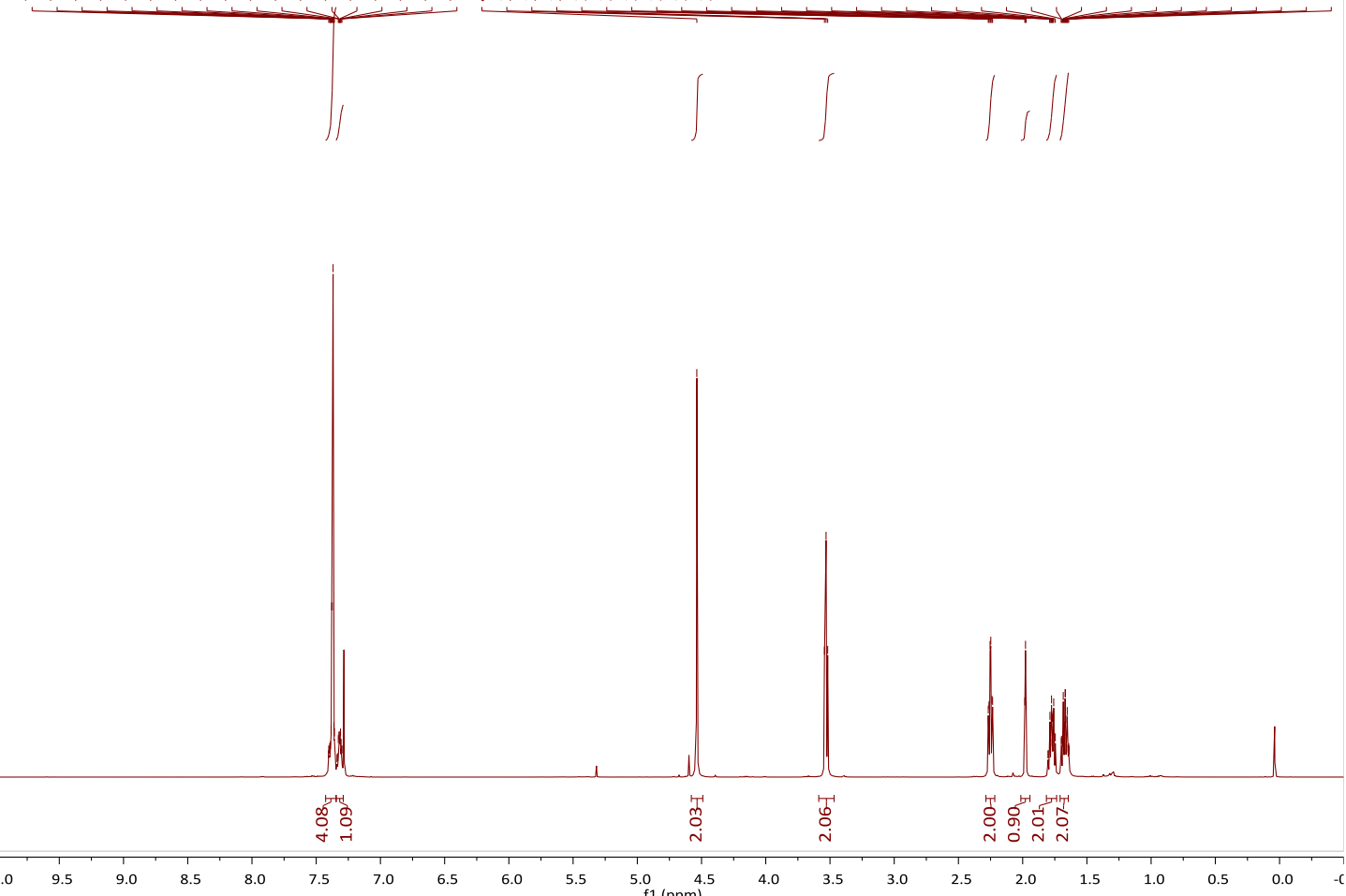

${ }^{13} \mathbf{C}$ NMR-spectrum $\left(125 \mathrm{MHz}, \mathrm{CDCl}_{3}\right)$ of $\mathbf{S 5 b}$

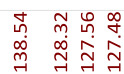

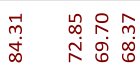

‥

๗

$1>1$

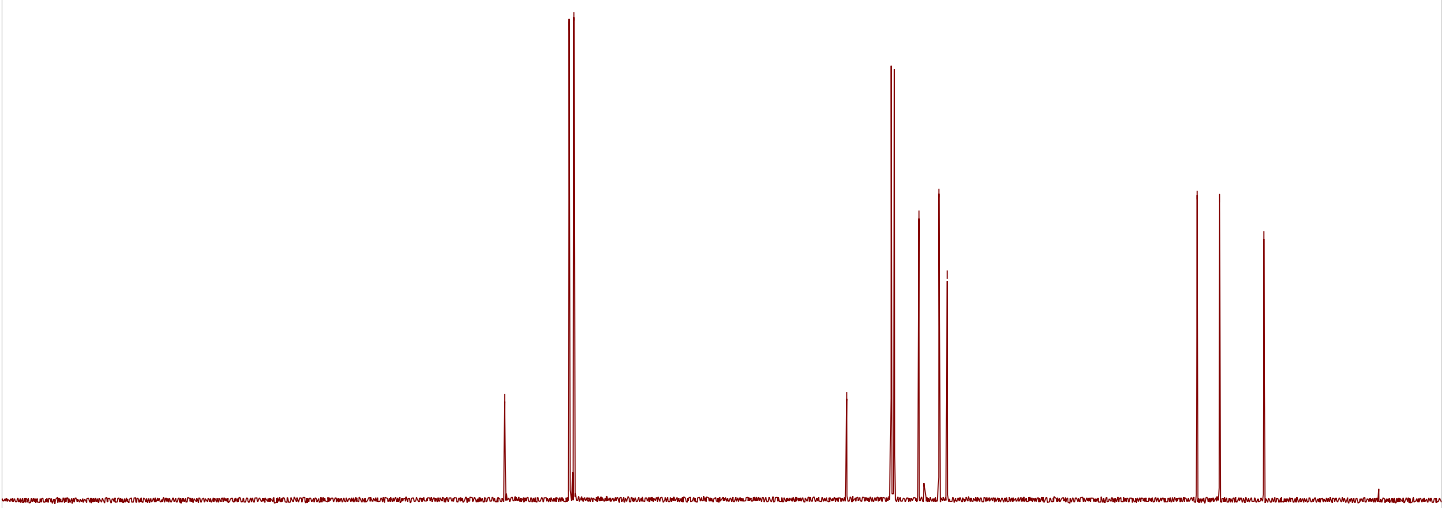

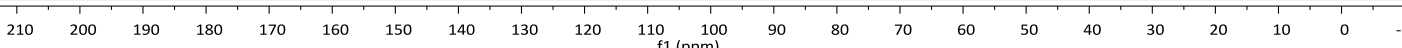


TBSO

${ }^{\mathbf{1}} \mathbf{H} \mathbf{N M R}$-spectrum $\left(500 \mathrm{MHz}, \mathrm{CDCl}_{3}\right)$ of $\mathbf{S 6 a}$

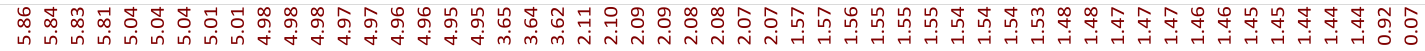

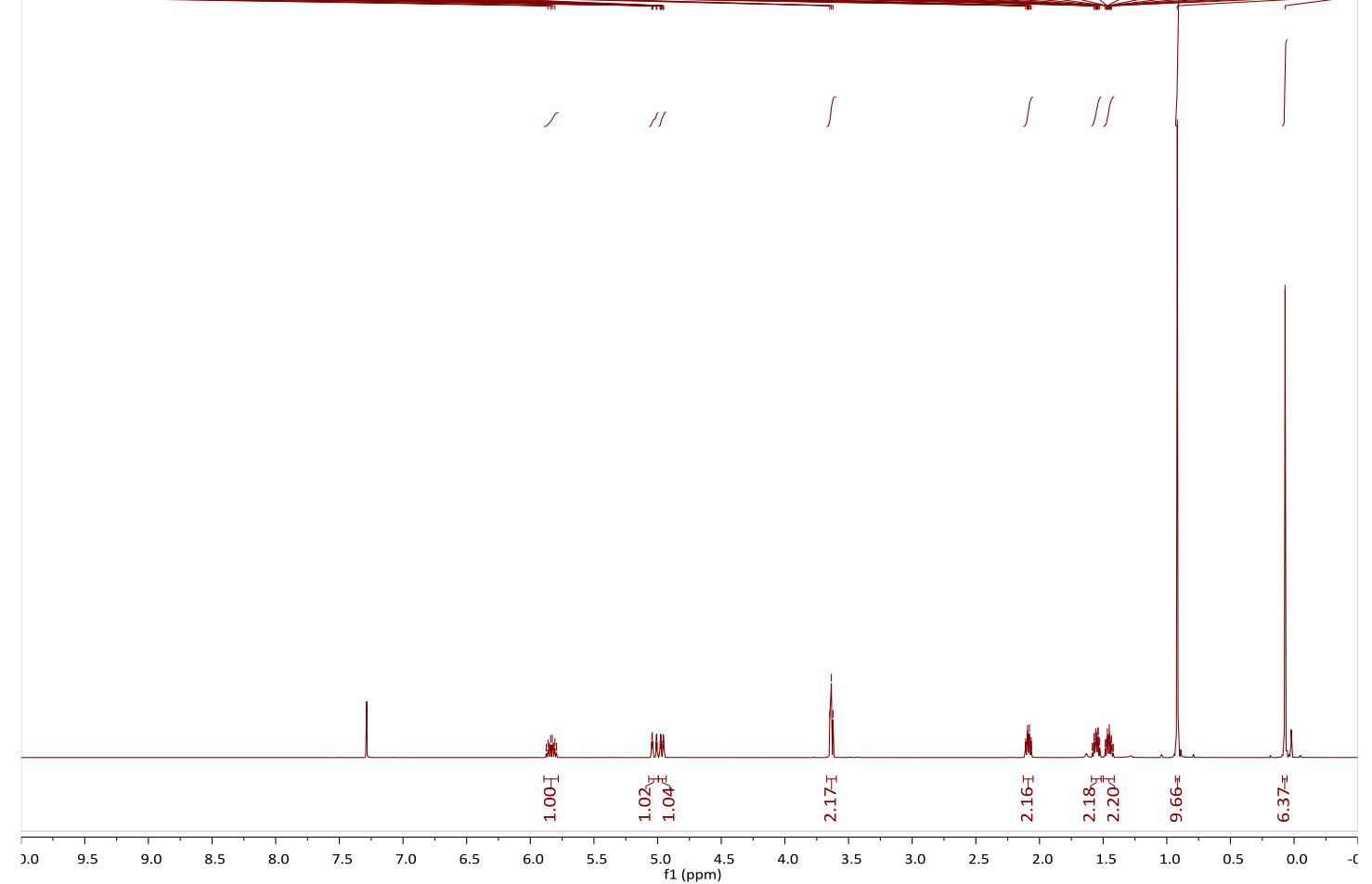

${ }^{13} \mathrm{C}$ NMR-spectrum (125 MHz, $\left.\mathrm{CDCl}_{3}\right)$ of $\mathbf{S 6 a}$

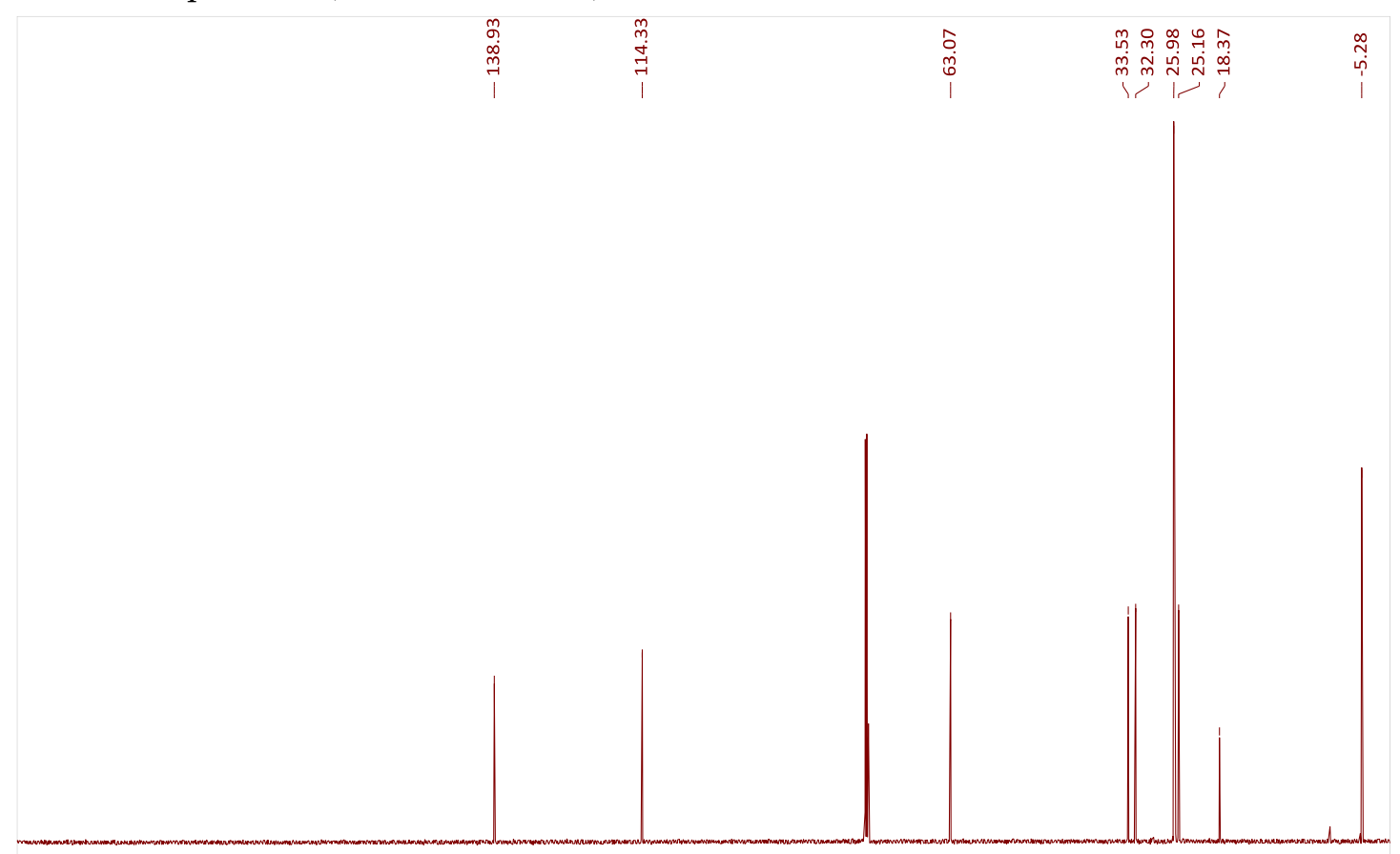

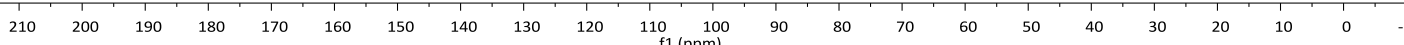


TBSO

${ }^{\mathbf{1}} \mathbf{H} \mathbf{N M R}$-spectrum $\left(500 \mathrm{MHz}, \mathrm{CDCl}_{3}\right)$ of $\mathbf{S 6 b}$

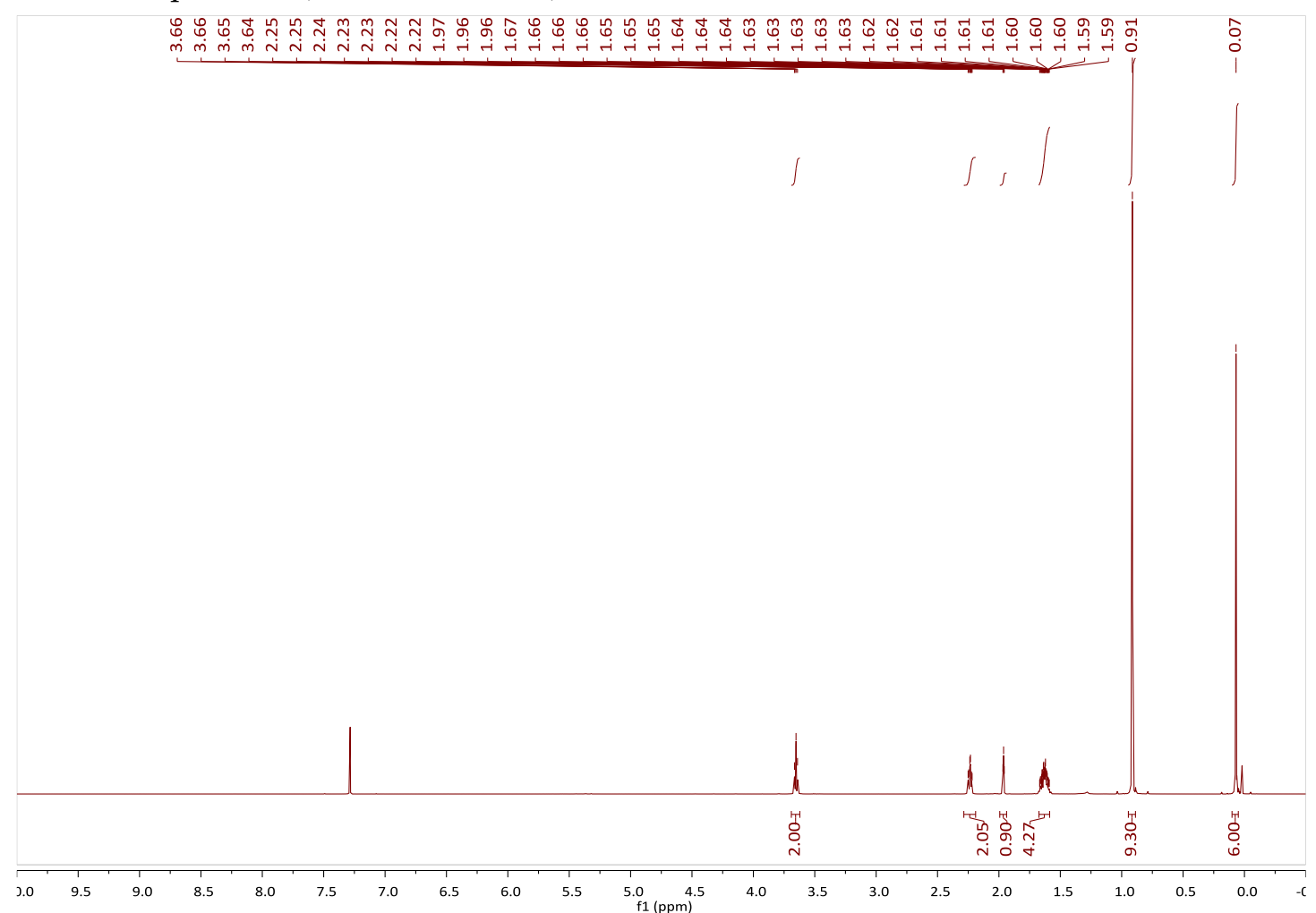

${ }^{13} \mathbf{C}$ NMR-spectrum $\left(125 \mathrm{MHz}, \mathrm{CDCl}_{3}\right)$ of $\mathbf{S 6 b}$

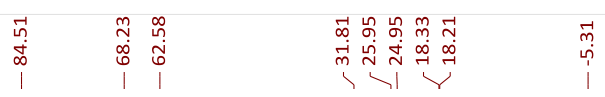

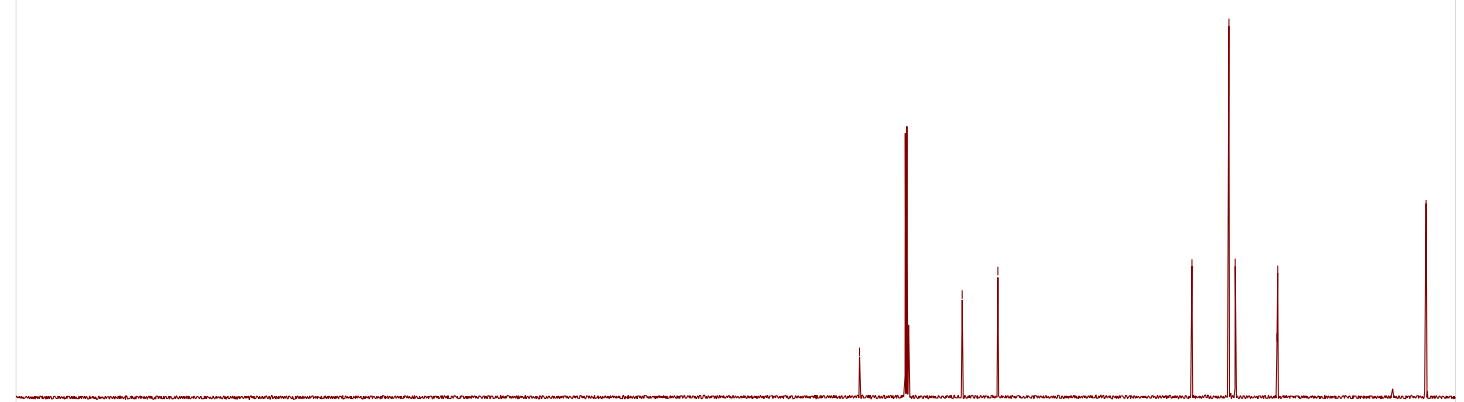

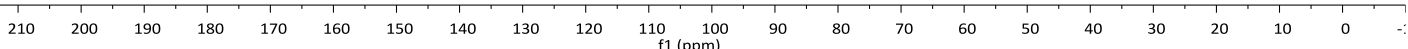


$\mathrm{Ph}_{\mathrm{O}} \sim \mathrm{N}$

${ }^{1} \mathbf{H}$ NMR-spectrum $\left(500 \mathrm{MHz}, \mathrm{CDCl}_{3}\right)$ of $\mathbf{S 8}$

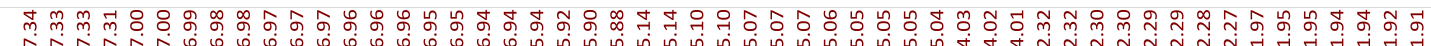
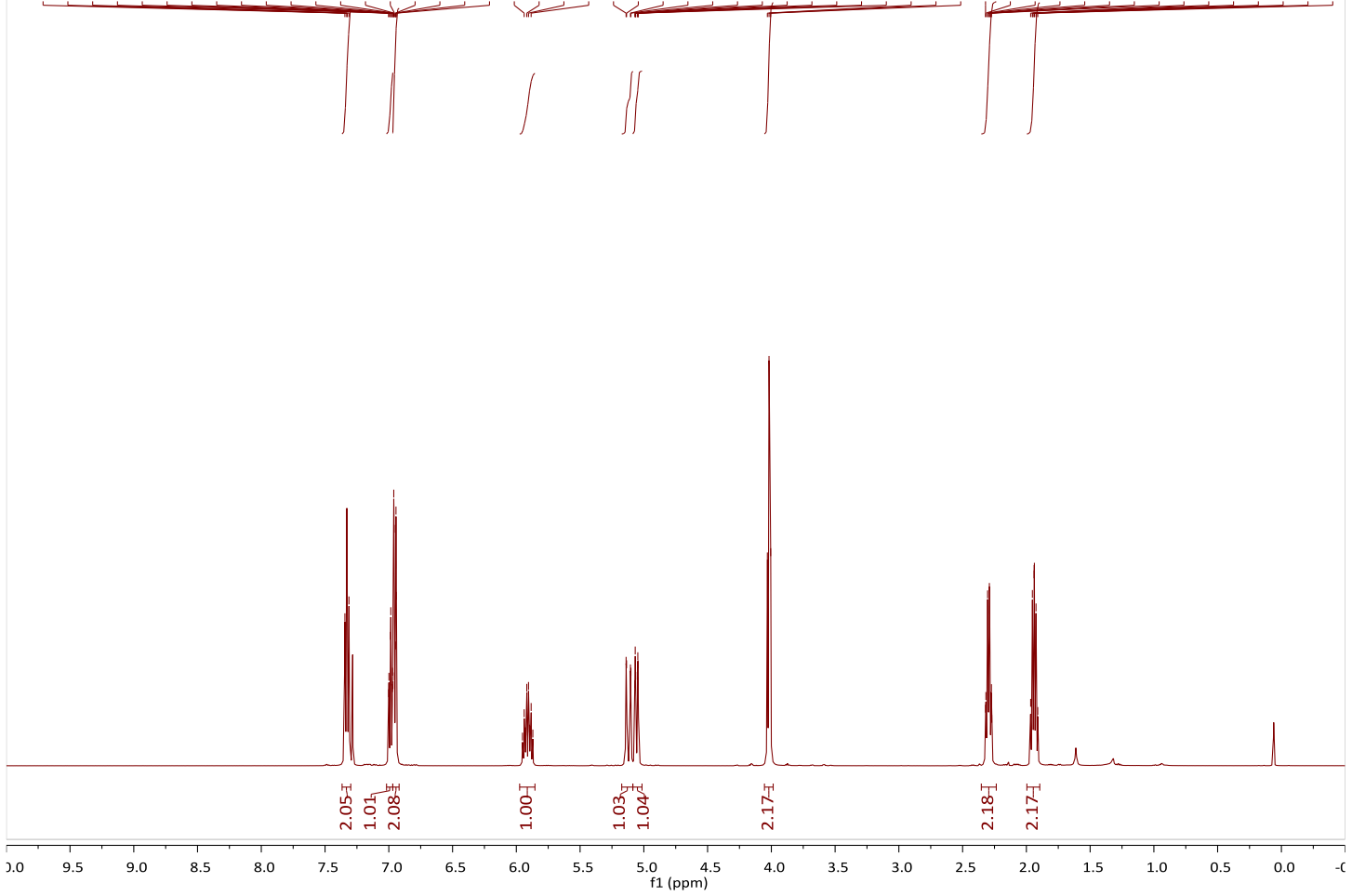

${ }^{13} \mathrm{C}$ NMR-spectrum $\left(125 \mathrm{MHz}, \mathrm{CDCl}_{3}\right)$ of $\mathbf{S 8}$

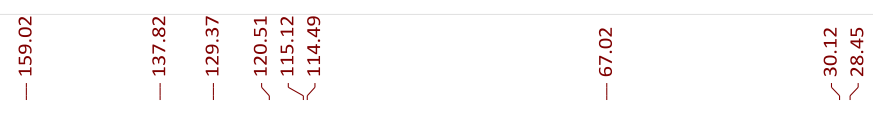

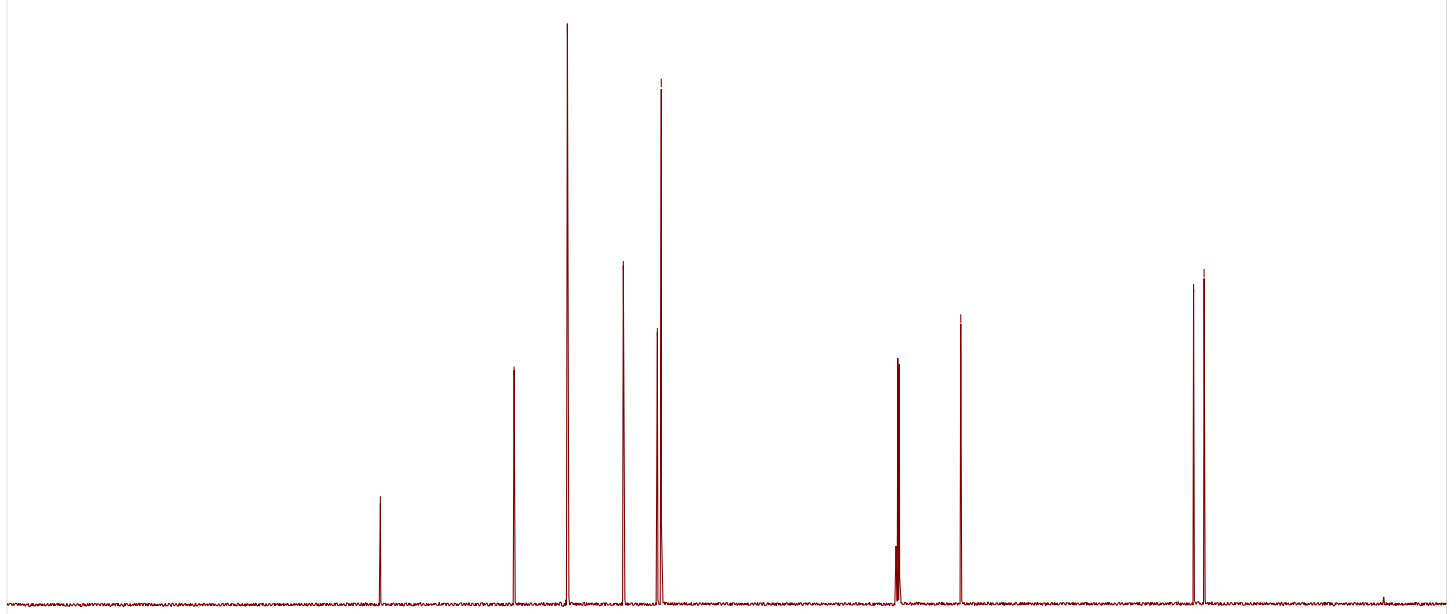

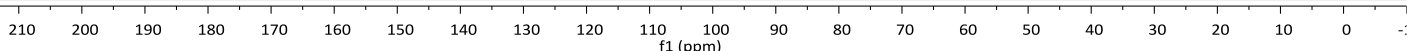




$$
\mathrm{N}_{3} \sim
$$

${ }^{1} \mathbf{H}$ NMR-spectrum $\left(500 \mathrm{MHz}, \mathrm{CDCl}_{3}\right)$ of $\mathbf{S 1 1 a}$

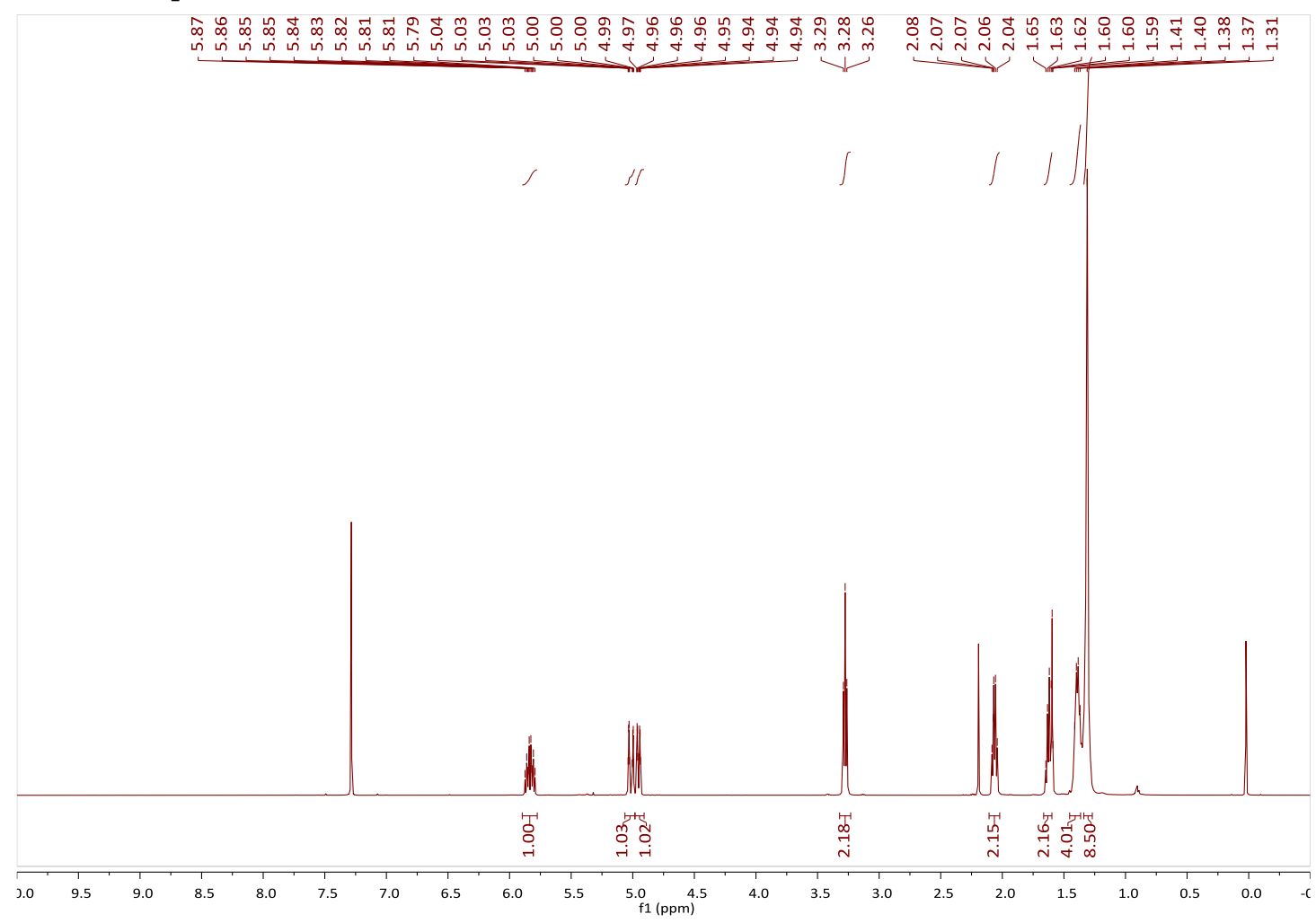

${ }^{13} \mathrm{C}$ NMR-spectrum $\left(125 \mathrm{MHz}, \mathrm{CDCl}_{3}\right)$ of S11a

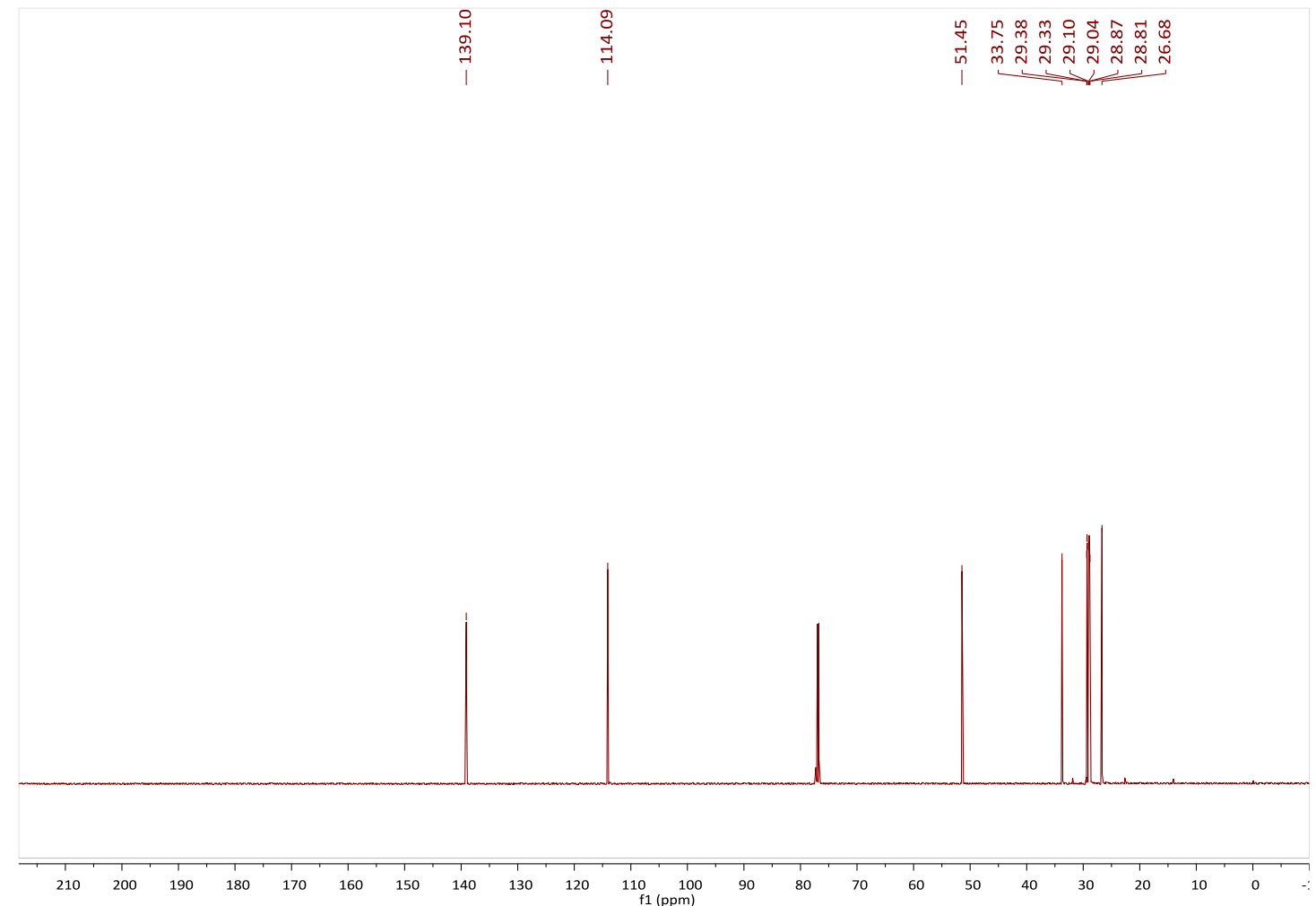




$$
\mathrm{N}_{3} \sim
$$

${ }^{1} \mathbf{H}$ NMR-spectrum $\left(500 \mathrm{MHz}, \mathrm{CDCl}_{3}\right)$ of $\mathbf{S 1 1} \mathbf{b}$

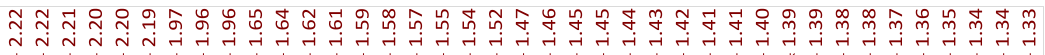

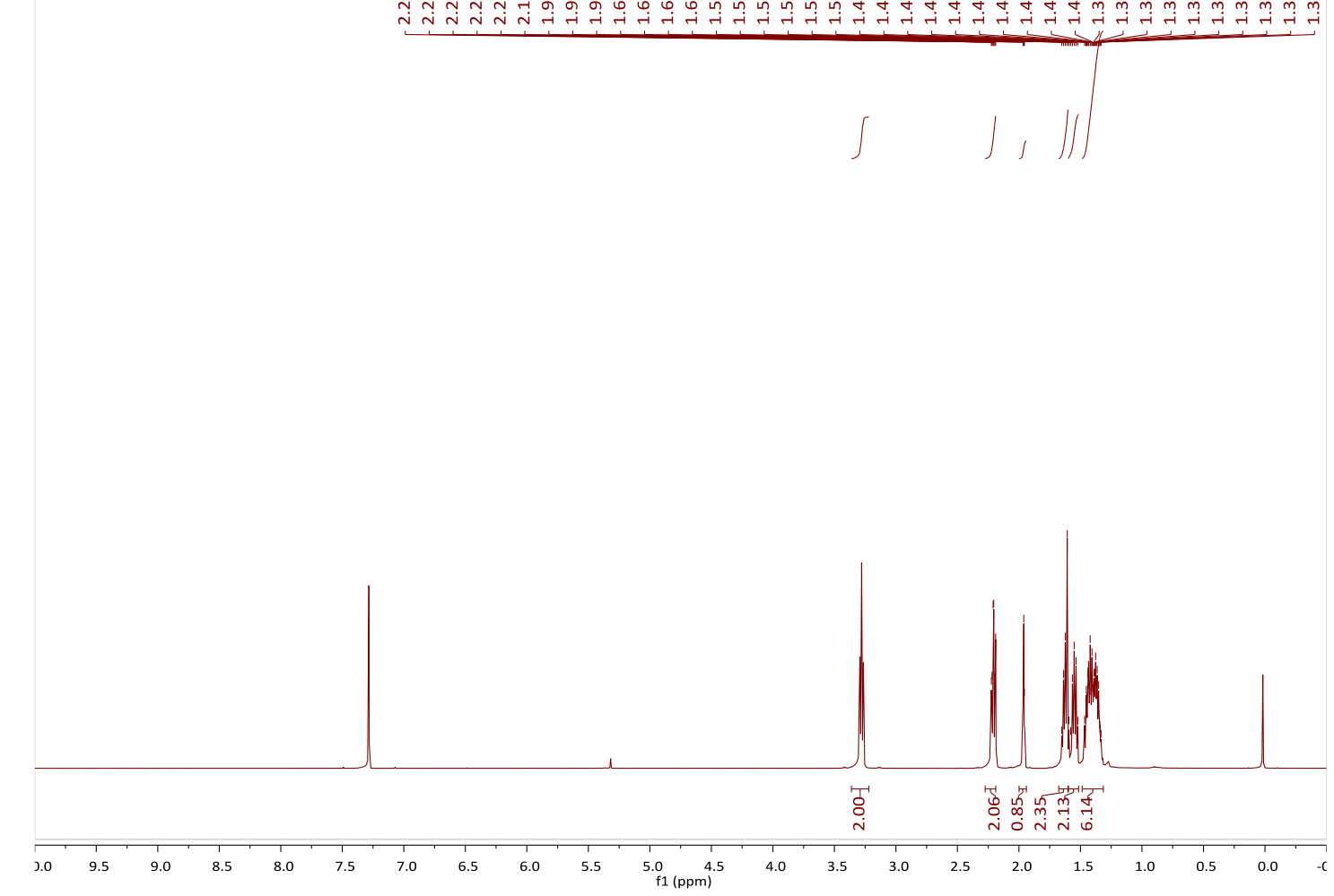

${ }^{13} \mathbf{C}$ NMR-spectrum $\left(125 \mathrm{MHz}, \mathrm{CDCl}_{3}\right)$ of $\mathbf{S 1 1} \mathbf{b}$

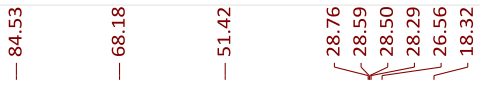
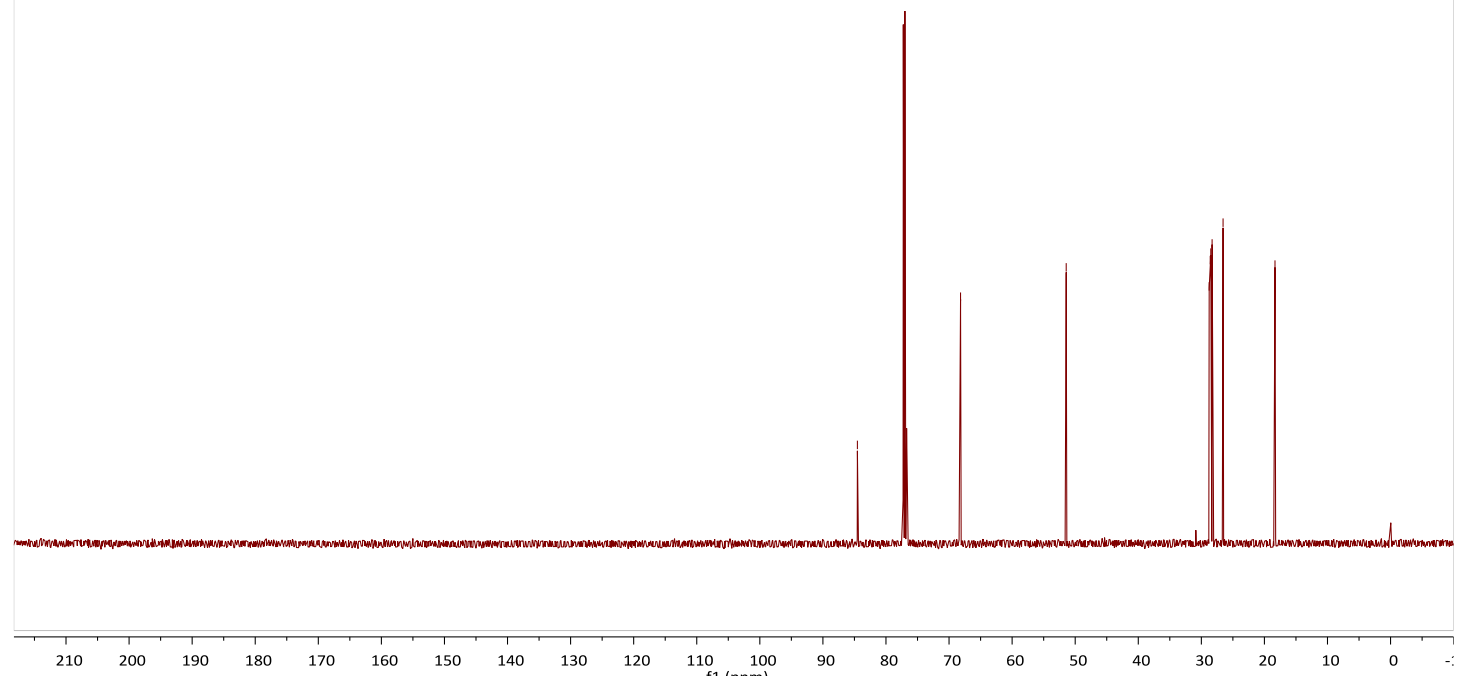
PhthN

${ }^{1} \mathbf{H}$ NMR-spectrum $\left(500 \mathrm{MHz}, \mathrm{CDCl}_{3}\right)$ of $\mathbf{S 1 2}$

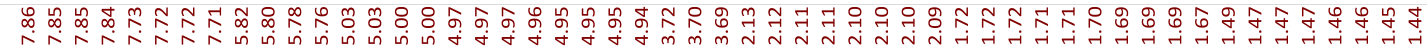

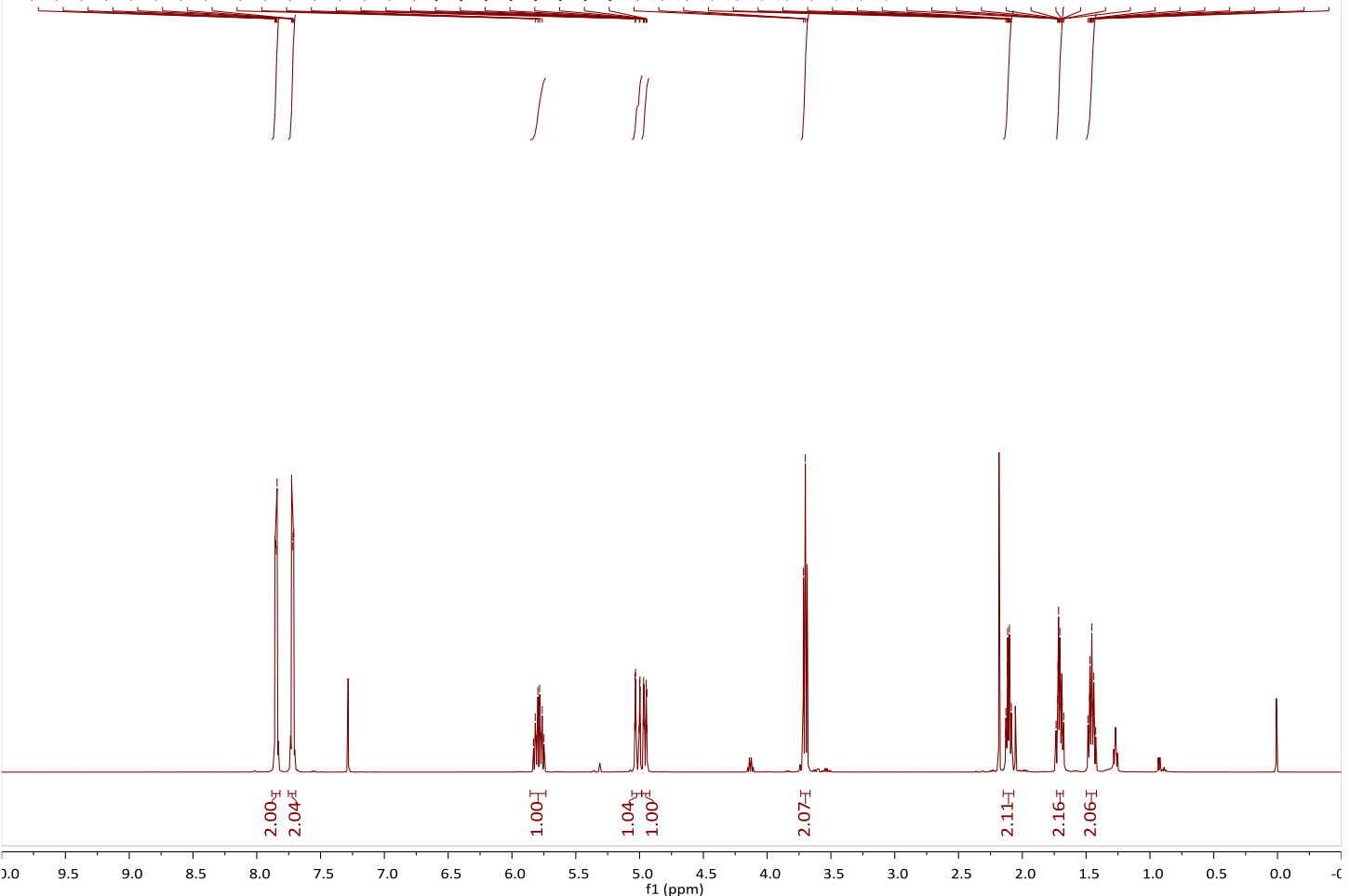

${ }^{13} \mathrm{C}$ NMR-spectrum (125 MHz, $\left.\mathrm{CDCl}_{3}\right)$ of $\mathbf{S 1 2}$

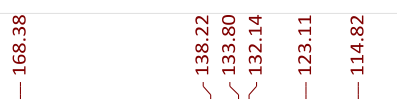

œ용요

$\hat{m}$

$1<11$

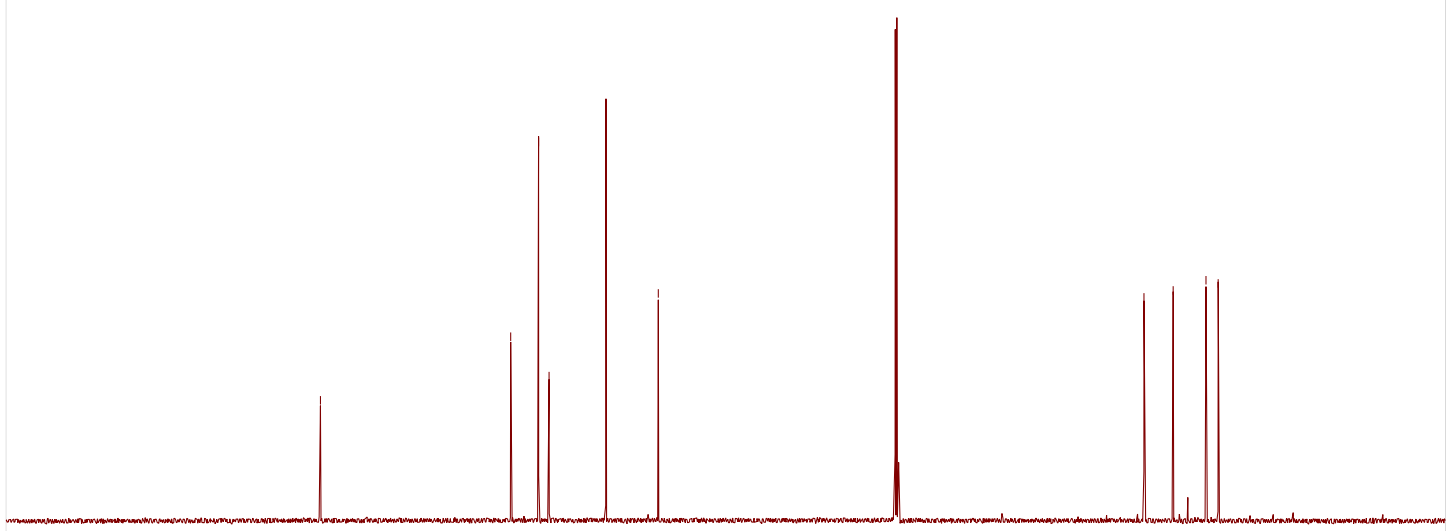

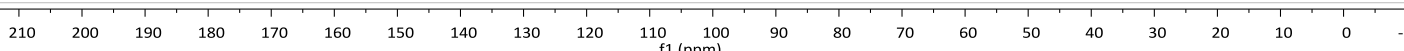




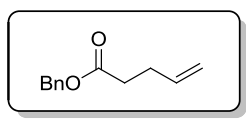

${ }^{1} \mathbf{H}$ NMR-spectrum $\left(500 \mathrm{MHz}, \mathrm{CDCl}_{3}\right)$ of $\mathbf{S 1 4 a}$

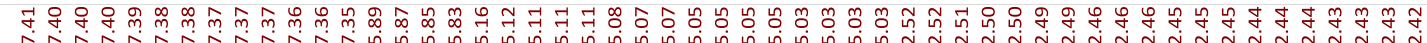
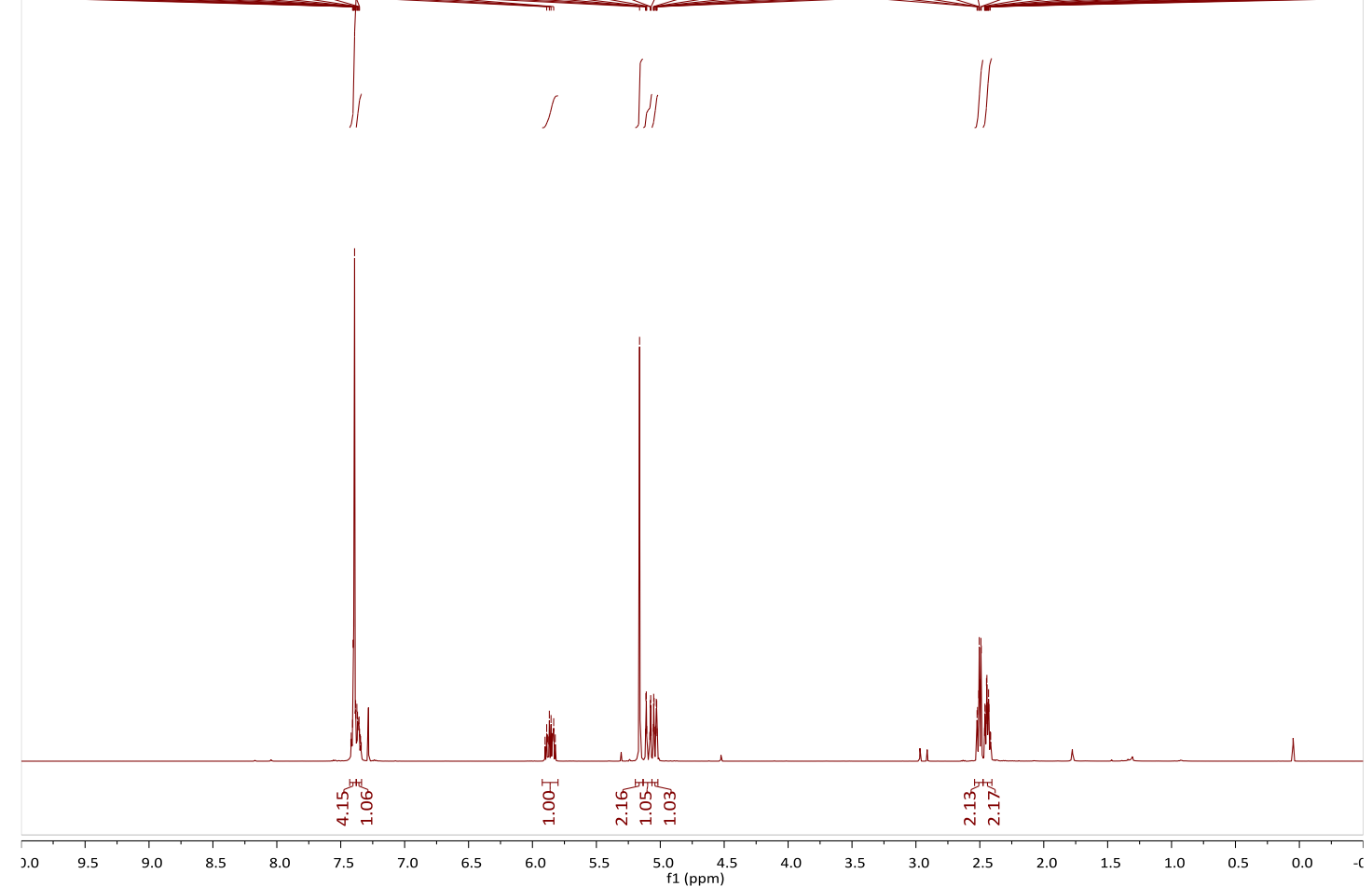

${ }^{13} \mathrm{C}$ NMR-spectrum $\left(125 \mathrm{MHz}, \mathrm{CDCl}_{3}\right)$ of S14a

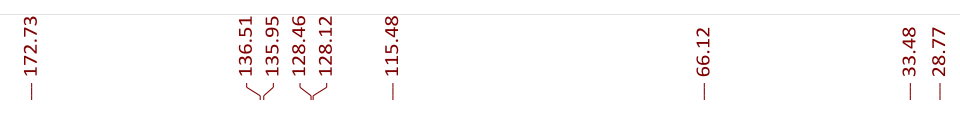

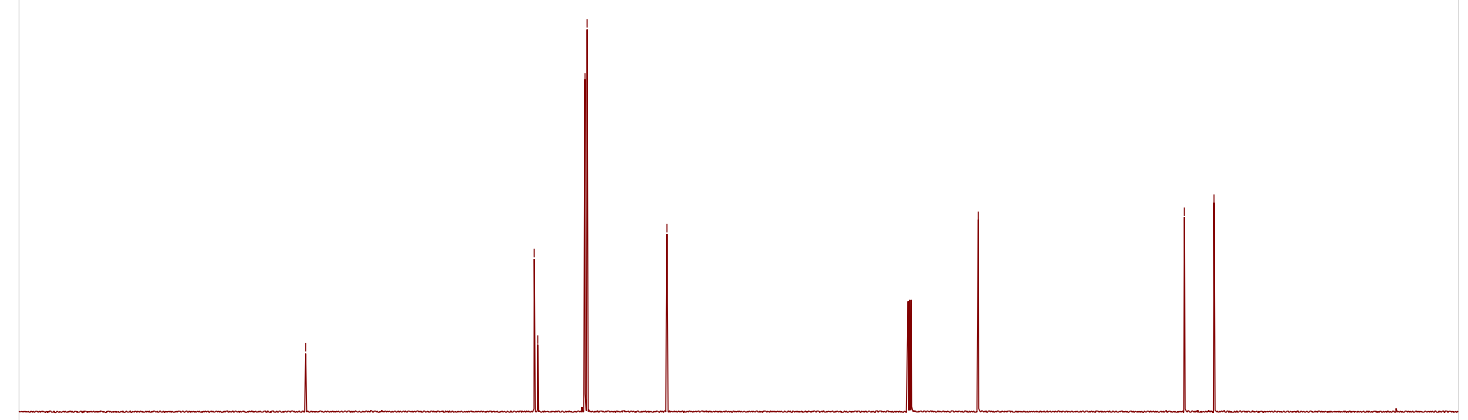

$\begin{array}{llllllllllllllllllllllllllllllllll}210 & 200 & 190 & 180 & 170 & 160 & 150 & 140 & 130 & 120 & 110 & 100 & 90 & 80 & 70 & 60 & 50 & 40 & 30 & 20 & 10 & 0 & \because\end{array}$ 


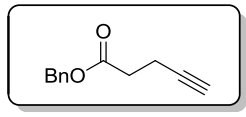

${ }^{1} \mathbf{H}$ NMR-spectrum $\left(500 \mathrm{MHz}, \mathrm{CDCl}_{3}\right)$ of $\mathbf{S 1 4 b}$

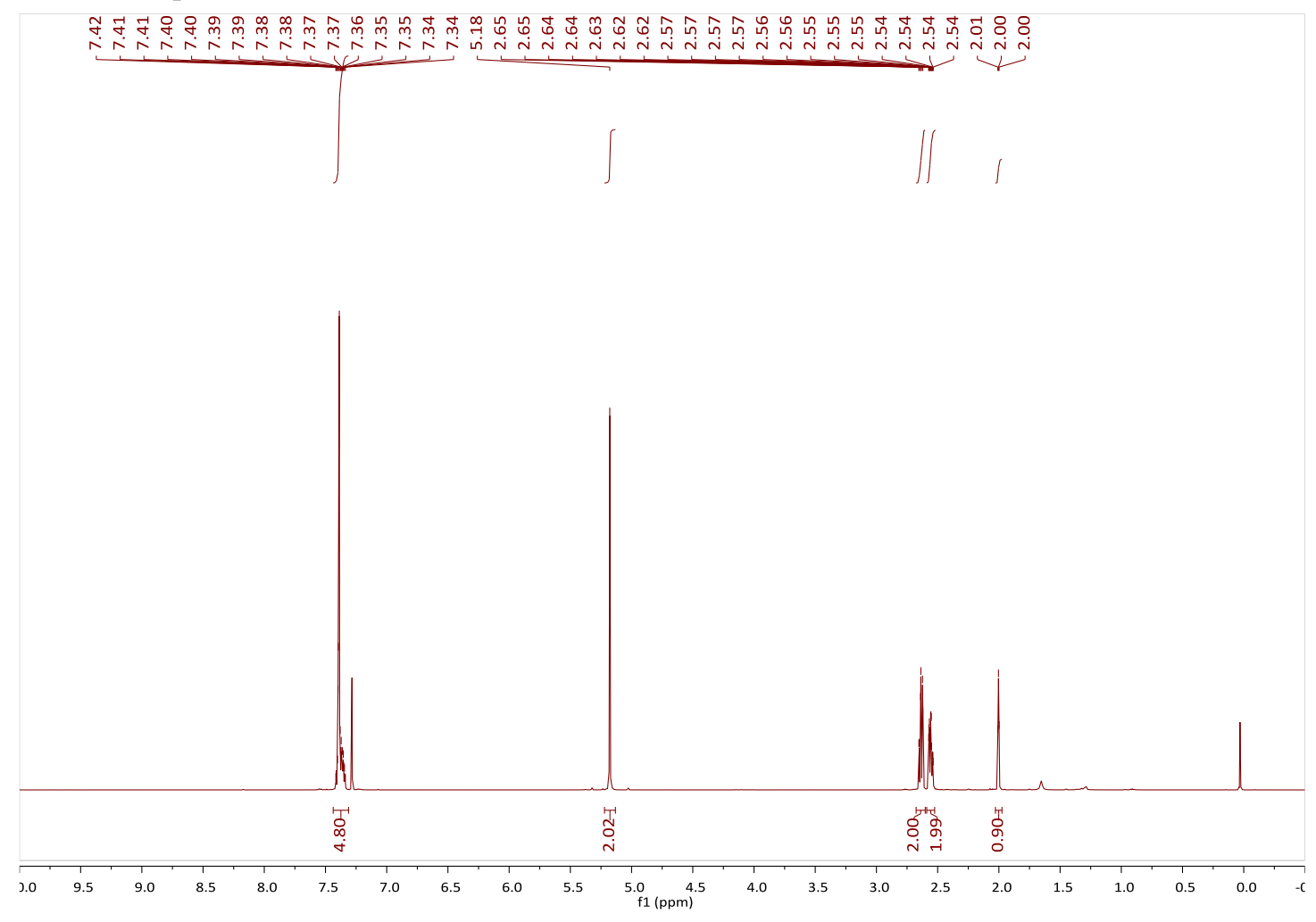

${ }^{13} \mathbf{C} \mathbf{N M R}$-spectrum $\left(125 \mathrm{MHz}, \mathrm{CDCl}_{3}\right)$ of $\mathbf{S 1 4} \mathbf{b}$

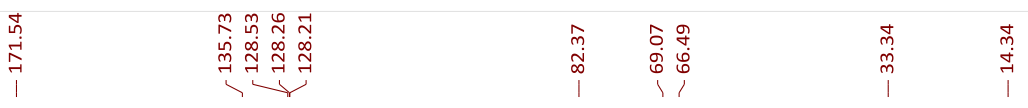

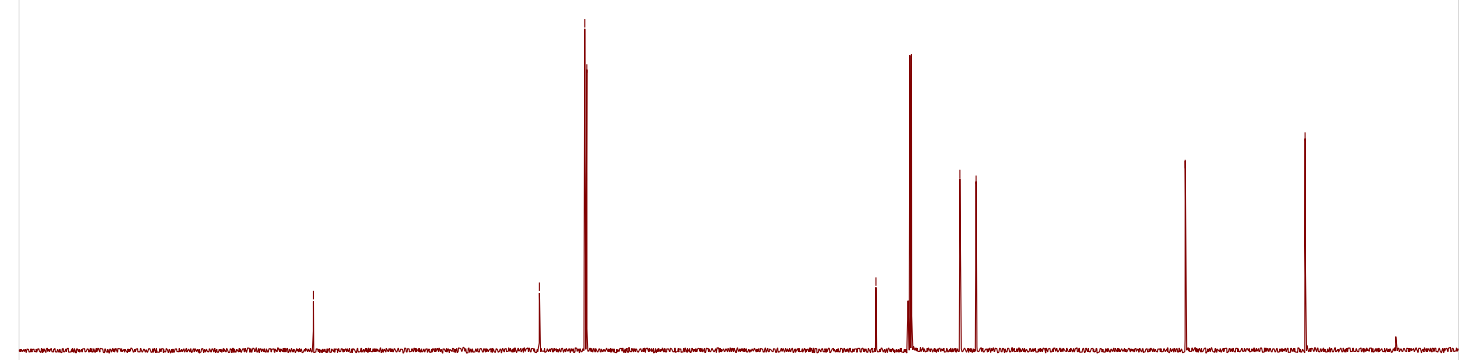

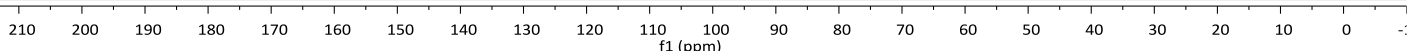




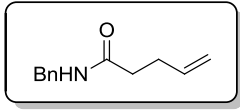

${ }^{1} \mathbf{H}$ NMR-spectrum $\left(500 \mathrm{MHz}, \mathrm{CDCl}_{3}\right)$ of $\mathbf{S 1 5 a}$

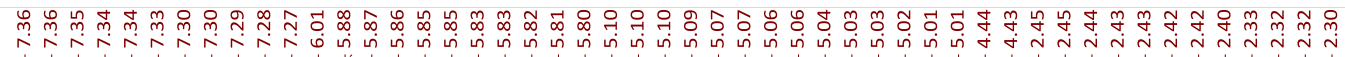

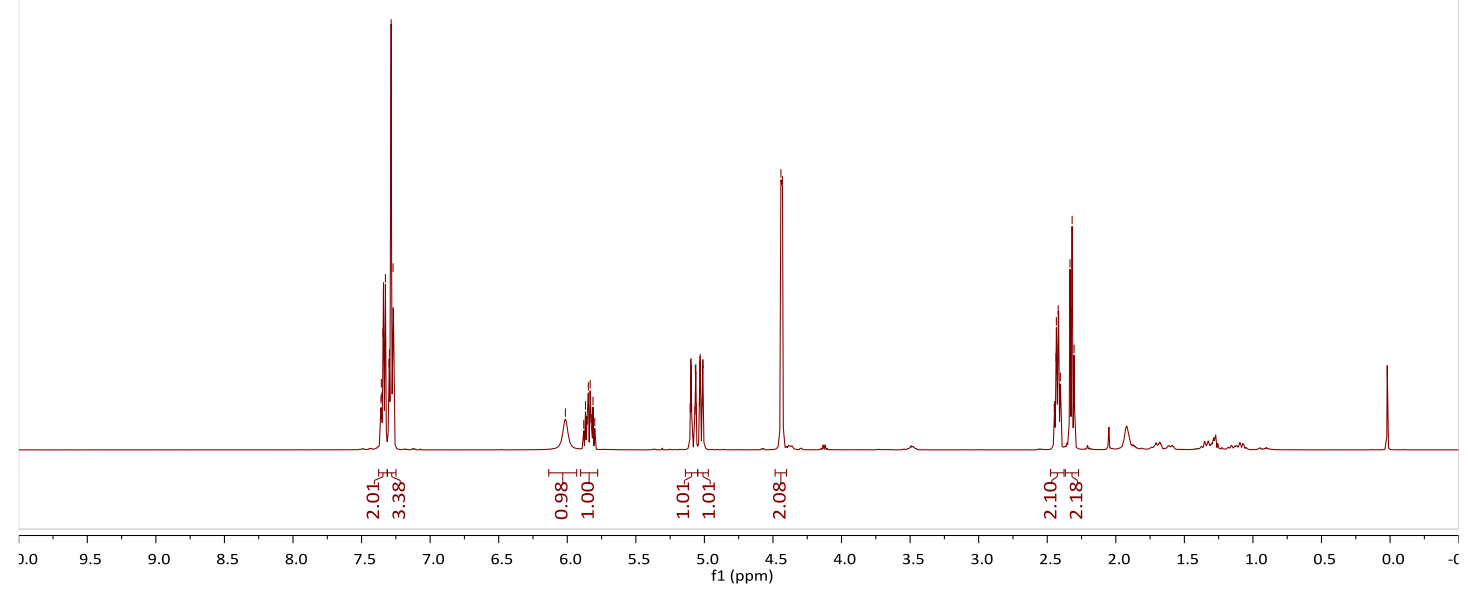

${ }^{13} \mathbf{C}$ NMR-spectrum (125 MHz, $\left.\mathrm{CDCl}_{3}\right)$ of $\mathbf{S 1 5 a}$

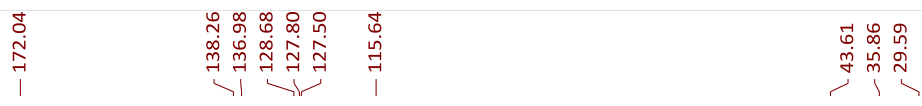

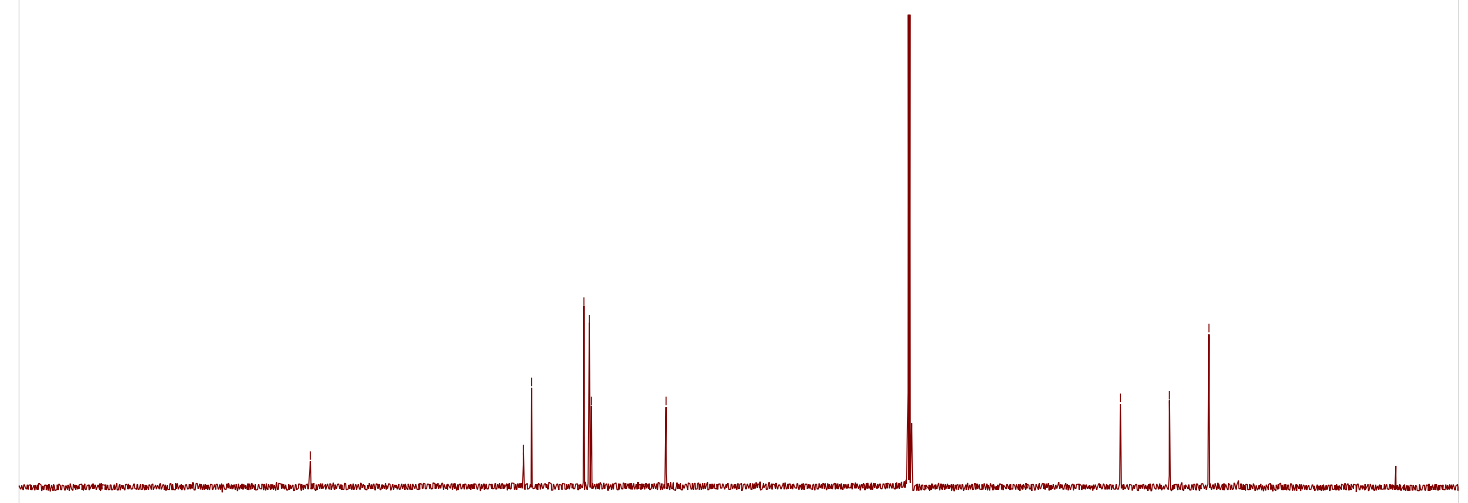

$\begin{array}{lllllllllllllllllllllllllllllllll}210 & 200 & 190 & 180 & 170 & 160 & 150 & 140 & 130 & 120 & 110 & 100 & 90 & 80 & 70 & 60 & 50 & 40 & 30 & 20 & 10 & 0 & =\end{array}$ 


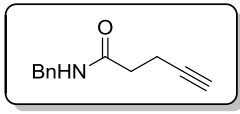

${ }^{1} \mathbf{H}$ NMR-spectrum (500 MHz, $\left.\mathrm{CDCl}_{3}\right)$ of $\mathbf{S 1 5 b}$

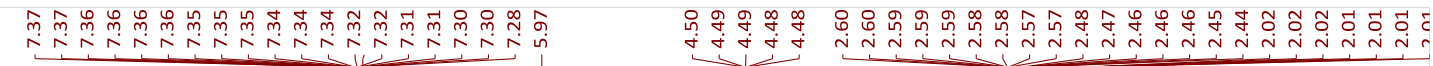

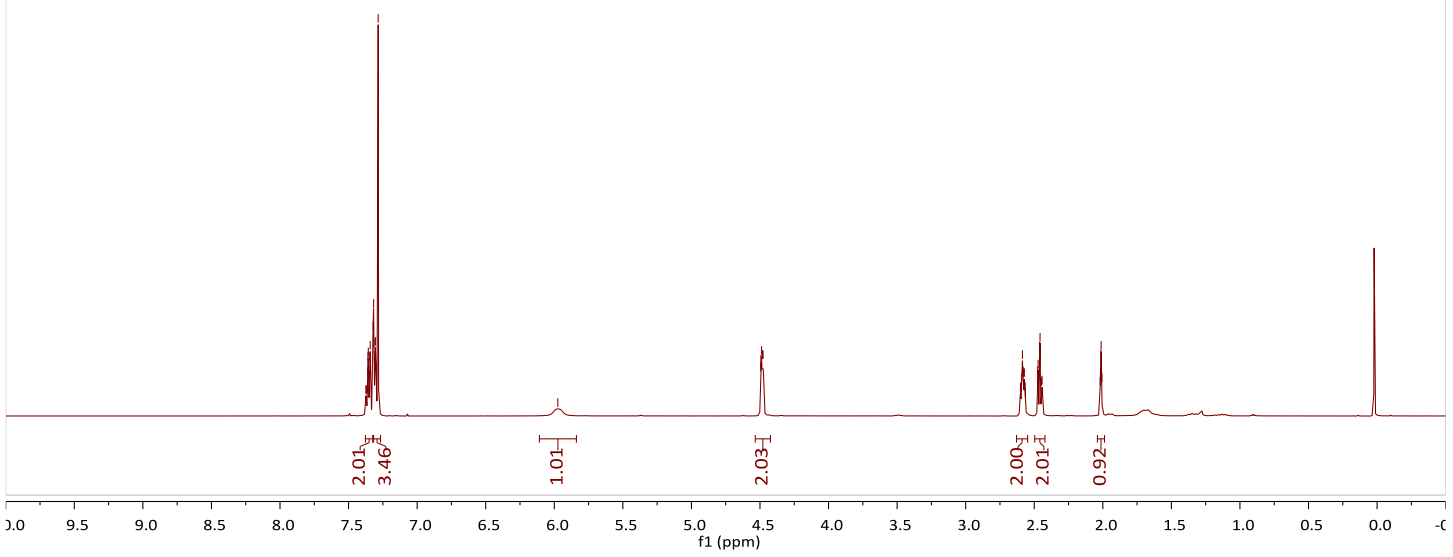

${ }^{13} \mathbf{C}$ NMR-spectrum $\left(125 \mathrm{MHz}, \mathrm{CDCl}_{3}\right)$ of $\mathbf{S 1 5 b}$

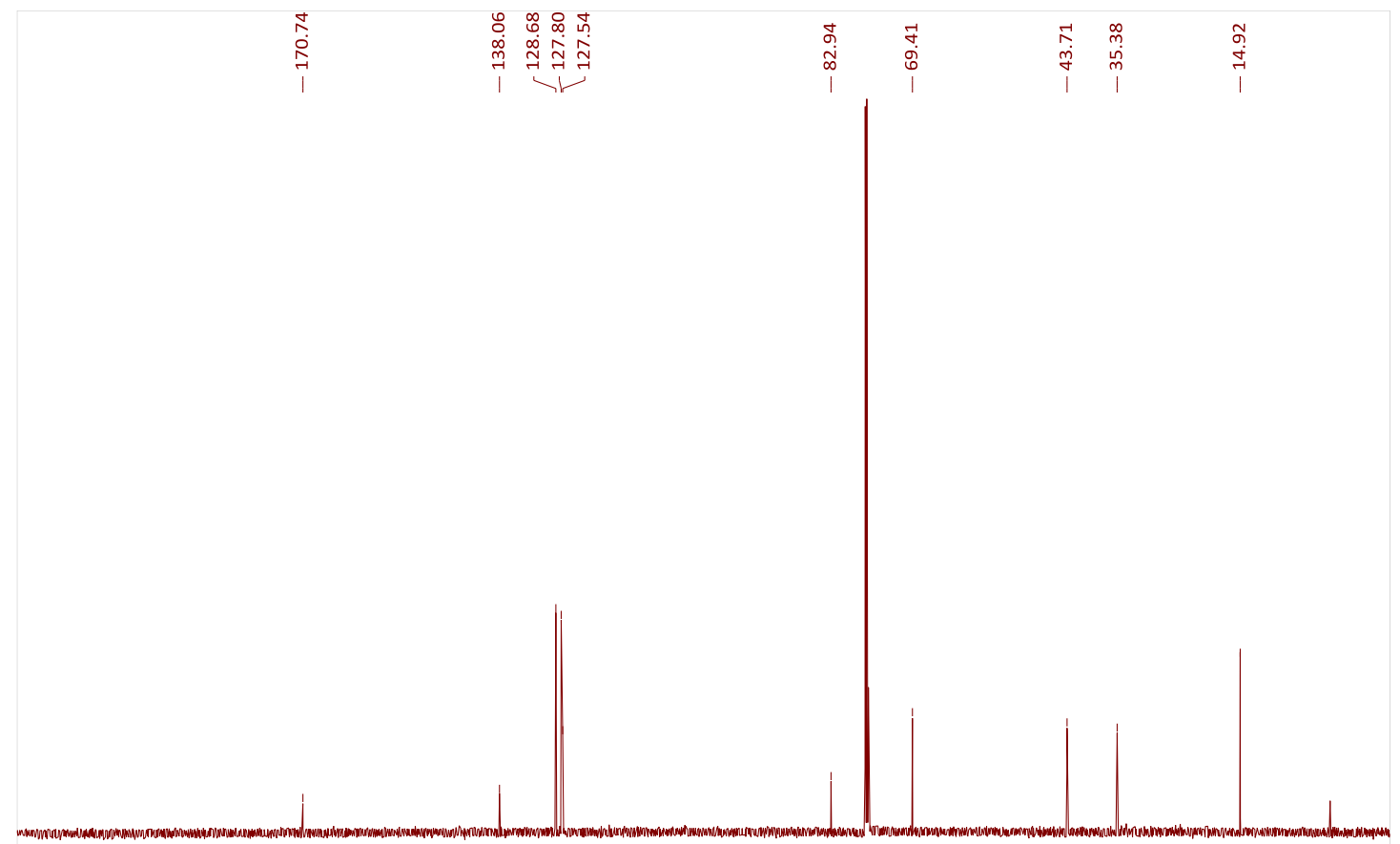

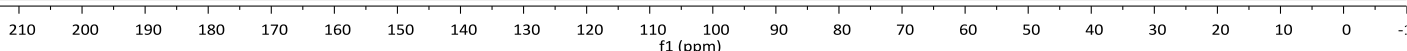




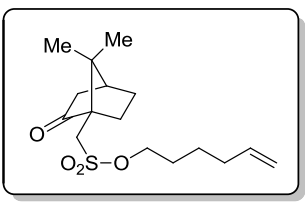

${ }^{1} \mathbf{H}$ NMR-spectrum $\left(500 \mathrm{MHz}, \mathrm{CDCl}_{3}\right)$ of $\mathbf{S 1 7}$

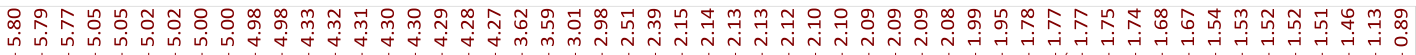

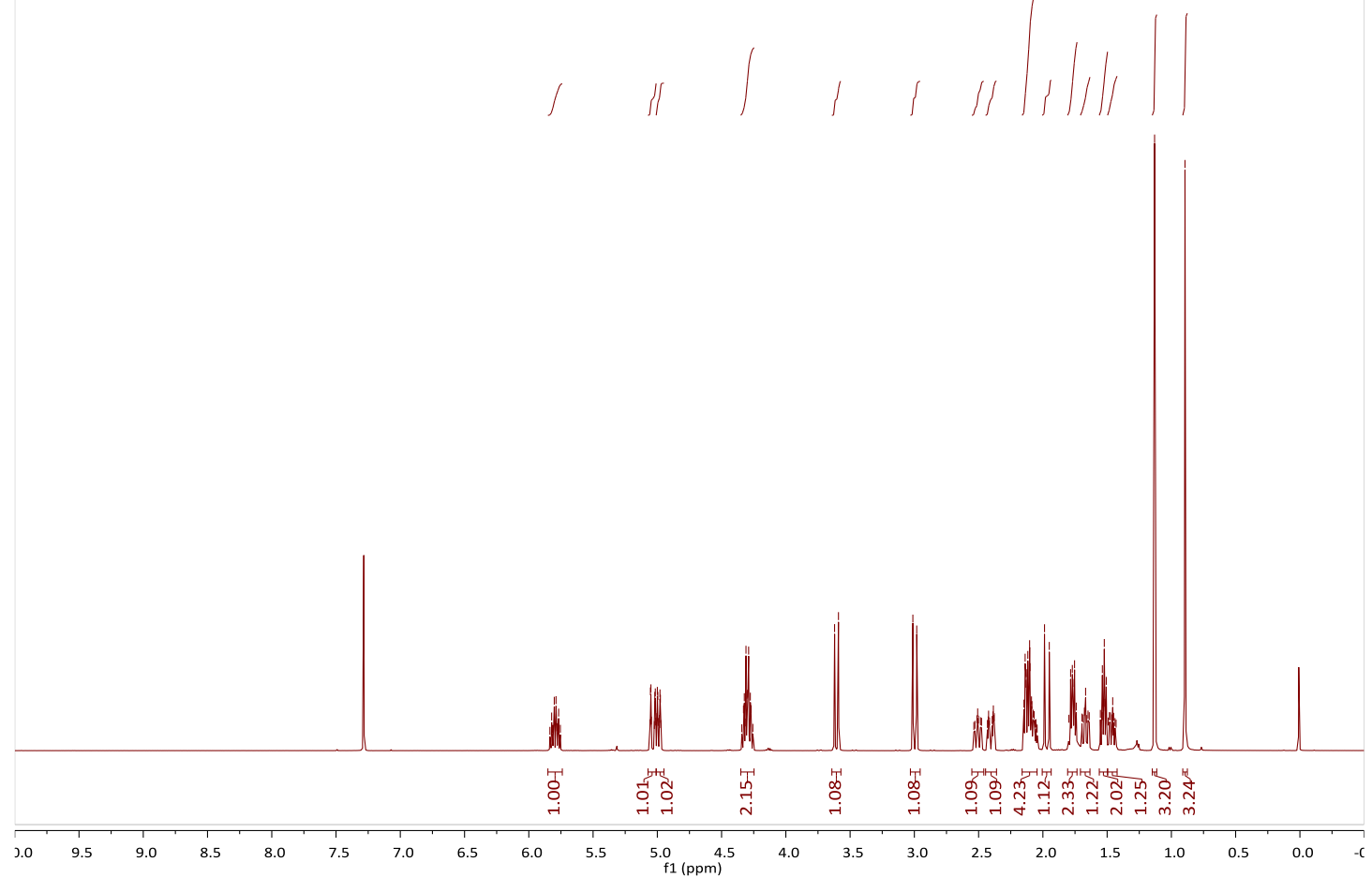

${ }^{13} \mathrm{C}$ NMR-spectrum (125 MHz, $\left.\mathrm{CDCl}_{3}\right)$ of $\mathbf{S 1 7}$
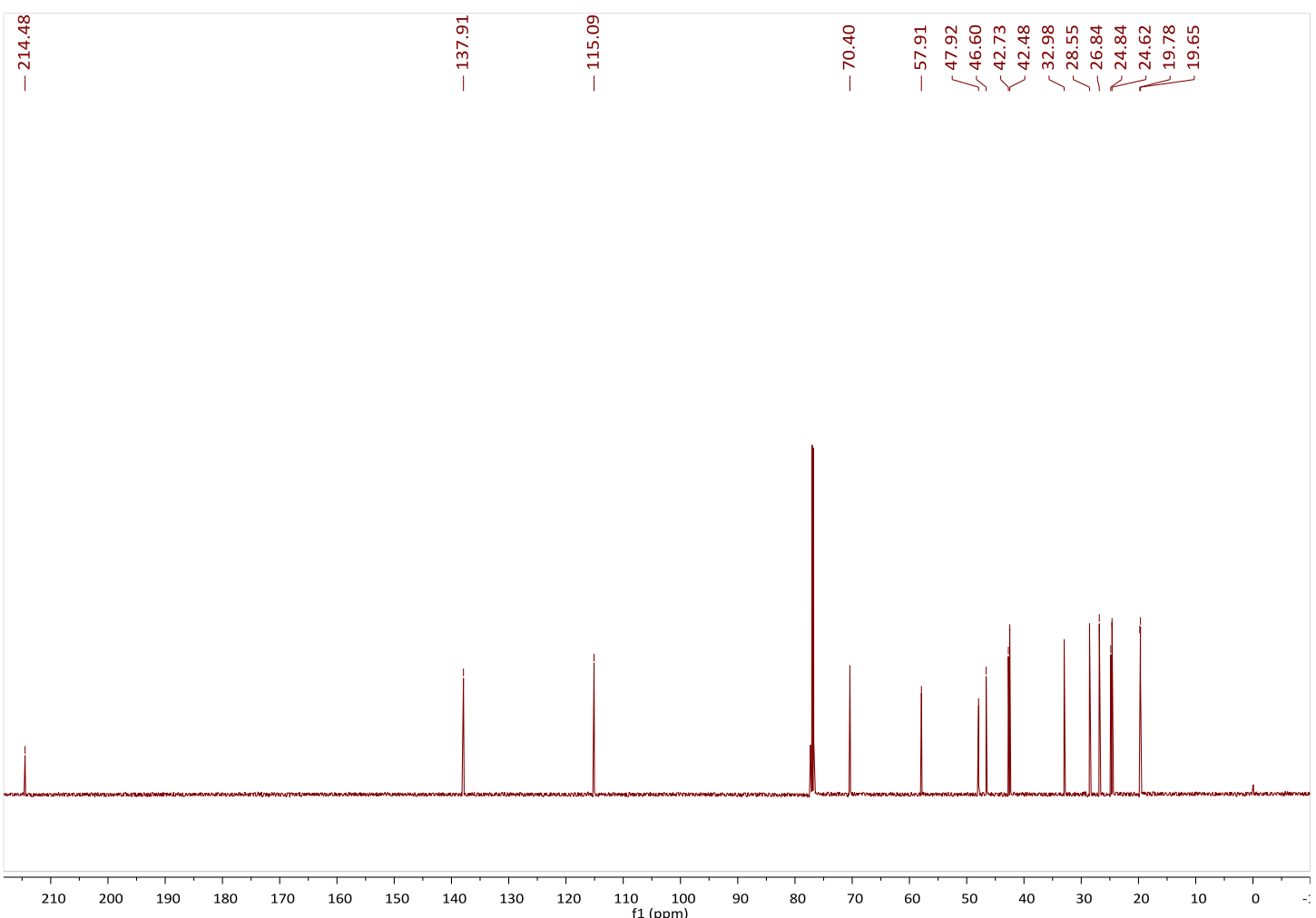


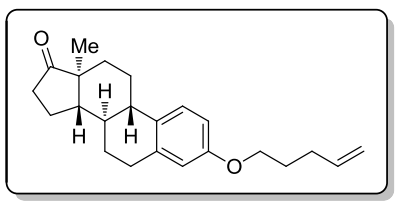

${ }^{1} \mathbf{H}$ NMR-spectrum $\left(500 \mathrm{MHz}, \mathrm{CDCl}_{3}\right)$ of $\mathbf{S 1 9}$

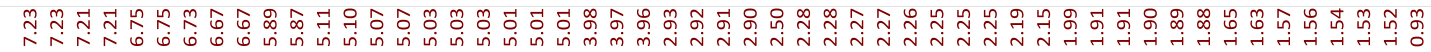

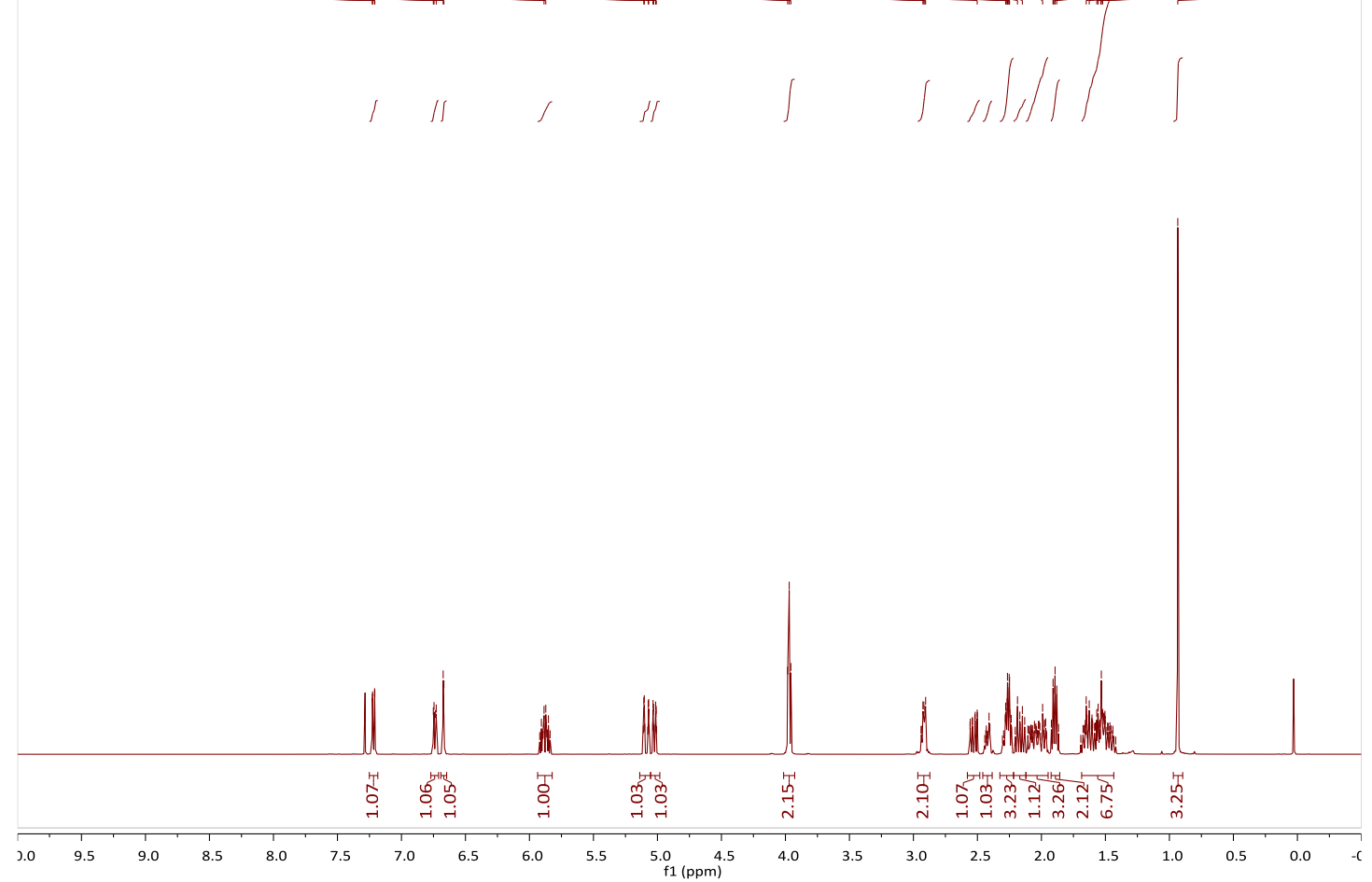

${ }^{13} \mathrm{C}$ NMR-spectrum (125 MHz, $\left.\mathrm{CDCl}_{3}\right)$ of $\mathbf{S 1 9}$

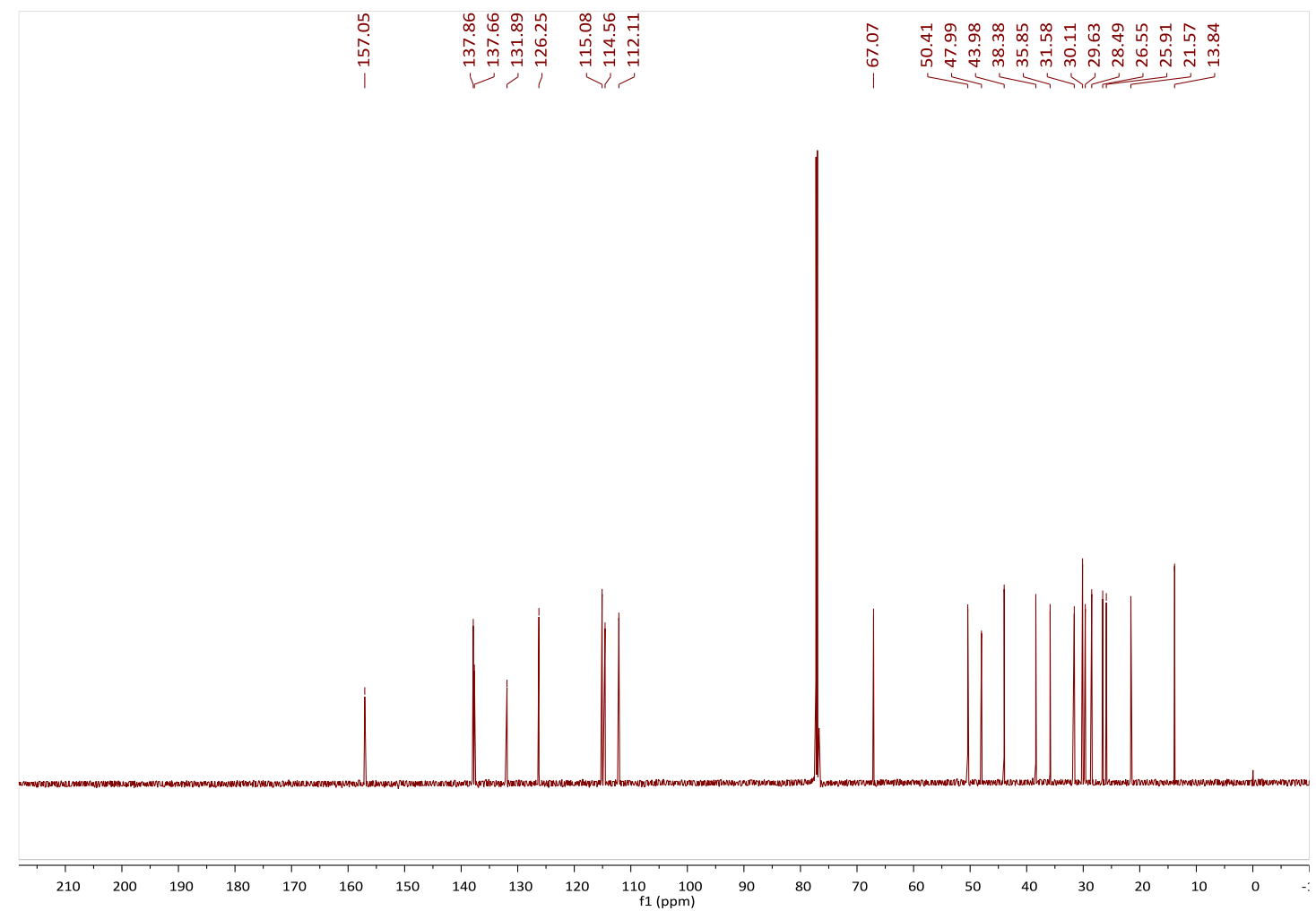




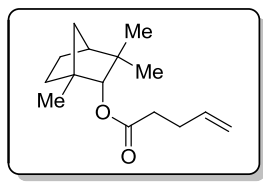

${ }^{1} \mathbf{H}$ NMR-spectrum $\left(500 \mathrm{MHz}, \mathrm{CDCl}_{3}\right)$ of $\mathbf{S 2 1}$

舫

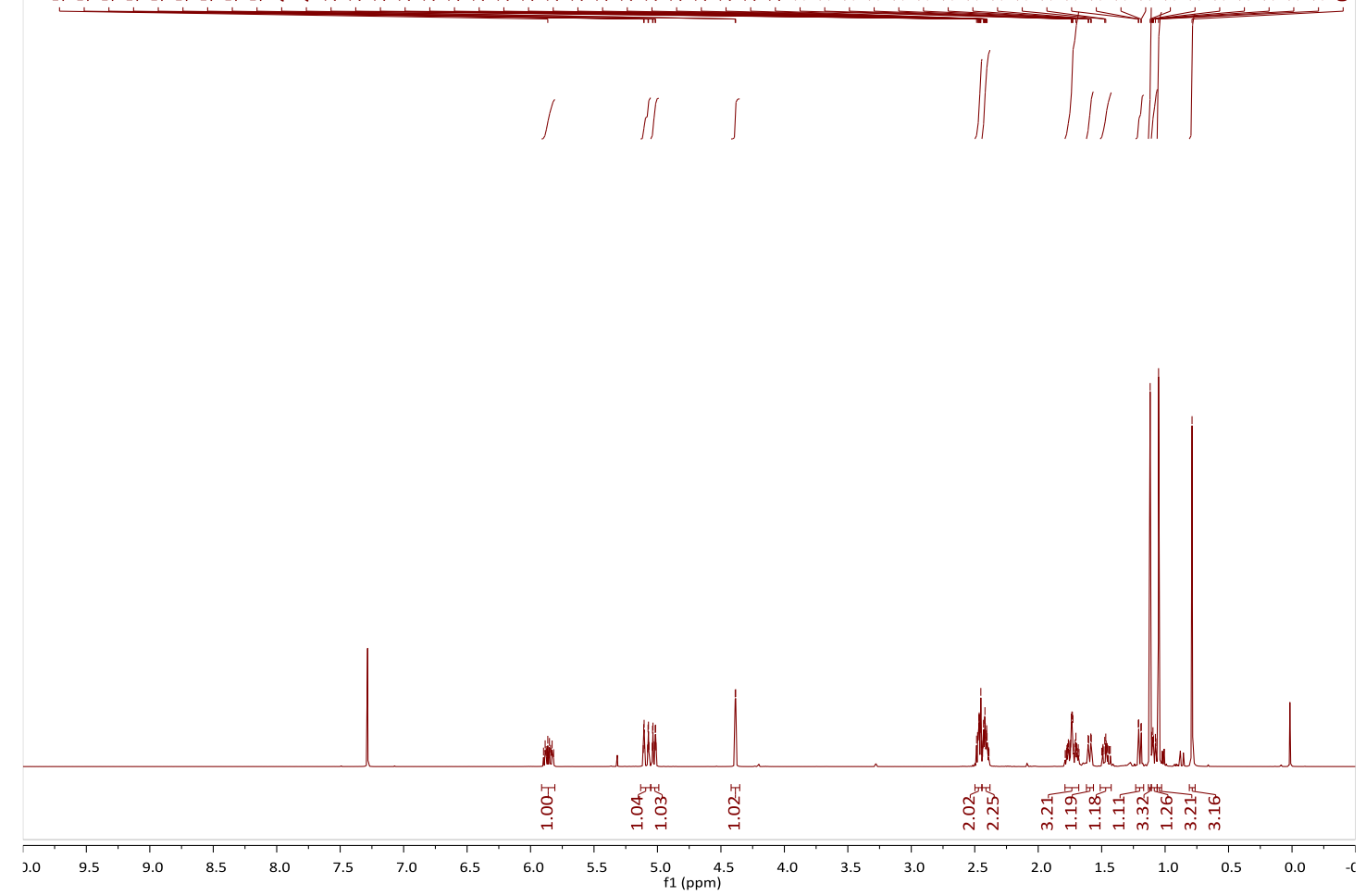

${ }^{13} \mathrm{C}$ NMR-spectrum (125 MHz, $\left.\mathrm{CDCl}_{3}\right)$ of $\mathbf{S 2 1}$

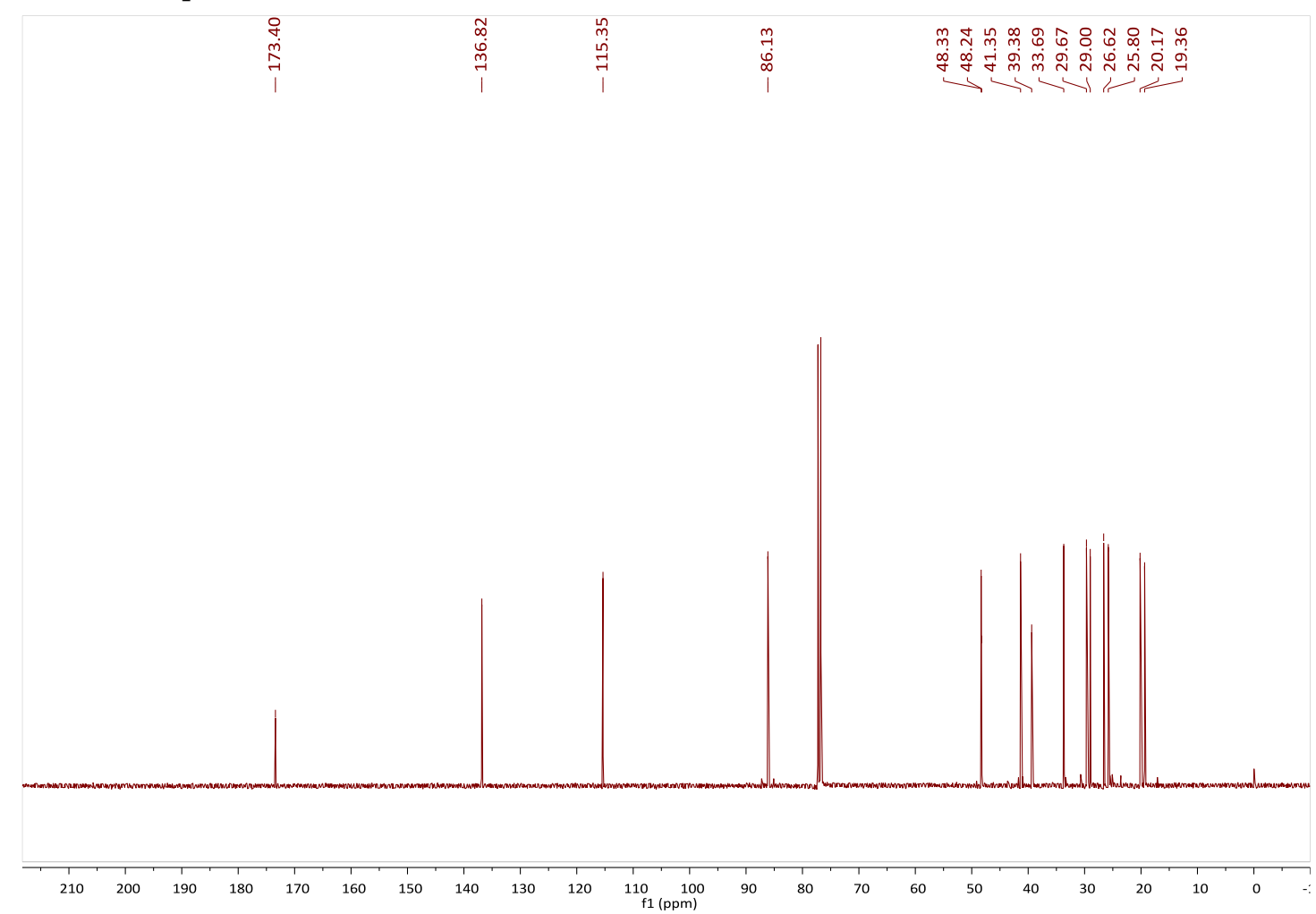




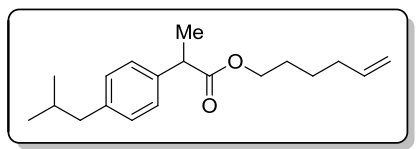

${ }^{1} \mathbf{H}$ NMR-spectrum $\left(500 \mathrm{MHz}, \mathrm{CDCl}_{3}\right)$ of $\mathbf{S 2 3}$

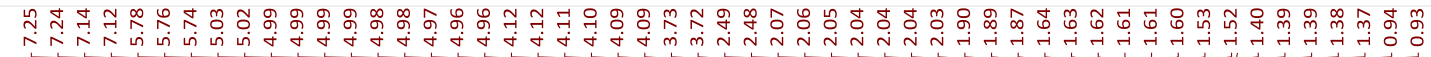

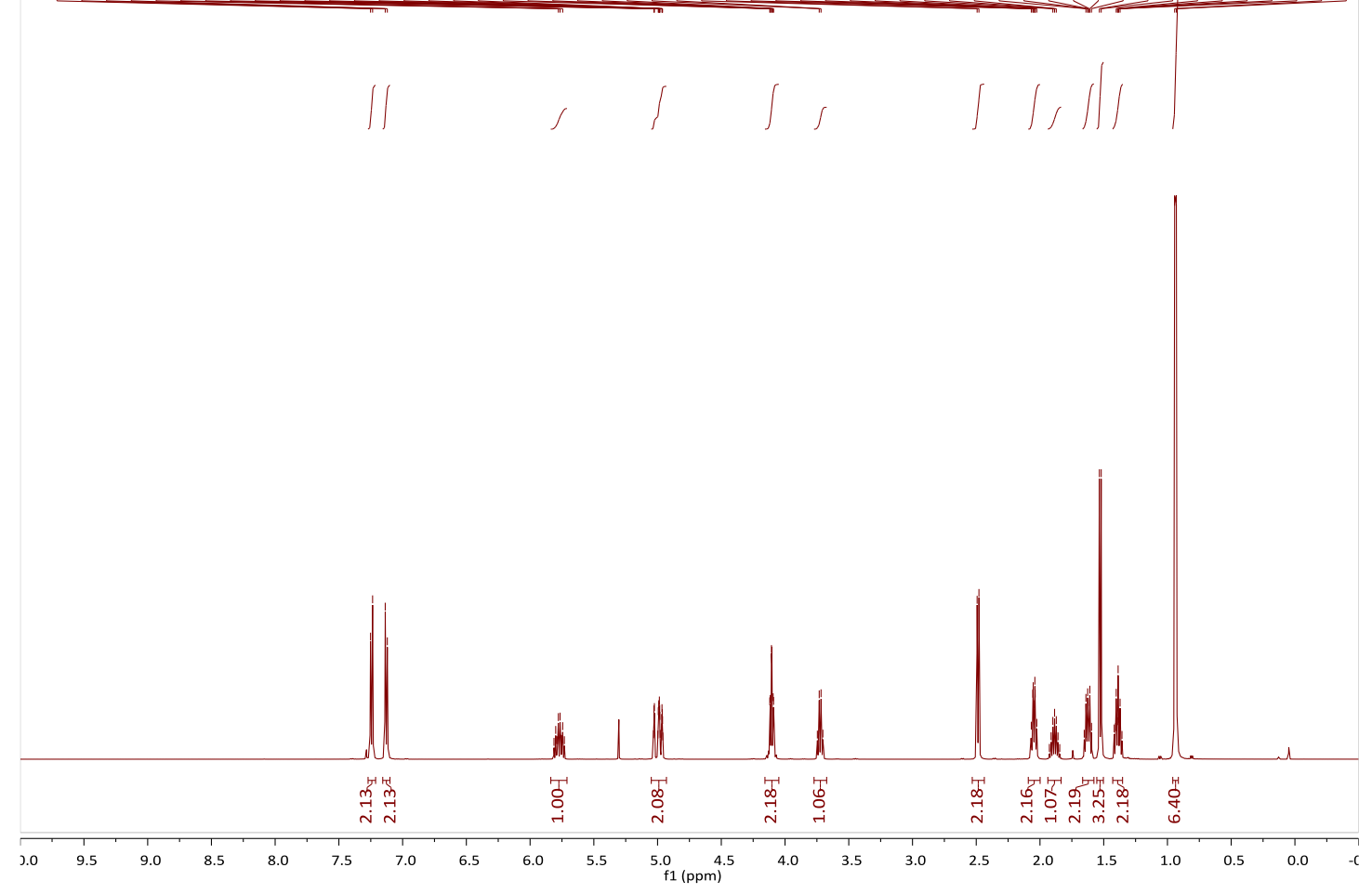

${ }^{13} \mathbf{C}$ NMR-spectrum (125 MHz, $\left.\mathrm{CDCl}_{3}\right)$ of $\mathbf{S 2 3}$

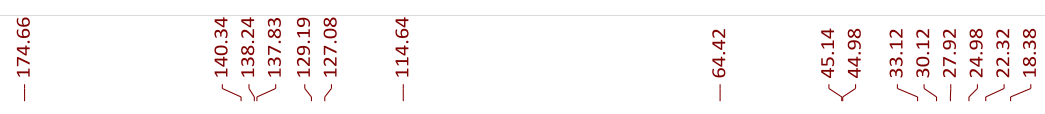

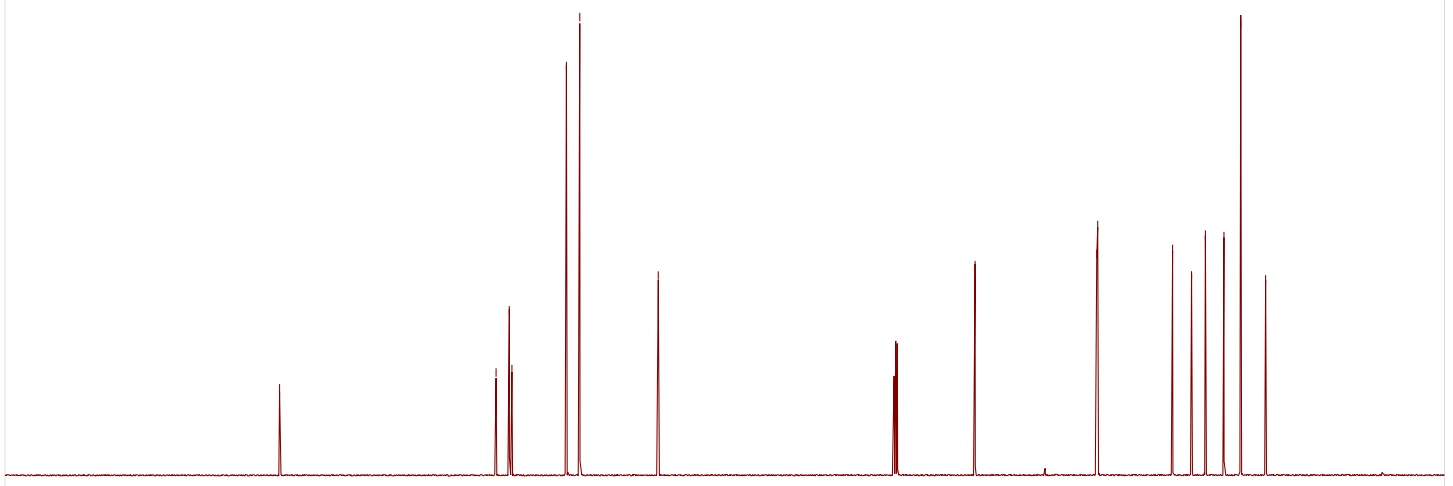

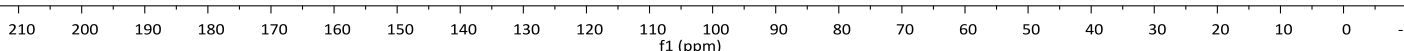




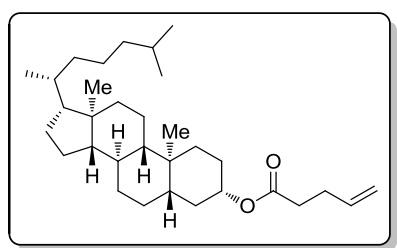

${ }^{1} \mathbf{H}$ NMR-spectrum $\left(500 \mathrm{MHz}, \mathrm{CDCl}_{3}\right)$ of $\mathbf{S 2 5}$

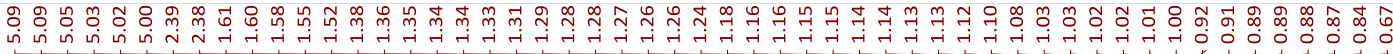

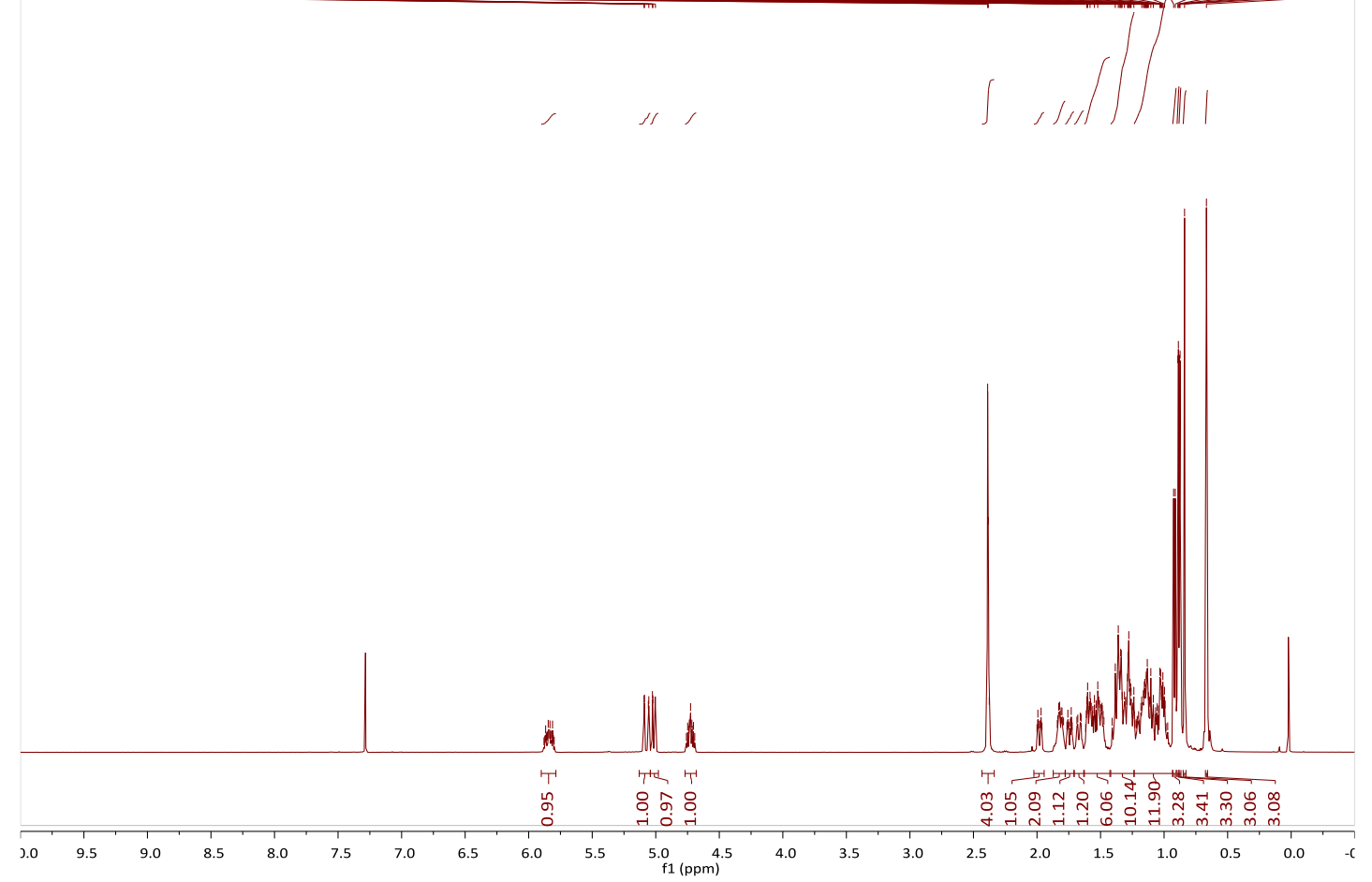

${ }^{13} \mathrm{C}$ NMR-spectrum (125 MHz, $\left.\mathrm{CDCl}_{3}\right)$ of $\mathbf{S 2 5}$
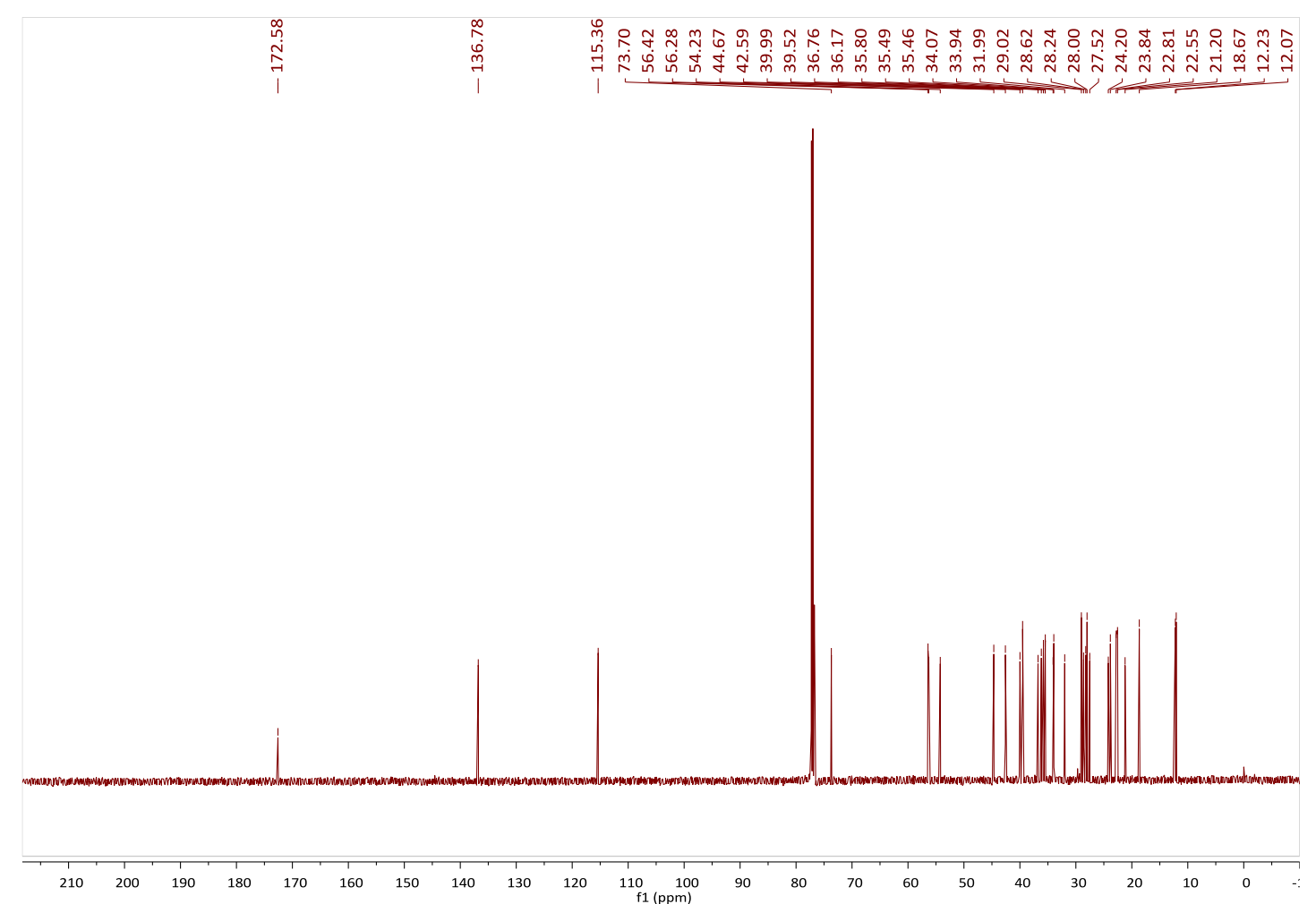


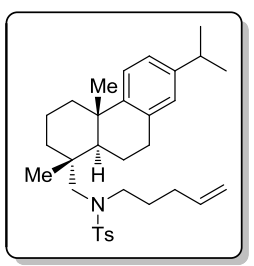

${ }^{1} \mathbf{H}$ NMR-spectrum $\left(500 \mathrm{MHz}, \mathrm{CDCl}_{3}\right)$ of $\mathbf{S 2 8}$

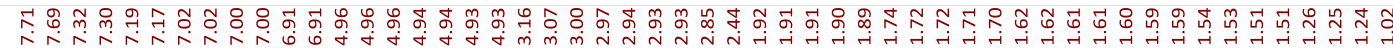

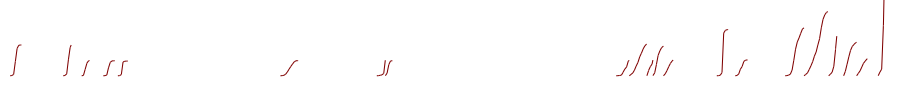

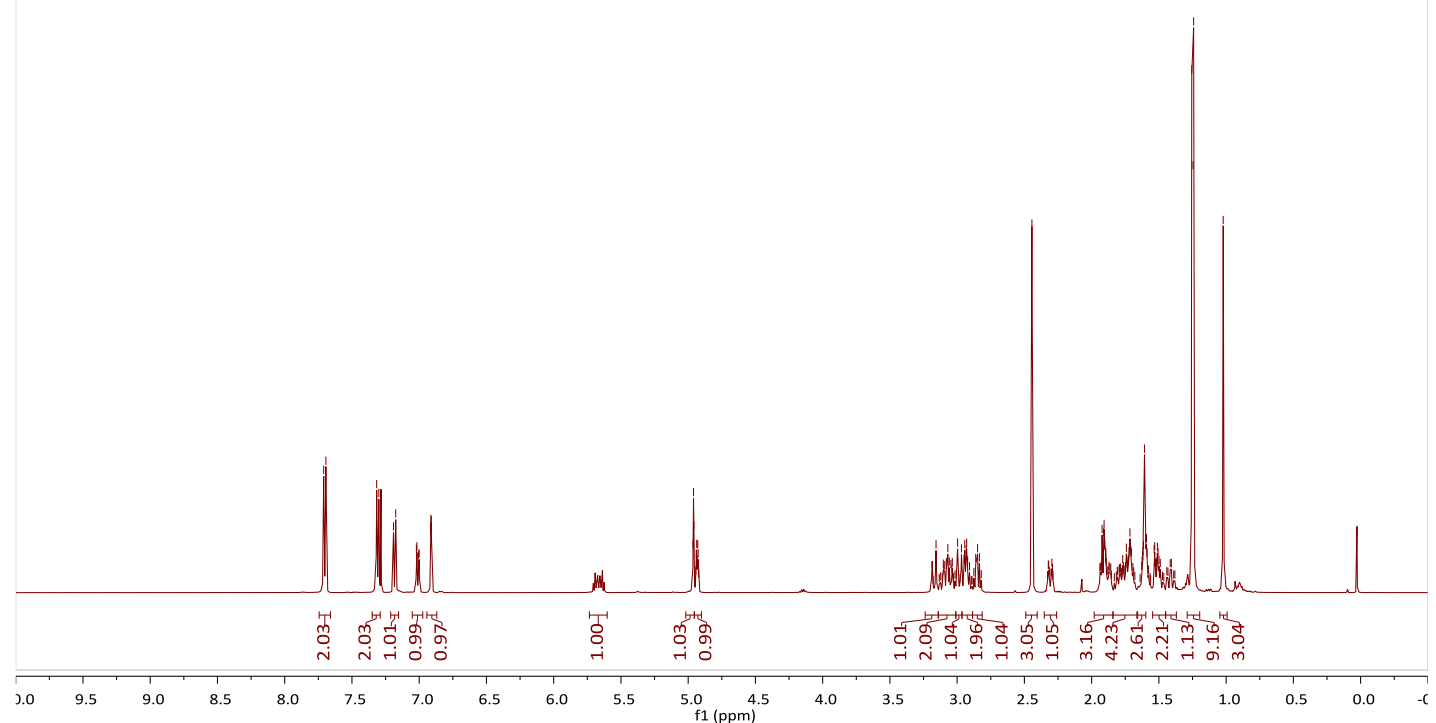

${ }^{13} \mathrm{C}$ NMR-spectrum (125 MHz, $\left.\mathrm{CDCl}_{3}\right)$ of $\mathbf{S 2 8}$

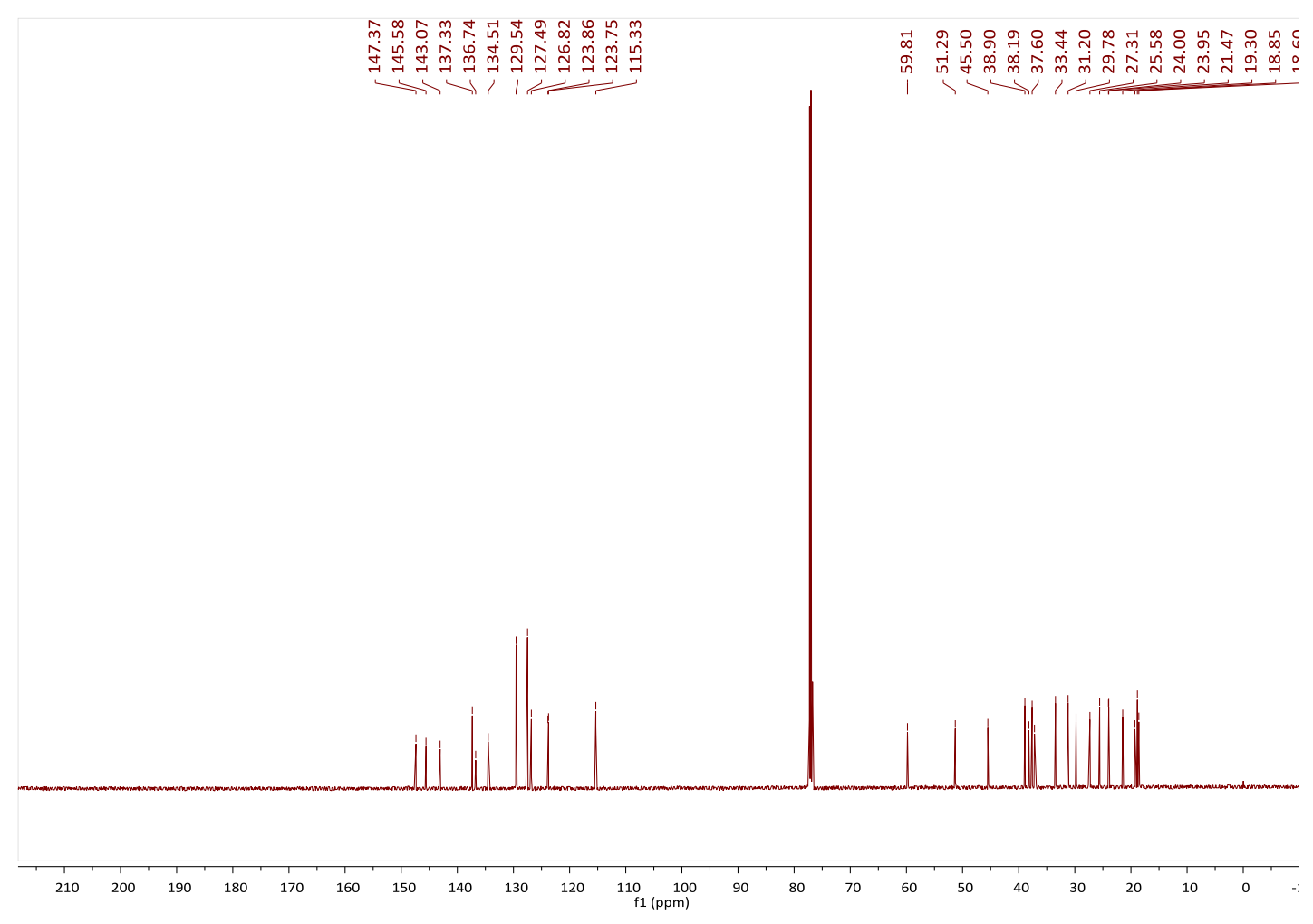




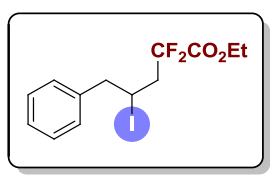

${ }^{1} \mathbf{H}$ NMR-spectrum $\left(500 \mathrm{MHz}, \mathrm{CDCl}_{3}\right)$ of $3 \mathbf{a}$

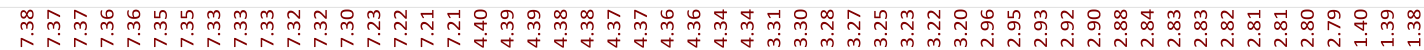
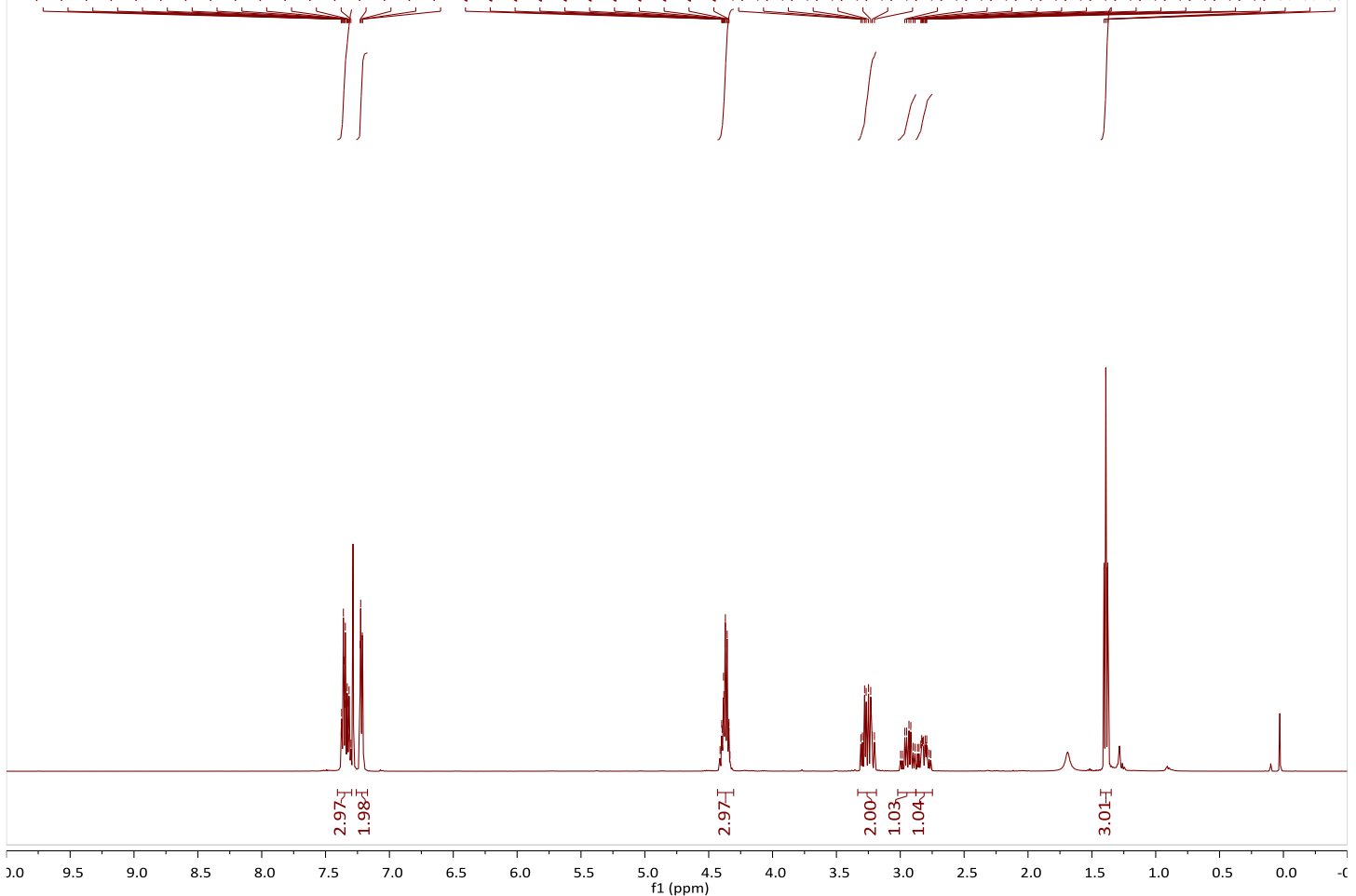

${ }^{19} \mathbf{F}$ NMR-spectrum $\left(471 \mathrm{MHz}, \mathrm{CDCl}_{3}\right)$ of $\mathbf{3 a}$

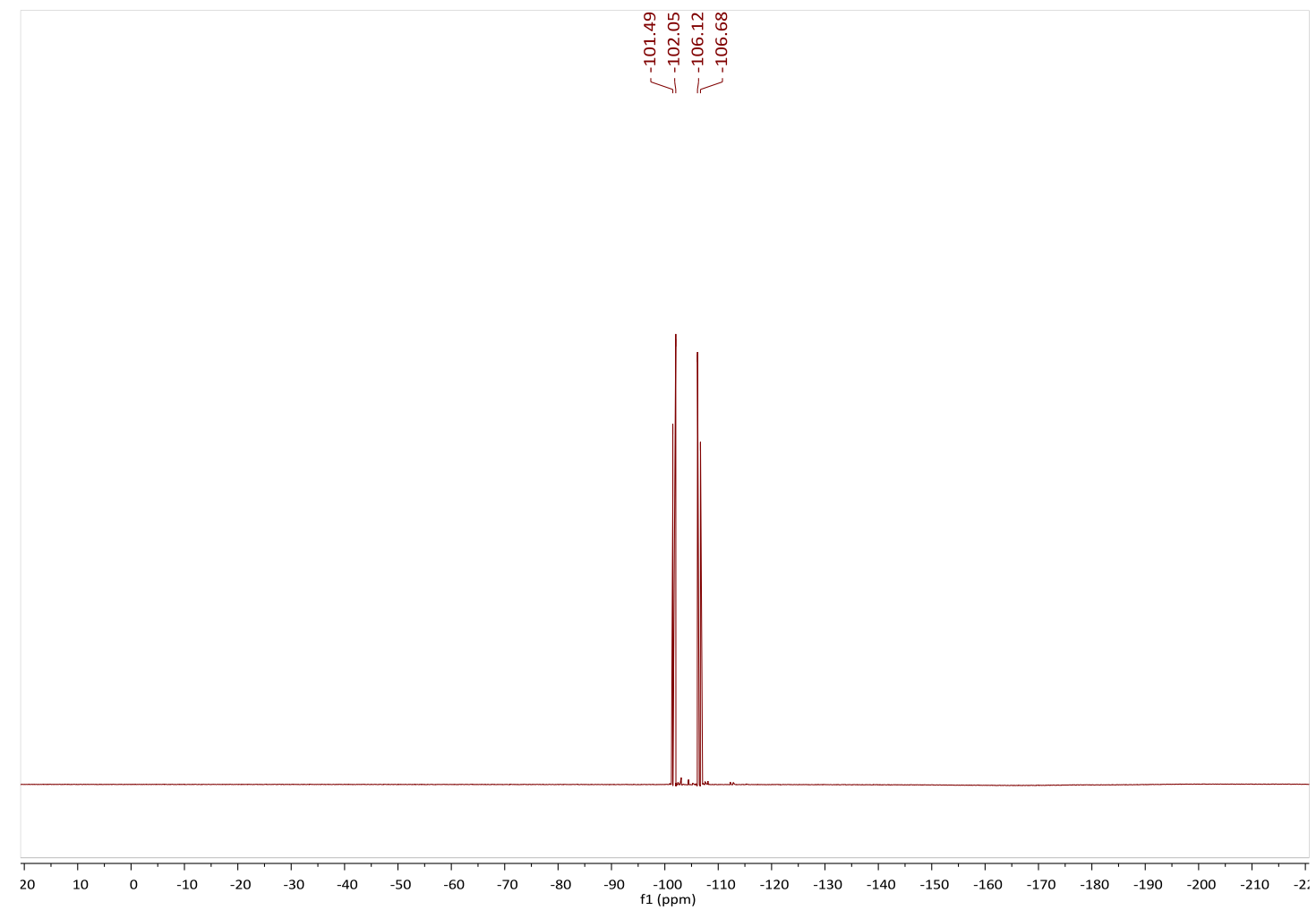




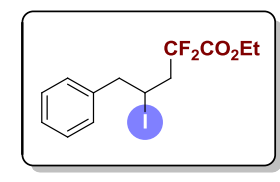

${ }^{13} \mathrm{C}$ NMR-spectrum (125 MHz, $\left.\mathrm{CDCl}_{3}\right)$ of 3a

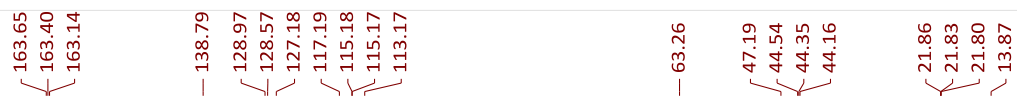
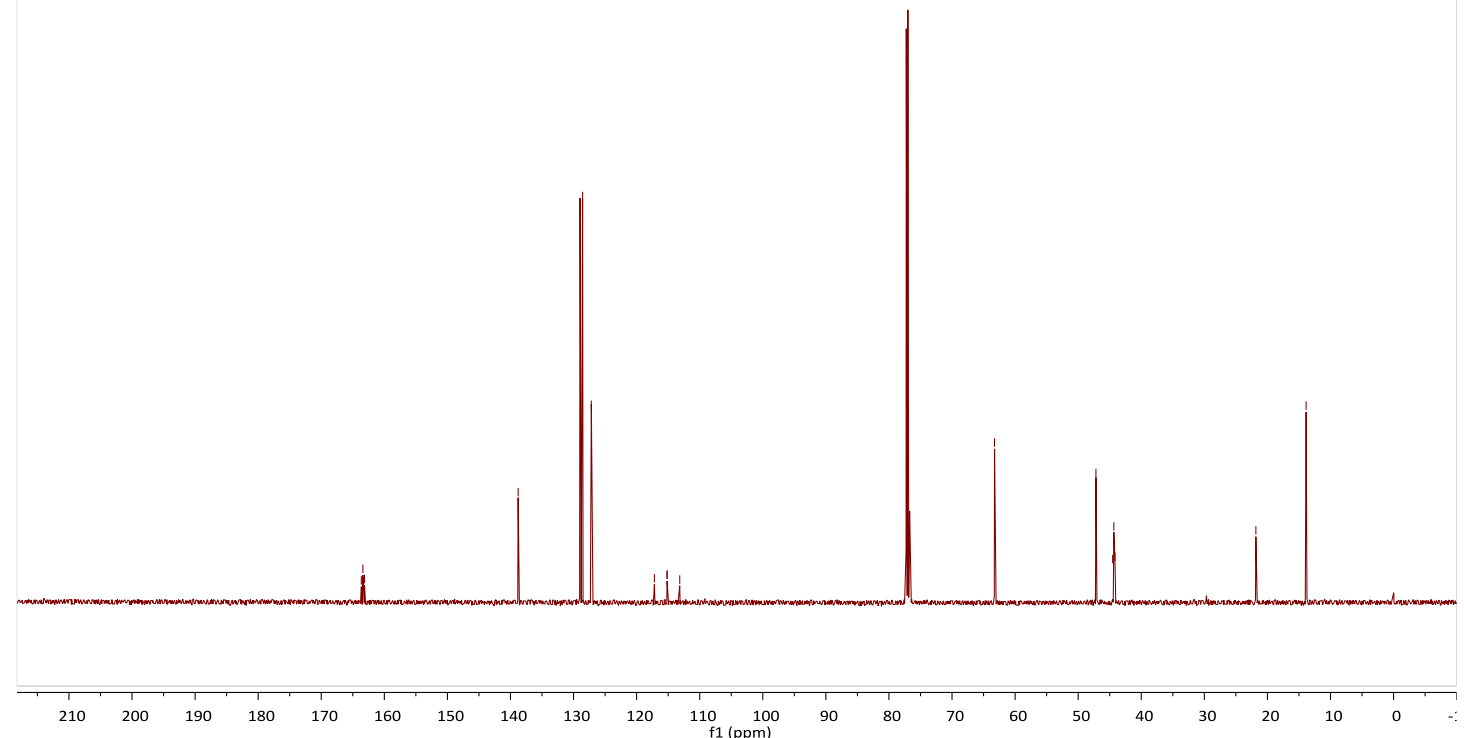


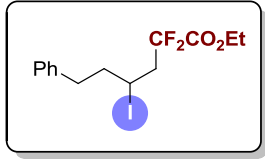

${ }^{1} \mathbf{H}$ NMR-spectrum $\left(500 \mathrm{MHz}, \mathrm{CDCl}_{3}\right)$ of $\mathbf{3 b}$

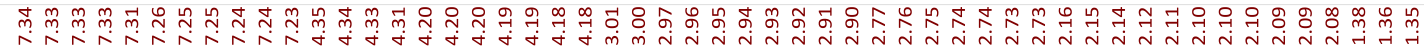

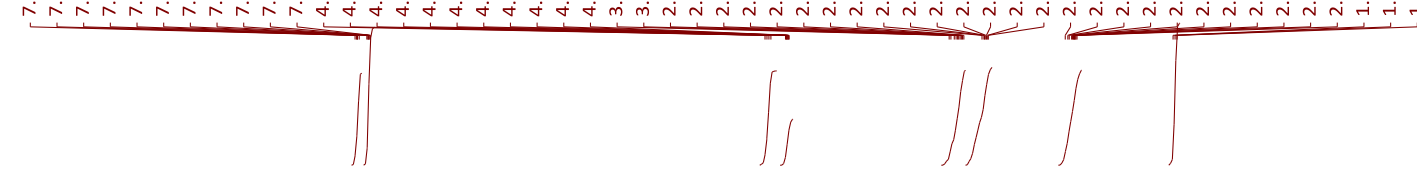

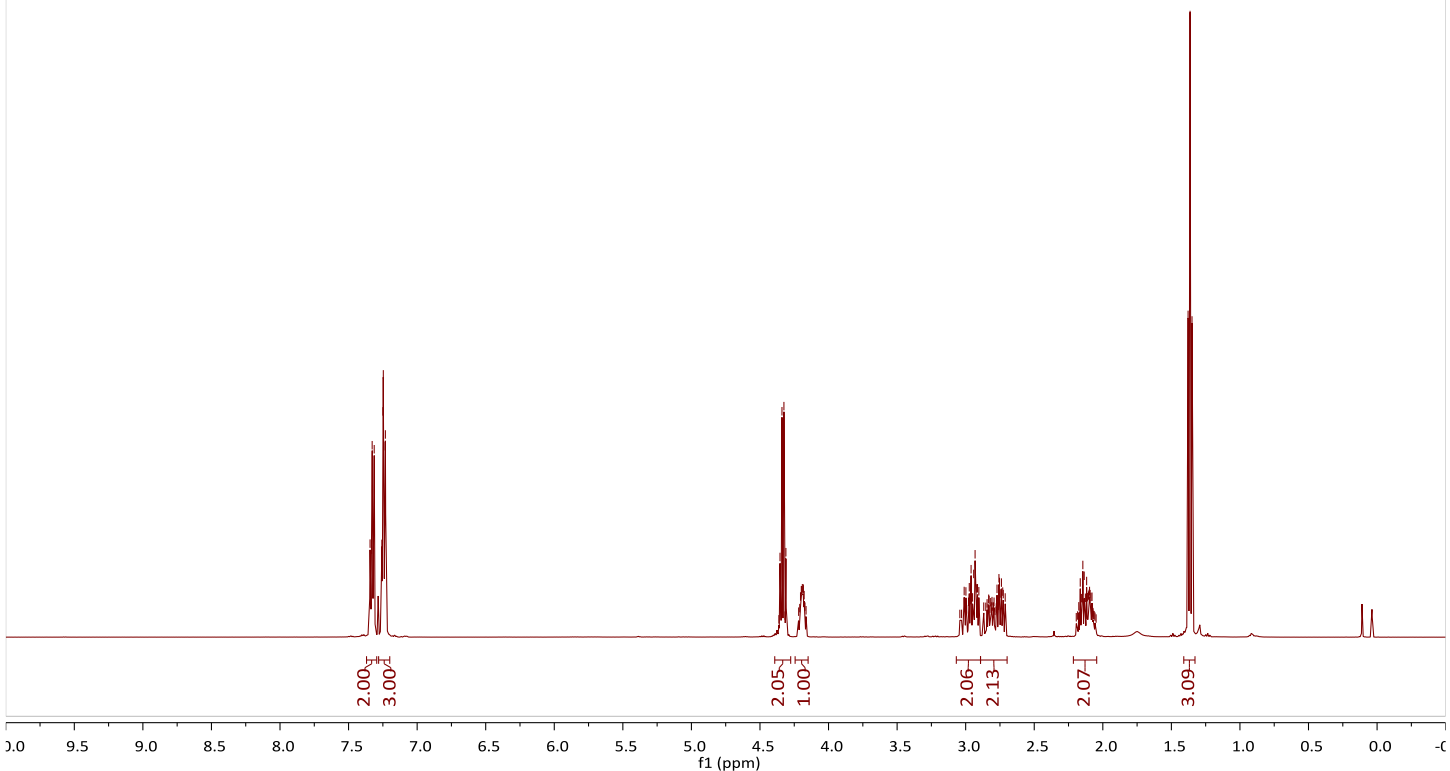

${ }^{19} \mathbf{F}$ NMR-spectrum $\left(471 \mathrm{MHz}, \mathrm{CDCl}_{3}\right)$ of $\mathbf{3 b}$

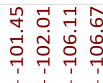

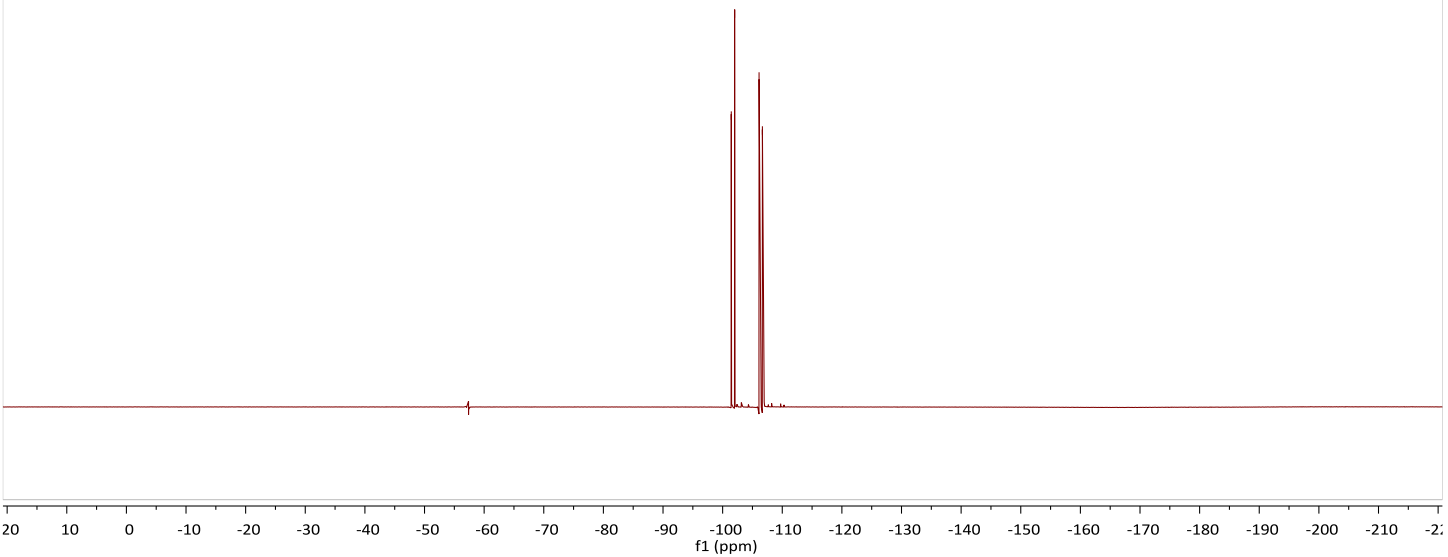




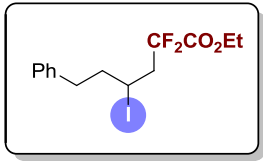

${ }^{13} \mathbf{C ~ N M R}$-spectrum $\left(125 \mathrm{MHz}, \mathrm{CDCl}_{3}\right)$ of $\mathbf{3 b}$

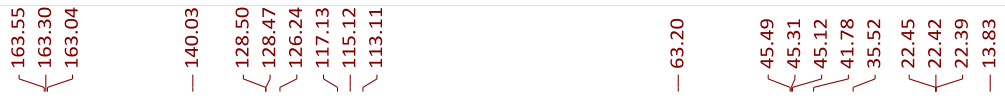

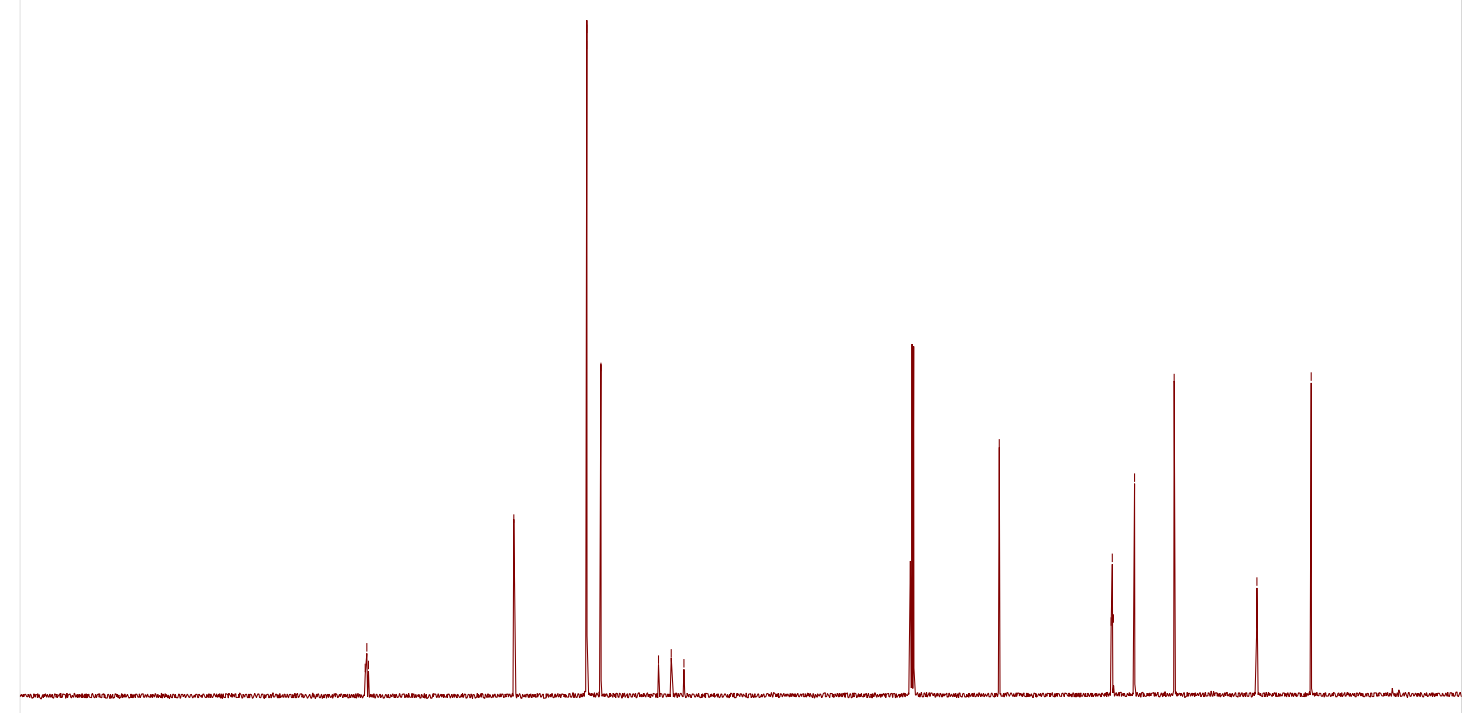

$\begin{array}{lllllllllllllllllllllllllllllllllllll}210 & 200 & 190 & 180 & 170 & 160 & 150 & 140 & 130 & 120 & 110 & 100 & 90 & 80 & 70 & 60 & 50 & 40 & 30 & 20 & 10 & 0 & -\end{array}$ 


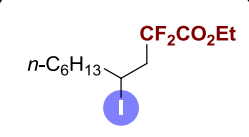

${ }^{1} \mathbf{H}$ NMR-spectrum $\left(500 \mathrm{MHz}, \mathrm{CDCl}_{3}\right)$ of $3 \mathbf{c}$

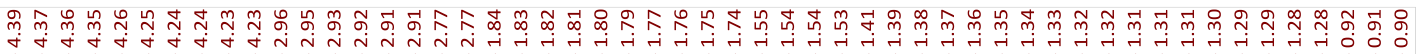

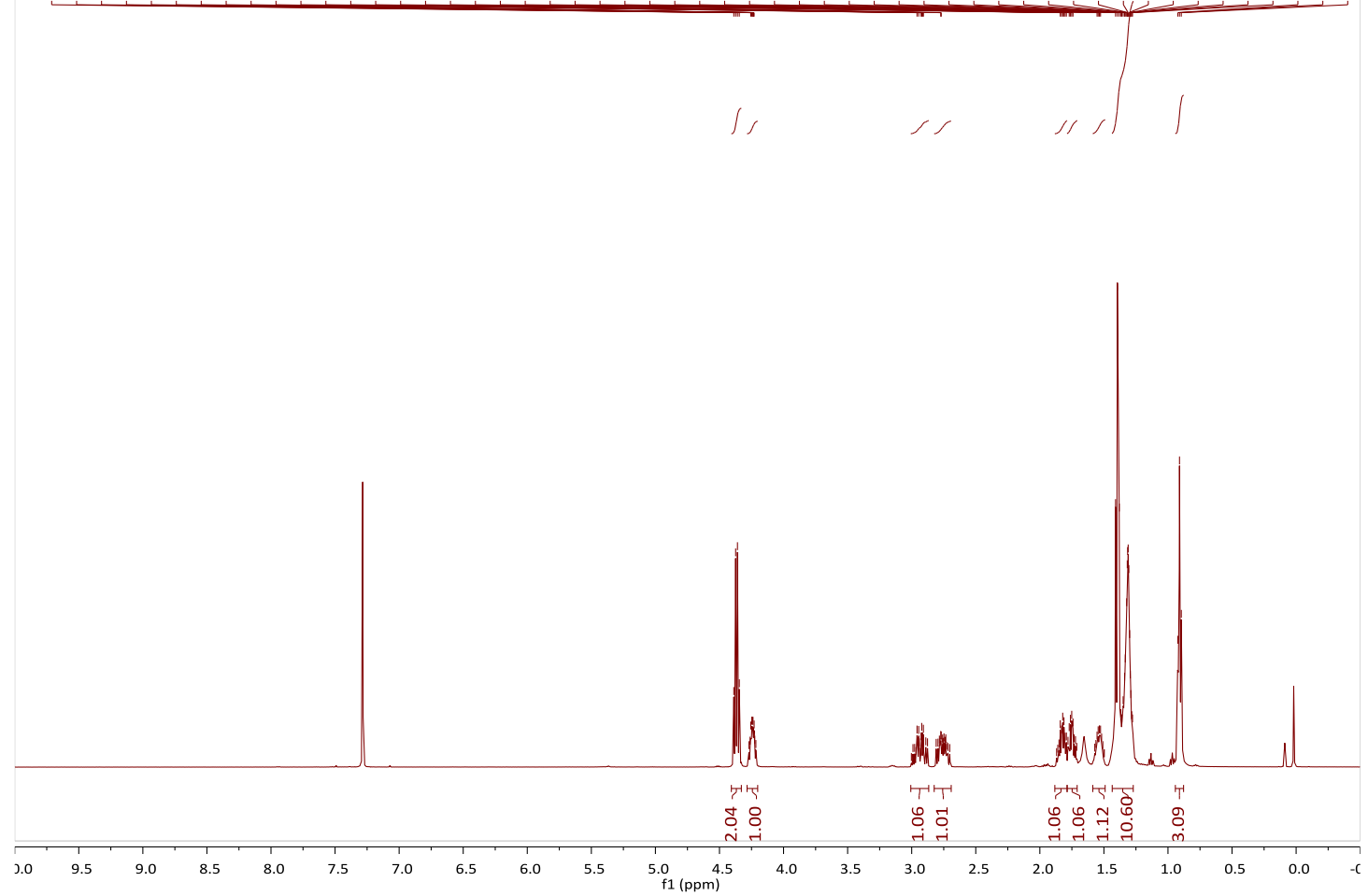

${ }^{19}$ F NMR-spectrum $\left(471 \mathrm{MHz}, \mathrm{CDCl}_{3}\right)$ of $3 \mathrm{c}$

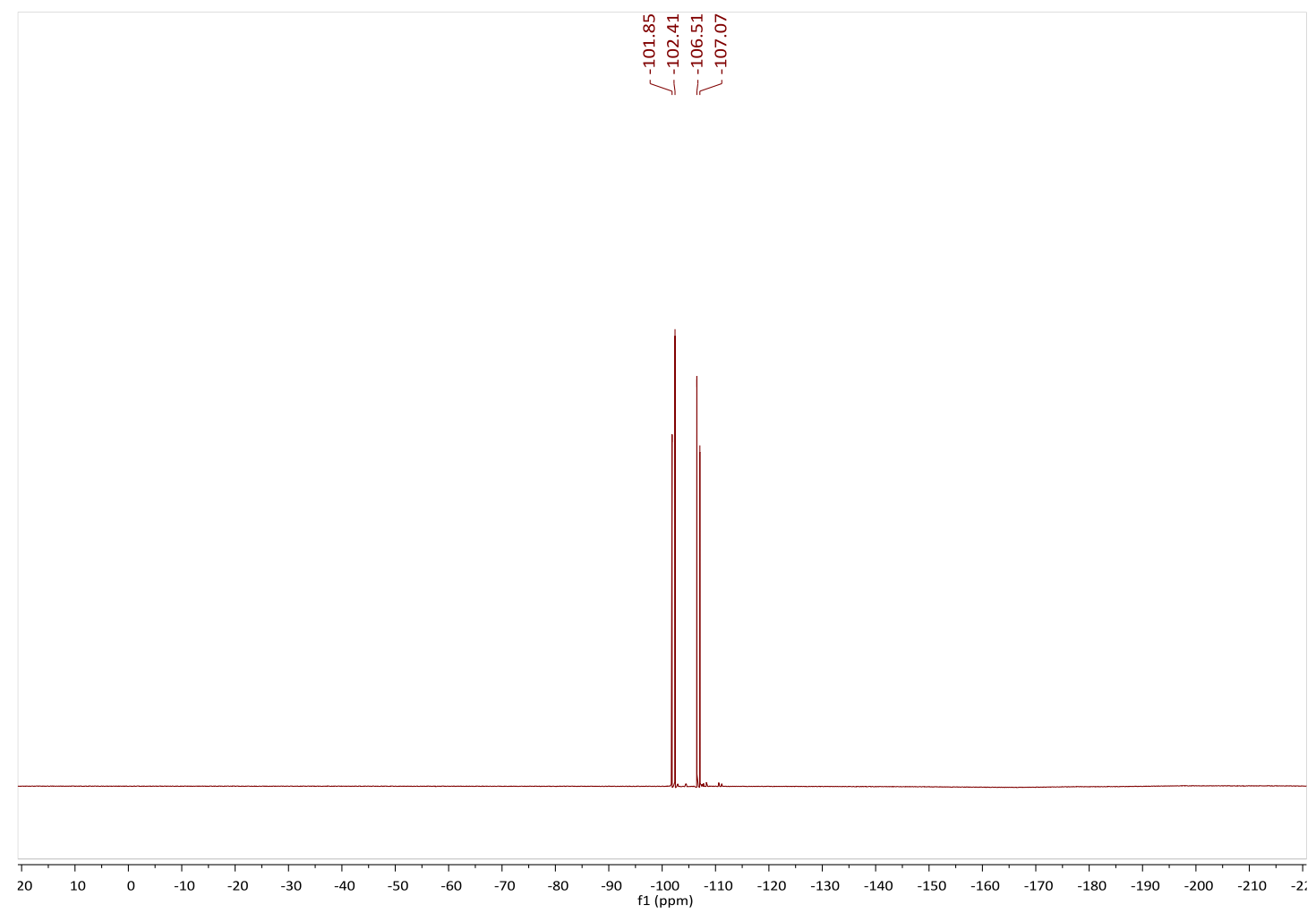




$$
{ }_{n-\mathrm{C}_{6} \mathrm{H}_{13}}^{\mathrm{CF}_{2} \mathrm{CO}_{2} \mathrm{Et}}
$$

${ }^{13} \mathrm{C}$ NMR-spectrum (125 MHz, $\left.\mathrm{CDCl}_{3}\right)$ of 3c

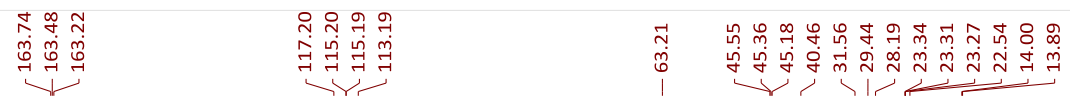

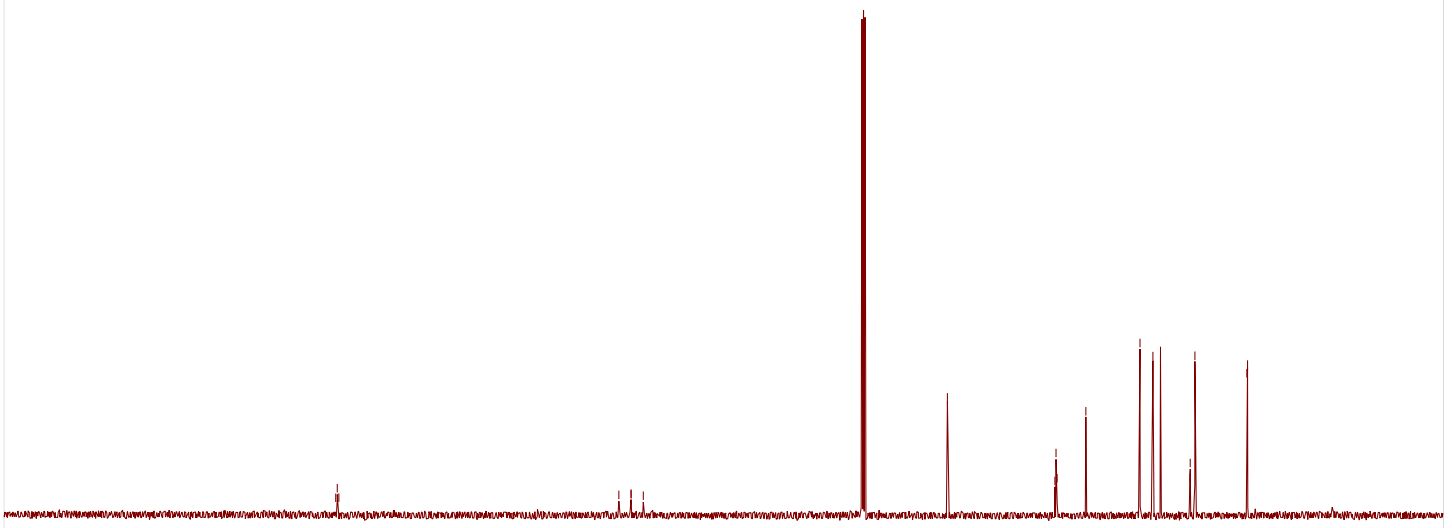

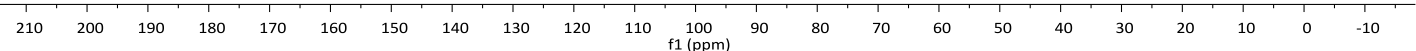




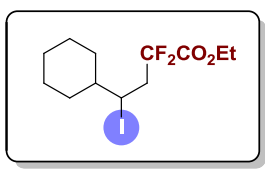

${ }^{1} \mathbf{H}$ NMR-spectrum $\left(500 \mathrm{MHz}, \mathrm{CDCl}_{3}\right)$ of $\mathbf{3 d}$

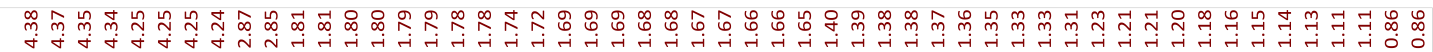

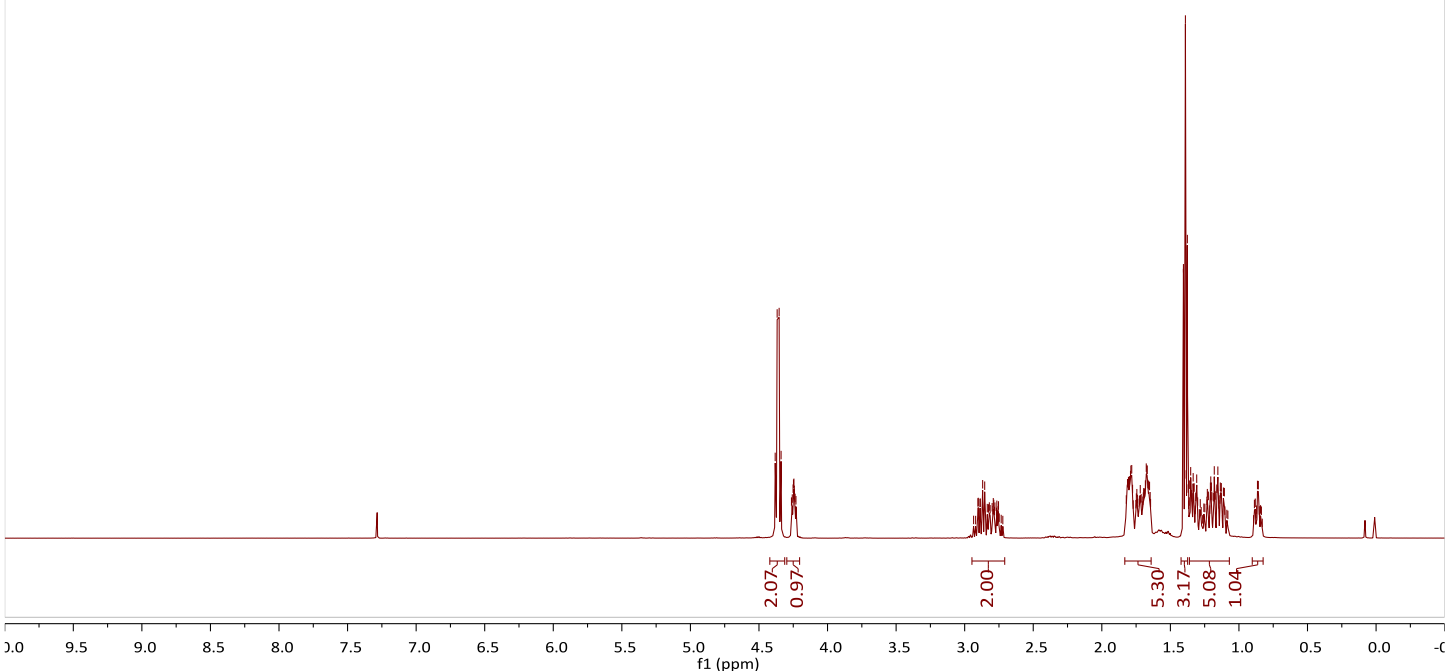

${ }^{19}$ F NMR-spectrum $\left(471 \mathrm{MHz}, \mathrm{CDCl}_{3}\right)$ of $\mathbf{3 d}$

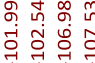

$\checkmark 1$

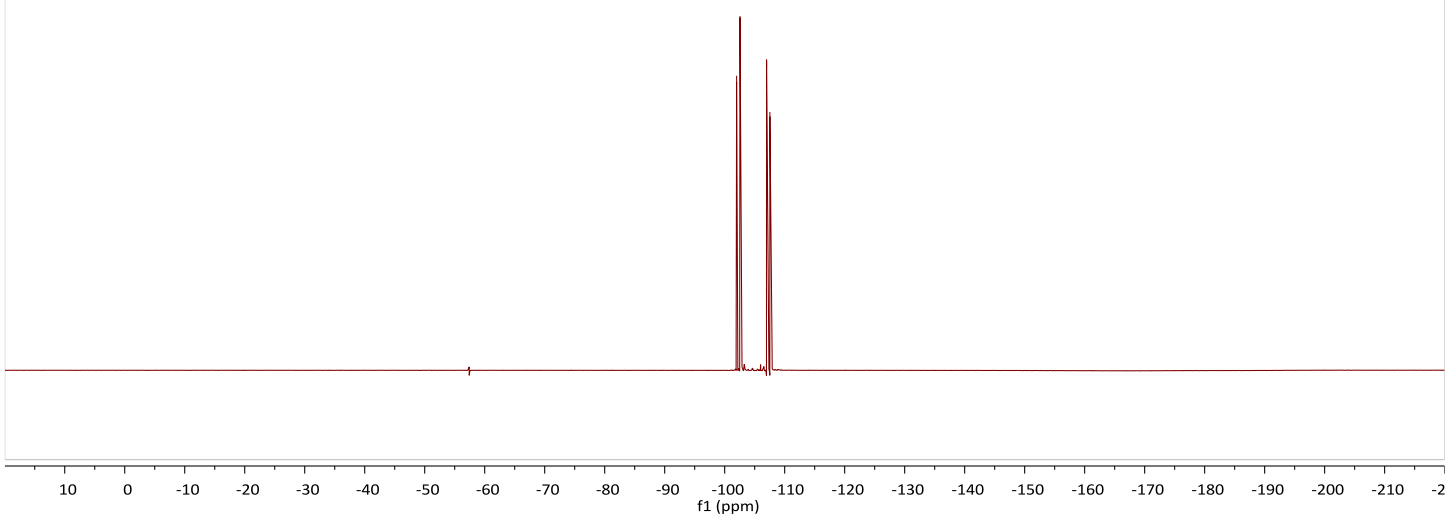




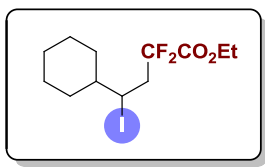

${ }^{13} \mathrm{C}$ NMR-spectrum $\left(125 \mathrm{MHz}, \mathrm{CDCl}_{3}\right)$ of $\mathbf{3 d}$

둔

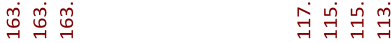

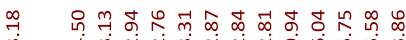

걱

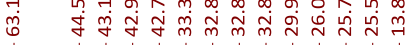

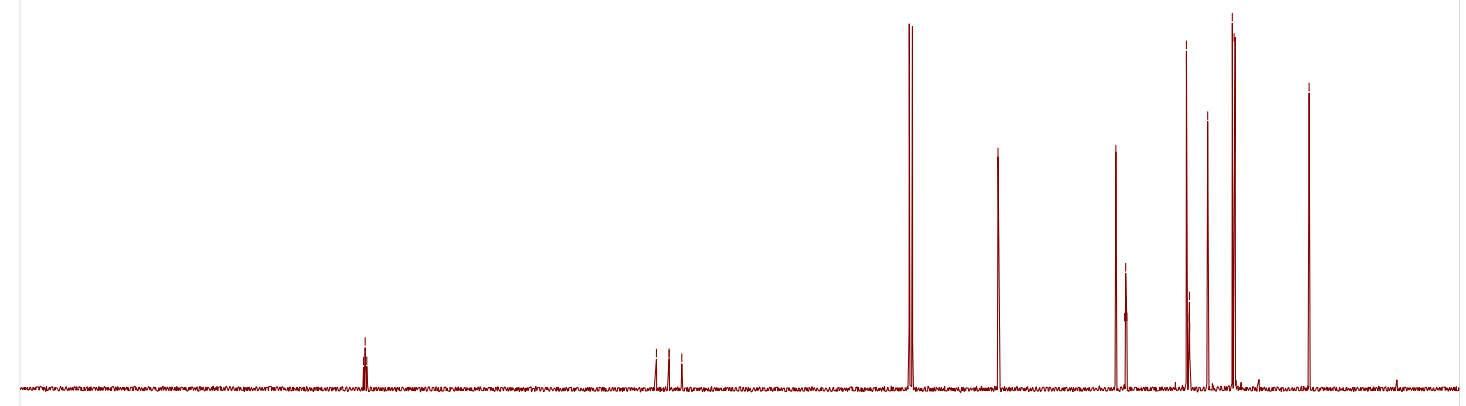

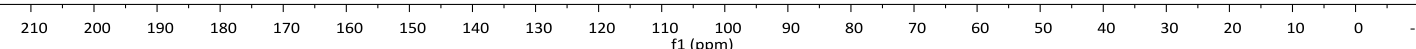




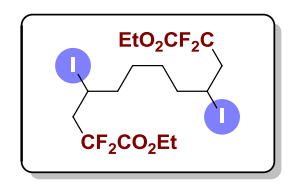

${ }^{1} \mathbf{H}$ NMR-spectrum $\left(500 \mathrm{MHz}, \mathrm{CDCl}_{3}\right)$ of $3 \mathbf{e}$

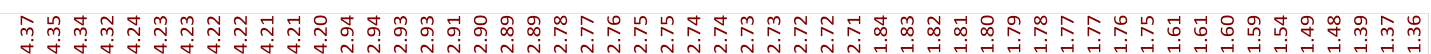

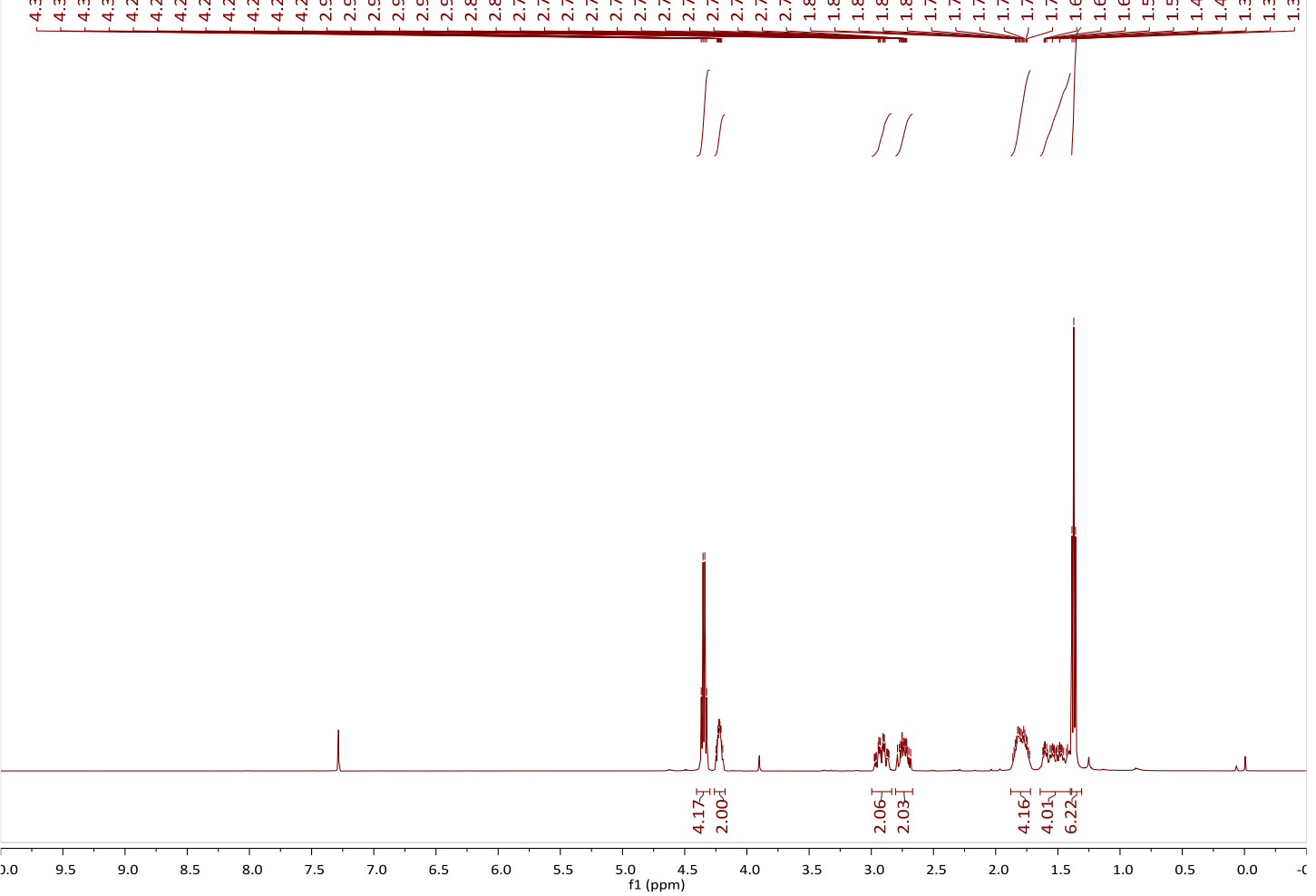

${ }^{19} \mathbf{F}$ NMR-spectrum $\left(471 \mathrm{MHz}, \mathrm{CDCl}_{3}\right)$ of $3 \mathbf{e}$

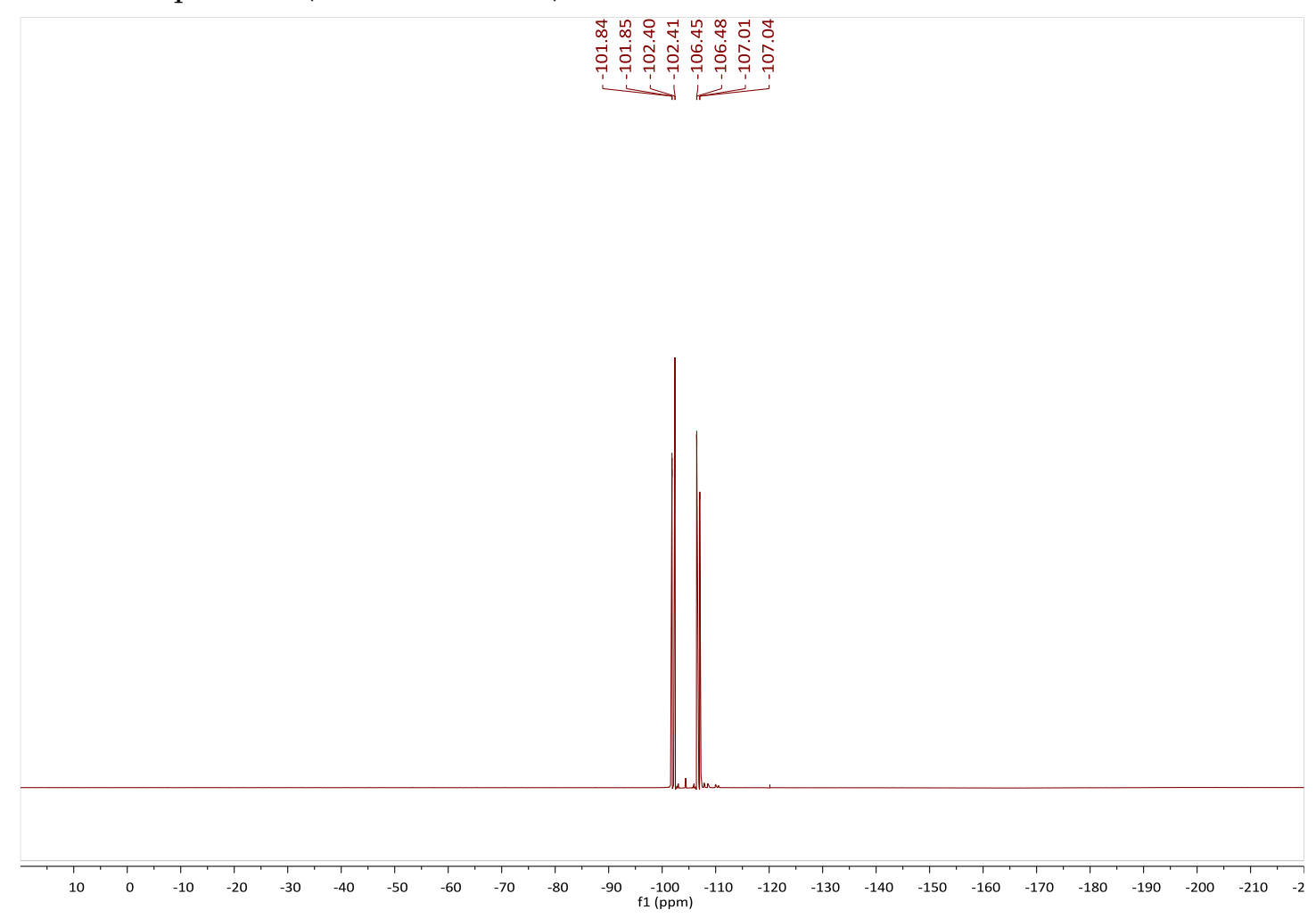




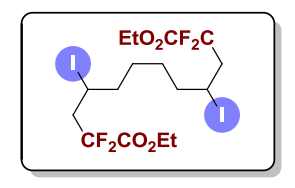

${ }^{13} \mathrm{C}$ NMR-spectrum $\left(125 \mathrm{MHz}, \mathrm{CDCl}_{3}\right)$ of $3 \mathbf{e}$

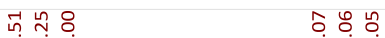

象兽兽 当当

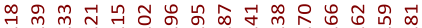

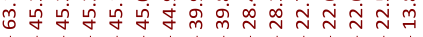

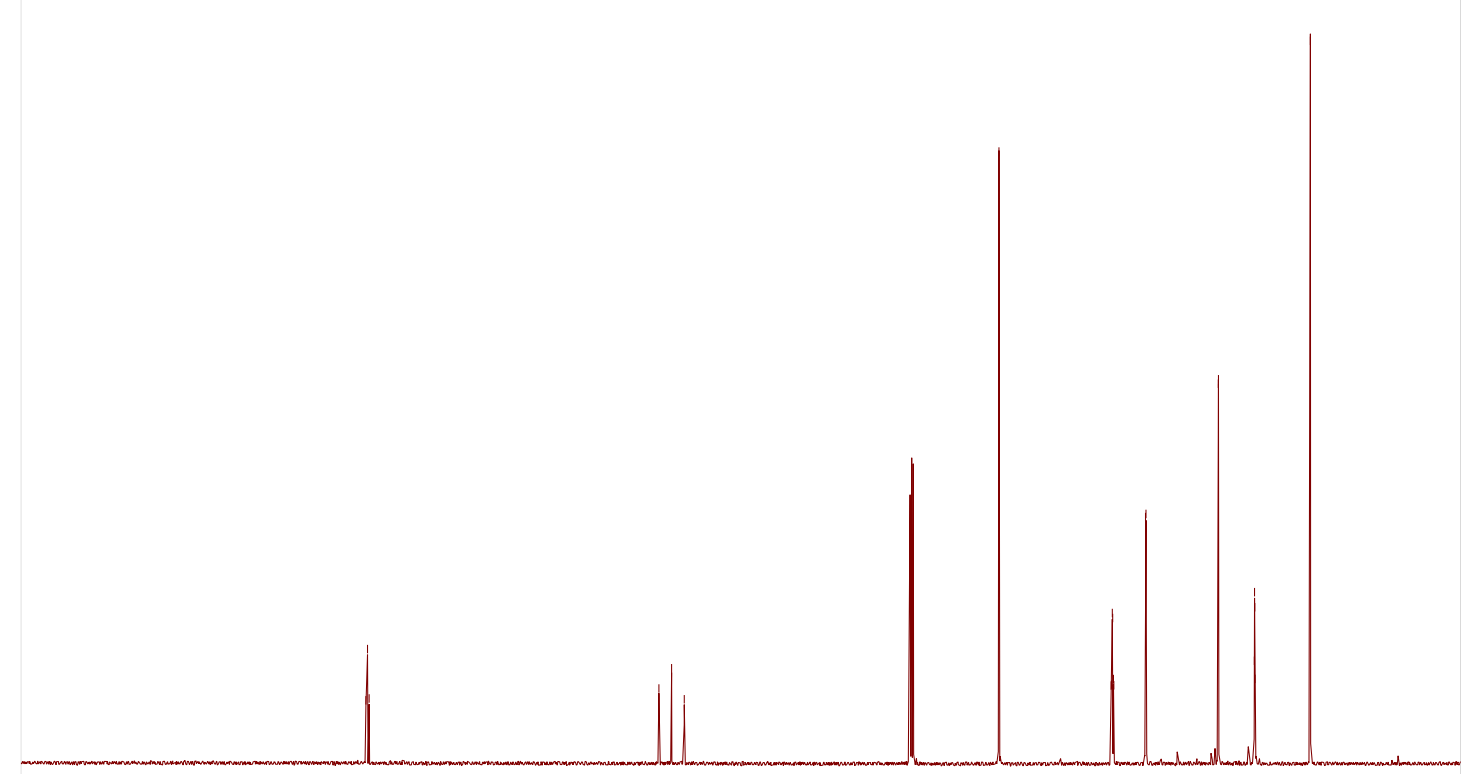

$\begin{array}{lllllllllllllllllllllllllllllllllllllll}210 & 200 & 190 & 180 & 170 & 160 & 150 & 140 & 130 & 120 & 110 & 100 & 90 & 80 & 70 & 60 & 50 & 40 & 30 & 20 & 10 & 0 & -\end{array}$ 


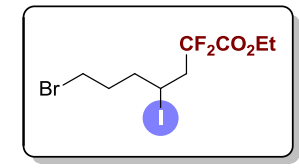

${ }^{1} \mathbf{H}$ NMR-spectrum $\left(500 \mathrm{MHz}, \mathrm{CDCl}_{3}\right)$ of $\mathbf{3 f}$

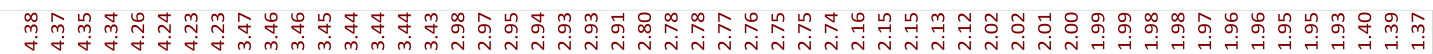
$\iiint \int \mid$

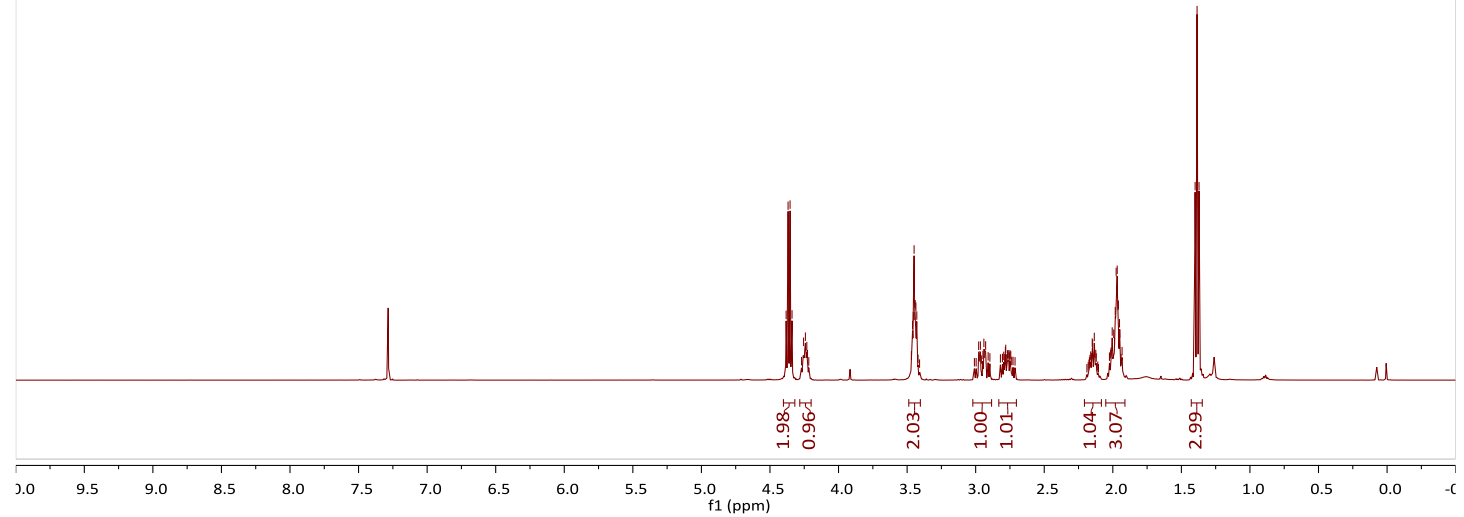

${ }^{19} \mathrm{~F}$ NMR-spectrum $\left(471 \mathrm{MHz}, \mathrm{CDCl}_{3}\right)$ of $3 \mathrm{f}$

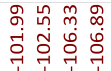

ir

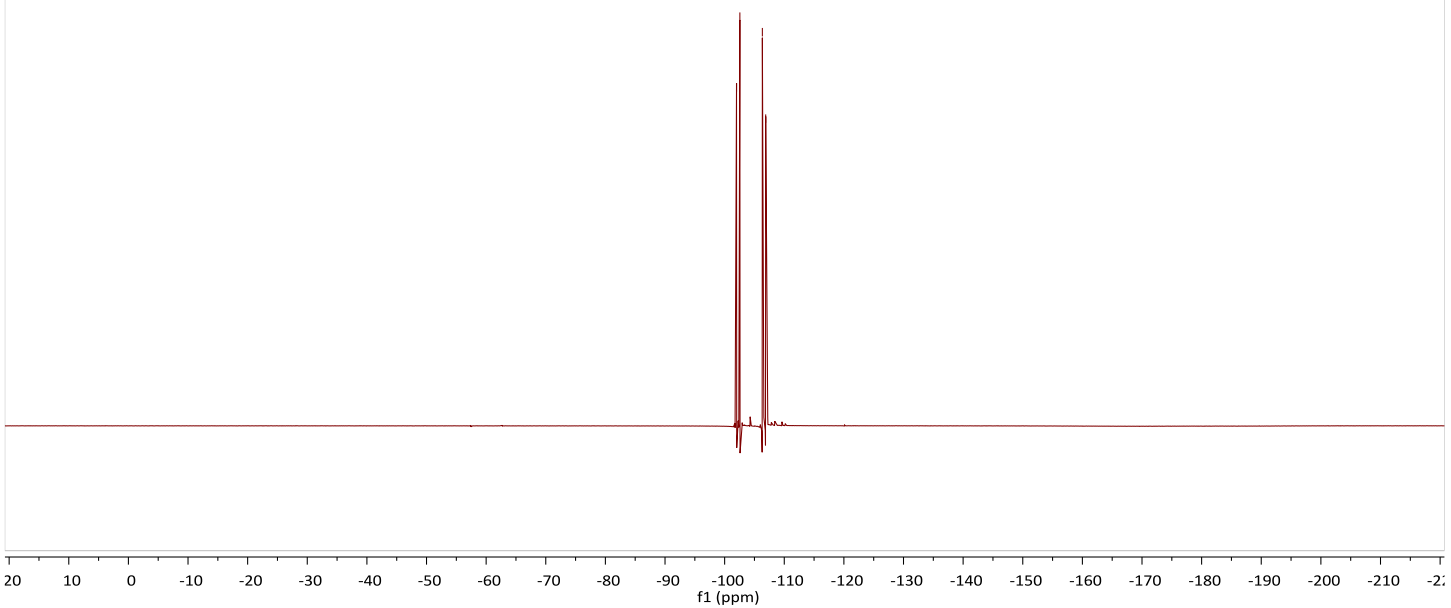




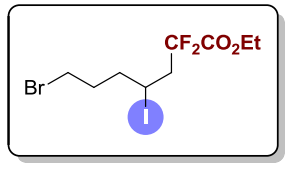

${ }^{13} \mathrm{C}$ NMR-spectrum (125 MHz, $\left.\mathrm{CDCl}_{3}\right)$ of $\mathbf{3 f}$

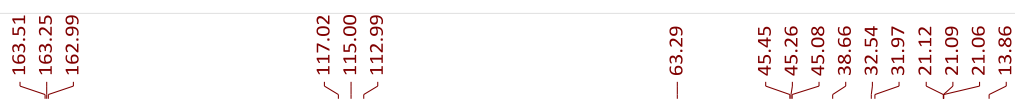
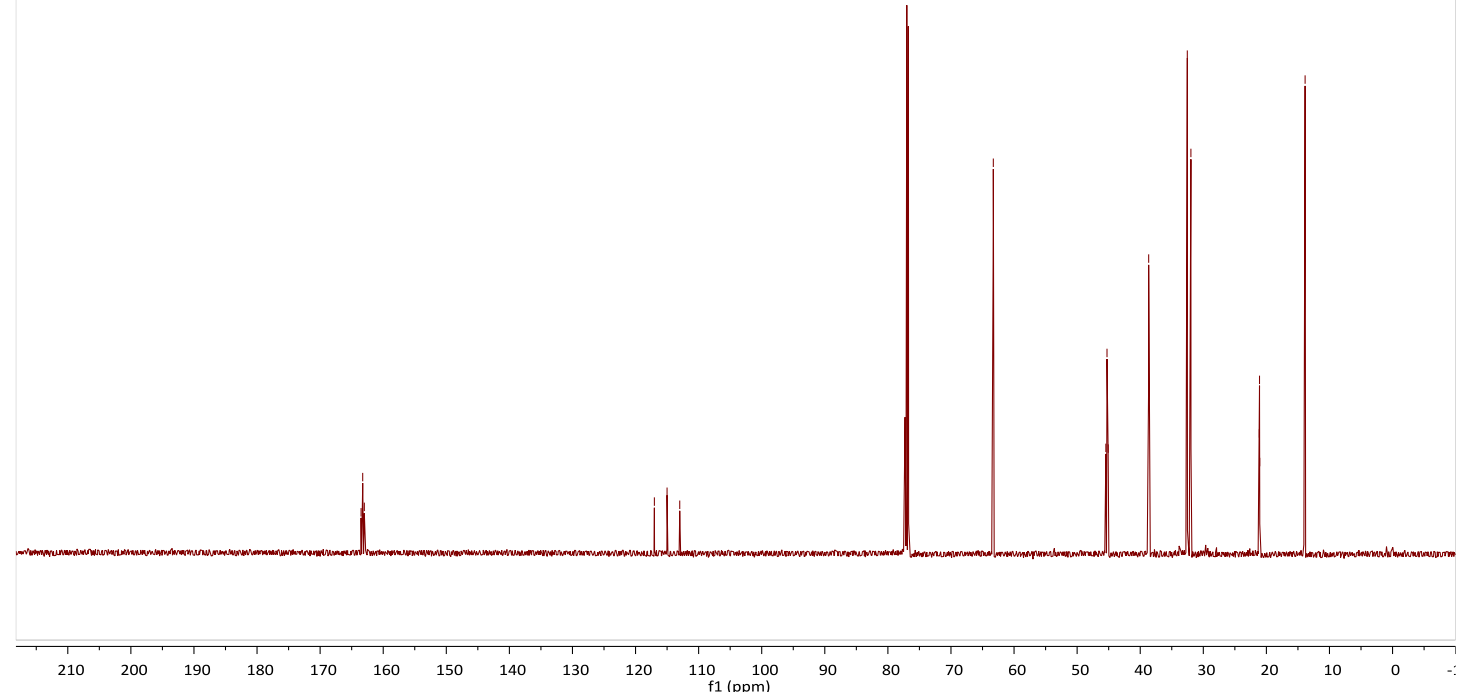


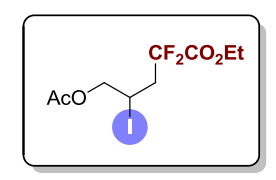

${ }^{1} \mathbf{H}$ NMR-spectrum $\left(500 \mathrm{MHz}, \mathrm{CDCl}_{3}\right)$ of $\mathbf{3 g}$

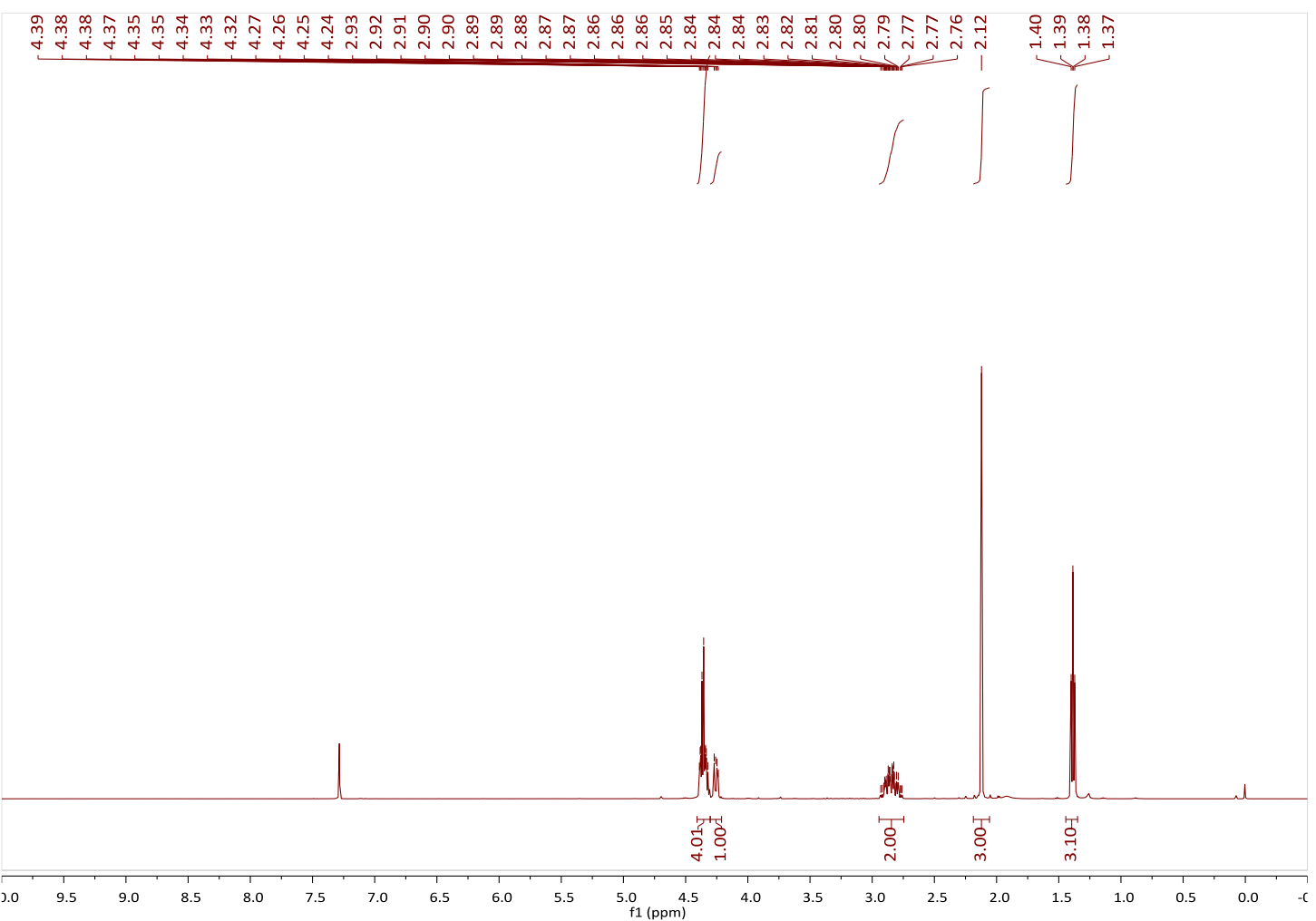

${ }^{19}$ F NMR-spectrum $\left(471 \mathrm{MHz}, \mathrm{CDCl}_{3}\right)$ of $\mathbf{3 g}$

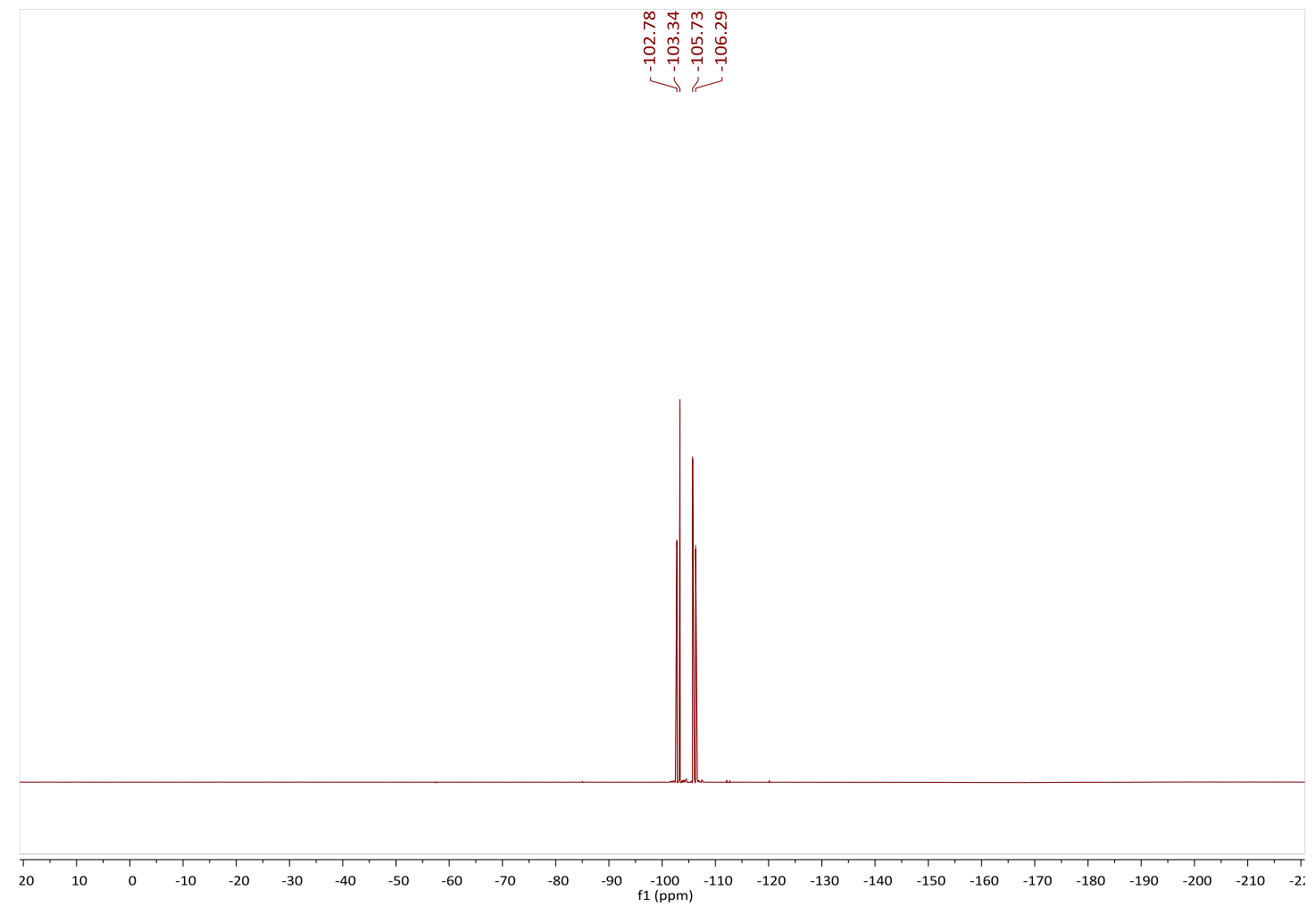




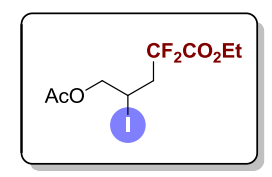

${ }^{13} \mathrm{C}$ NMR-spectrum (125 MHz, $\left.\mathrm{CDCl}_{3}\right)$ of $\mathbf{3 g}$

육요

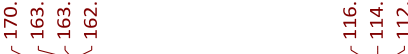

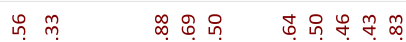

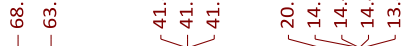

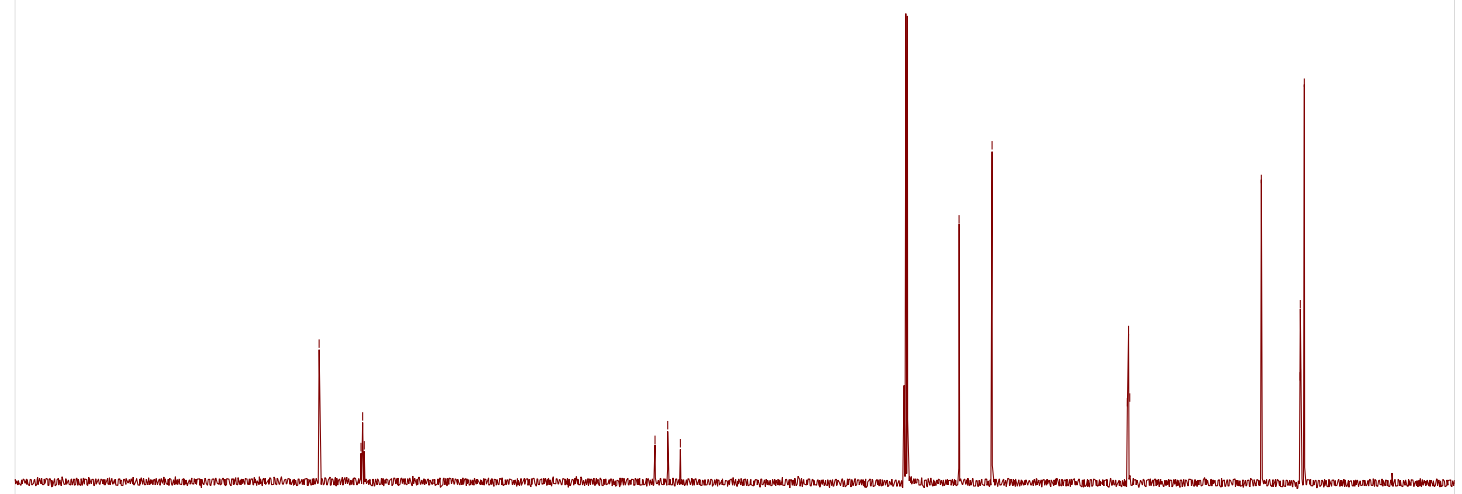

$\begin{array}{lllllllllll}210 & 200 & 190 & 180 & 170 & 160 & 150 & 140 & 130 & 120 & \begin{array}{l}110 \\ \mathrm{f} 1(\mathrm{ppm})\end{array}\end{array}$ 


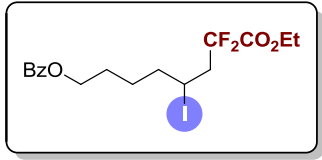

${ }^{1} \mathbf{H}$ NMR-spectrum $\left(500 \mathrm{MHz}, \mathrm{CDCl}_{3}\right)$ of $3 \mathbf{h}$

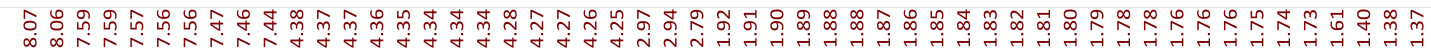
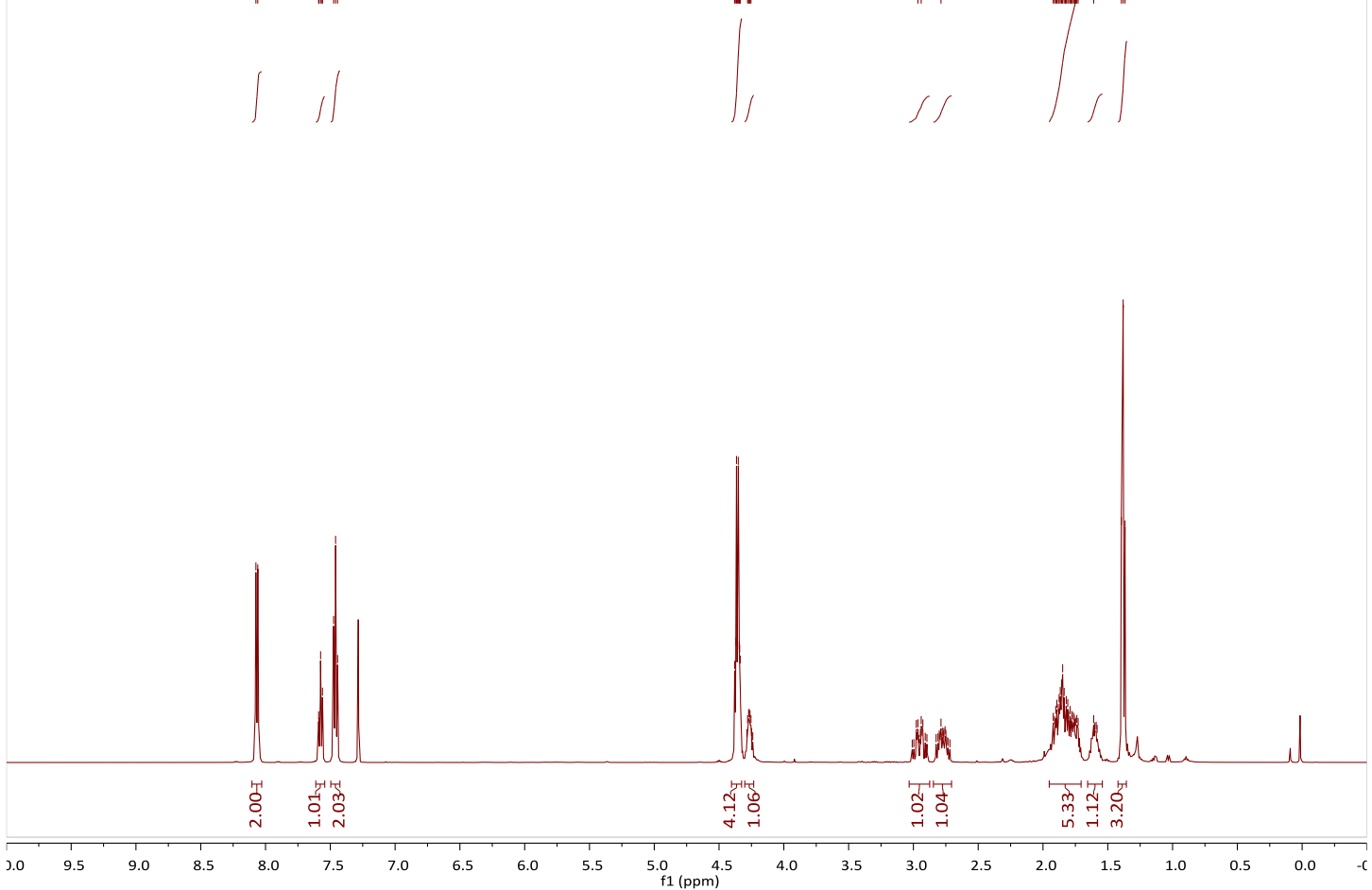

${ }^{19}$ F NMR-spectrum $\left(471 \mathrm{MHz}, \mathrm{CDCl}_{3}\right)$ of $3 \mathbf{h}$

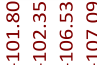

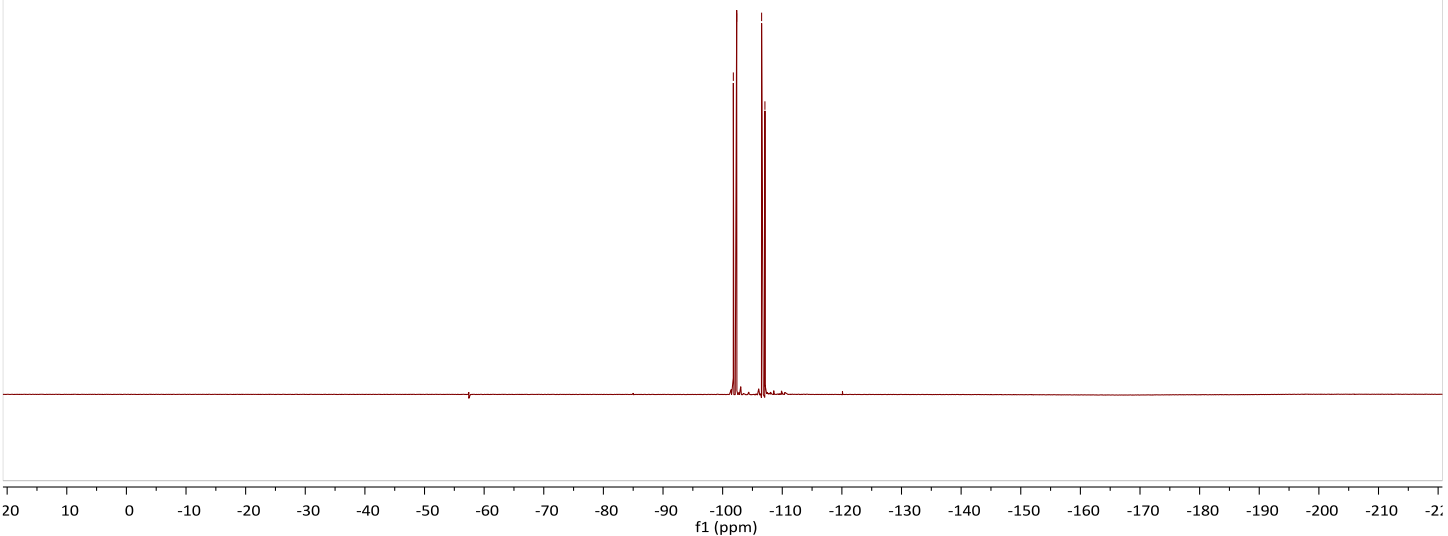




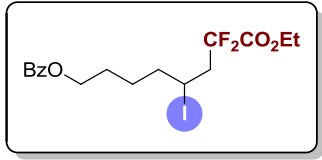

${ }^{13} \mathrm{C}$ NMR-spectrum (125 MHz, $\left.\mathrm{CDCl}_{3}\right)$ of $\mathbf{3 h}$

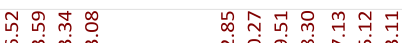

过

ま స

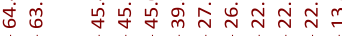

नै

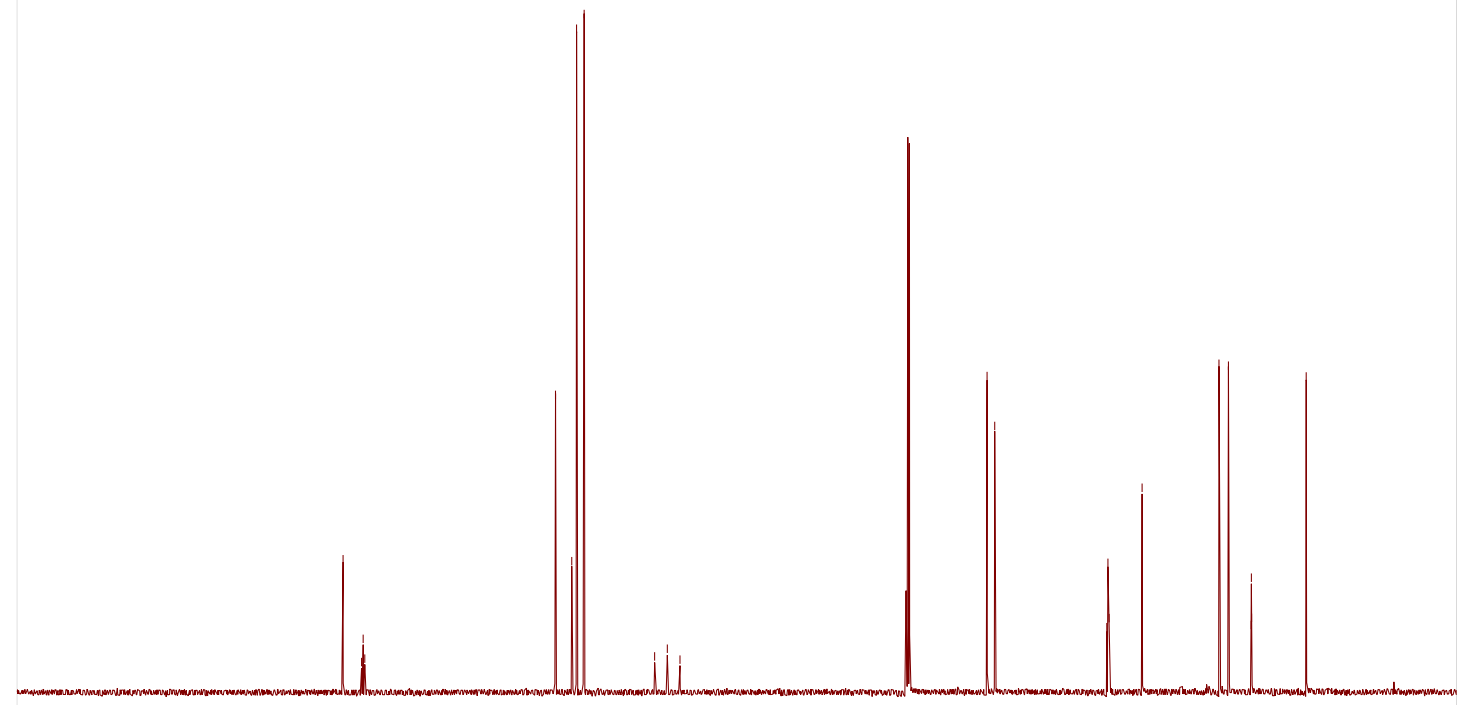

$\begin{array}{llllllllllllllllllllllllllllllll}210 & 200 & 190 & 180 & 170 & 160 & 150 & 140 & 130 & 120 & 110 & 100 & 90 & 80 & 70 & 60 & 50 & 40 & 30 & 20 & 10 & 0 & 0\end{array}$ 


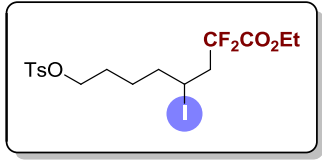

${ }^{1} \mathbf{H}$ NMR-spectrum $\left(500 \mathrm{MHz}, \mathrm{CDCl}_{3}\right)$ of $\mathbf{3 i}$

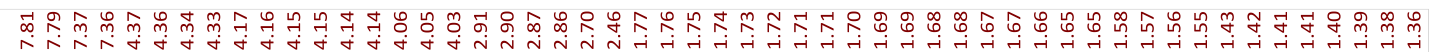<smiles>C1CCCCC1</smiles><smiles>CC1(C)C2C=CC(C2)C1(C)C</smiles>

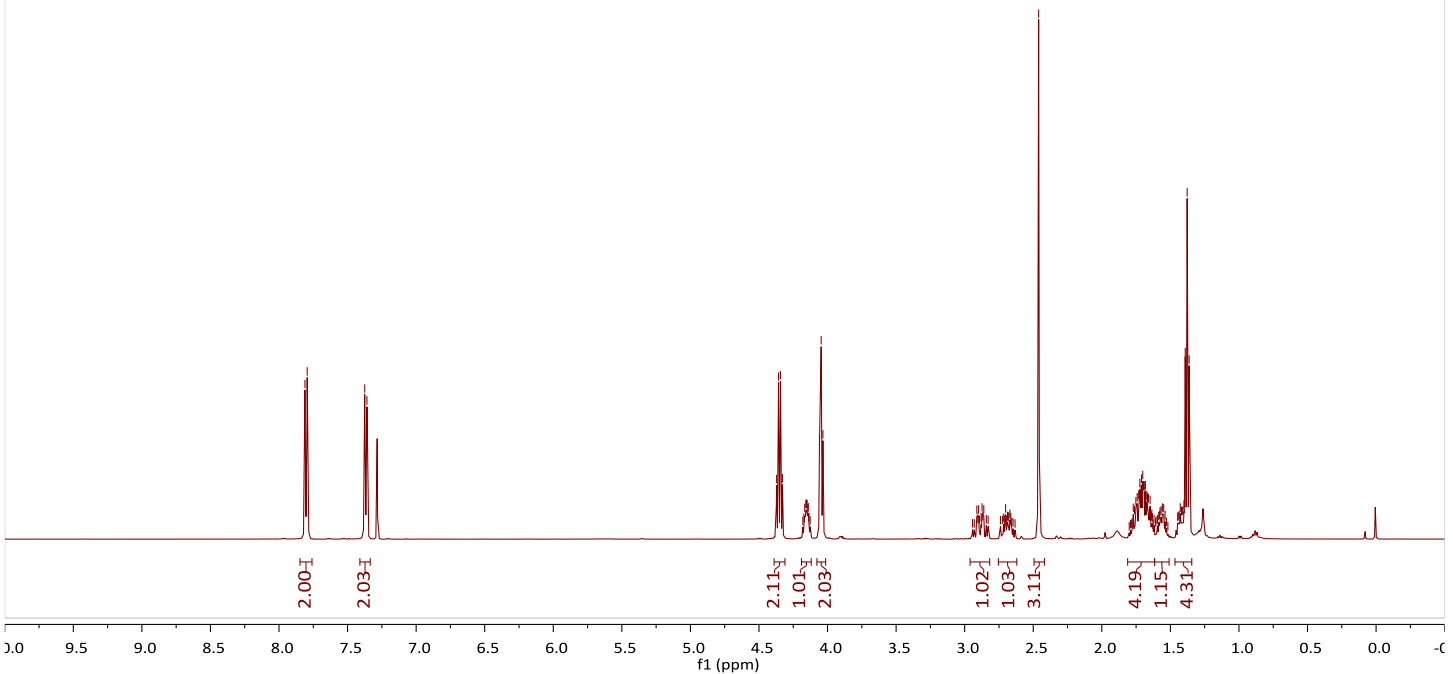

${ }^{19}$ F NMR-spectrum $\left(471 \mathrm{MHz}, \mathrm{CDCl}_{3}\right)$ of $3 \mathbf{i}$

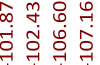

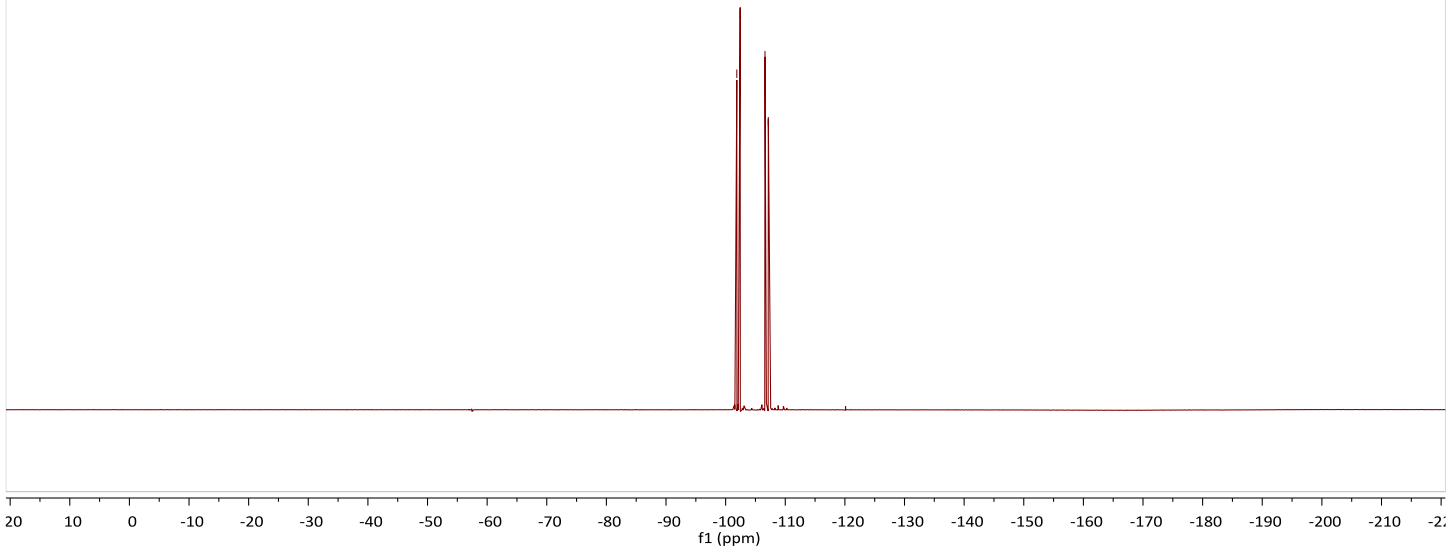




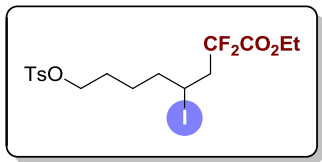

${ }^{13} \mathrm{C}$ NMR-spectrum (125 MHz, $\left.\mathrm{CDCl}_{3}\right)$ of $3 \mathbf{i}$

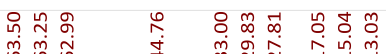

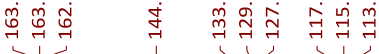

นู

月

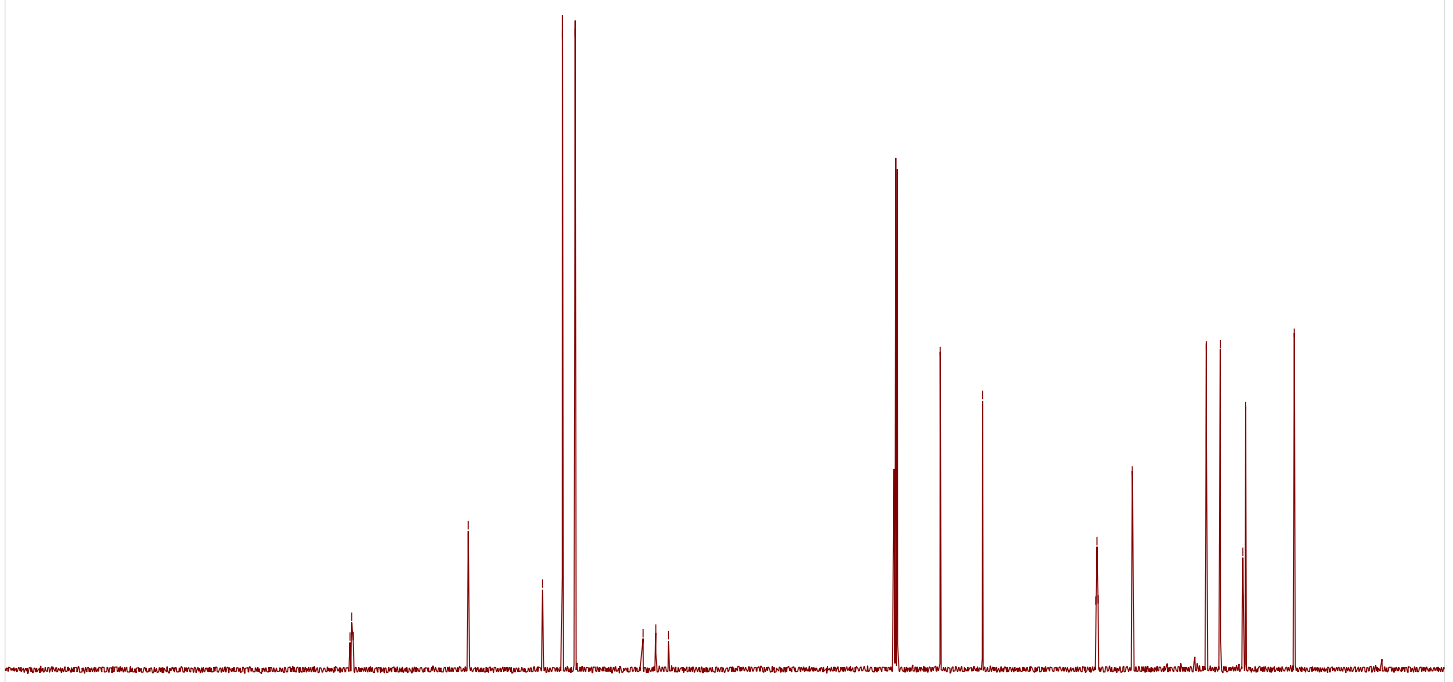

$\begin{array}{lllllllllllllllllllllllllllllllllllll}210 & 200 & 190 & 180 & 170 & 160 & 150 & 140 & 130 & 120 & 110 & 100 & 90 & 80 & 70 & 60 & 50 & 40 & 30 & 20 & 10 & 0 & -\end{array}$ 


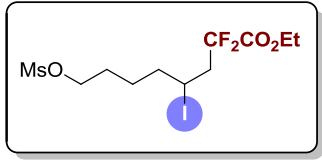

${ }^{1} \mathbf{H}$ NMR-spectrum $\left(500 \mathrm{MHz}, \mathrm{CDCl}_{3}\right)$ of $\mathbf{3 j}$

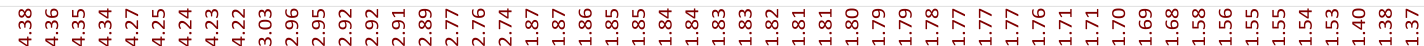
$\| \int_{/ 1} \mid$

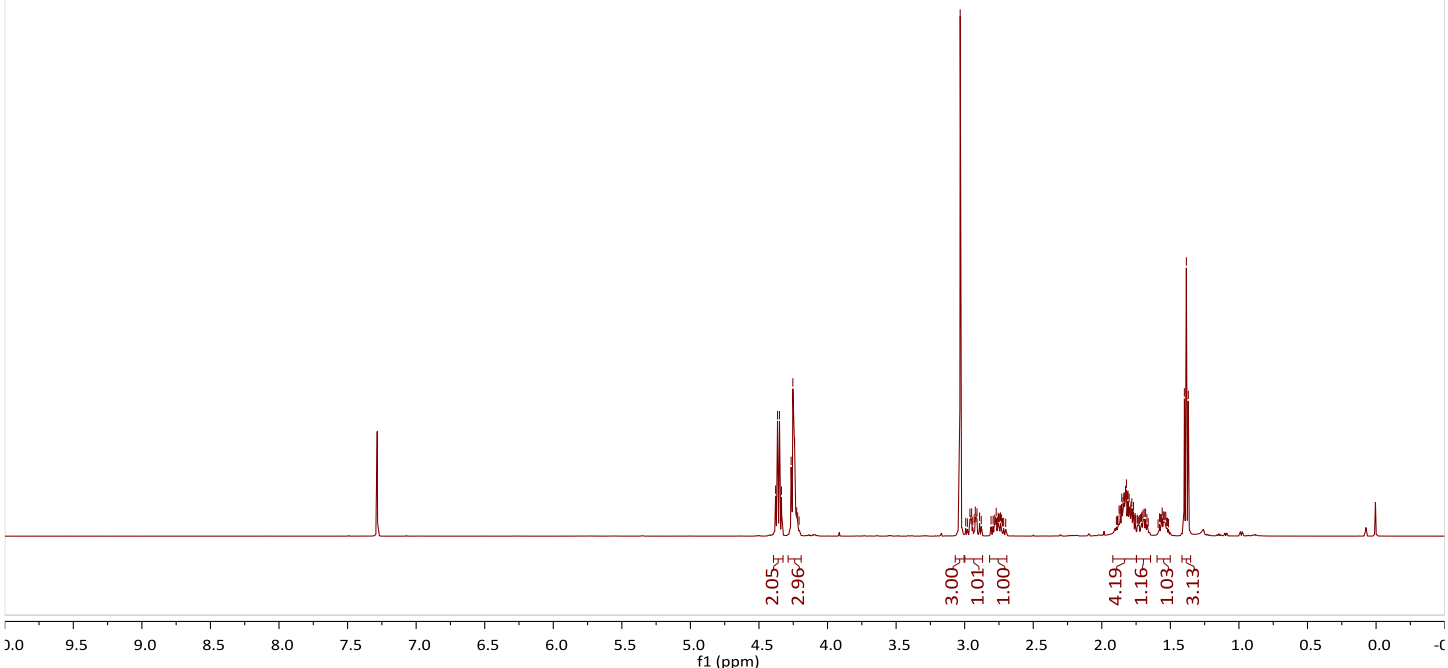

${ }^{19} \mathbf{F}$ NMR-spectrum $\left(471 \mathrm{MHz}, \mathrm{CDCl}_{3}\right)$ of $\mathbf{3 j}$

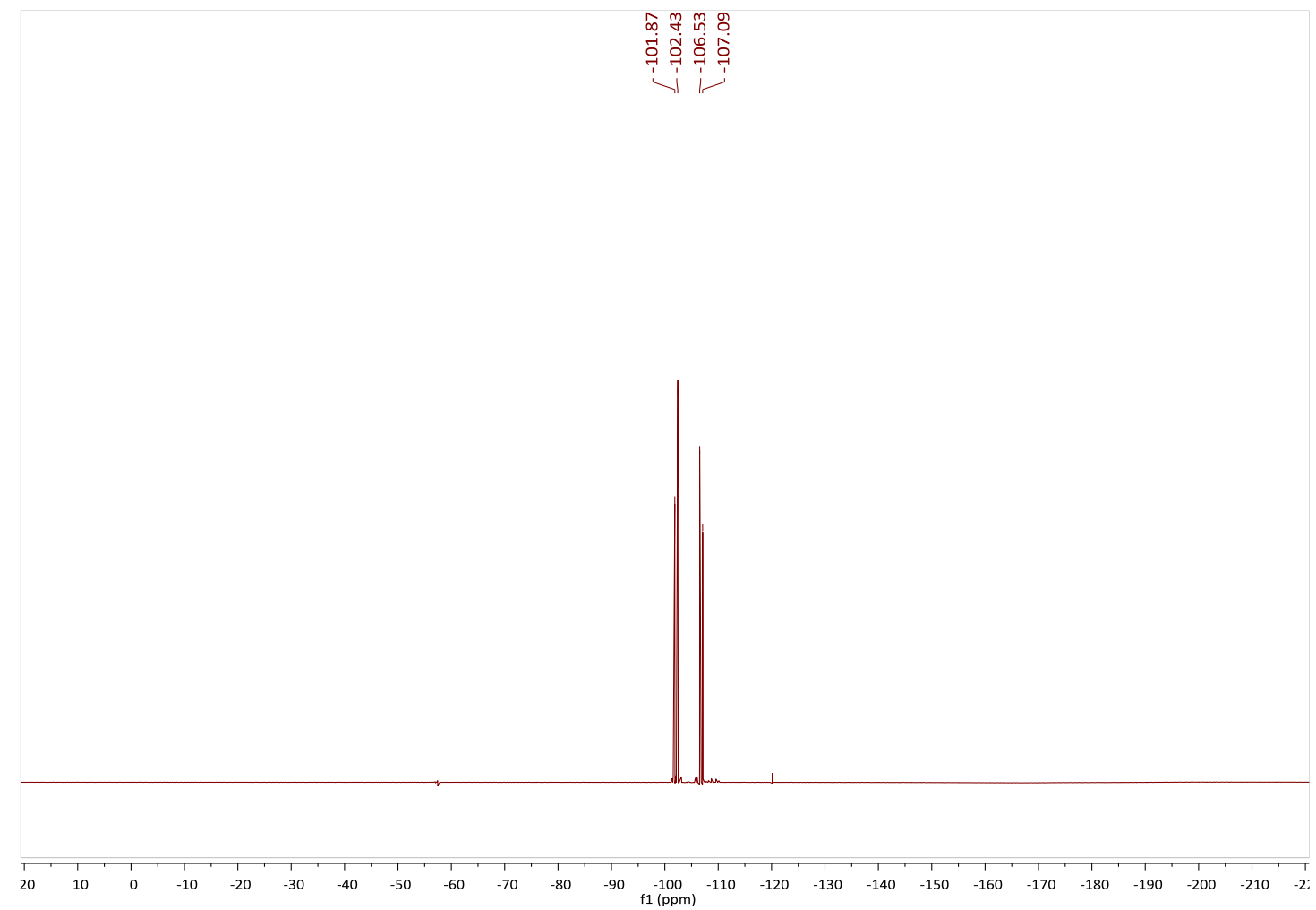




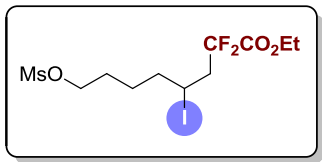

${ }^{13} \mathrm{C}$ NMR-spectrum $\left(125 \mathrm{MHz}, \mathrm{CDCl}_{3}\right)$ of $\mathbf{3 j}$

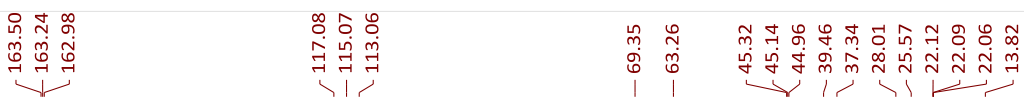

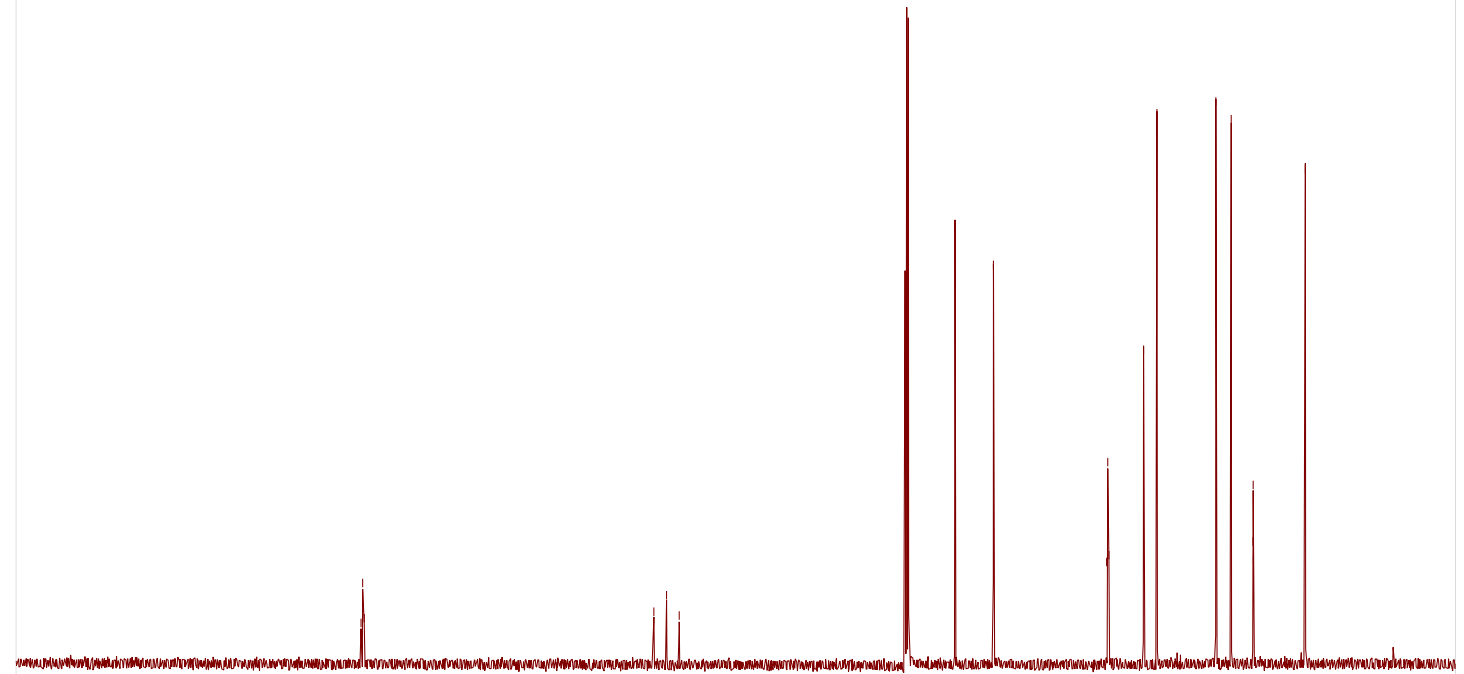

$\begin{array}{llllllllllllllllllllllllllllllllllllll}1 & 210 & 200 & 190 & 180 & 170 & 160 & 150 & 140 & 130 & 120 & 110 & 100 & 90 & 80 & 70 & 60 & 50 & 40 & 30 & 20 & 10 & 0 & -\end{array}$ 


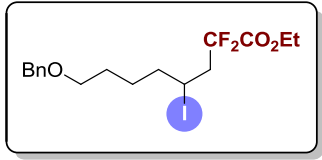

${ }^{1} \mathbf{H}$ NMR-spectrum $\left(500 \mathrm{MHz}, \mathrm{CDCl}_{3}\right)$ of $\mathbf{3 k}$

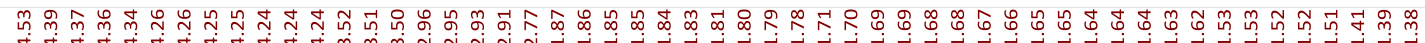

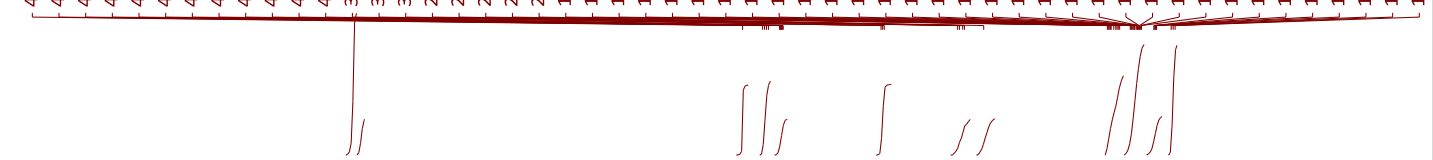

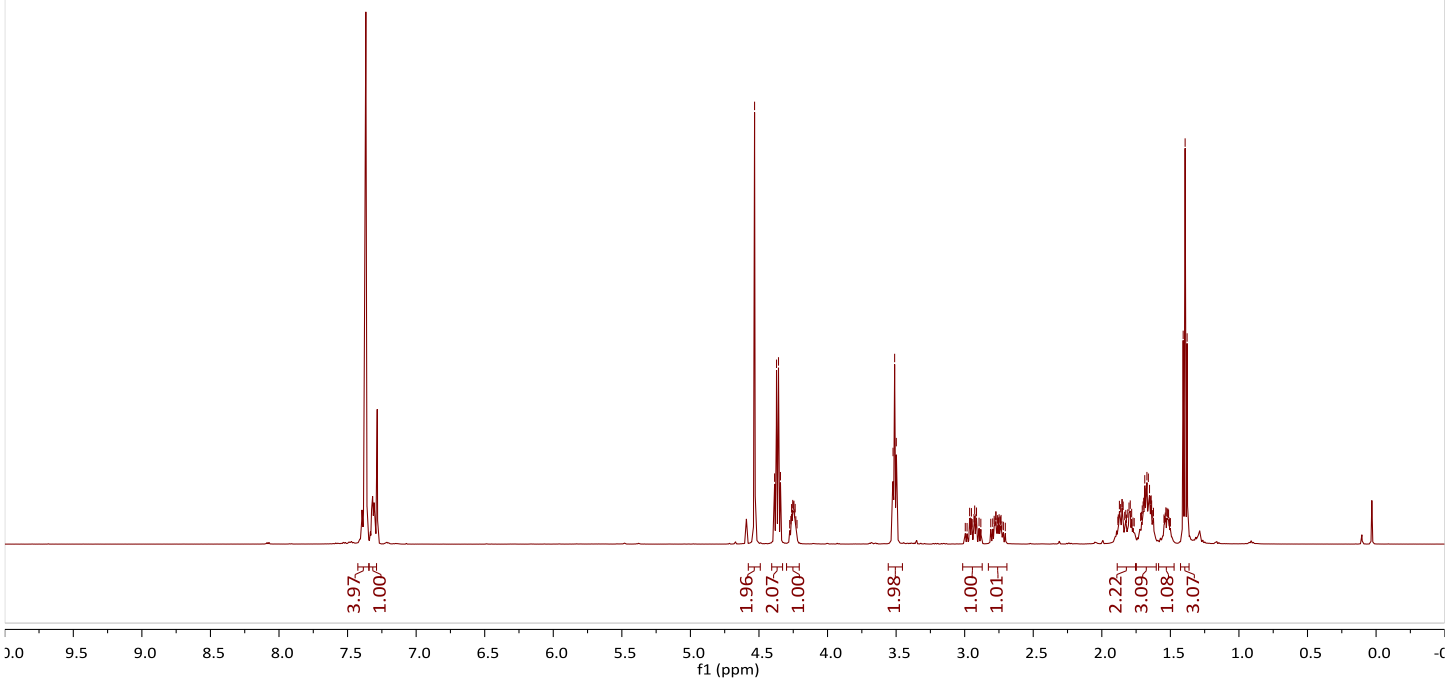

${ }^{19}$ F NMR-spectrum $\left(471 \mathrm{MHz}, \mathrm{CDCl}_{3}\right)$ of $3 \mathbf{k}$

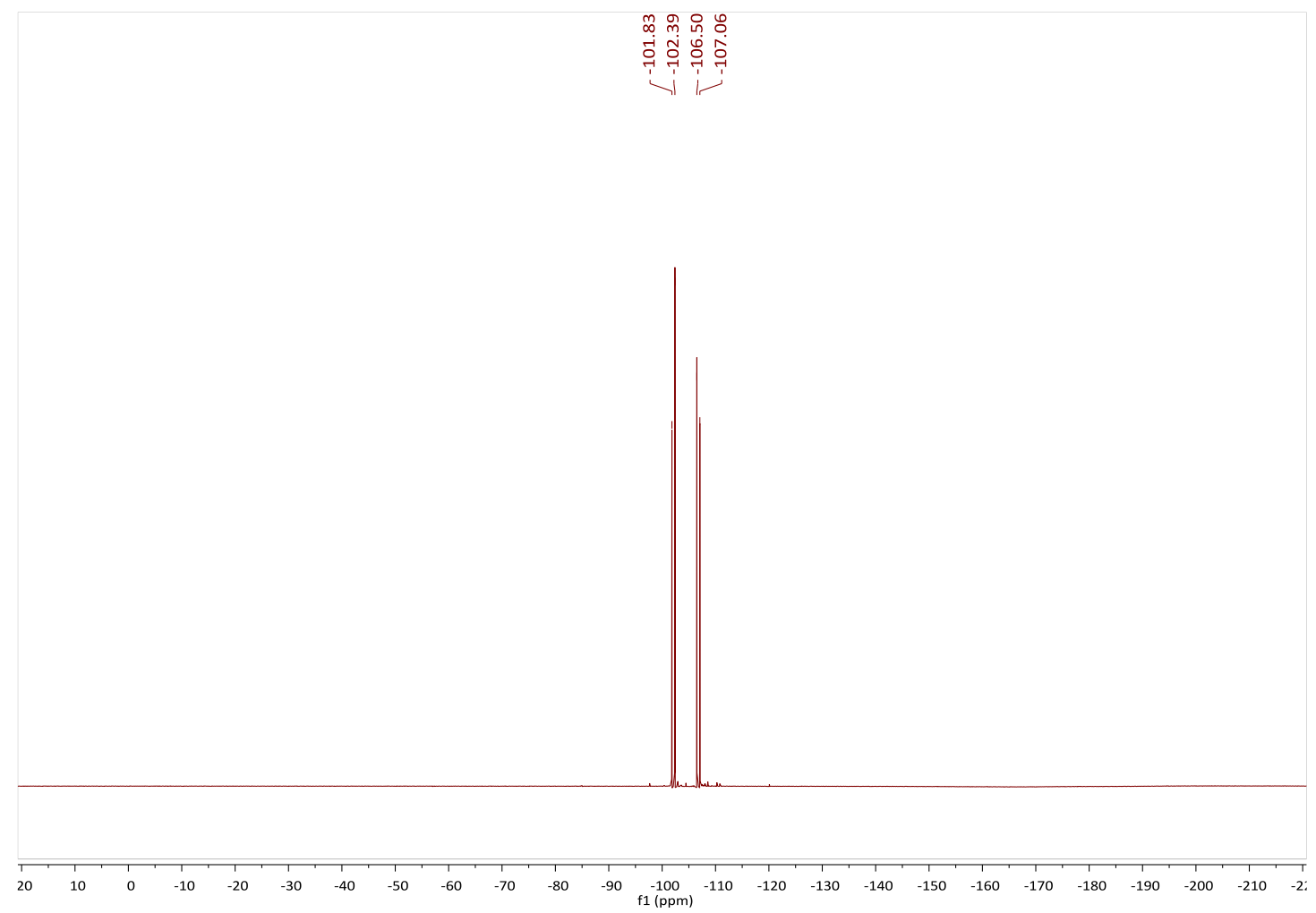




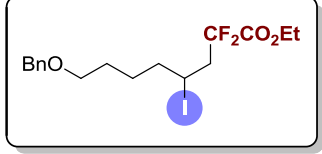

${ }^{13} \mathrm{C}$ NMR-spectrum (125 MHz, $\left.\mathrm{CDCl}_{3}\right)$ of $3 \mathbf{k}$

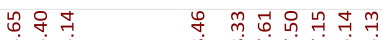

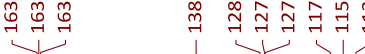

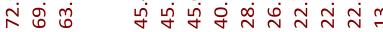

$1,1 \underbrace{1,13}$

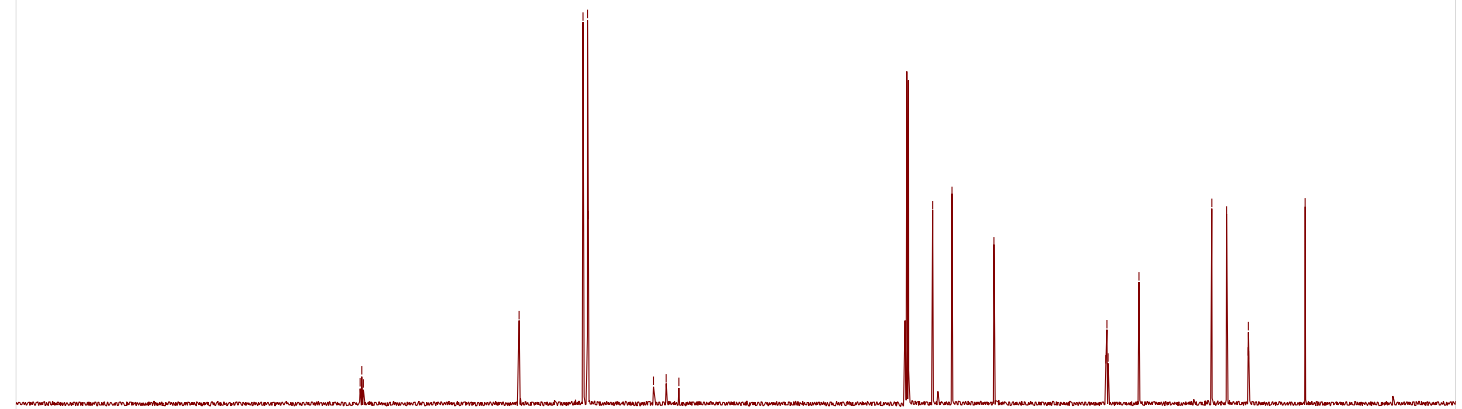

$\begin{array}{lllllllllllllllllllllllllllllllllllll}210 & 200 & 190 & 180 & 170 & 160 & 150 & 140 & 130 & 120 & 110 & 100 & 90 & 80 & 70 & 60 & 50 & 40 & 30 & 20 & 10 & 0 & -10\end{array}$ 


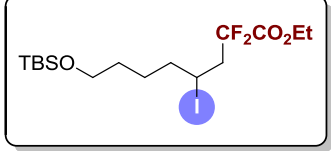

${ }^{1} \mathbf{H}$ NMR-spectrum $\left(500 \mathrm{MHz}, \mathrm{CDCl}_{3}\right)$ of 31

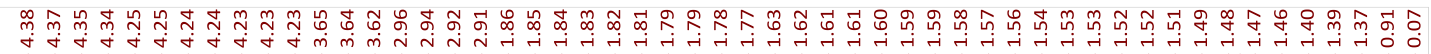

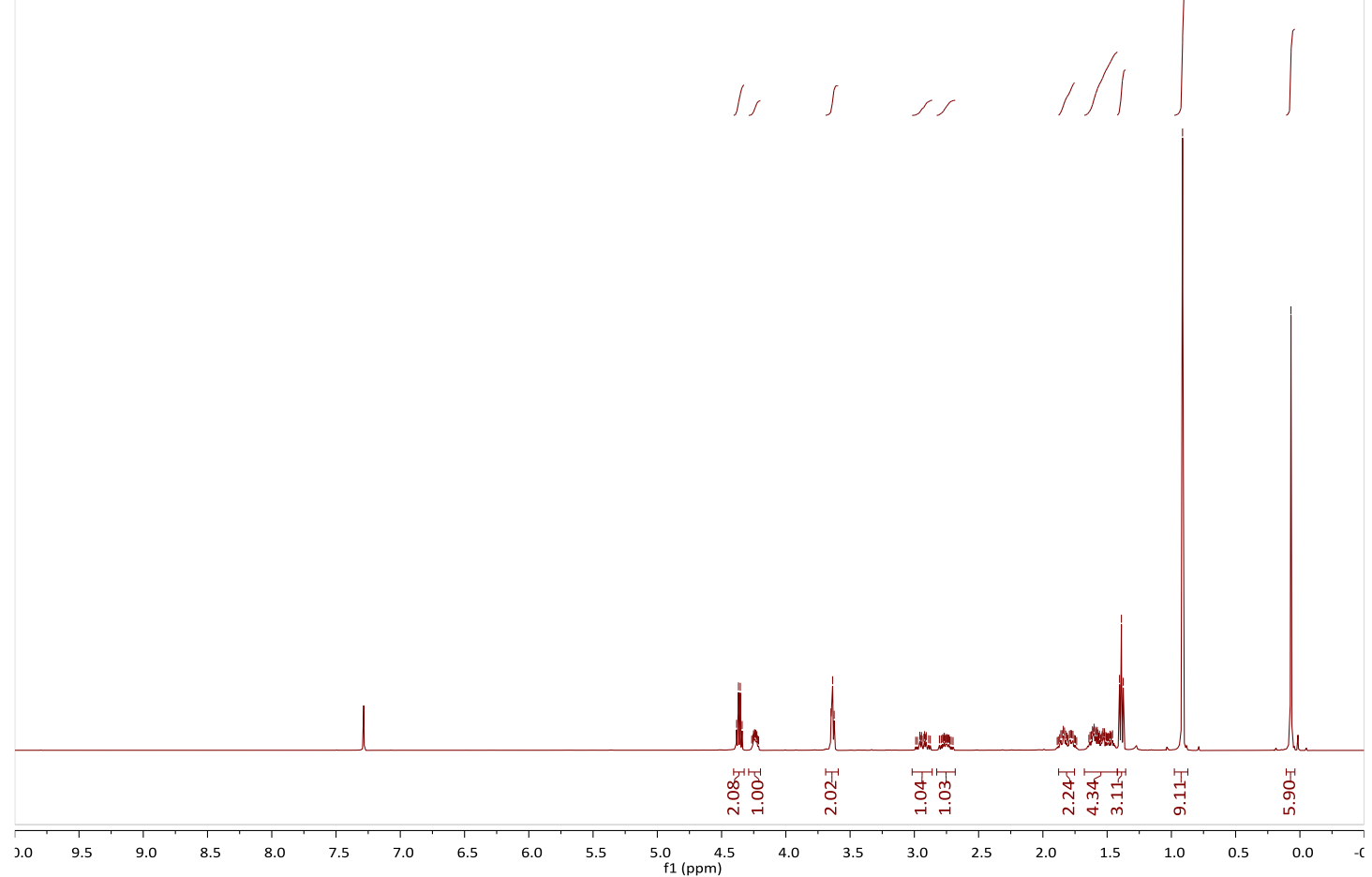

${ }^{19} \mathrm{~F}$ NMR-spectrum $\left(471 \mathrm{MHz}, \mathrm{CDCl}_{3}\right)$ of 31

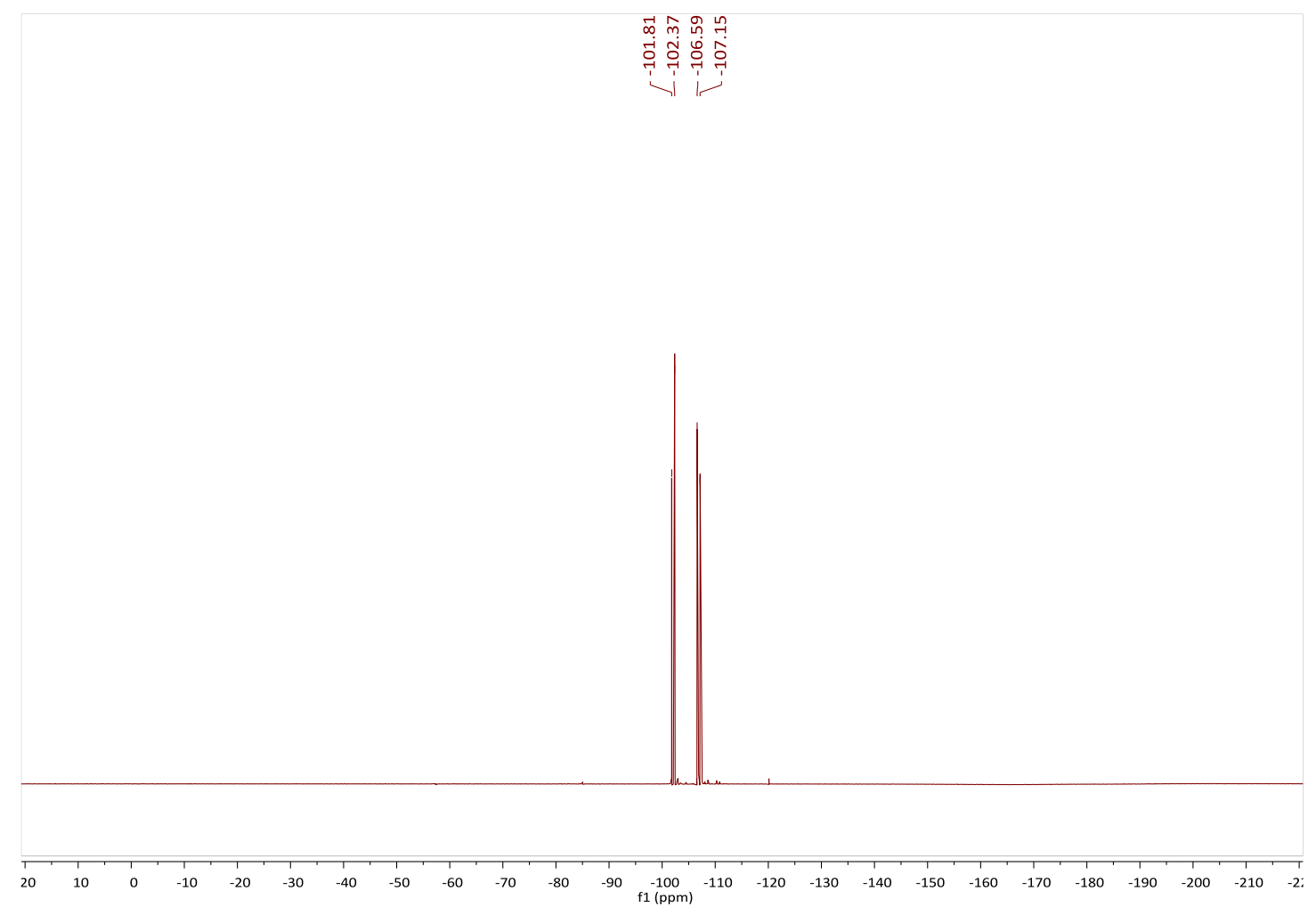




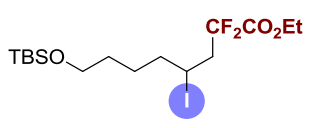

${ }^{13} \mathrm{C}$ NMR-spectrum (125 MHz, $\left.\mathrm{CDCl}_{3}\right)$ of 31

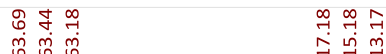

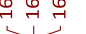

ᄀి 0ิ

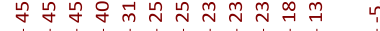

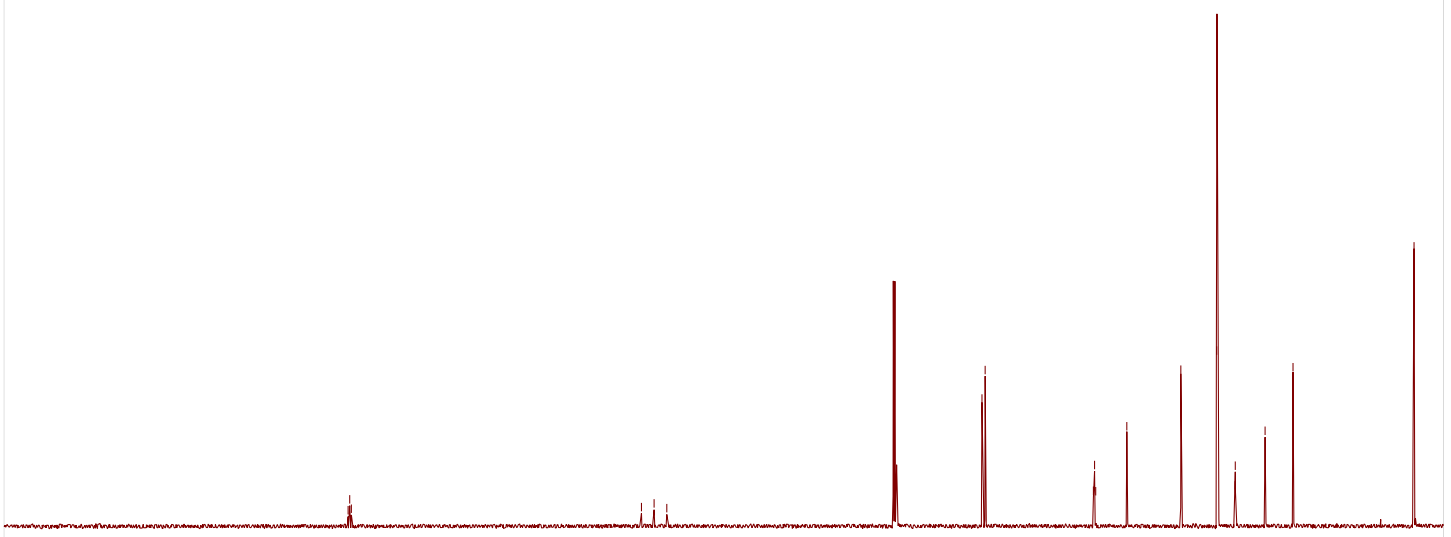

$\begin{array}{llllllllllll}210 & 200 & 190 & 180 & 170 & 160 & 150 & 140 & 130 & 120 & 110 & 100\end{array}$ 


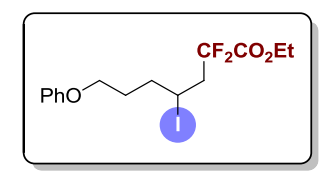

${ }^{1} \mathbf{H}$ NMR-spectrum $\left(500 \mathrm{MHz}, \mathrm{CDCl}_{3}\right)$ of $3 \mathbf{m}$

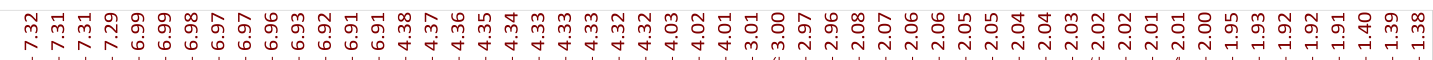
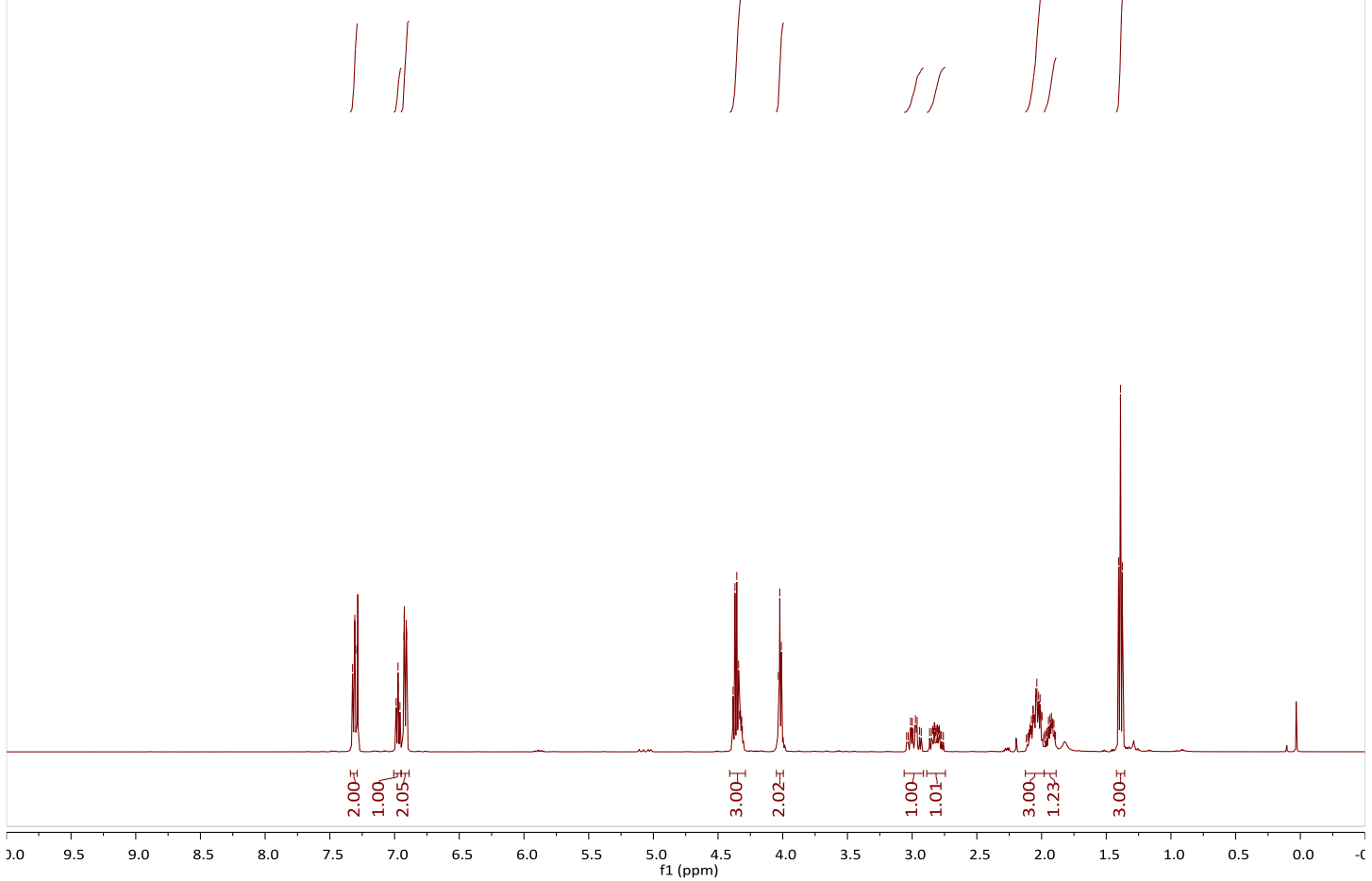

${ }^{19}$ F NMR-spectrum $\left(471 \mathrm{MHz}, \mathrm{CDCl}_{3}\right)$ of $3 \mathbf{m}$

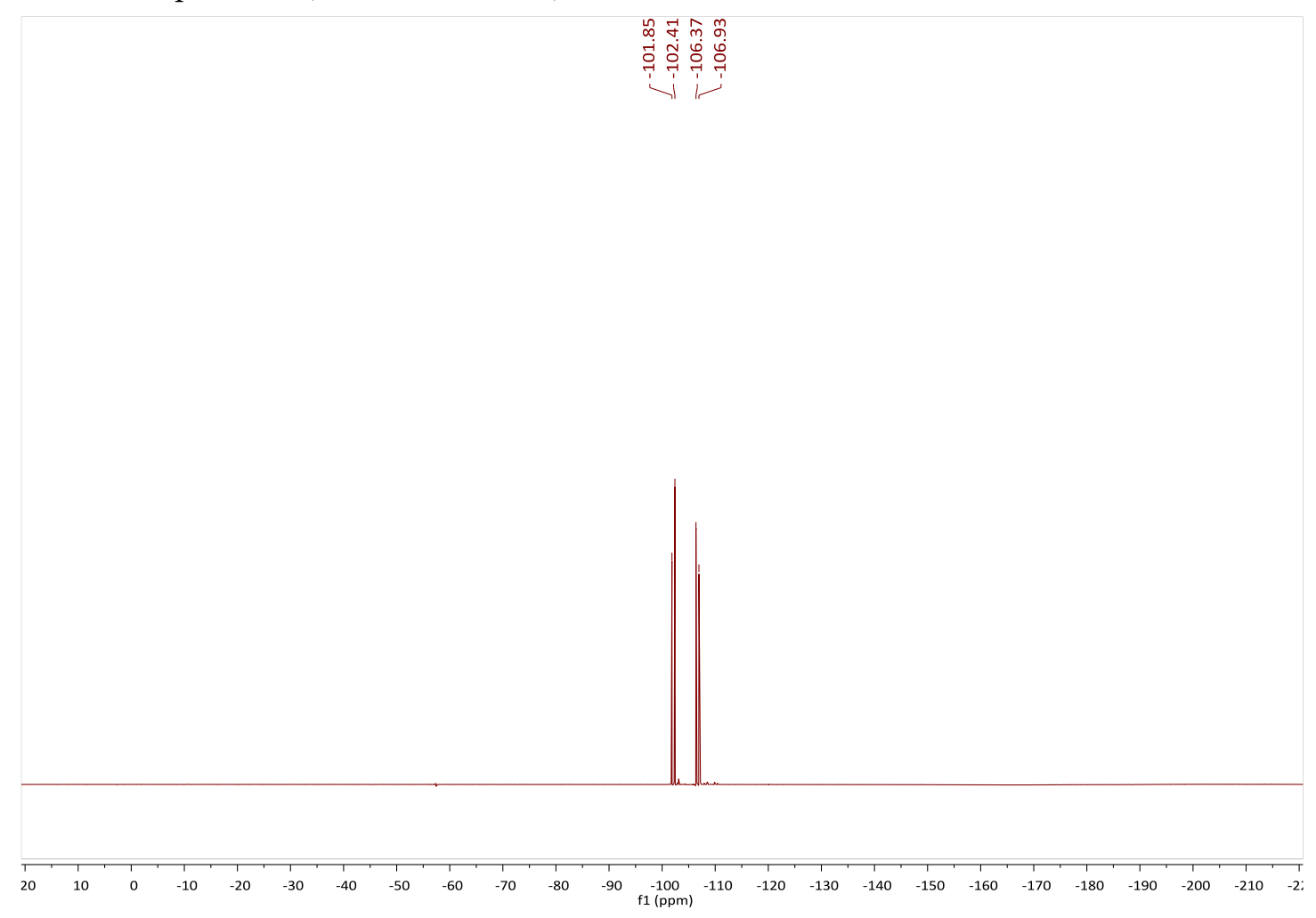




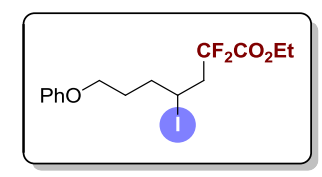

${ }^{13} \mathrm{C}$ NMR-spectrum (125 MHz, $\left.\mathrm{CDCl}_{3}\right)$ of $\mathbf{3 m}$

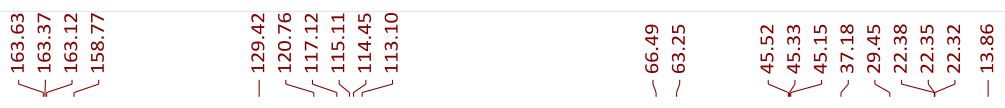

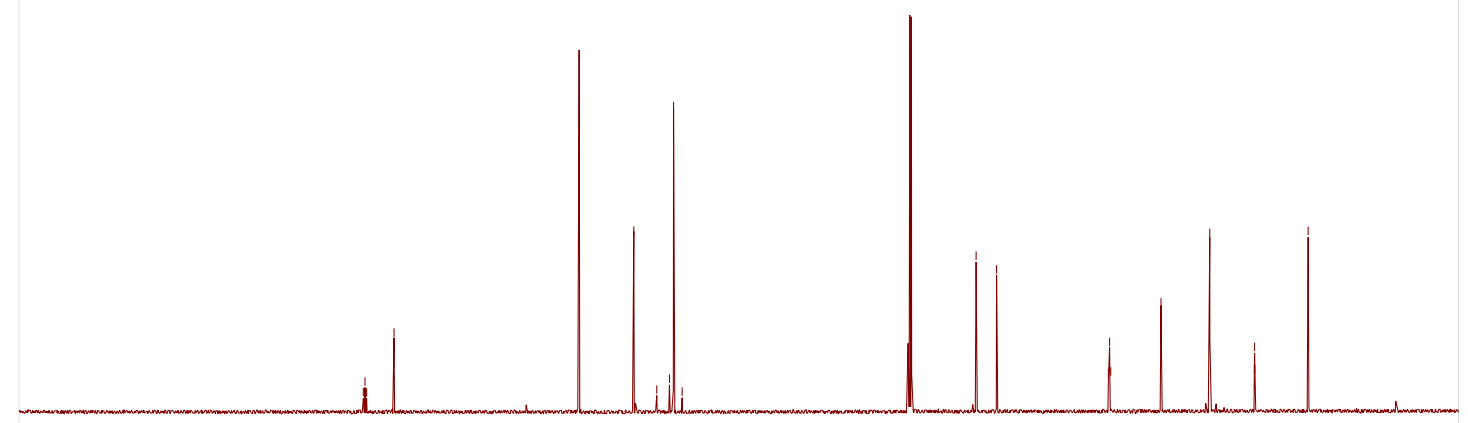

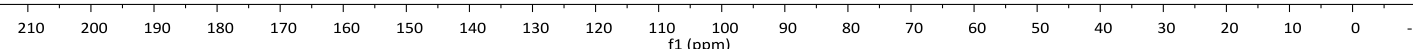




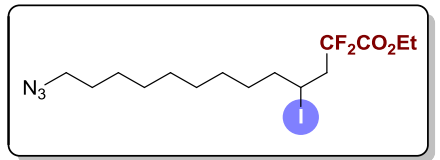

${ }^{1} \mathbf{H}$ NMR-spectrum $\left(500 \mathrm{MHz}, \mathrm{CDCl}_{3}\right)$ of $\mathbf{3 n}$

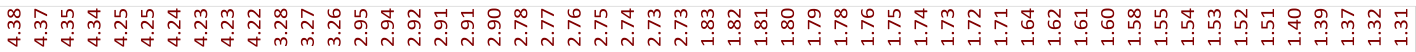

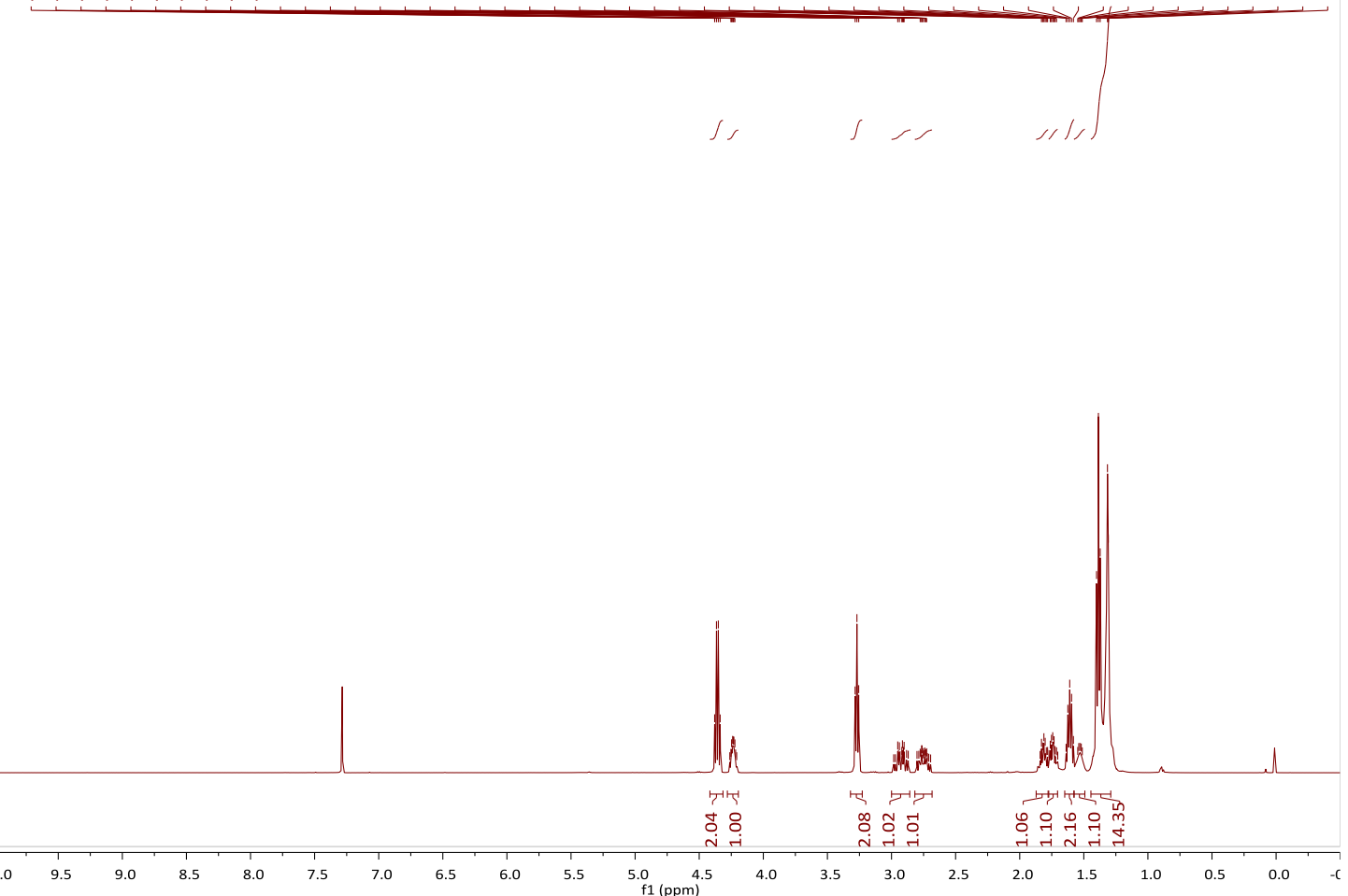

${ }^{19}$ F NMR-spectrum $\left(471 \mathrm{MHz}, \mathrm{CDCl}_{3}\right)$ of $\mathbf{3 n}$

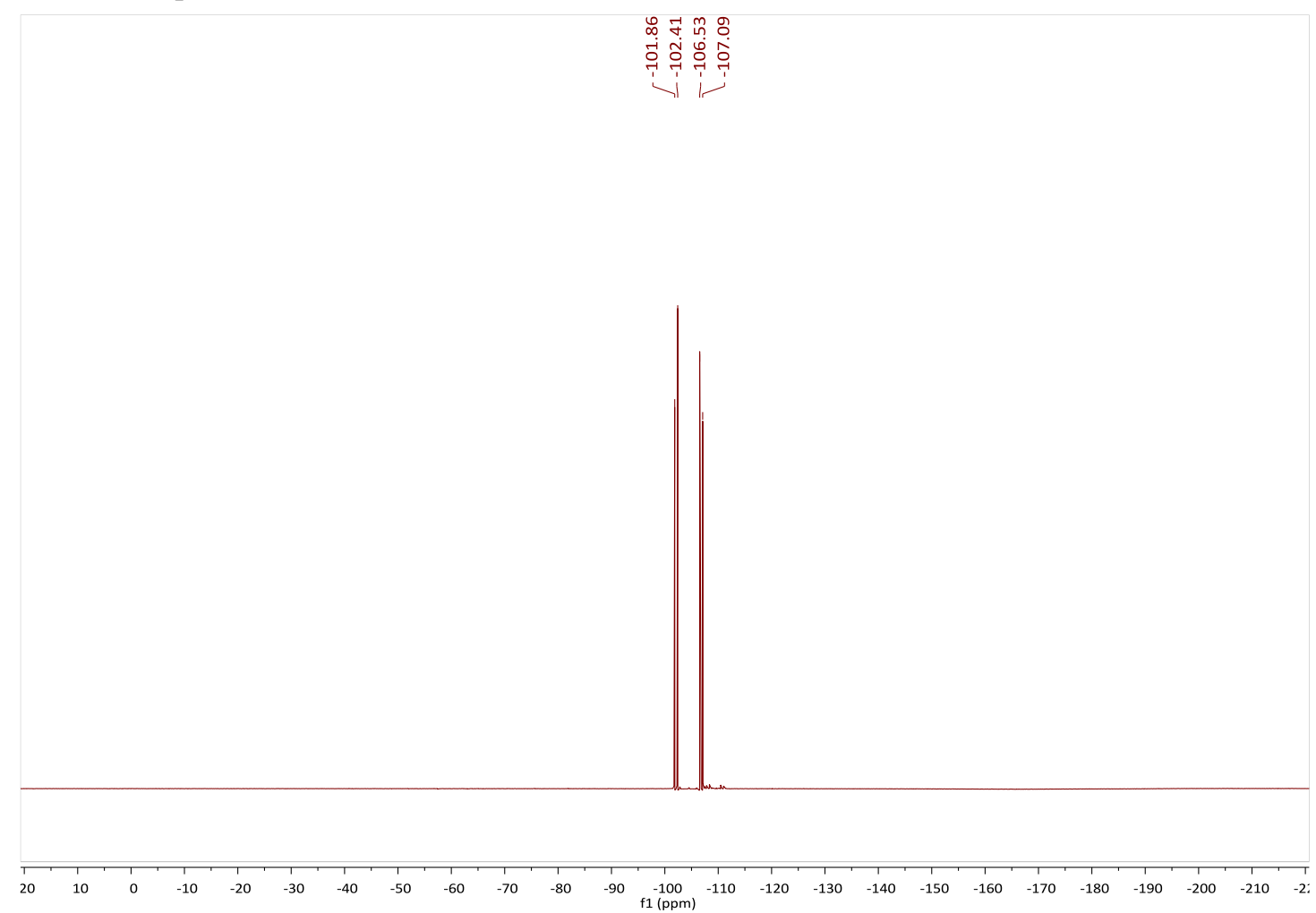




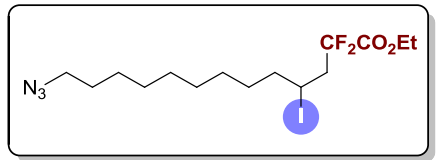

${ }^{13} \mathrm{C}$ NMR-spectrum (125 MHz, $\left.\mathrm{CDCl}_{3}\right)$ of $\mathbf{3 n}$

\begin{tabular}{|c|c|c|c|c|}
\hline 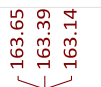 & 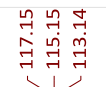 & $\begin{array}{l}0 \\
\stackrel{7}{n} \\
0 \\
1\end{array}$ & 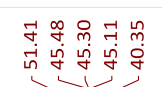 & 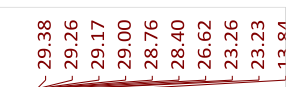 \\
\hline
\end{tabular}
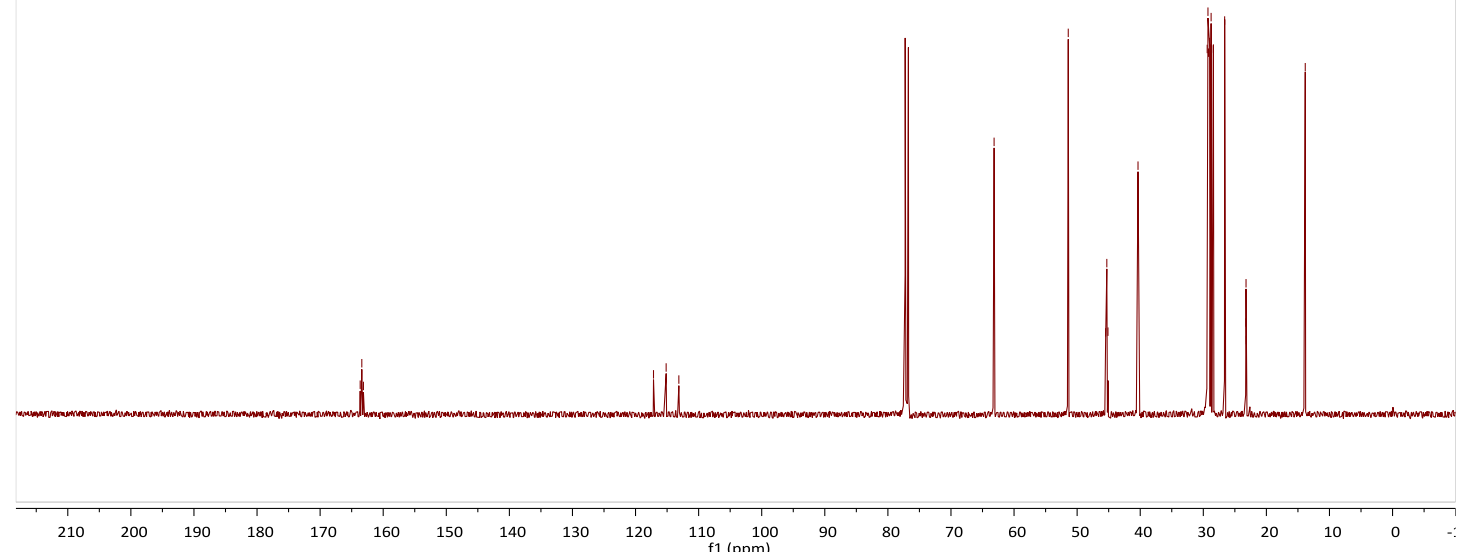


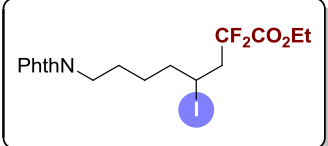

${ }^{1} \mathbf{H}$ NMR-spectrum $\left(500 \mathrm{MHz}, \mathrm{CDCl}_{3}\right)$ of $3 \mathbf{o}$

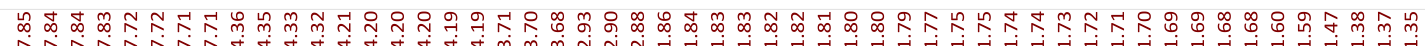

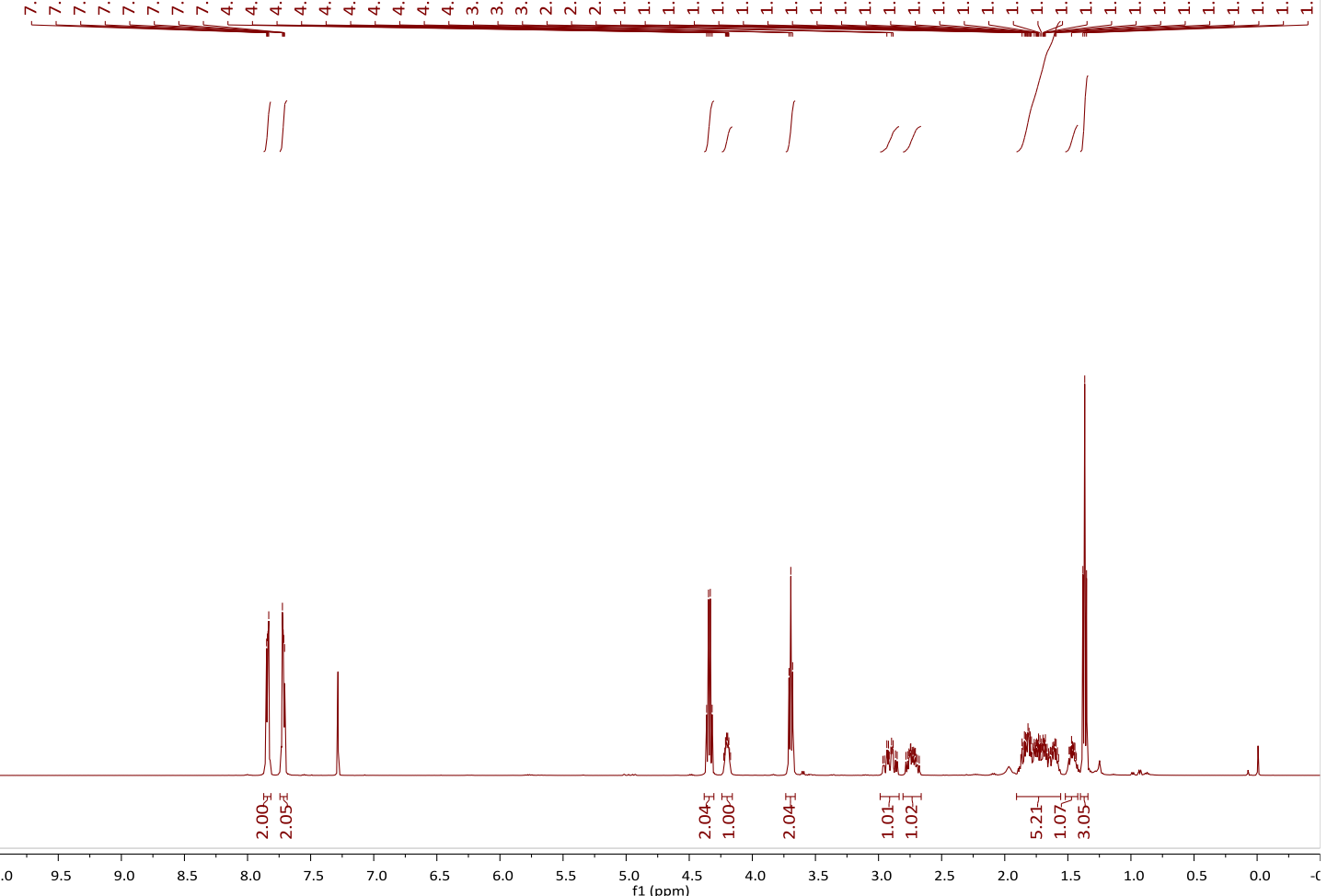

${ }^{19}$ F NMR-spectrum $\left(471 \mathrm{MHz}, \mathrm{CDCl}_{3}\right)$ of $3 \mathbf{o}$

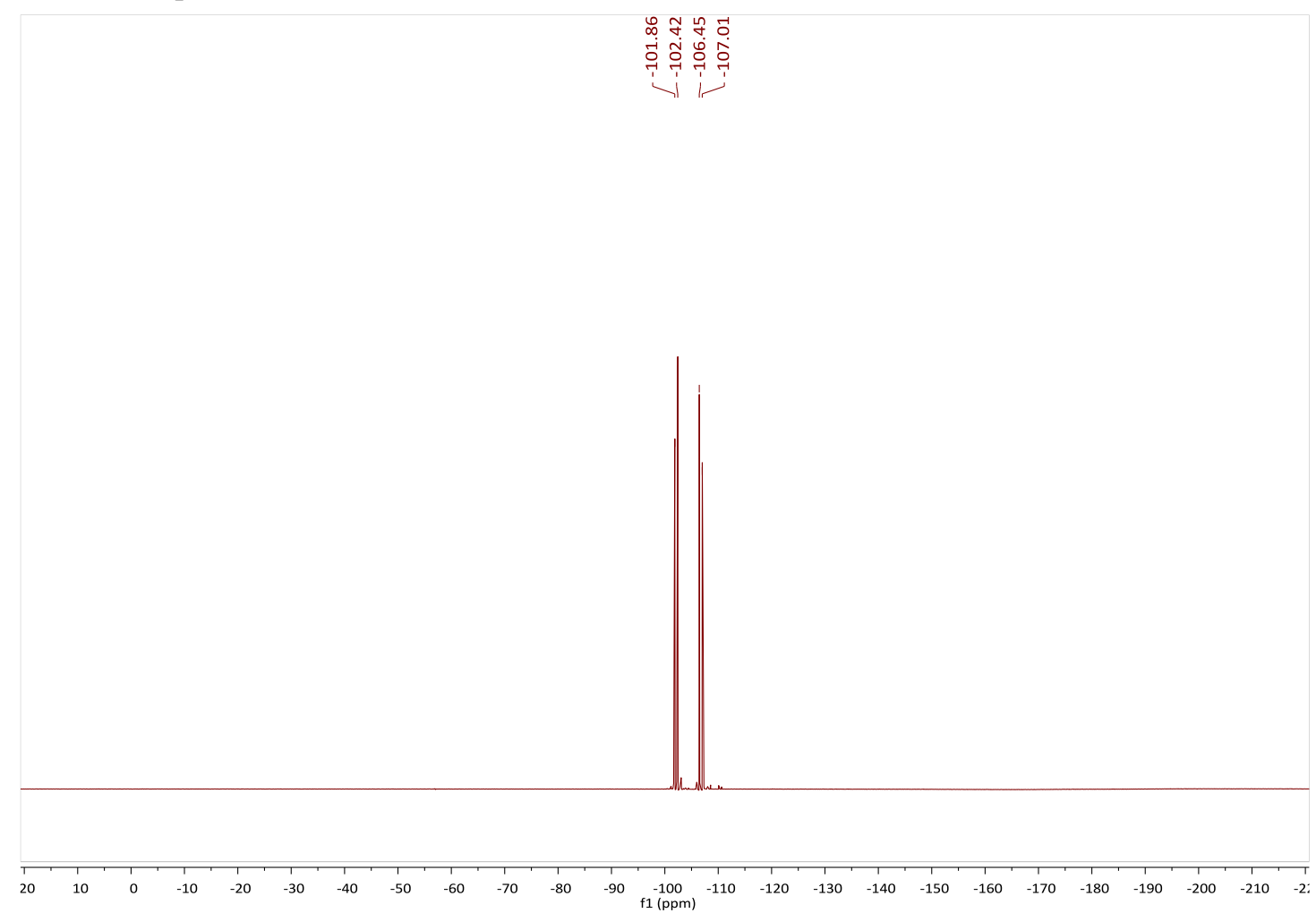




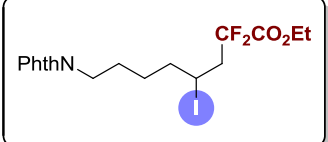

${ }^{13} \mathrm{C}$ NMR-spectrum (125 MHz, $\left.\mathrm{CDCl}_{3}\right)$ of $3 \mathbf{o}$

กับ

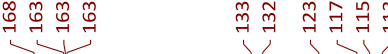

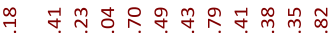

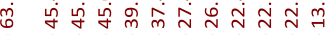

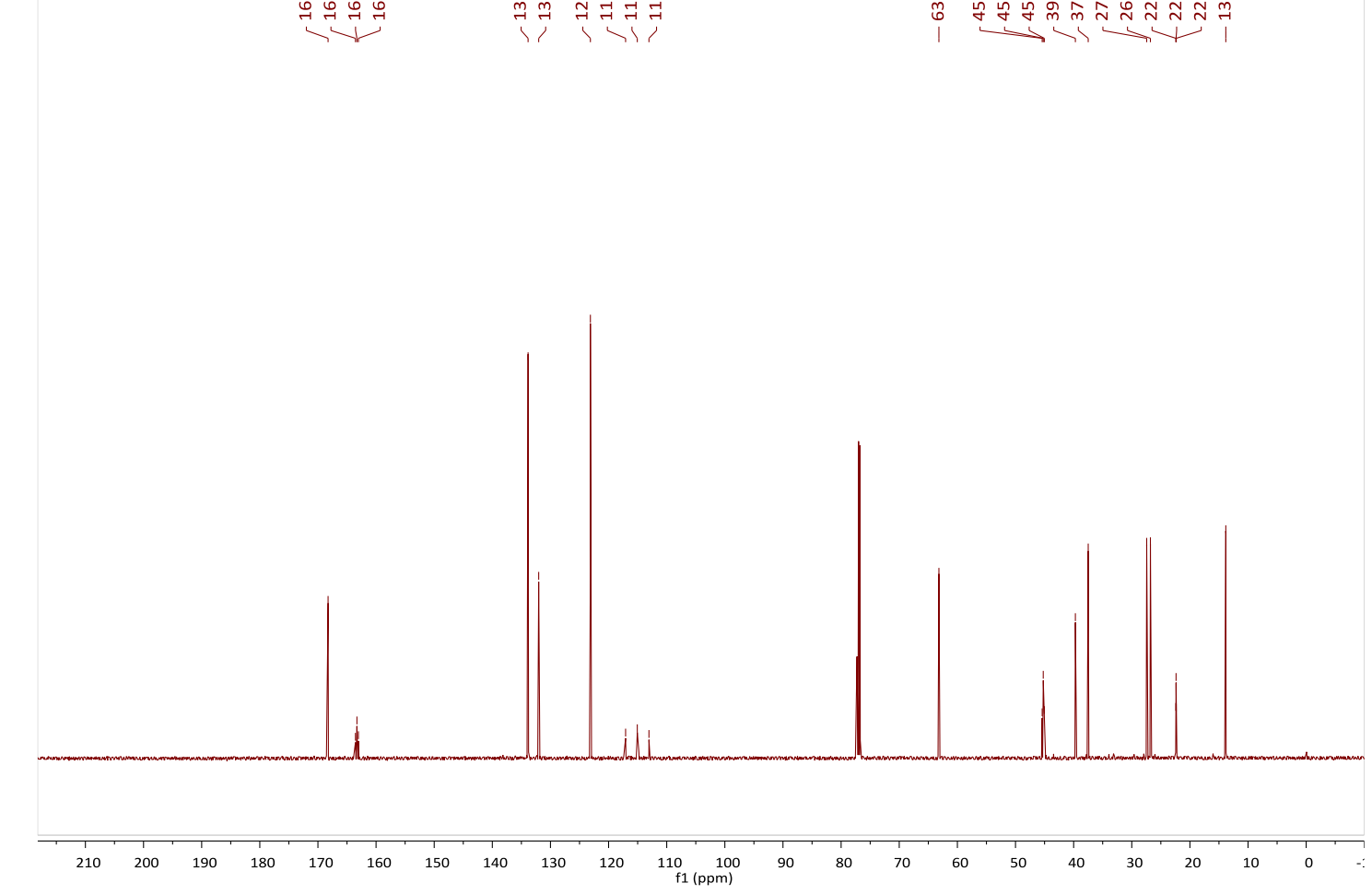




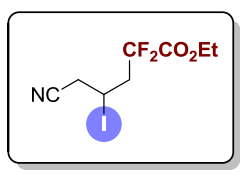

${ }^{1} \mathbf{H}$ NMR-spectrum $\left(500 \mathrm{MHz}, \mathrm{CDCl}_{3}\right)$ of $3 \mathbf{p}$

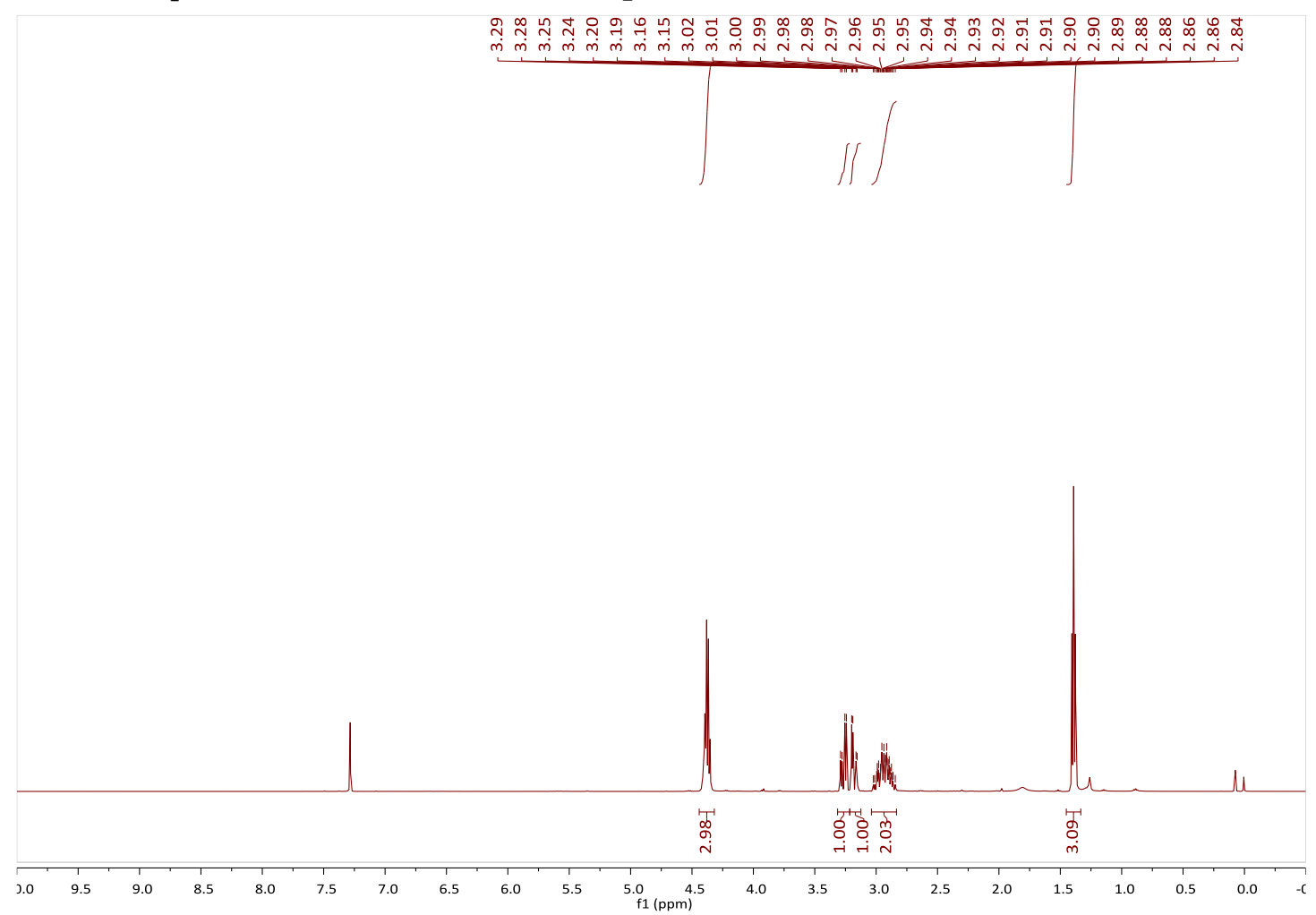

${ }^{19} \mathbf{F}$ NMR-spectrum $\left(471 \mathrm{MHz}, \mathrm{CDCl}_{3}\right)$ of $3 \mathbf{p}$

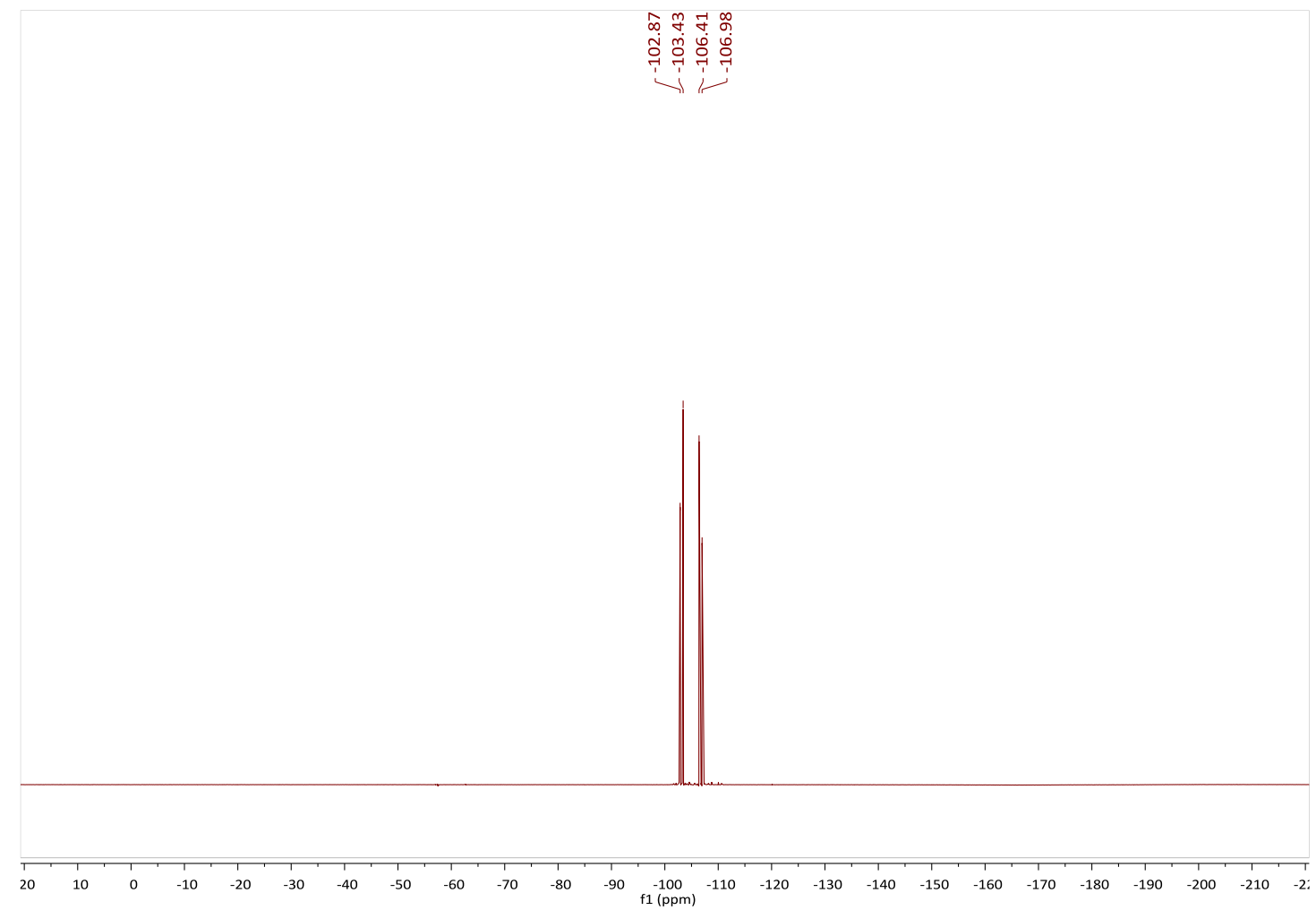




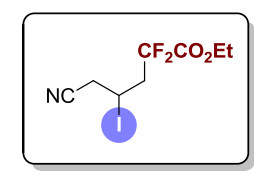

${ }^{13} \mathrm{C}$ NMR-spectrum (125 MHz, $\left.\mathrm{CDCl}_{3}\right)$ of $3 \mathbf{p}$

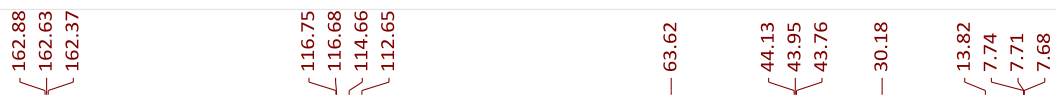

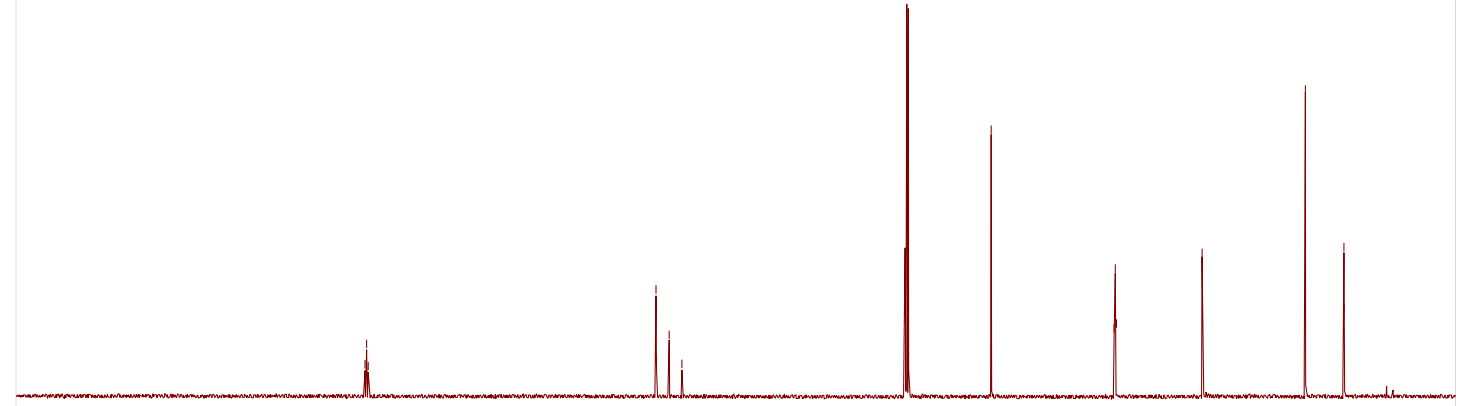

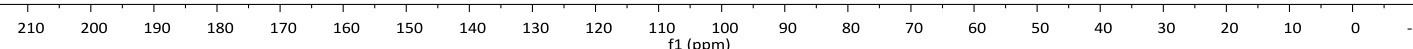




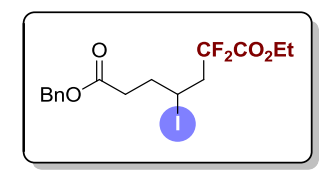

${ }^{1} \mathbf{H}$ NMR-spectrum $\left(500 \mathrm{MHz}, \mathrm{CDCl}_{3}\right)$ of $\mathbf{3 q}$

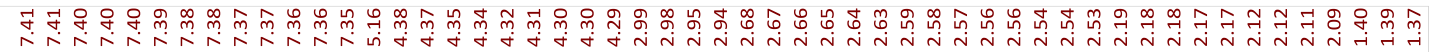

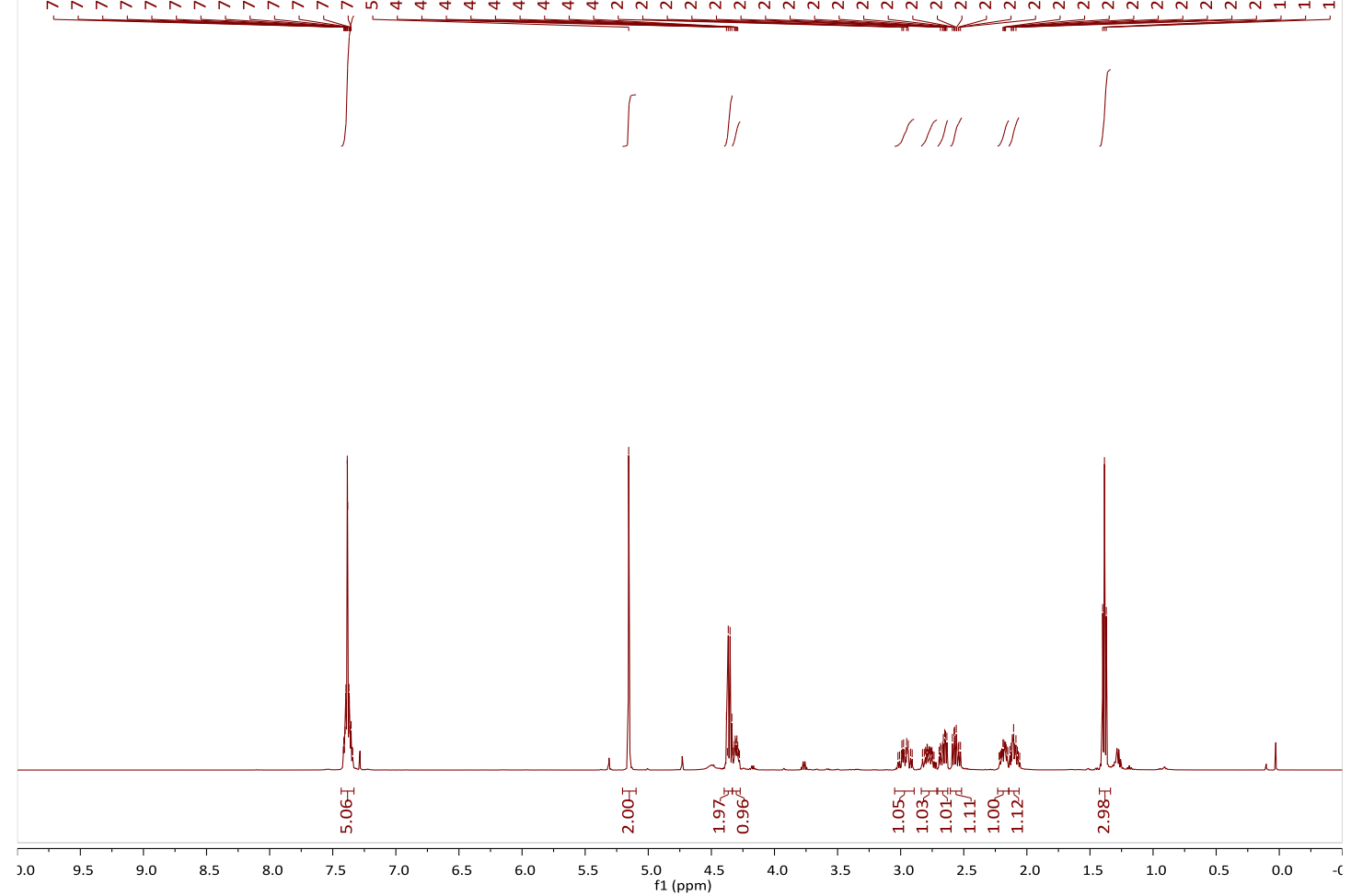

${ }^{19}$ F NMR-spectrum $\left(471 \mathrm{MHz}, \mathrm{CDCl}_{3}\right)$ of $\mathbf{3 q}$

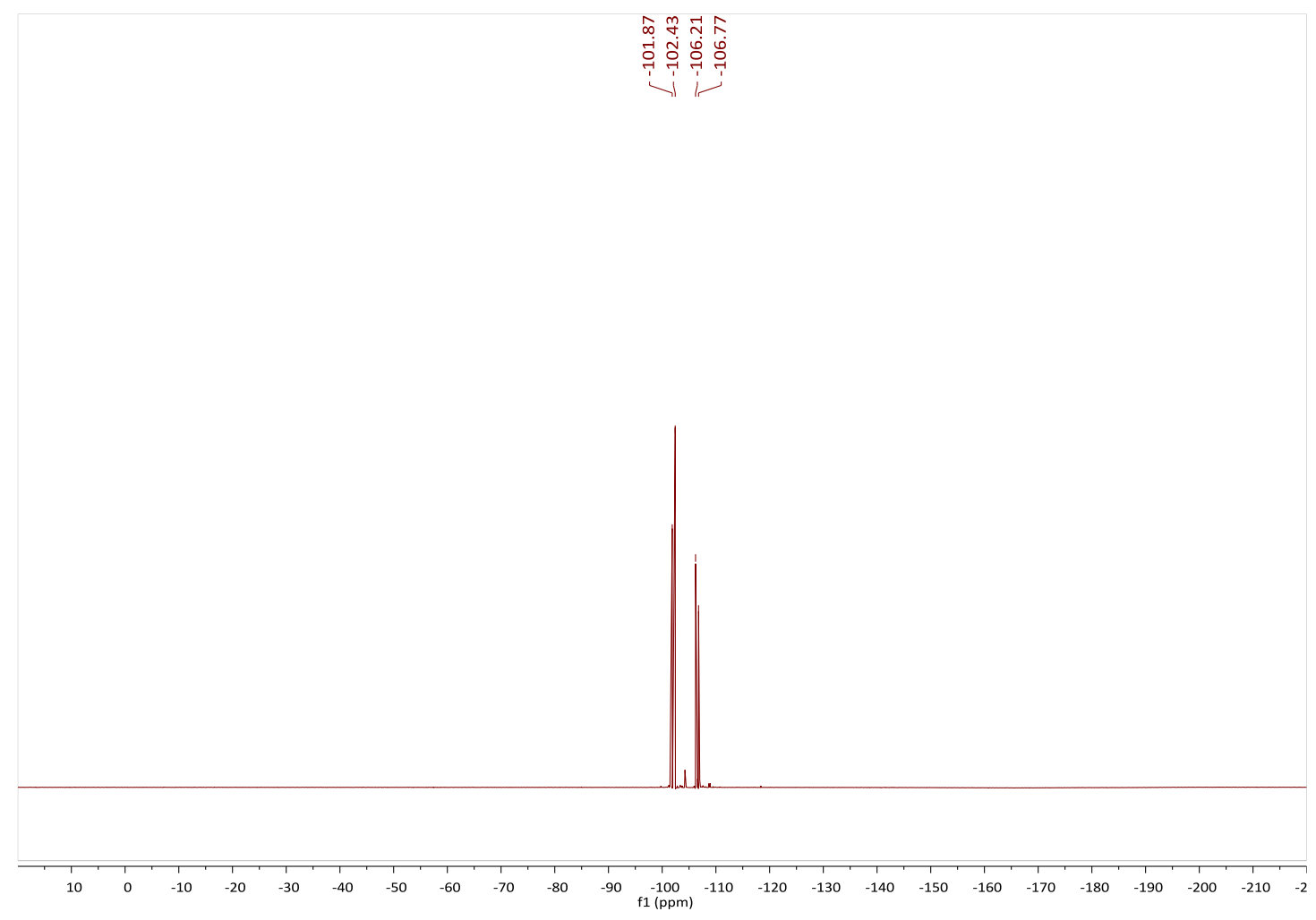




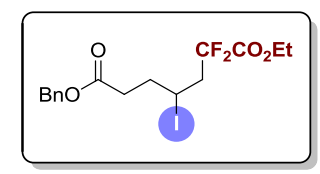

${ }^{13} \mathbf{C}$ NMR-spectrum (125 MHz, $\left.\mathrm{CDCl}_{3}\right)$ of $\mathbf{3 q}$

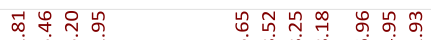

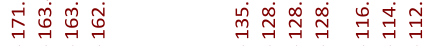

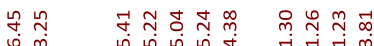

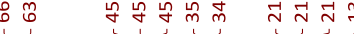

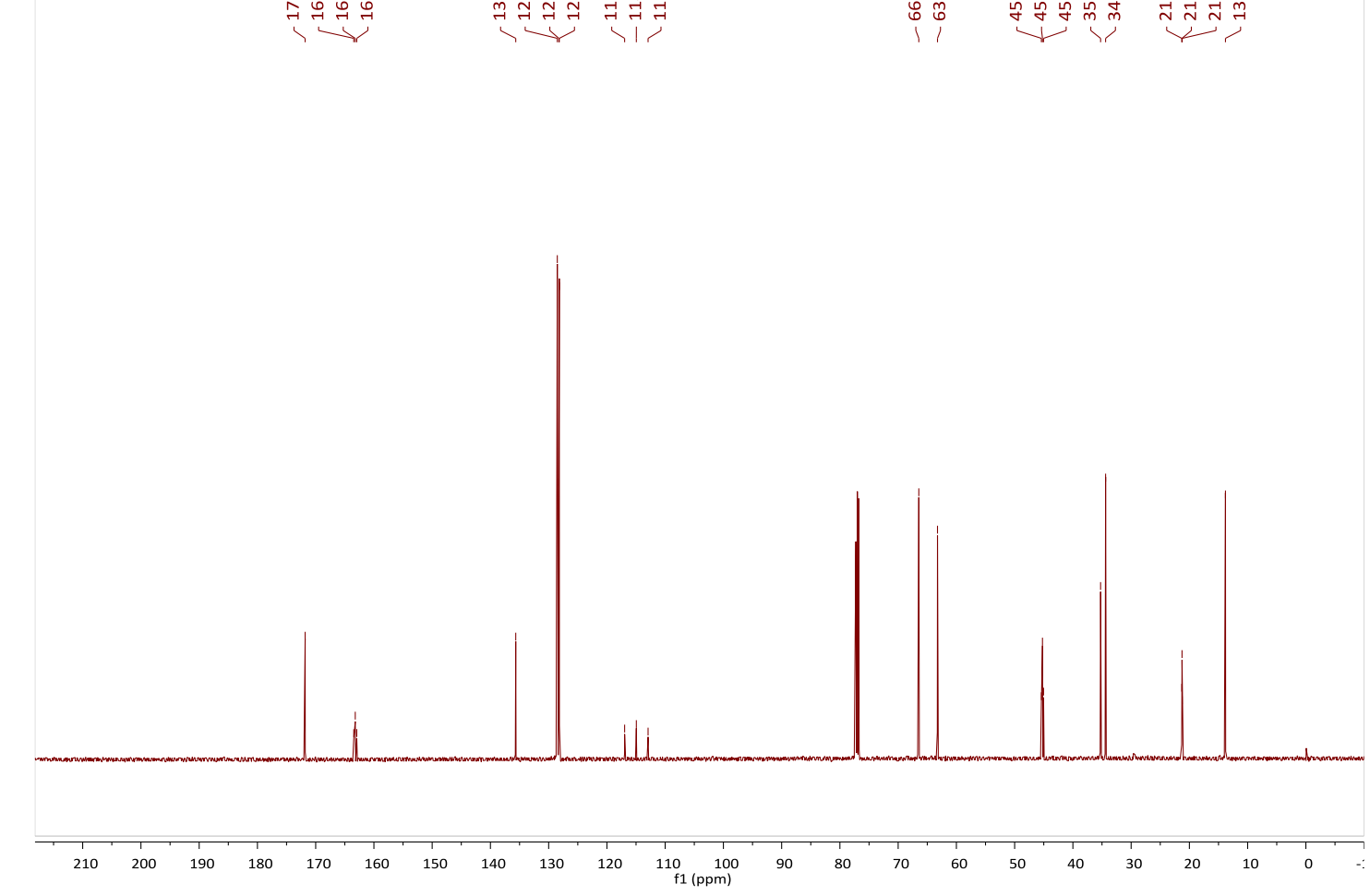




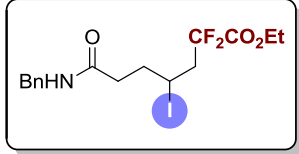

${ }^{1} \mathbf{H}$ NMR-spectrum $\left(500 \mathrm{MHz}, \mathrm{CDCl}_{3}\right)$ of $3 \mathbf{r}$

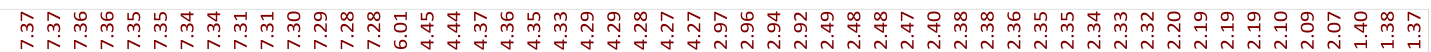
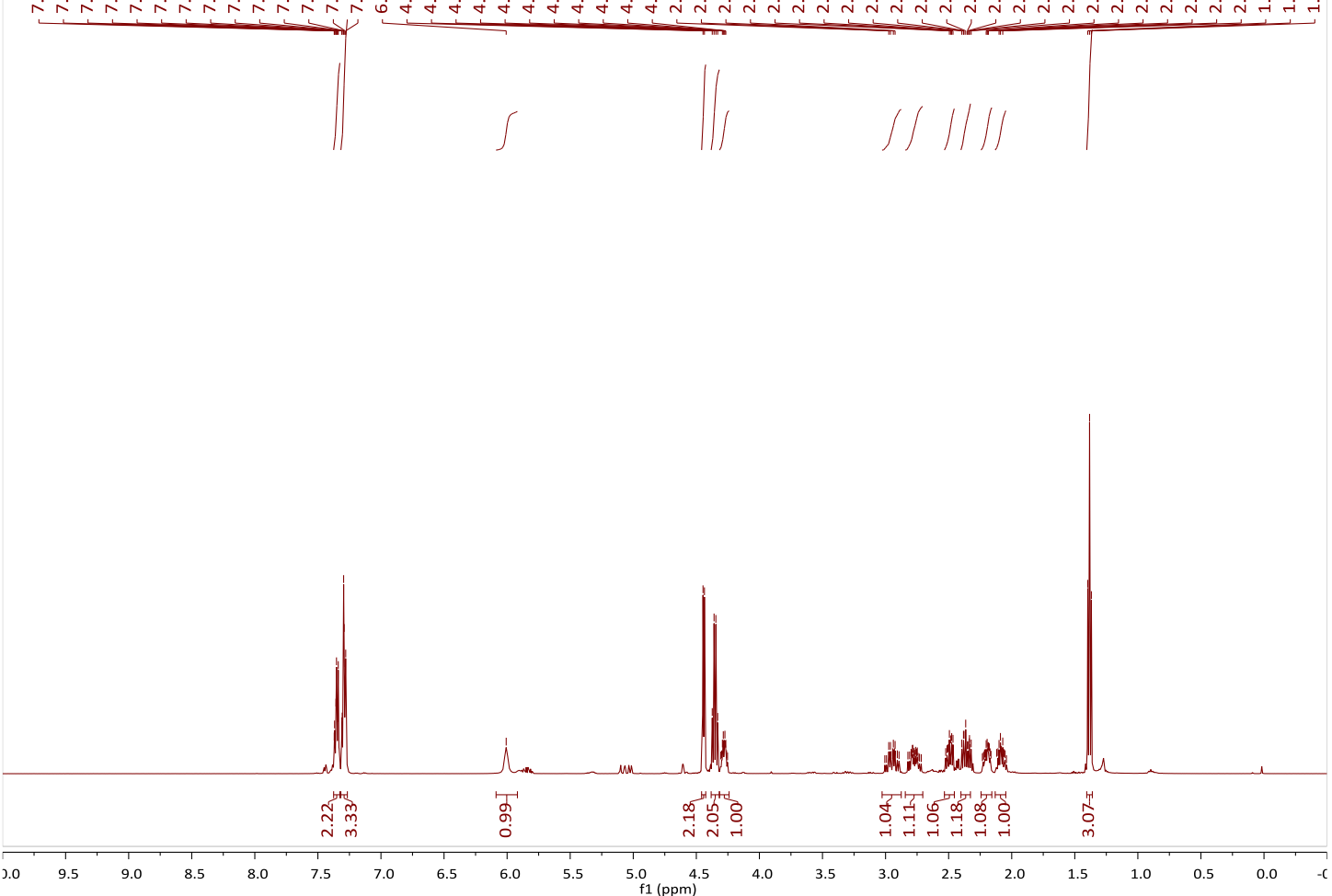

${ }^{19}$ F NMR-spectrum $\left(471 \mathrm{MHz}, \mathrm{CDCl}_{3}\right)$ of $3 \mathbf{r}$

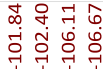

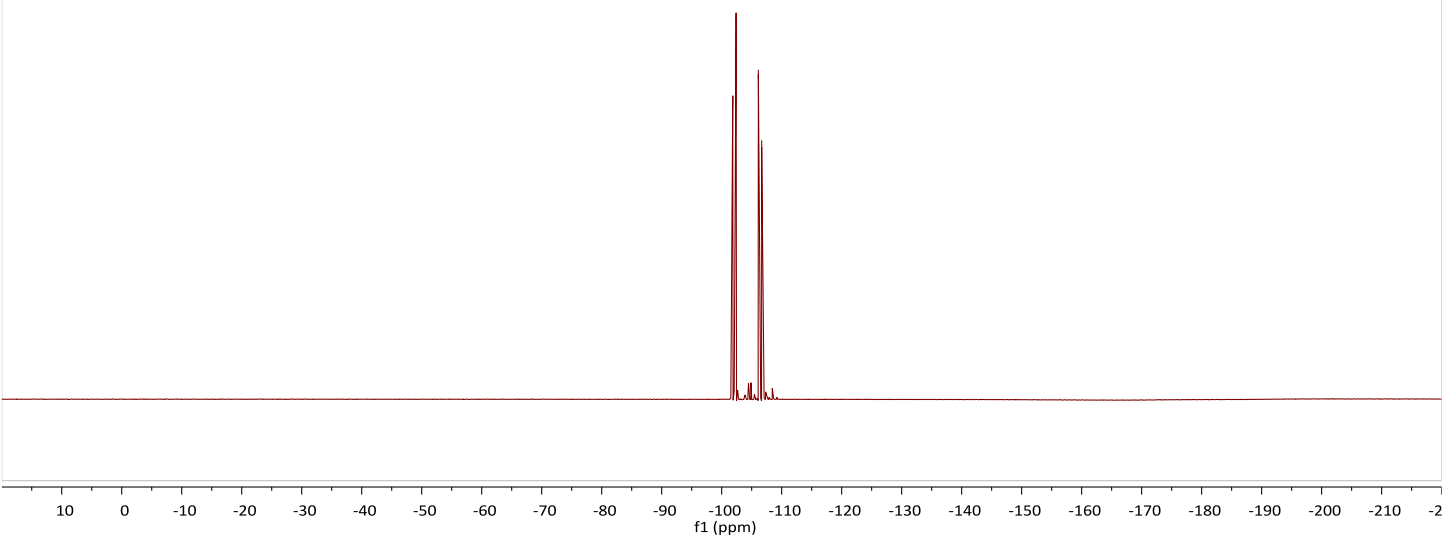




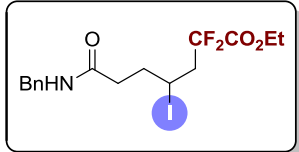

${ }^{13} \mathrm{C}$ NMR-spectrum (125 MHz, $\left.\mathrm{CDCl}_{3}\right)$ of $3 \mathbf{r}$

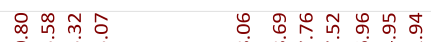

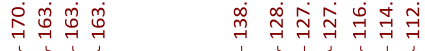

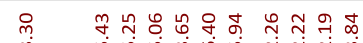

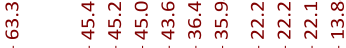

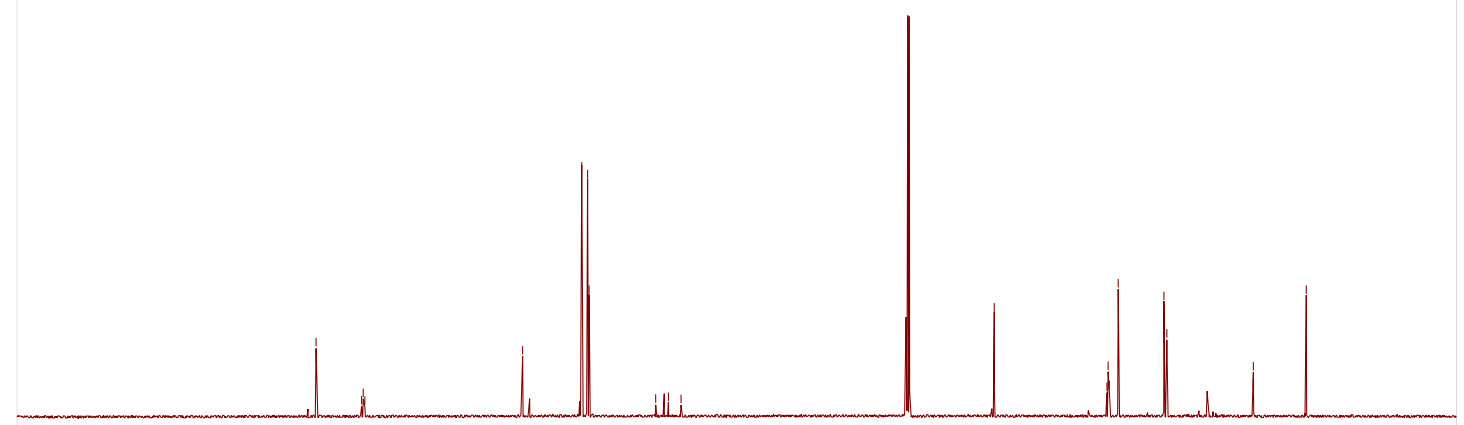

$\begin{array}{llllllllllllllllllllllllllllllllll}210 & 200 & 190 & 180 & 170 & 160 & 150 & 140 & 130 & 120 & 110 & 100 & 90 & 80 & 70 & 60 & 50 & 40 & 30 & 20 & 10 & 0 & -\end{array}$ 


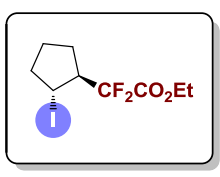

${ }^{1} \mathbf{H}$ NMR-spectrum $\left(500 \mathrm{MHz}, \mathrm{CDCl}_{3}\right)$ of $3 \mathbf{s}$

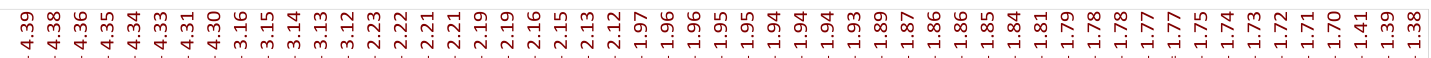
$\|\quad\| \| \mid$
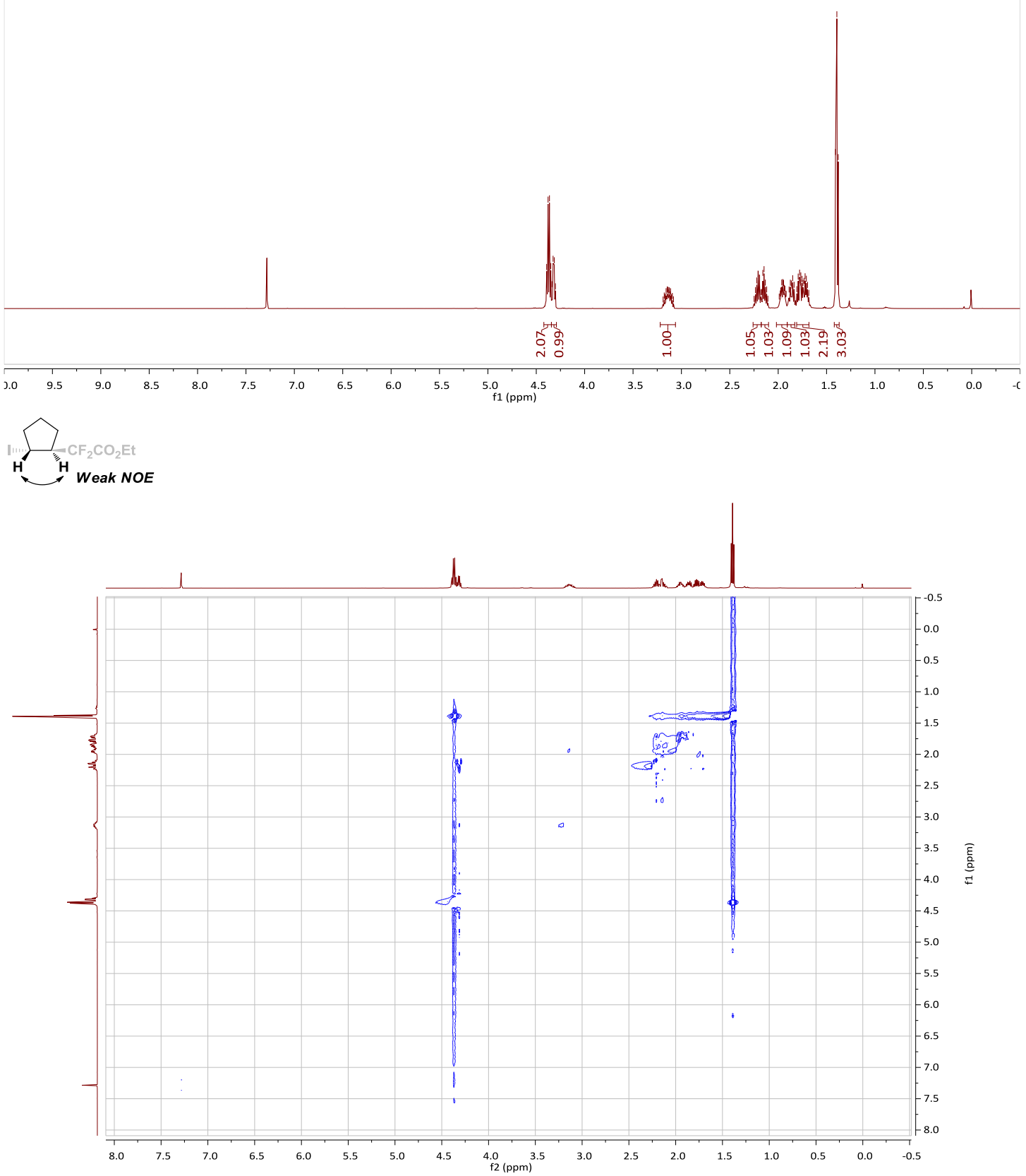


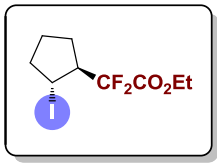

${ }^{19} \mathbf{F}$ NMR-spectrum $\left(471 \mathrm{MHz}, \mathrm{CDCl}_{3}\right)$ of $3 \mathbf{s}$

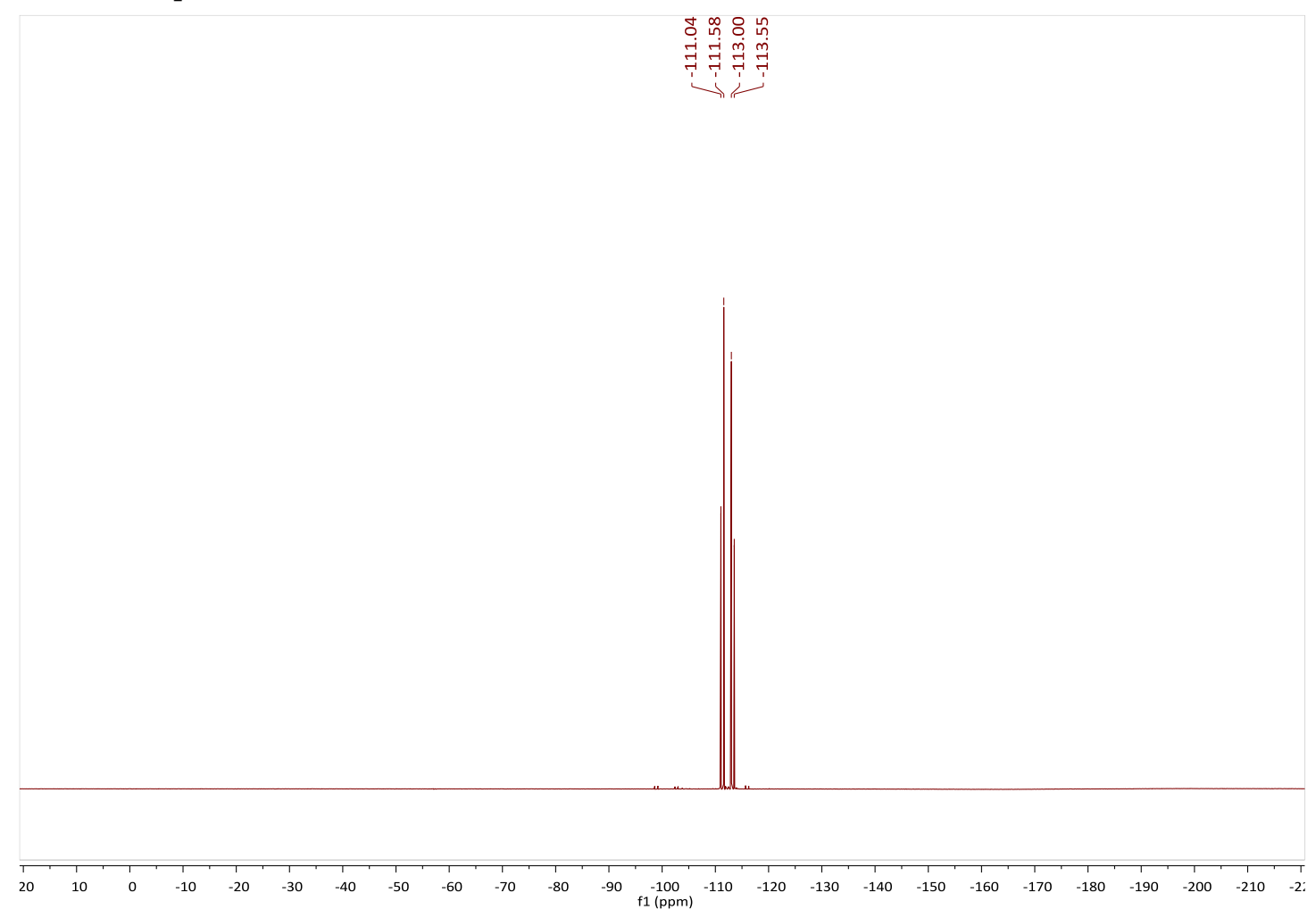

${ }^{13} \mathrm{C}$ NMR-spectrum $\left(125 \mathrm{MHz}, \mathrm{CDCl}_{3}\right)$ of $3 \mathbf{s}$

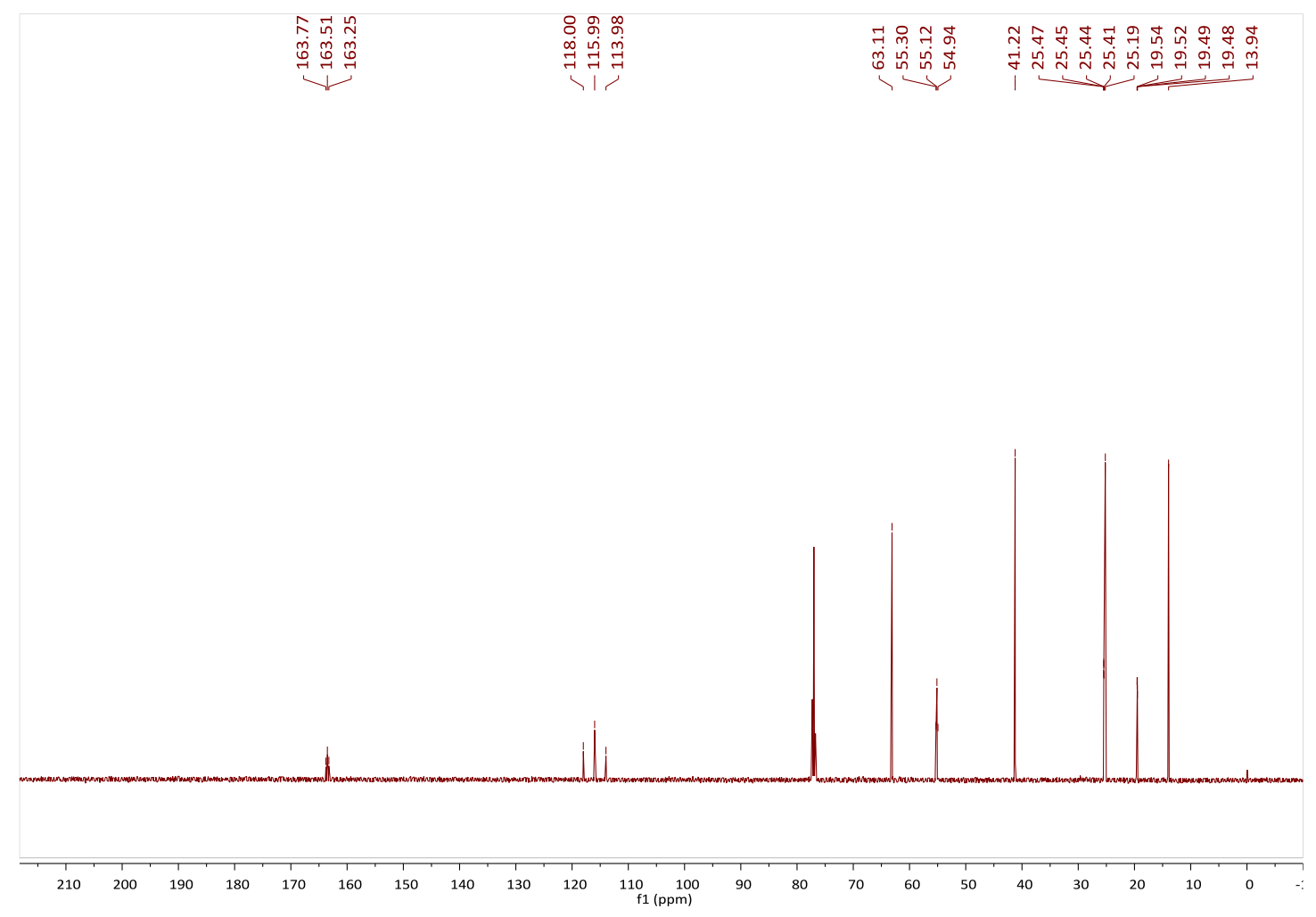




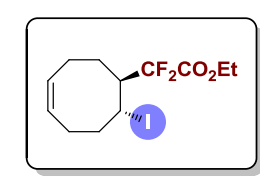

${ }^{1} \mathbf{H}$ NMR-spectrum $\left(500 \mathrm{MHz}, \mathrm{CDCl}_{3}\right)$ of $\mathbf{3 t}$

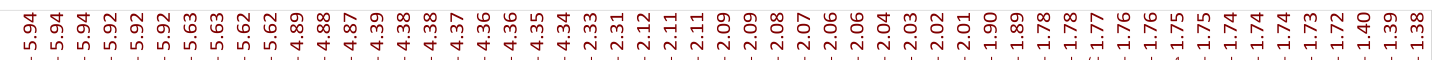

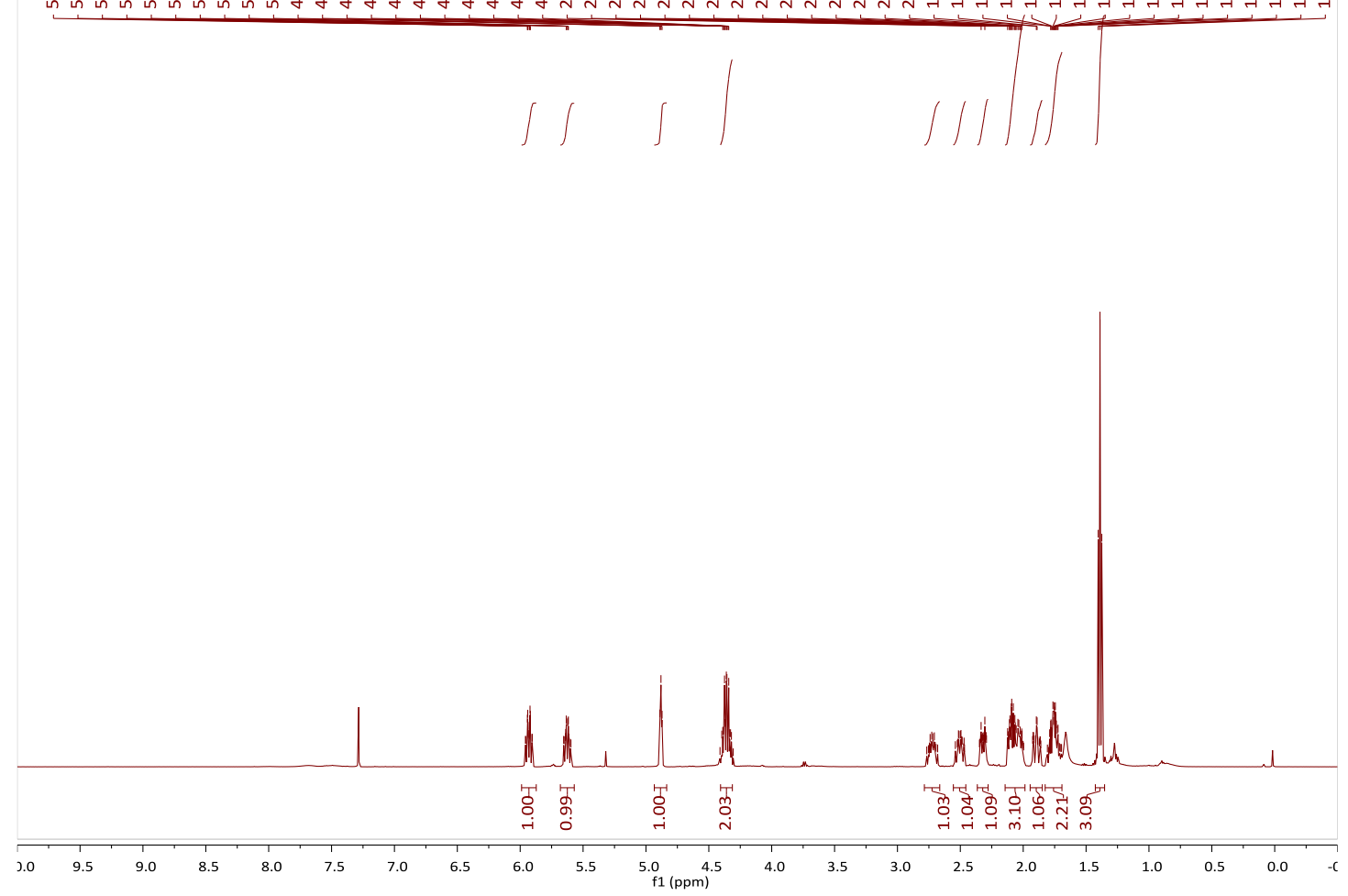

${ }^{19} \mathbf{F}$ NMR-spectrum $\left(471 \mathrm{MHz}, \mathrm{CDCl}_{3}\right)$ of $\mathbf{3 t}$

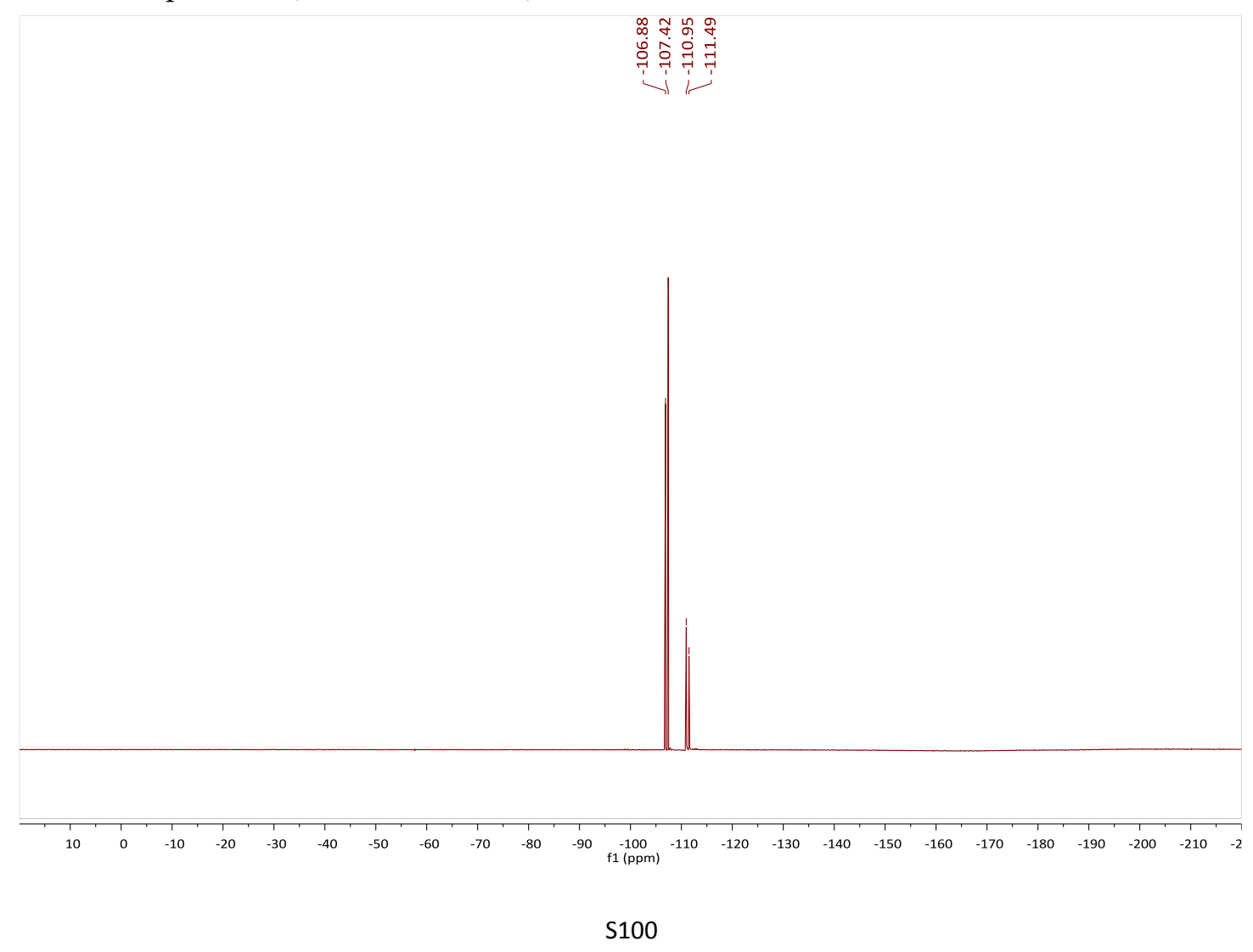




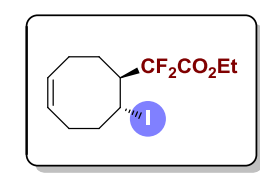

${ }^{13} \mathrm{C}$ NMR-spectrum (125 MHz, $\left.\mathrm{CDCl}_{3}\right)$ of $\mathbf{3 t}$

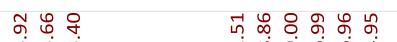

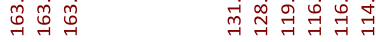

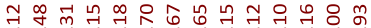

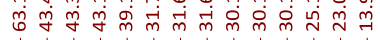

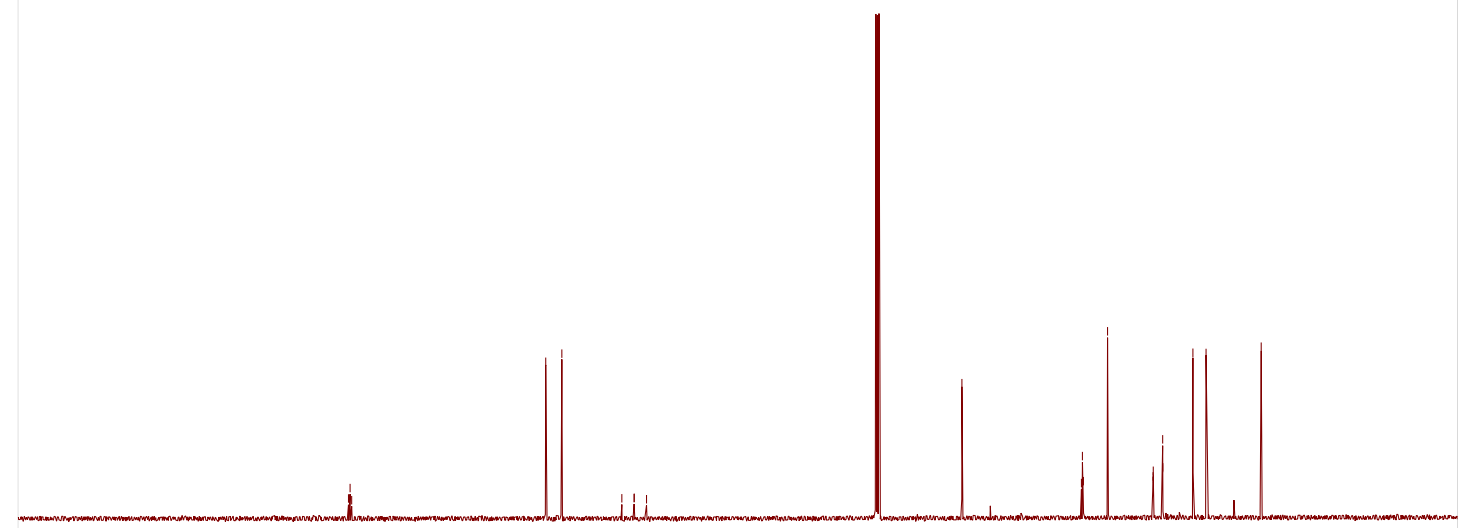

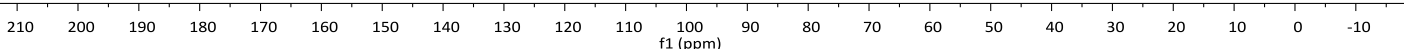




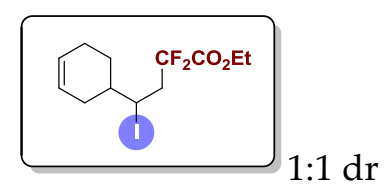

${ }^{1} \mathrm{H}$ NMR-spectrum $\left(500 \mathrm{MHz}, \mathrm{CDCl}_{3}\right)$ of $3 \mathbf{u}$

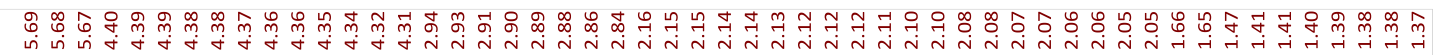

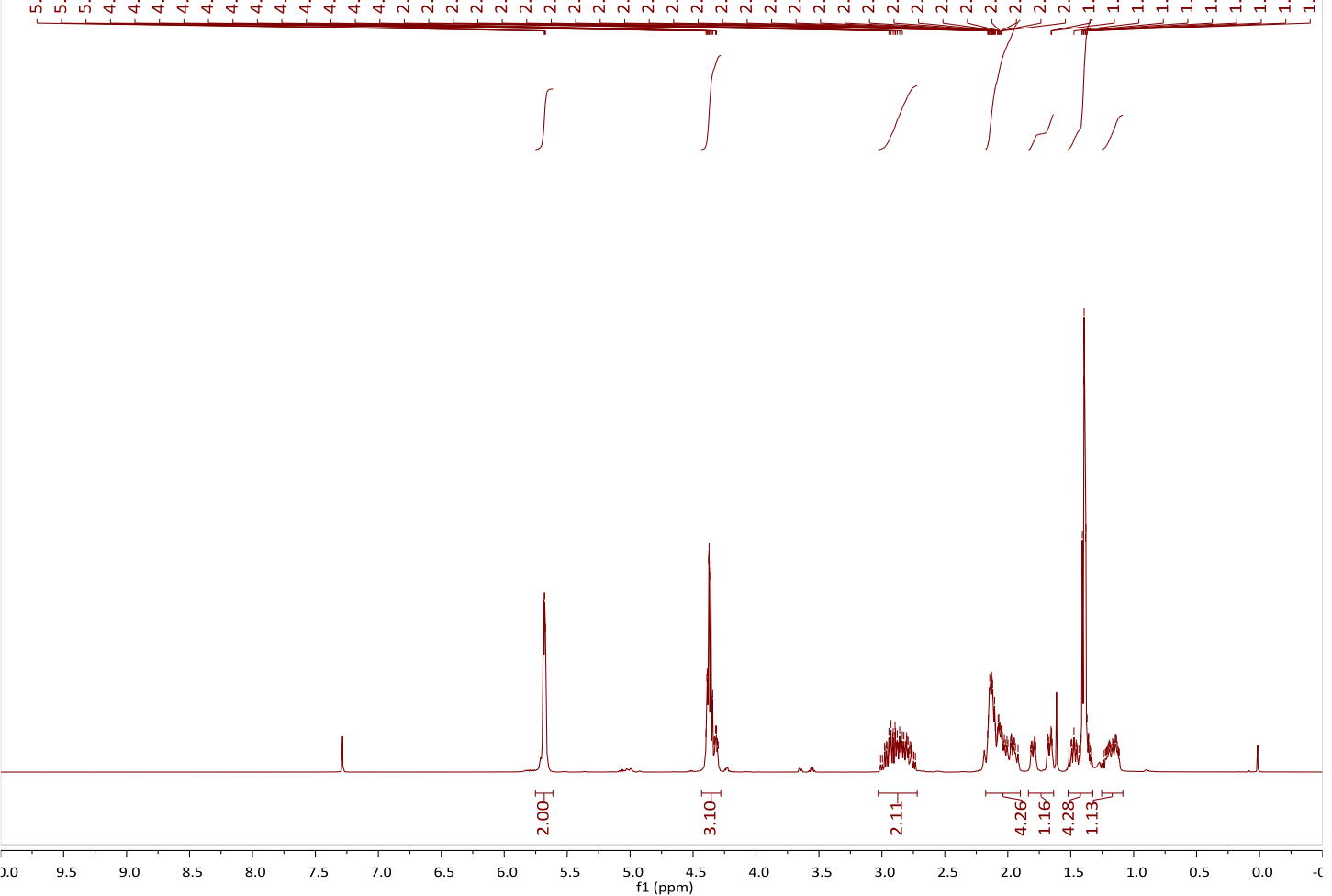

${ }^{19}$ F NMR-spectrum $\left(471 \mathrm{MHz}, \mathrm{CDCl}_{3}\right)$ of $\mathbf{3 u}$

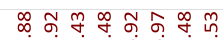

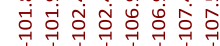
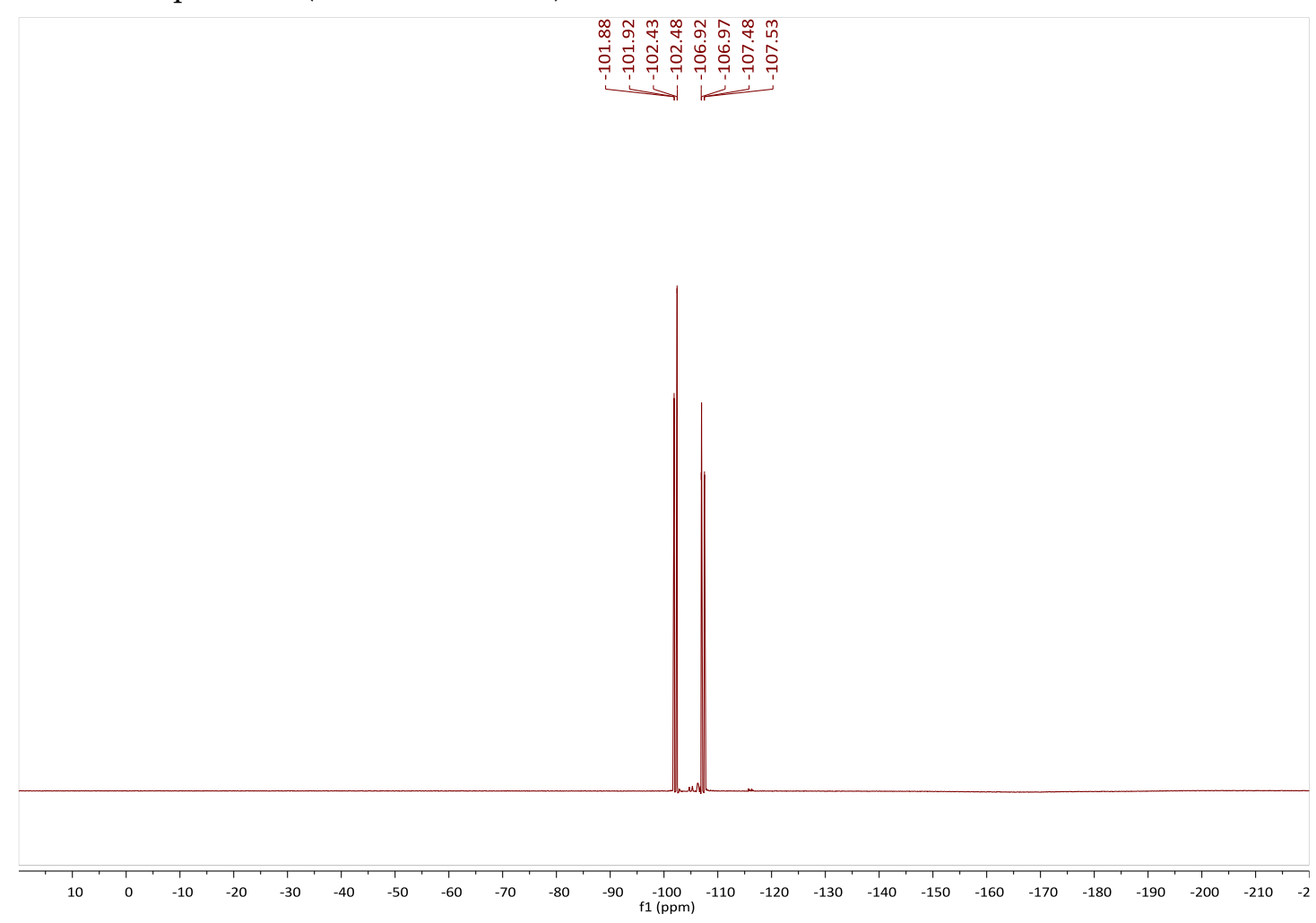


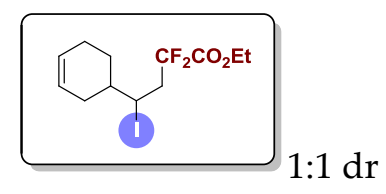

${ }^{13} \mathrm{C}$ NMR-spectrum $\left(125 \mathrm{MHz}, \mathrm{CDCl}_{3}\right)$ of $\mathbf{3 u}$

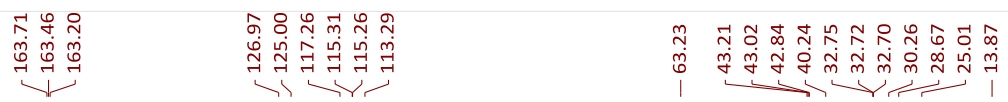

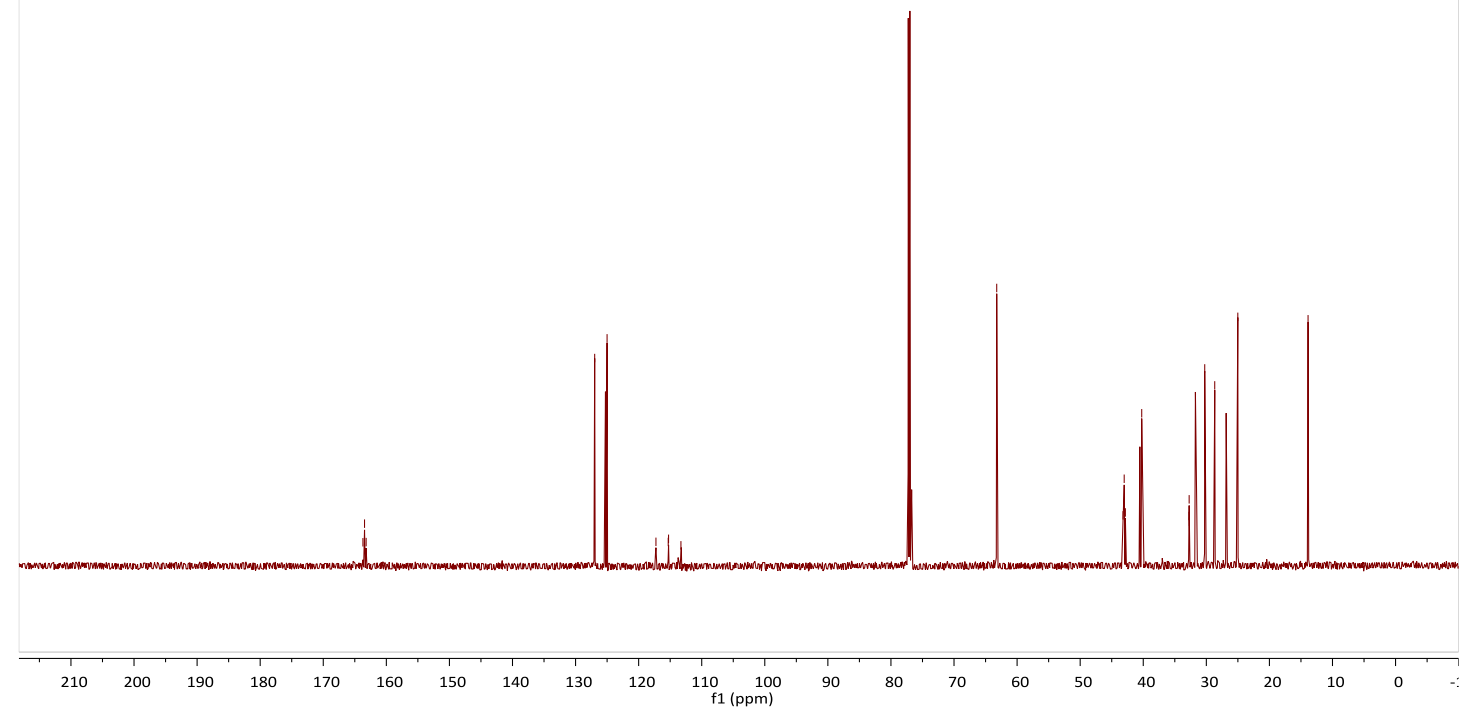




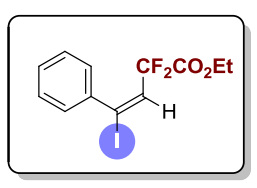

${ }^{1} \mathbf{H}$ NMR-spectrum $\left(500 \mathrm{MHz}, \mathrm{CDCl}_{3}\right)$ of $\mathbf{5 a}$
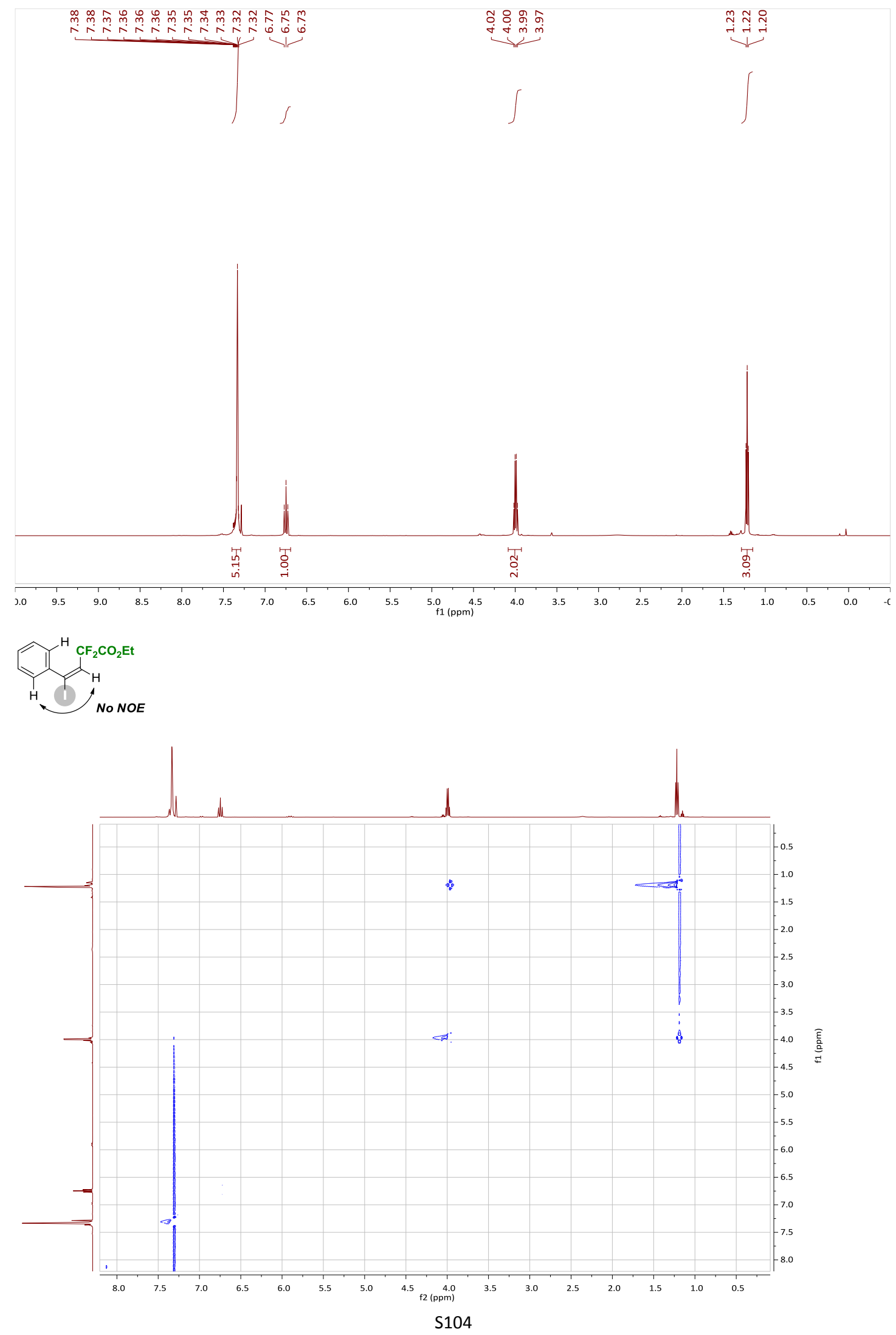


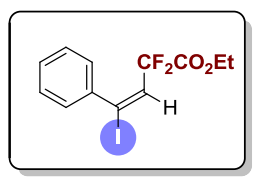

${ }^{19} \mathbf{F}$ NMR-spectrum $\left(471 \mathrm{MHz}, \mathrm{CDCl}_{3}\right)$ of $\mathbf{5 a}$

$\infty$
ñ
1
1

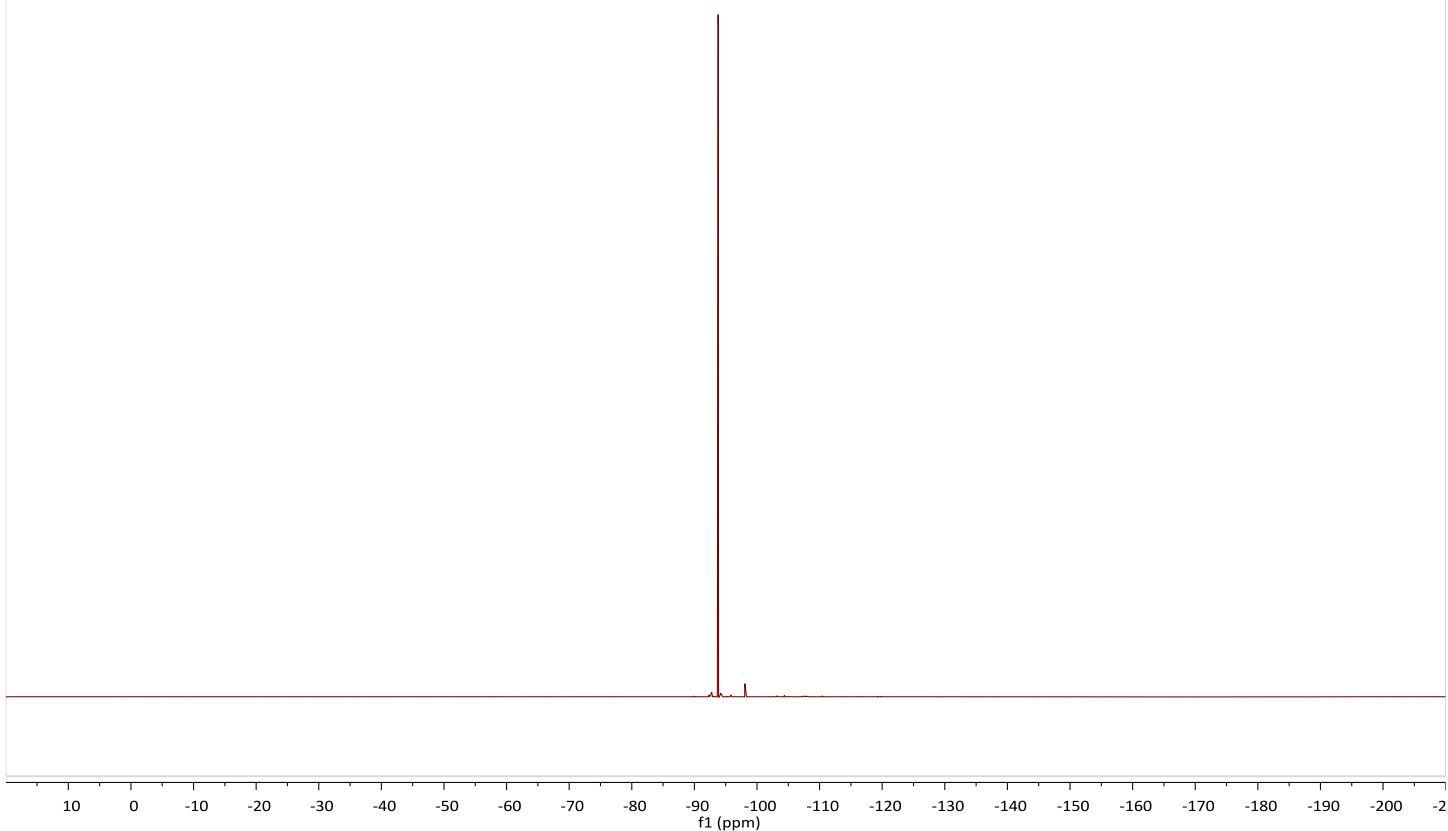

${ }^{13} \mathrm{C}$ NMR-spectrum $\left(125 \mathrm{MHz}, \mathrm{CDCl}_{3}\right)$ of $\mathbf{5 a}$

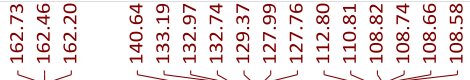

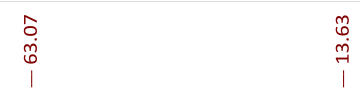

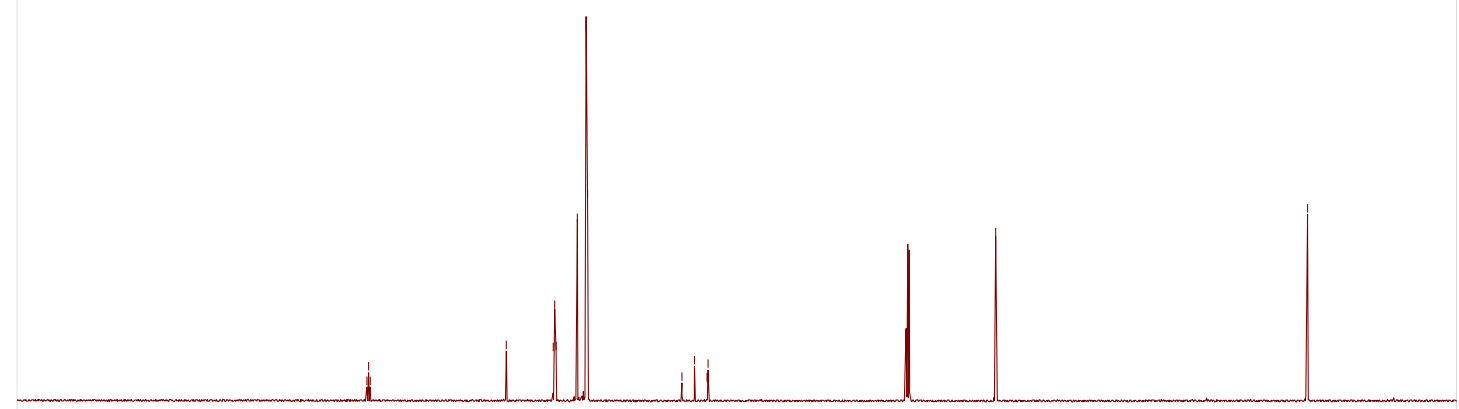

$\begin{array}{lllllllllll}210 & 200 & 190 & 180 & 170 & 160 & 150 & 140 & 130 & 120 & 110 \quad \begin{array}{l}100 \\ \mathrm{f} 1(\mathrm{ppm})\end{array}\end{array}$ 


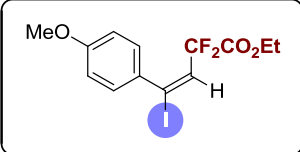

${ }^{1} \mathbf{H}$ NMR-spectrum $\left(500 \mathrm{MHz}, \mathrm{CDCl}_{3}\right)$ of $5 \mathbf{b}$
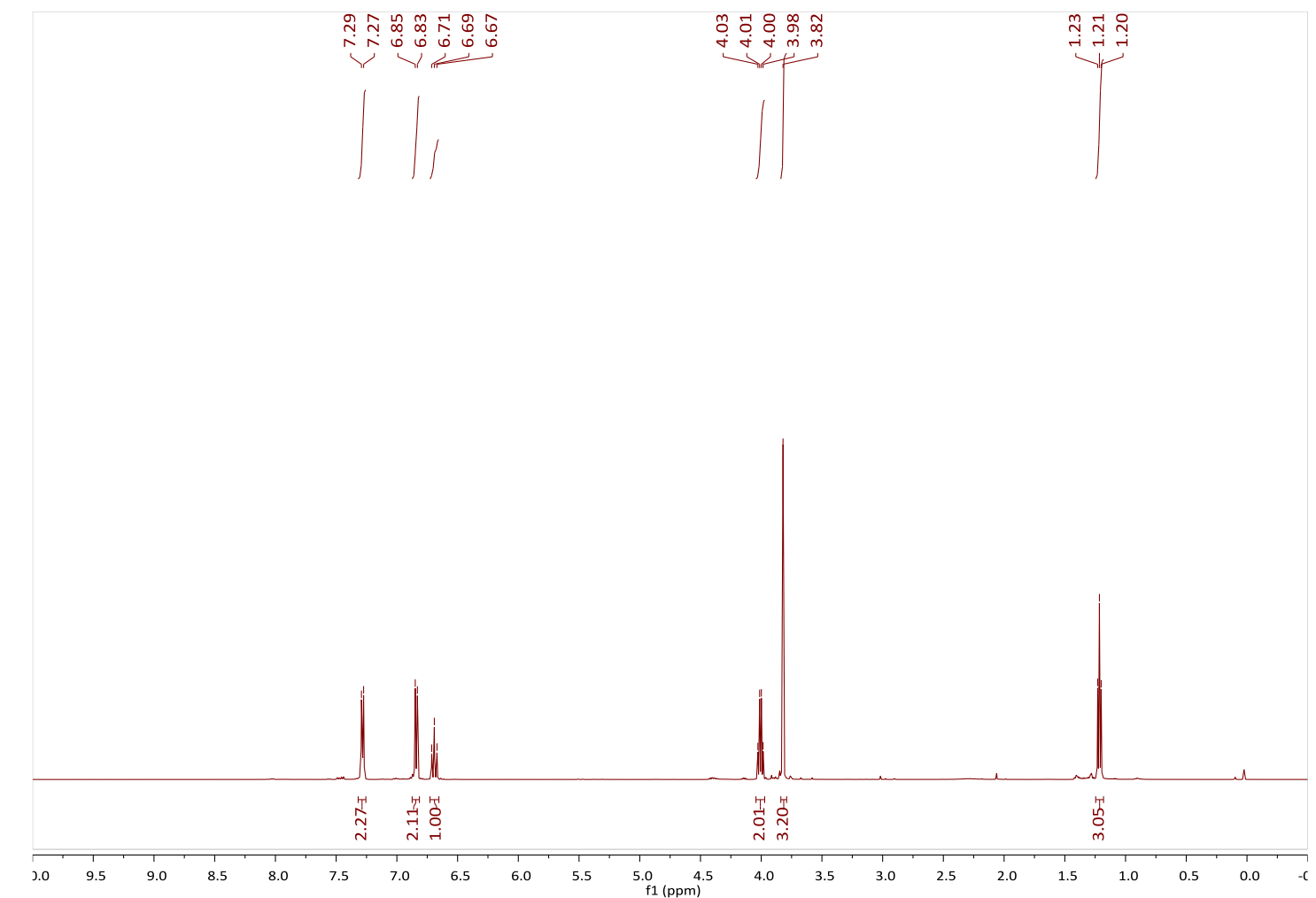

$\overbrace{}^{-1}$

${ }^{19} \mathbf{F}$ NMR-spectrum $\left(471 \mathrm{MHz}, \mathrm{CDCl}_{3}\right)$ of $\mathbf{5 b}$

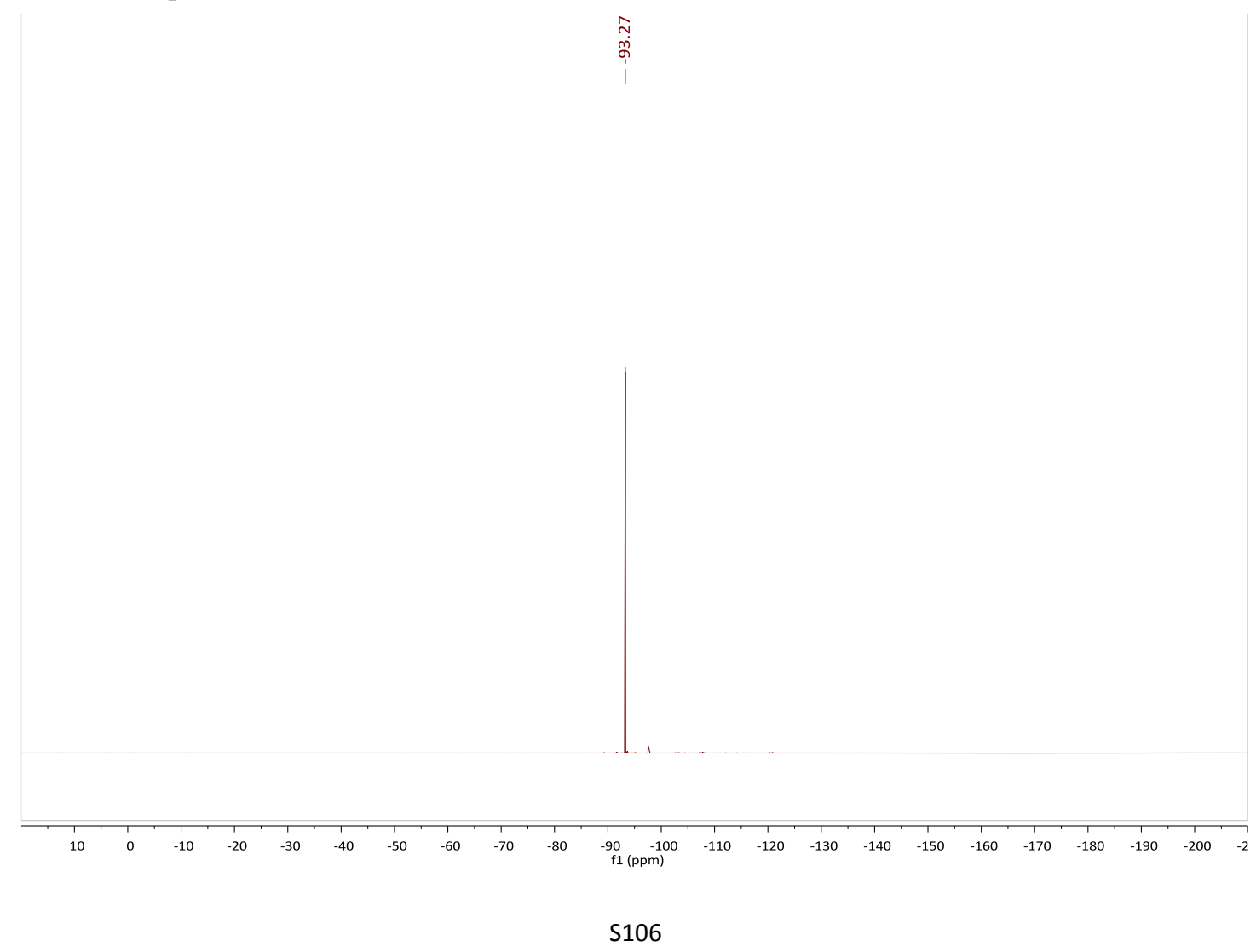




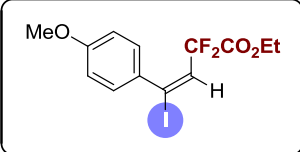

${ }^{13} \mathbf{C}$ NMR-spectrum (125 MHz, $\left.\mathrm{CDCl}_{3}\right)$ of $\mathbf{5 b}$

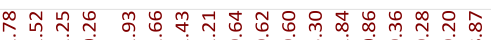

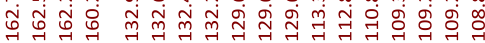

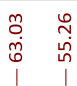

$\stackrel{\substack{0 \\ \dot{m}}}{+}$

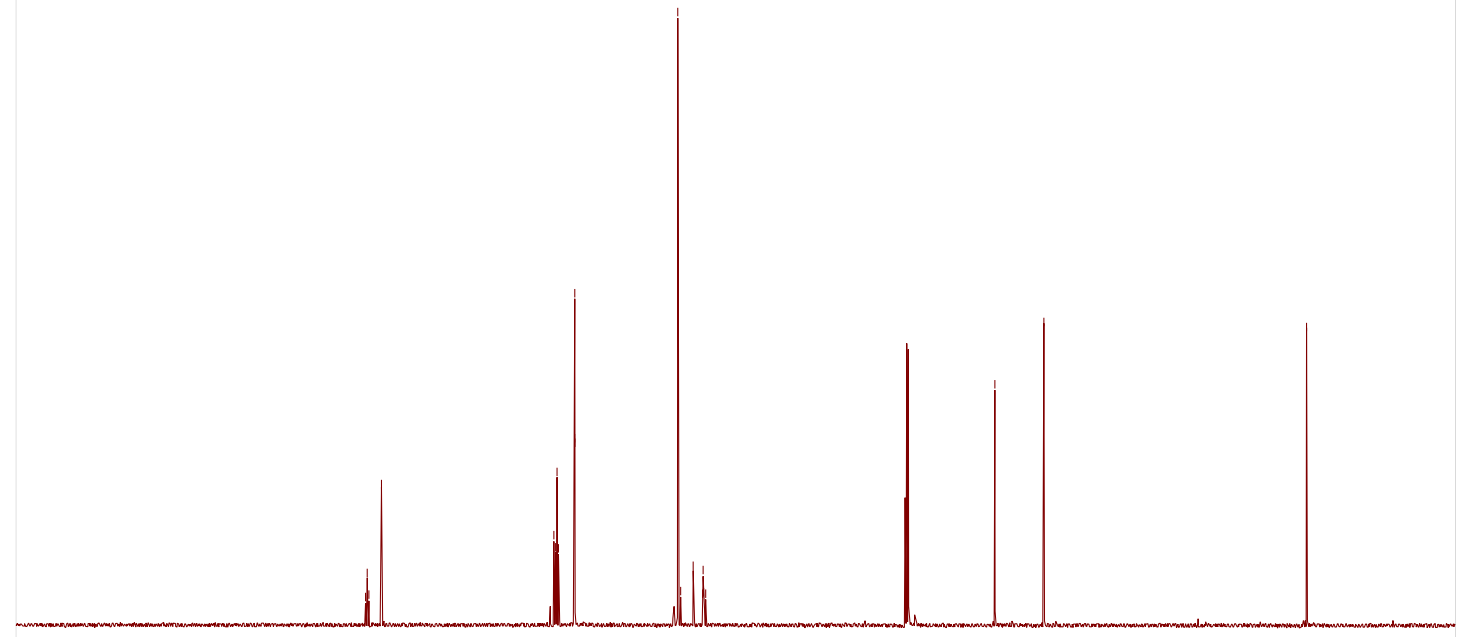

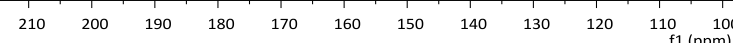




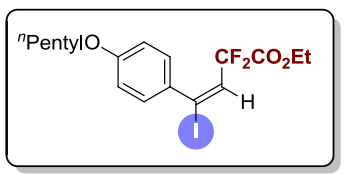

${ }^{1} \mathbf{H}$ NMR-spectrum $\left(500 \mathrm{MHz}, \mathrm{CDCl}_{3}\right)$ of $5 \mathbf{c}$

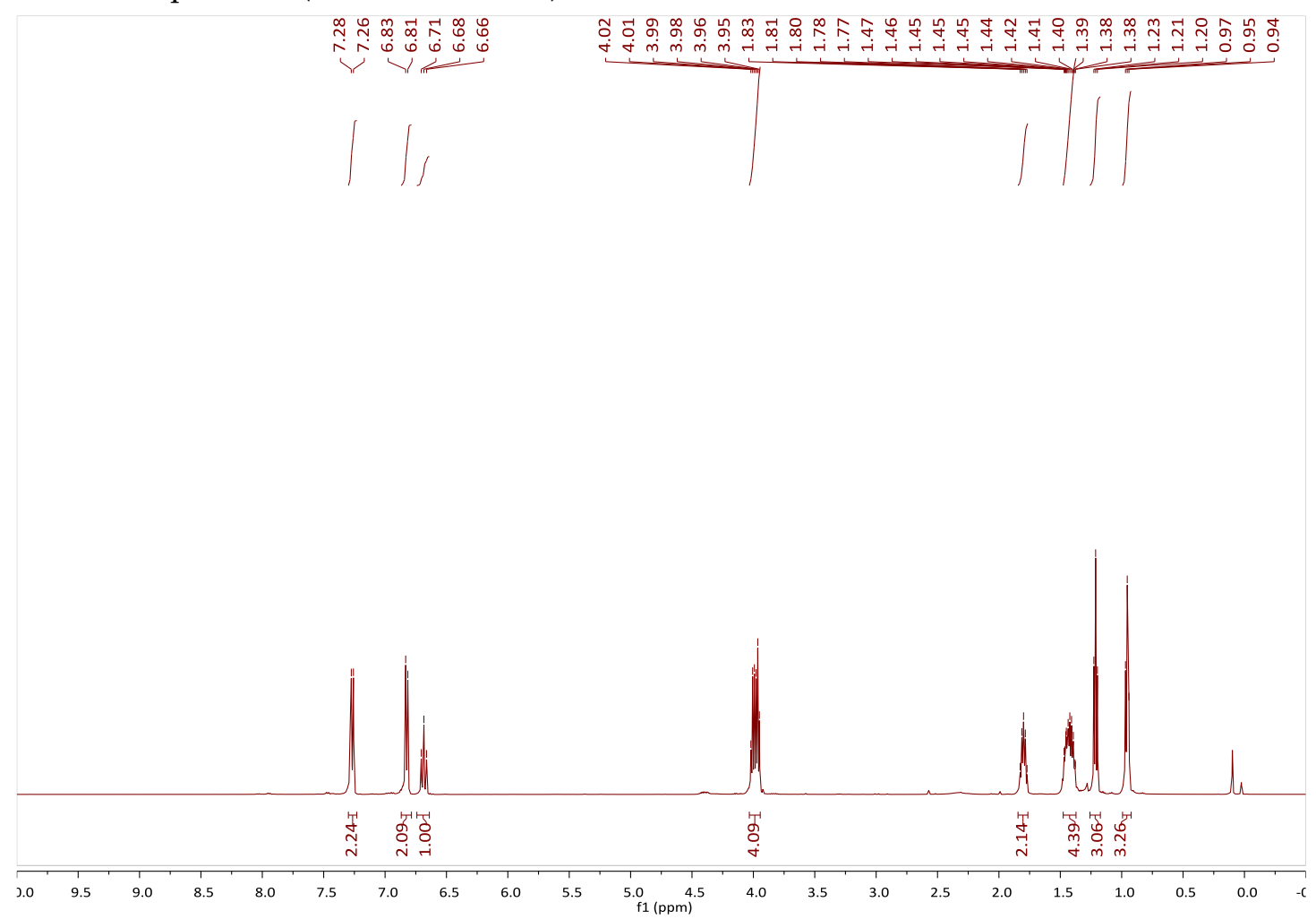

${ }^{19} \mathbf{F}$ NMR-spectrum $\left(471 \mathrm{MHz}, \mathrm{CDCl}_{3}\right)$ of $5 \mathbf{c}$

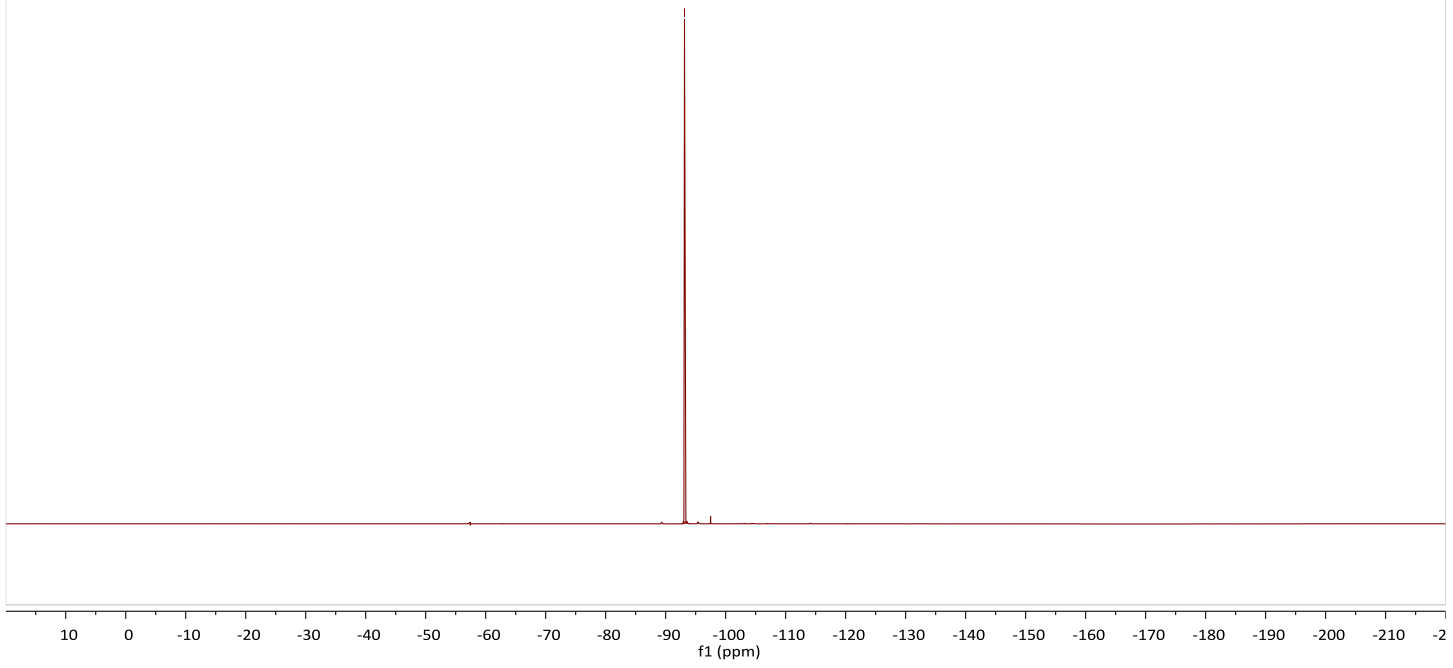




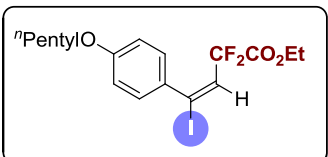

${ }^{13} \mathrm{C}$ NMR-spectrum (125 MHz, $\left.\mathrm{CDCl}_{3}\right)$ of $5 \mathbf{c}$

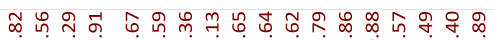

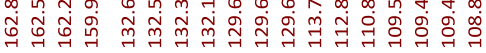

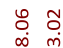

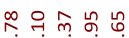

일

Wt

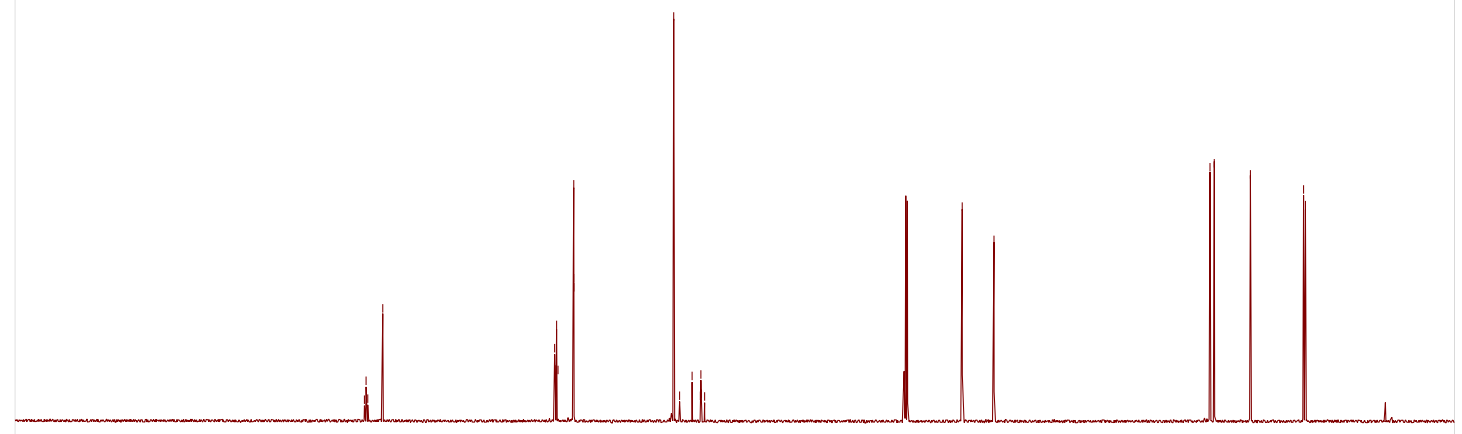

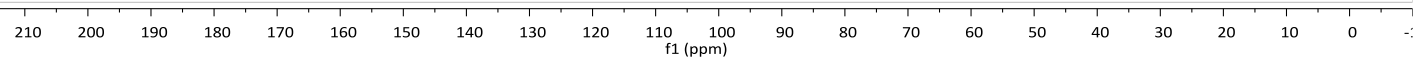




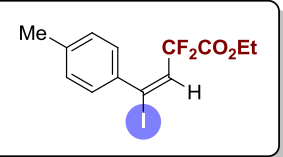

${ }^{1} \mathbf{H} \mathbf{N M R}$-spectrum $\left(500 \mathrm{MHz}, \mathrm{CDCl}_{3}\right)$ of $\mathbf{5 d}$

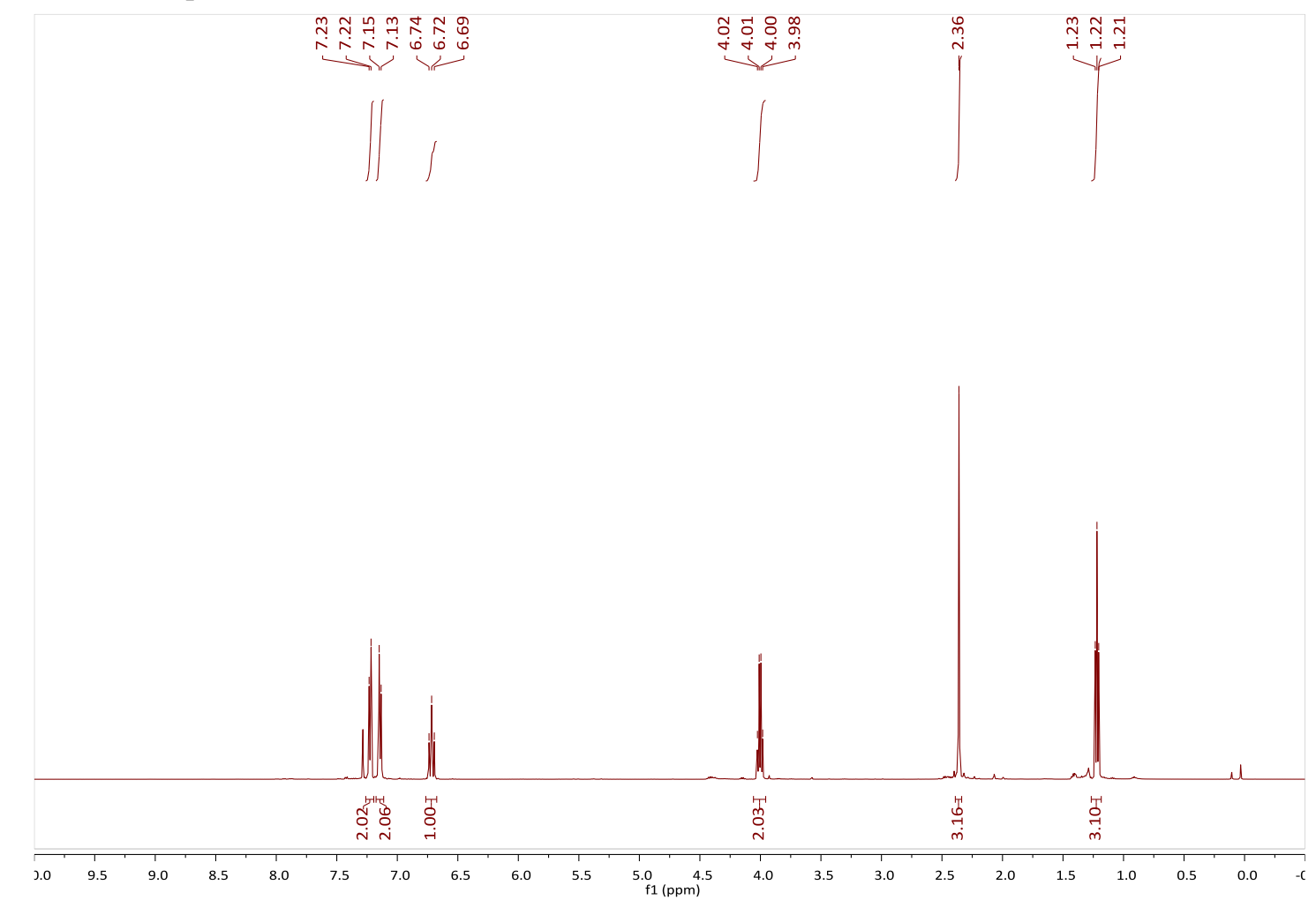

${ }^{19}$ F NMR-spectrum $\left(471 \mathrm{MHz}, \mathrm{CDCl}_{3}\right)$ of $\mathbf{5 d}$

$$
\begin{aligned}
& \infty \\
& 0 \\
& m \\
& \dot{p} \\
& i
\end{aligned}
$$

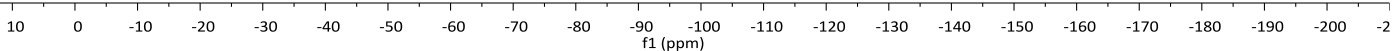




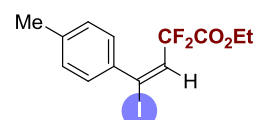

${ }^{13} \mathrm{C}$ NMR-spectrum $\left(125 \mathrm{MHz}, \mathrm{CDCl}_{3}\right)$ of $\mathbf{5 d}$

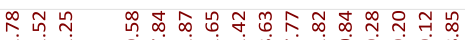

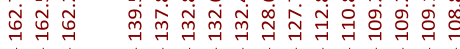

ָָ.

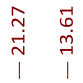

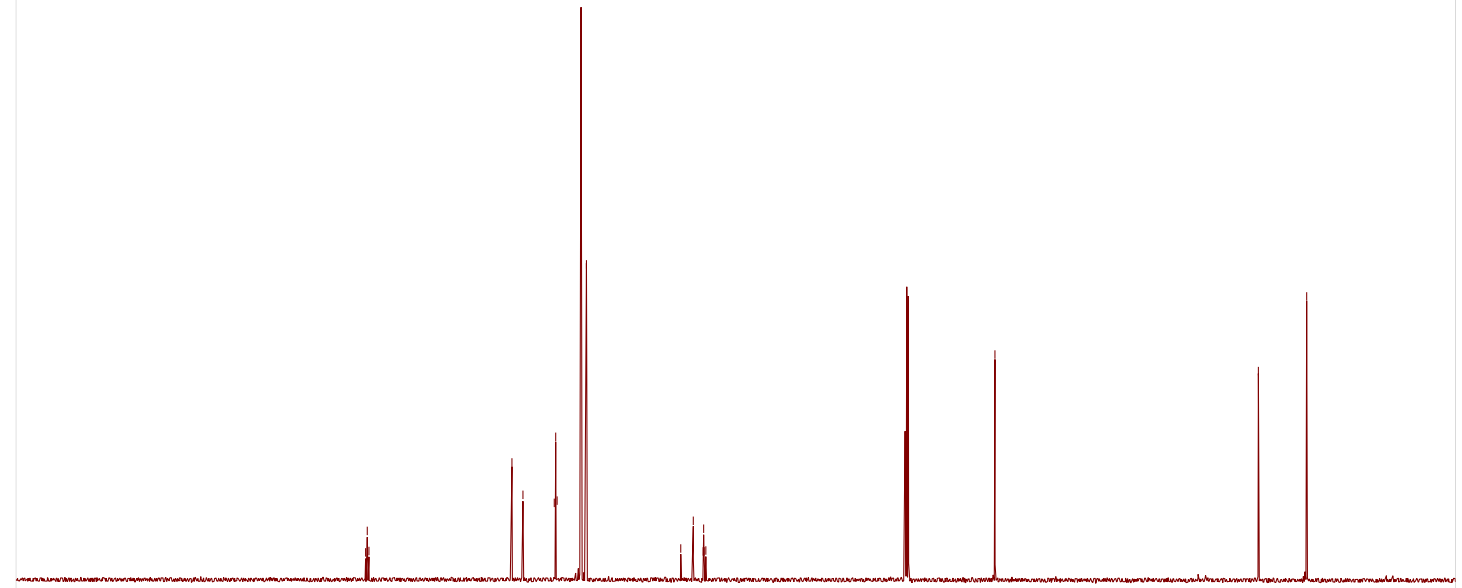

$\begin{array}{llllllllllll}210 & 200 & 190 & 180 & 170 & 160 & 150 & 140 & 130 & 120 & 110 & 100\end{array}$ 


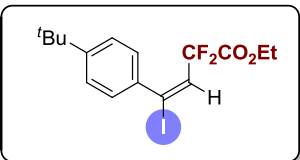

${ }^{1} \mathbf{H}$ NMR-spectrum $\left(500 \mathrm{MHz}, \mathrm{CDCl}_{3}\right)$ of $\mathbf{5 e}$

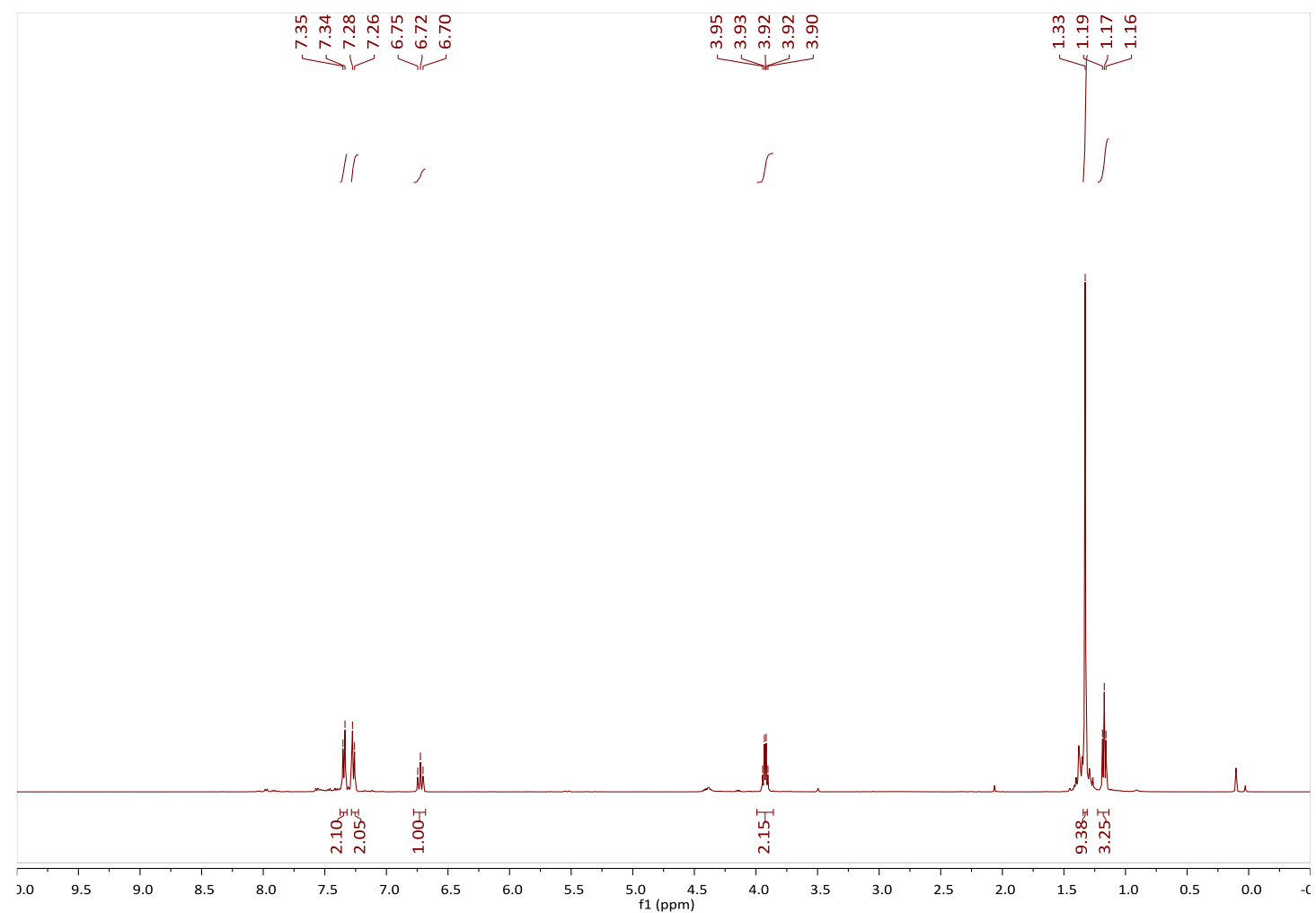

${ }^{19} \mathbf{F}$ NMR-spectrum $\left(471 \mathrm{MHz}, \mathrm{CDCl}_{3}\right)$ of $\mathbf{5 e}$

$$
\text { ō }
$$

$\begin{array}{ccccccccccc}10 & 0 & -10 & -20 & -30 & -40 & -50 & -60 & -70 & -80 & \begin{array}{c}-90 \\ \mathrm{f} 1(\mathrm{ppm})\end{array}\end{array}$ 


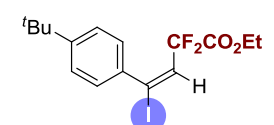

${ }^{13} \mathrm{C}$ NMR-spectrum $\left(125 \mathrm{MHz}, \mathrm{CDCl}_{3}\right)$ of $\mathbf{5 e}$

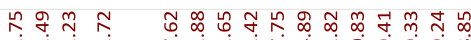

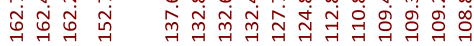

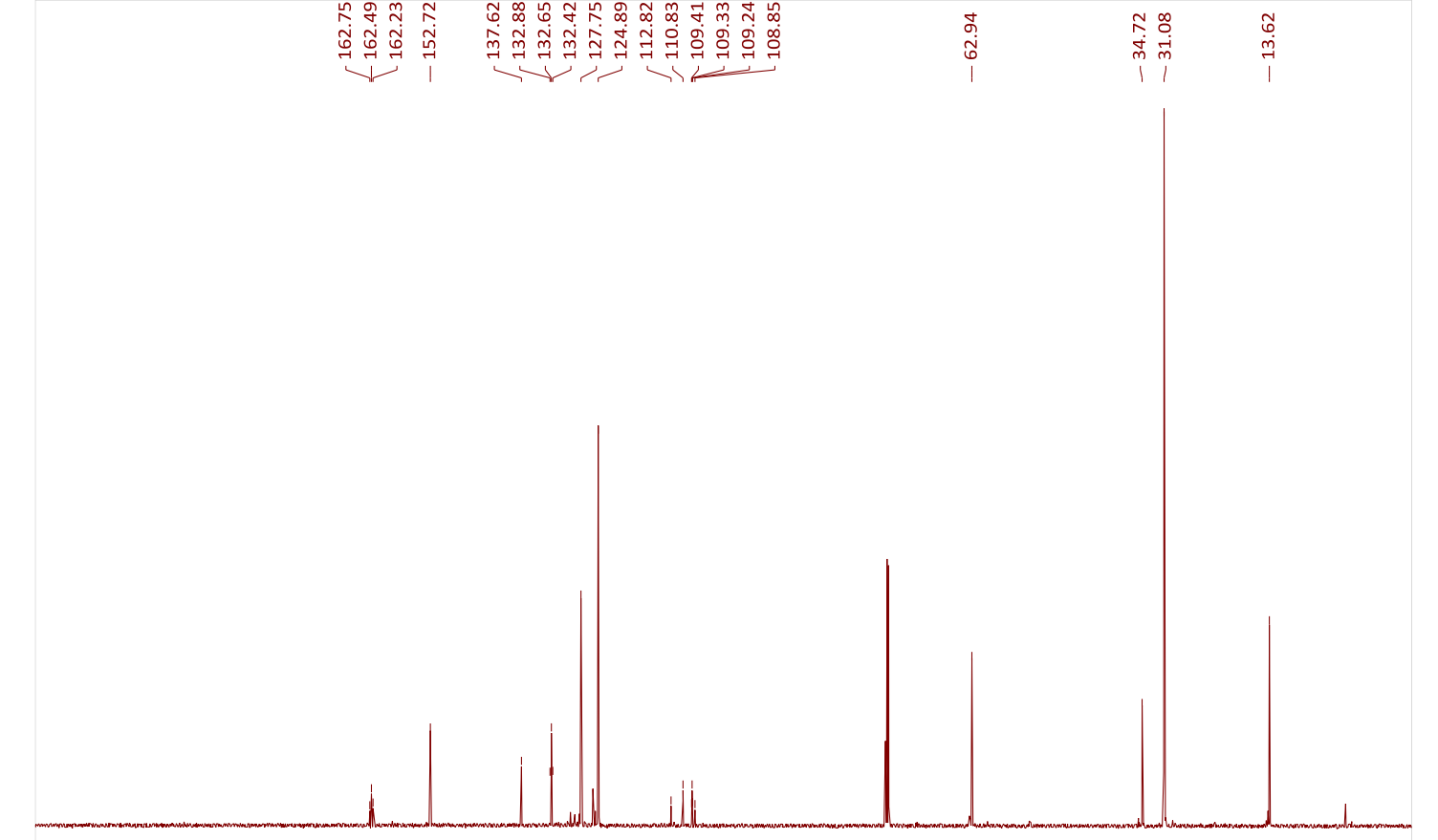

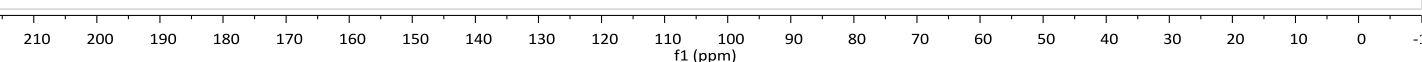




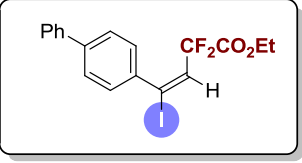

${ }^{1} \mathbf{H}$ NMR-spectrum $\left(500 \mathrm{MHz}, \mathrm{CDCl}_{3}\right)$ of $\mathbf{5 f}$

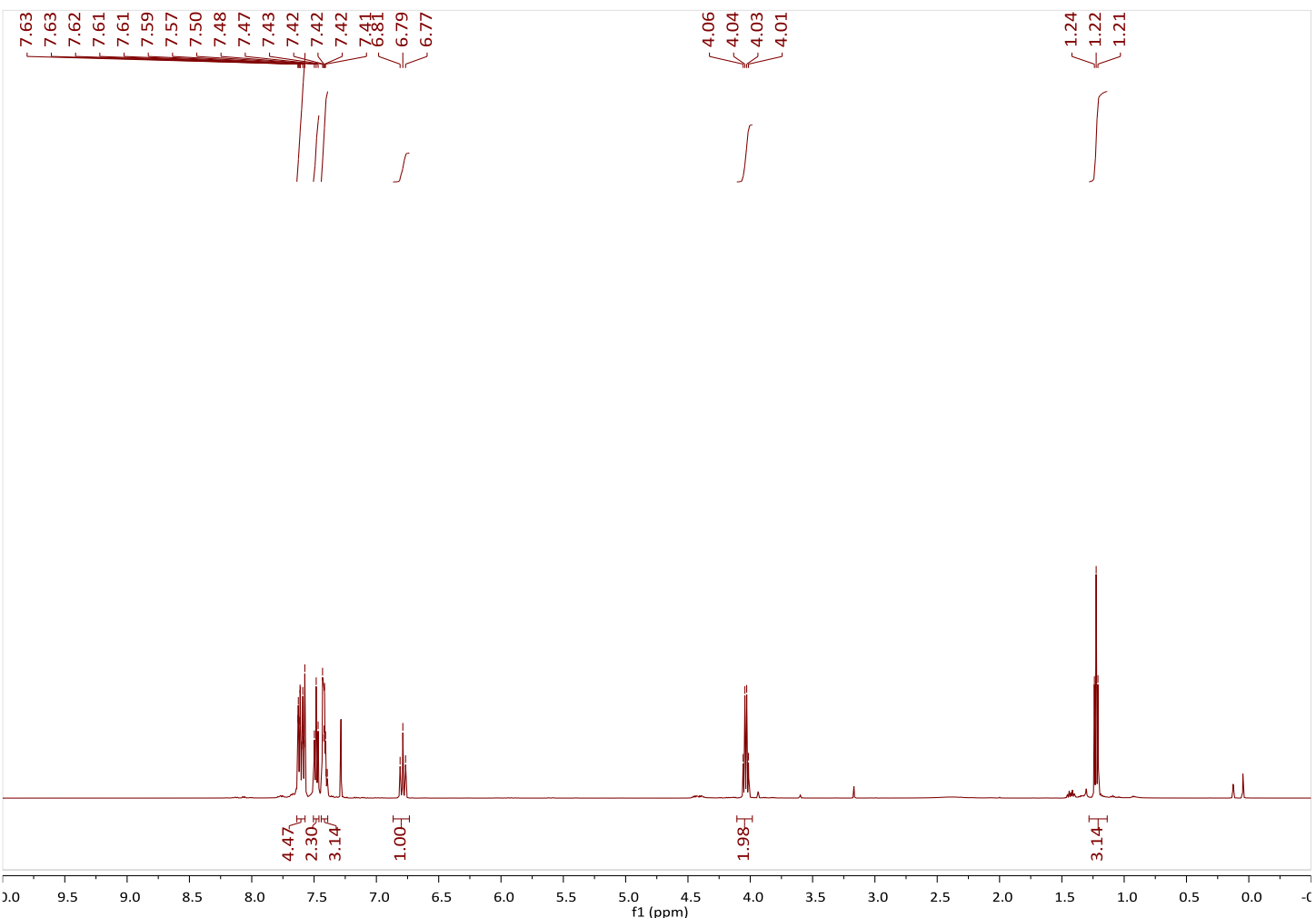

${ }^{19} \mathbf{F}$ NMR-spectrum $\left(471 \mathrm{MHz}, \mathrm{CDCl}_{3}\right)$ of $\mathbf{5 f}$

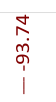

$\begin{array}{lllllllllllllllllllllllllllll}10 & 0 & -10 & -20 & -30 & -40 & -50 & -60 & -70 & -80 & -90 & -100 & -110 & -120 & -130 & -140 & -150 & -160 & -170 & -180 & -190 & -200 & -210\end{array}$ 


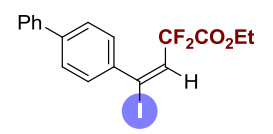

${ }^{13} \mathrm{C}$ NMR-spectrum $\left(125 \mathrm{MHz}, \mathrm{CDCl}_{3}\right)$ of $\mathbf{5 f}$

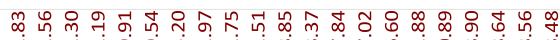

¿্ర

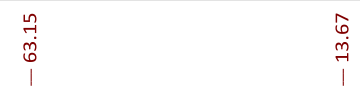

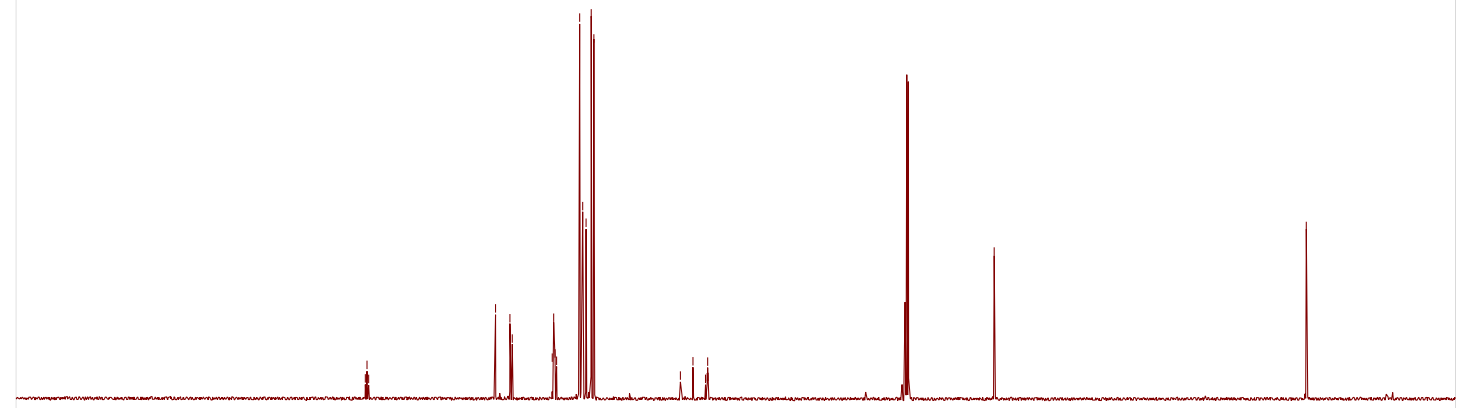

$\begin{array}{lllllllllllllllllllllllllll}210 & 200 & 190 & 180 & 170 & 160 & 150 & 140 & 130 & 120 & 110 & 100 & 90 & 80 & 70 & 60 & 50 & 40 & 30 & 20 & 10 & 0\end{array}$ 


$$
\text { ( }
$$

${ }^{1} \mathbf{H}$ NMR-spectrum $\left(500 \mathrm{MHz}, \mathrm{CDCl}_{3}\right)$ of $\mathbf{5 g}$
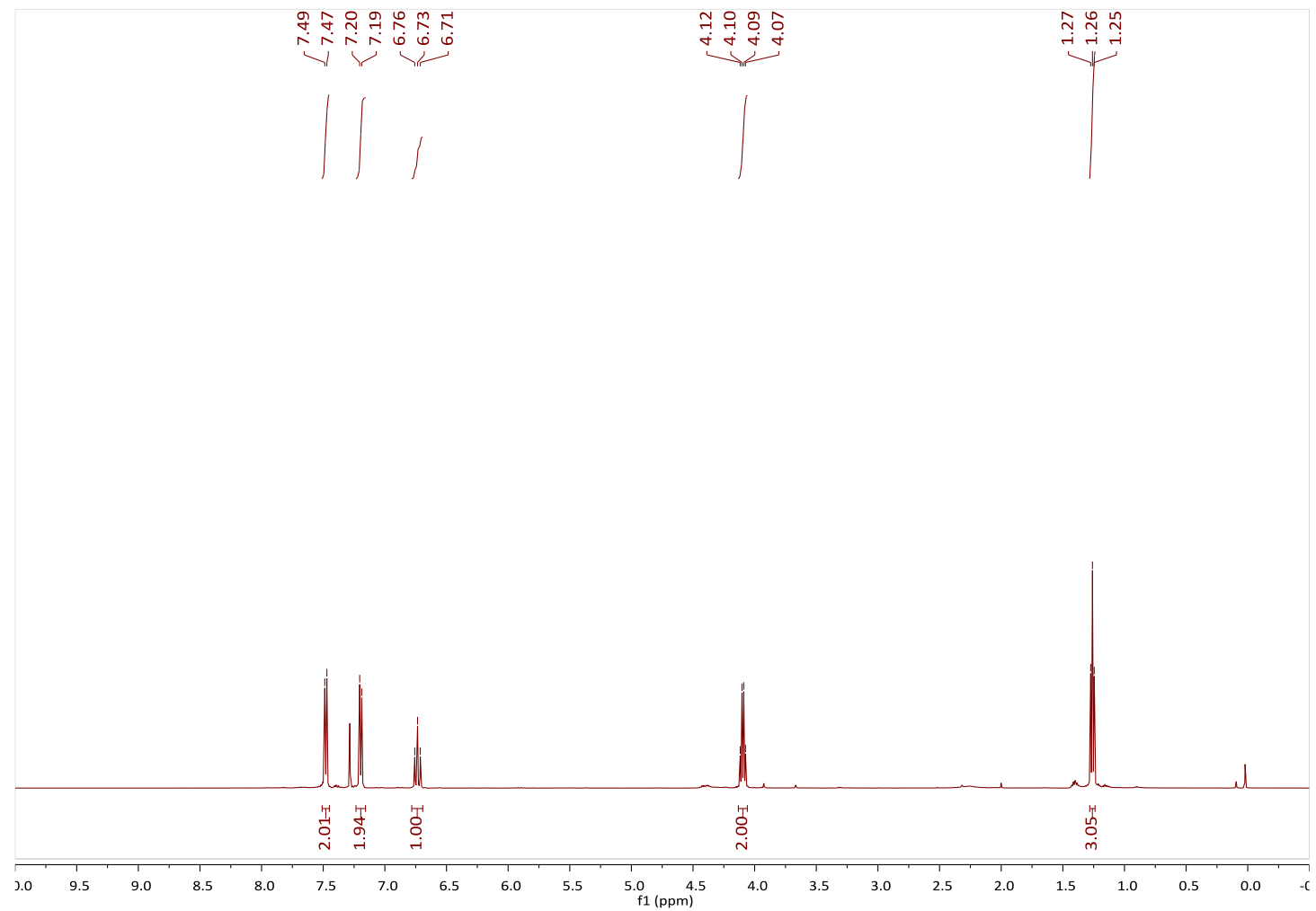

${ }^{19} \mathbf{F}$ NMR-spectrum $\left(471 \mathrm{MHz}, \mathrm{CDCl}_{3}\right)$ of $\mathbf{5 g}$ 


\section{$\mathrm{CF}_{2} \mathrm{CO}_{2} \mathrm{Et}$ (1)}

${ }^{13} \mathrm{C}$ NMR-spectrum $\left(125 \mathrm{MHz}, \mathrm{CDCl}_{3}\right)$ of $\mathbf{5 g}$

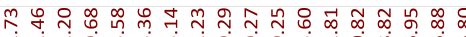

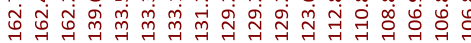

$\underset{\substack{m \\ \dot{m}}}{\substack{p \\ \dot{m}}}$

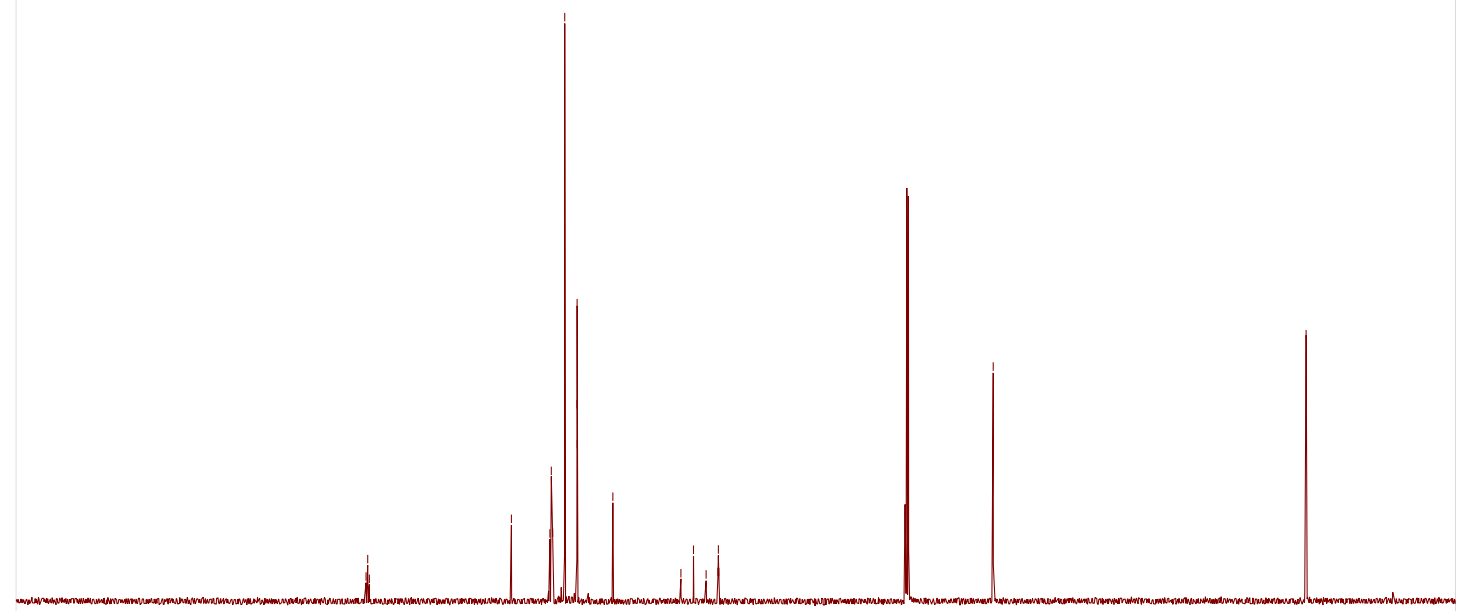

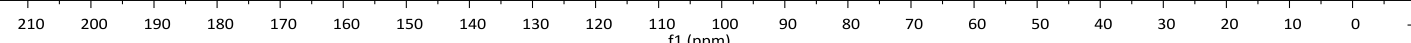




$$
{ }_{\mathrm{H}}^{\mathrm{Cl}}
$$

${ }^{1} \mathbf{H}$ NMR-spectrum $\left(500 \mathrm{MHz}, \mathrm{CDCl}_{3}\right)$ of $5 \mathbf{h}$
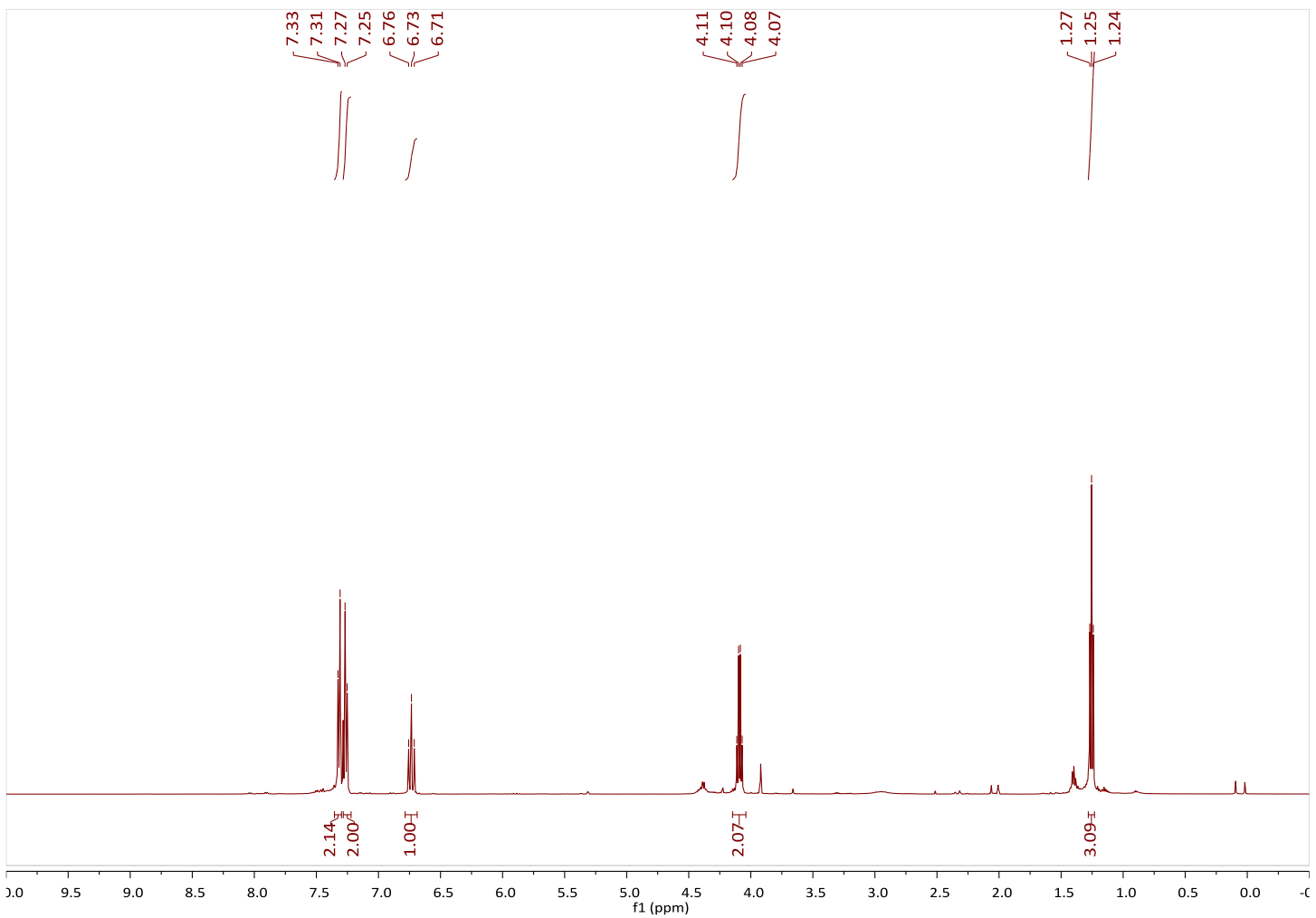

${ }^{19}$ F NMR-spectrum $\left(471 \mathrm{MHz}, \mathrm{CDCl}_{3}\right)$ of $\mathbf{5 h}$ 


$$
\text { Cl }
$$

${ }^{13} \mathrm{C}$ NMR-spectrum (125 MHz, $\left.\mathrm{CDCl}_{3}\right)$ of $5 \mathbf{h}$

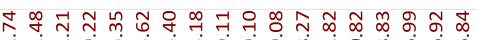

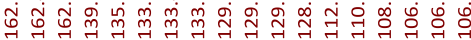

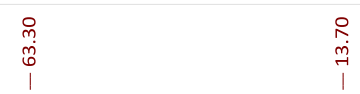

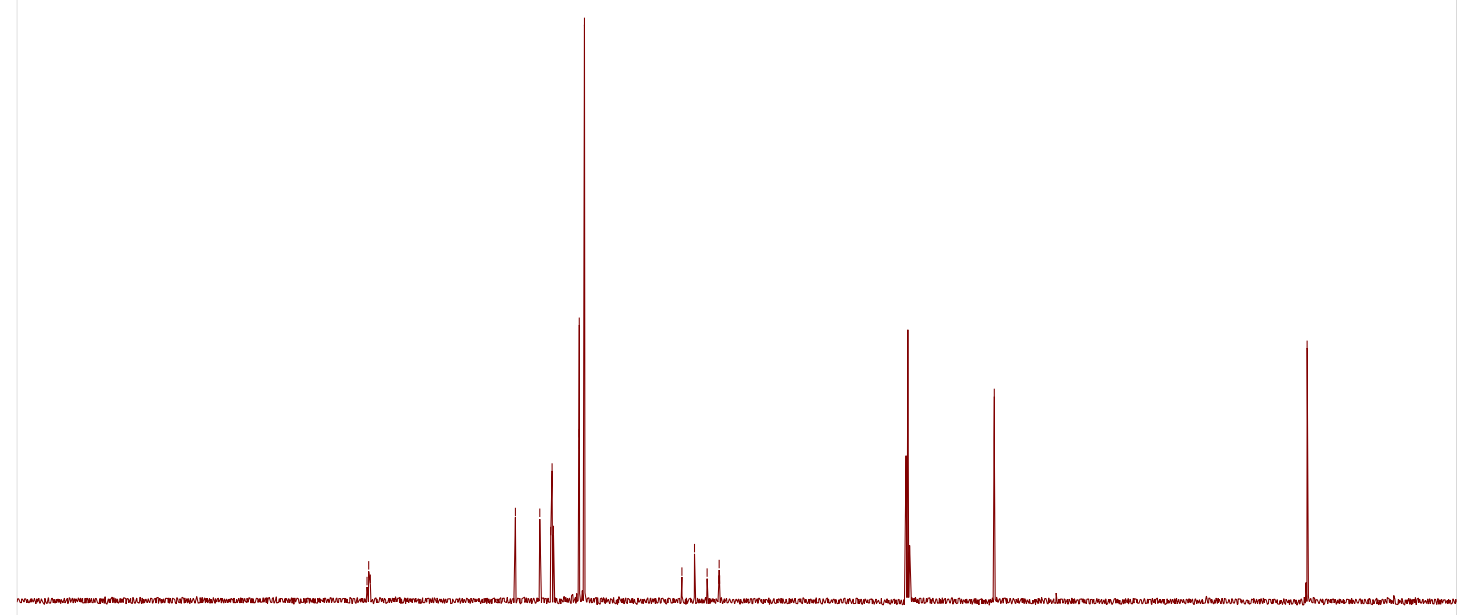

$\begin{array}{lllllllllll}210 & 200 & 190 & 180 & 170 & 160 & 150 & 140 & 130 & 120 & 110 \\ f 1(\mathrm{pmm}) & 100\end{array}$ 


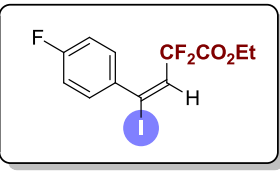

${ }^{1} \mathbf{H}$ NMR-spectrum $\left(500 \mathrm{MHz}, \mathrm{CDCl}_{3}\right)$ of $5 \mathbf{i}$

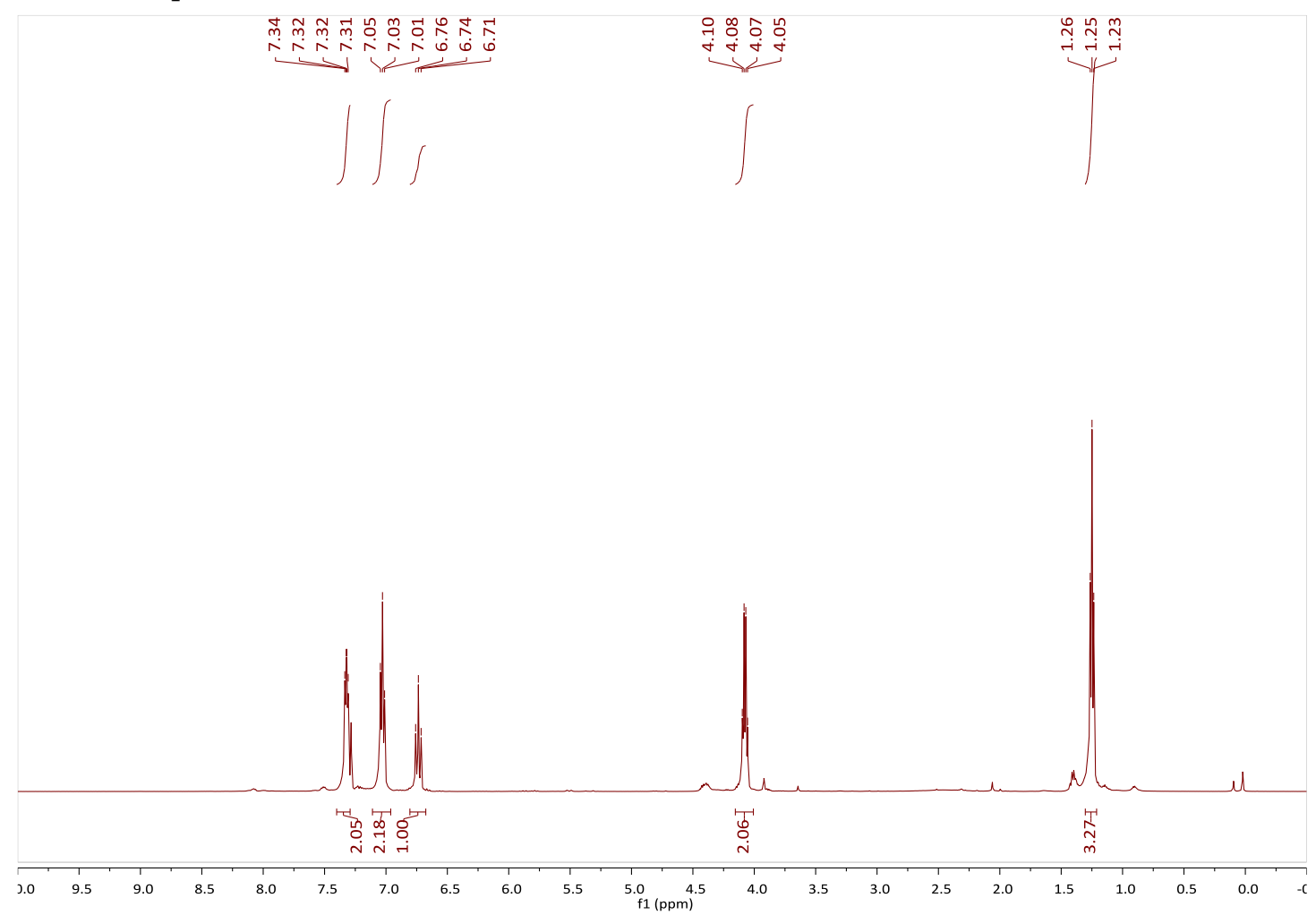

${ }^{19} \mathbf{F}$ NMR-spectrum $\left(471 \mathrm{MHz}, \mathrm{CDCl}_{3}\right)$ of $5 \mathbf{i}$

$$
\begin{array}{ll}
0 & 0 \\
0 & 0 \\
0 & 0 \\
0 & i \\
i & i
\end{array}
$$

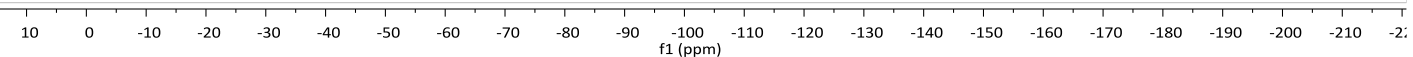




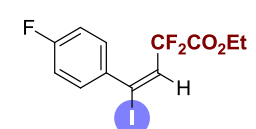

${ }^{13} \mathrm{C}$ NMR-spectrum $\left(125 \mathrm{MHz}, \mathrm{CDCl}_{3}\right)$ of $5 \mathbf{i}$

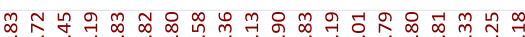

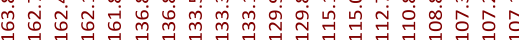

ํㅜㅇ
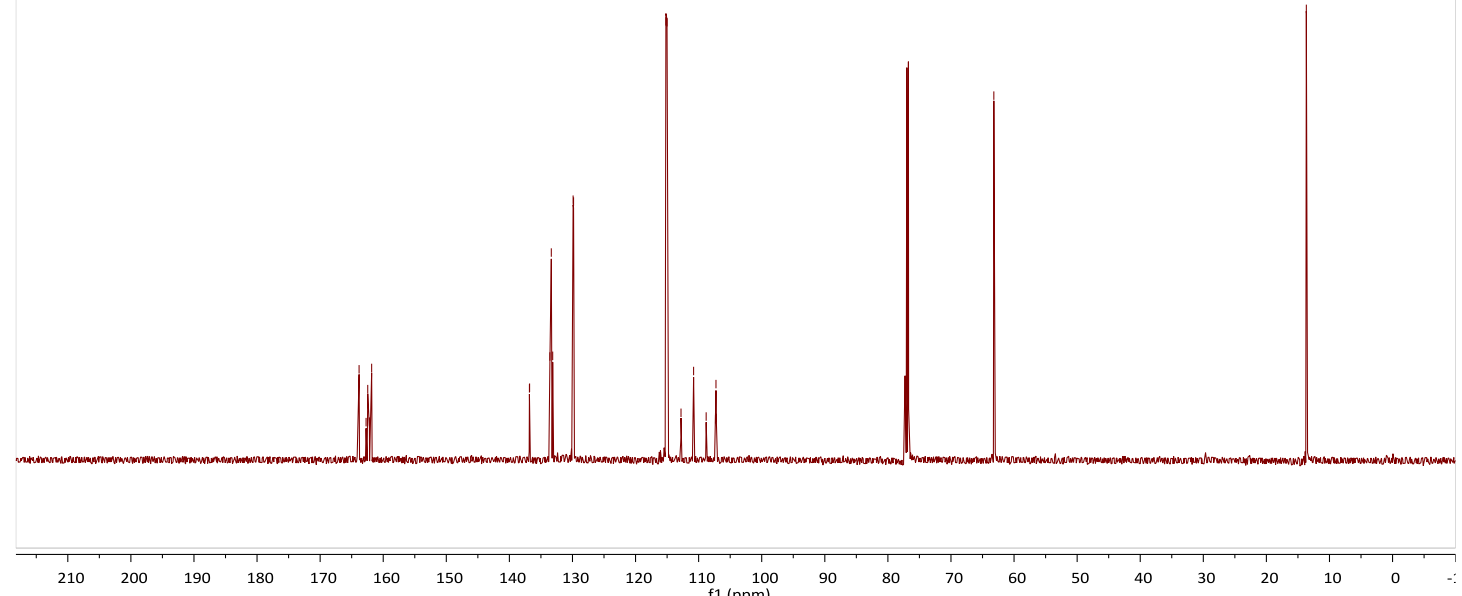


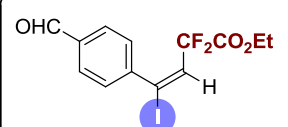

${ }^{1} \mathrm{H}$ NMR-spectrum $\left(500 \mathrm{MHz}, \mathrm{CDCl}_{3}\right)$ of $5 \mathbf{j}$
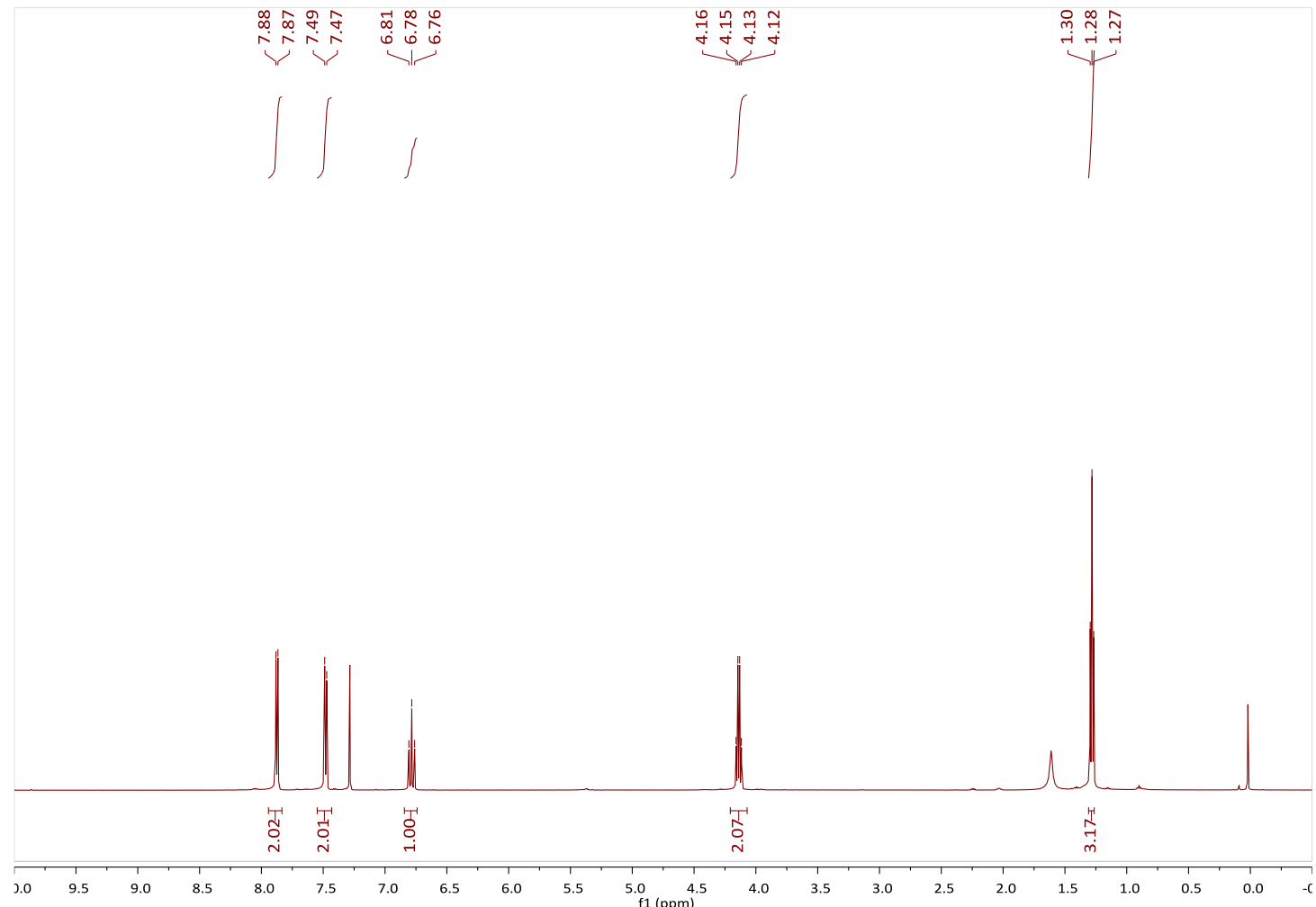

${ }^{19}$ F NMR-spectrum $\left(471 \mathrm{MHz}, \mathrm{CDCl}_{3}\right)$ of $5 \mathbf{j}$

$\begin{array}{lllllllllllllllllllllllll}20 & 10 & 0 & -10 & -20 & -30 & -40 & -50 & -60 & -70 & -80 & -90 & -100 & -110 & -120 & -130 & -140 & -150 & -160 & -170 & -180 & -190 & -200 & -210 & -2\end{array}$ 


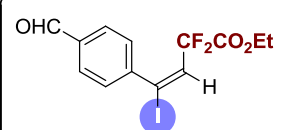

${ }^{13} \mathrm{C}$ NMR-spectrum $\left(125 \mathrm{MHz}, \mathrm{CDCl}_{3}\right)$ of $5 \mathbf{j}$

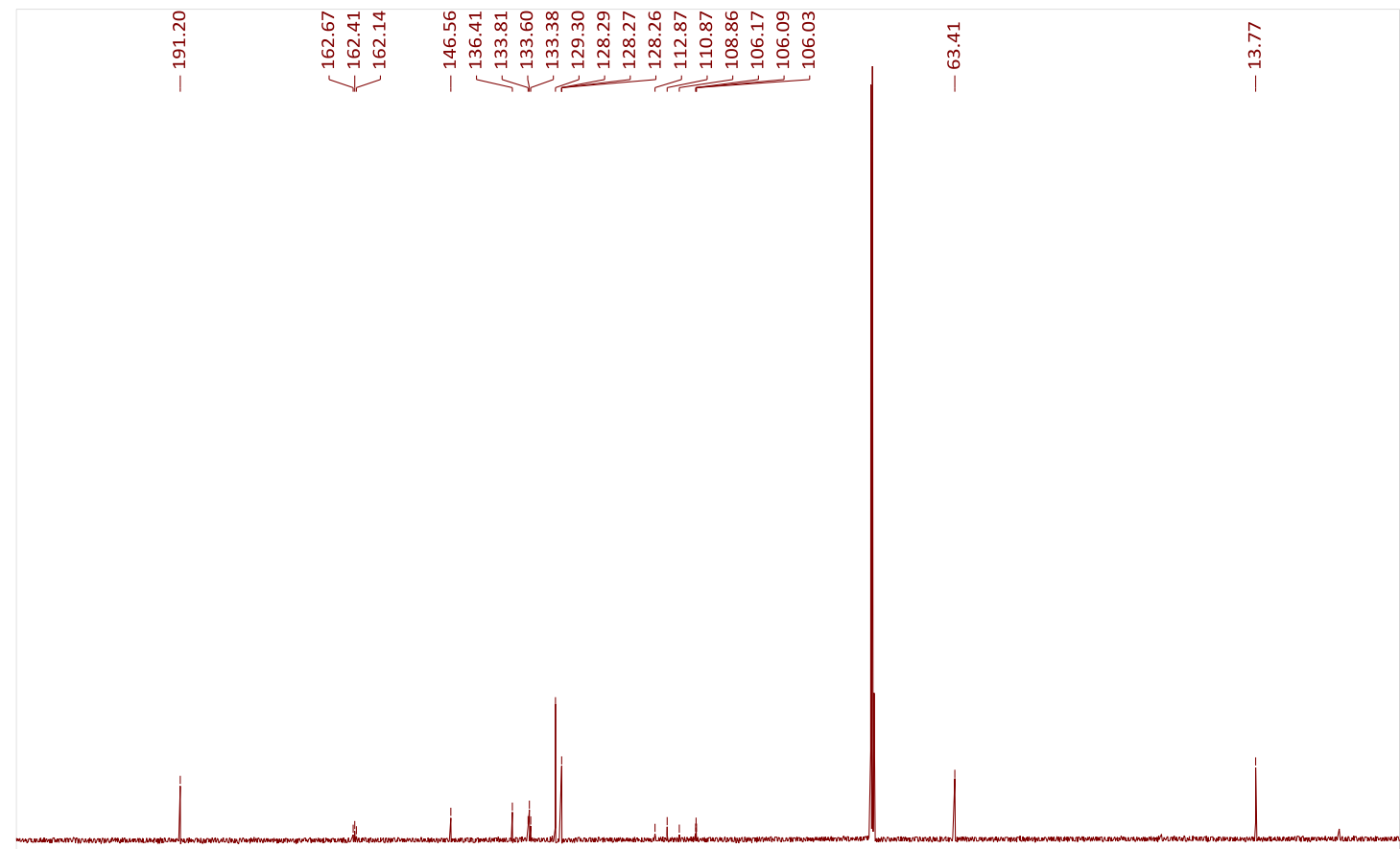

$\begin{array}{llllllllllllllllllllllllll}210 & 200 & 190 & 180 & 170 & 160 & 150 & 140 & 130 & 120 & 110 & 100 & 90 & 80 & 70 & 60 & 50 & 40 & 30 & 20 & 10 & 0 & -\end{array}$ 


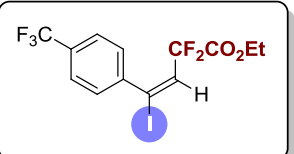

${ }^{1} \mathbf{H}$ NMR-spectrum $\left(500 \mathrm{MHz}, \mathrm{CDCl}_{3}\right)$ of $5 \mathbf{k}$
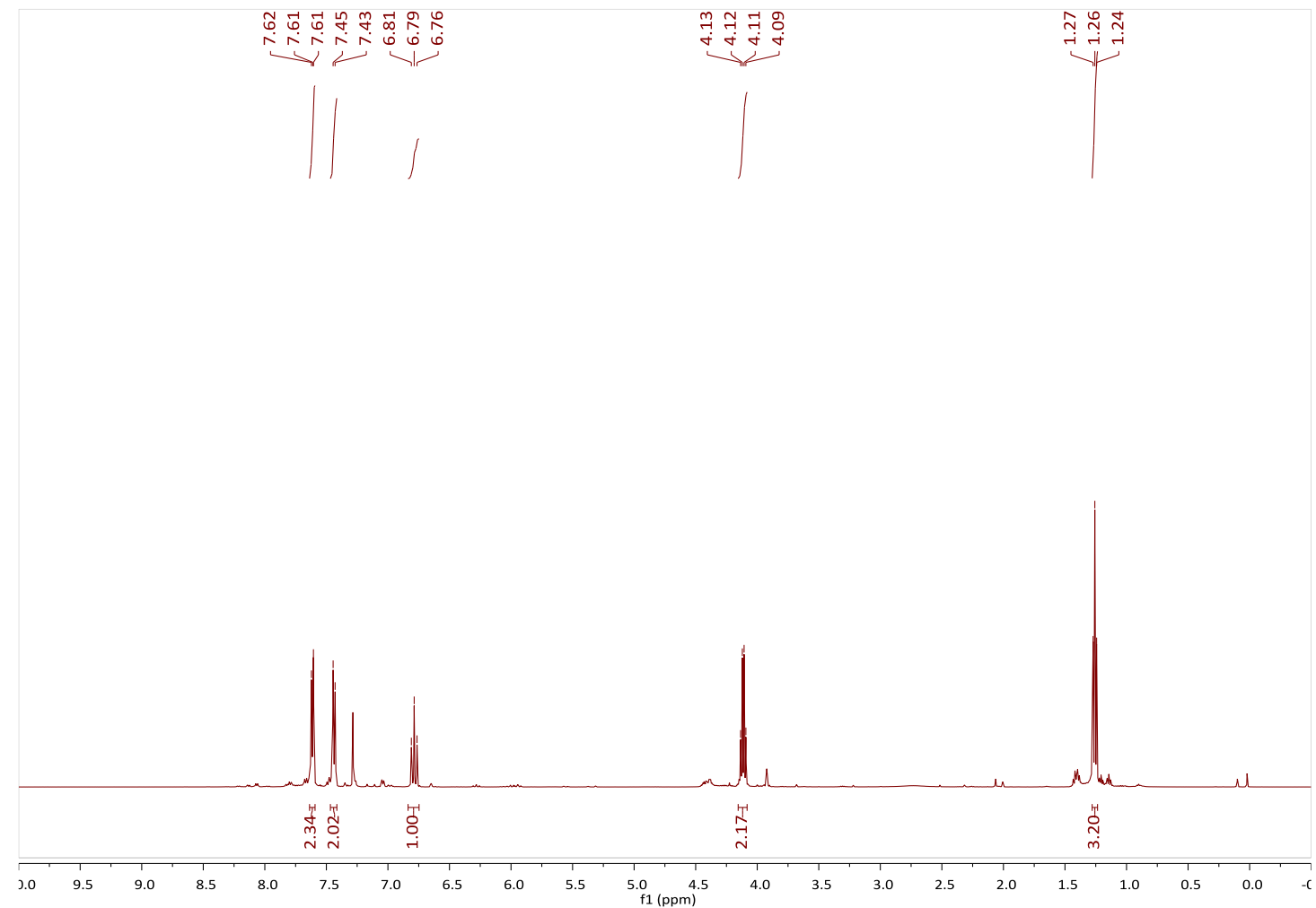

${ }^{19} \mathbf{F}$ NMR-spectrum $\left(471 \mathrm{MHz}, \mathrm{CDCl}_{3}\right)$ of $\mathbf{5 k}$

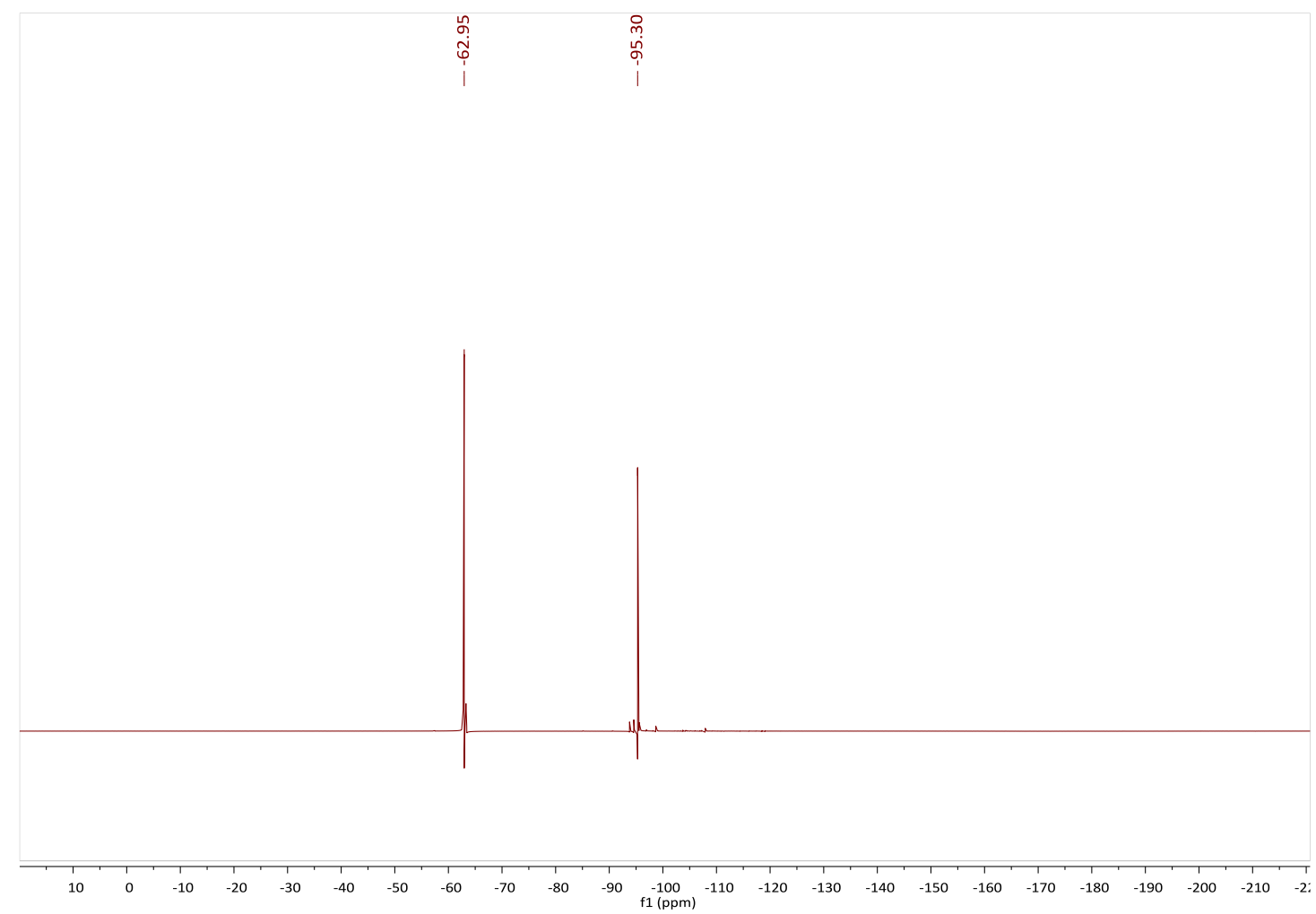




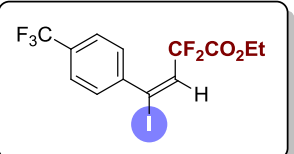

${ }^{13} \mathrm{C}$ NMR-spectrum (125 MHz, $\left.\mathrm{CDCl}_{3}\right)$ of $5 \mathbf{k}$

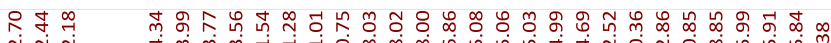

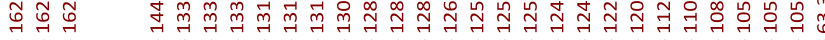
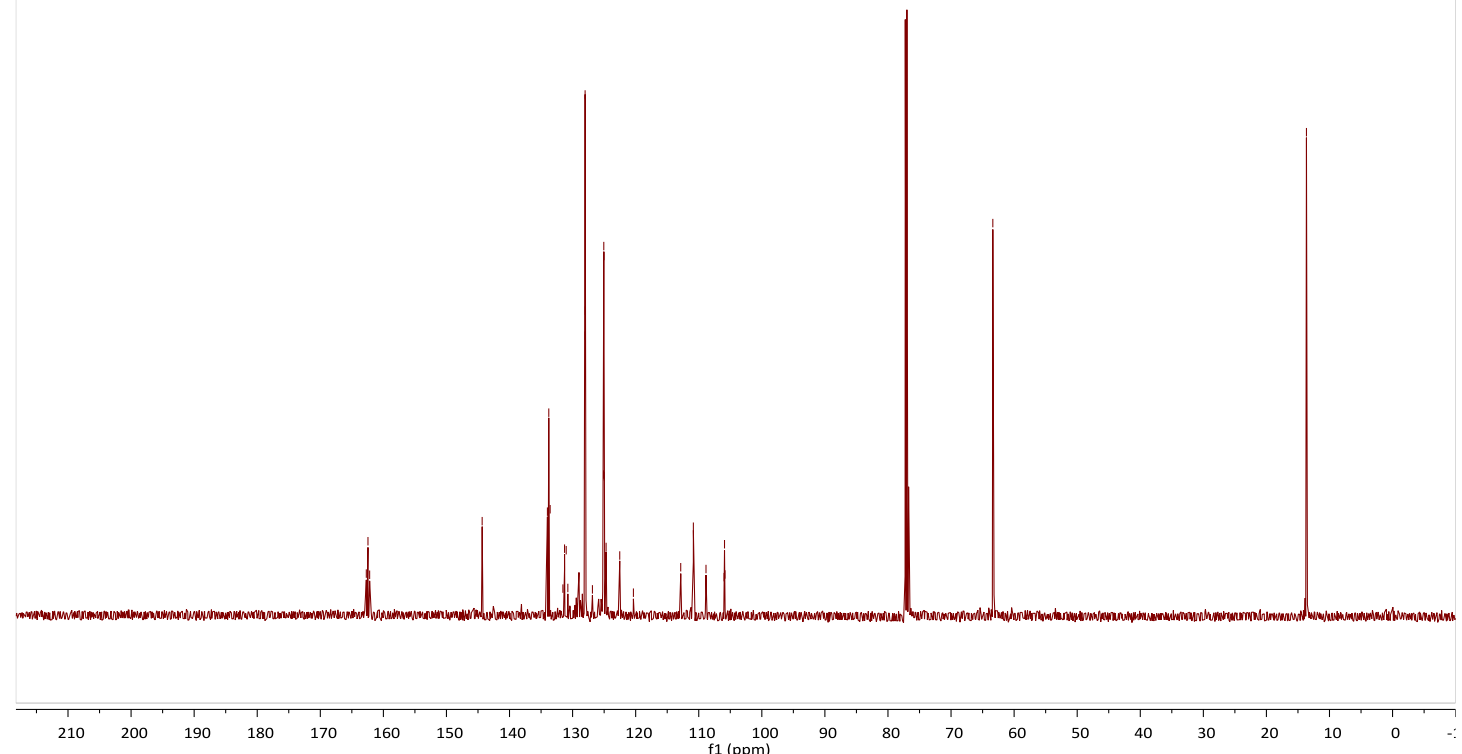


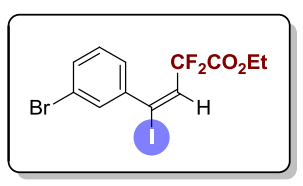

${ }^{1} \mathbf{H}$ NMR-spectrum $\left(500 \mathrm{MHz}, \mathrm{CDCl}_{3}\right)$ of 51

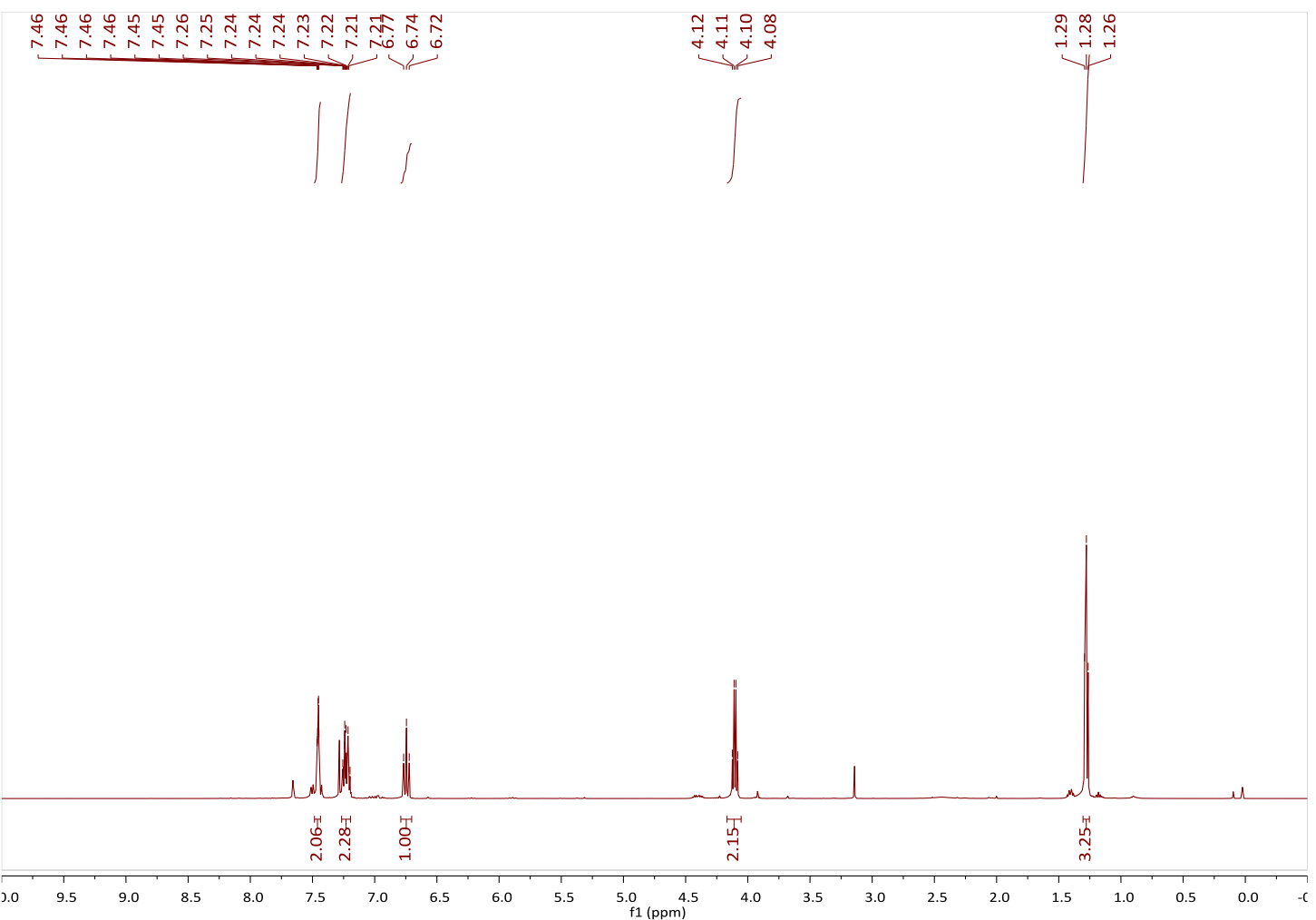

${ }^{19}$ F NMR-spectrum $\left(471 \mathrm{MHz}, \mathrm{CDCl}_{3}\right)$ of 51

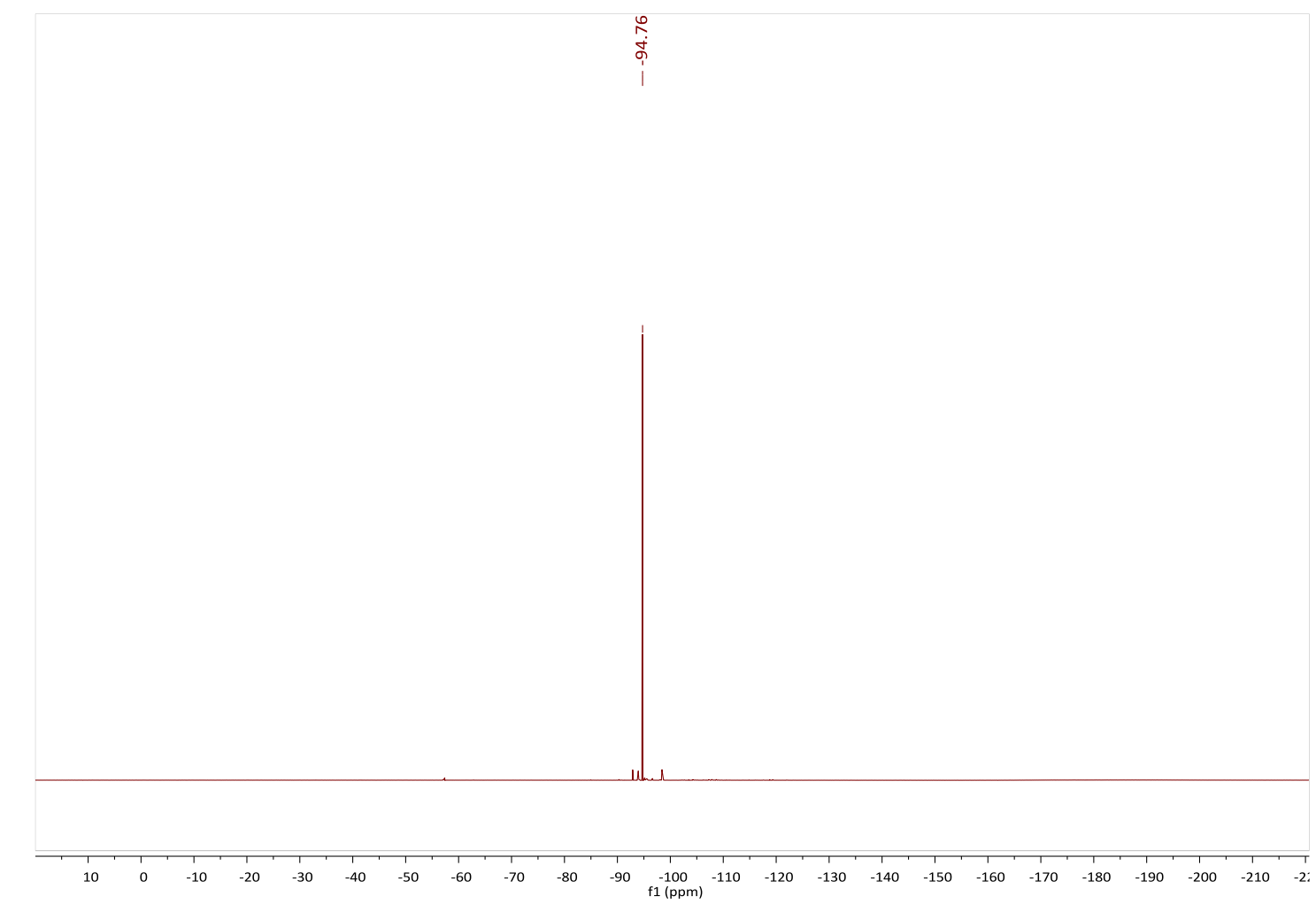




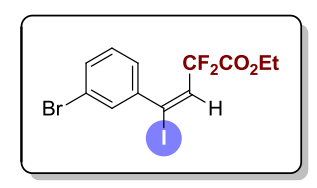

${ }^{13} \mathrm{C}$ NMR-spectrum $\left(125 \mathrm{MHz}, \mathrm{CDCl}_{3}\right)$ of 51

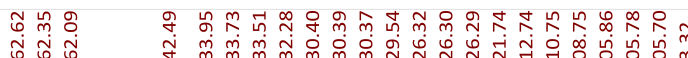

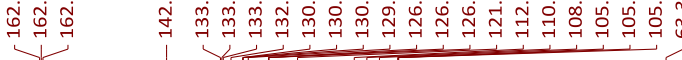
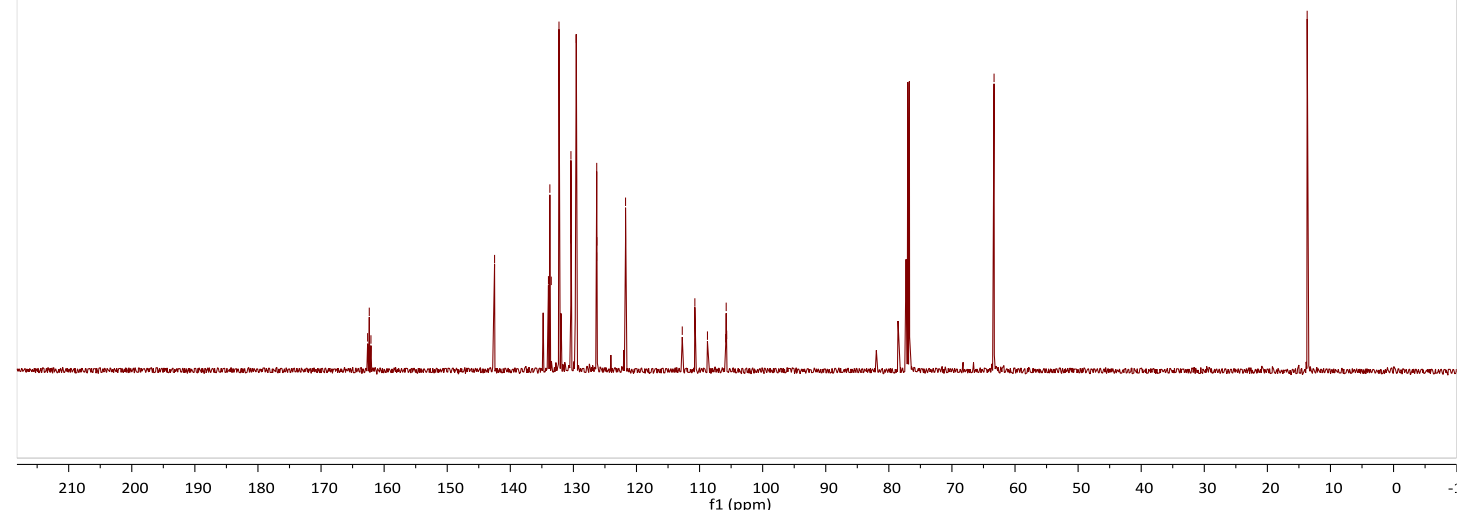


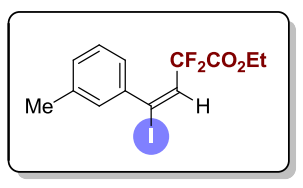

${ }^{1} \mathbf{H}$ NMR-spectrum $\left(500 \mathrm{MHz}, \mathrm{CDCl}_{3}\right)$ of $5 \mathbf{m}$

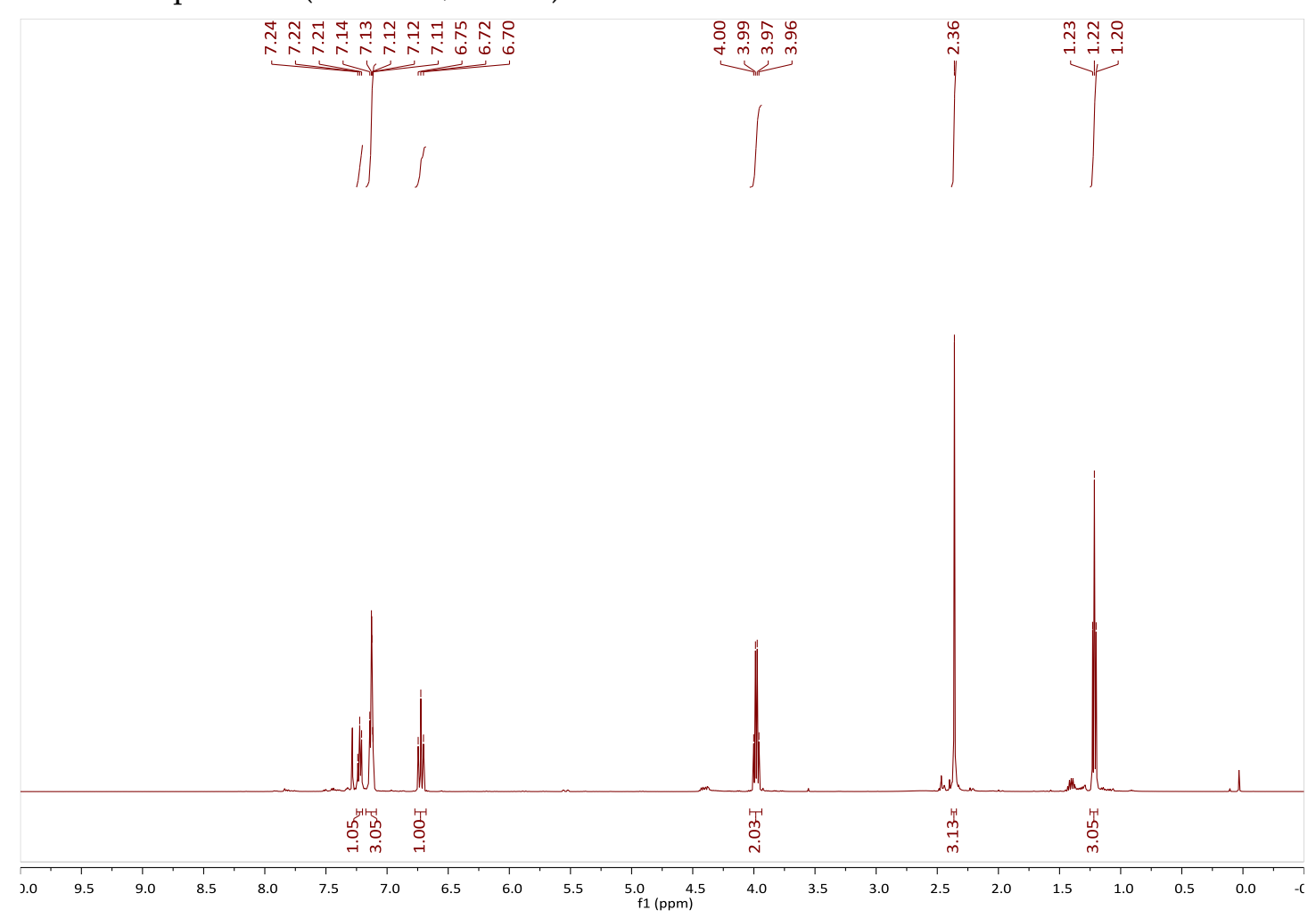

${ }^{19}$ F NMR-spectrum $\left(471 \mathrm{MHz}, \mathrm{CDCl}_{3}\right)$ of $5 \mathbf{m}$

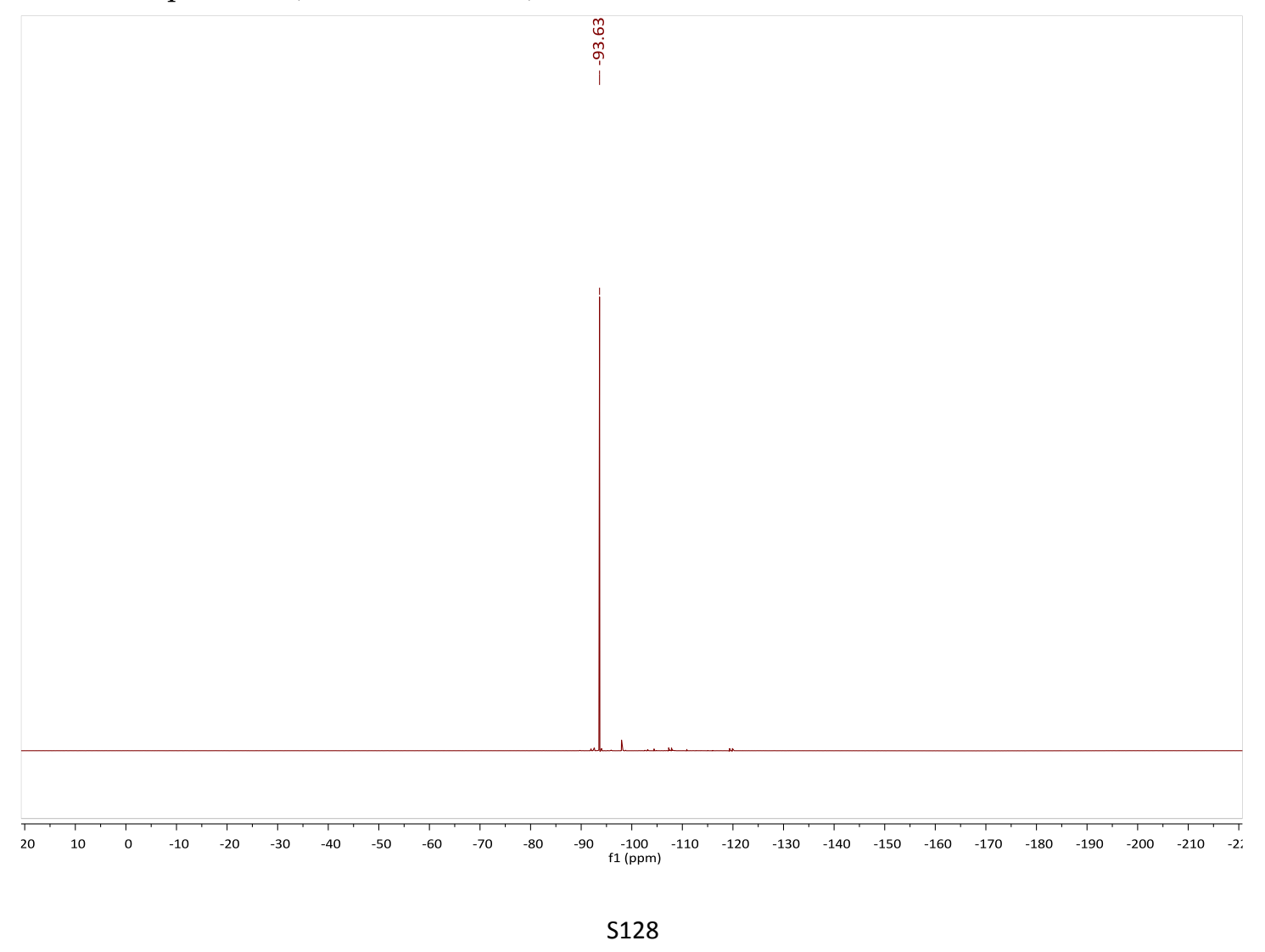




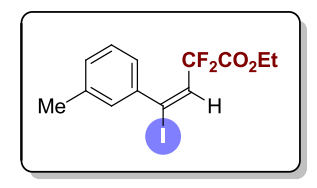

${ }^{13} \mathrm{C} \mathrm{NMR}$-spectrum (125 MHz, $\left.\mathrm{CDCl}_{3}\right)$ of $5 \mathrm{~m}$

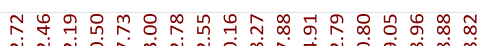

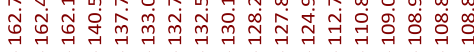

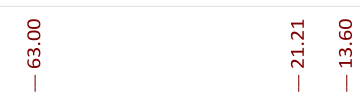

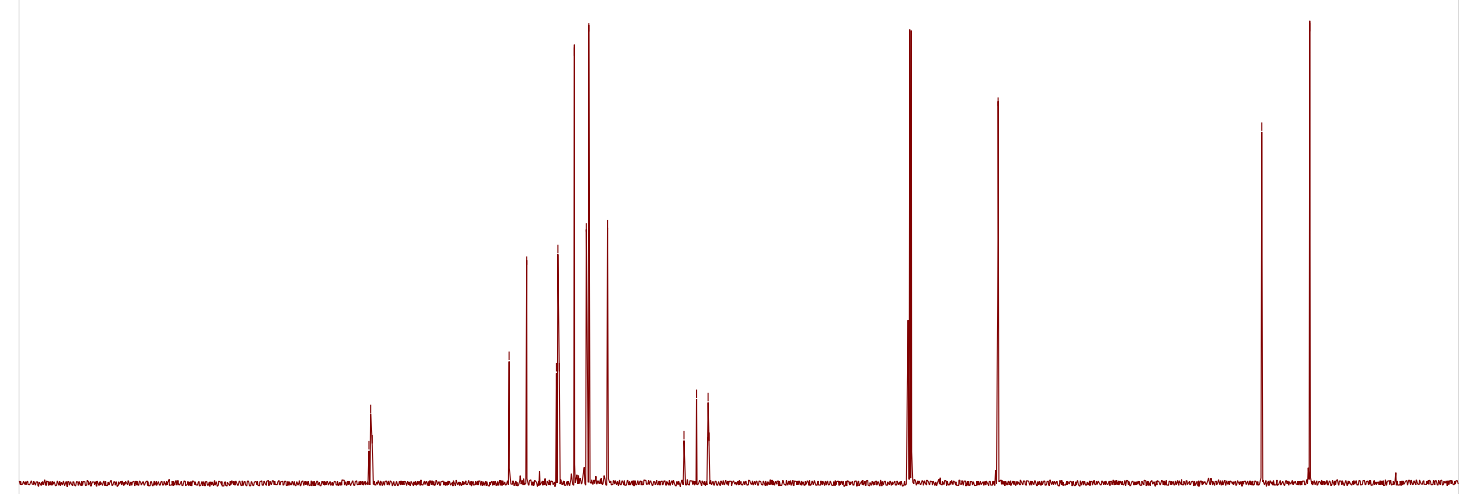

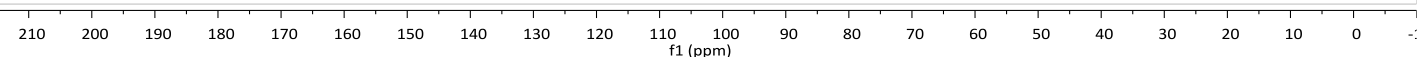




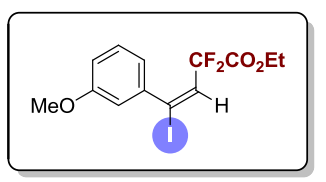

${ }^{1} \mathbf{H}$ NMR-spectrum $\left(500 \mathrm{MHz}, \mathrm{CDCl}_{3}\right)$ of $\mathbf{5 n}$

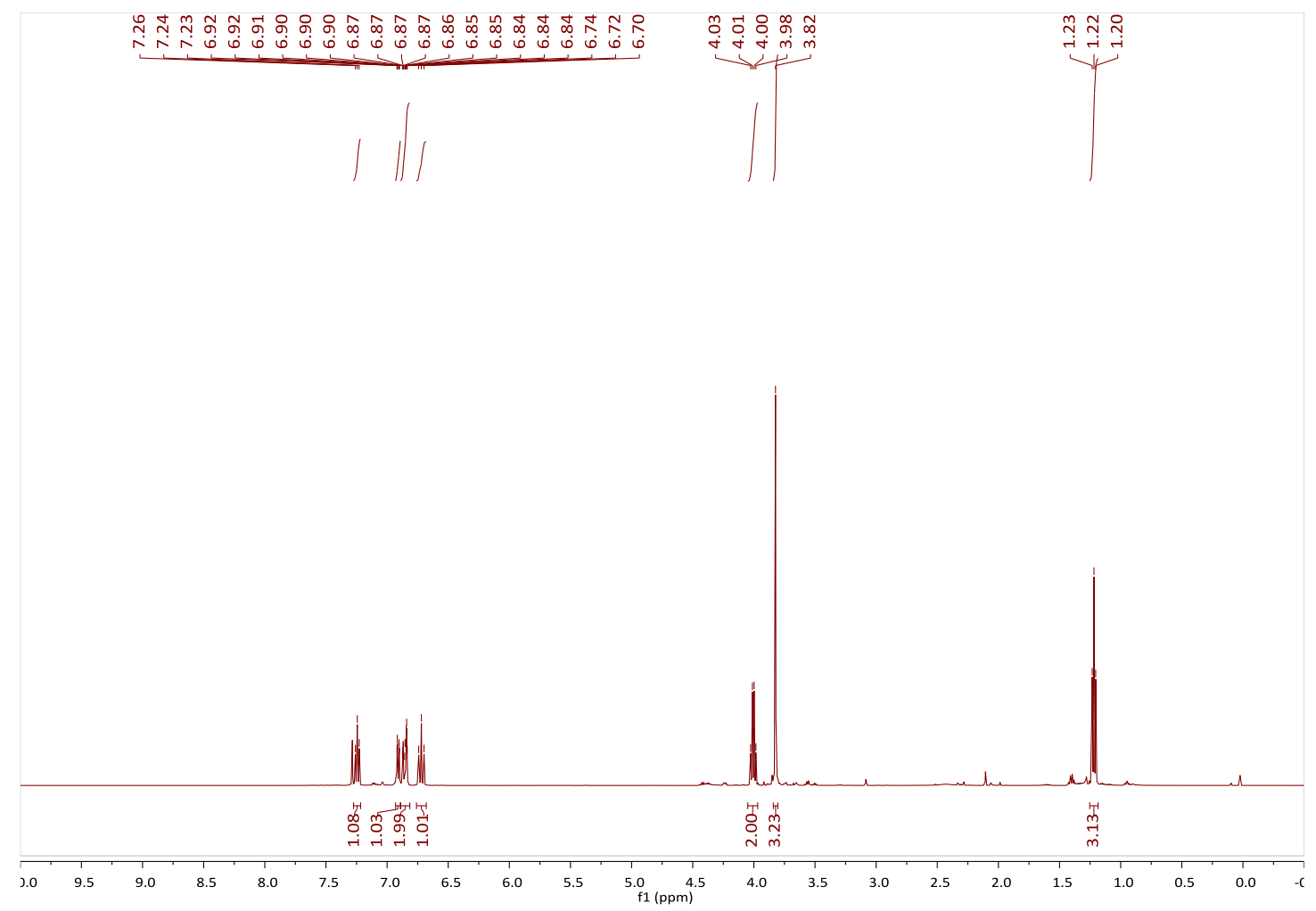

${ }^{19}$ F NMR-spectrum $\left(471 \mathrm{MHz}, \mathrm{CDCl}_{3}\right)$ of 5 n

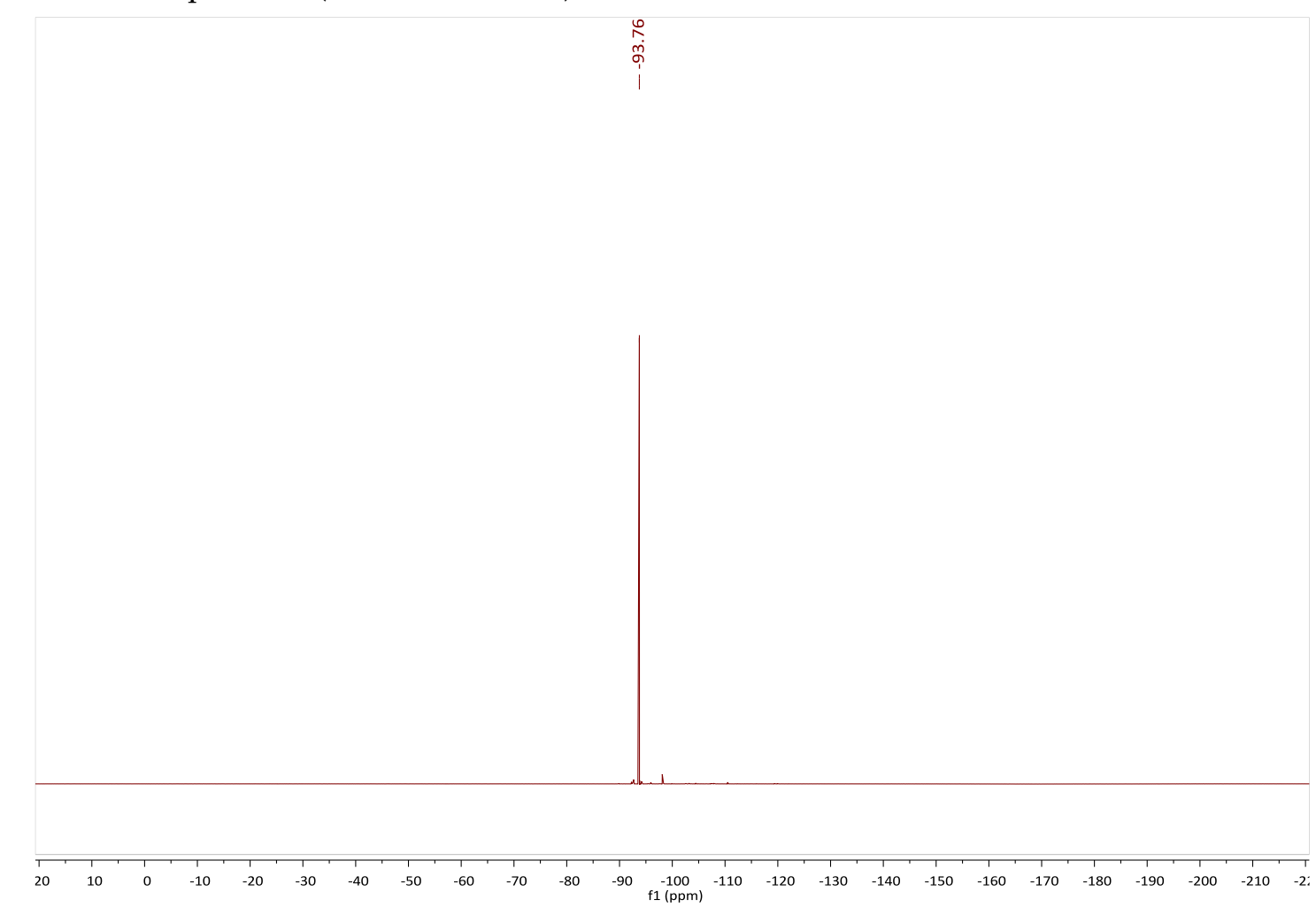




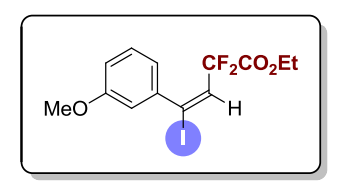

${ }^{13} \mathrm{C}$ NMR-spectrum (125 MHz, $\left.\mathrm{CDCl}_{3}\right)$ of 5 n

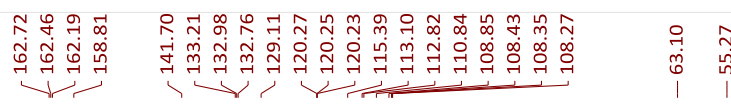

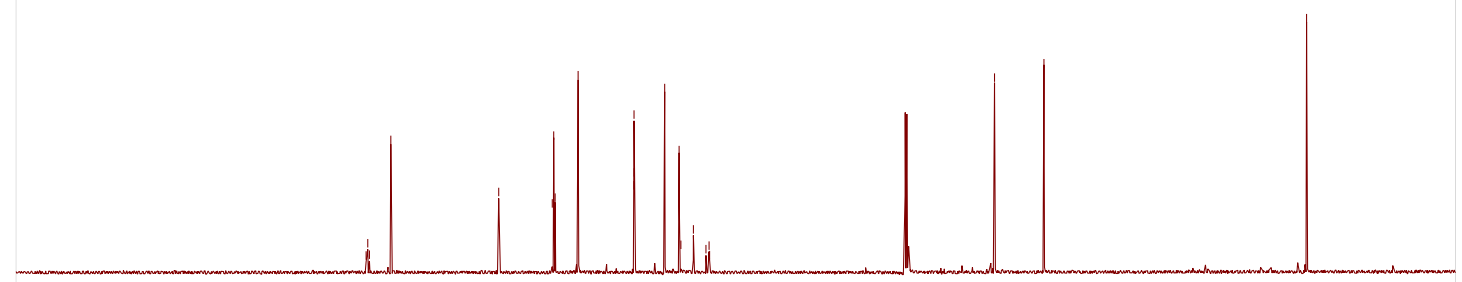

$\begin{array}{llllllllllllllllllllllllllllllllllllll}210 & 200 & 190 & 180 & 170 & 160 & 150 & 140 & 130 & 120 & 110 & 100 & 90 & 80 & 70 & 60 & 50 & 40 & 30 & 20 & 10 & 0 & -\end{array}$ 


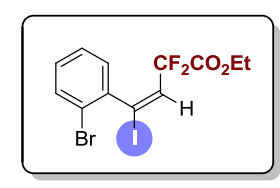

${ }^{1} \mathbf{H}$ NMR-spectrum $\left(500 \mathrm{MHz}, \mathrm{CDCl}_{3}\right)$ of $\mathbf{5 o}$

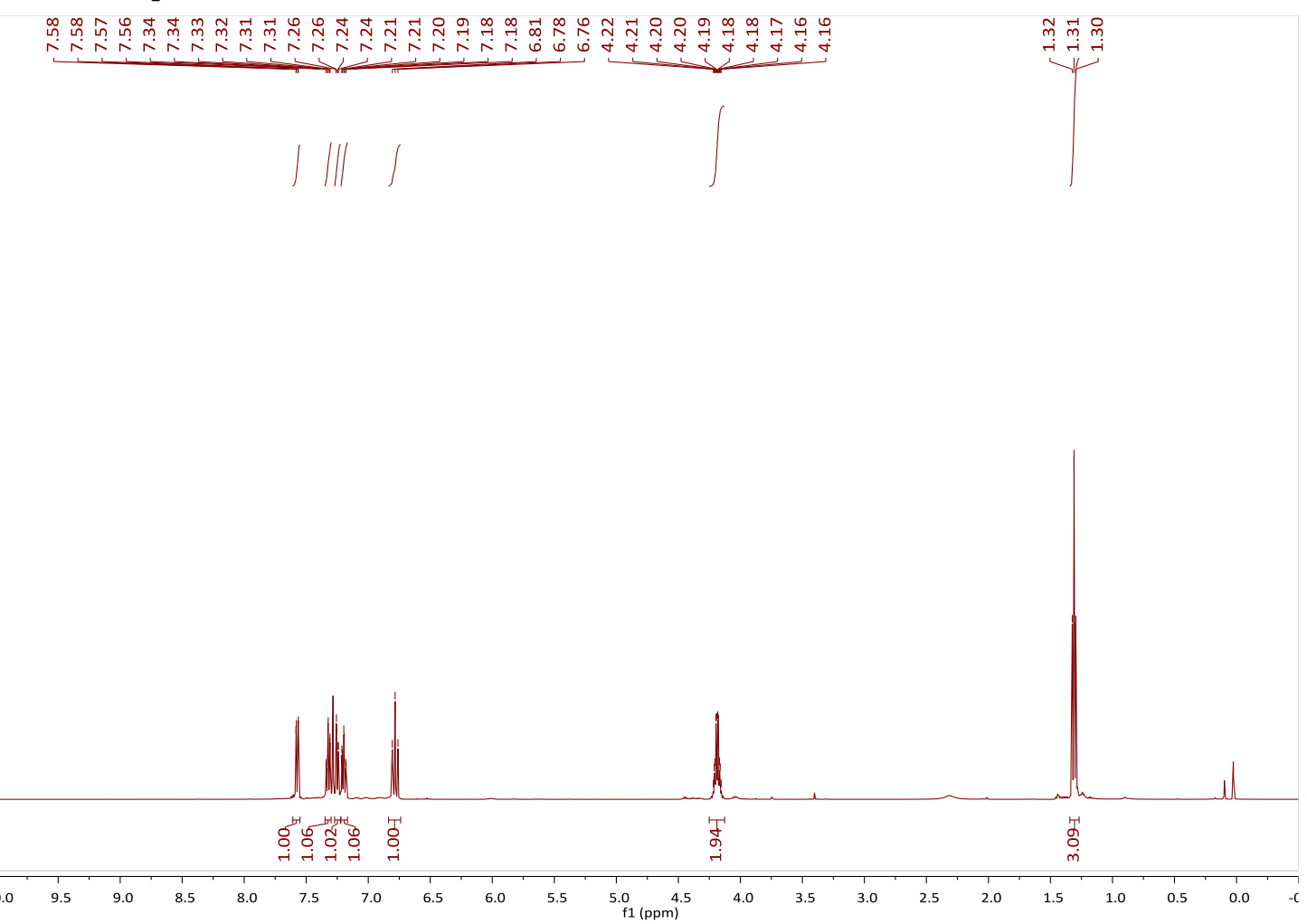

${ }^{19} \mathrm{~F}$ NMR-spectrum $\left(471 \mathrm{MHz}, \mathrm{CDCl}_{3}\right)$ of $\mathbf{5 o}$

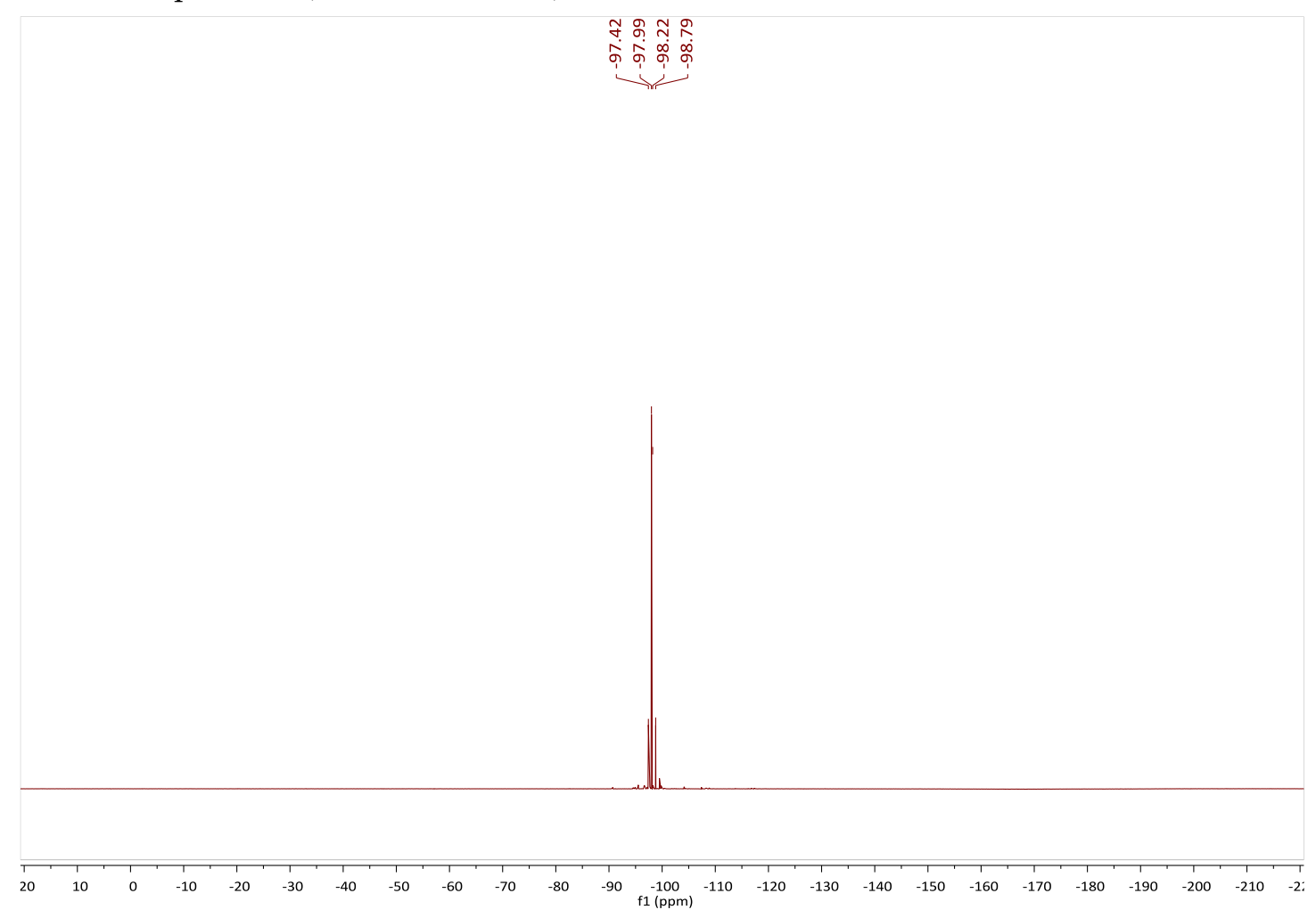




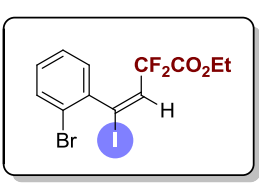

${ }^{13} \mathrm{C}$ NMR-spectrum (125 MHz, $\left.\mathrm{CDCl}_{3}\right)$ of $5 \mathbf{o}$

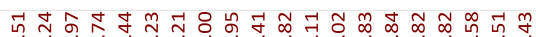

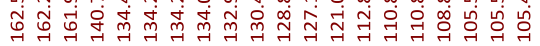

$\underset{\substack{m \\ 0 \\ 0}}{1}$

$\stackrel{\infty}{\stackrel{\infty}{m}}$

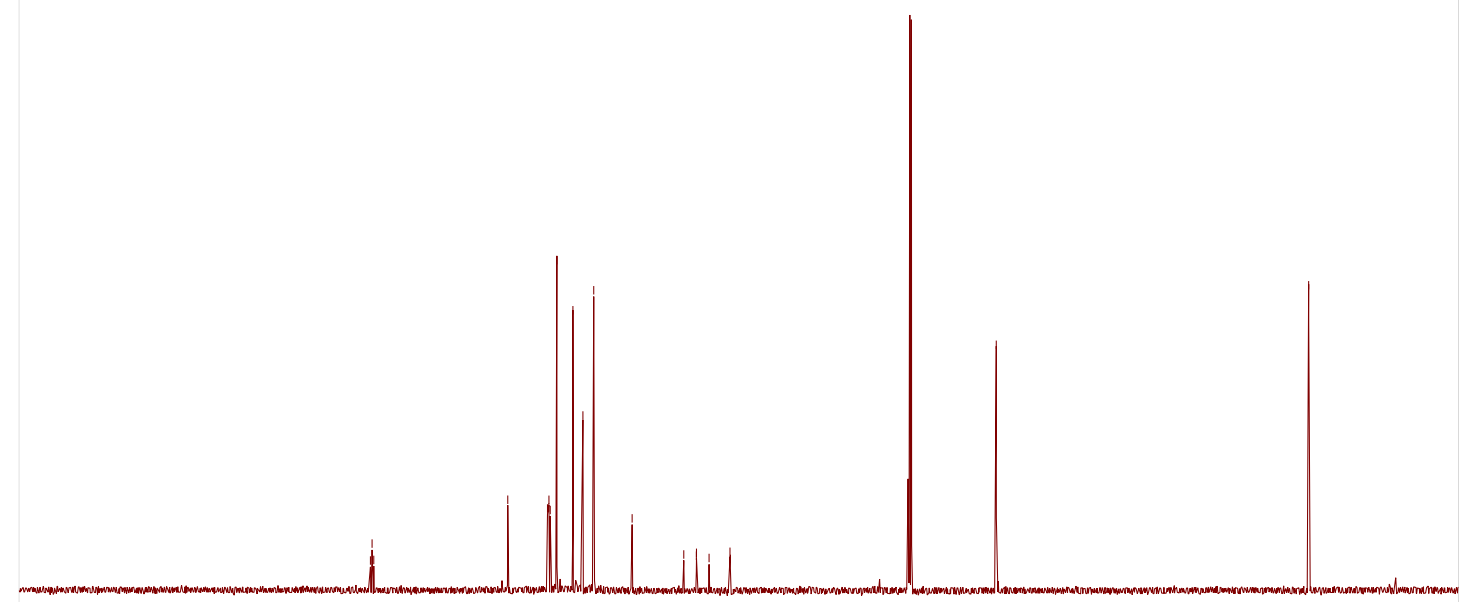

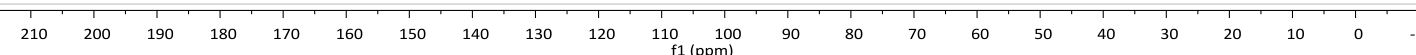




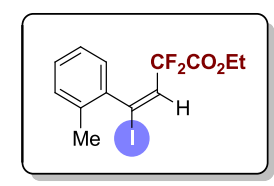

${ }^{1} \mathbf{H}$ NMR-spectrum $\left(500 \mathrm{MHz}, \mathrm{CDCl}_{3}\right)$ of $\mathbf{5 p}$

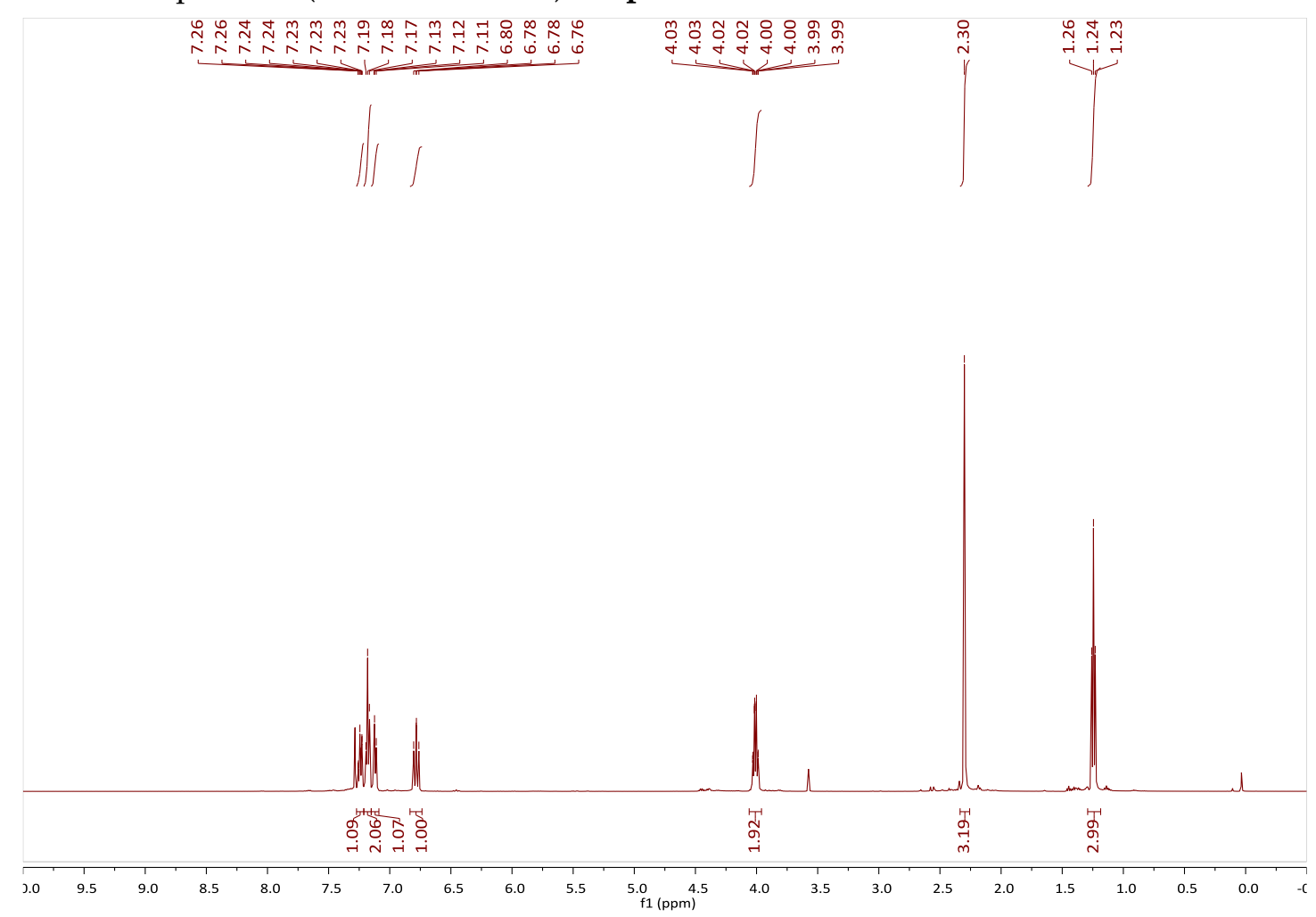

${ }^{19}$ F NMR-spectrum (471 MHz, $\left.\mathrm{CDCl}_{3}\right)$ of 5 p

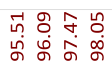

1

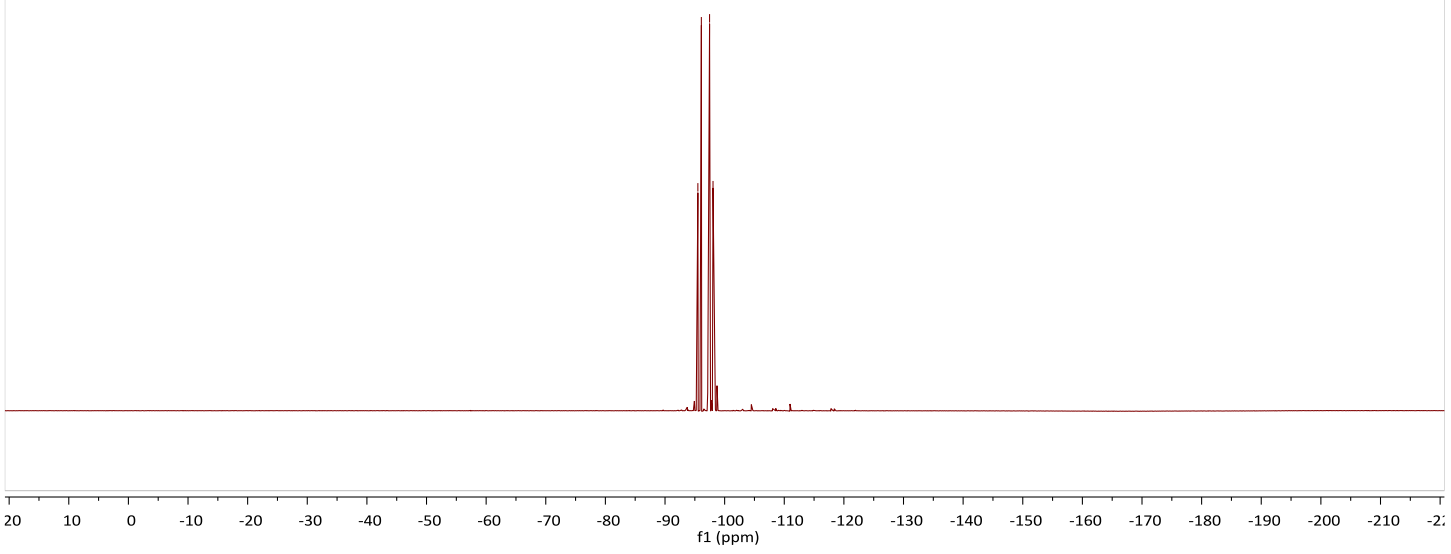




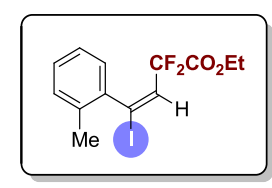

${ }^{13} \mathrm{C} \mathrm{NMR}$-spectrum (125 MHz, $\left.\mathrm{CDCl}_{3}\right)$ of $5 p$

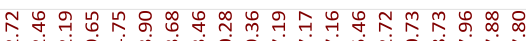

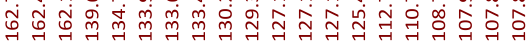

$\infty$

ㅇำ

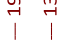

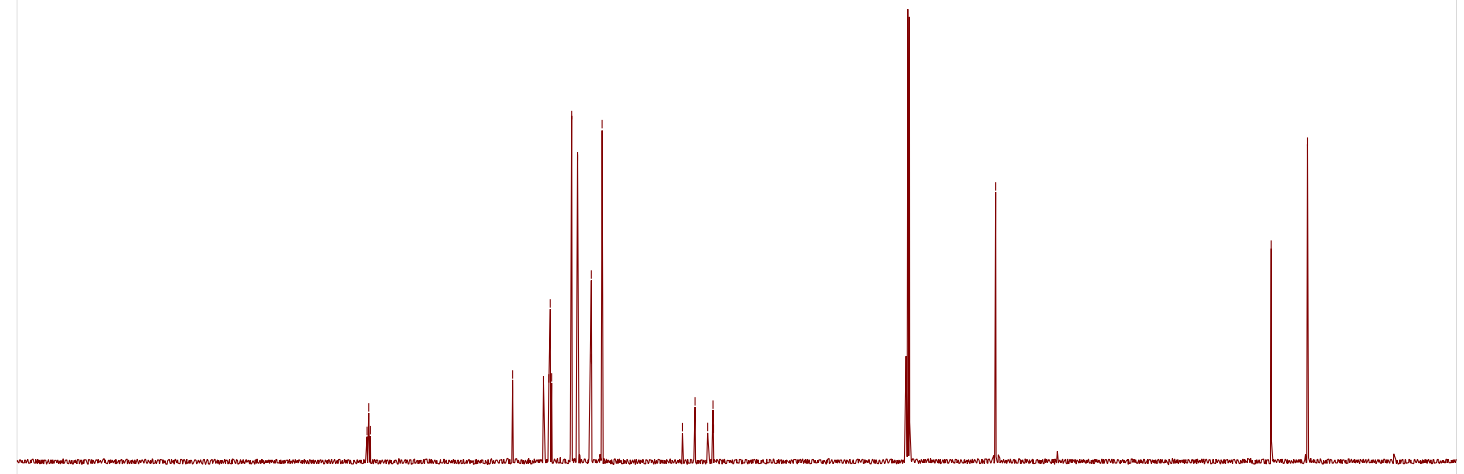

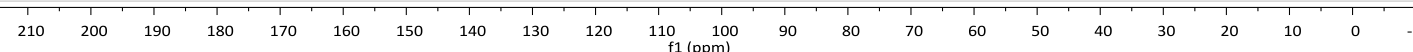




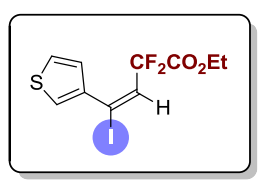

${ }^{1} \mathbf{H}$ NMR-spectrum $\left(500 \mathrm{MHz}, \mathrm{CDCl}_{3}\right)$ of $\mathbf{5 q}$

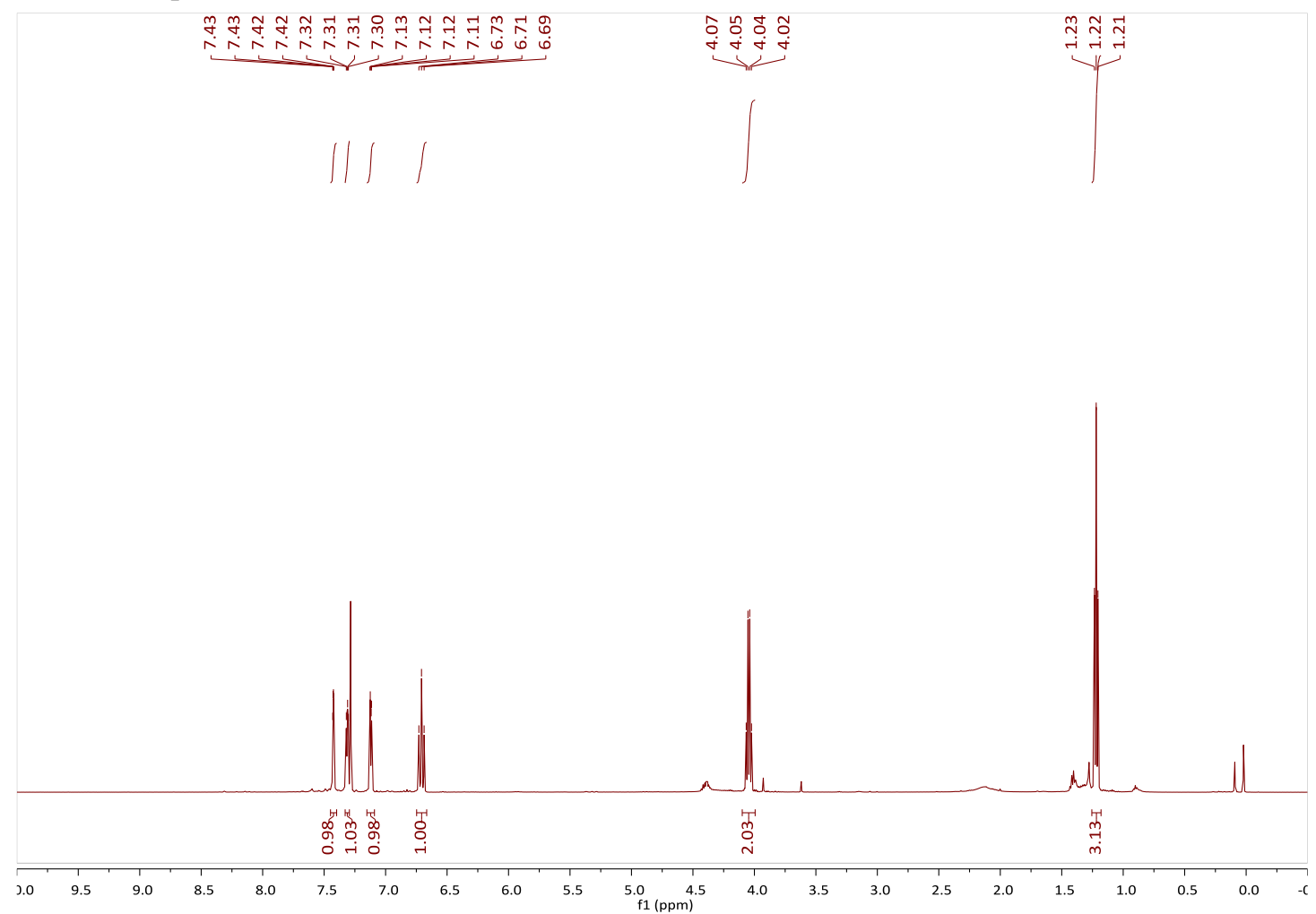

${ }^{19} \mathbf{F}$ NMR-spectrum $\left(471 \mathrm{MHz}, \mathrm{CDCl}_{3}\right)$ of $\mathbf{5 q}$

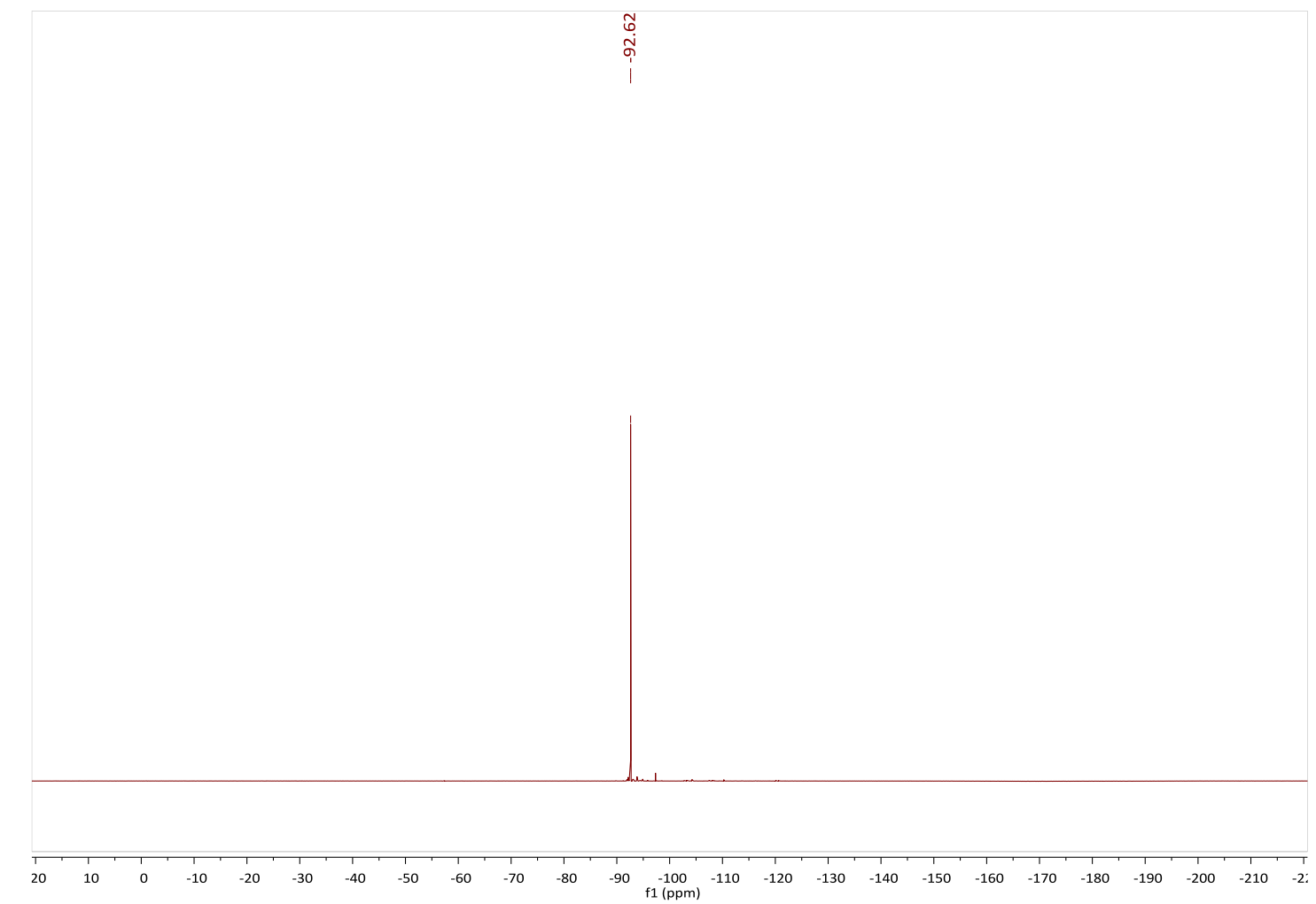




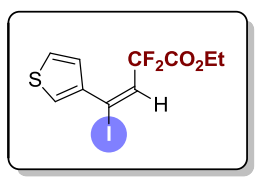

${ }^{13} \mathrm{C}$ NMR-spectrum (125 MHz, $\left.\mathrm{CDCl}_{3}\right)$ of $\mathbf{5 q}$

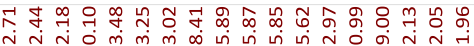

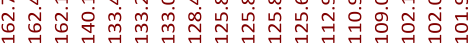

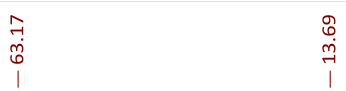

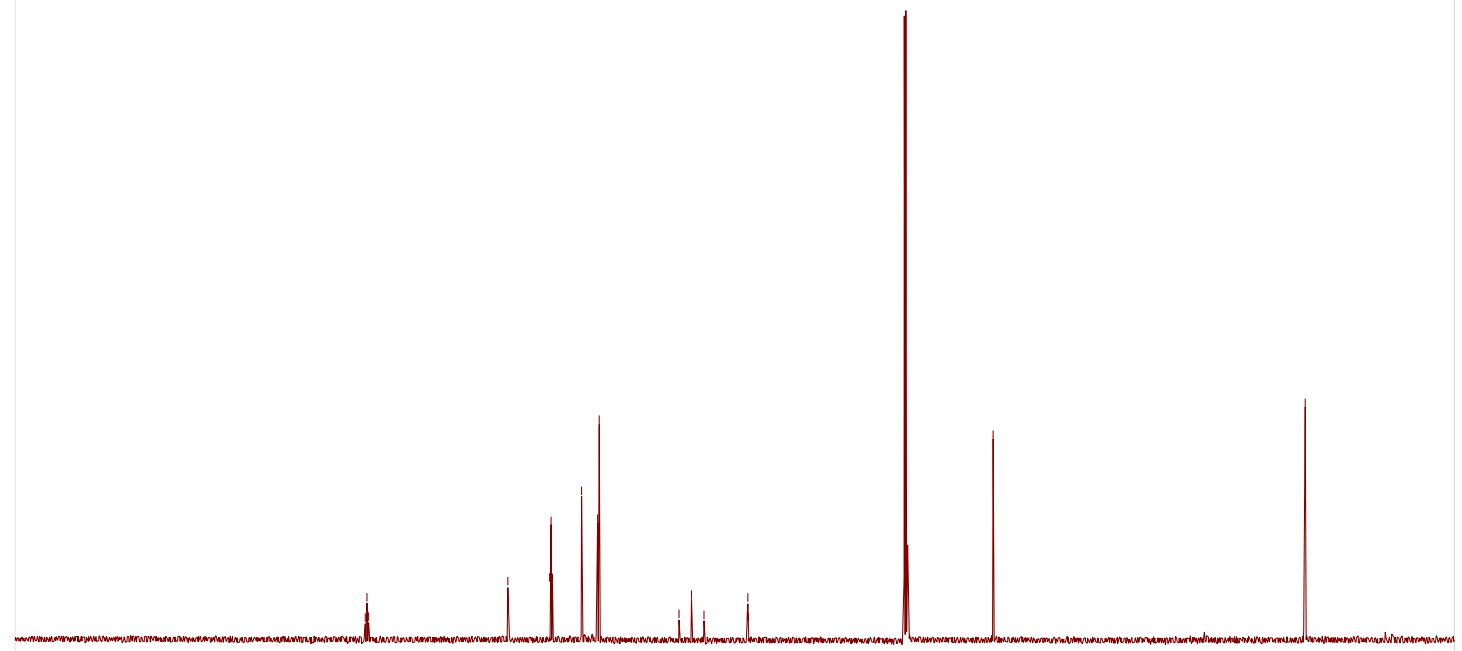

$\begin{array}{llllllllllll}210 & 200 & 190 & 180 & 170 & 160 & 150 & 140 & 130 & 120 & 110 & 100 \\ f 1(p p m)\end{array}$ 
${ }^{1} \mathbf{H}$ NMR-spectrum $\left(500 \mathrm{MHz}, \mathrm{CDCl}_{3}\right)$ of $5 \mathbf{r}$

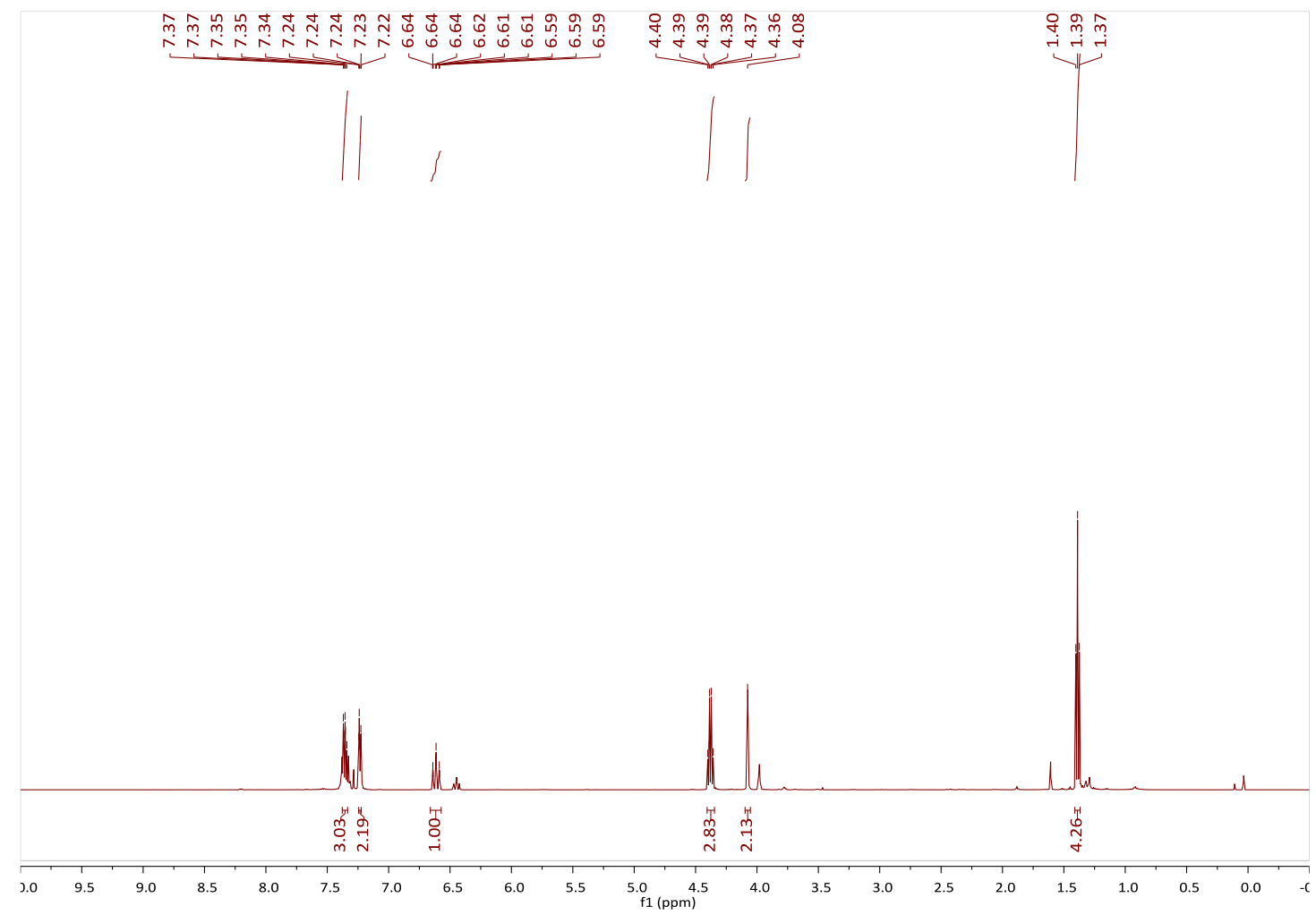

${ }^{19} \mathbf{F}$ NMR-spectrum $\left(471 \mathrm{MHz}, \mathrm{CDCl}_{3}\right)$ of $5 \mathbf{r}$

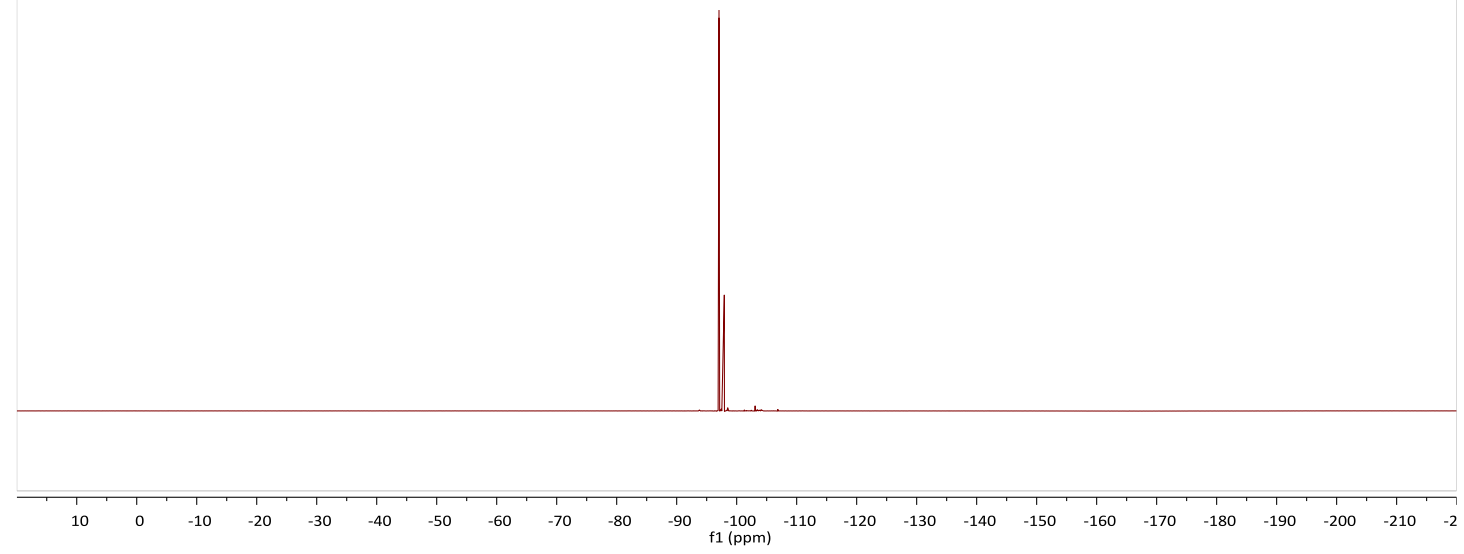




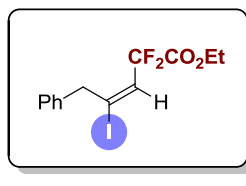

$E / Z=3: 1$

${ }^{13} \mathrm{C}$ NMR-spectrum $\left(125 \mathrm{MHz}, \mathrm{CDCl}_{3}\right)$ of $5 \mathbf{r}$

舟

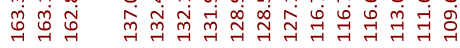

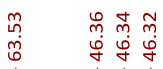

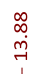

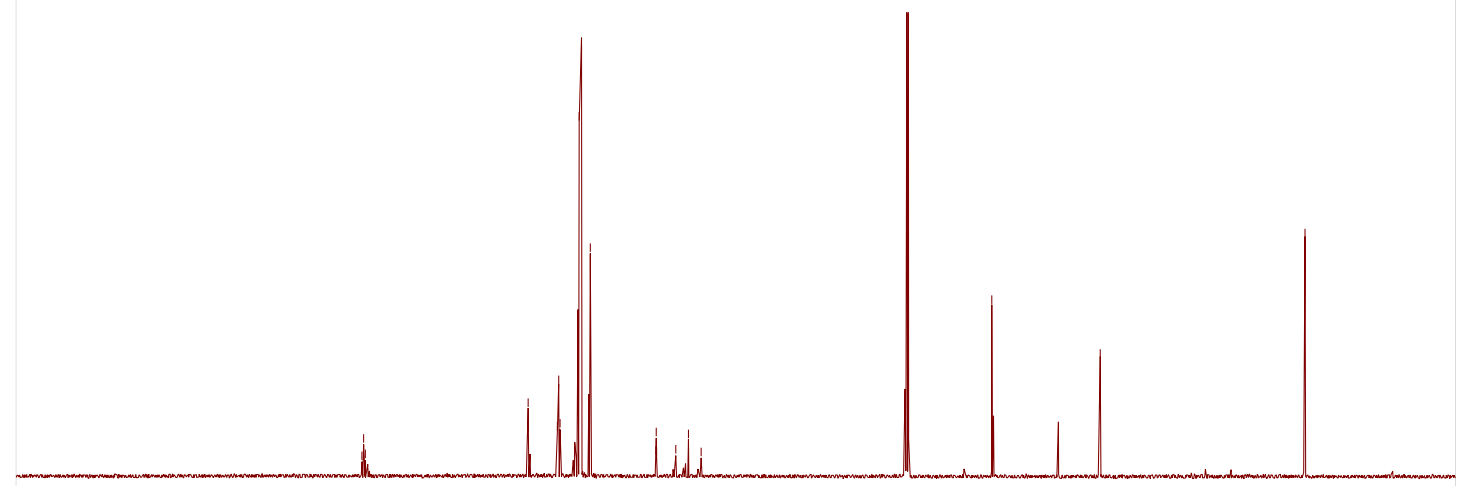

$\begin{array}{llllllllllllllllllllllllllllllllllllll}210 & 200 & 190 & 180 & 170 & 160 & 150 & 140 & 130 & 120 & 110 & 100 & 90 & 80 & 70 & 60 & 50 & 40 & 30 & 20 & 10 & 0 & -\end{array}$ 


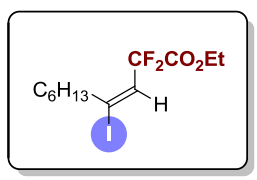

${ }^{1} \mathbf{H}$ NMR-spectrum $\left(500 \mathrm{MHz}, \mathrm{CDCl}_{3}\right)$ of $5 \mathbf{s}$

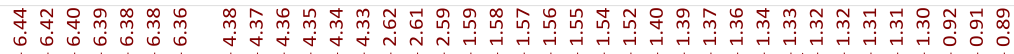

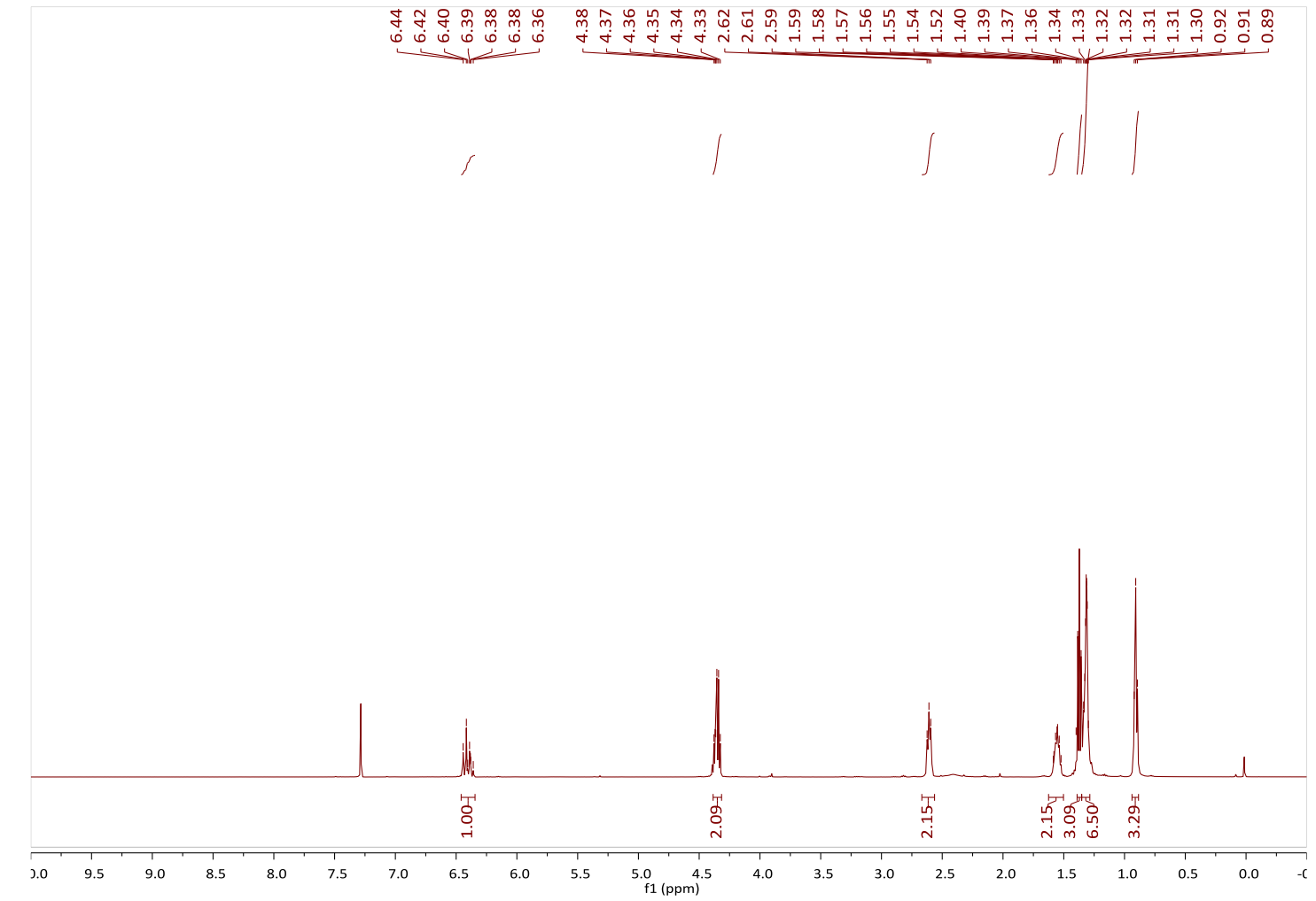

${ }^{19} \mathrm{~F}$ NMR-spectrum $\left(471 \mathrm{MHz}, \mathrm{CDCl}_{3}\right)$ of $5 \mathrm{~s}$

$$
\text { ำ }
$$

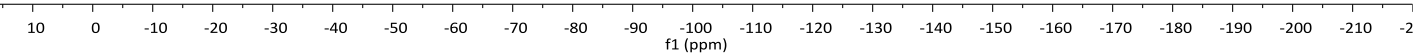




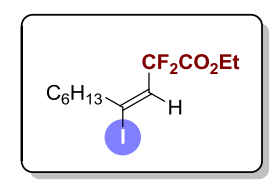

${ }^{13} \mathrm{C}$ NMR-spectrum $\left(125 \mathrm{MHz}, \mathrm{CDCl}_{3}\right)$ of $5 \mathbf{s}$

舟

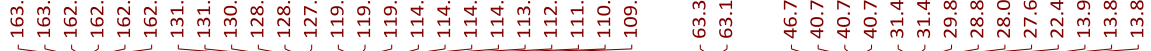

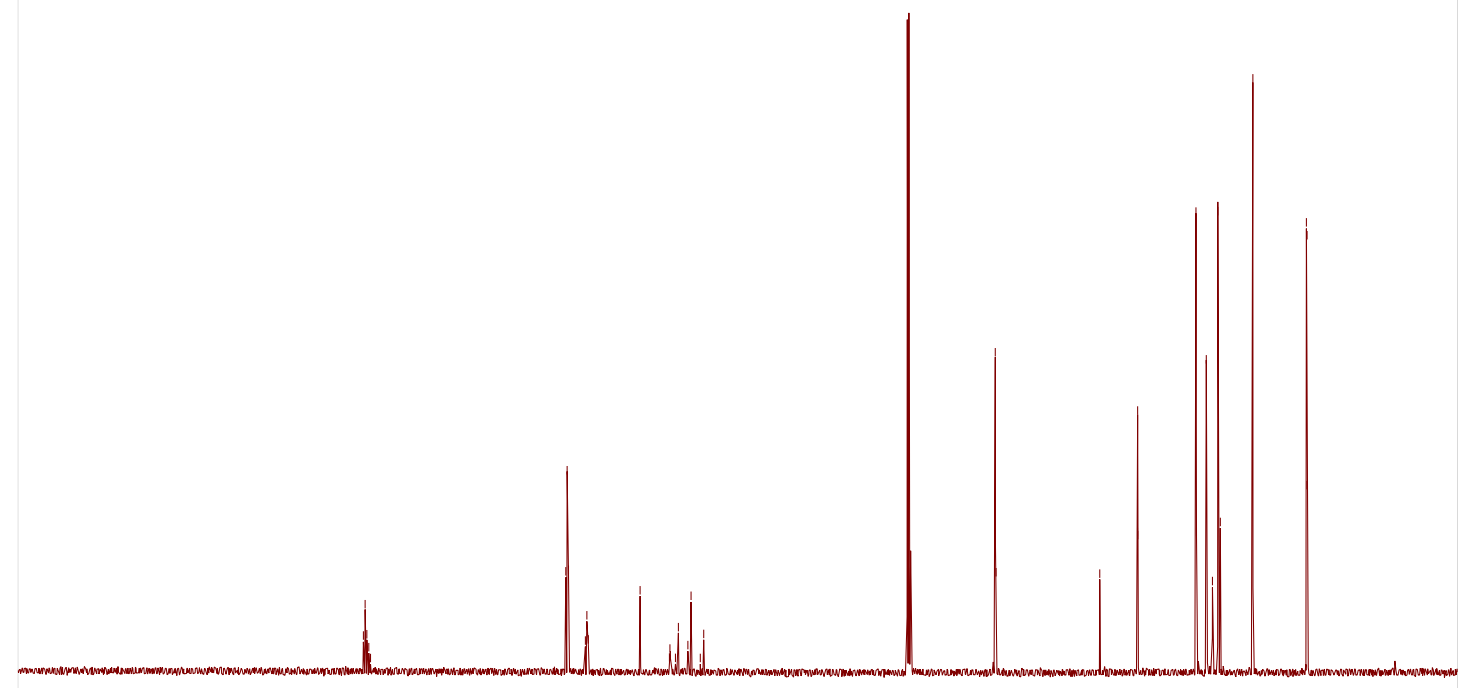

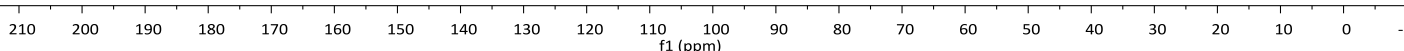




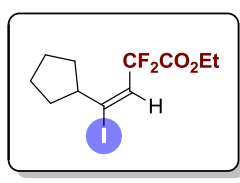

$\mathrm{E} / \mathrm{Z}=2.0: 1$

${ }^{1} \mathbf{H}$ NMR-spectrum $\left(500 \mathrm{MHz}, \mathrm{CDCl}_{3}\right)$ of $\mathbf{5 t}$

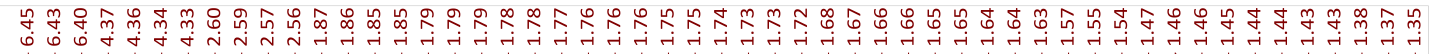
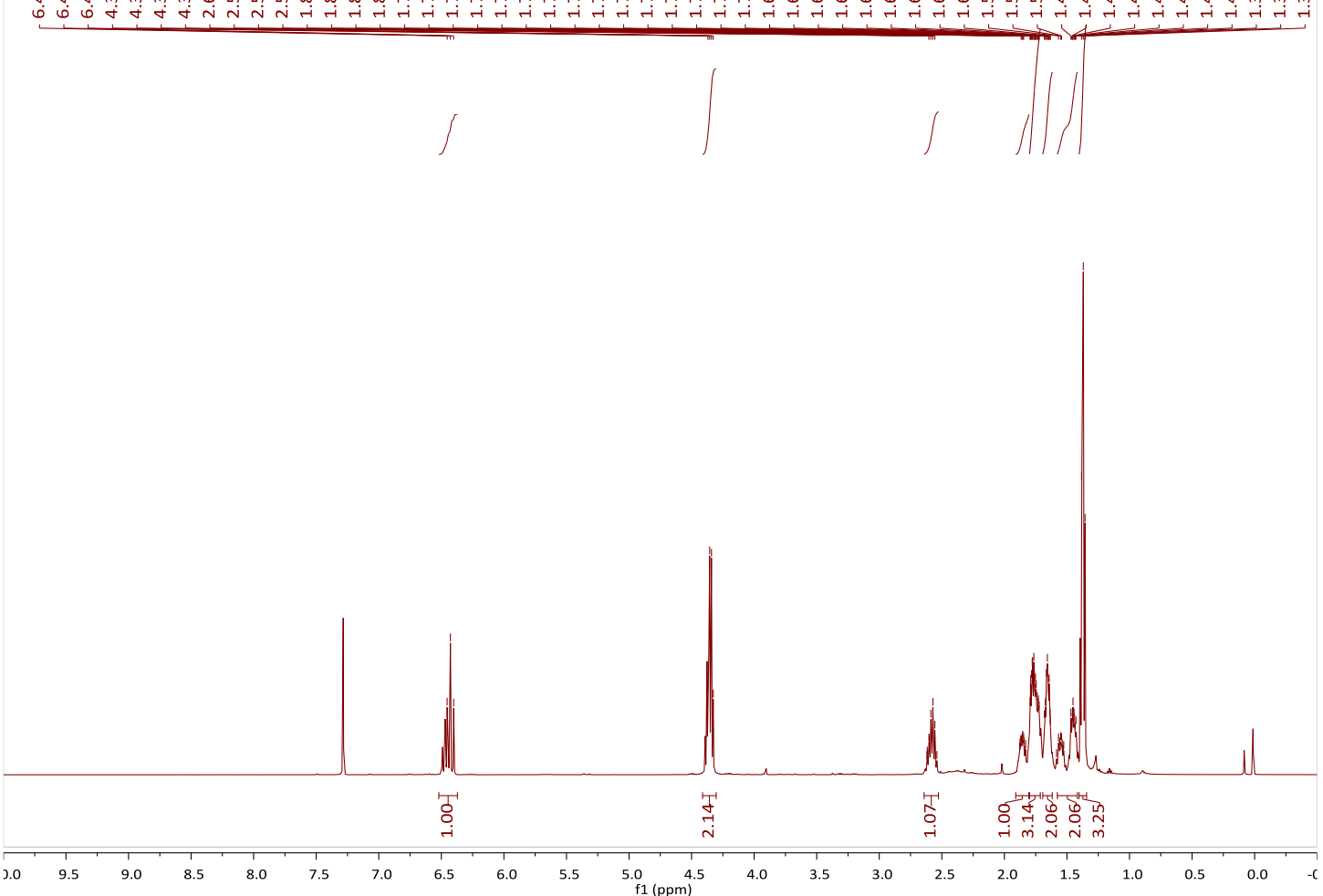

${ }^{19} \mathbf{F}$ NMR-spectrum $\left(471 \mathrm{MHz}, \mathrm{CDCl}_{3}\right)$ of $5 \mathbf{t}$

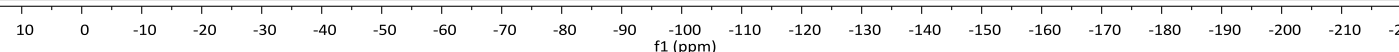




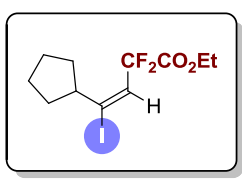

$\mathrm{E} / \mathrm{Z}=2.0: 1$

${ }^{13} \mathrm{C}$ NMR-spectrum (125 MHz, $\left.\mathrm{CDCl}_{3}\right)$ of $\mathbf{5 t}$

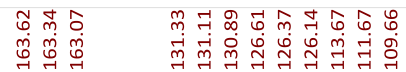

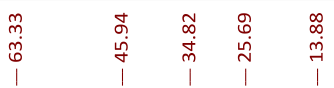

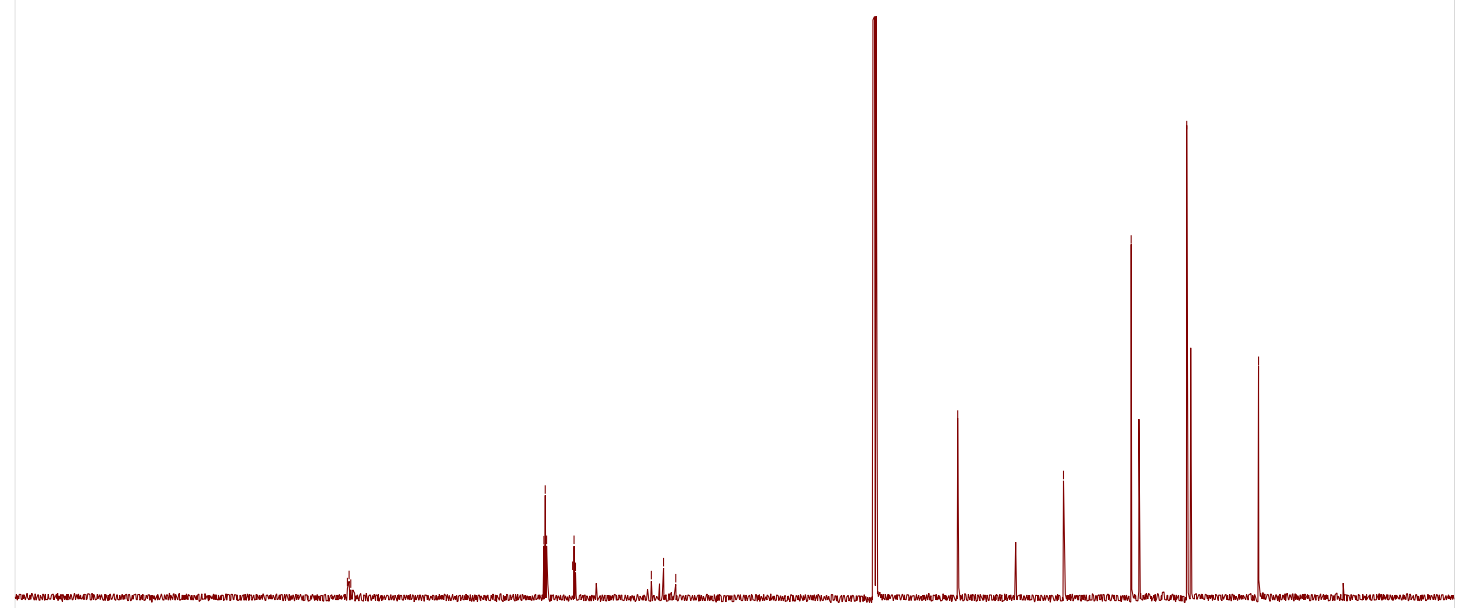

$\begin{array}{llllllllllllllllllllllllllllllllllll}210 & 200 & 190 & 180 & 170 & 160 & 150 & 140 & 130 & 120 & 110 & 100 & 90 & 80 & 70 & 60 & 50 & 40 & 30 & 20 & 10 & 0 & -10\end{array}$ 


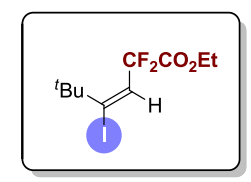

${ }^{1} \mathbf{H}$ NMR-spectrum $\left(500 \mathrm{MHz}, \mathrm{CDCl}_{3}\right)$ of $\mathbf{5 u}$

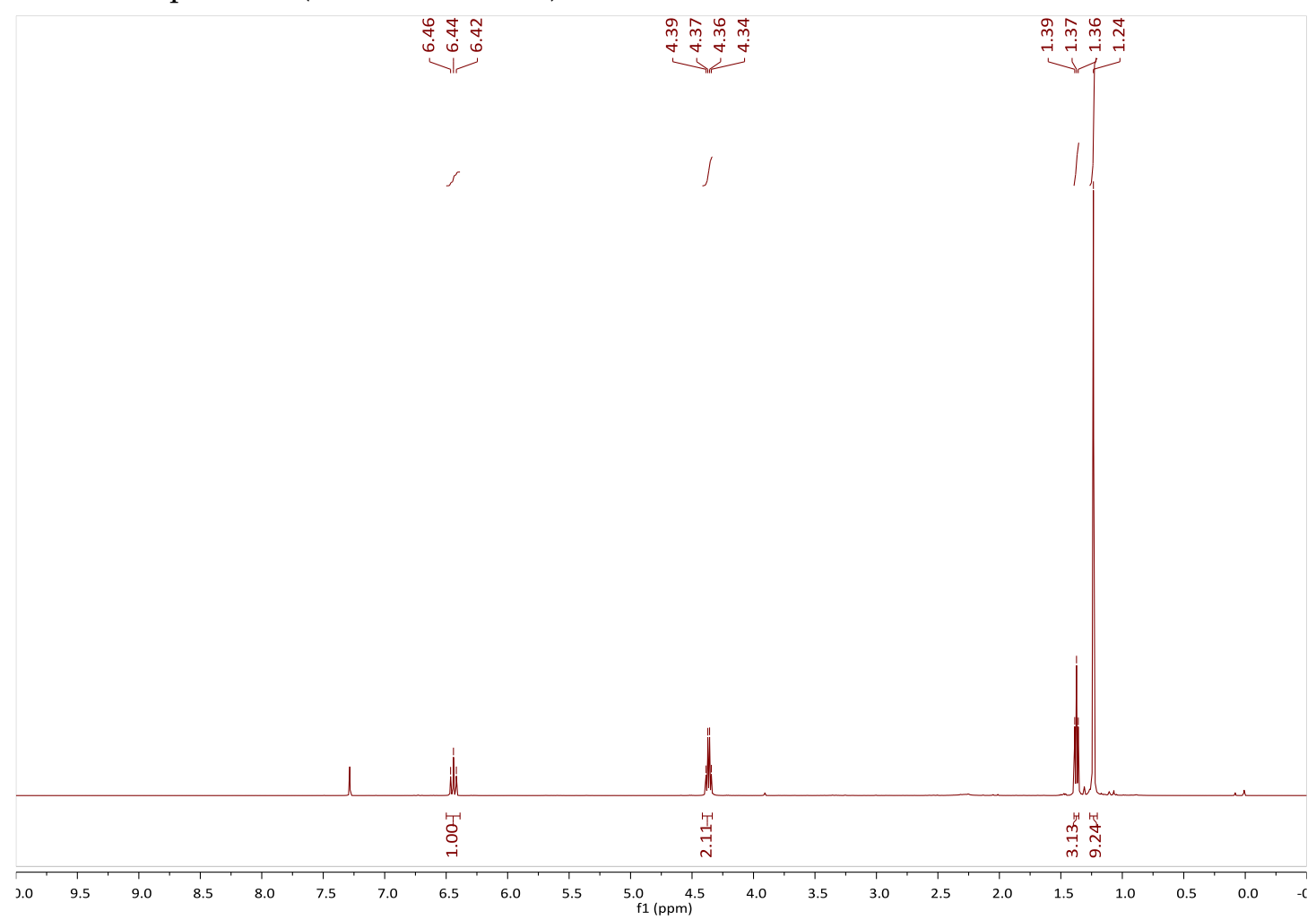

${ }^{19} \mathbf{F}$ NMR-spectrum $\left(471 \mathrm{MHz}, \mathrm{CDCl}_{3}\right)$ of $5 \mathbf{u}$ 


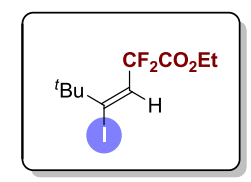

${ }^{13} \mathbf{C}$ NMR-spectrum $\left(125 \mathrm{MHz}, \mathrm{CDCl}_{3}\right)$ of $\mathbf{5 u}$

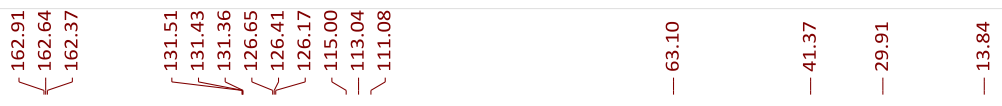

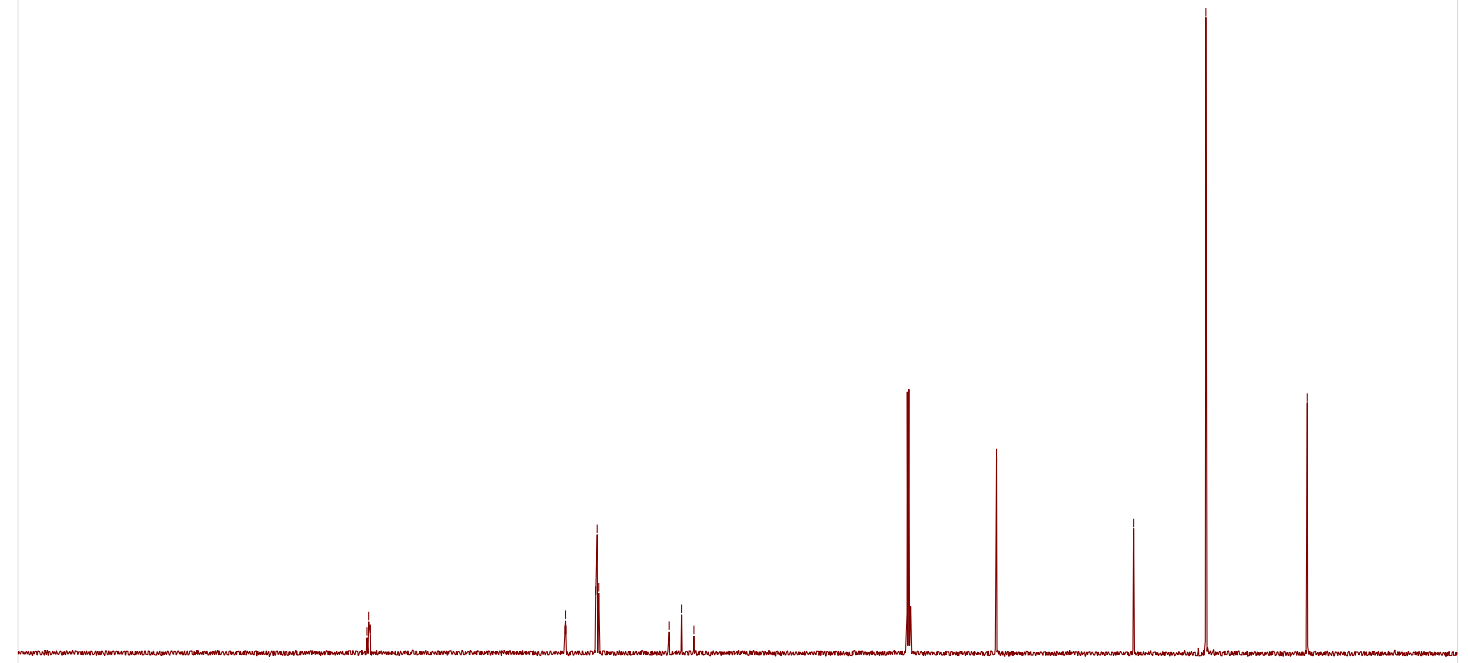

$\begin{array}{llllllllllllllllllllllllllllllllllllllll}210 & 200 & 190 & 180 & 170 & 160 & 150 & 140 & 130 & 120 & 110 & 100 & 90 & 80 & 70 & 60 & 50 & 40 & 30 & 20 & 10 & 0 & -\end{array}$ 


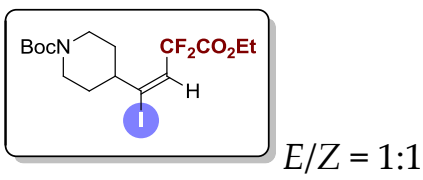

${ }^{1} \mathbf{H}$ NMR-spectrum $\left(500 \mathrm{MHz}, \mathrm{CDCl}_{3}\right)$ of $5 \mathbf{v}$

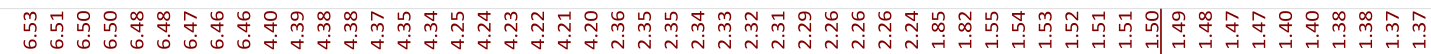

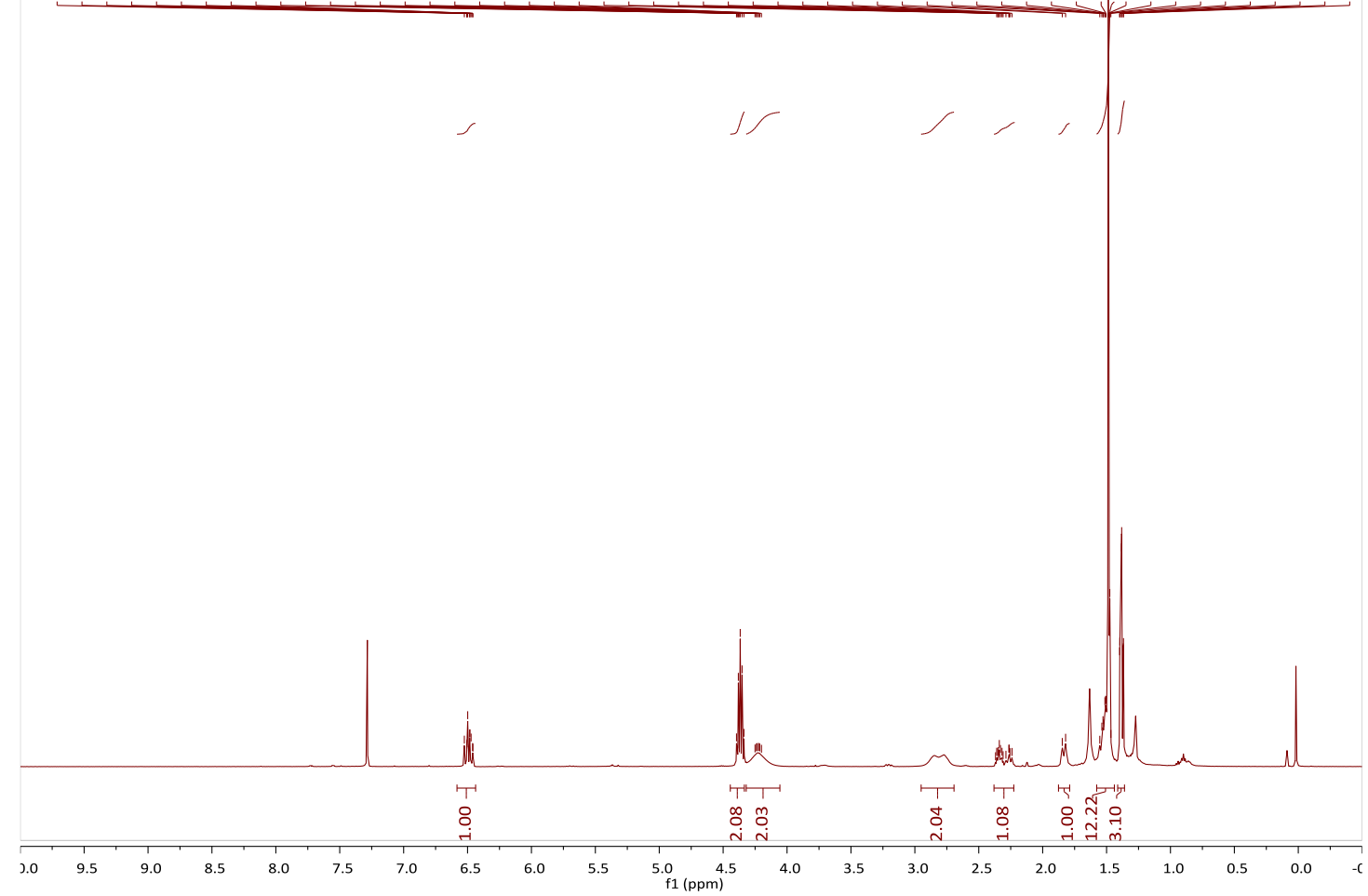

${ }^{19}$ F NMR-spectrum $\left(471 \mathrm{MHz}, \mathrm{CDCl}_{3}\right)$ of $\mathbf{5 v}$

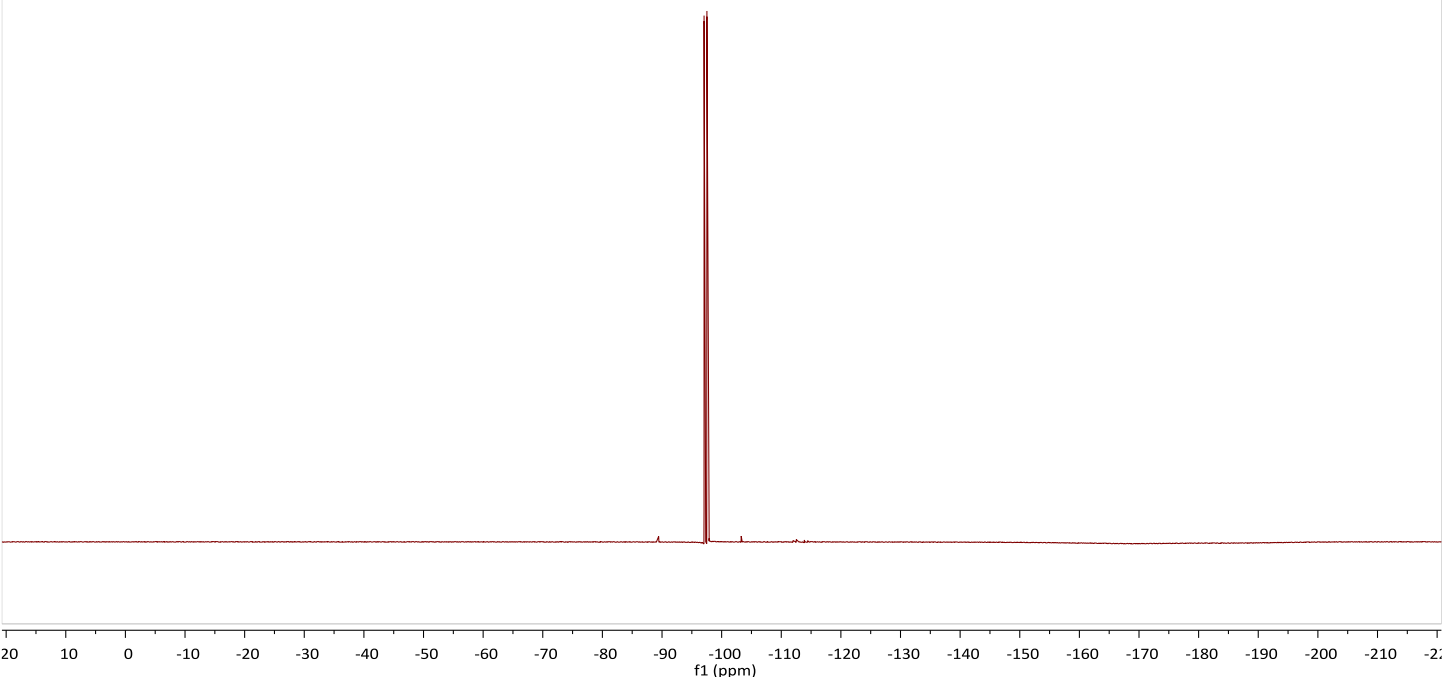




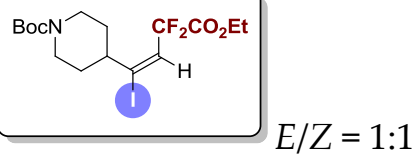

${ }^{13} \mathrm{C}$ NMR-spectrum $\left(125 \mathrm{MHz}, \mathrm{CDCl}_{3}\right)$ of $\mathbf{5 v}$

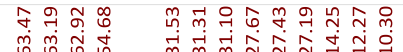

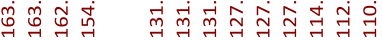

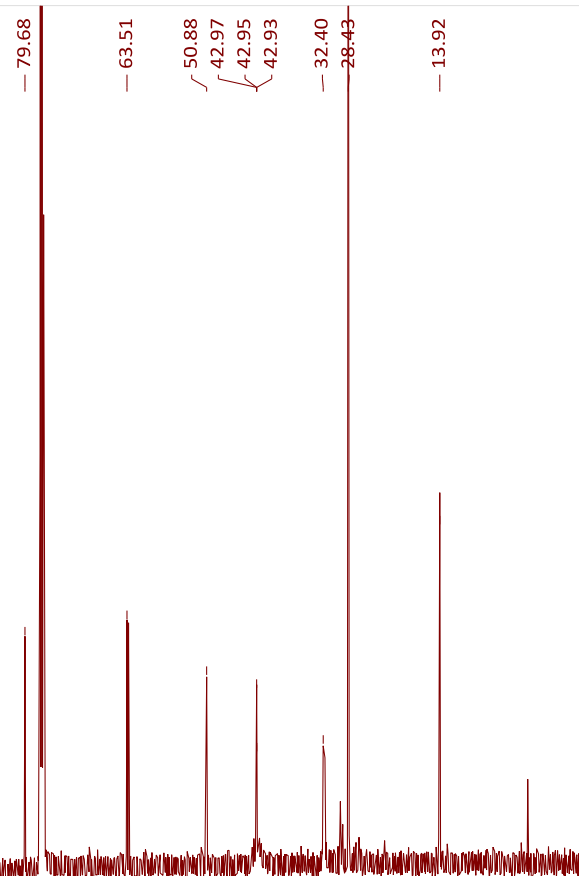

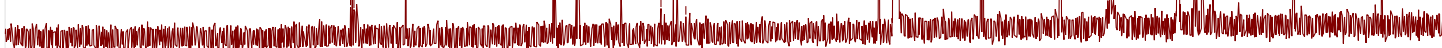

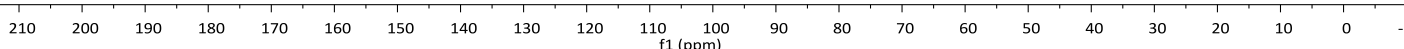




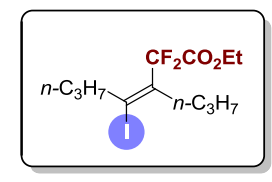

${ }^{1} \mathbf{H}$ NMR-spectrum $\left(500 \mathrm{MHz}, \mathrm{CDCl}_{3}\right)$ of $\mathbf{5 w}$

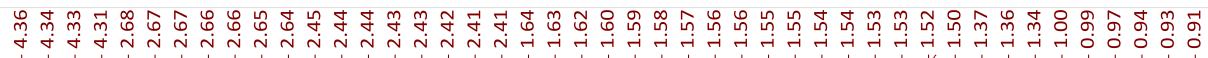

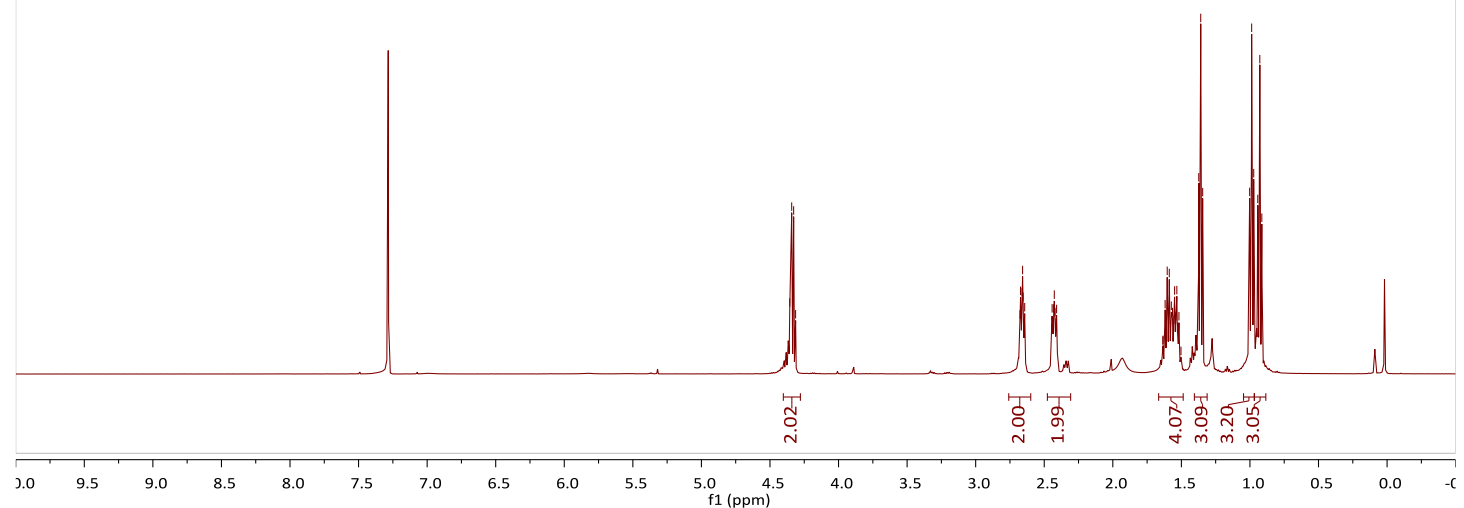

${ }^{19} \mathbf{F}$ NMR-spectrum $\left(471 \mathrm{MHz}, \mathrm{CDCl}_{3}\right)$ of $5 \mathbf{w}$

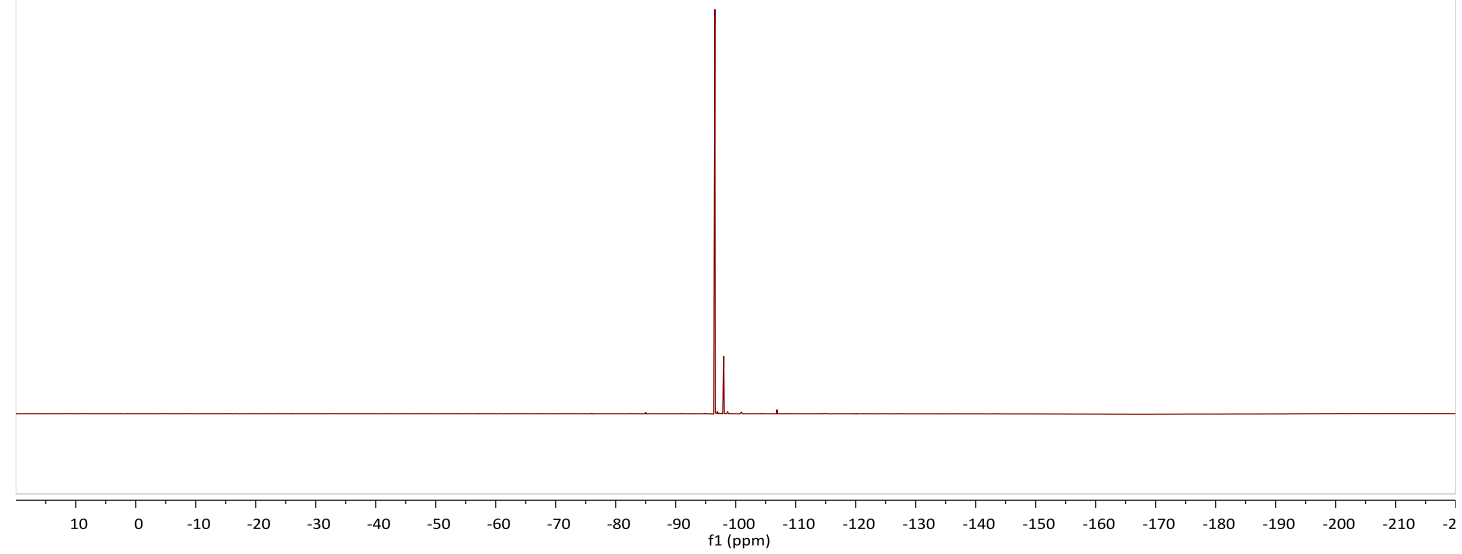




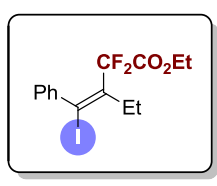

${ }^{1} \mathbf{H}$ NMR-spectrum $\left(500 \mathrm{MHz}, \mathrm{CDCl}_{3}\right)$ of $\mathbf{5 x}$

m
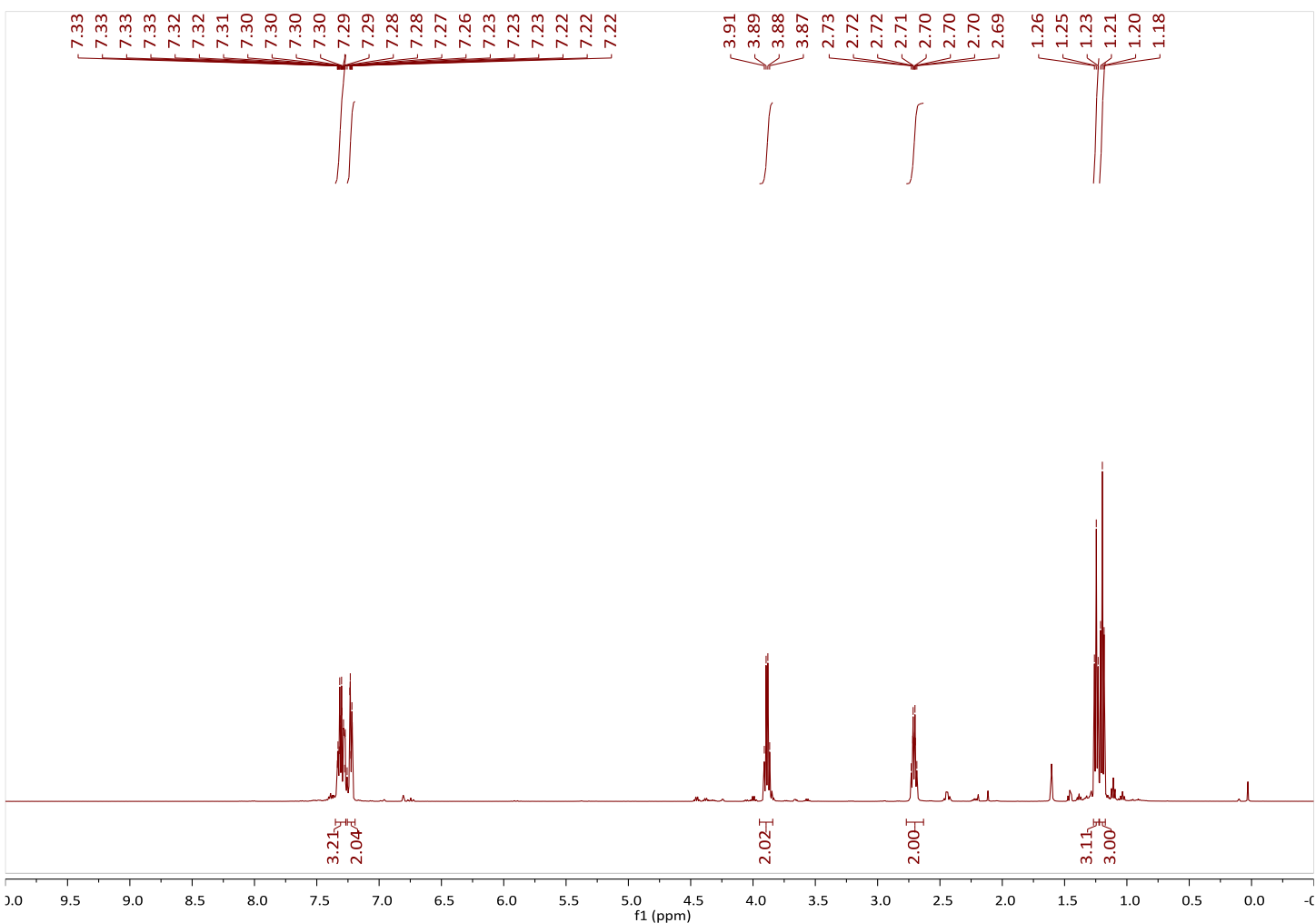

${ }^{19}$ F NMR-spectrum $\left(471 \mathrm{MHz}, \mathrm{CDCl}_{3}\right)$ of $\mathbf{5 x}$ 


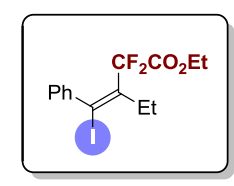

${ }^{13} \mathrm{C}$ NMR-spectrum $\left(125 \mathrm{MHz}, \mathrm{CDCl}_{3}\right)$ of $\mathbf{5 x}$

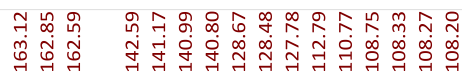

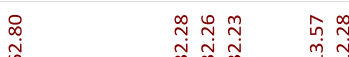

실

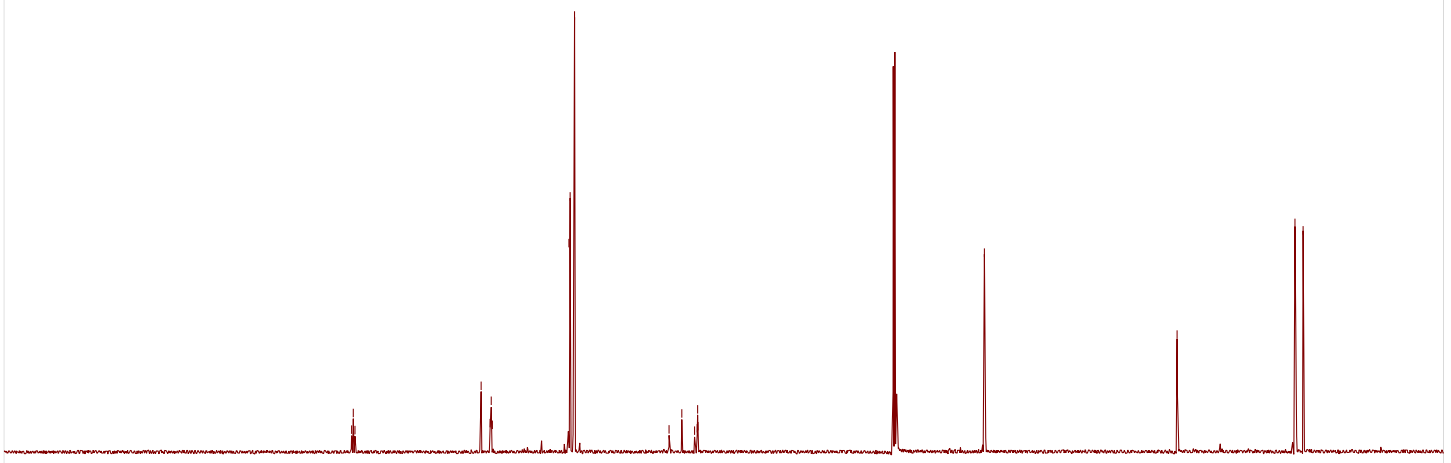

$\begin{array}{llllllllllllllllllllllllllllllllllll}210 & 200 & 190 & 180 & 170 & 160 & 150 & 140 & 130 & 120 & 110 & 100 & 90 & 80 & 70 & 60 & 50 & 40 & 30 & 20 & 10 & 0 & -\end{array}$ 


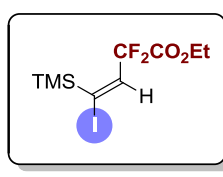

$E / Z=2: 1$

${ }^{1} \mathbf{H}$ NMR-spectrum $\left(500 \mathrm{MHz}, \mathrm{CDCl}_{3}\right)$ of $5 \mathbf{y}$

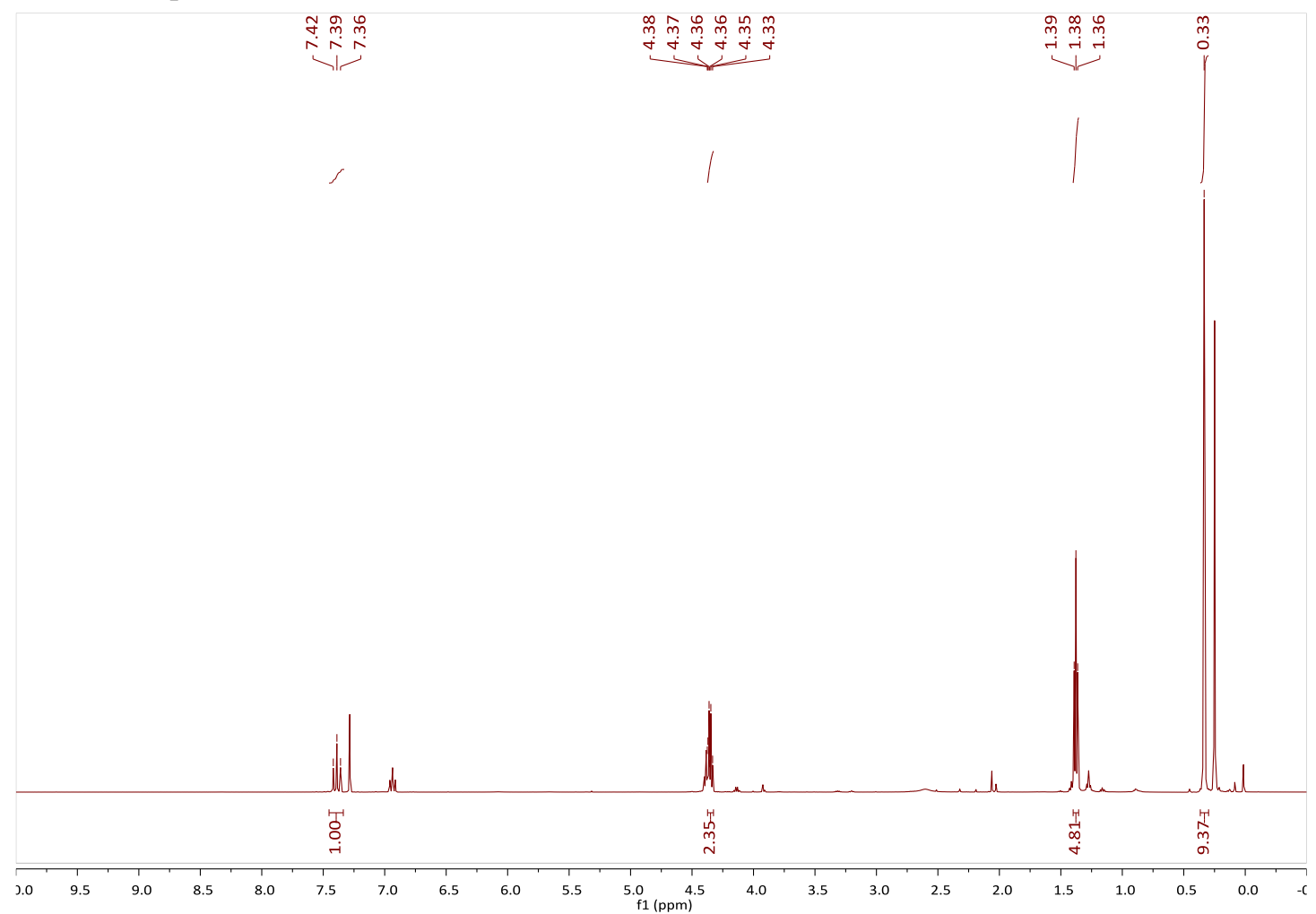

${ }^{19}$ F NMR-spectrum $\left(471 \mathrm{MHz}, \mathrm{CDCl}_{3}\right)$ of $5 \mathbf{y}$

$$
\stackrel{\substack{0 \\ \stackrel{\leftrightarrow}{i}}}{i}
$$

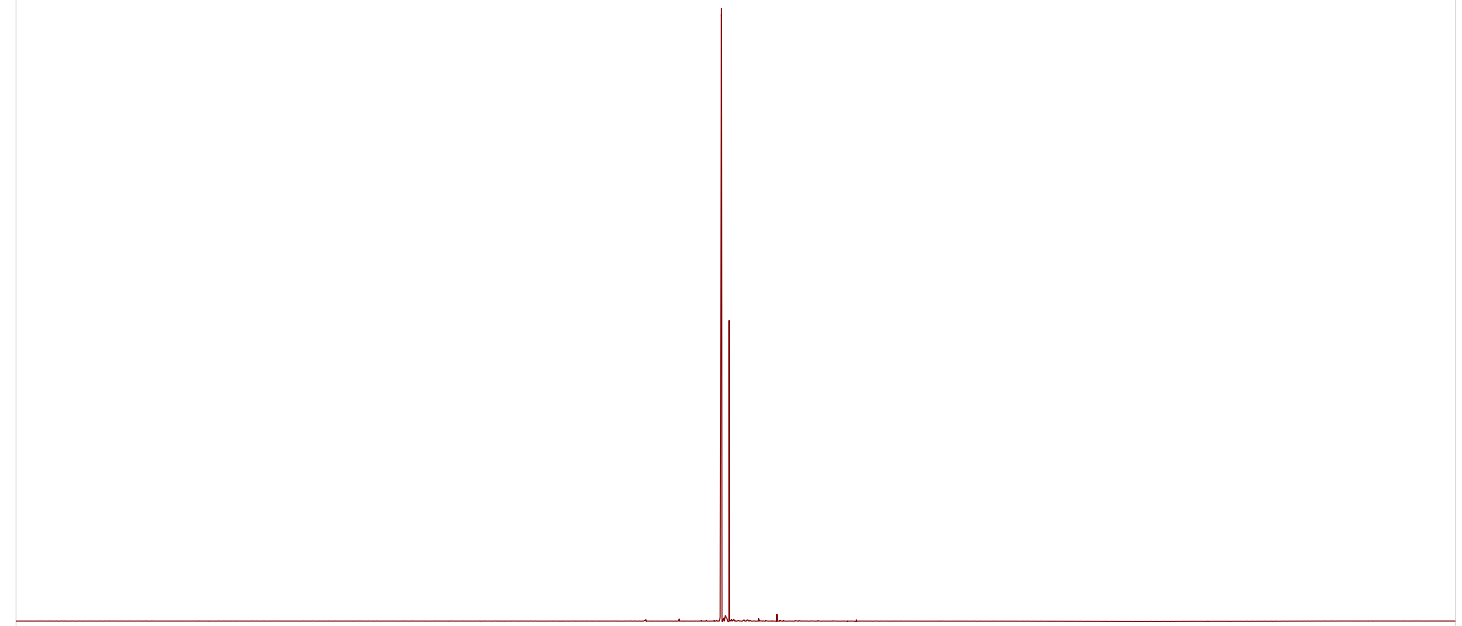

$\begin{array}{lllllllllllllllllllllllllllllllll}10 & 0 & -10 & -20 & -30 & -40 & -50 & -60 & -70 & -80 & -90 & -100 & -110 & -120 & -130 & -140 & -150 & -160 & -170 & -180 & -190 & -200 & -210 & -2\end{array}$ 


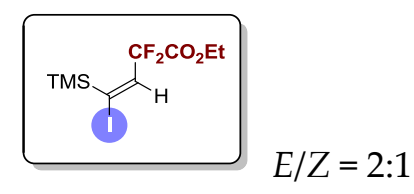

${ }^{13} \mathbf{C ~ N M R}$-spectrum (125 MHz, $\left.\mathrm{CDCl}_{3}\right)$ of $5 \mathbf{y}$

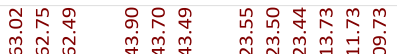

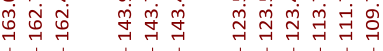

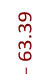

$\stackrel{\substack{\infty \\ \stackrel{0}{i}}}{\stackrel{\sim}{1}}$

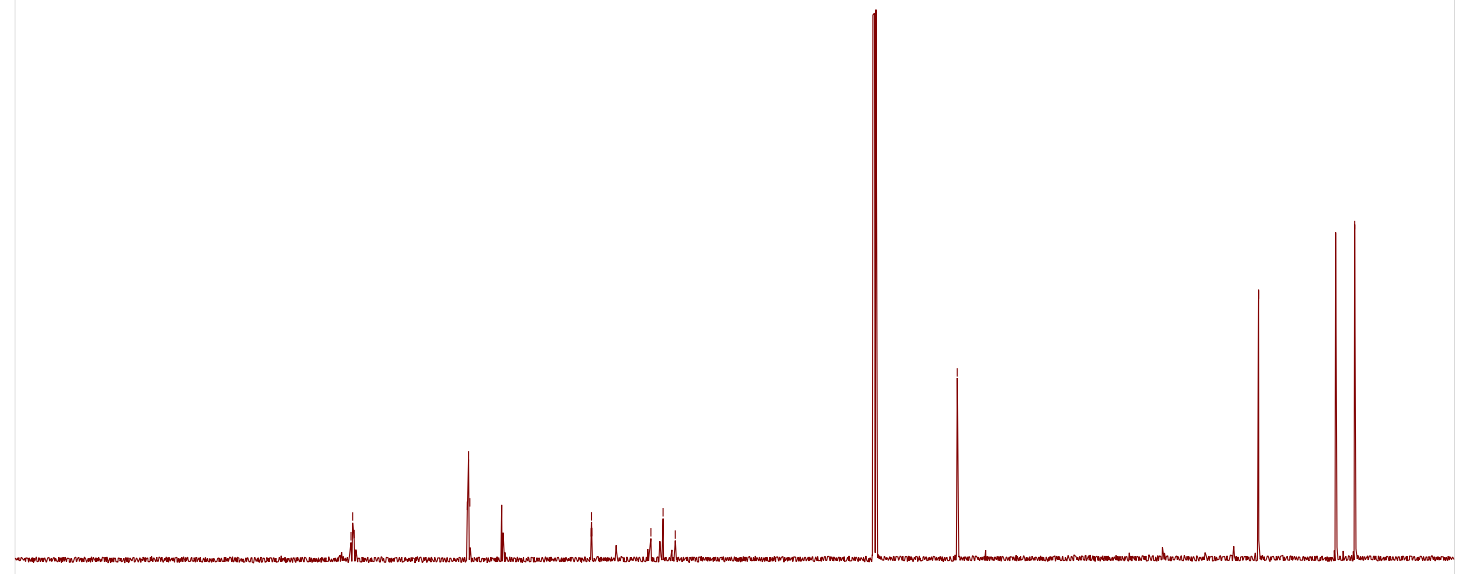

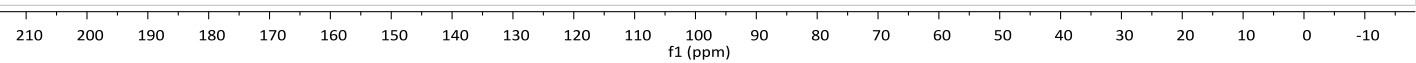




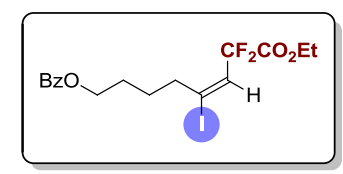

$E / Z=5: 1$

${ }^{1} \mathbf{H}$ NMR-spectrum $\left(500 \mathrm{MHz}, \mathrm{CDCl}_{3}\right)$ of $5 \mathbf{z}$

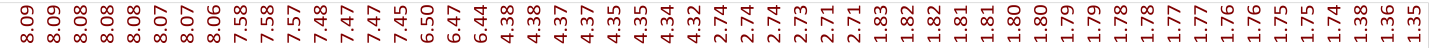
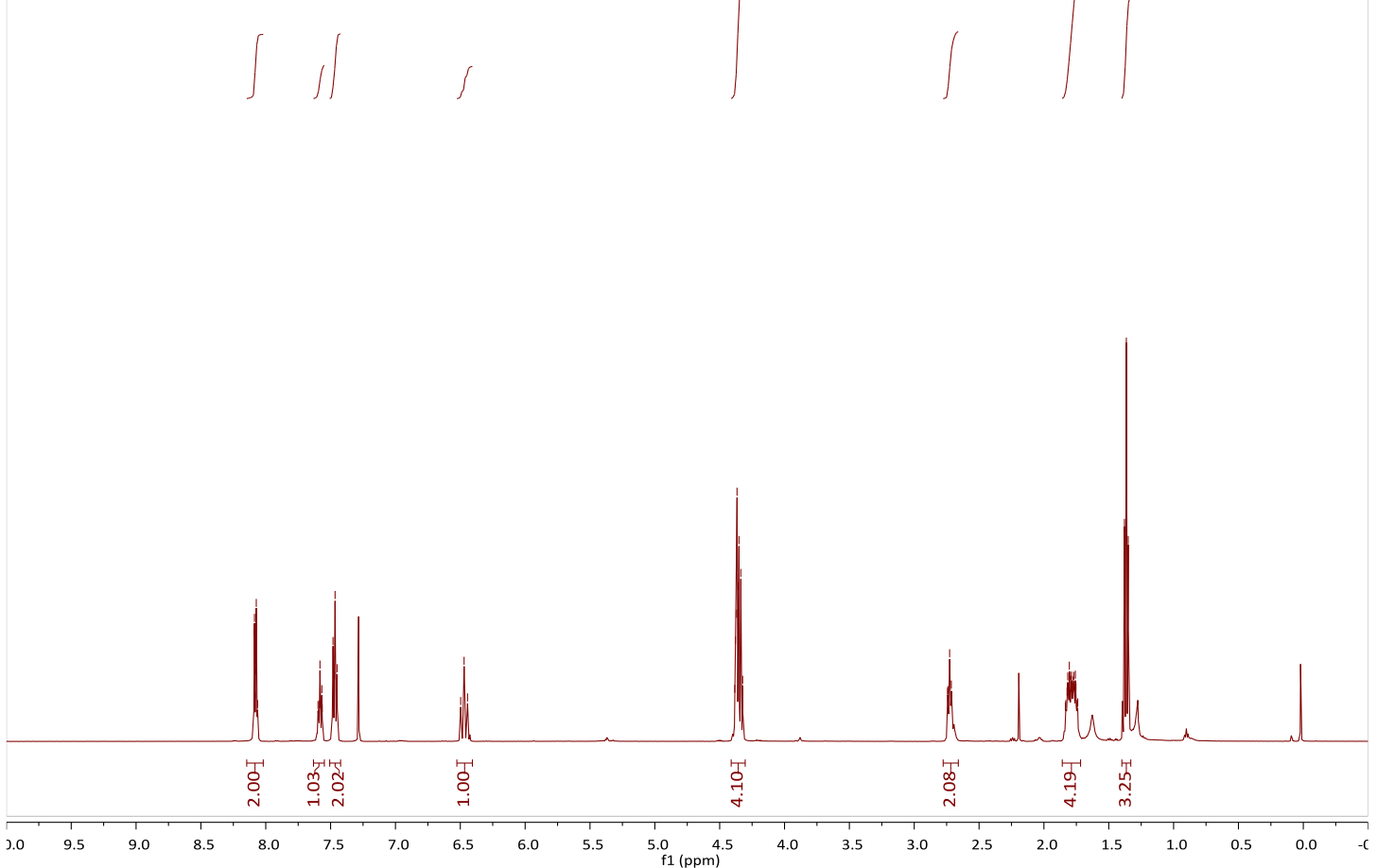

${ }^{19} \mathbf{F}$ NMR-spectrum $\left(471 \mathrm{MHz}, \mathrm{CDCl}_{3}\right)$ of $\mathbf{5 z}$

$$
\text { ํ. }
$$

$\begin{array}{llllllllllllllllllllllll}10 & 0 & -10 & -20 & -30 & -40 & -50 & -60 & -70 & -80 & -90 & -100 & -110 & -120 & -130 & -140 & -150 & -160 & -170 & -180 & -190 & -200 & -210 & -2\end{array}$ 


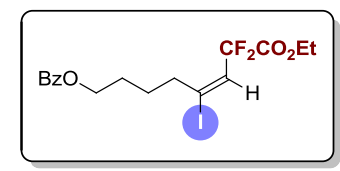

$E / Z=5: 1$

${ }^{13} \mathrm{C}$ NMR-spectrum (125 MHz, $\left.\mathrm{CDCl}_{3}\right)$ of $\mathbf{5 z}$

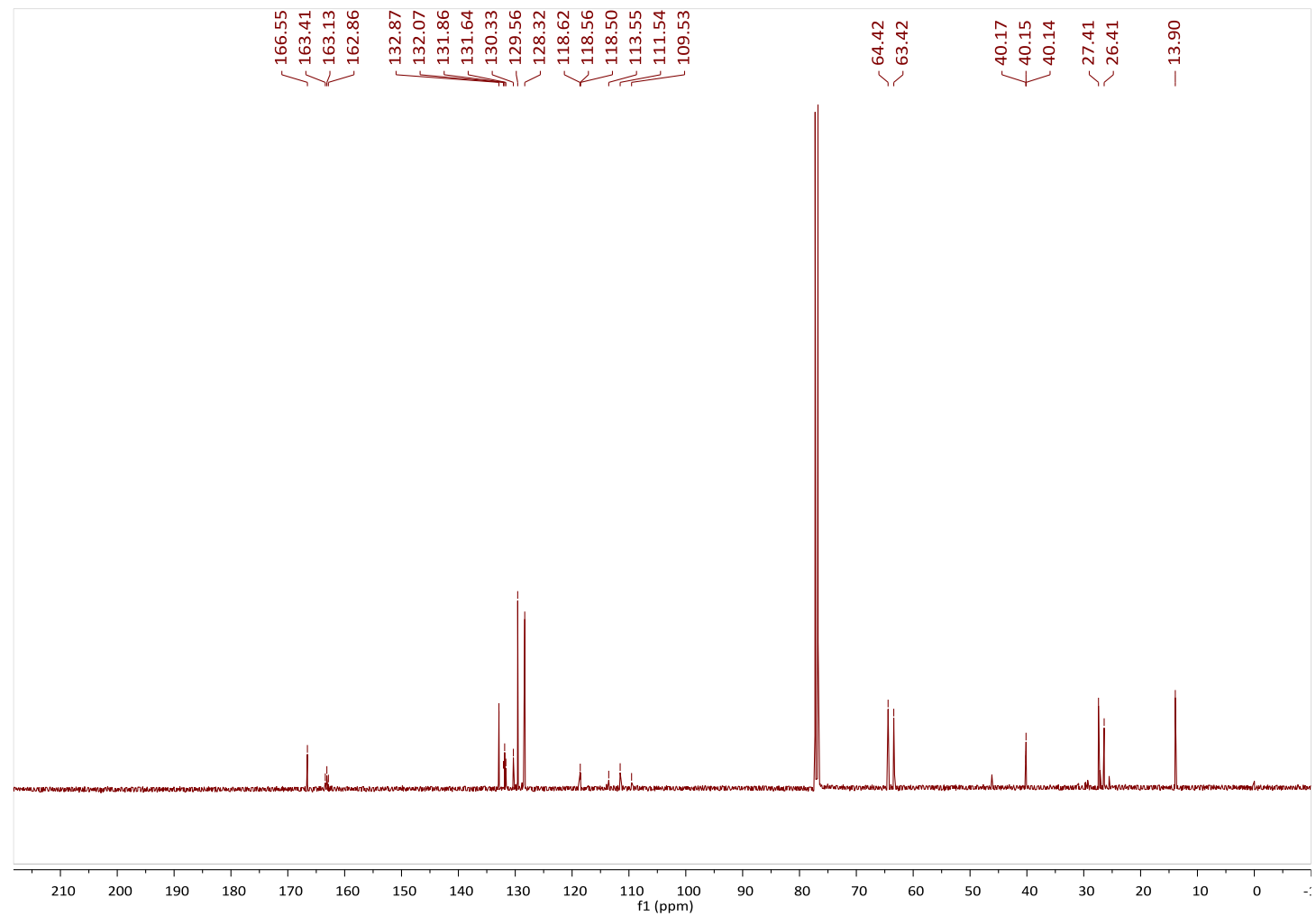




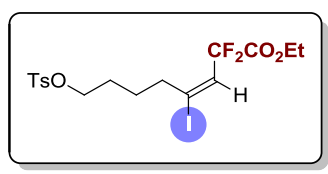

$E / Z=4: 1$

${ }^{1} \mathbf{H}$ NMR-spectrum $\left(500 \mathrm{MHz}, \mathrm{CDCl}_{3}\right)$ of $5 \mathbf{a a}$

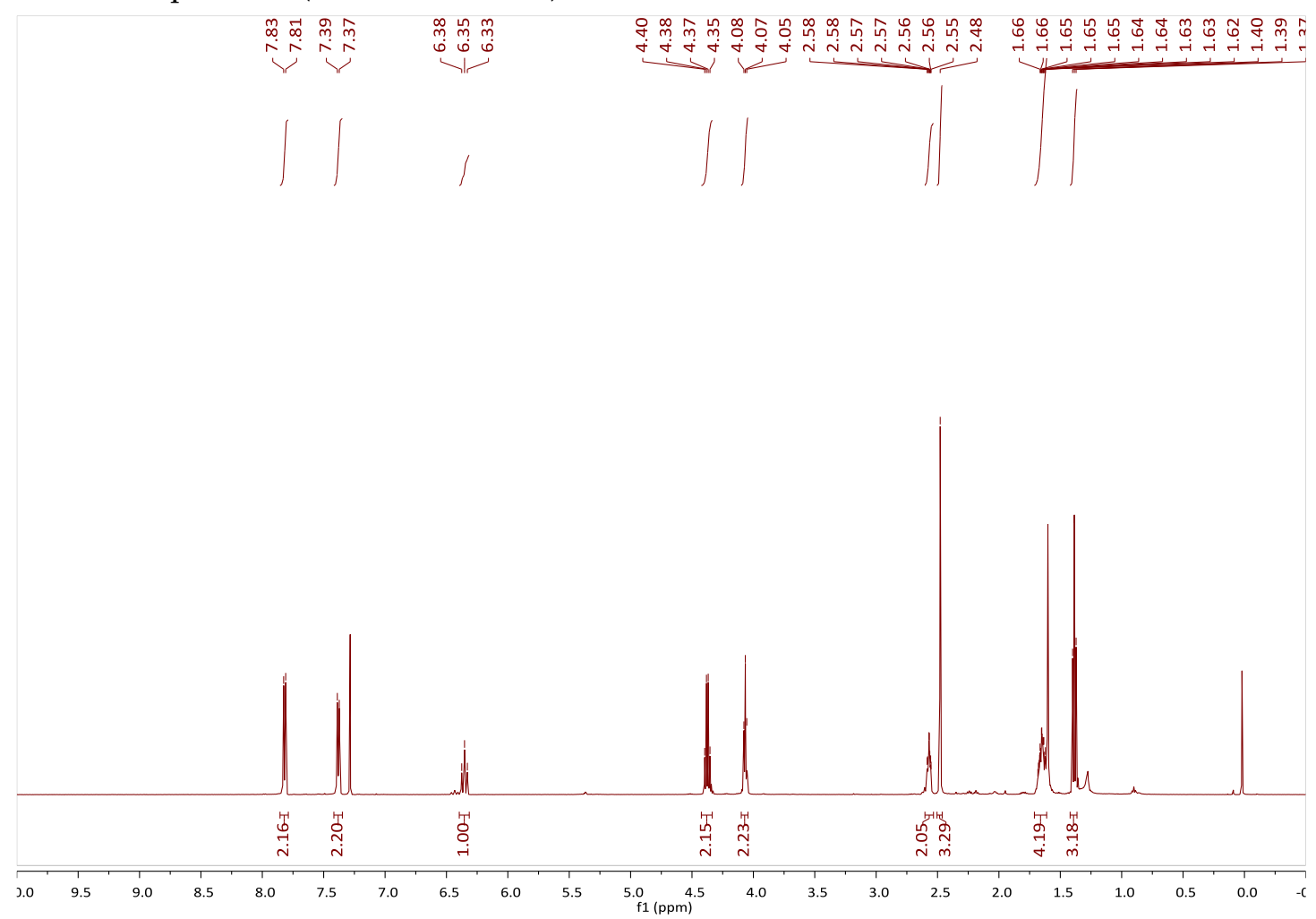

${ }^{19} \mathbf{F}$ NMR-spectrum $\left(471 \mathrm{MHz}, \mathrm{CDCl}_{3}\right)$ of $\mathbf{5 a a}$ 


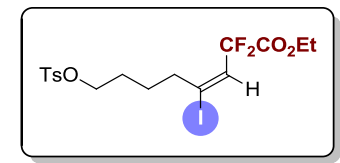

$E / Z=4: 1$

${ }^{13} \mathrm{C}$ NMR-spectrum (125 MHz, $\left.\mathrm{CDCl}_{3}\right)$ of $\mathbf{5 a a}$

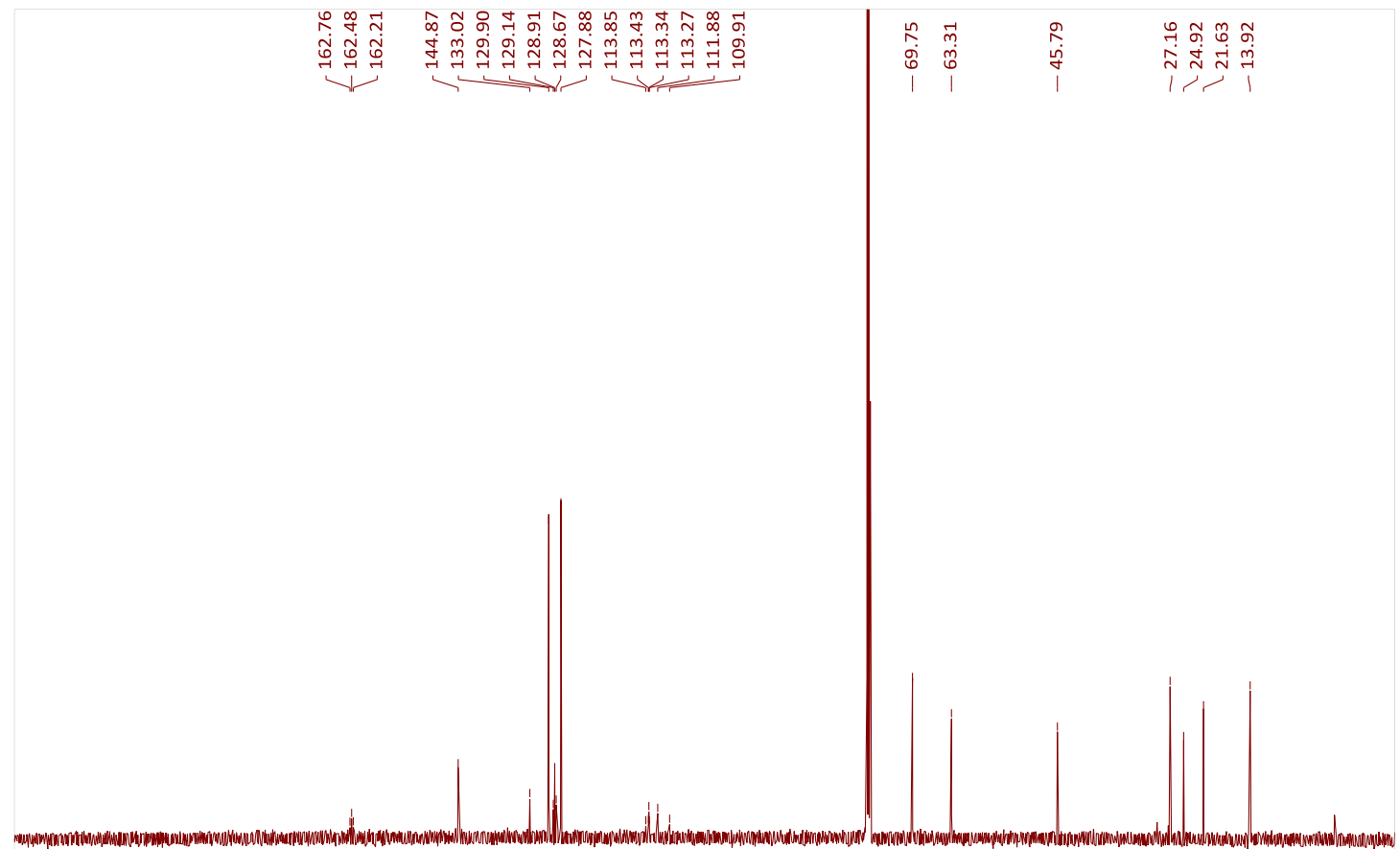

$\begin{array}{llllllllllll}210 & 200 & 190 & 180 & 170 & 160 & 150 & 140 & 130 & 120 & 110 & 100\end{array}$ 


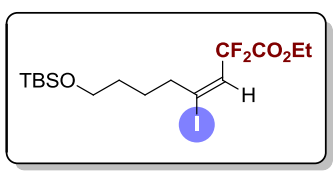

$$
E / Z=3: 1
$$

${ }^{1} \mathbf{H}$ NMR-spectrum $\left(500 \mathrm{MHz}, \mathrm{CDCl}_{3}\right)$ of $5 \mathbf{a b}$

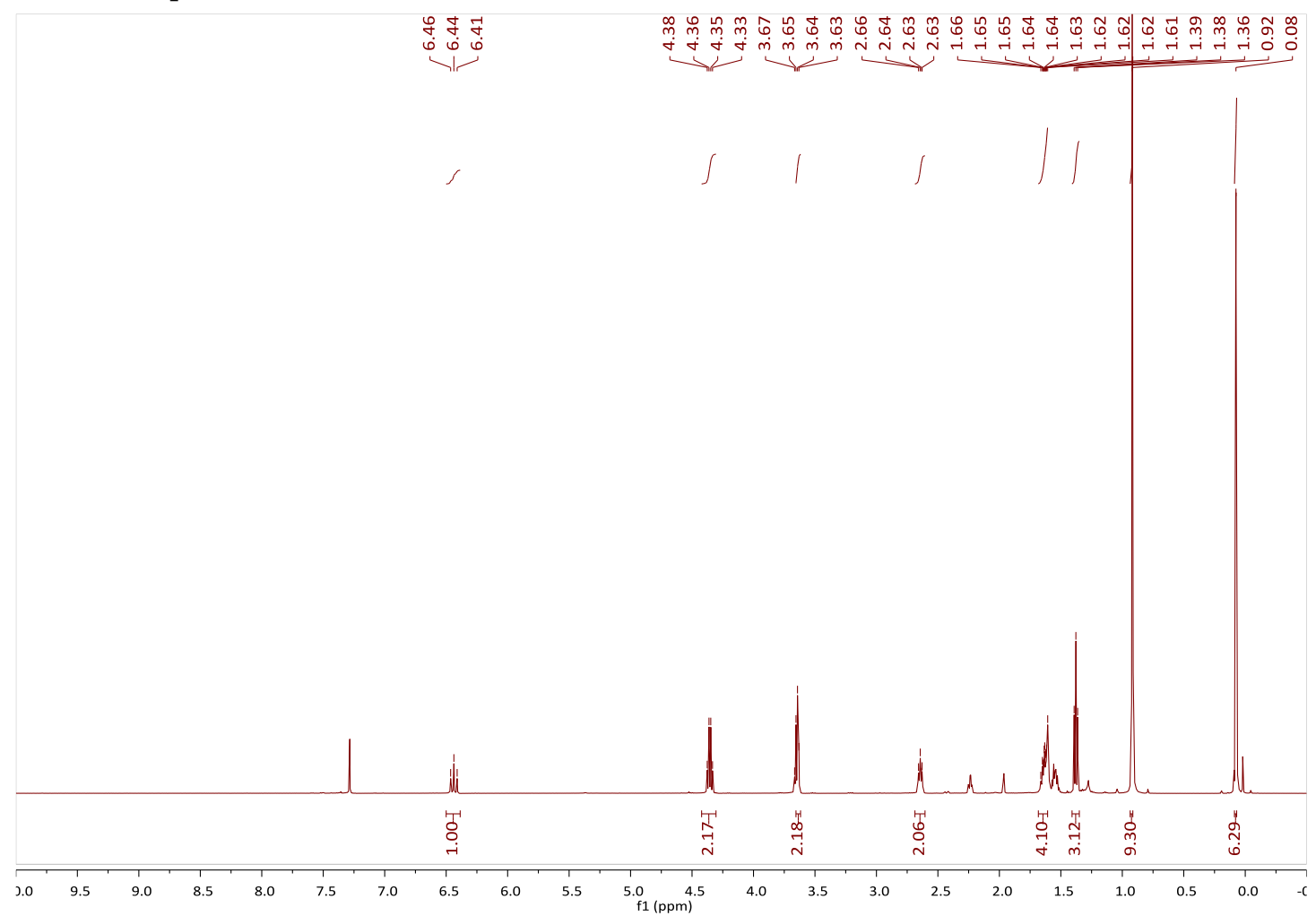

${ }^{19}$ F NMR-spectrum $\left(471 \mathrm{MHz}, \mathrm{CDCl}_{3}\right)$ of $5 \mathbf{a b}$

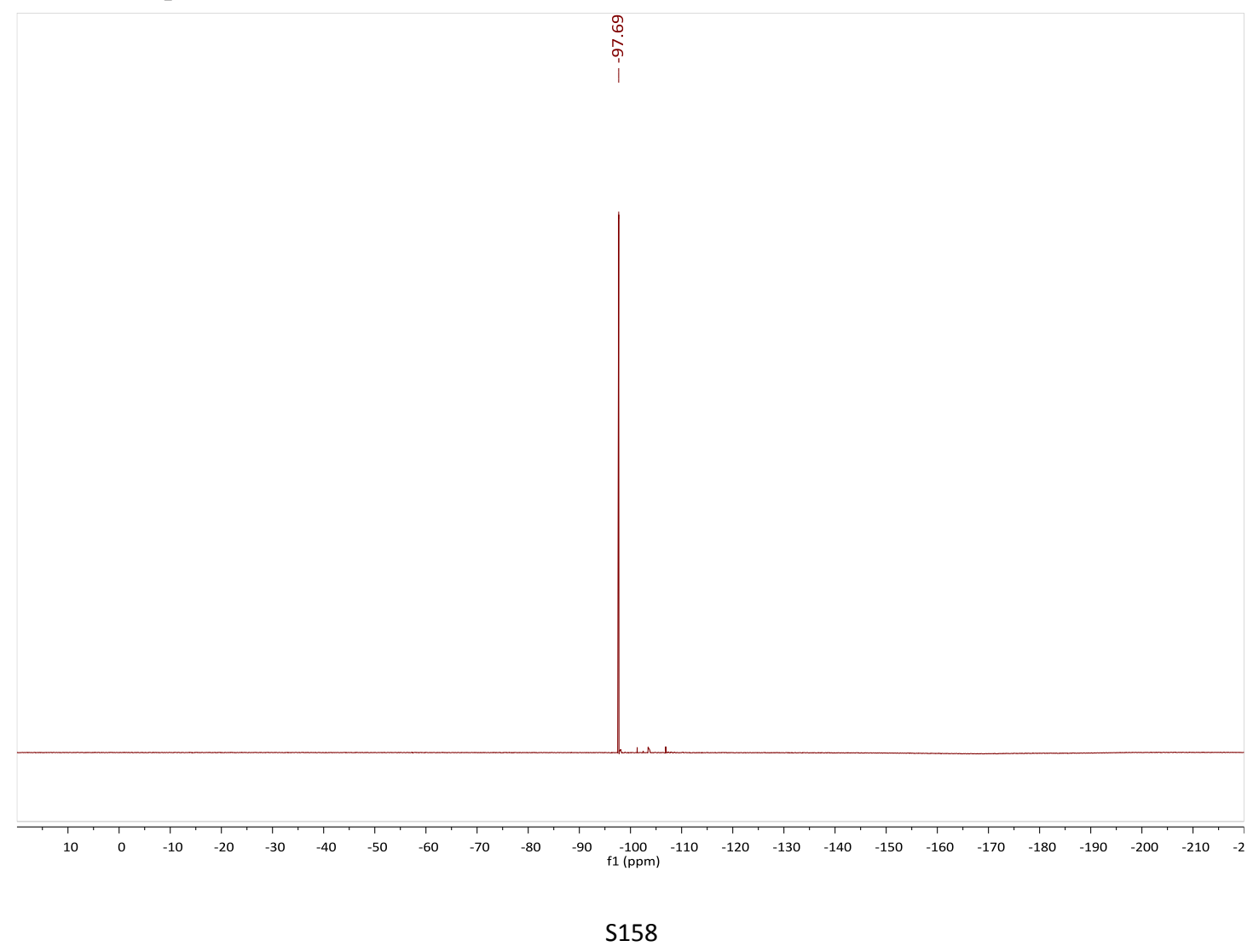




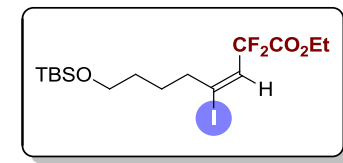

$E / Z=3: 1$

${ }^{13} \mathrm{C}$ NMR-spectrum (125 MHz, $\left.\mathrm{CDCl}_{3}\right)$ of $5 \mathbf{a b}$

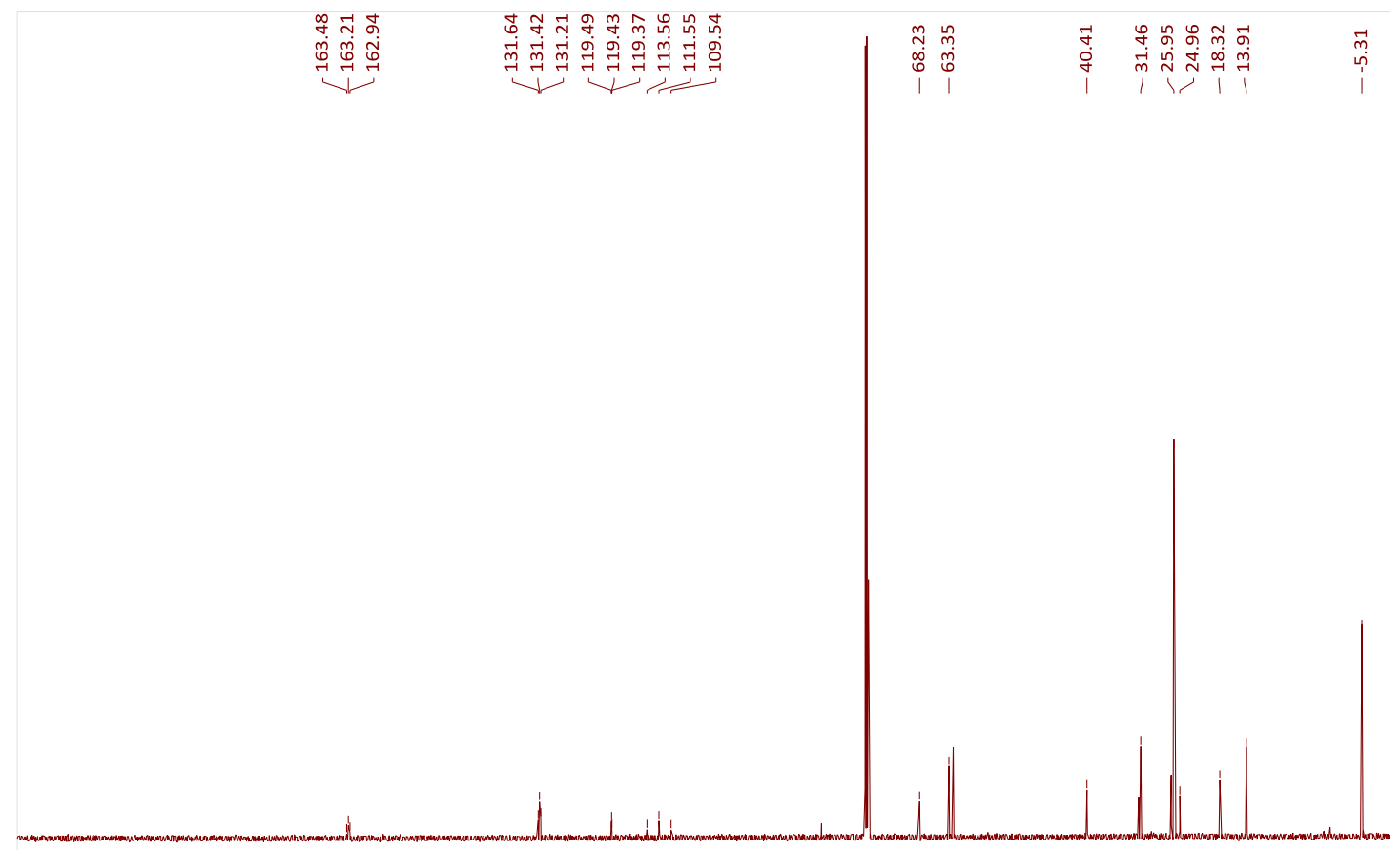

$\begin{array}{lllllllllllllllllllllllllllll}210 & 200 & 190 & 180 & 170 & 160 & 150 & 140 & 130 & 120 & 110 & 100 & 90 & 80 & 70 & 60 & 50 & 40 & 30 & 20 & 10 & 0 & \end{array}$ 


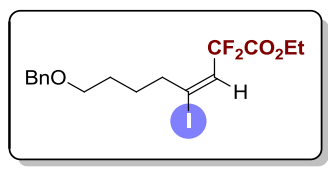

$E / Z=3: 1$

${ }^{1} \mathbf{H}$ NMR-spectrum $\left(500 \mathrm{MHz}, \mathrm{CDCl}_{3}\right)$ of $5 \mathbf{a c}$

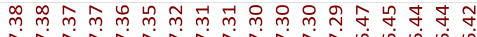

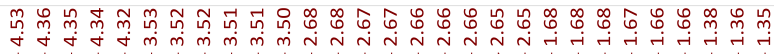

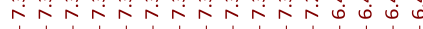
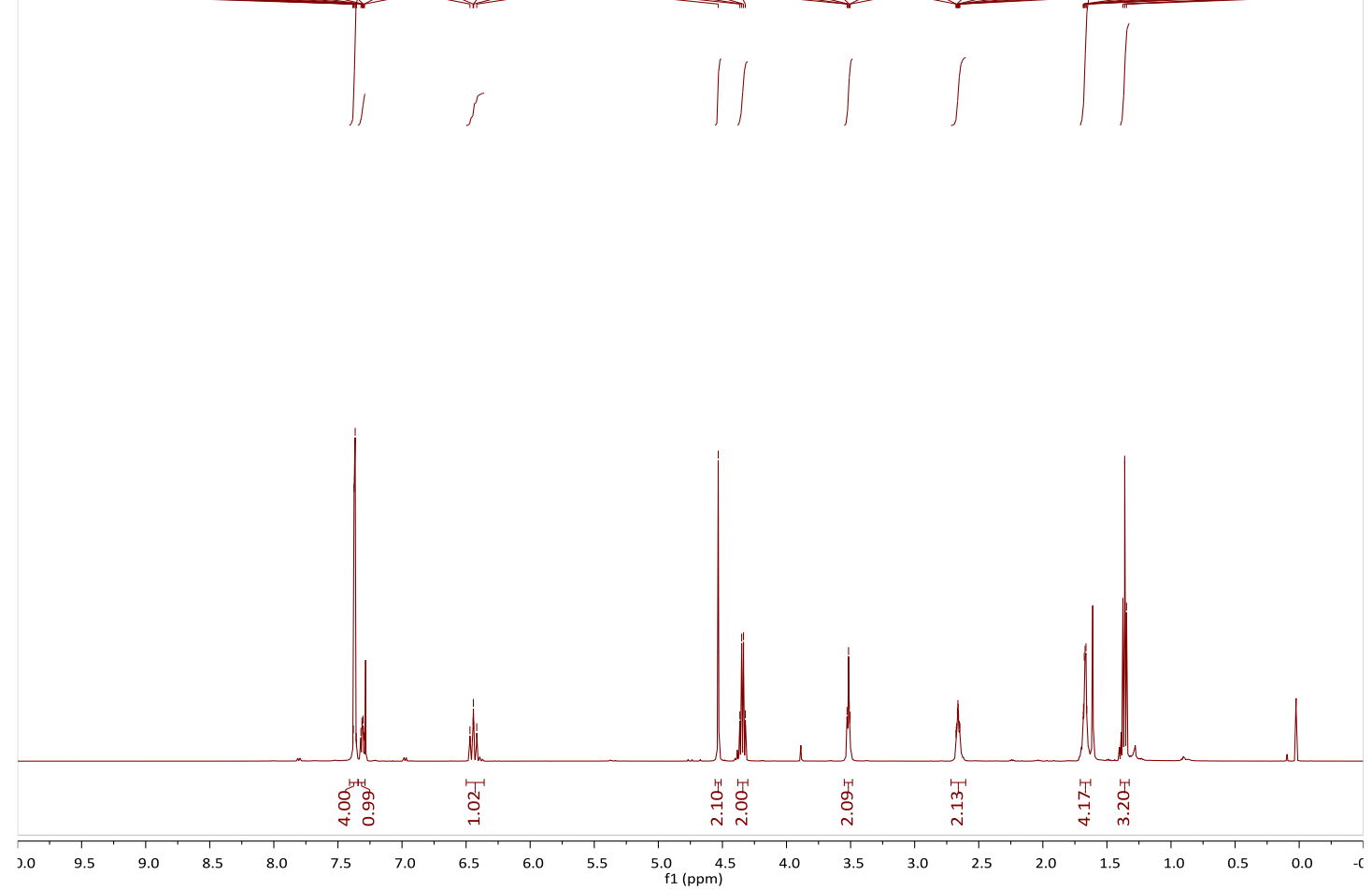

${ }^{19}$ F NMR-spectrum $\left(471 \mathrm{MHz}, \mathrm{CDCl}_{3}\right)$ of $5 \mathbf{a c}$

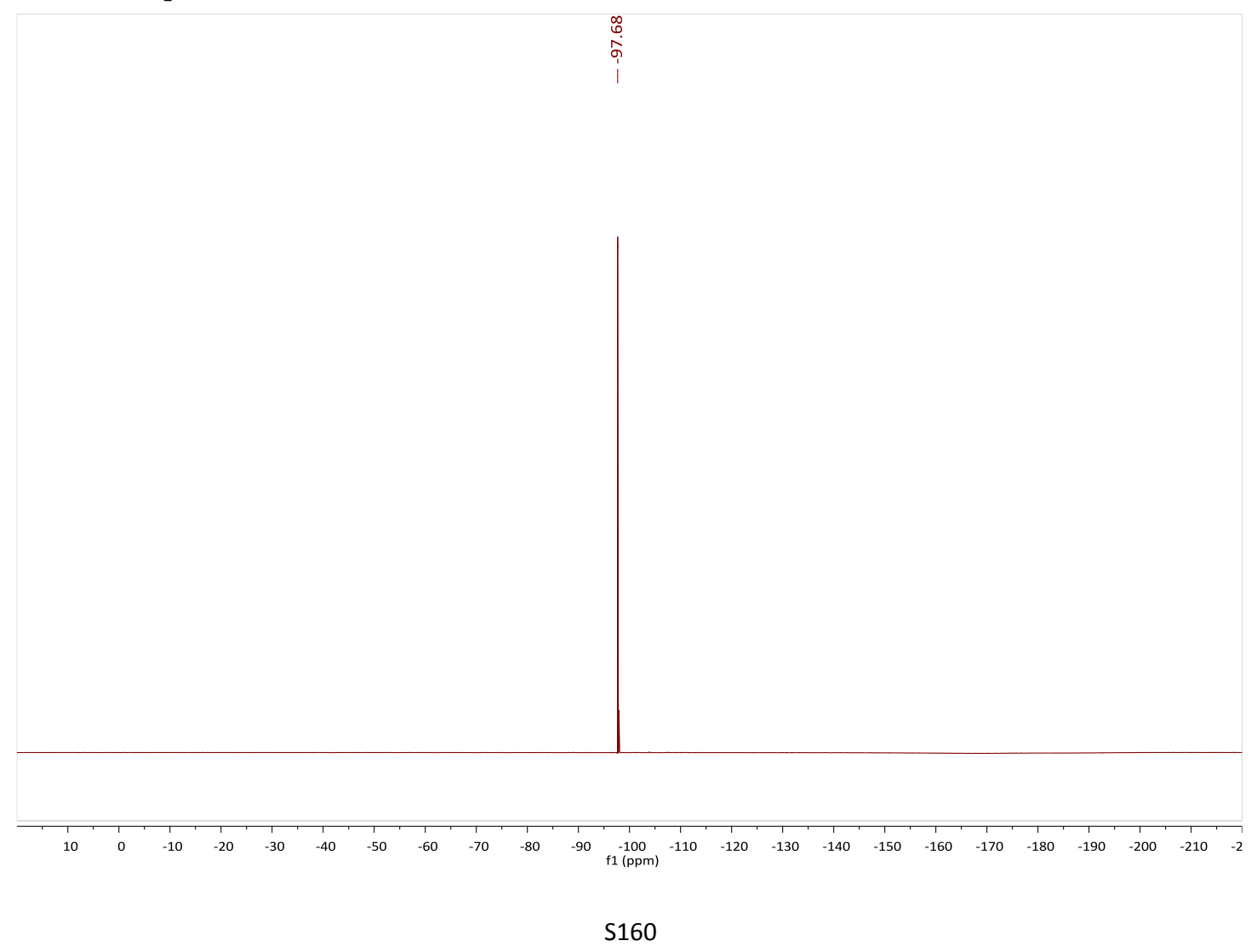




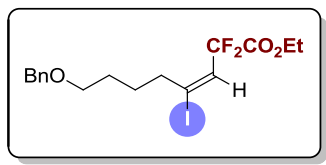

$E / Z=3: 1$

${ }^{13} \mathrm{C}$ NMR-spectrum $\left(125 \mathrm{MHz}, \mathrm{CDCl}_{3}\right)$ of $5 \mathrm{ac}$

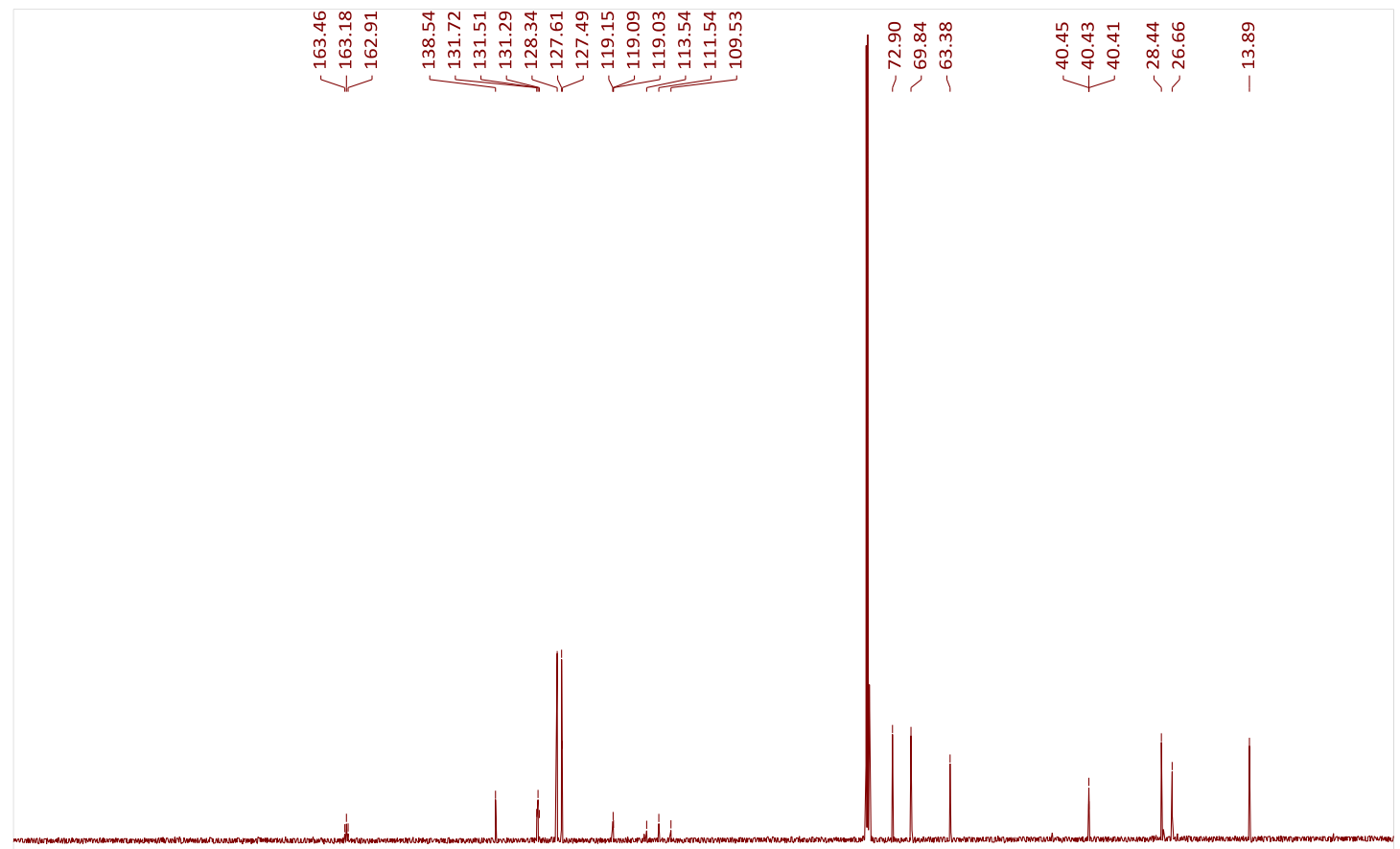

$\begin{array}{lllllllllllllllllllllllll}210 & 200 & 190 & 180 & 170 & 160 & 150 & 140 & 130 & 120 & 110 & 100 & 90 & 80 & 70 & 60 & 50 & 40 & 30 & 20 & 10 & 0 & \end{array}$ 


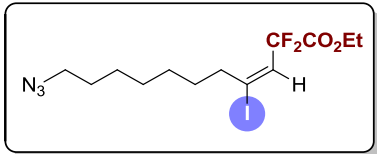

${ }^{1} \mathbf{H}$ NMR-spectrum $\left(500 \mathrm{MHz}, \mathrm{CDCl}_{3}\right)$ of $\mathbf{5 a d}$

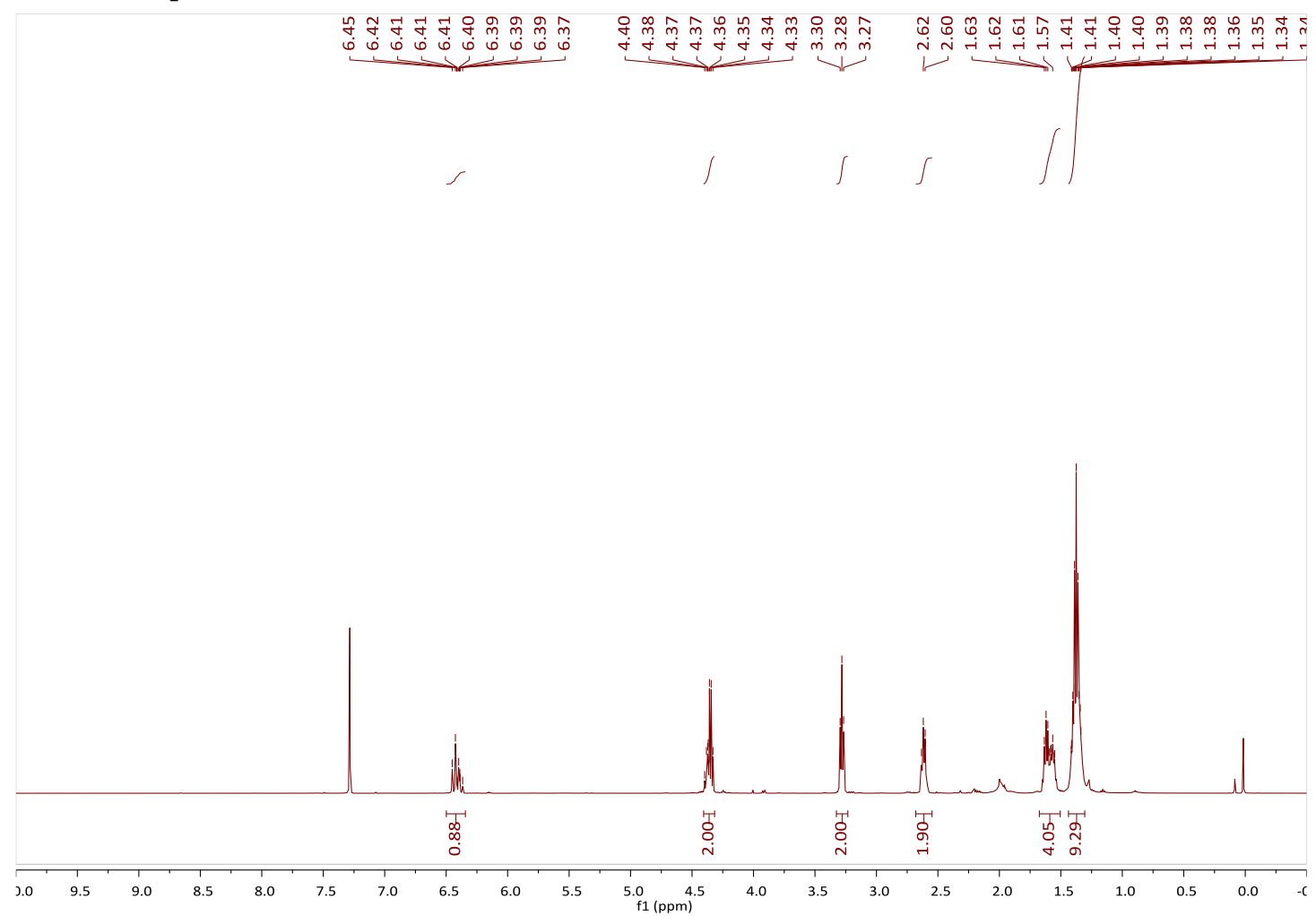

${ }^{19} \mathbf{F}$ NMR-spectrum $\left(471 \mathrm{MHz}, \mathrm{CDCl}_{3}\right)$ of $\mathbf{5 a d}$

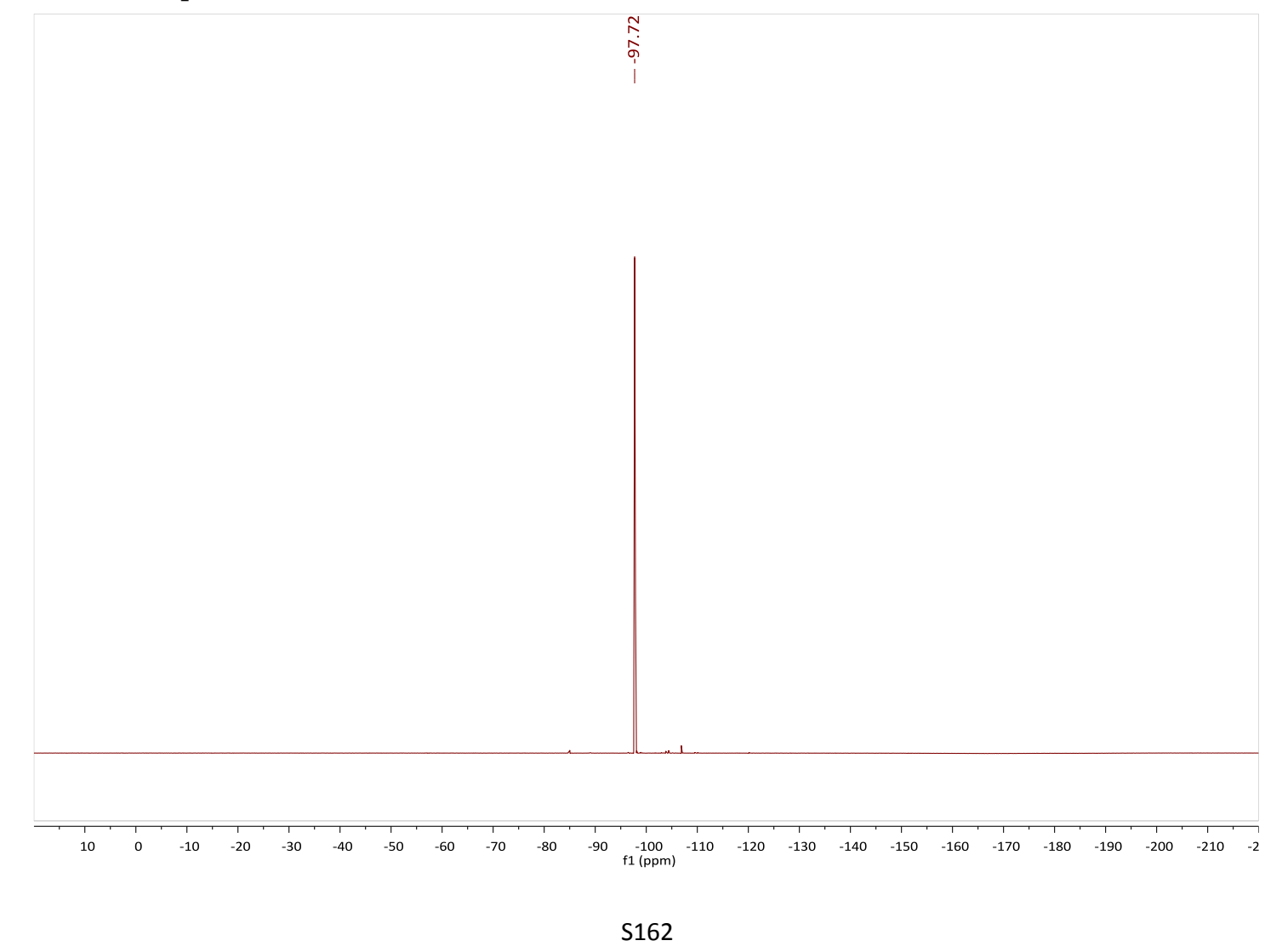




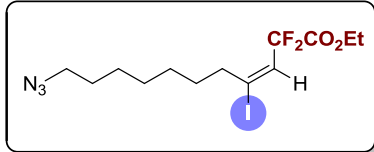

${ }^{13} \mathrm{C}$ NMR-spectrum (125 MHz, $\left.\mathrm{CDCl}_{3}\right)$ of 5 ad

莳示昌

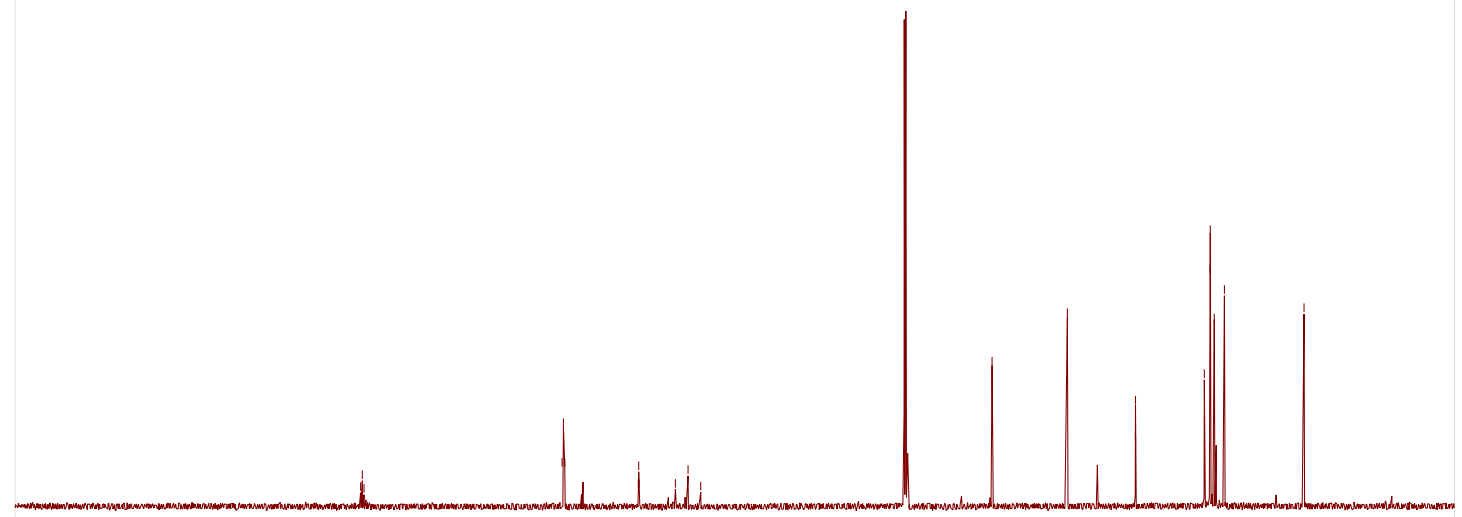

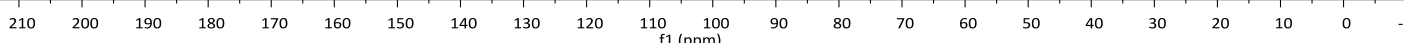




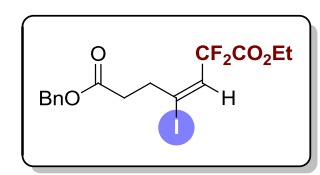

$E / Z=3: 1$

${ }^{1} \mathbf{H}$ NMR-spectrum $\left(500 \mathrm{MHz}, \mathrm{CDCl}_{3}\right)$ of $5 \mathbf{a e}$

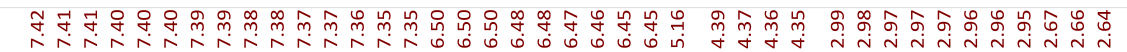

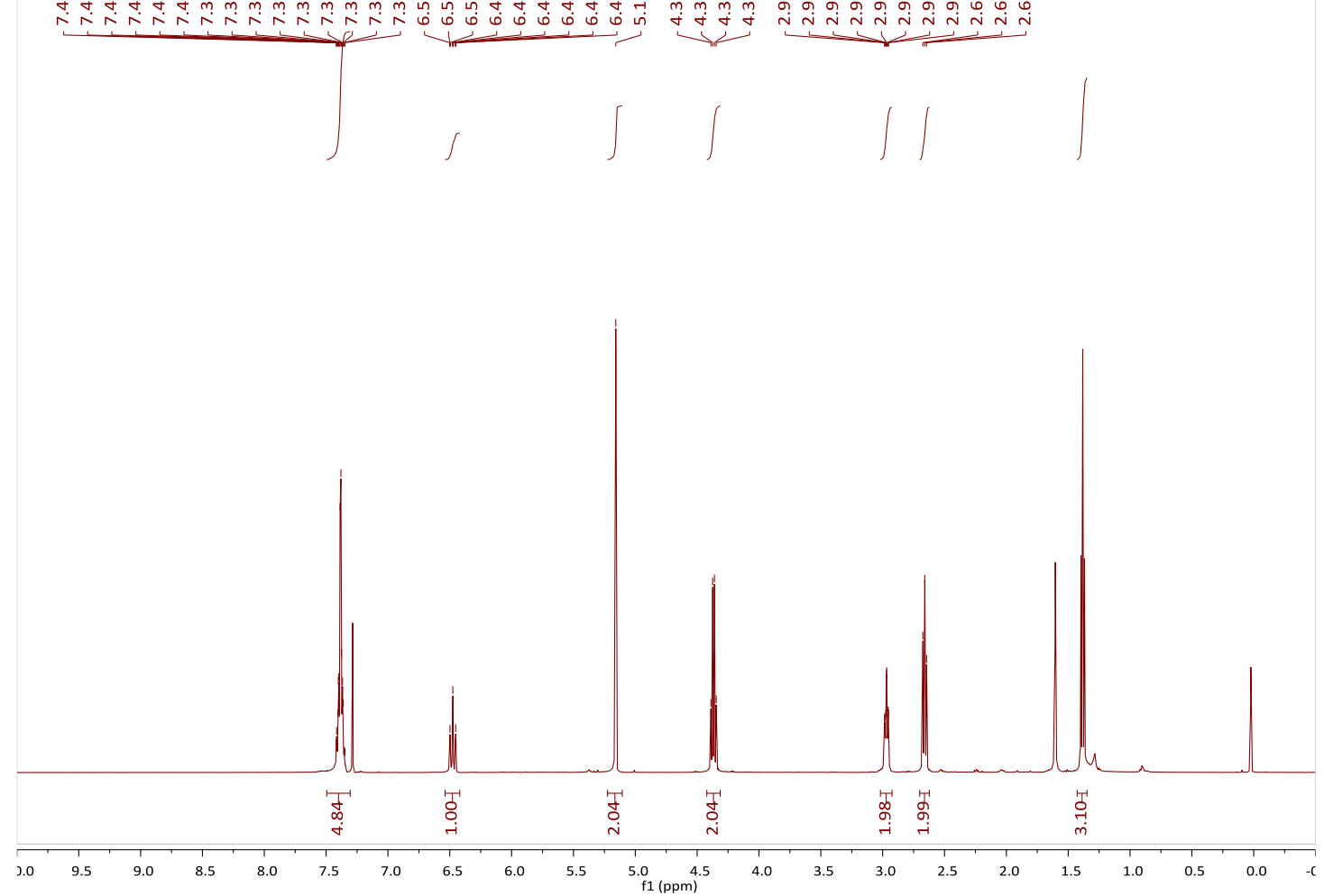

${ }^{19} \mathrm{~F}$ NMR-spectrum $\left(471 \mathrm{MHz}, \mathrm{CDCl}_{3}\right)$ of $\mathbf{5 a e}$

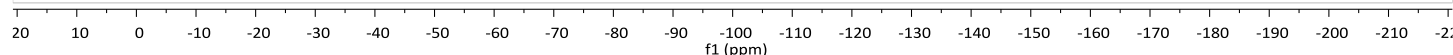




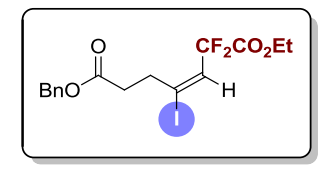

$E / Z=3: 1$

${ }^{13} \mathrm{C}$ NMR-spectrum $\left(125 \mathrm{MHz}, \mathrm{CDCl}_{3}\right)$ of $5 \mathbf{a e}$

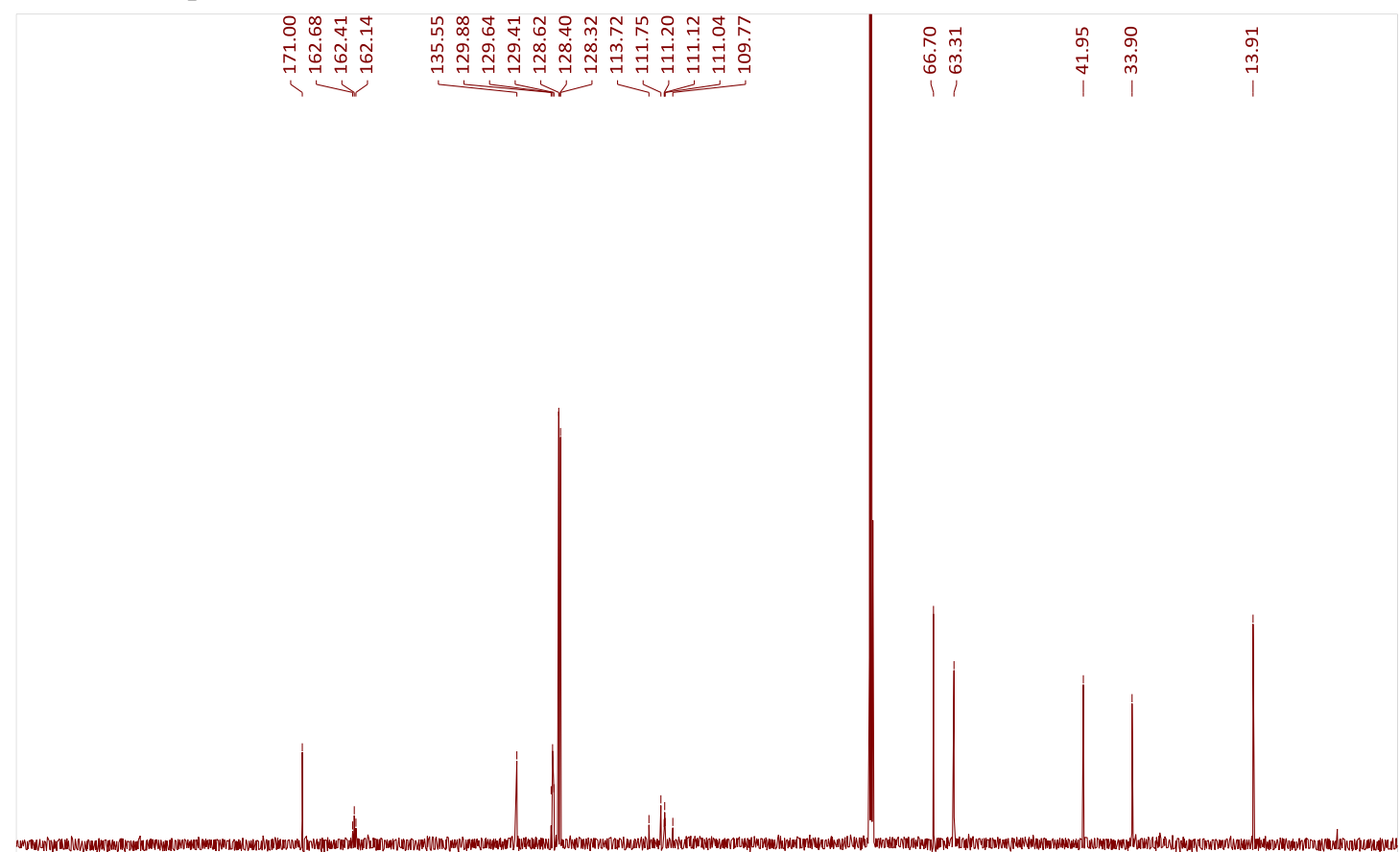

$\begin{array}{llllllllllllllllllllllllllll}210 & 200 & 190 & 180 & 170 & 160 & 150 & 140 & 130 & 120 & 110 & 100 & 90 & 80 & 70 & 60 & 50 & 40 & 30 & 20 & 10 & 0\end{array}$ 


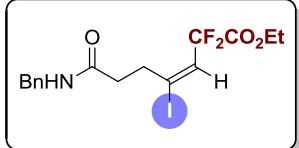

${ }^{1} \mathbf{H}$ NMR-spectrum $\left(500 \mathrm{MHz}, \mathrm{CDCl}_{3}\right)$ of 5 af

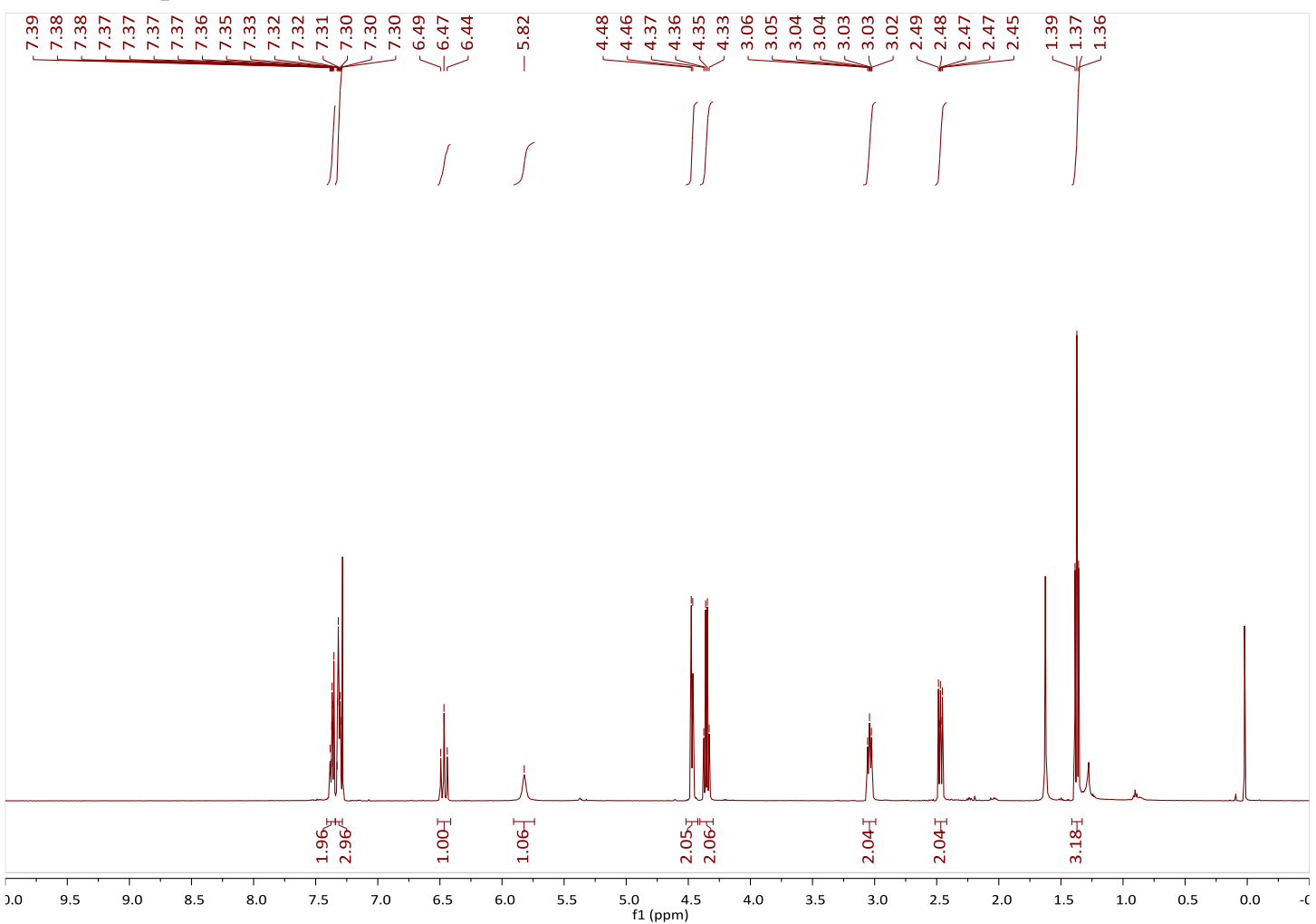

${ }^{19}$ F NMR-spectrum $\left(471 \mathrm{MHz}, \mathrm{CDCl}_{3}\right)$ of 5 af

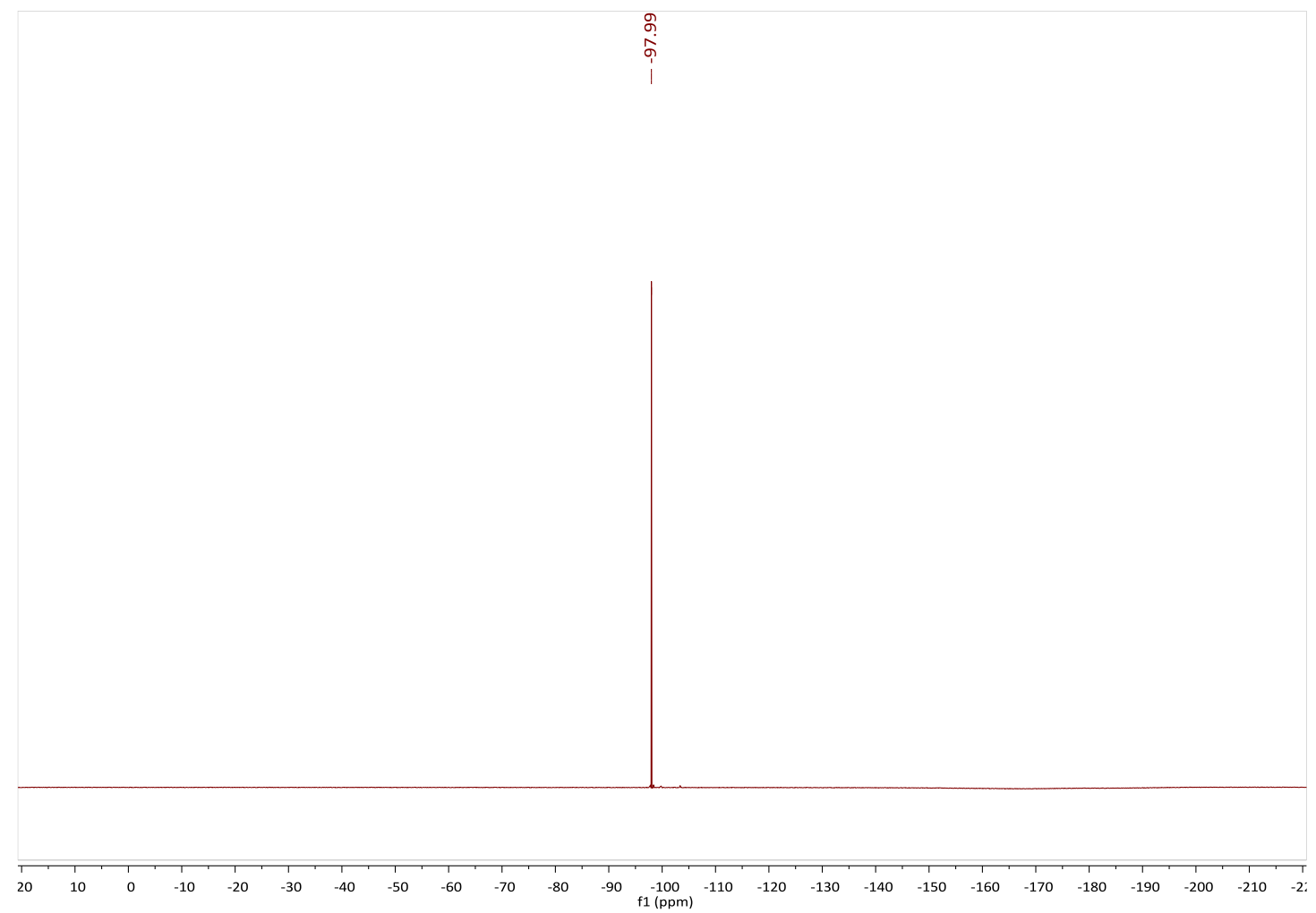




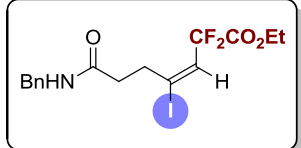

${ }^{13} \mathrm{C}$ NMR-spectrum (125 MHz, $\left.\mathrm{CDCl}_{3}\right)$ of 5 af

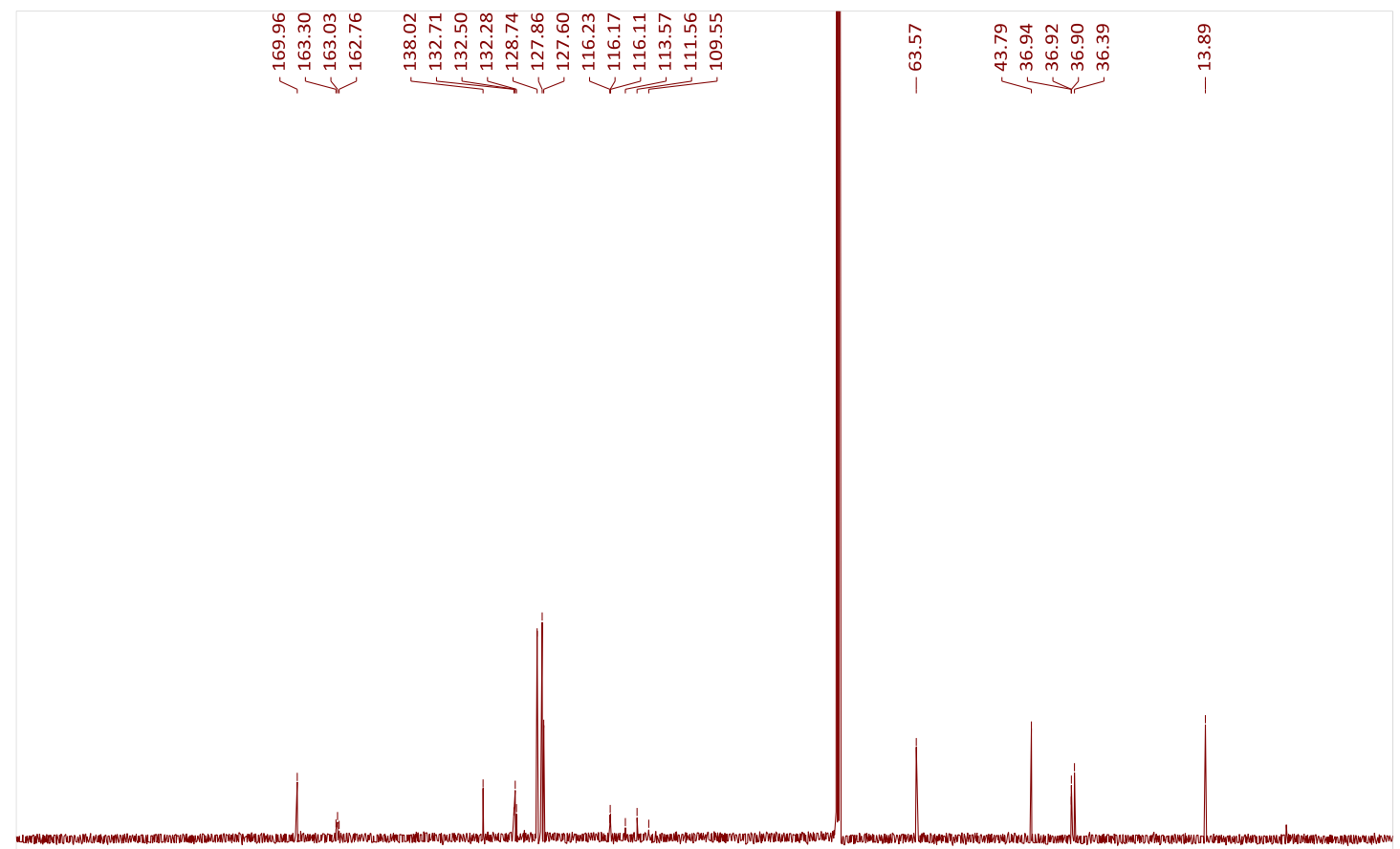

$\begin{array}{llllllllllllllllllllllllllllllllllll}210 & 200 & 190 & 180 & 170 & 160 & 150 & 140 & 130 & 120 & 110 & 100 & 90 & 80 & 70 & 60 & 50 & 40 & 30 & 20 & 10 & 0 & -10\end{array}$ 


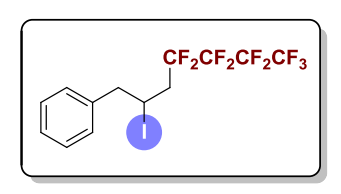

${ }^{1} \mathbf{H}$ NMR-spectrum $\left(500 \mathrm{MHz}, \mathrm{CDCl}_{3}\right)$ of $\mathbf{6 a}$

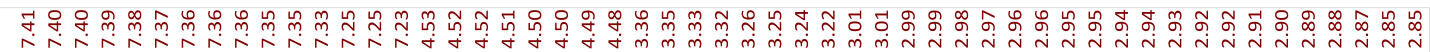

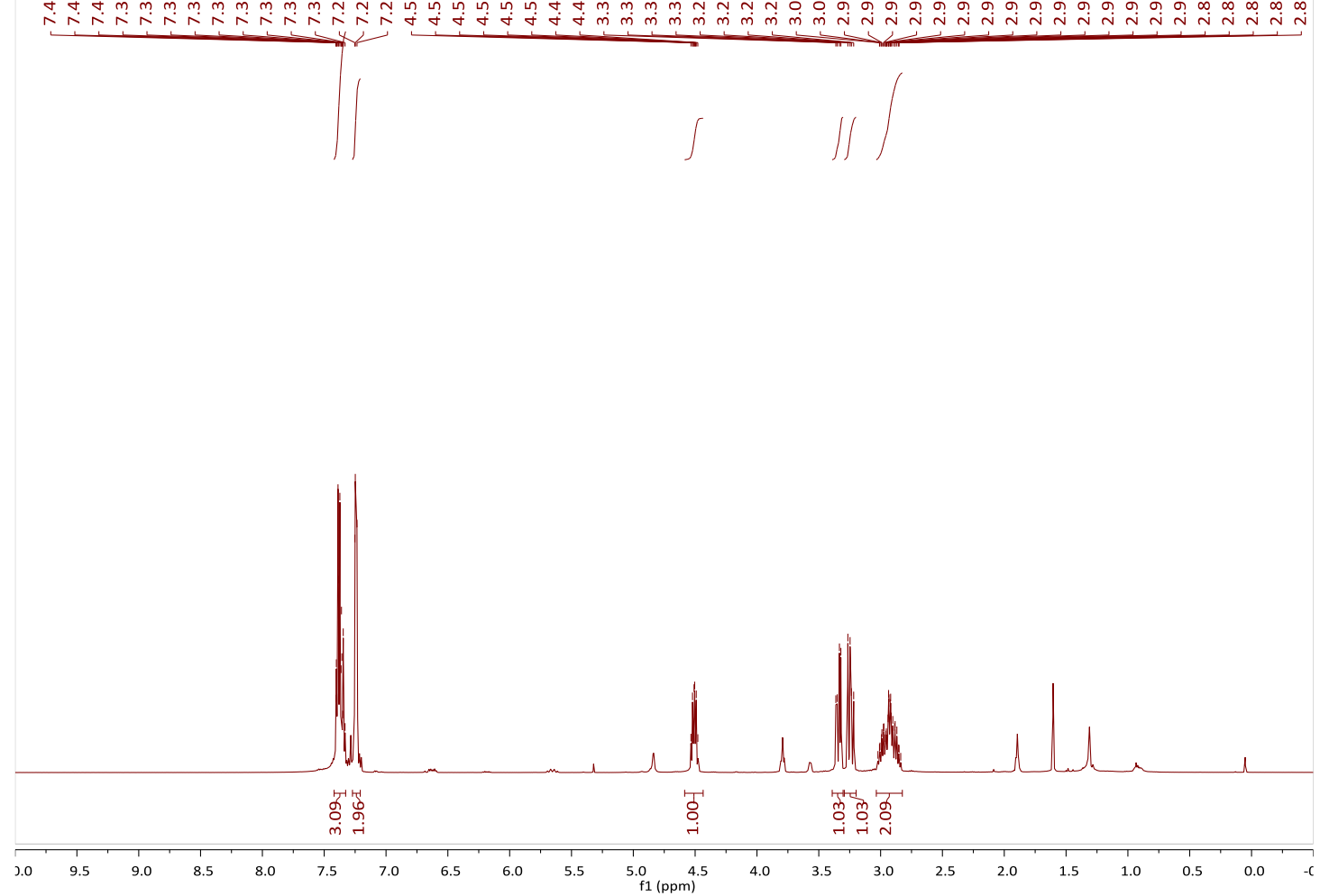

${ }^{19} \mathbf{F}$ NMR-spectrum $\left(471 \mathrm{MHz}, \mathrm{CDCl}_{3}\right)$ of $\mathbf{6 a}$

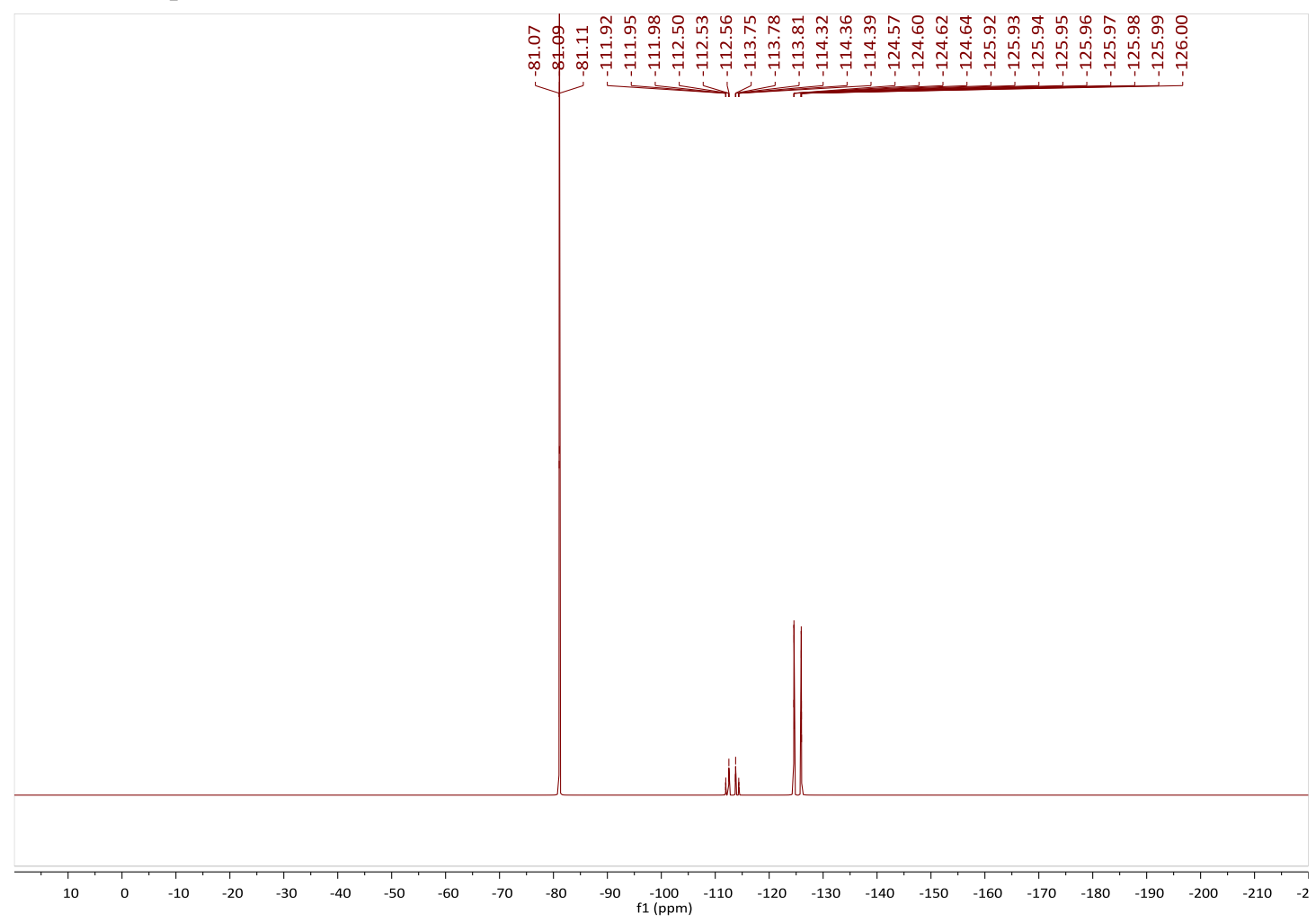




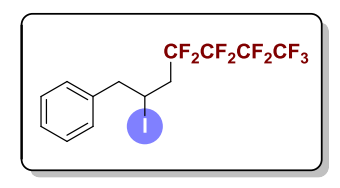

${ }^{13} \mathrm{C}$ NMR-spectrum $\left(125 \mathrm{MHz}, \mathrm{CDCl}_{3}\right)$ of $\mathbf{6 a}$

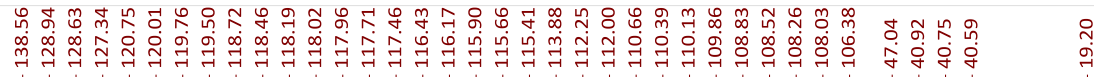

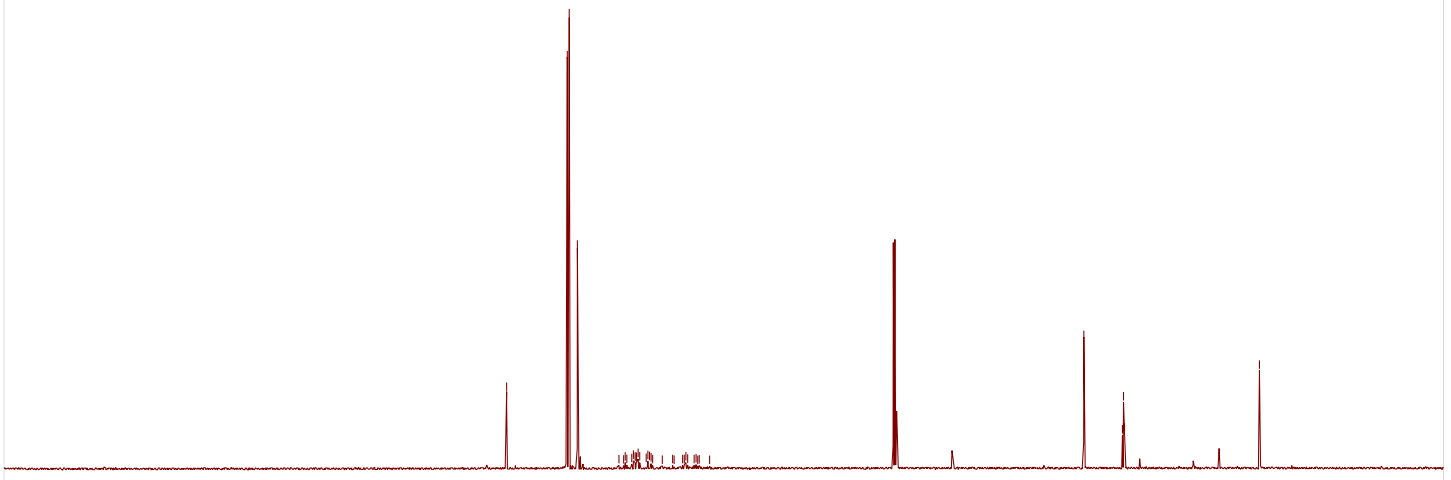

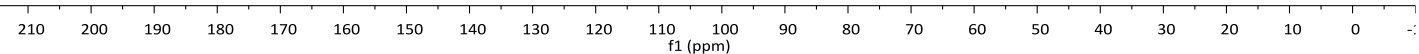




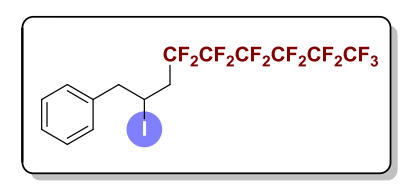

${ }^{1} \mathbf{H}$ NMR-spectrum $\left(500 \mathrm{MHz}, \mathrm{CDCl}_{3}\right)$ of $\mathbf{6 b}$

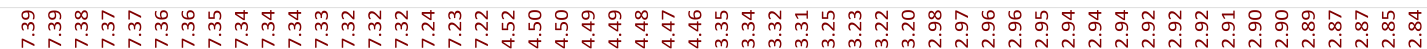
$\underbrace{1}_{1}$

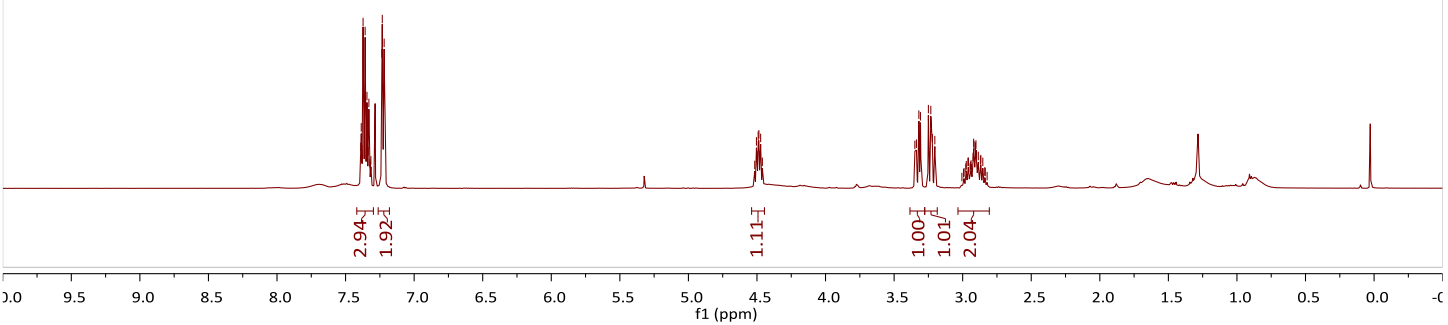

${ }^{19}$ F NMR-spectrum $\left(471 \mathrm{MHz}, \mathrm{CDCl}_{3}\right)$ of $\mathbf{6 b}$

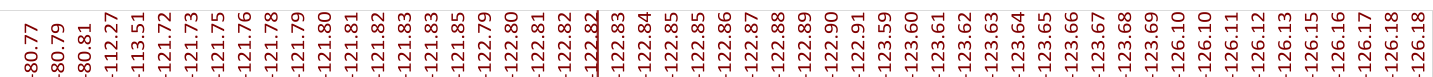

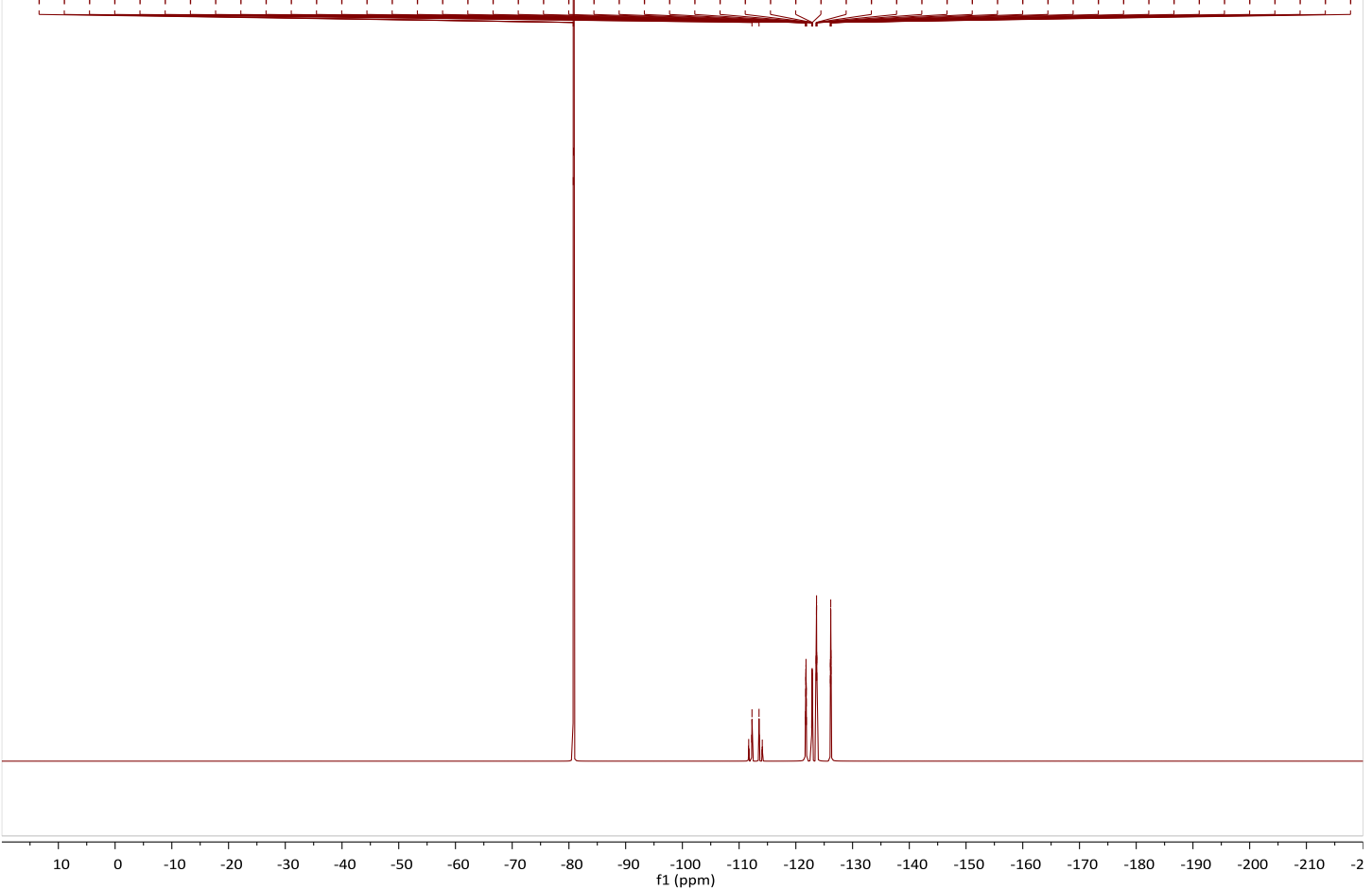




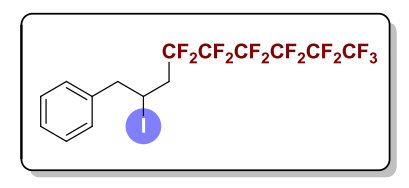

${ }^{13} \mathrm{C}$ NMR-spectrum $\left(125 \mathrm{MHz}, \mathrm{CDCl}_{3}\right)$ of $\mathbf{6 b}$

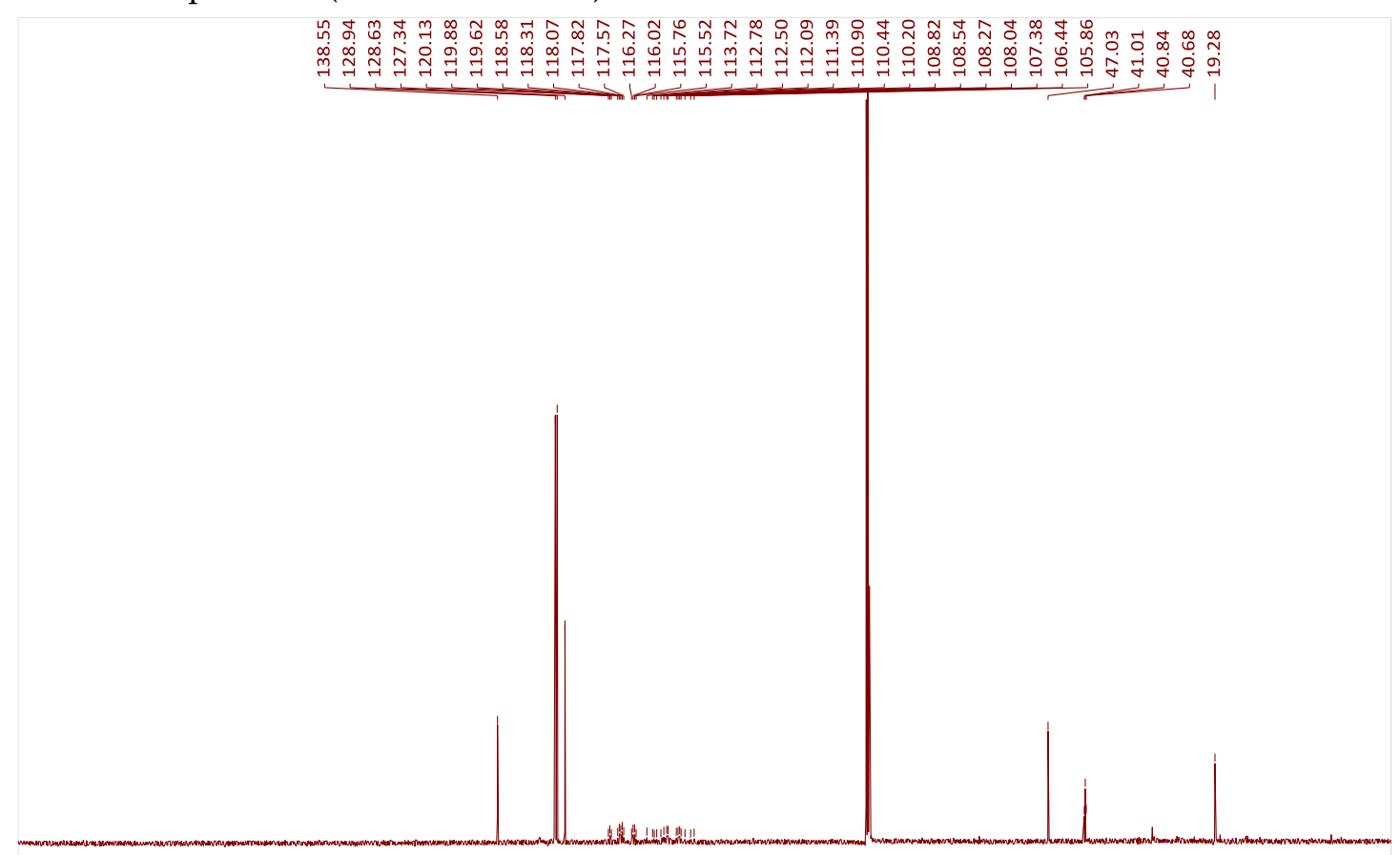

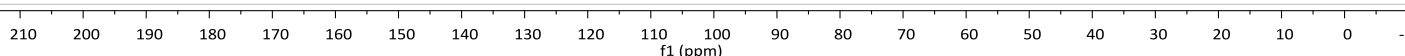




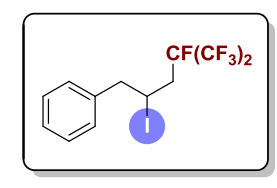

${ }^{1} \mathbf{H}$ NMR-spectrum $\left(500 \mathrm{MHz}, \mathrm{CDCl}_{3}\right)$ of $\mathbf{6 c}$

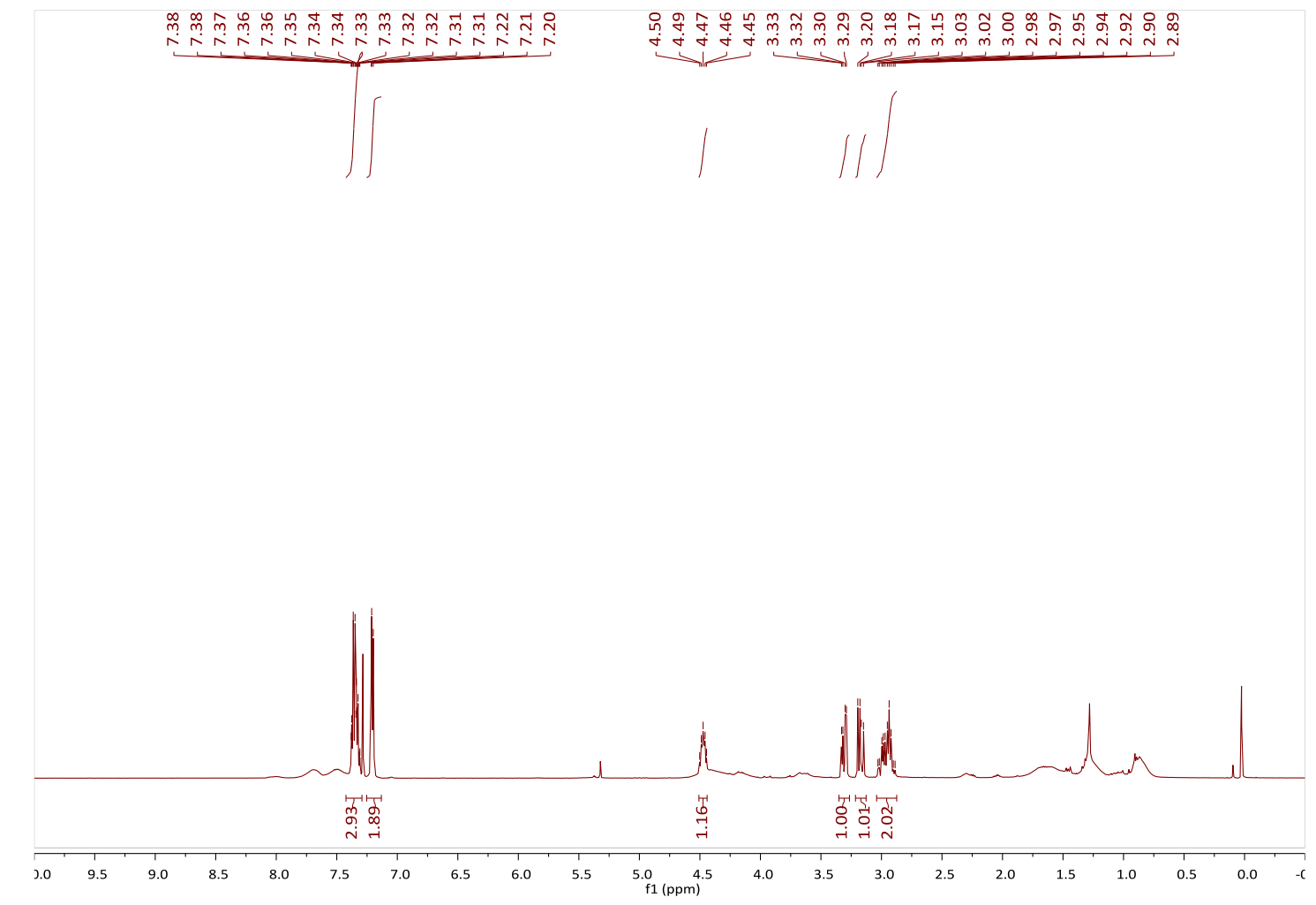

${ }^{19} \mathrm{~F}$ NMR-spectrum $\left(471 \mathrm{MHz}, \mathrm{CDCl}_{3}\right)$ of $6 \mathrm{c}$

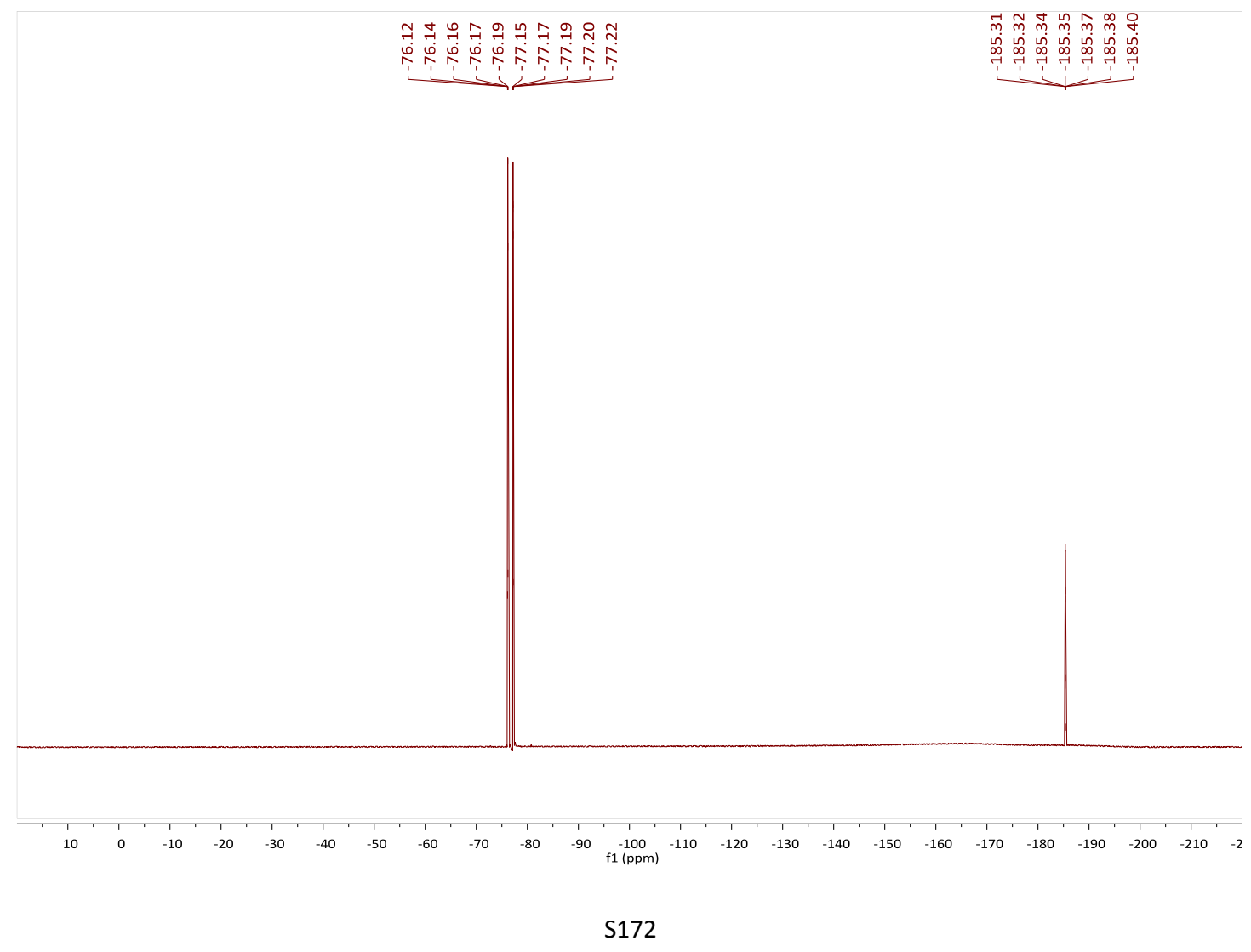




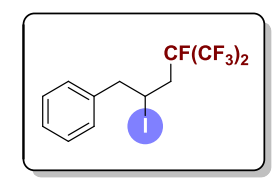

${ }^{13} \mathrm{C}$ NMR-spectrum $\left(125 \mathrm{MHz}, \mathrm{CDCl}_{3}\right)$ of $6 \mathbf{c}$

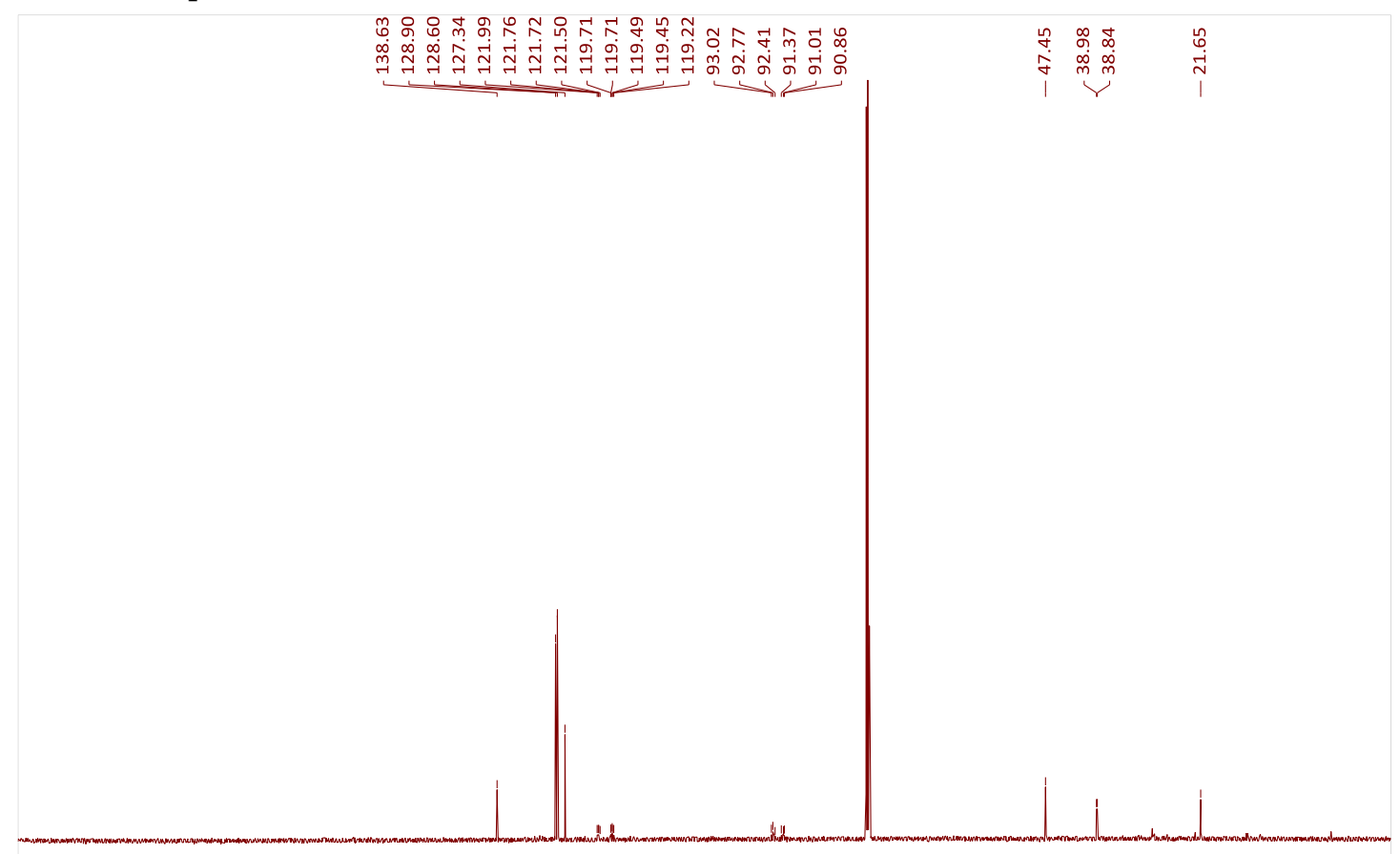

$\begin{array}{lllllllllllllllllllllllllllllllllllll} & 210 & 200 & 190 & 180 & 170 & 160 & 150 & 140 & 130 & 120 & 110 & 100 & 90 & 80 & 70 & 60 & 50 & 40 & 30 & 20 & 10 & 0 & -\end{array}$ 


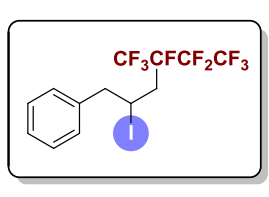

${ }^{1} \mathbf{H}$ NMR-spectrum $\left(500 \mathrm{MHz}, \mathrm{CDCl}_{3}\right)$ of $\mathbf{6 d}$

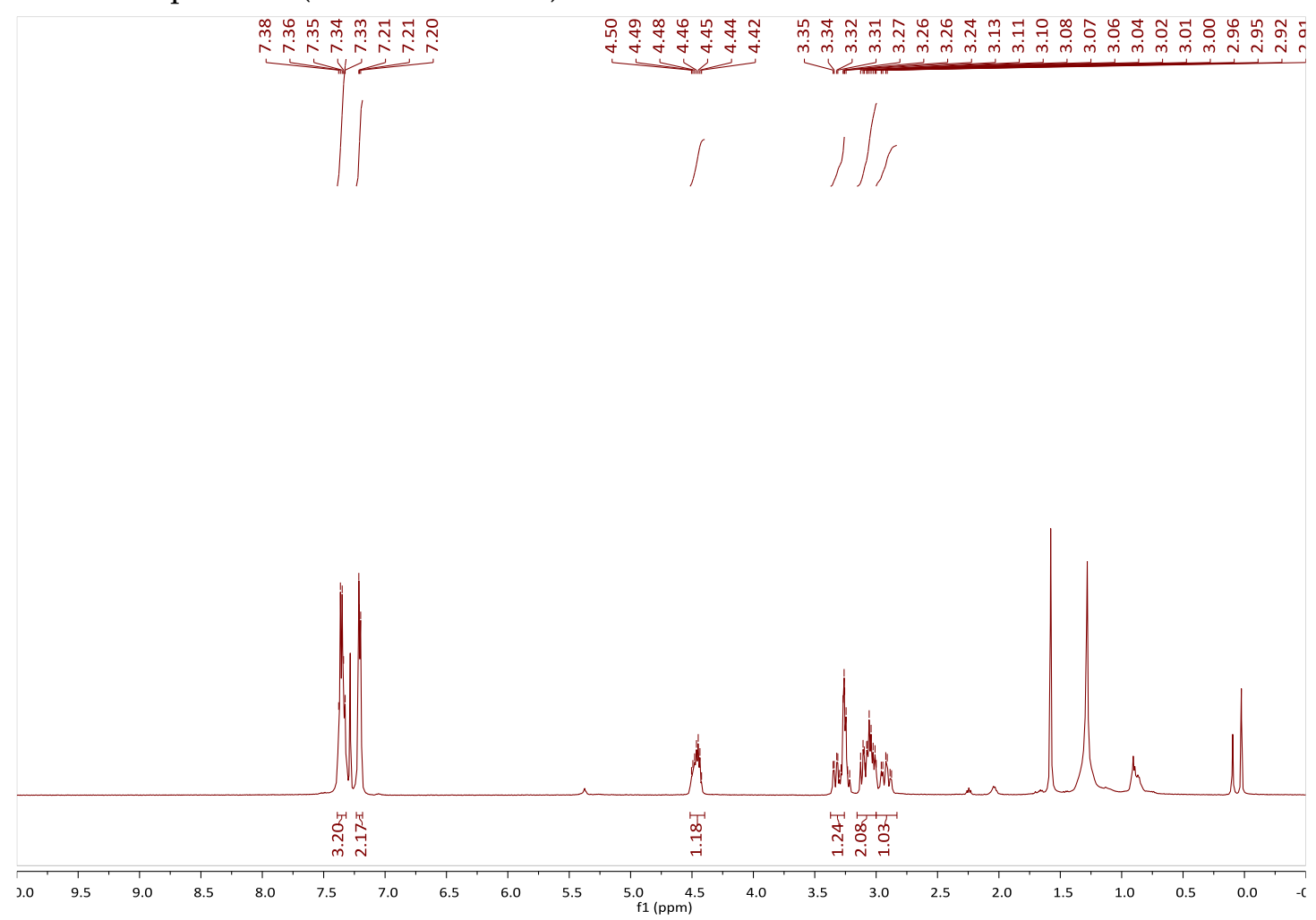

${ }^{19}$ F NMR-spectrum $\left(471 \mathrm{MHz}, \mathrm{CDCl}_{3}\right)$ of $\mathbf{6} \mathbf{d}$

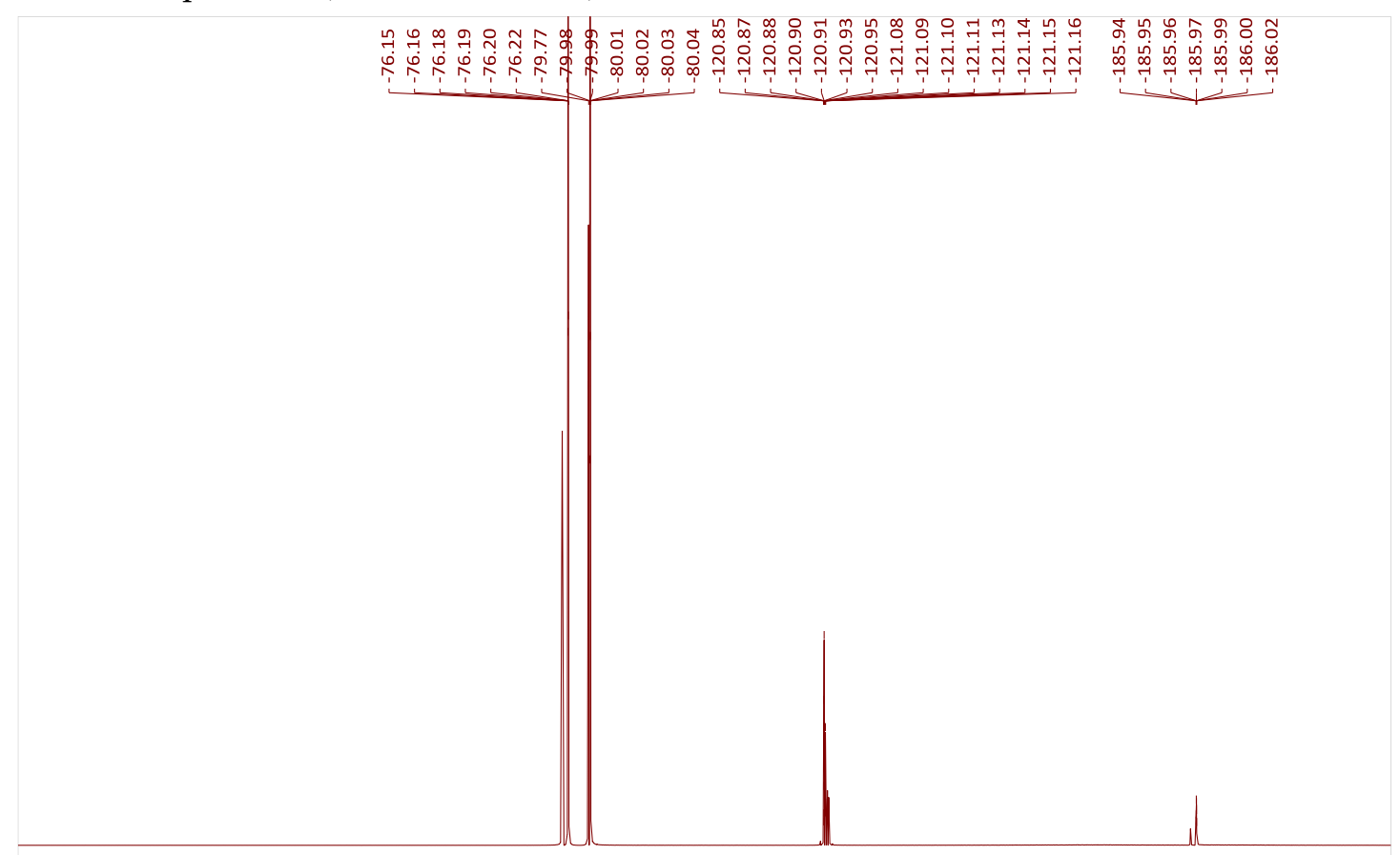

$\begin{array}{llllllllllllllllllllllllllllll}10 & 0 & -10 & -20 & -30 & -40 & -50 & -60 & -70 & -80 & -90 & -100 & -110 & -120 & -130 & -140 & -150 & -160 & -170 & -180 & -190 & -200 & -210 & -2\end{array}$ 


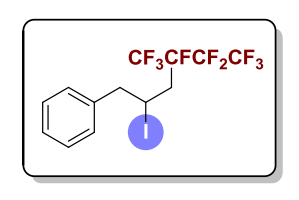

${ }^{13} \mathrm{C}$ NMR-spectrum (125 MHz, $\left.\mathrm{CDCl}_{3}\right)$ of $\mathbf{6 d}$

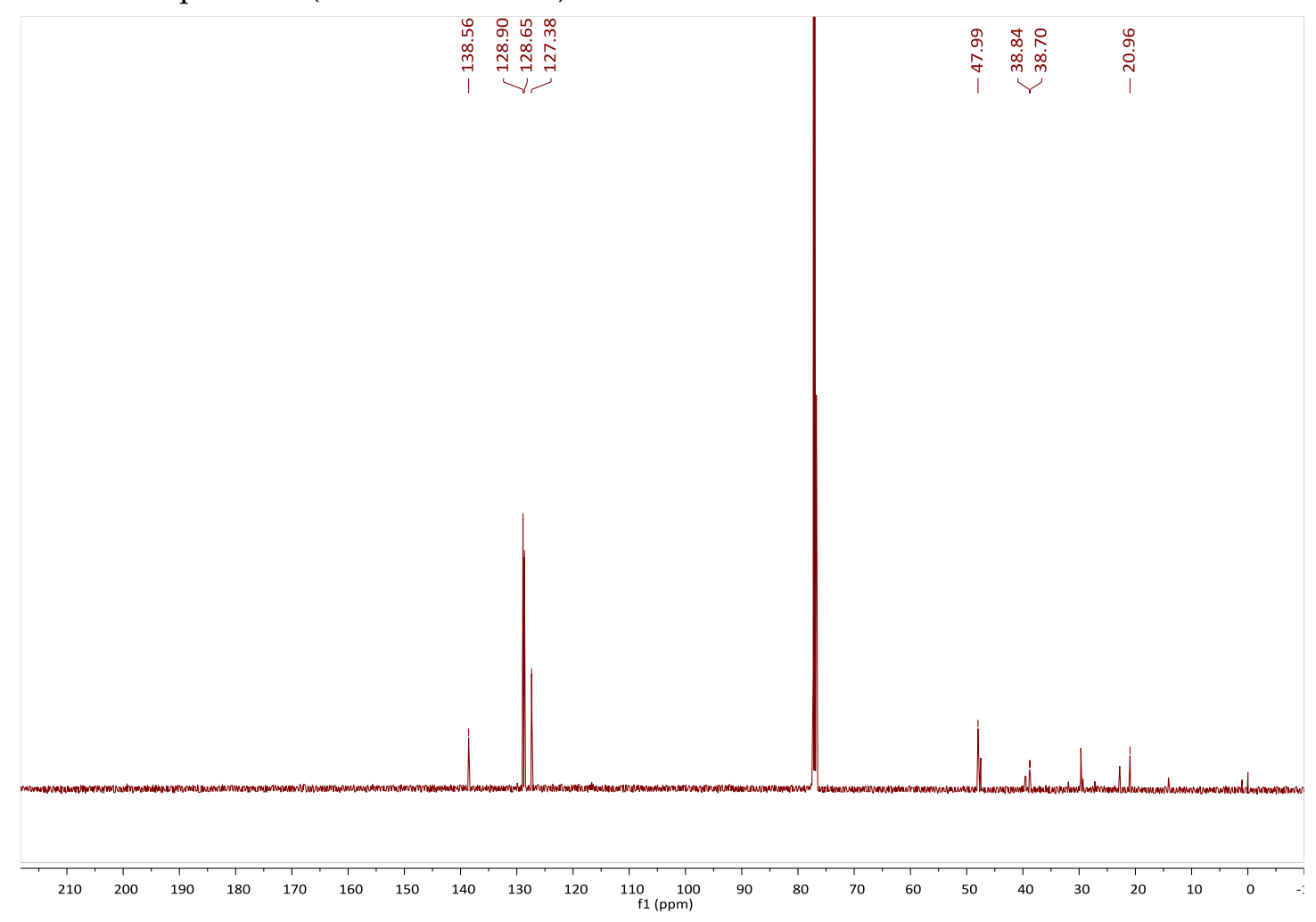




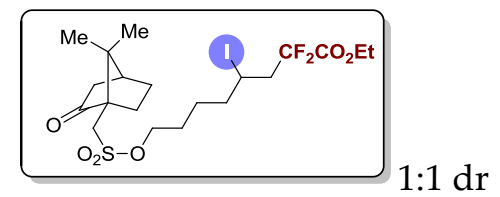

${ }^{1} \mathbf{H}$ NMR-spectrum $\left(500 \mathrm{MHz}, \mathrm{CDCl}_{3}\right)$ of $7 \mathbf{a}$

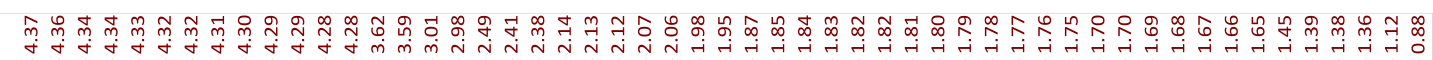

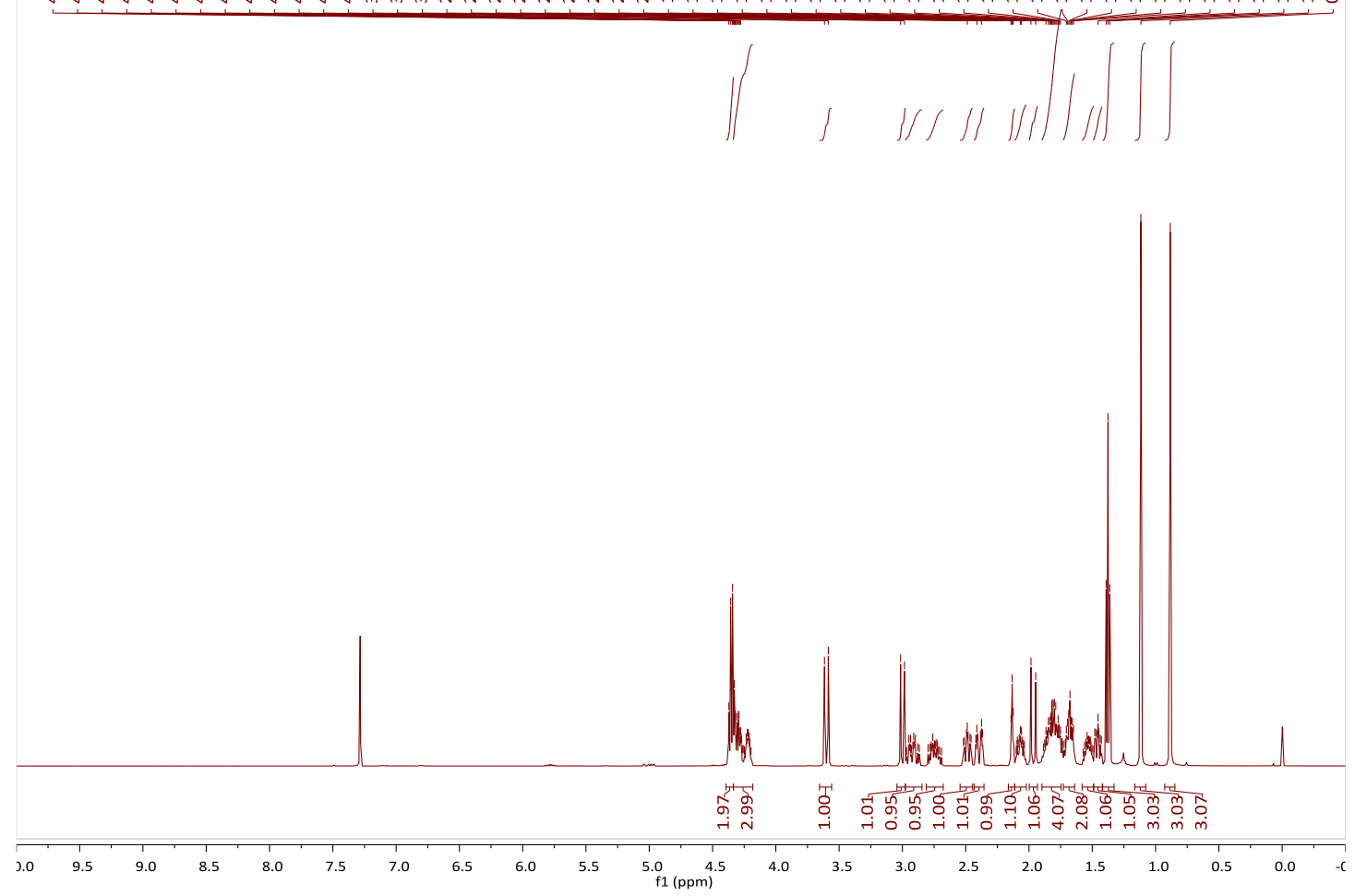

${ }^{19} \mathbf{F}$ NMR-spectrum $\left(471 \mathrm{MHz}, \mathrm{CDCl}_{3}\right)$ of $7 \mathbf{a}$

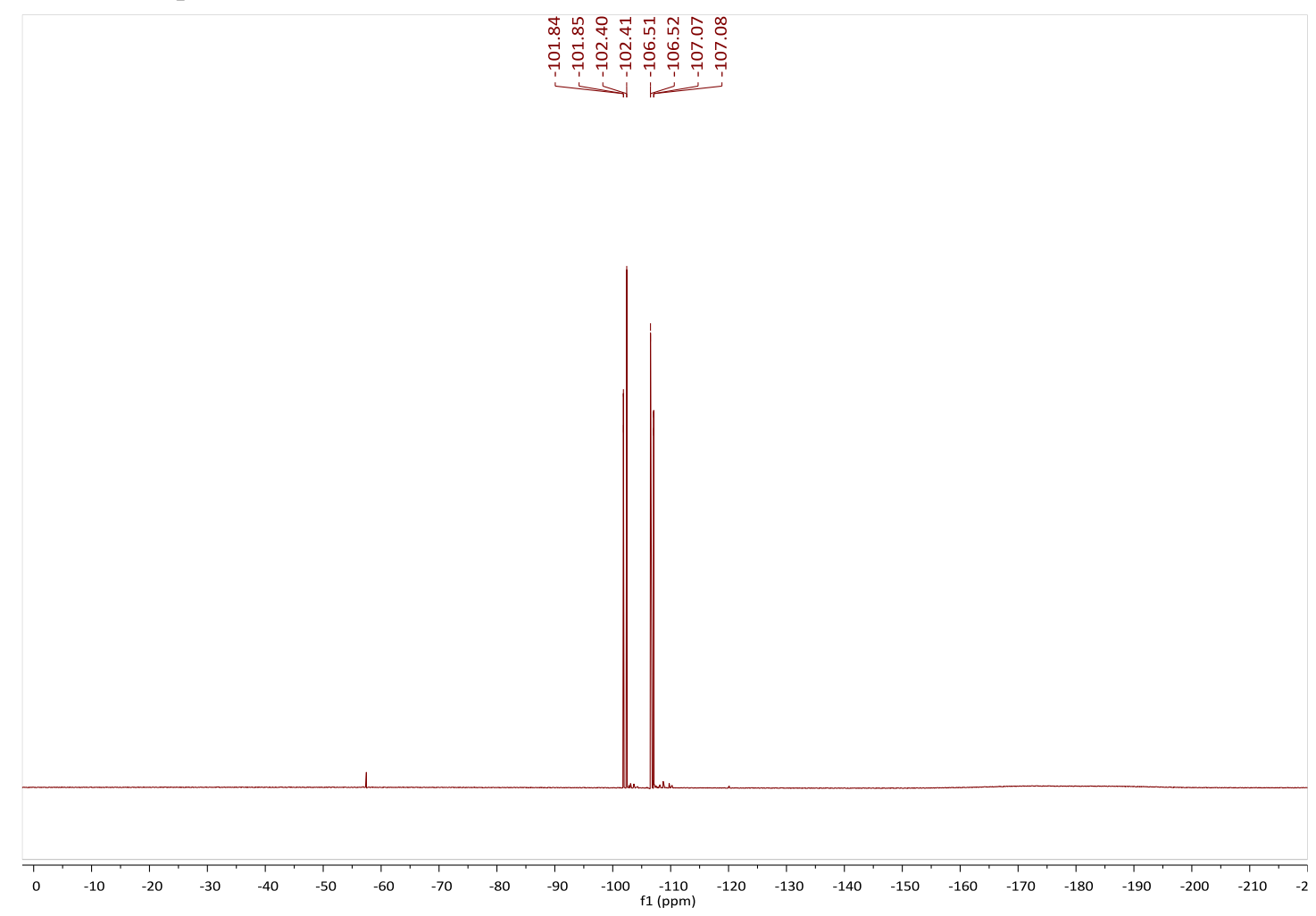




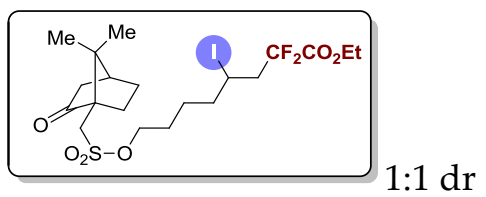

${ }^{13} \mathrm{C}$ NMR-spectrum (125 MHz, $\left.\mathrm{CDCl}_{3}\right)$ of $7 \mathbf{a}$

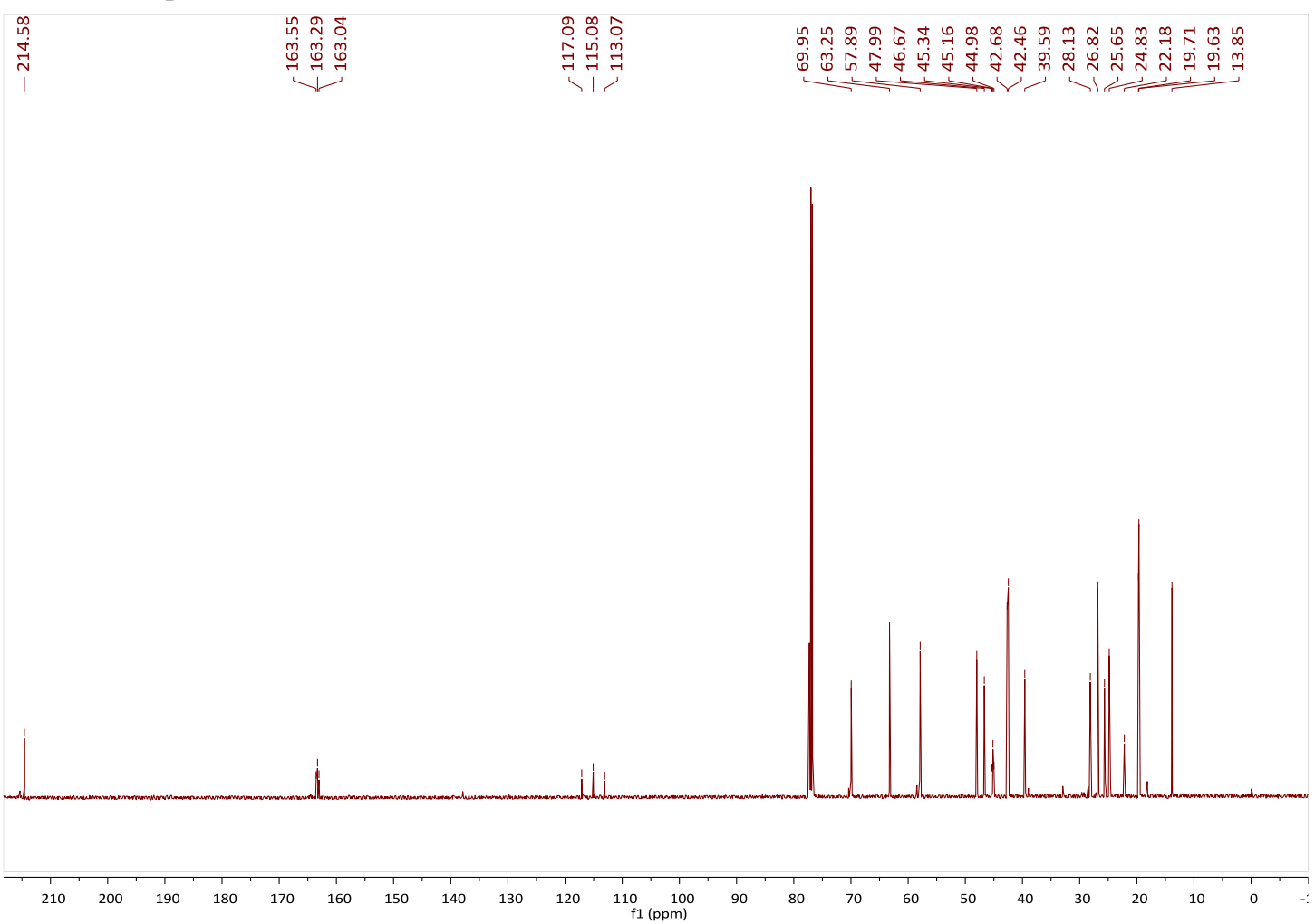




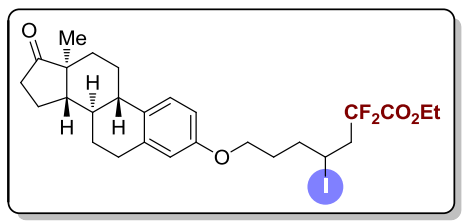

${ }^{1} \mathbf{H}$ NMR-spectrum $\left(500 \mathrm{MHz}, \mathrm{CDCl}_{3}\right)$ of $\mathbf{7 b}$

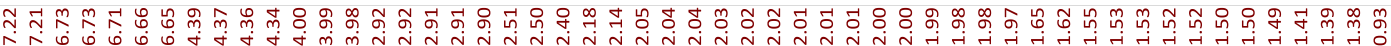

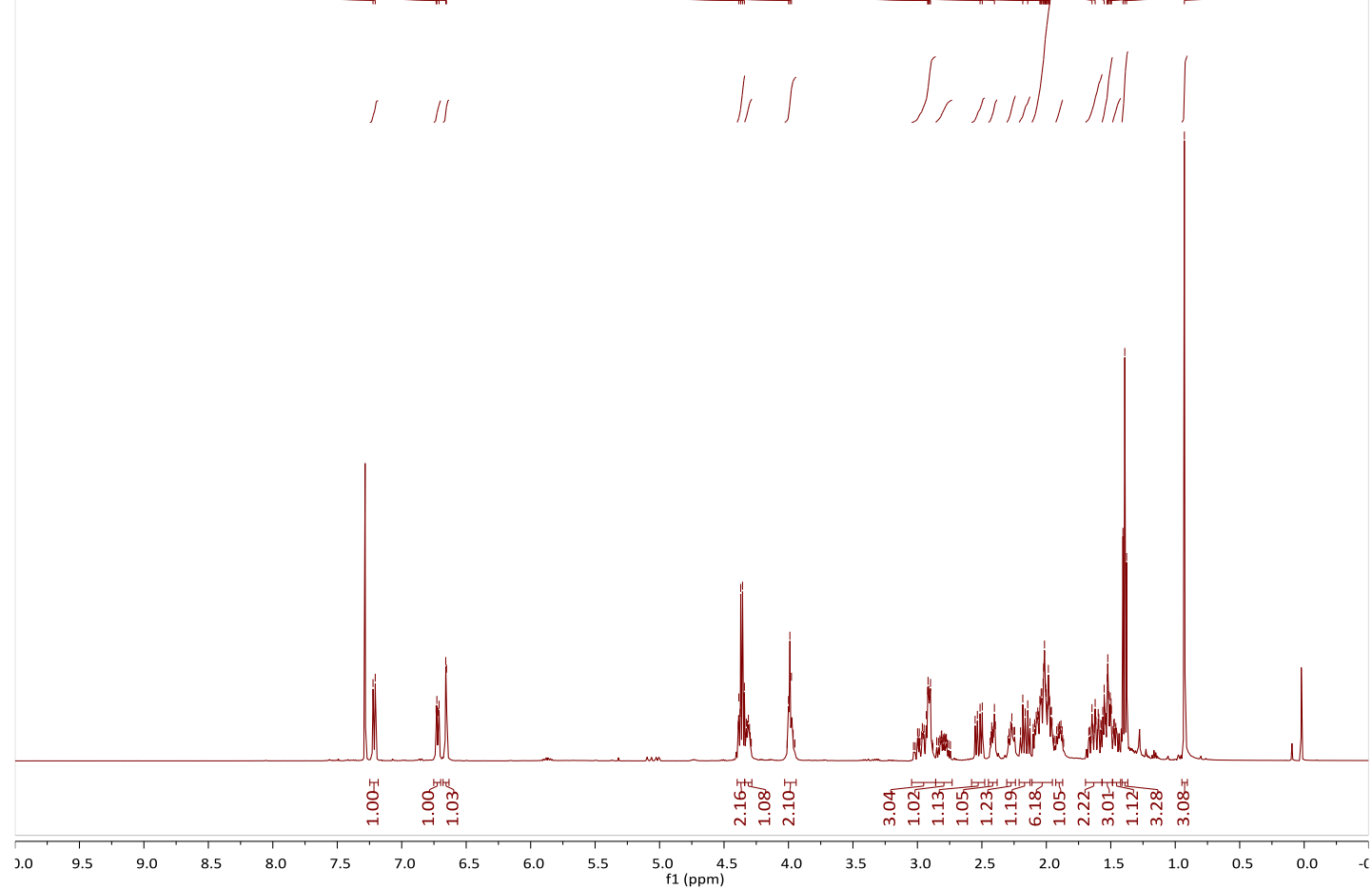

${ }^{19}$ F NMR-spectrum $\left(471 \mathrm{MHz}, \mathrm{CDCl}_{3}\right)$ of $7 \mathbf{b}$

œ 융ำ

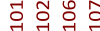

i i

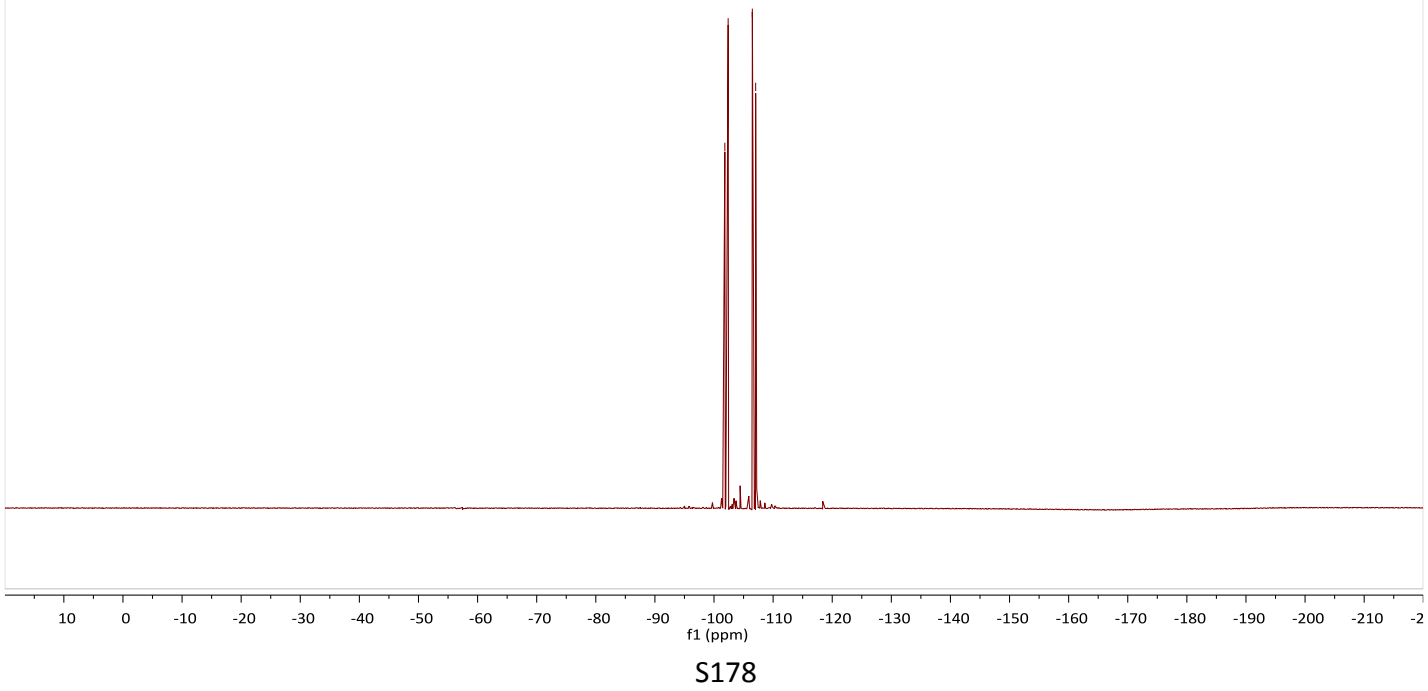




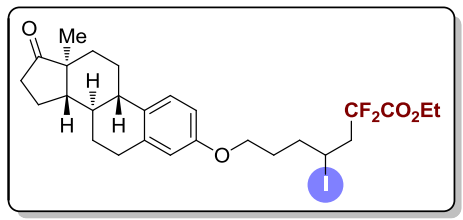

${ }^{13} \mathbf{C}$ NMR-spectrum (125 MHz, $\left.\mathrm{CDCl}_{3}\right)$ of $7 \mathbf{b}$

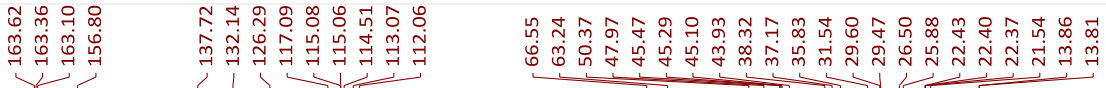
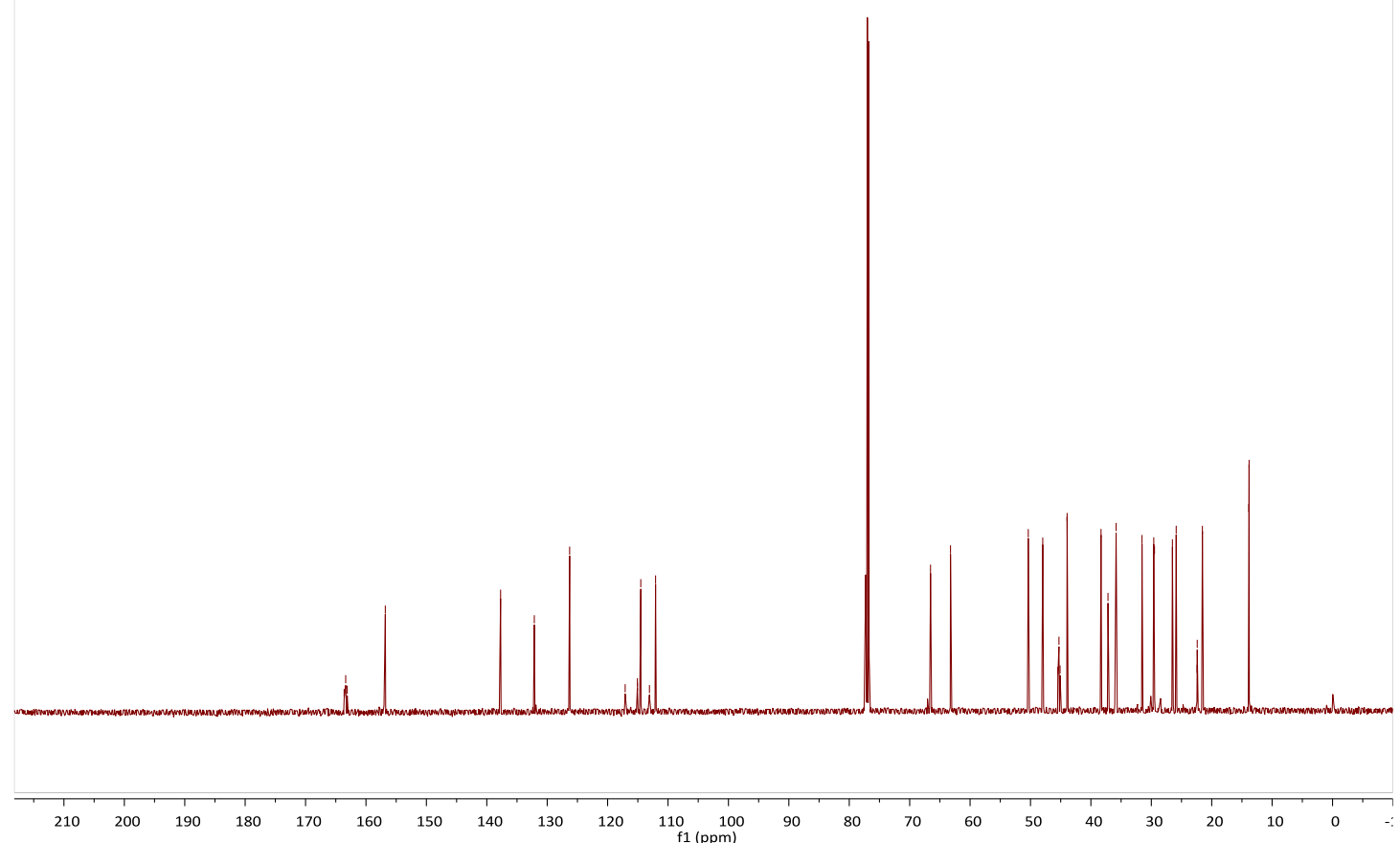


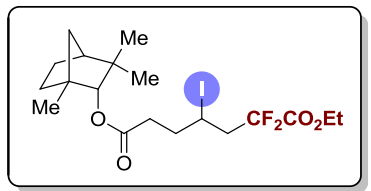

$1: 1 \mathrm{dr}$

${ }^{1} \mathbf{H}$ NMR-spectrum $\left(500 \mathrm{MHz}, \mathrm{CDCl}_{3}\right)$ of $7 \mathrm{c}$

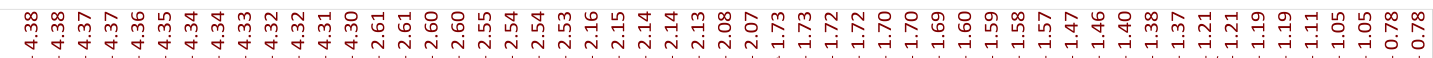
$\iiint \iiint \int / \| \mid$

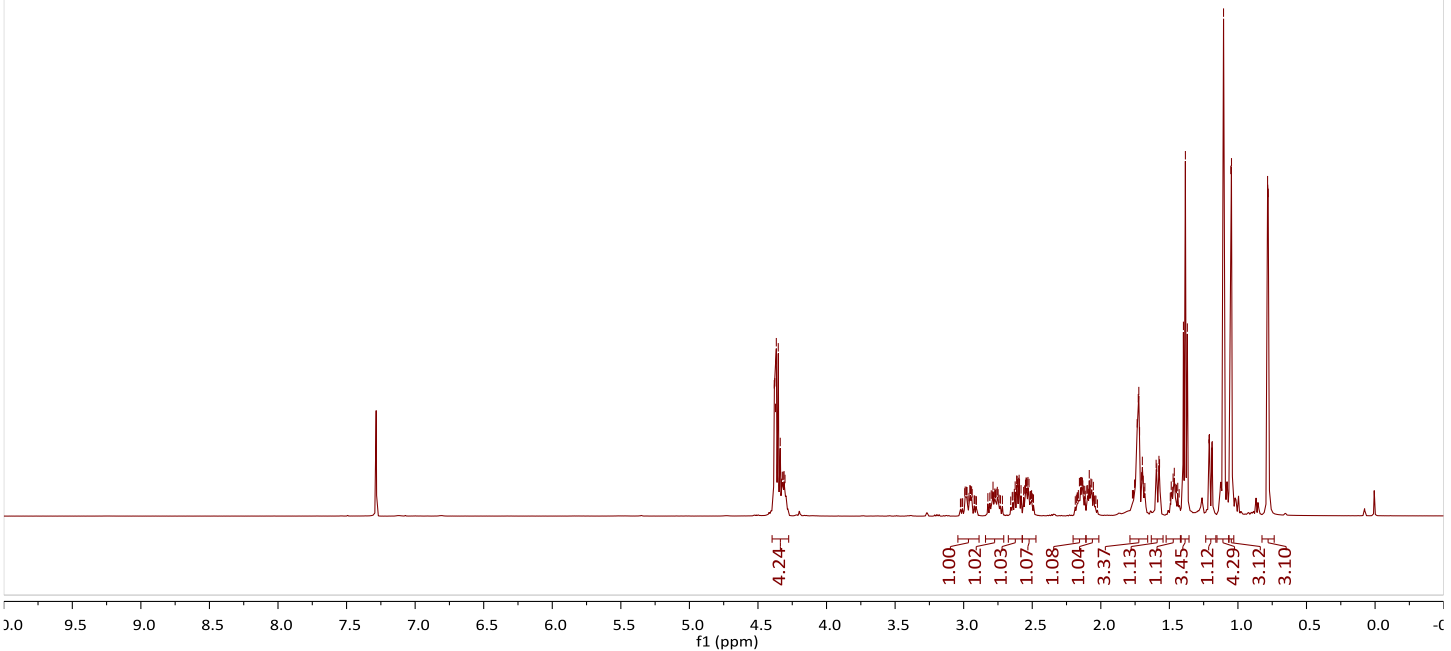

${ }^{19}$ F NMR-spectrum $\left(471 \mathrm{MHz}, \mathrm{CDCl}_{3}\right)$ of $7 \mathrm{c}$

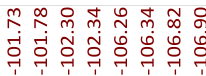

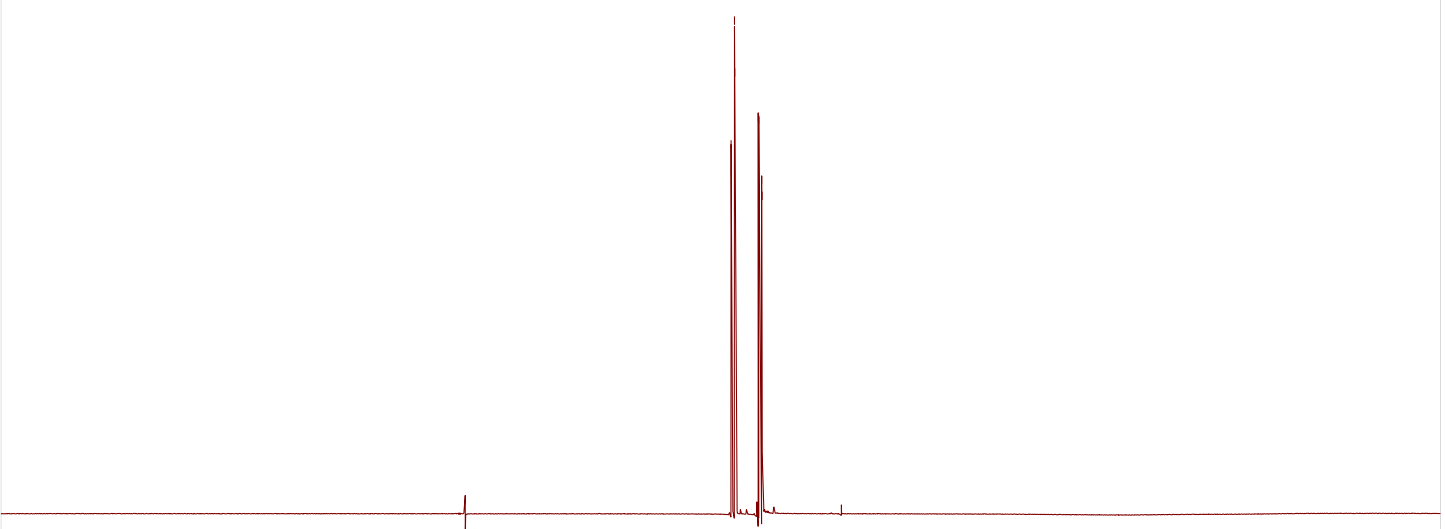

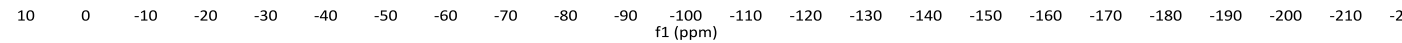




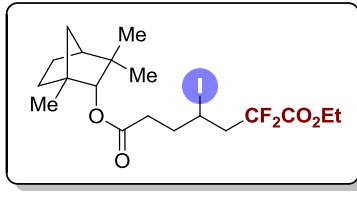

$1: 1 \mathrm{dr}$

${ }^{13} \mathrm{C}$ NMR-spectrum (125 MHz, $\left.\mathrm{CDCl}_{3}\right)$ of $7 \mathrm{c}$

กำ กำ

궁

ดั के

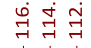

$\stackrel{\sim}{\leftrightarrow}$
$\dot{\infty}$

กิ

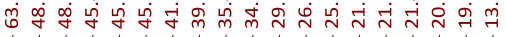

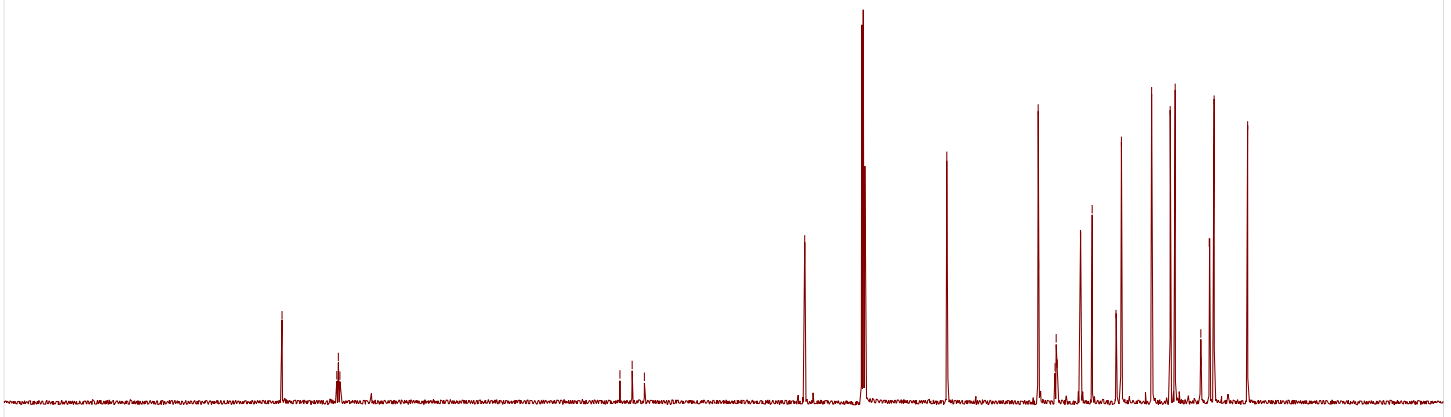

$\begin{array}{lllllllllllllllllllllllllll}210 & 200 & 190 & 180 & 170 & 160 & 150 & 140 & 130 & 120 & 110 & 100 & 90 & 80 & 70 & 60 & 50 & 40 & 30 & 20 & 10 & 0 & -10\end{array}$ 


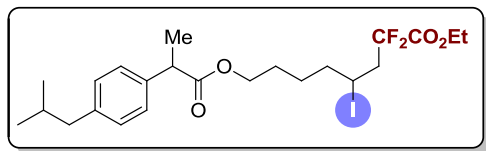

${ }^{1} \mathrm{H}$ NMR-spectrum $\left(500 \mathrm{MHz}, \mathrm{CDCl}_{3}\right)$ of $\mathbf{7 d}$

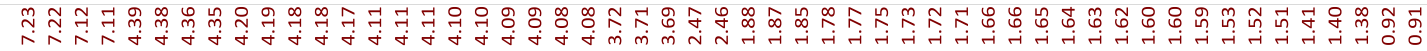

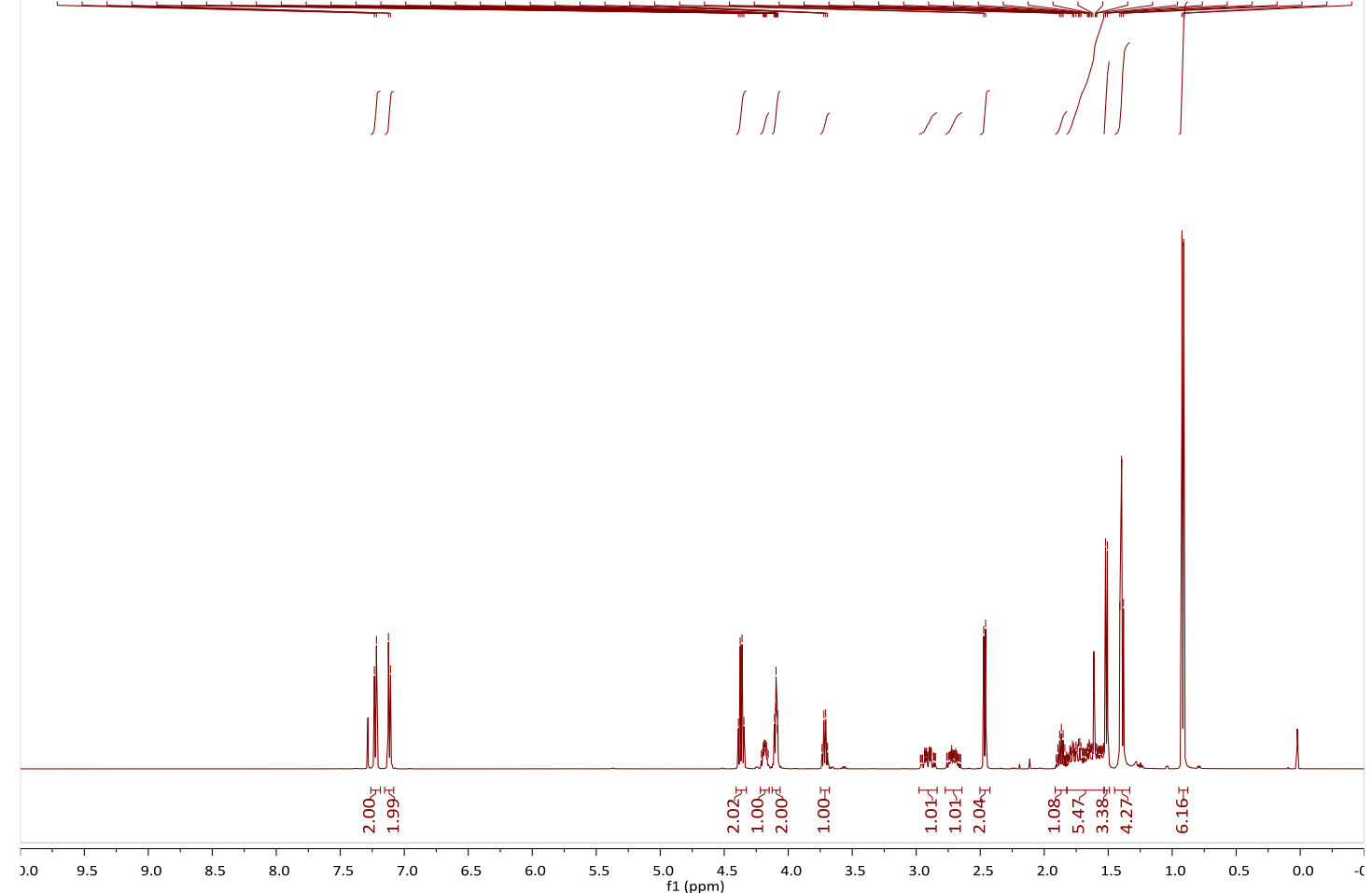

${ }^{19}$ F NMR-spectrum $\left(471 \mathrm{MHz}, \mathrm{CDCl}_{3}\right)$ of $\mathbf{7 d}$

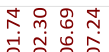

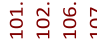

i if

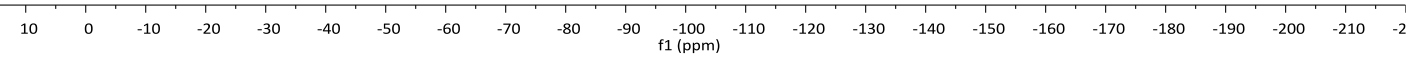




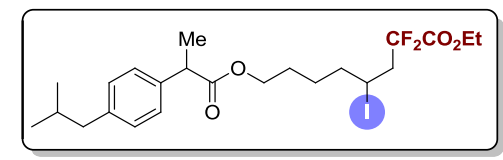

${ }^{13} \mathrm{C}$ NMR-spectrum $\left(125 \mathrm{MHz}, \mathrm{CDCl}_{3}\right)$ of $\mathbf{7 d}$

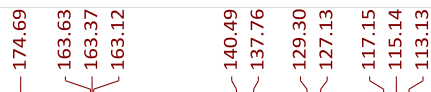

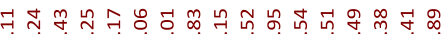

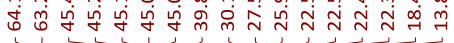

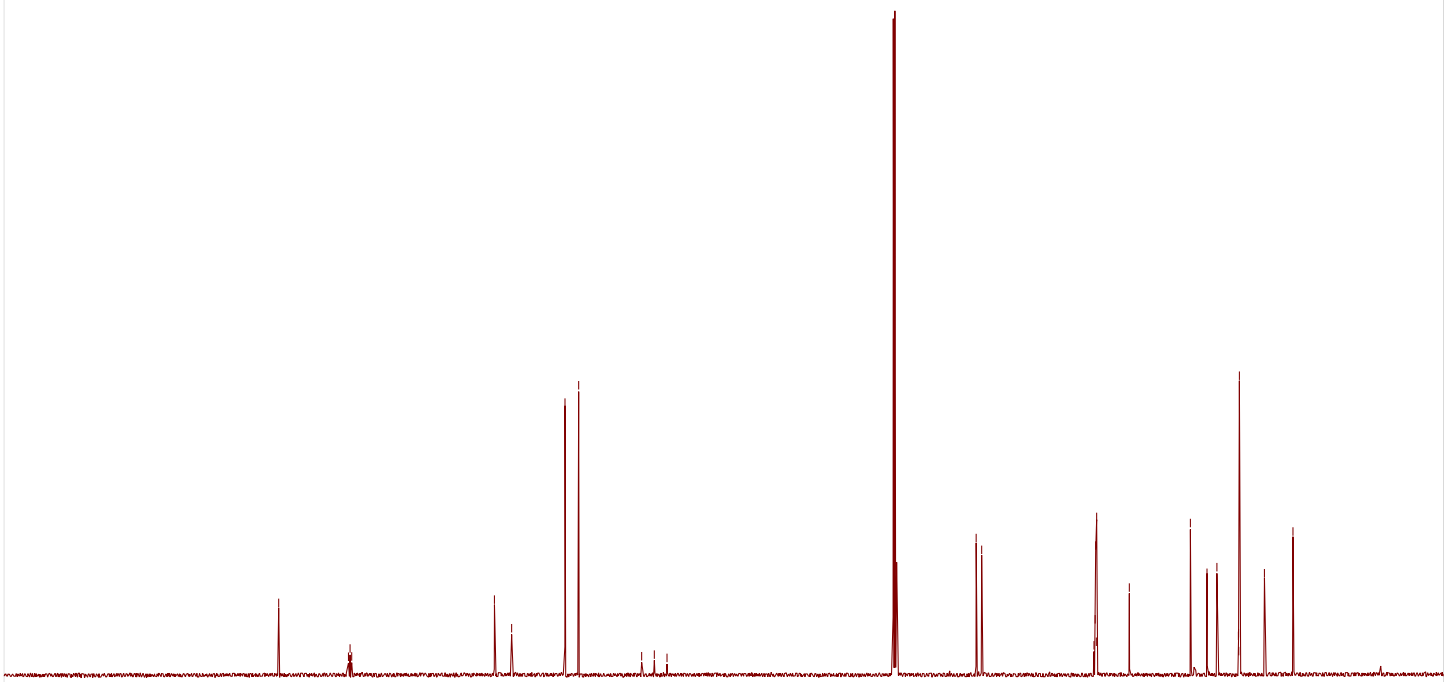

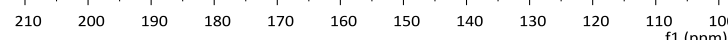




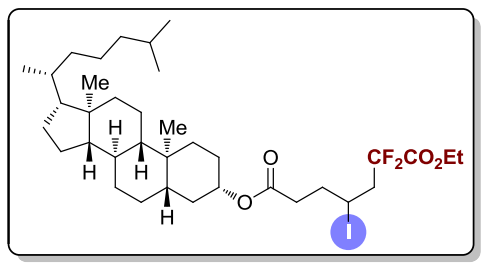

${ }^{1} \mathbf{H}$ NMR-spectrum $\left(500 \mathrm{MHz}, \mathrm{CDCl}_{3}\right)$ of $7 \mathbf{e}$

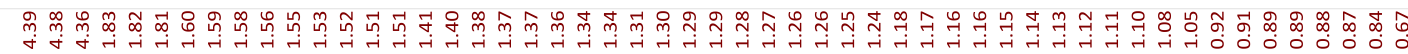

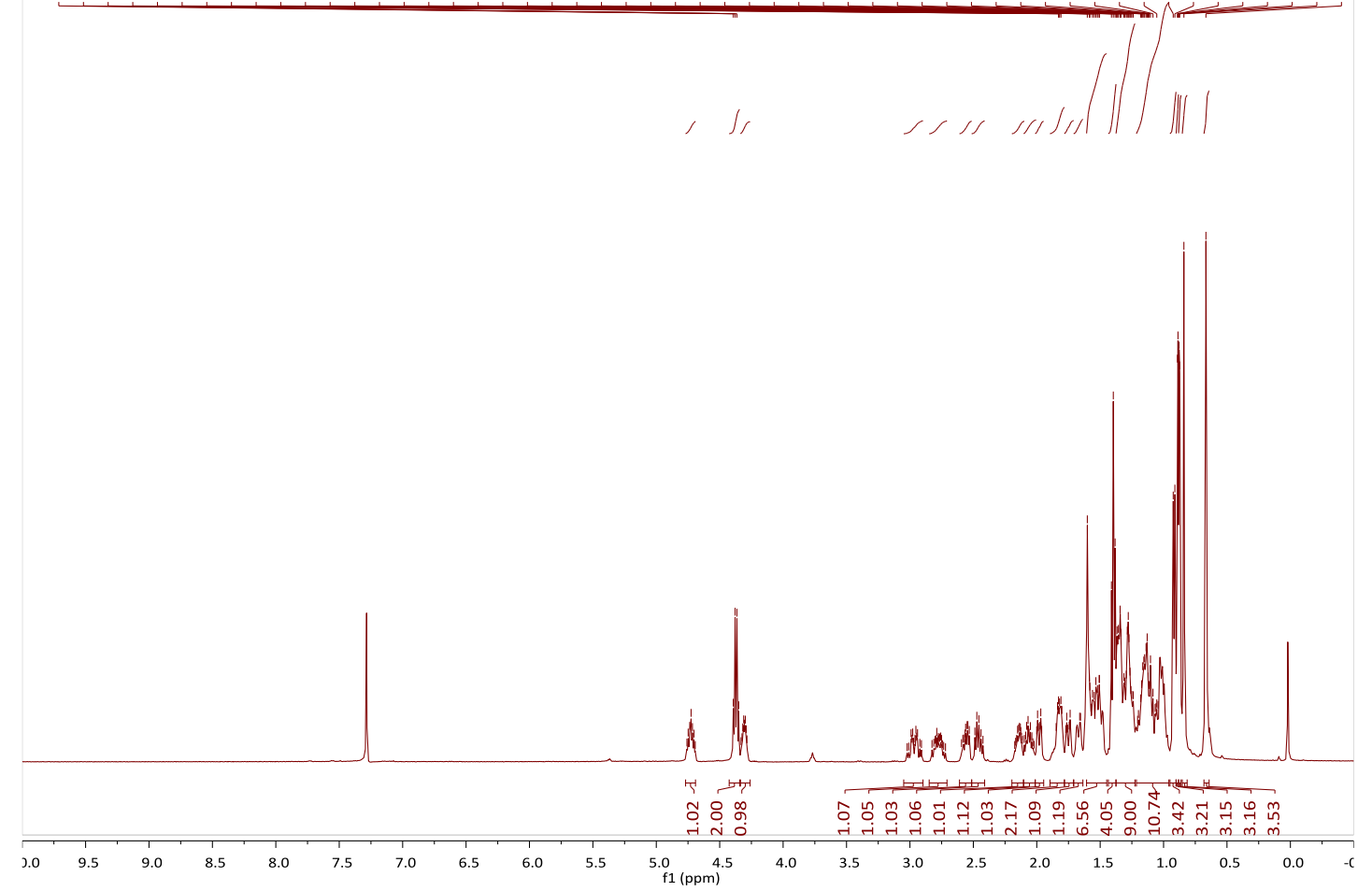

${ }^{19} \mathbf{F}$ NMR-spectrum $\left(471 \mathrm{MHz}, \mathrm{CDCl}_{3}\right)$ of $7 \mathbf{e}$

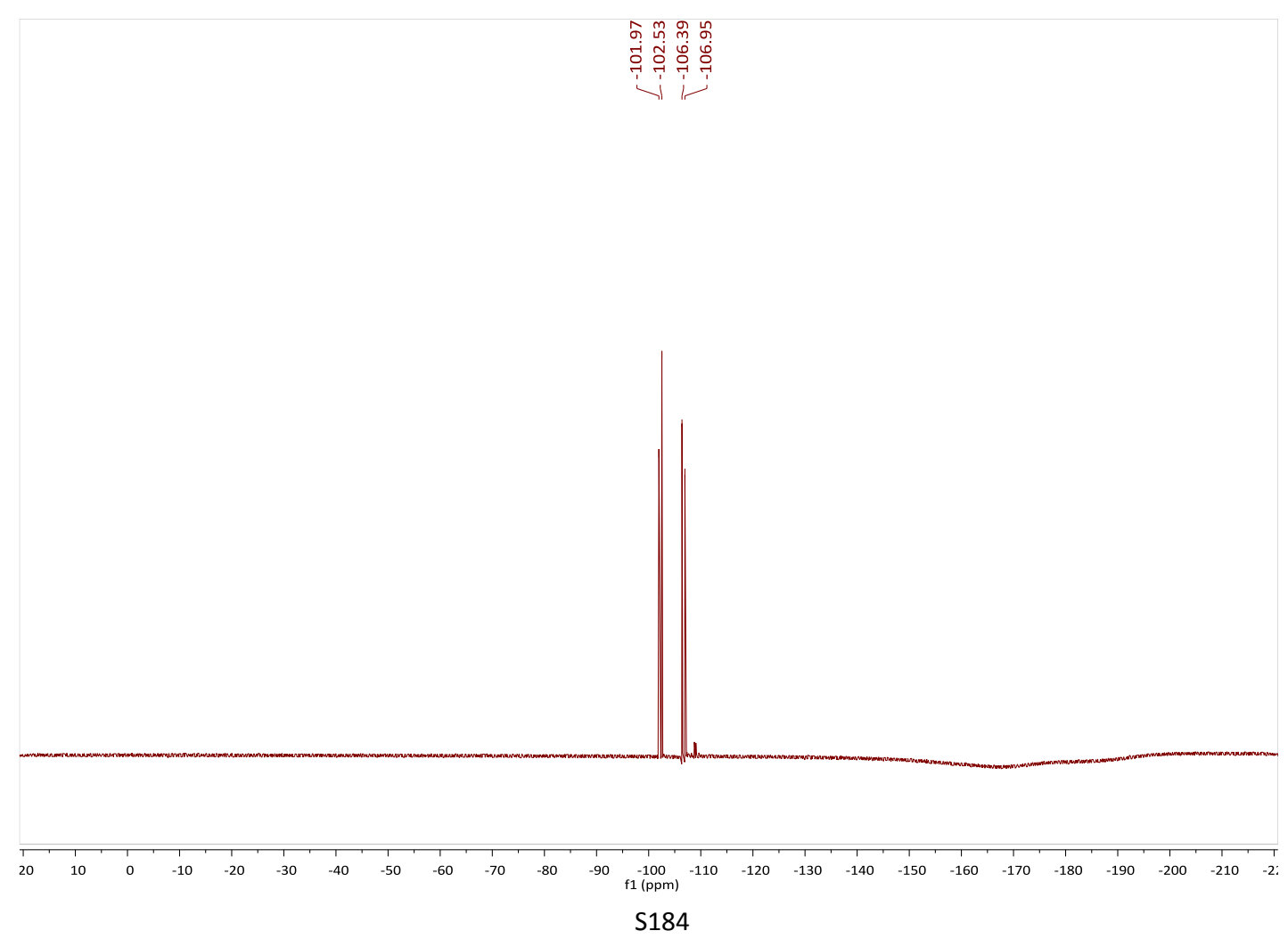




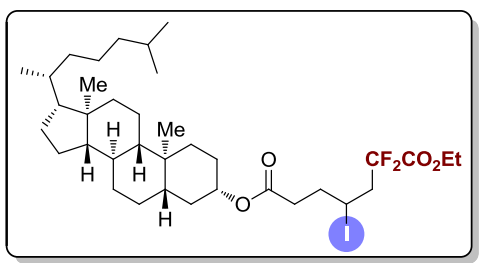

${ }^{13} \mathrm{C}$ NMR-spectrum $\left(125 \mathrm{MHz}, \mathrm{CDCl}_{3}\right)$ of $7 \mathbf{e}$

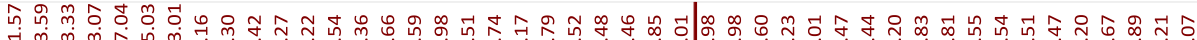

స్

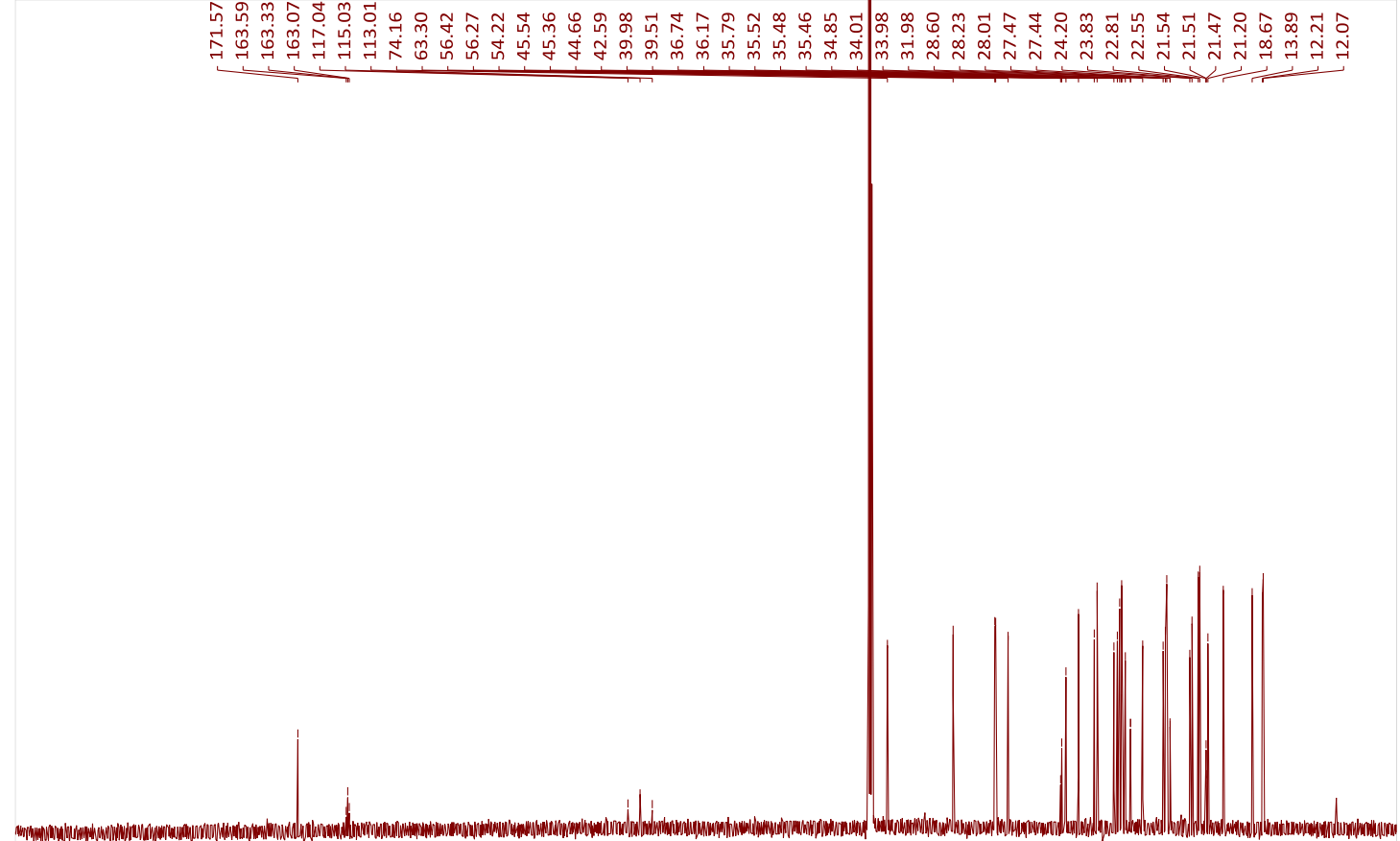

$\begin{array}{lllllllllllllllllllllll}210 & 200 & 190 & 180 & 170 & 160 & 150 & 140 & 130 & 120 & \underset{\mathrm{f} 1(\mathrm{ppm})}{110} & 90 & 80 & 70 & 60 & 50 & 40 & 30 & 20 & 10 & 0 & -\end{array}$ 


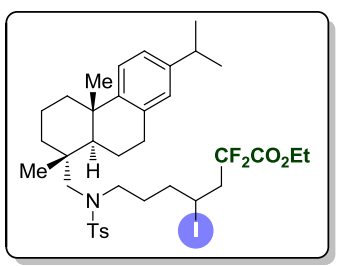

${ }^{1} \mathrm{H}$ NMR-spectrum $\left(500 \mathrm{MHz}, \mathrm{CDCl}_{3}\right)$ of $\mathbf{7 f}$

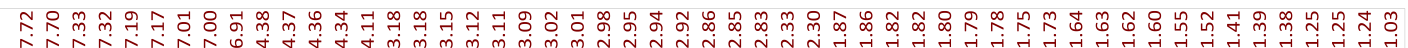
$\iint S S$ $\int_{, j}, \int 11$

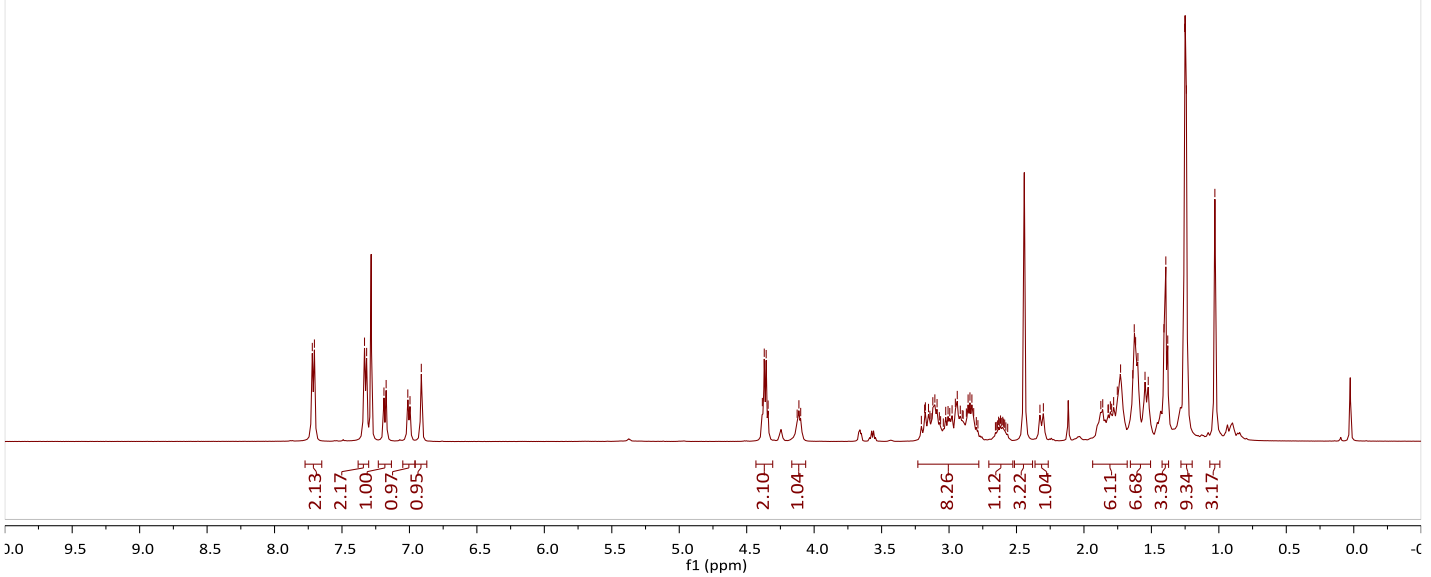

${ }^{19} \mathrm{~F}$ NMR-spectrum $\left(471 \mathrm{MHz}, \mathrm{CDCl}_{3}\right)$ of $\mathbf{7 f}$

bo m

实高宫

i i

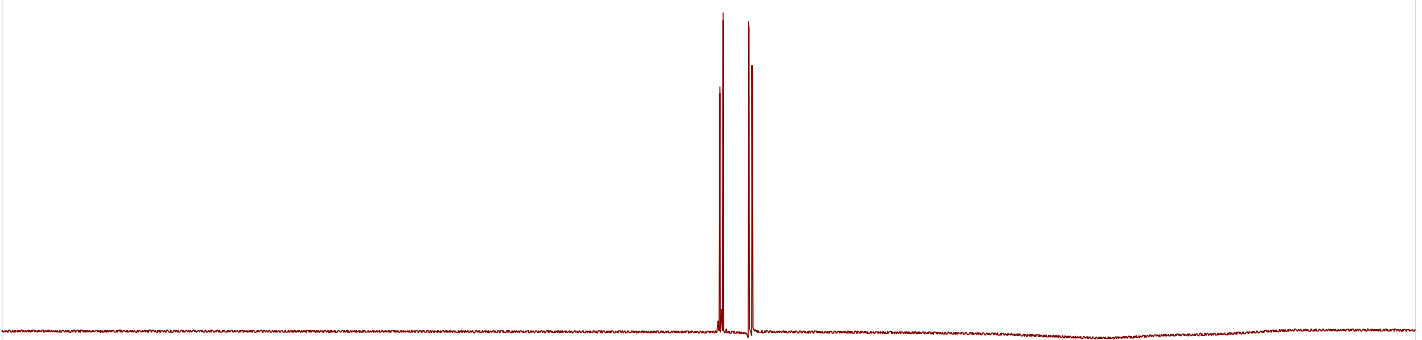

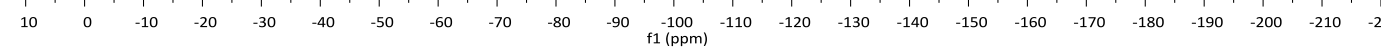
S186 


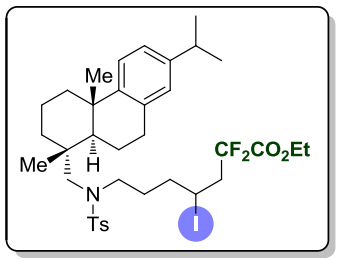

${ }^{13} \mathrm{C}$ NMR-spectrum $\left(125 \mathrm{MHz}, \mathrm{CDCl}_{3}\right)$ of $\mathbf{7 f}$

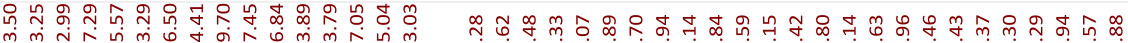

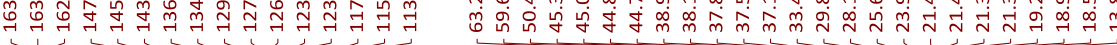
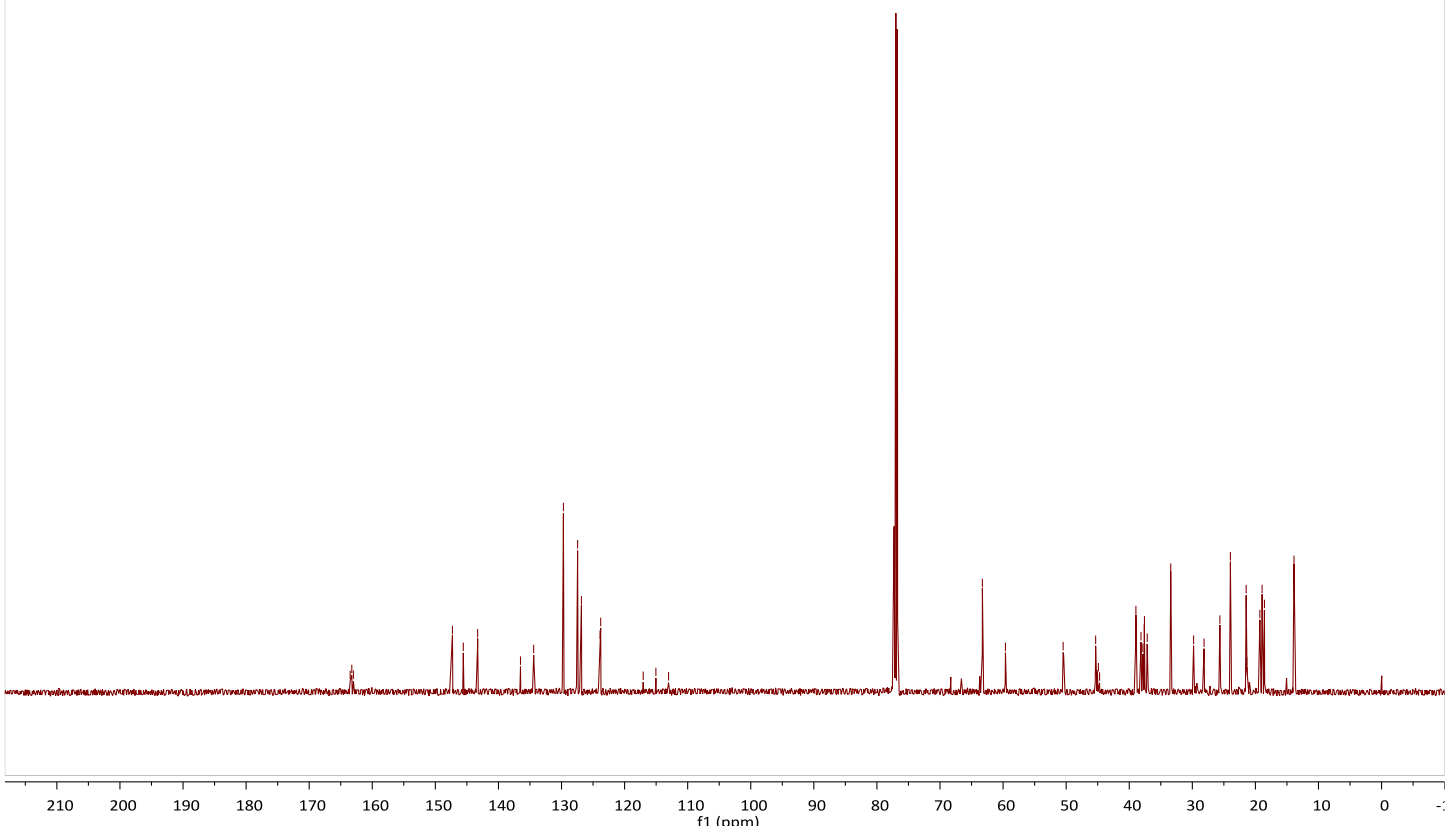


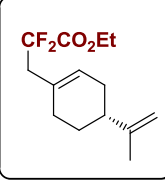

${ }^{1} \mathrm{H}$ NMR-spectrum $\left(500 \mathrm{MHz}, \mathrm{CDCl}_{3}\right)$ of 9

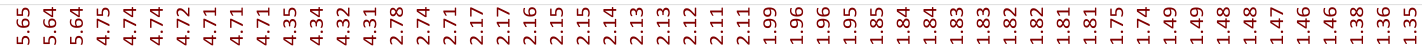

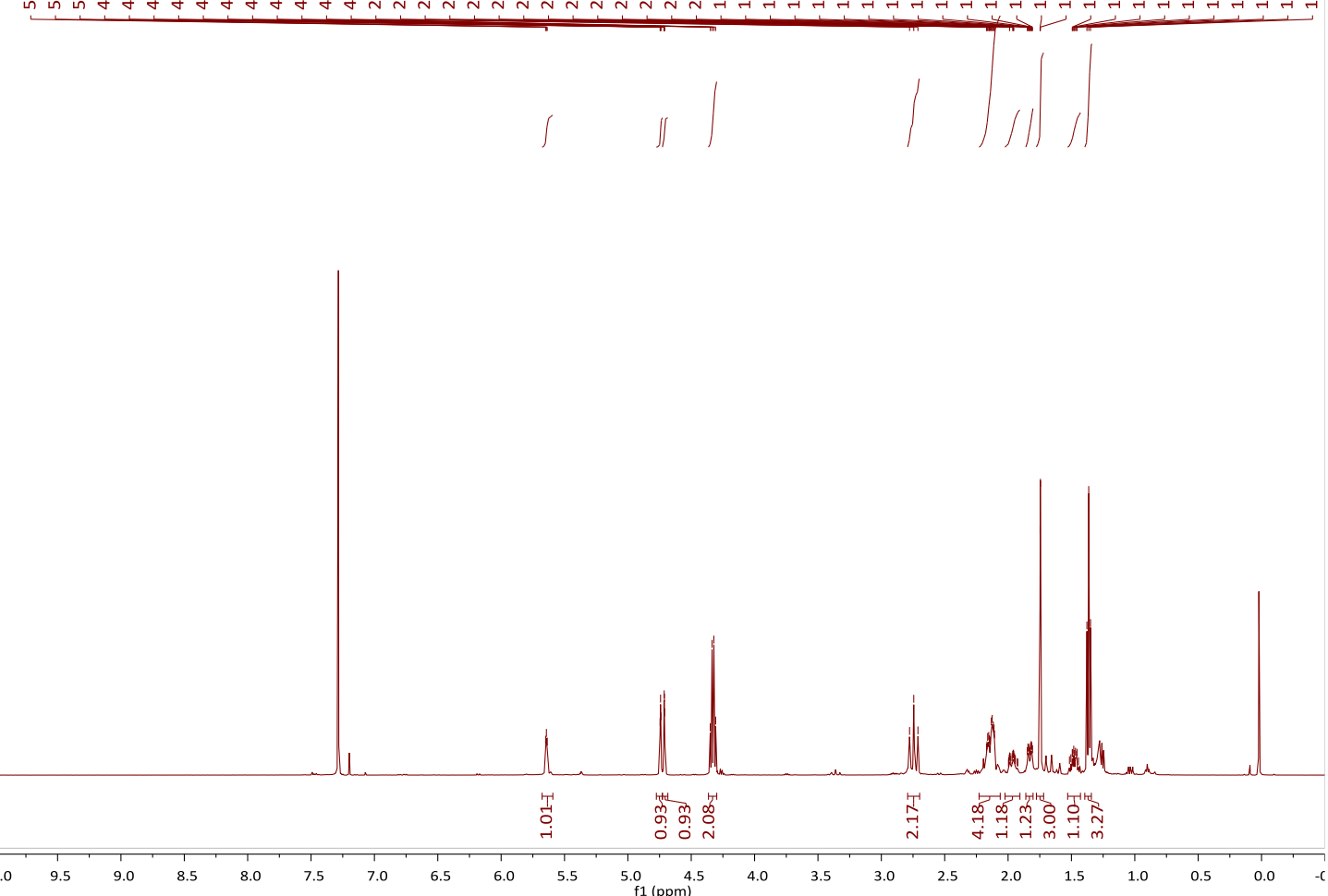

${ }^{19}$ F NMR-spectrum $\left(471 \mathrm{MHz}, \mathrm{CDCl}_{3}\right)$ of 9

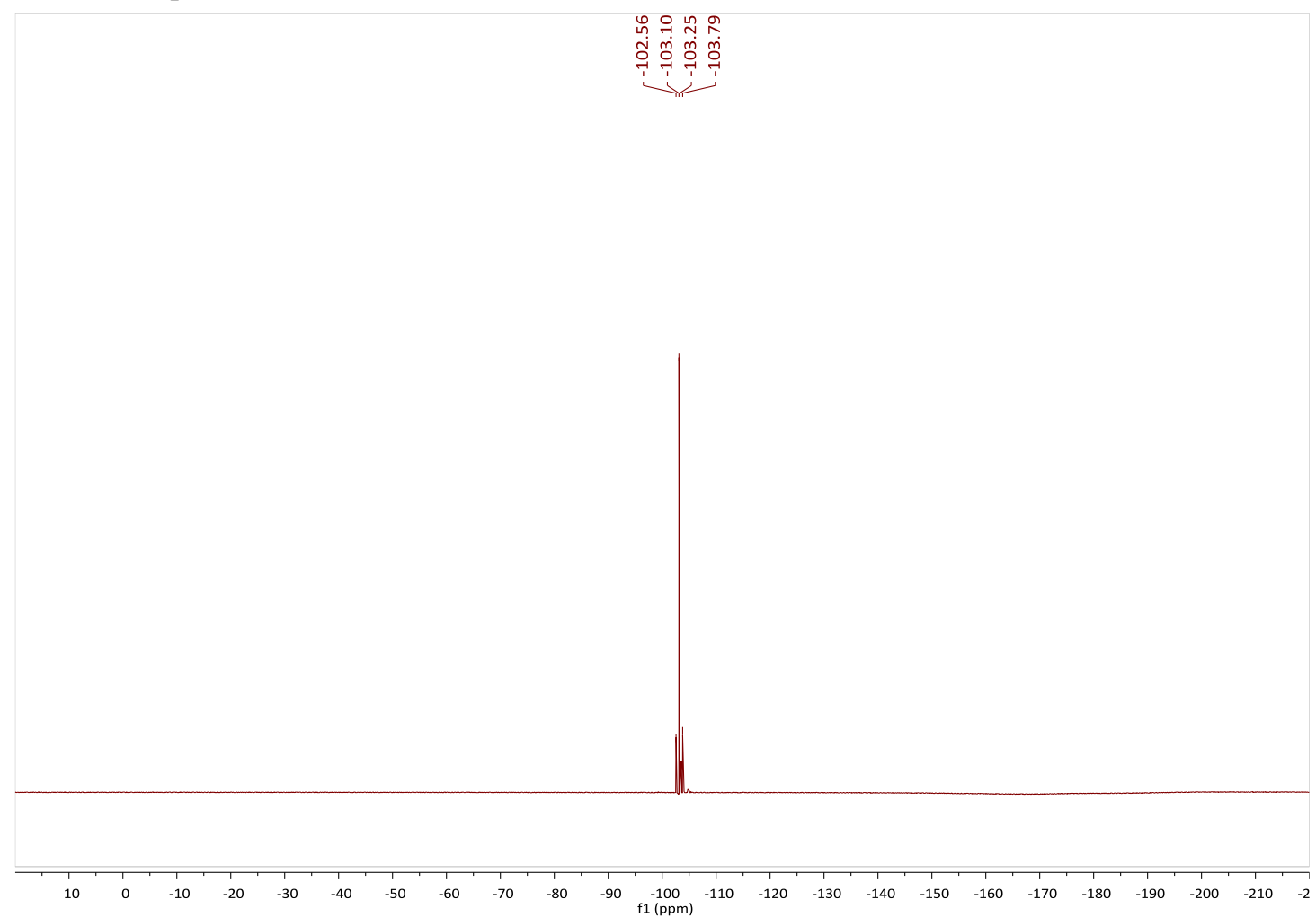




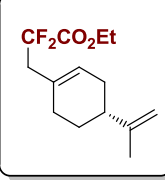

${ }^{13} \mathrm{C}$ NMR-spectrum $\left(125 \mathrm{MHz}, \mathrm{CDCl}_{3}\right)$ of 9

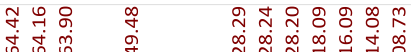

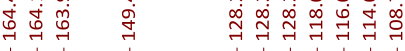

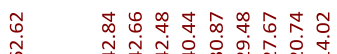

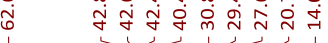

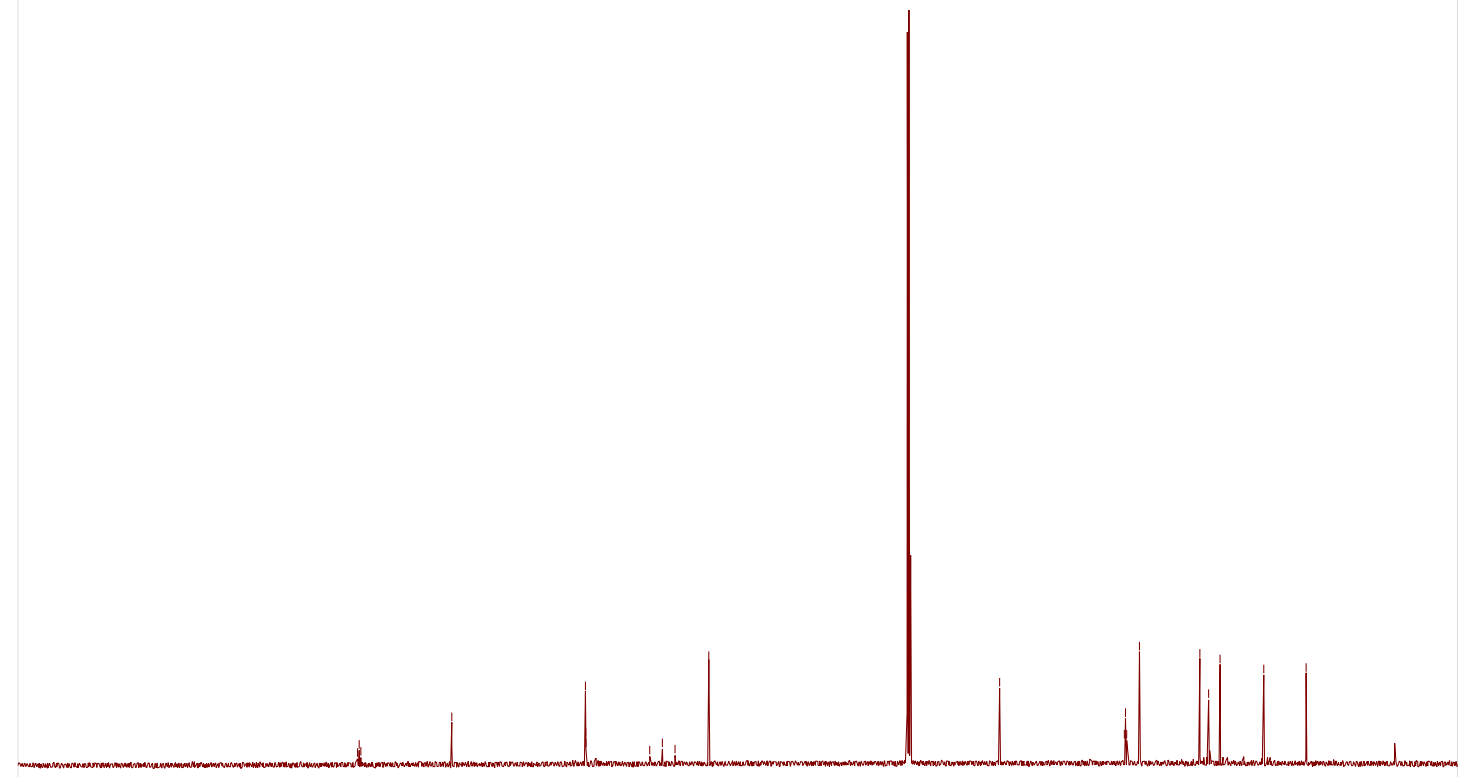

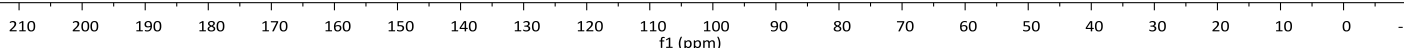




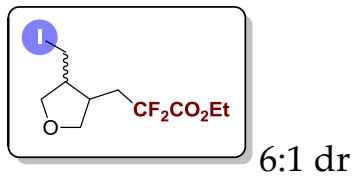

${ }^{1} \mathbf{H}$ NMR-spectrum $\left(500 \mathrm{MHz}, \mathrm{CDCl}_{3}\right)$ of $\mathbf{1 0 a}$

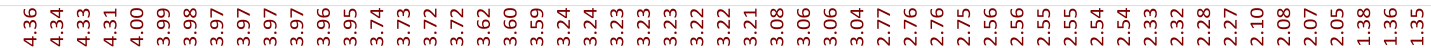
$\int 1111111$

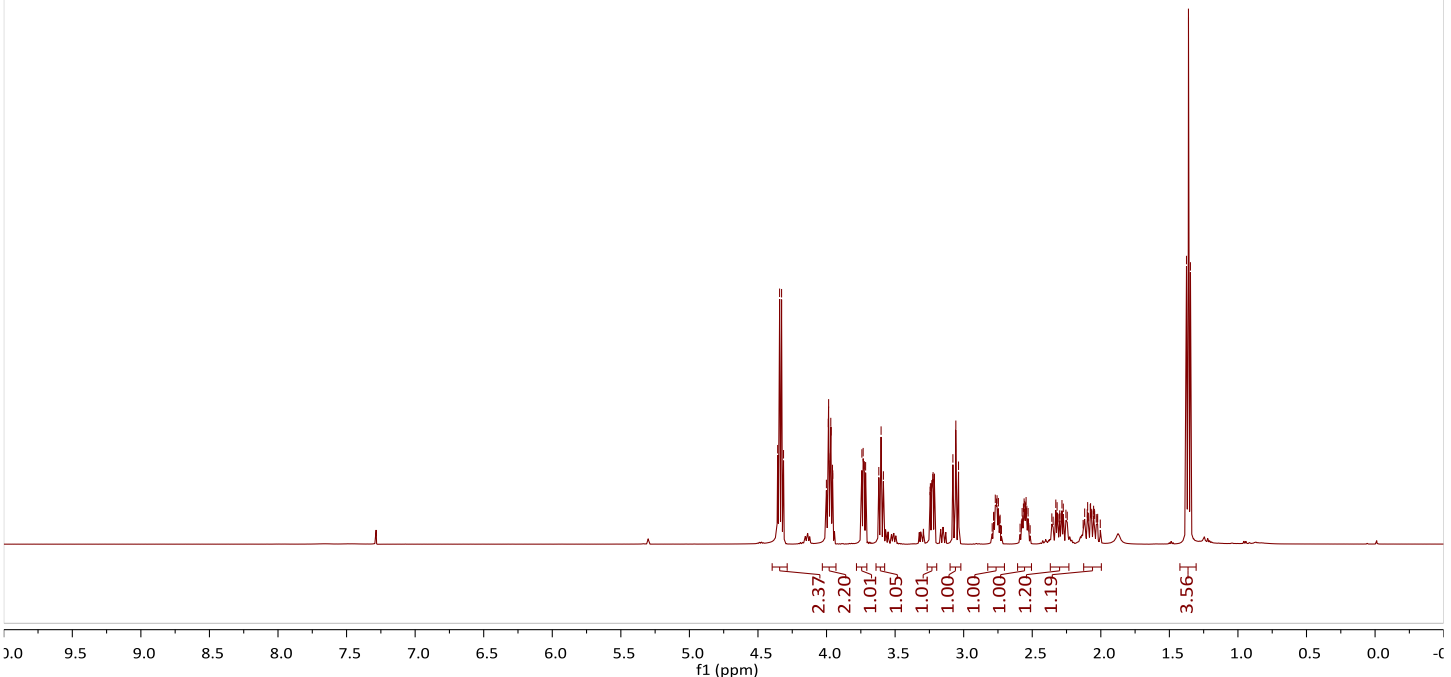

${ }^{19}$ F NMR-spectrum $\left(471 \mathrm{MHz}, \mathrm{CDCl}_{3}\right)$ of $10 a$

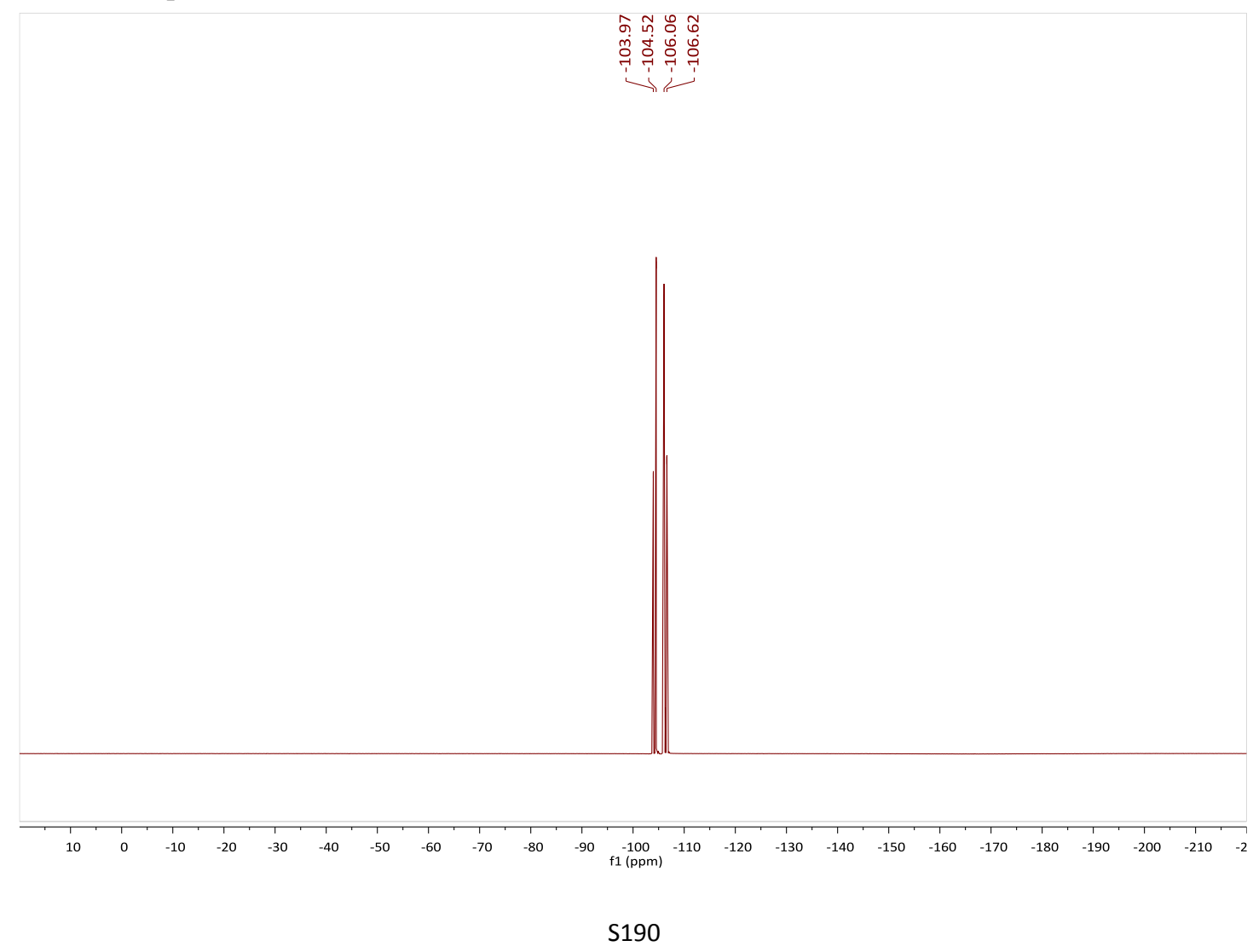




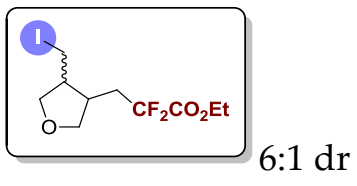

${ }^{13} \mathrm{C}$ NMR-spectrum (125 MHz, $\left.\mathrm{CDCl}_{3}\right)$ of $\mathbf{1 0 a}$

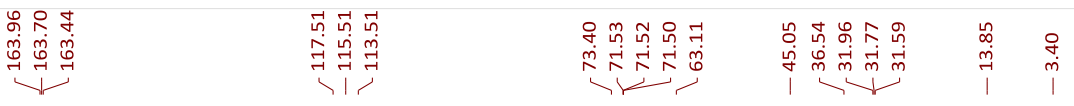

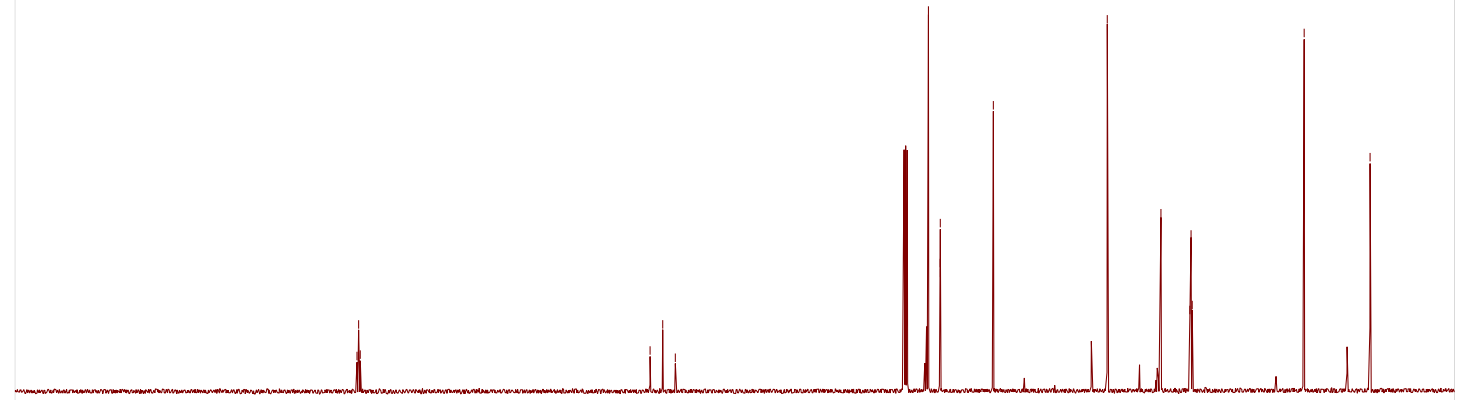

$\begin{array}{llllllllllllllllllllllllllllllllllll}210 & 200 & 190 & 180 & 170 & 160 & 150 & 140 & 130 & 120 & 110 & 100 & 90 & 80 & 70 & 60 & 50 & 40 & 30 & 20 & 10 & 0 & -\end{array}$ 


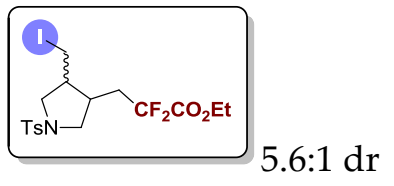

${ }^{1} \mathrm{H}$ NMR-spectrum $\left(500 \mathrm{MHz}, \mathrm{CDCl}_{3}\right)$ of $\mathbf{1 0 b}$

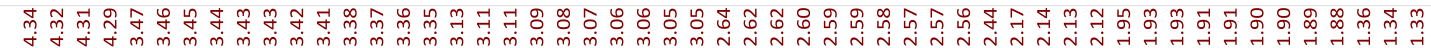
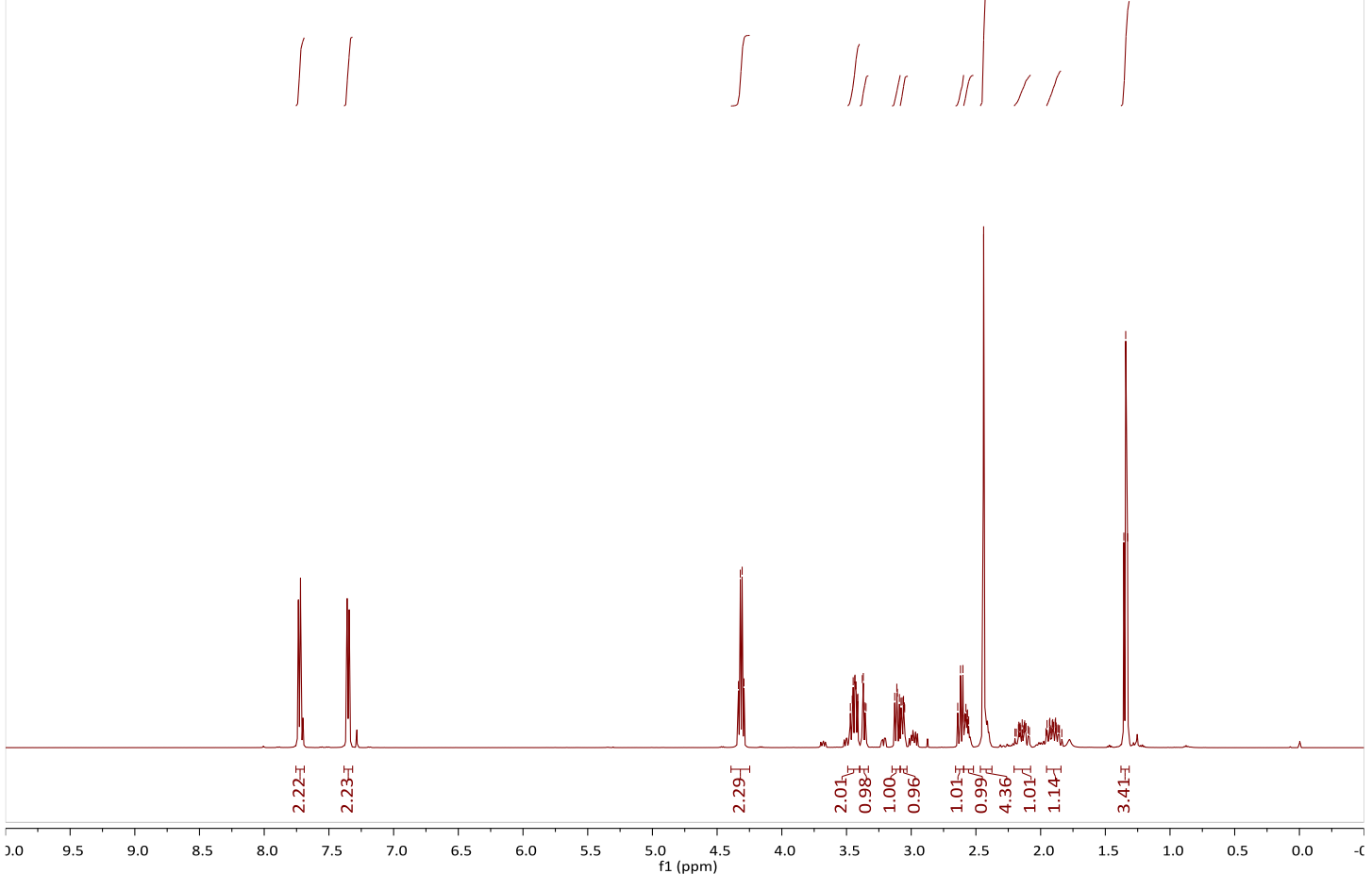

${ }^{19}$ F NMR-spectrum $\left(471 \mathrm{MHz}, \mathrm{CDCl}_{3}\right)$ of $\mathbf{1 0 b}$

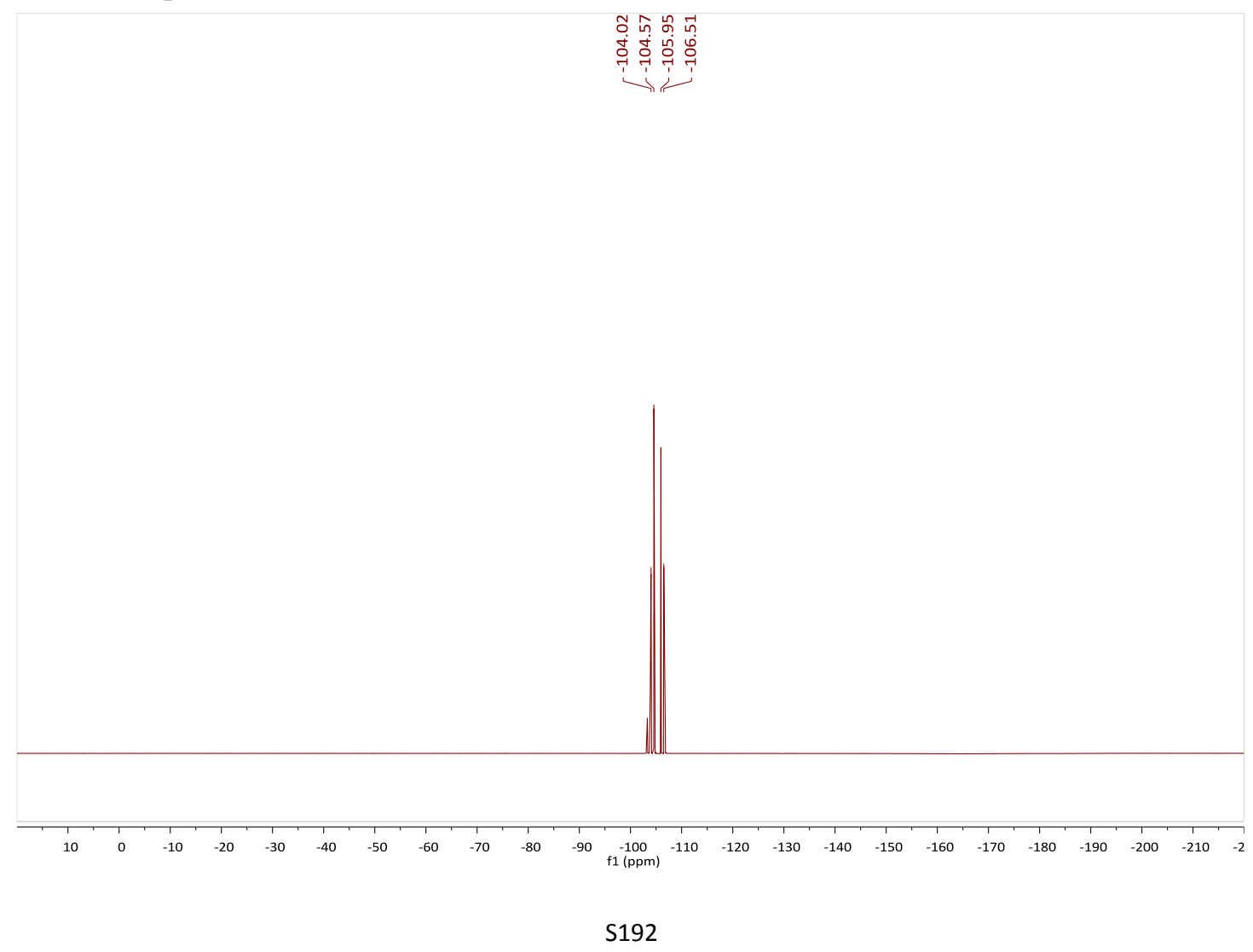




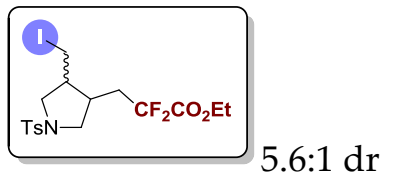

${ }^{13} \mathrm{C} \mathrm{NMR}$-spectrum (125 MHz, $\left.\mathrm{CDCl}_{3}\right)$ of $\mathbf{1 0 b}$

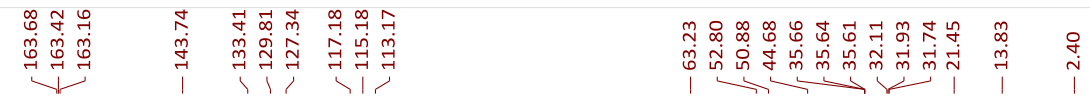

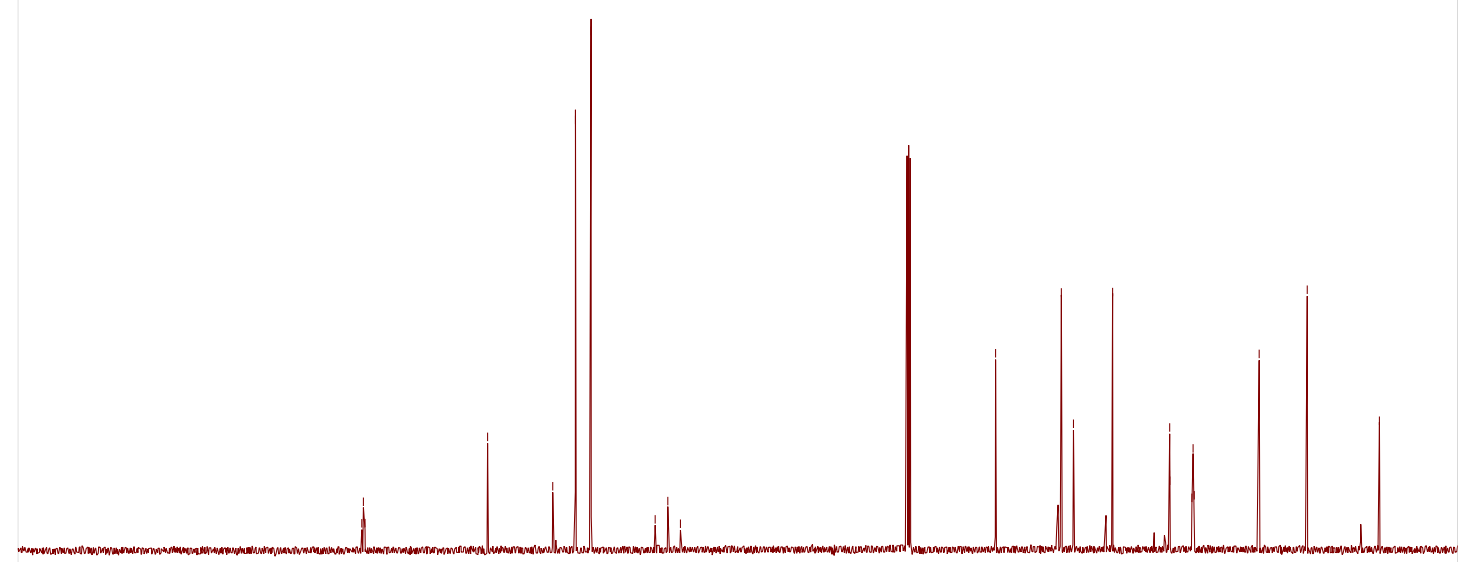

$\begin{array}{llllllllllllllllllllllllllllllllllllllllll}210 & 200 & 190 & 180 & 170 & 160 & 150 & 140 & 130 & 120 & 110 & 100 & 90 & 80 & 70 & 60 & 50 & 40 & 30 & 20 & 10 & 0 & \therefore\end{array}$ 


$$
\underbrace{\mathrm{CF}_{2} \mathrm{CO}_{2} \mathrm{Et}}_{\mathbf{N}_{3}}
$$

${ }^{1} \mathbf{H}$ NMR-spectrum $\left(500 \mathrm{MHz}, \mathrm{CDCl}_{3}\right)$ of $\mathbf{1 2}$

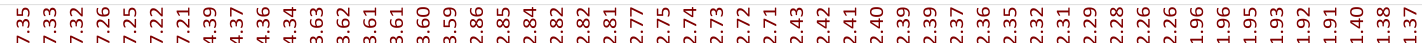
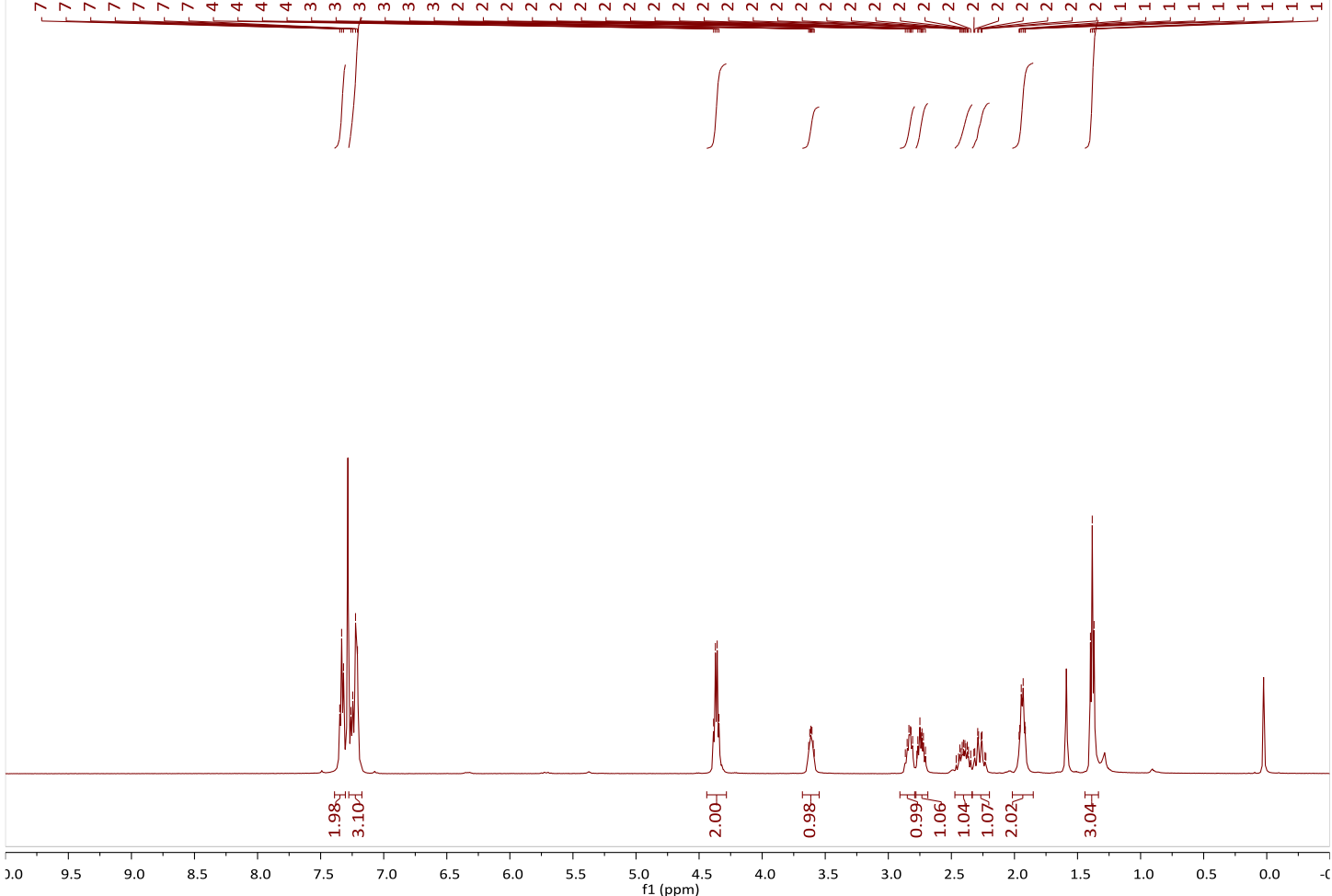

${ }^{19}$ F NMR-spectrum $\left(471 \mathrm{MHz}, \mathrm{CDCl}_{3}\right)$ of $\mathbf{1 2}$

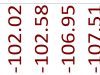

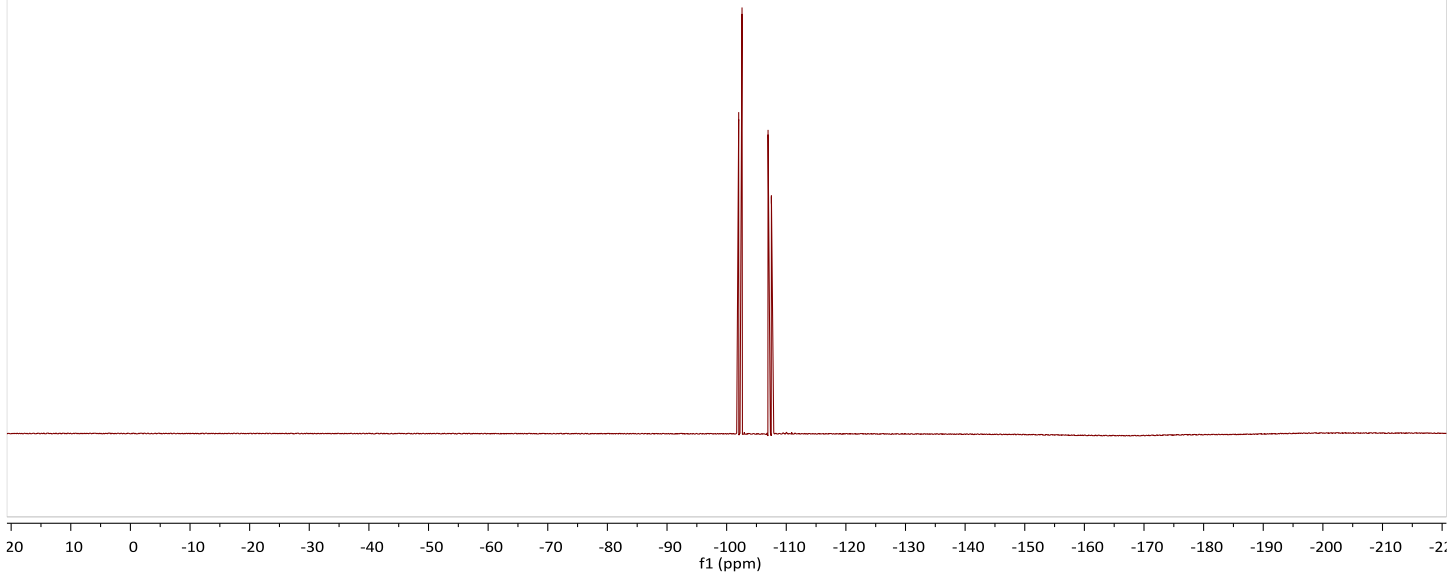




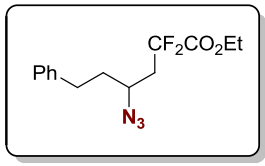

${ }^{13} \mathrm{C}$ NMR-spectrum (125 MHz, $\left.\mathrm{CDCl}_{3}\right)$ of 12

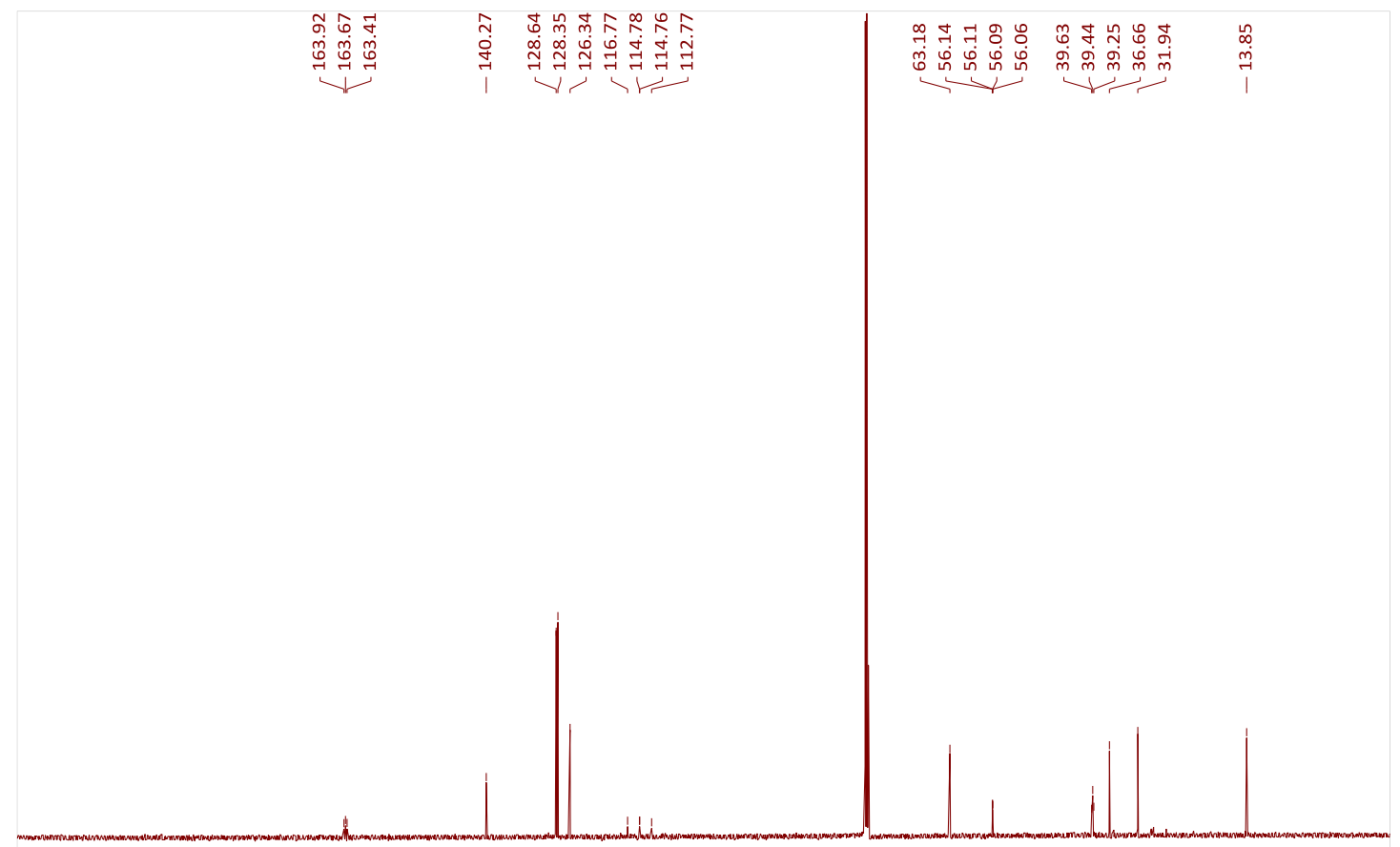

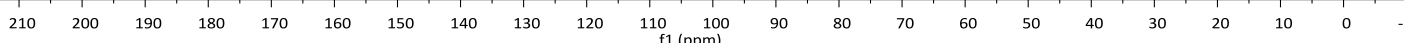




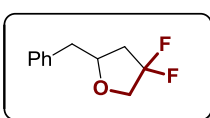

${ }^{1}$ H NMR-spectrum (500 MHz, $\left.\mathrm{CDCl}_{3}\right)$ of $\mathbf{1 3}$

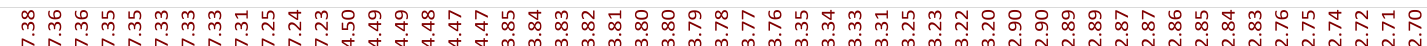

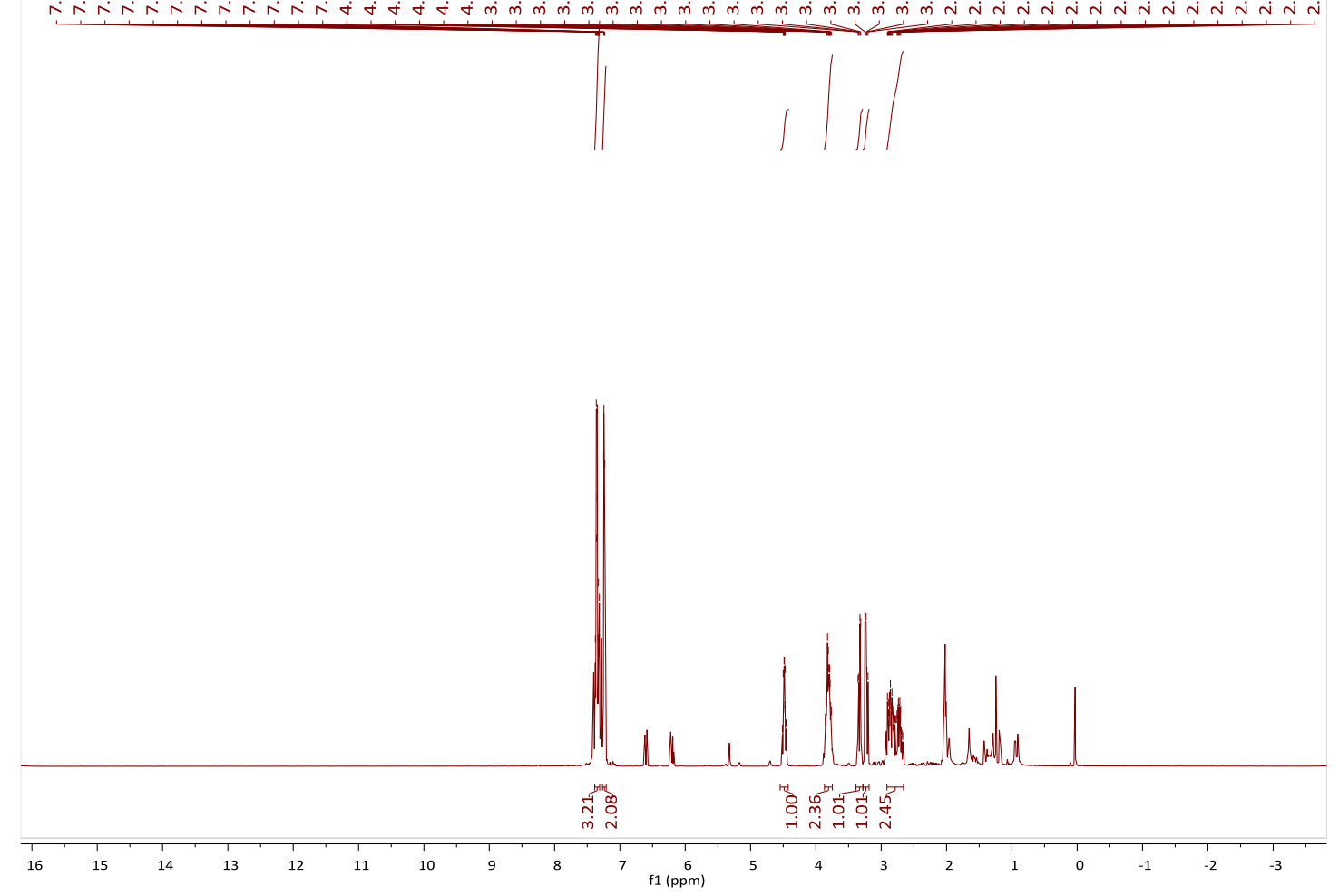

${ }^{19} \mathrm{~F}$ NMR-spectrum $\left(471 \mathrm{MHz}, \mathrm{CDCl}_{3}\right)$ of $\mathbf{1 3}$

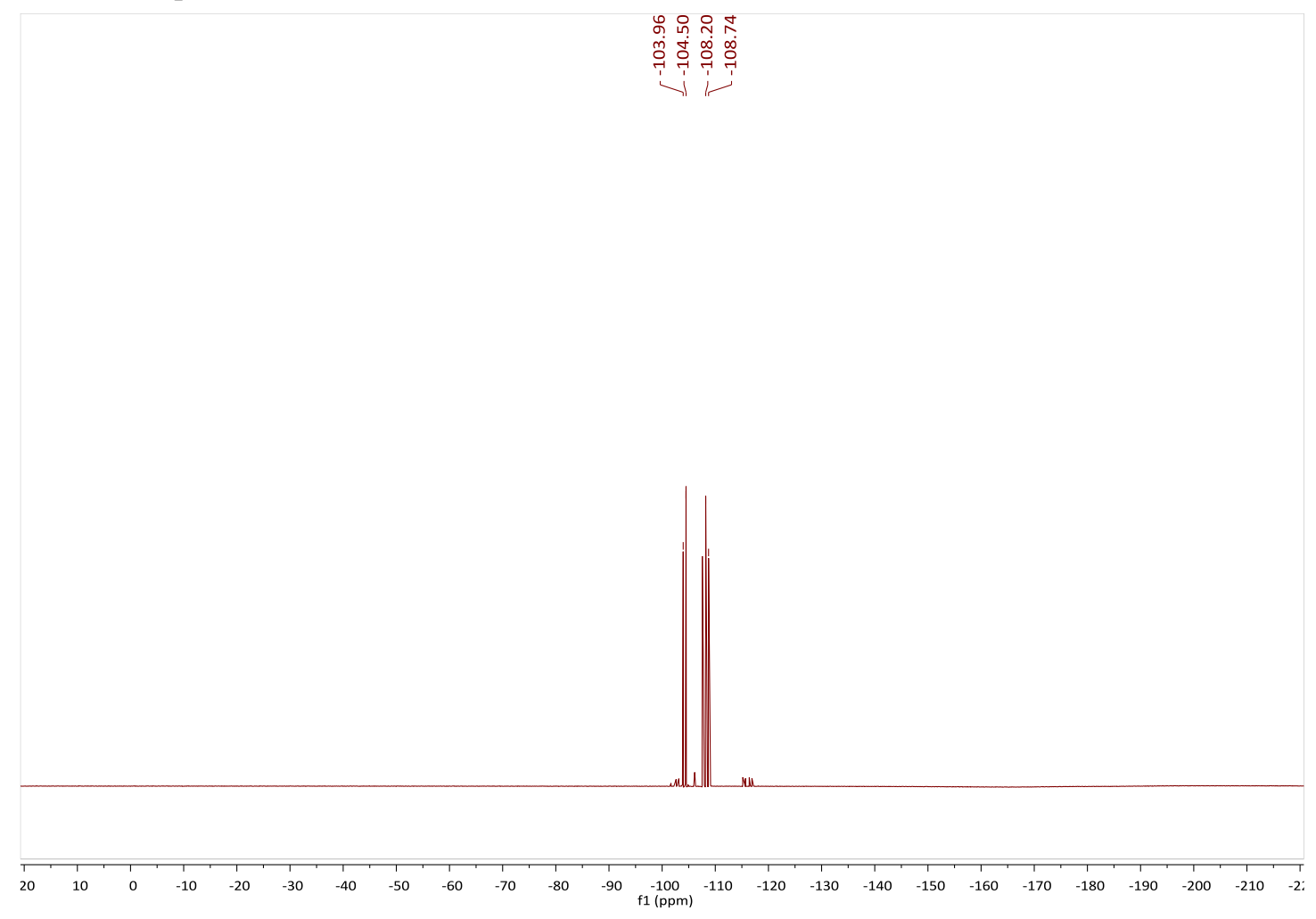




$$
\overbrace{0}^{F}
$$

${ }^{13} \mathrm{C}$ NMR-spectrum $\left(125 \mathrm{MHz}, \mathrm{CDCl}_{3}\right)$ of $\mathbf{1 3}$

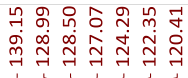

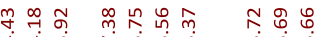

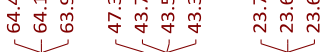

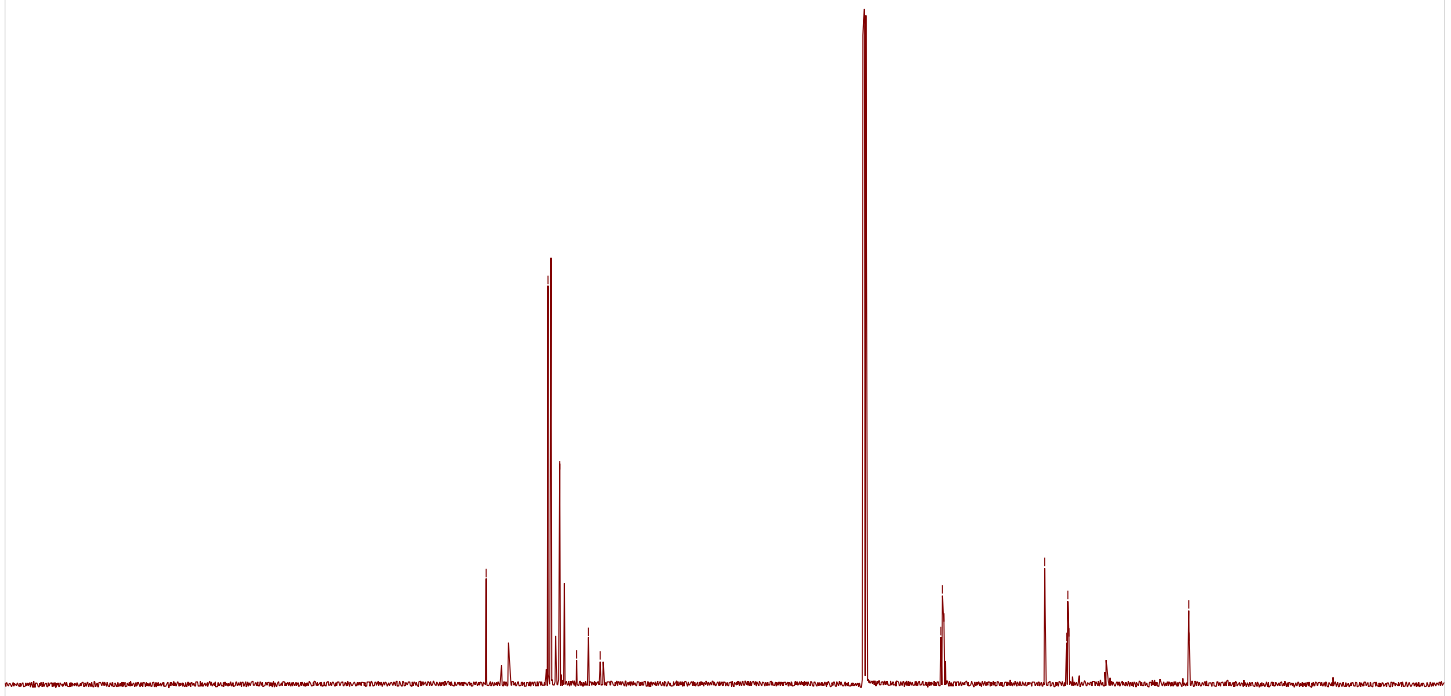

$\begin{array}{llllllllllllllllllllllllllllllllllllllll}210 & 200 & 190 & 180 & 170 & 160 & 150 & 140 & 130 & 120 & 110 & 100 & 90 & 80 & 70 & 60 & 50 & 40 & 30 & 20 & 10 & 0 & -10\end{array}$ 


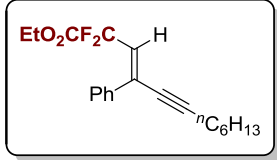

${ }^{1} \mathbf{H}$ NMR-spectrum $\left(500 \mathrm{MHz}, \mathrm{CDCl}_{3}\right)$ of $\mathbf{1 4}$

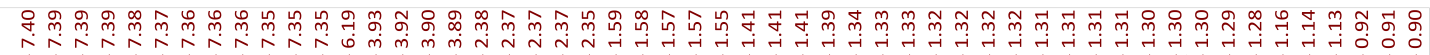

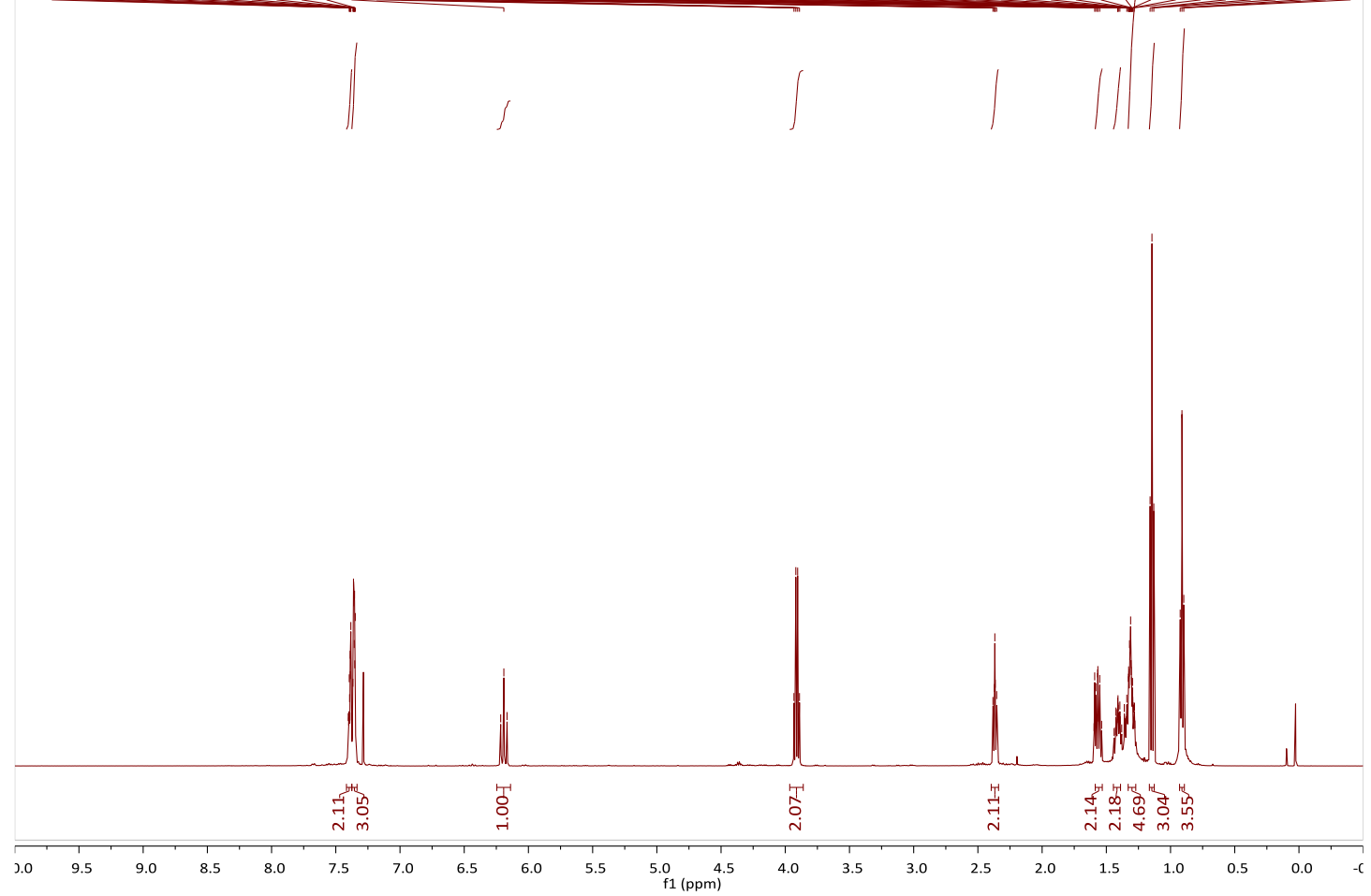

${ }^{19}$ F NMR-spectrum $\left(471 \mathrm{MHz}, \mathrm{CDCl}_{3}\right)$ of $\mathbf{1 4}$

$\begin{array}{lllllllllllllllllllllllllllllllllllllll}10 & 0 & -10 & -20 & -30 & -40 & -50 & -60 & -70 & -80 & -90 & -100 & -110 & -120 & -130 & -140 & -150 & -160 & -170 & -180 & -190 & -200 & -210 & -2\end{array}$ 


$$
{ }^{\mathrm{EtO}_{2} \mathrm{CF}_{2} \mathrm{C}}{ }^{\mathrm{H}}
$$

${ }^{13} \mathrm{C}$ NMR-spectrum (125 MHz, $\left.\mathrm{CDCl}_{3}\right)$ of $\mathbf{1 4}$

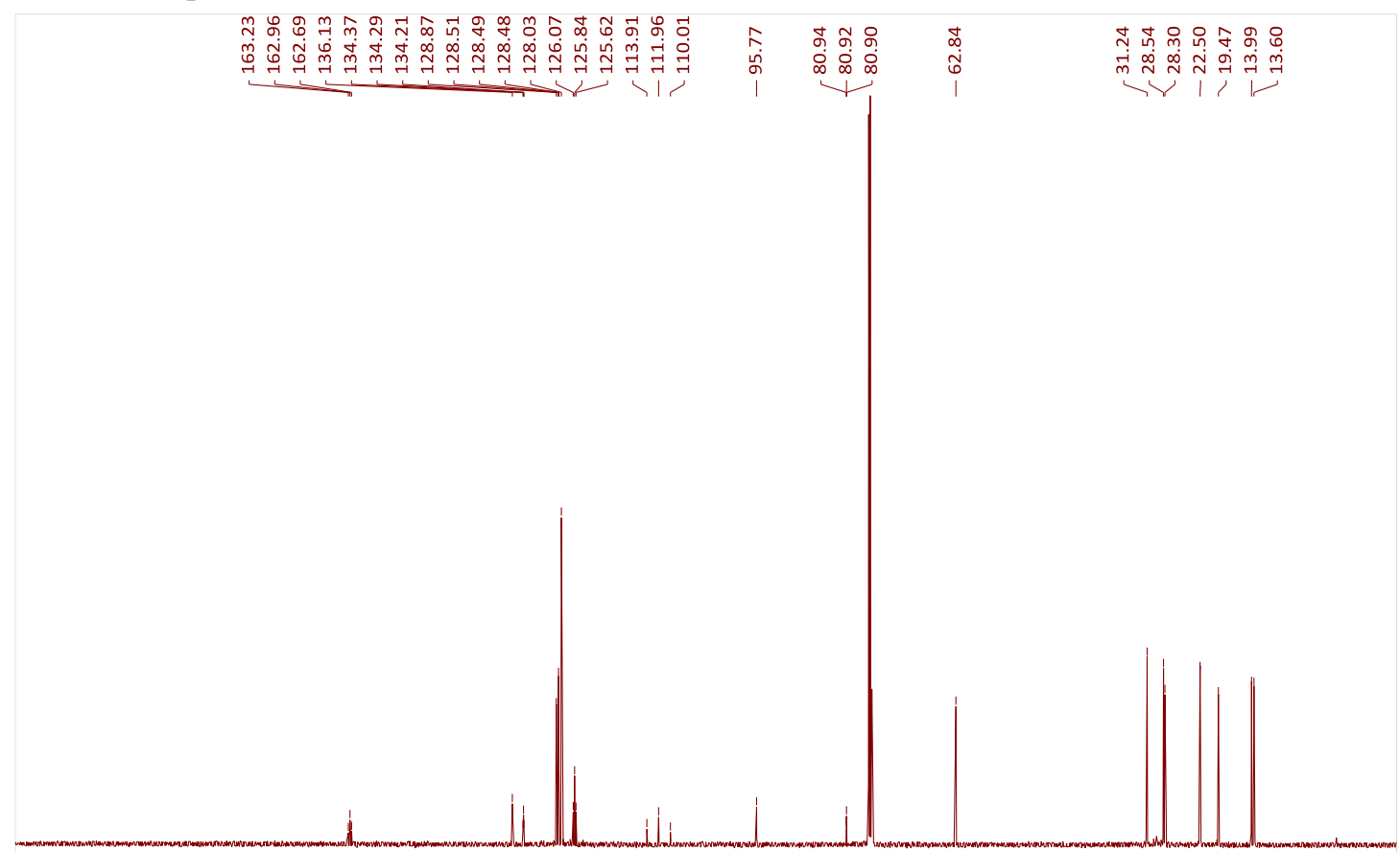

$\begin{array}{llllllllllllllllllllllll}210 & 200 & 190 & 180 & 170 & 160 & 150 & 140 & 130 & 120 & 110 & 100 & 90 & 80 & 70 & 60 & 50 & 40 & 30 & 20 & 10 & 0 & \end{array}$ 


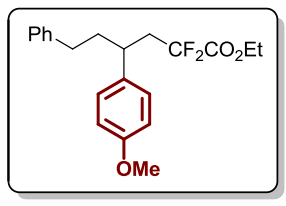

${ }^{1} \mathrm{H}$ NMR-spectrum $\left(500 \mathrm{MHz}, \mathrm{CDCl}_{3}\right)$ of $\mathbf{1 5}$

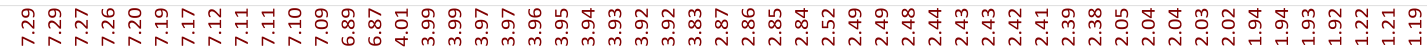
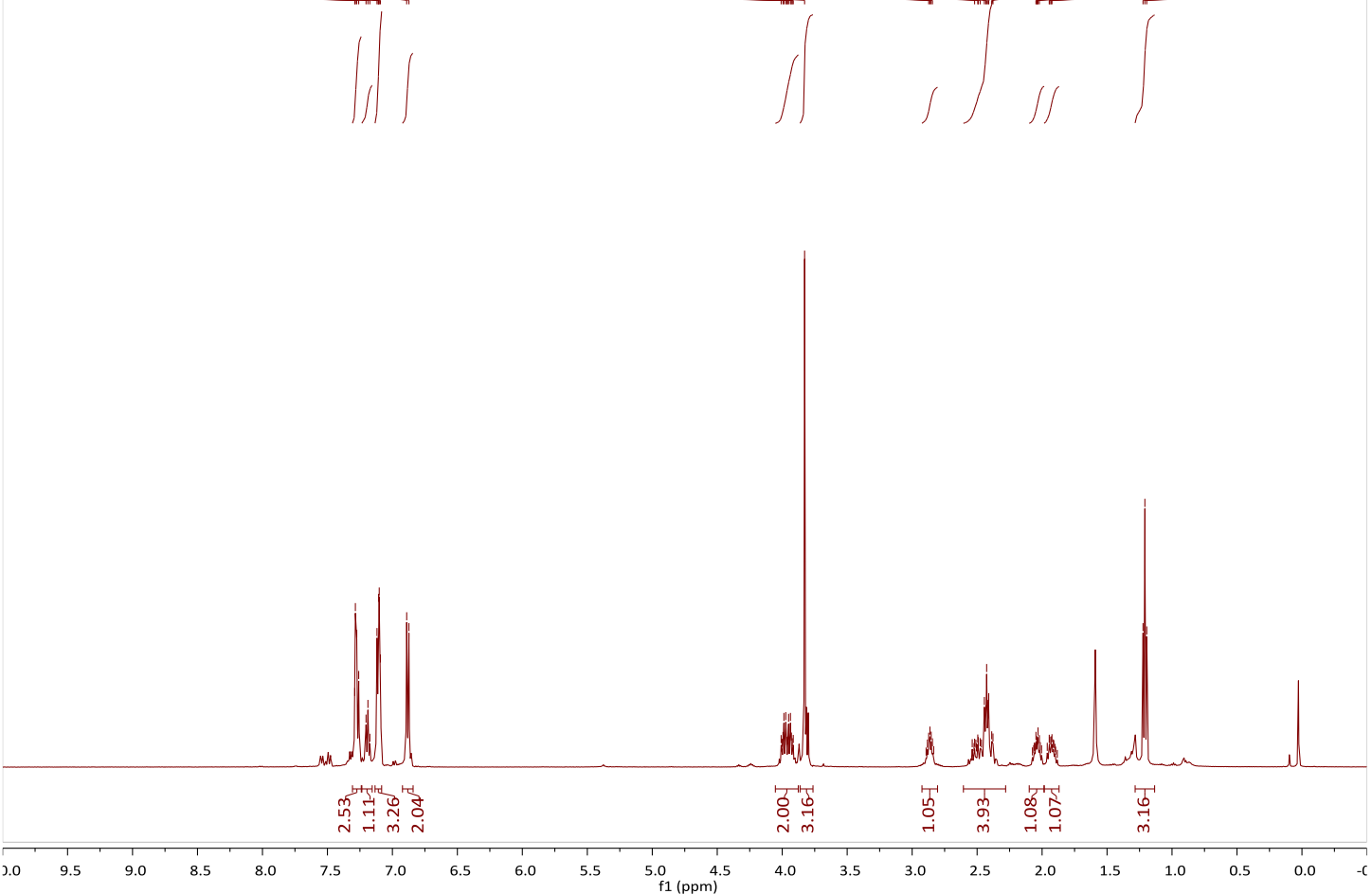

${ }^{19}$ F NMR-spectrum $\left(471 \mathrm{MHz}, \mathrm{CDCl}_{3}\right)$ of $\mathbf{1 5}$

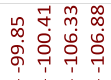

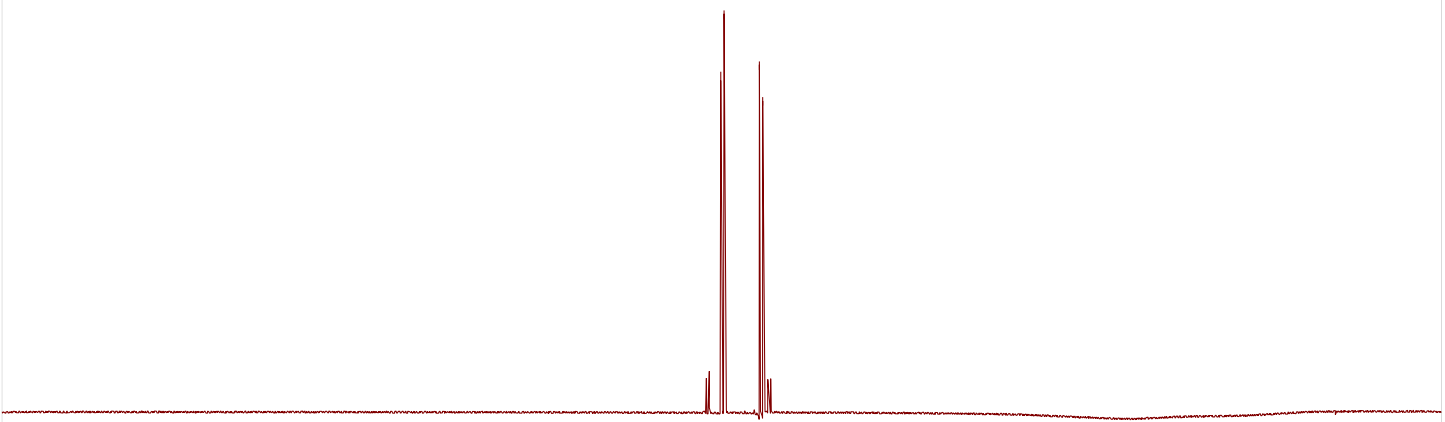

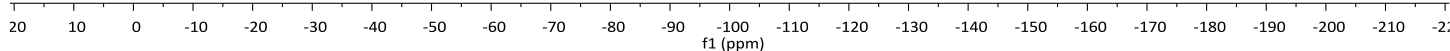




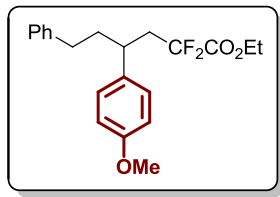

${ }^{13} \mathrm{C}$ NMR-spectrum $\left(125 \mathrm{MHz}, \mathrm{CDCl}_{3}\right)$ of $\mathbf{1 5}$

의

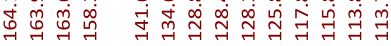

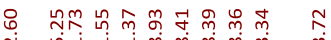

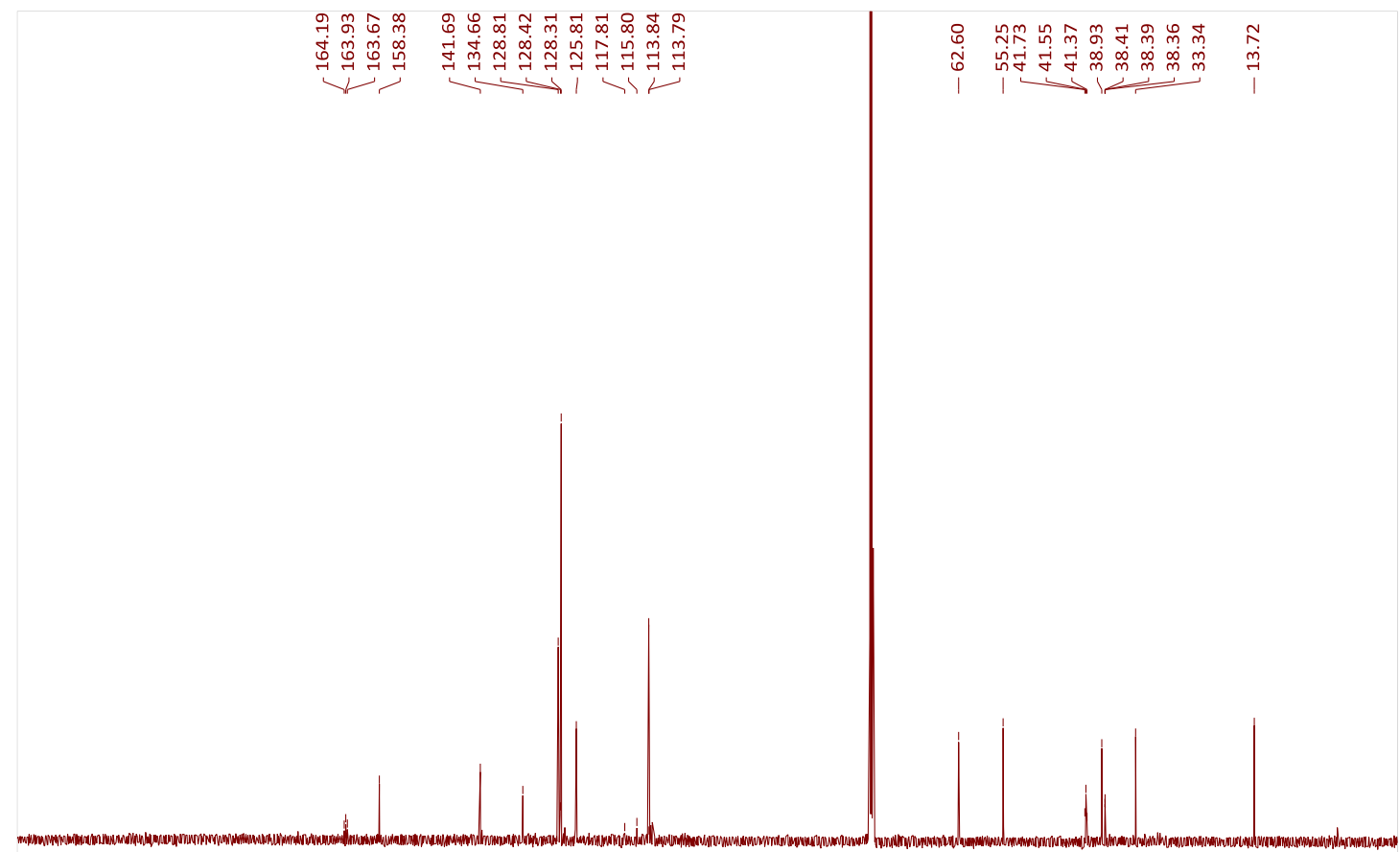

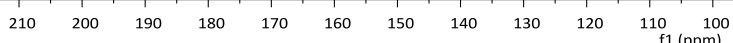

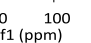

